\title{
Molecular Biogeochemistry of Modern ANd AnCiEnt Marine Microbes
}

\author{
by \\ Jacob Richard Waldbauer \\ A.B., Dartmouth College, 2001 \\ Submitted in partial fulfillment of the requirements for the degree of \\ Doctor of Philosophy \\ at the \\ MASSACHUSETTS INSTITUTE OF TECHNOLOGY \\ and the \\ WOODS HOLE OCEANOGRAPHIC INSTITUTION
}

February 2010

(C) 2010 Jacob R. Waldbauer. All rights reserved.

The author hereby grants to MIT and WHOI permission to reproduce and to distribute publicly paper and electronic copies of this thesis document in whole or in part in any medium now known or hereafter created.

Signature of Author:

Joint Program in Oceanography/Applied Ocean Science and Engineering January 8,2010

Certified by:

Sallie W. Chisholm

Lee and Geraldine Martin Professor of Environmental Studies Professor of Civil and Environmental Engineering and of Biology

Thesis Supervisor

Roger E. Summons

Professor of Earth, Atmospheric and Planetary Sciences

Thesis Supervisor

Accepted by:

Roger E. Summons

Chair, Joint Committee for Chemical Oceanography 


\title{
Molecular Biogeochemistry of Modern and Ancient Marine Microbes
}

\author{
by \\ Jacob Richard Waldbauer \\ Submitted to the Joint Program in Oceanography/Applied Ocean Science \\ and Engineering in partial fulfillment of the requirements for the degree of \\ Doctor of Philosophy in Chemical Oceanography
}

\begin{abstract}
Biological activity has shaped the surface of the earth in numerous ways, but life's most pervasive and persistent global impact has been the secular oxidation of the surface environment. Through primary production - the biochemical reduction of carbon dioxide to synthesize biomass - large amounts of oxidants such as molecular oxygen, sulfate and ferric iron have accumulated in the ocean, atmosphere and crust, fundamentally altering the chemical environment of the earth's surface. This thesis addresses aspects of the role of marine microorganisms in driving this process. In the first section of the thesis, biomarkers (hydrocarbon molecular fossils) are used to investigate the early history of microbial diversity and biogeochemistry. Molecular fossils from the Transvaal Supergroup, South Africa, document the presence in the oceans of a diverse microbiota, including eukaryotes, as well as oxygenic photosynthesis and aerobic biochemistry, by ca. 2.7Ga. Experimental study of the oxygen requirements of steroid biosynthesis suggests that sterane biomarkers in late Archean rocks are consistent with the persistence of microaerobic surface ocean environments long before the initial oxygenation of the atmosphere. In the second part, using Prochlorococcus (a marine cyanobacterium that is the most abundant primary producer on earth today) as a model system, we explored how microbes use the limited nutrient resources available in the marine environment to make the protein catalysts that enable primary production. Quantification of the Prochlorococcus proteome over the diel cell-division cycle reveals that protein abundances are distinct from transcript-level dynamics, and that small temporal shifts in enzyme levels can redirect metabolic fluxes. This thesis illustrates how molecular techniques can contribute to a systems-level understanding of biogeochemical processes, which will aid in reconstructing the past of, and predicting future change in, earth surface environments.
\end{abstract}

Thesis supervisor: Sallie W. Chisholm

Title: Lee and Geraldine Martin Professor of Environmental Studies Professor of Civil and Environmental Engineering and of Biology

Thesis supervisor: Roger E. Summons

Title: Professor of Earth, Atmospheric and Planetary Sciences 


\section{ACKNOWLEDGMENTS}

I have incurred many debts of gratitude and appreciation over the course of this work. None are greater than those to my advisors, Penny Chisholm and Roger Summons, who allowed and enabled me to pursue such a wide range of scientific interests and offered their help and advice every step of the way. I have been very lucky to have had the chance to learn so much during my Ph.D., and none of that would have been possible without their guidance and support.

I have also had the great fortune to know and work with all the members of the Chisholm and Summons labs, who have made this an enjoyable and rewarding place to be. I am especially grateful to Laura Sherman for her skill and dedication in analyzing biomarkers in the late Archean cores - it was truly getting blood from a stone. I was lucky to collaborate with Sébastien Rodrigue, whose expertise and collegiality made the diel experiment even more fruitful than I could have hoped. Many others, including Matt Sullivan, Anne Thompson, Alex Bradley, Gordon Love, Rex Malmstrom, Libusha Kelly, Luke Thompson, Jason Bragg and Adam Rivers, shared advice, ideas, reagents, humor and beer. Thanks as well to Carolyn Colonero, Allison Coe, Marcia Osburne, Alla Skorokhod, Mary Elliff and Deborah Fullerton for the wonderful job they have done in keeping the labs running.

Early in my graduate career, John Hayes generously shared his time and insights into the mysteries of the carbon cycle. Our discussions shaped my view of the earth system and the role of life within it. I am also thankful for both the scientific opportunities and warm hospitality offered to me by Dianne Newman and her lab. Paula Welander, Alexa Price-Whelan, Alex Poulain and Lars Dietrich patiently taught me anaerobic culturing and microelectrode techniques.

Further scientific support came from many quarters. My thesis committee members, Liz Kujawinski and Mak Saito, offered guidance and helpful feedback. Melissa Soule managed the FTMS facility at WHOI, and the proteomics dataset would not have been collected without her help. Bryan Krastins and David Sarracino provided invaluable bench training. David Shtyenberg at ISB enabled much of the proteomics data analysis. The Agouron Griqualand Drilling Project and core sampling were coordinated by Andy Knoll, Nic Beukes and Joe Kirschvink, and Alex Birch and Francis McDonald oversaw the difficult clean drilling operation.

A special community is assembled around the Joint Program, EAPS and the Parsons Lab. The EAPS and APO staff, including Ronni Schwartz, Valerie Caron, Julia Westwater, Carol Sprague and especially Marsha Gomes, were unfailingly knowledgeable and helpful. Vicki Murphy kept me employed, funded, and, on some days, sane. Numerous friends - Elizabeth Tuckerman, Frédéric Chagnon, Sora Kim, Alyson Santoro, Seth Newsome, Alison Cohen, Nurit Bloom, Virginia Rich, Tracy Mincer, Janelle Thompson, Gajan Sivandran and Rebecca Giannotti, to name only a few - shared good times and laughter. The Klepac-Ceraj clan, Vanja, Ivan, Zara and Mirta, became treasured family; hvala lijepa. Ray Gilliar, Quang Truong, Adam Bisig and Jeff Kinkaid have provided a welcome yearly diversion at the annual conference. 
My parents have helped, supported, and inspired me in innumerable ways through the years, for which I will always be grateful.

The second sentence wasn't true. My greatest debt is to Maureen. Thank you.

THIS WORK WAS SUPPORTED BY THE FOLLOWING FUNDING SOURCES:

Office of Naval Research National Defense Science \& Engineering Graduate Fellowship (to JRW)

National Science Foundation Graduate Research Fellowship (to JRW)

NASA Exobiology Program Award NNG05GN62G (to RES)

NASA Astrobiology Institute Award NNA08CN84A (to RES)

National Science Foundation Award EAR0418619 (to RES)

Agouron Institute Geobiology Award AI-GB4.02.3Extn06.2bMIT (to RES)

Gordon and Betty Moore Foundation Marine Microbiology Award 495 (to SWC)

National Science Foundation Award OCE0425602 (to SWC)

National Science Foundation Award EF0424599 (to SWC)

Department of Energy Award DE-FG02-07ER64506 (to SWC)

Department of Energy Award DE-FG02-08ER64516 (to SWC) 


\section{TABLE OF CONTENTS}

CHAPTER 1: Introduction

CHAPTER 2: Late Archean molecular fossils from the Transvaal Supergroup record the antiquity of microbial diversity and aerobiosis

Waldbauer et al. (2009) Precambrian Research 169:28-47.

ChAPTER 3: Microaerobic steroid biosynthesis and the molecular fossil record of Archean life

ChAPTER 4: A multilevel view of gene expression and regulation in the diel cell cycle of Prochlorococcus

CHAPTER 5: Biogeochemical insights from Prochlorococcus systems biology

ChaPTER 6: Conclusions and Future Directions

APPEndix A: Use of stable isotope labeled cells to identify active grazers of picocyanobacteria in ocean surface waters

Frias-Lopez et al. (2009) Environ Microbiol 11: 512-525.

APPENDIX B: Improved methods for isolating and validating indigenous biomarkers in Precambrian rocks

Sherman et al. (2007) Org Geochem 38:1987-2000.

APPENDix C: Proteorhodopsin photosystem gene expression enables photophosphorylation in a heterologous host

Martínez et al. (2007) PNAS 104:5590-5595.

APPENDix D: The Geological Succession of Primary Producers in the Oceans

Knoll et al. (2007) In The Evolution of Primary Producers in the Sea, Falkowski and Knoll, eds. Academic Press, p.133-163.

APPENDIX E: The carbon cycle and associated redox processes through time

Hayes \& Waldbauer (2006) Phil Trans Roy Soc B 361:931-950.

APPENDIX F: Steroids, triterpenoids and molecular oxygen

Summons et al. (2006) Phil Trans Roy Soc B 361:951-968. 
Chapter One

Introduction 


\section{Introduction}

The coevolution of life and earth surface environments is central to the history and functioning of our planet. Life and its habitats coevolve through a wide array of reciprocal interactions, constantly applying pressures to and driving changes in one another. Only life evolves in a Darwinian sense and has a distinct mechanism for heritable transmission of information. But the impact of biology on the geochemistry of the oceans, atmosphere and crust is so pervasive that the present distributions of even inorganic chemicals are inexplicable without acknowledging life's influence. When the changes in geochemical distributions over time, evident in the rock record, are taken into account, biology's role appears even more central.

The most enduring and globally significant change that life has driven over the course of earth history has been the progressive oxidation of the surface environment. As a result of biological activity, particularly photosynthesis, oxidizing power has been continuously exported by the carbon cycle and has accumulated in reservoirs such as molecular oxygen, sulfate, and ferric iron. This process has increased redox disequilibrium between the surface environment (including the atmosphere, oceans and upper crust) and the deeper solid earth, with broad consequences for the cycling of nearly all elements. Progressive oxidation has also been a defining control on the ecological distribution of microbes and on the biogeochemistry that results from their activities.

\section{HOW DID LIFE OXIDIZE THE EARTH'S SURFACE?}

The secular oxidation of the surface environment has been driven in large part by primary production. Life, of course, does not create or destroy electrons; "secular oxidation" means the redistribution of reducing equivalents, principally from a variety of electrondonating species to carbon. (The only recognized process that truly changes the redox state of the planet as a whole is the escape of hydrogen to space, which, while in itself a 
purely physical phenomenon, has also been strongly influenced by biology (Catling et al., 2001).) In the process, oxidized species such as $\mathrm{O}_{2}, \mathrm{Fe}(\mathrm{III})$ and $\mathrm{SO}_{4}{ }^{2-}$ accumulate, while carbon undergoes net reduction. It is in molecular action of the biochemical machinery of autotrophy that the sum total of this redistribution takes place. The other various and complex components of the carbon cycle - respiration, burial, weathering, subduction and so on - act to reverse autotrophs' accomplishments and make the net redistribution only a tiny fraction of the gross.

The planetary-scale workings of the carbon cycle as a whole have determined the rate of secular oxidation. But what controls the gross rate of autotrophic transfer of electrons from donors to carbon? Most basically, this process requires a source of carbon and a suitable source of reducing power. Carbon has generally not been a limiting nutrient for primary production over earth history, as attested by the consistent ${ }^{13} \mathrm{C}$ depletion of sedimentary organic carbon below that of marine carbonates, which suggests that enzymatic discrimination between $\mathrm{C}$ isotopes has generally been expressed (in contrast, for example, to the situation for $\mathrm{S}$ isotopes (Canfield, 2001). Notable exceptions include photosynthesis by some terrestrial and aquatic $\mathrm{C}_{3}$ plants in the modern, low- $\mathrm{CO}_{2}$ atmosphere (Wullschleger, 1993) and certain geochemically extraordinary habitats such as the Lost City hydrothermal system (Bradley et al., 2009). The former is a geologically recent phenomenon of the late Cenozoic, while the latter is a spatially-constrained phenomenon. During the "snowball earth" episodes of the Neoproterozoic, marine primary producers may also have been limited by some combination of carbon- and lightstarvation due to permanent sea ice cover, which may have influenced the evolution of carbon concentrating mechanisms (Riding, 2006). For the vast majority of times and places in earth history, though, autotrophs in sunlit aquatic habitats have not been carbonlimited.

Primary electron donors have a greater potential to limit primary production, though whether they have truly done so on a global scale is unclear. Supplies of reductants such 
as sulfide, ferrous iron and molecular hydrogen are ultimately dependent on volcanism and rock weathering and hardly ever approach the availability of inorganic carbon. The invention of oxygenic photosynthesis, which uses water as an electron donor, might have relieved reductant limitation; if it did, oxygenic primary producers ought to have proliferated rapidly. Water is in unlimited supply in aquatic environments, and has only become growth-limiting in geologically-recent periods as vascular plants have colonized drier parts of the land surface. Examining the pace and character of the global ecological succession from anoxygenic to oxygenic primary production would tell us much about the limitations faced, and the adaptive strategies employed, by photoautotrophs.

\section{WHEN - AND HOW QUICKLY - DID OXYGENIC PHOTOSYNTHESIS ARISE?}

Knowing when life developed the ability to produce $\mathrm{O}_{2}$ would provide a valuable landmark for reconstructions of biological diversity and environmental conditions over earth history. In particular, assessing the relative timing of the origin of oxygenic photosynthesis and the oxygenation of various parts of the surface environment (the atmosphere, surface and deep oceans, pore waters, etc.) would lend insight into the pacing and feedbacks involved in coevolutionary processes. Chapters 2 and 3 of this thesis address the timing and biogeochemical consequences of the evolution of oxygenic photosynthesis using biomarkers - hydrocarbon molecular fossils of membrane lipids produced by microorganisms and preserved in sedimentary rocks.

Chapter 2 presents findings of a molecular fossil investigation of late Archean marine sedimentary rocks from the Transvaal Supergroup, South Africa dating from ca. 2.672.46Ga. Previous molecular fossil studies had been carried out on rocks of similar age from Western Australia (Brocks et al., 1999; Brocks et al., 2003; Eigenbrode et al., 2008), and documented a diverse microbiota, including the oldest molecular evidence for oxygenic cyanobacteria. However, those findings have remained controversial (Rasmussen et al., 2008), due in part to the rock samples having been recovered many 
years earlier as part of a resource-exploration drilling project, and thus potentially compromised by hydrocarbon contamination during core recovery and/or storage. The work presented here is the first to have been conducted on freshly-extracted core material that had been drilled without the usual hydrocarbon lubricants for the express purpose of organic geochemical investigation. Furthermore, exceptional care was taken and novel analytical methods developed (described in Appendix B) to ensure that syngenetic molecular fossils were not compromised or overprinted with exogenous contaminants.

To the first-order question, is there reliable evidence of oxygenic photosynthesis in late Archean sedimentary rocks?, the answer is clearly affirmative. Multiple types of molecular fossils found in the Transvaal rocks are indicative of both the presence of cyanobacteria and the utilization of molecular oxygen by other microorganisms in the community. This result, in accord with other recently-described lines of evidence (Anbar et al., 2007; Bosak et al., 2009; Frei et al., 2009), suggests that oxygen production was occurring in some marine ecosystems at least 250-300 million years before the first signs of widespread atmospheric oxygenation. Beyond aerobiosis, what types of organisms and metabolisms were present in the ocean before the oxygenation of the atmosphere? The molecular fossil record documents a broad taxonomic range of microbes - including Eubacteria, Archaea, and Eukaryotes - implying that the three Domains of cellular life had all originated by the late Archean. The broader evidence for active biogeochemical cycling of most elements also suggests that much of higher-level microbial diversification had taken place by this time.

Chapter 3 of this thesis addresses the biogeochemical implications of one class of molecular fossils: the steranes. These hydrocarbons are the diagenetic products of steroids, and finding them in late Archean rocks is particularly significant because steroid biosynthesis requires molecular oxygen. Thus their presence in marine sediments of this era implies that dissolved $\mathrm{O}_{2}$ was available in biochemically significant concentrations in at least some parts of the ocean. Simultaneously, multiple geochemical proxies suggest 
an atmosphere with an $\mathrm{O}_{2}$ content $<10^{-6}$ of the present level until ca. 2.4Ga. Are prevailing interpretations of the molecular fossil and geochemical proxies for oxygenation mutually inconsistent? To answer this question, we investigated the oxygen requirements of de novo steroid biosynthesis. By growing the facultatively anaerobic eukaryote Saccharomyces cerevisiae under well-defined, microaerobic conditions and tracking the incorporation of isotopically-labeled carbon into sterols, we found that steroid biosynthesis is enabled by oxygen concentrations as low as nanomolar. Using constraints from Precambrian atmospheric evolution models, we show that such microaerobic regions of the surface ocean could have been persistent features long before the oxygenation of the atmosphere.

Taken together, these results contribute to understanding of the nature and tempo of biogeochemical change in the early Precambrian. Rather than the evolution of cyanobacteria suddenly relieving global reductant limitation and driving rapid oxygenation of the atmosphere and oceans, the process appears to have been much more gradual. The origination of cyanobacteria (along with most other high-level microbial diversity) before $2.7 \mathrm{Ga}$ was followed by a extended period of close coexistence between oxygenic and anoxygenic primary producers (Johnston et al., 2009).

\section{HOW DO PRIMARY PRODUCERS USE NUTRIENTS?}

If carbon has not been growth limiting until perhaps the late Cenozoic, and reductants have not been limiting even since before the invention of oxygenic photosynthesis (and certainly not afterwards), what has limited primary production over most of earth history? With both reactants (inorganic carbon and electron donors) in abundant supply but the inherent kinetics terribly slow, the rate of reaction is limited by the availability and activity of catalyst. Life provides catalysts for autotrophy in the form of protein-based enzymes. These enzymes are made of nitrogen-rich amino acids, are encoded and expressed by phosphate-rich nucleic acids, and require a wide variety of metals as 
cofactors. Thus the kinetic limitation of the gross rate of carbon fixation is also limitation by nutrients.

The second part of this thesis explores how a particular marine primary producer, Prochlorococcus, utilizes nutrients to make the biochemicals it needs to grow. A cyanobacterium abundant in the tropical and subtropical epipelagic ocean, Prochlorococcus contributes significantly to global primary production while living in one of the most nutrient-deplete habitats on earth (Partensky et al., 1999; Coleman and Chisholm, 2007). How it accomplishes this feat is intriguing from a metabolic biochemistry standpoint and central to our understanding of the functioning of nutrient limited ecosystems in the past and present.

The time scale of this investigation is rather shorter than the geologic spans considered in Chapters 2 and 3: we focus on the dynamics of gene expression over the 24-hour diel cycle, to which cell division in Prochlorococcus is closely synchronized (Vaulot et al., 1995). Chapter 4 presents results of a proteomic, transcriptomic and regulatory investigation of the diel cell cycle. In particular, we sought to address the question, what are the differences in, and controls on, gene expression patterns between mRNA and proteins during the diel cell cycle? We found that temporal variations in protein abundance are substantially smaller than the oscillations in their respective transcripts (Zinser et al., 2009). Physiologically-essential shifts in metabolic fluxes occurring over the diel cycle appear to be driven by fairly small changes in the relative abundance of enzymes. This includes central pathways of carbon fixation and respiration, suggesting that Prochlorococcus' role as a primary producer hinges on precise control of a metabolic network poised near a flux balancing point.

The whole-cell, quantitative portrait of gene expression generated in this experiment also enabled us to ask, which proteins constitute the greatest proportion of the proteome, and is the composition of the proteome strongly remodeled over the diel cycle? 
Given the phototrophic lifestyle of Prochlorococcus, we might expect that the cells bear a quite distinct complement of enzymes in the light and dark periods of the day. Somewhat surprisingly, we found that the overall composition of the proteome, as gauged by the fractional abundance of various gene products, remained generally constant. The set of highly abundant proteins included expected enzymes involved in photosynthesis, $\mathrm{C}$ fixation, nutrient acquisition and cell growth, but also some unexpected oxidative-stress and biosynthetic systems. There did not appear to be evidence for large-scale redistributions of cellular resources (at least in terms of protein) to different metabolic systems over the course of the day-night cycle. Since many, if not most, physiological processes in Prochlorococcus are temporally variable to some extent, the stability of its proteome composition underlines the well-tuned nature of cellular metabolic networks.

Chapter 5 presents a model of Prochlorococcus cellular composition based on this systems-level view of metabolism and gene product abundances. To provide initial bounds on problem, we asked, how are the major elemental complements of Prochlorococcus cells apportioned among various pools of biochemicals? Using a combination of experimental and genomic data, we calculated carbon, nitrogen and phosphorus distributions among the major biochemical constituents of a hypothetical Prochlorococcus cell. From these budgets we infer that almost half of cellular P is in the chromosome, that cells contain only a few hundred ribosomes, and that protein copy numbers probably span about four orders of magnitude.

We then used these cellular elemental and biochemical budgets to inform two outstanding questions regarding the ecology and evolution of Prochlorococcus. First, is genome streamlining in Prochlorococcus primarily driven by adaptation to nutrient limitation? The small, A+T-rich genomes, especially of high-light-adapted strains, have been proposed to result from selection for lowered nutrient requirements (Dufresne et al., 2005; Partensky and Garczarek, 2010). Instead, we found that the effects of genome streamlining on cellular nutrient budgets were likely marginal, and some of the more 
significant nutrient economies actually oppose the streamlining trend. It seems more plausible that a combination of genetic- and population-level processes are responsible for many of the large-scale features of genome evolution in marine picocyanobacteria.

\section{Second, how might stochasticity in gene expression affect the growth of}

Prochlorococcus cells? As small, nutrient-poor cells with low-integer number of copies of most gene products, Prochlorococcus could be susceptible to strong stochastic effects in expressing its genes, which could lead to metabolic and phenotypic instability (Raj and van Oudenaarden, 2008). However, the slow translation rate in Prochlorococcus would act to damp expression noise at the protein level, and we develop a hypothesis for the potential of stochastic effects to indirectly limit the growth of small cells.

\section{REFERENCES}

Anbar, AD, Y Duan, TW Lyons, GL Arnold, B Kendall, RA Creaser, AJ Kaufman, GW Gordon, C Scott, J Garvin, and R Buick. 2007. A whiff of oxygen before the great oxidation event? Science 317, no. 5846: 1903-1906.

Bosak, T, B Liang, MS Sim, and AP Petroff. 2009. Morphological record of oxygenic photosynthesis in conical stromatolites. Proc Natl Acad Sci USA 106, no. 27: 10939-10943.

Bradley, AS, JM Hayes, and RE Summons. 2009. Extraordinary 13C enrichment of diether lipids at the Lost City Hydrothermal Field indicates a carbon-limited ecosystem. Geochimica et Cosmochimica Acta 73, 102-118.

Brocks, JJ, GA Logan, R Buick, and RE Summons. 1999. Archean molecular fossils and the early rise of eukaryotes. Science 285, no. 5430: 1033-1036.

Brocks, JJ, R Buick, RE Summons, and GA Logan. 2003. A reconstruction of Archean biological diversity based on molecular fossils from the 2.78 to 2.45 billion-yearold Mount Bruce Supergroup, Hamersley Basin, Western Australia. Geochimica et Cosmochimica Acta 67, no. 22: 4321-4335.

Canfield, DE. 2001. Biogeochemistry of sulfur isotopes. Reviews in Mineralogy \& Geochemistry 43, 607-636.

Catling, DC, KJ Zahnle, and C McKay. 2001. Biogenic methane, hydrogen escape, and the irreversible oxidation of early Earth. Science 293, no. 5531: 839-843.

Coleman, ML, and SW Chisholm. 2007. Code and context: Prochlorococcus as a model for cross-scale biology. Trends Microbiol 15, no. 9: 398-407.

Dufresne, A, L Garczarek, and F Partensky. 2005. Accelerated evolution associated with genome reduction in a free-living prokaryote. Genome Biol 6, no. 2:

Eigenbrode, JL, KH Freeman, and RE Summons. 2008. Methylhopane biomarker hydrocarbons in Hamersley Province sediments provide evidence for Neoarchean aerobiosis. Earth and Planetary Science Letters 273, no. 3-4: 323-331. 
Frei, R, C Gaucher, SW Poulton, and DE Canfield. 2009. Fluctuations in Precambrian atmospheric oxygenation recorded by chromium isotopes. Nature 461, no. 7261: 250-253.

Johnston, D, F Wolfe-Simon, A Pearson, and AH Knoll. 2009. Anoxygenic photosynthesis modulated Proterozoic oxygen and sustained Earth's middle age. Proceedings of the National Academy of Sciences

Partensky, F, and L Garczarek. 2010. Prochlorococcus: Advantages and Limits of Minimalism. Annual Review of Marine Science 2, 211-237.

Partensky, F, WR Hess, and D Vaulot. 1999. Prochlorococcus, a marine photosynthetic prokaryote of global significance. Microbiol Mol Biol Rev 63, no. 1: 106-127.

Raj, A, and A van Oudenaarden. 2008. Nature, nurture, or chance: stochastic gene expression and its consequences. Cell 135, no. 2: 216-226.

Rasmussen, B, IR Fletcher, JJ Brocks, and MR Kilburn. 2008. Reassessing the first appearance of eukaryotes and cyanobacteria. Nature 455, 1101-1104.

Riding, R. 2006. Cyanobacterial calcification, carbon diodixe concentrating mechanisms, and Proterozoic-Cambrian changes in atmospheric composition. Geobiology 4, 299-316.

Vaulot, D, D Marie, RJ Olson, and SW Chisholm. 1995. Growth of Prochlorococcus, a Photosynthetic Prokaryote, in the Equatorial Pacific Ocean. Science 268, no. 5216: 1480-1482.

Wullschleger, SD. 1993. Biochemical limitations to carbon assimilation in C3 plants -- a retrospective analysis of the $\mathrm{A} / \mathrm{Ci}$ curves from 109 species. Journal of Experimental Botany 44, 907-920.

Zinser, ER, D Lindell, ZI Johnson, ME Futschik, C Steglich, ML Coleman, MA Wright, T Rector, R Steen, N McNulty, LR Thompson, and SW Chisholm. 2009. Choreography of the transcriptome, photophysiology, and cell cycle of a minimal photoautotroph, Prochlorococcus. PLoS ONE 4, no. 4: e5135. 


\title{
Chapter Two
}

\section{Late Archean molecular fossils from the Transvaal Supergroup record the antiquity of microbial diversity and aerobiosis}

\author{
Jacob R. Waldbauer, Laura S. Sherman, Dawn Y. Sumner and \\ Roger E. Summons
}

Reprinted with permission from Precambrian Research

(C) 2009 Elsevier B.V.

Waldbauer, J.R., Sherman, L.S., Sumner, D.Y. and Summons, R.E. (2009) Late Archean molecular fossils from the Transvaal Supergroup record the antiquity of microbial diversity and aerobiosis. Precambrian Research 169: 28-47. 


\title{
Late Archean molecular fossils from the Transvaal Supergroup record the antiquity of microbial diversity and aerobiosis
}

\author{
Jacob R. Waldbauer ${ }^{\mathrm{a}}$, Laura S. Sherman ${ }^{\mathrm{b}, 1}$, Dawn Y. Sumner ${ }^{\mathrm{c}}$, Roger E. Summons ${ }^{\mathrm{b}, *}$ \\ a Joint Program in Chemical Oceanography, Massachusetts Institute of Technology and Woods Hole Oceanographic Institution, Cambridge, MA 02139, United States \\ ${ }^{\mathrm{b}}$ Department of Earth, Atmospheric, and Planetary Sciences, Massachusetts Institute of Technology, Cambridge, MA 02139, United States \\ ${ }^{\mathrm{c}}$ Department of Geology, University of California, Davis, CA 95616, United States
}

\section{A R T I C L E I N F O}

\section{Article history:}

Received 5 July 2007

Received in revised form 21 December 2007

Accepted 23 October 2008

\section{Keywords:}

Biomarker

Archean

Molecular fossil

Oxygen

Sterane

Triterpane

Transvaal

\begin{abstract}
A B S T R A C T
Cores recovered during the Agouron Griqualand Drilling Project contain over $2500 \mathrm{~m}$ of well-preserved late Archean Transvaal Supergroup sediments, dating from ca. 2.67 to $2.46 \mathrm{Ga}$. Bitumen extracts of these strata were obtained using clean drilling, sampling and analysis protocols that avoided overprinting syngenetic molecular fossil signatures with contaminant hydrocarbons. Comparisons of biomarker contents in stratigraphically correlated intervals from diverse lithofacies in two boreholes separated by $24 \mathrm{~km}$, as well as across a $\sim 2 \mathrm{Gyr}$ unconformity, provide compelling support for their syngenetic nature. The suite of molecular fossils identified in the late Archean bitumens includes hopanes attributable to bacteria, potentially including cyanobacteria and methanotrophs, and steranes of eukaryotic origin. This molecular fossil record supports an origin in the Archean Eon of the three Domains of cellular life, as well as of oxygenic photosynthesis and the anabolic use of $\mathrm{O}_{2}$.
\end{abstract}

(c) 2009 Elsevier B.V. All rights reserved.

\section{Introduction}

Widely accepted evidence for an active microbial biosphere during the Archean Eon (3.8-2.5 Ga) includes physically preserved objects, such as microfossils and stromatolites, and a range of chemical, isotopic and geologic signatures of biogeochemical processes (Schopf and Walter, 1983; Knoll, 2003; Schopf, 2006). Shales bearing abundant organic matter attest to vigorous primary production in marine ecosystems by the middle Archean, and biotic activity may have also played a role in deposition of the massive iron formations of the period (Cloud and Licari, 1968; Cloud, 1973). Although there is little doubt that life had established itself throughout much of the oceans no later than ca. 3.4 Ga (Allwood et al., 2006), there is scant information about what types of organisms were present in Archean marine environments, or what sorts of metabolic processes they relied on.

While there are numerous reports of microfossils in Archean sediments (Schopf, 2006), it is generally agreed that morphology cannot consistently document the phylogenetic affinities or physiological capabilities of Archean microbes. Several sets of criteria for judging the biogenicity of microstructures have been proposed (Schopf, 2006). Archean stromatolites are also controversial bio-

\footnotetext{
* Corresponding author.

E-mail address: rsummons@mit.edu (R.E. Summons).

${ }^{1}$ Present address: Department of Geological Sciences, University of Michigan, Ann Arbor, MI 48109, United States.
}

genic remains (Walter et al., 1980; Walter, 1983; Grotzinger and Rothman, 1996; Hofmann et al., 1999). Their occurrence and diverse morphologies tend to be associated with shallow water depositional settings (Allwood et al., 2006), and some authors attribute particular deposits to microbes capable of oxygenic (Buick, 1992; Altermann et al., 2006) or anoxygenic (Bosak et al., 2007) photosynthesis.

Chemical and isotopic traces of Archean life are widespread and may sometimes be directly associated with particular microfossils (House et al., 2000). Sulfur isotopic data provide indirect evidence for the early evolution of sulfate reduction (Shen et al., 2001; Shen and Buick, 2004). Carbon isotopes of total organic carbon in Archean rocks provide more general information about biogeochemical processes such as carbon assimilation, methanogenesis, methanotrophy and aerobiosis (Hayes, 1983; Eigenbrode and Freeman, 2006). This sedimentary organic matter is the direct geological legacy of microbial activity and, if it is of sufficiently low thermal grade, there is potential for a far more detailed evaluation of the microbiota present at the time of deposition using particular kinds of hydrocarbons, or biomarkers, preserved therein (Brocks and Summons, 2003).

Fossil biomarkers are chemically stable molecules that derive from the carbon skeletons of precursor lipids. Biomarkers become incorporated into sediments, either freely as bitumen or bound into macromolecular organic matter (kerogen), where they may be preserved for billions of years (Eglinton et al., 1964; Brocks et al., $1999,2003 b)$. Where these compounds occur intact and uncontam- 
inated, they represent a direct avenue for ancient organisms to leave identifiable traces of themselves in the fossil record. In contrast to bulk chemical and isotopic data, which only carry circumstantial evidence of the metabolic attributes of their sources, biomarkers can carry specific information about the identities and physiologies of organisms because they were, in their original state, functioning components of living cells. In their preserved state, they have chemical structures derived from the original biomolecules through reasonably well-known pathways of diagenetic alteration (Peters et al., 2005). Most paleobiologically informative biomarkers are structurally related to steroids, triterpenoids and photosynthetic pigments of various types (Ourisson and Albrecht, 1992; Brocks and Summons, 2003; Volkman, 2005).

For a biomarker extracted from a rock to be considered a molecular fossil, we must be able to assess its syngeneity: that is, to discern whether or not a particular molecule derives from the original input of organic matter to a sediment. There are two principal routes for non-syngenetic biomarker hydrocarbons to be introduced into sedimentary rocks. First, under certain conditions, hydrocarbons can be widely mobile in sedimentary basins, so bitumen (operationally defined as the solvent-extractable portion of the organic matter) in a particular rock can potentially include material that has migrated between hydraulically connected strata of very different ages. This phenomenon is central to the accumulation of massive bitumen deposits - for example, oil reservoirs - in many petroleum systems. Second, human activity has suffused much of the surface environment with petroleum-derived hydrocarbons, rendering outcrop samples of bitumen-poor, thermally mature Precambrian rocks unsuitable for biomarker analysis. The heavy weathering experienced by most Archean terrains and their low bitumen contents mean that surface exposures are generally compromised. Sampling the subsurface by drilling affords long stretches of pristine stratigraphy, but necessitates contact of the core samples with drilling equipment and fluids. The trace quantities of biomarker molecules extractable from even the best-preserved Archean strata mean that attention to the possibility of even low-level contamination is essential to establishing a genuine molecular fossil record (Sherman et al., 2007).

To date there have been few detailed studies of biomarkers from Archean deposits. Two in recent years (Brocks, 2001; Eigenbrode, 2004) focused on biomarker analysis of resource-exploration cores drilled in the ca. 2.7-2.5 Ga Hamersley Basin, Western Australia. In both cases the syngeneity of the proposed Archean hydrocarbons was carefully assessed, although the approaches and methods differed. Both authors referred to the geological isolation of the basin, the structural integrity of the sediments studied and the absence of younger petroleum source rocks from the Hamersley Basin as valid reasons for discounting contamination from hydrocarbons migrated long distances from adjacent petroleum-prone basins. Brocks (2001) established that the identified Hamersley Basin hydrocarbons were associated with kerogenous shales, that they were at concentrations significantly above procedural blanks and that they showed maturity patterns, especially in respect to aromatic steroids, adamantanes and polyaromatic hydrocarbons, that were consistent with the prehnite-pumpellyite to lower greenschist metamorphic grade of the host rocks. He also showed that there was significant stratigraphic variation in biomarker compositions, that the biomarkers showed typical Precambrian patterns and that inappropriate compounds (e.g. plant terpanes) were not evident. Brocks et al. (2003a) concluded that the biomarkers from the Hamersley and Fortescue groups were 'probably syngenetic with their Archean host rock' although they could not absolutely rule out anthropogenic hydrocarbon contamination introduced during drilling, transport and storage of the cores.

Eigenbrode (2004) examined samples representing a wider suite of lithologies (and, therefore, paleoenvironments) and reached sim- ilar findings for those wells that were analyzed in common with Brocks (2001). Eigenbrode detected significant dispersion in the $\delta^{13} \mathrm{C}$ values of kerogen that was interpreted in the context of different paleoevironmental settings and a secular trend toward increasing apparent oxygenation of shallow water environments (Eigenbrode and Freeman, 2006). Further, it was shown that some biomarker ratios were strongly correlated to the $\delta^{13} \mathrm{C}$ values of associated kerogens or to dolomite abundance, supporting a syngenetic relationship with host sediments (Eigenbrode, 2004, 2008; Eigenbrode et al., 2008).

Another approach to molecular analysis of Precambrian organic matter is the study of hydrocarbons trapped in fluid inclusions. Hydrocarbon-bearing fluid inclusions in Proterozoic rocks from Australia (Dutkiewicz et al., 2003a,b, 2004; Volk et al., 2003; George et al., 2008), Gabon (Dutkiewicz et al., 2007) and Canada (Dutkiewicz et al., 2006) dating as far back as $>2.2 \mathrm{Ga}$ have yielded suites of biomarker compounds including steroids and triterpenoids. Fluid inclusions present unique bitumen trapping conditions, including high fluid pressures and the absence of clay minerals, and the opportunity to assess the inclusion entrapment in the context of the alteration history of the host rock. Fluid inclusion analysis has provided insight into both the molecular fossil record of Precambrian life and the chemical behavior of biomarker hydrocarbons at high temperatures and pressures (George et al., 2008).

In this study, we examined the characteristics of organic matter in two cores (GKF01 and GKP01) drilled as part of the Agouron Griqualand Drilling Project with the express purpose of obtaining fresh, minimally contaminated late Archean sediments for sedimentological, geochemical and paleontological analyses (Beukes et al., 2004; Schröder et al., 2006; Sumner and Beukes, 2006). Importantly, these cores represent some of the first to be recovered from late Archean strata using protocols specifically designed to minimize potential for organic contamination throughout the drilling, handling and storage process. Given the extremely low quantities of extractable hydrocarbons in even the best-preserved Archean sediments, minimization of contamination is essential to avoid overprinting the indigenous organic signatures. A detailed discussion of these measures and the biomarker analysis methods used in this work is presented elsewhere (Sherman et al., 2007). Here we report the results of analysis of Archean biomarkers and correlations of their patterns across the same formations in two cores drilled $24 \mathrm{~km}$ apart.

\section{Geological context}

The Transvaal Supergroup consists of a mixed siliciclasticcarbonate ramp that grades upward into an extensive carbonate platform overlain by banded iron formation. It was deposited on the Kaapvaal Craton between 2670 and $2460 \mathrm{Ma}$ (Armstrong et al., 1991; Barton et al., 1994; Walraven and Martini, 1995; Sumner and Bowring, 1996). The platform is up to $2 \mathrm{~km}$ thick, with predominantly peritidal facies in the north and east and mostly deeper facies to the south and west. Platform, slope and basinal sediments are preserved between Griquatown and Prieska (Beukes, 1987; Sumner and Beukes, 2006).

Two scientific cores, Agouron Institute cores GKP01 and GKF01 (hereafter referred to as GKP and GKF), were drilled through slope facies to provide geochemically fresh samples. The two cores are correlated to each other with 14 tie lines using volcanic and impact spherule layers, shale geochemistry, and distinctive facies distributions (Schröder et al., 2006). They are correlated to the shallower platform with five time lines using impact spherule layers and sequence stratigraphy (Sumner et al., unpublished). Water depths represented in the cores range from wave base to hundreds of meters, with GKP comprised of generally deeper-water facies than GKF. 
Depositional facies in the cores are well preserved. Most of the platform experienced sub-greenschist grade metamorphism at most (Button, 1973; Miyano and Beukes, 1984). However, supergene alteration during late fluid flow produced local $\mathrm{Pb}-\mathrm{Zn}$, fluorite, and gold deposits along fluid flow paths (Martini, 1976; Clay, 1986; Duane et al., 1991, 2004; Tyler and Tyler, 1996) and caused dolomitization along the platform margin, including rocks in GKP and GKF. Fluid flow was driven by the ca. 2 Ga Kheis orogeny to the west (Beukes and Smit, 1987; Duane and Kruger, 1991), and this event probably produced the peak alteration temperatures experienced by these rocks (Duane et al., 2004).

\section{Methods}

\subsection{Sample selection and correlation}

Samples were chosen to both span the full stratigraphic time represented by the cores and to represent the breadth of lithofacies. Sampling also focused on collecting temporally equivalent samples from the two cores, utilizing detailed inter-core stratigraphic correlations (Schröder et al., 2006; Sumner et al., unpublished). This sampling approach produced pairs of samples that can be used to compare biomarker preservation and composition in temporally equivalent but environmentally distinct samples. The depths of the samples analyzed, as well as their formations, rock types and correlations, are indicated in Table 1. Detailed images of the full lengths of the cores and stratigraphic columns are available as part of the AgouronGriqualand Paleoproterozoic Drilling Project Online Database at http://general.uj.ac.za/agouron/index.aspx. After recovery, cores were stored in aluminum trays and samples selected for biomarker analysis were wrapped in two layers of precombusted aluminum foil.

\subsection{Materials}

Solvents (hexane, dichloromethane, and methanol) used in sample extraction and equipment cleaning were high purity grade (OmniSolv, EMD Chemicals). De-ionized (DI) water and hydrochloric acid $(\mathrm{HCl})$ used to process samples were extracted five times with dichloromethane (DCM). Glassware, aluminum foil, silica gel, and glass wool were combusted at $550^{\circ} \mathrm{C}$ for $8 \mathrm{~h}$ and quartz sand (Accusand, Unimin Corp.) was combusted at $850^{\circ} \mathrm{C}$ for $12 \mathrm{~h}$ prior to use. Metal tools used to process samples were cleaned with DI water and then rinsed five times with methanol, DCM, and hexane. Crushing tools (described below) were cleaned of particulates with combusted quartz sand and then ultrasonicated for $30 \mathrm{~min}$ each in methanol and DCM.

\subsection{Sample preparation}

Quarter core samples (mostly $1 / 4 \mathrm{NQ}, 47.6 \mathrm{~mm}$ diameter) were approximately $20-50 \mathrm{~cm}$ in length and $50-200 \mathrm{~g}$ in weight. They were processed in batches of six with at least one procedural sand blank per batch. Through a series of experiments (Sherman et al., 2007), we found that foreign, less mature organic matter was present on the outsides of the cores and that this component could not be removed simply by ultrasonication in organic solvents. As a result, we found it necessary to remove the outer $3-5 \mathrm{~mm}$ of each exposed surface of the core pieces. Samples were cut using a sectioning saw with a water lubricated diamond-edged blade (UKAM). DCM-extracted water was used to lubricate the saw blade. Between samples, the saw was washed with DI water and the blade was washed and ultrasonicated in methanol and DCM. After cutting, the inner core pieces were ultrasonicated in DCM-cleaned water, methanol, and DCM to further clean their outer surfaces. The samples were then crushed to $<5 \mathrm{~mm}$ pieces using a stainless steel mortar and pestle. These pieces were then ground to a fine powder (sub-140 mesh) in a SPEX 8510 Shatterbox using a stainless steel puck mill that was modeled after a SPEX 8507 mill (Sherman et al., 2007).

\subsection{Extraction and fractionation}

Each powdered sample (40-90 g) was divided into $60 \mathrm{ml}$ vials ( $\sim 15 \mathrm{~g}$ per vial) and $25-30 \mathrm{ml}$ of DCM was added to each vial. The vials were then ultrasonicated for $30 \mathrm{~min}$ and the extraction solvent decanted after allowing the rock powder to settle. This process was repeated twice and the solvent from the three extractions was pooled. These total bitumen extracts were filtered in wide-bore columns over $\sim 3 \mathrm{~cm}$ of silica gel and then treated with acid-activated copper to remove elemental sulfur. The extracts were separated into saturated, aromatic, and polar fractions by liquid column chromatography in glass pipette columns packed with $\sim 8 \mathrm{~cm}$ of silica gel. Saturated hydrocarbons were eluted with hexane ( $3 / 8$ column volume), aromatic hydrocarbons with hexane:DCM (4:1 (v/v); 4 column volumes), and polars with DCM:methanol (7:3 (v/v); 4 column volumes).

\subsection{Preparation of bitumen II}

After the samples were extracted as described above, a subset of powders were demineralized by acidification. About $30 \mathrm{~g}$ of each sample was placed into aqua regia- and solvent-cleaned Teflon tubes ( $\sim 12 \mathrm{~g}$ per tube). Acids were then added to the samples and allowed to react as follows: $6 \mathrm{~N}$ DCM-extracted $\mathrm{HCl}$ for $24-48 \mathrm{~h}$ (to remove carbonates), $48 \% \mathrm{HF}$ for at least $72 \mathrm{~h}$ (to dissolve silicates), and $6 \mathrm{~N} \mathrm{DCM}$-extracted $\mathrm{HCl}$ again for $24 \mathrm{~h}$ (to remove fluoride precipitates). The samples were then washed several times in DCMextracted water. The resulting powders were re-extracted following the procedures described above and were analyzed as "bitumen II."

\subsection{GC-MS and GC-MS-MS (MRM) analyses}

The saturated and aromatic hydrocarbon fractions were then gently dried under a stream of nitrogen to a volume of roughly $80 \mu l .10 \mathrm{ng}$ of $\mathrm{D}_{4}\left(\mathrm{~d}_{4}-\mathrm{C}_{29}-\alpha \alpha \alpha\right.$-ethlycholestane; Chiron Laboratories, Inc.) and $1 \mu \mathrm{g}$ of $a_{i C_{22}}$ (3-methylheneicosane, 99+\% purity; ULTRA Scientific) was added to the saturated fraction and $413 \mathrm{ng}$ of $\mathrm{D}_{14}\left(\mathrm{~d}_{14}\right.$-para-terphenyl, 98 atom\% deuterium; Cambridge Isotope Laboratories, Inc.) was added to the aromatic fraction as internal standards.

The saturated and aromatic hydrocarbon fractions were analyzed by gas chromatography-mass spectrometry (GC-MS) in full scan mode and by selected ion monitoring respectively. Biomarker analyses of the saturated fraction were conducted by metastable reaction monitoring GC-MS (GC-MS-MS or MRM). Each of these analyses was conducted on a Micromass AutoSpec Ultima equipped with an Agilent 6890N gas chromatograph. The GC was fitted with a DB-1 fused silica capillary column $(60 \mathrm{~m} \times 0.25 \mathrm{~mm}$ i.d., $0.25 \mu \mathrm{m}$ film thickness; J\&W Scientific) and used $\mathrm{He}$ as the carrier gas. During each analysis, the GC ramped from 60 to $150^{\circ} \mathrm{C}$ at $10^{\circ} \mathrm{C} / \mathrm{min}$, then at $3{ }^{\circ} \mathrm{C} / \mathrm{min}$ to $315^{\circ} \mathrm{C}$, which was held for $24 \mathrm{~min}$. The AutoSpec source was operated in EI-mode at $250^{\circ} \mathrm{C}, 70 \mathrm{eV}$ ionization energy, and $8 \mathrm{kV}$ accelerating voltage. Full scan analyses were conducted over a mass range of $50-600 \mathrm{Da}$ at a rate of $0.8 \mathrm{~s} /$ decade with a $0.2 \mathrm{~s}$ inter-scan delay. Data were acquired and processed using MassLynx 4.0 (Micromass Ltd.). Compounds were quantified based on manual peak integration and comparison to the internal standards (using $m / z=85$ for full scan analyses). 


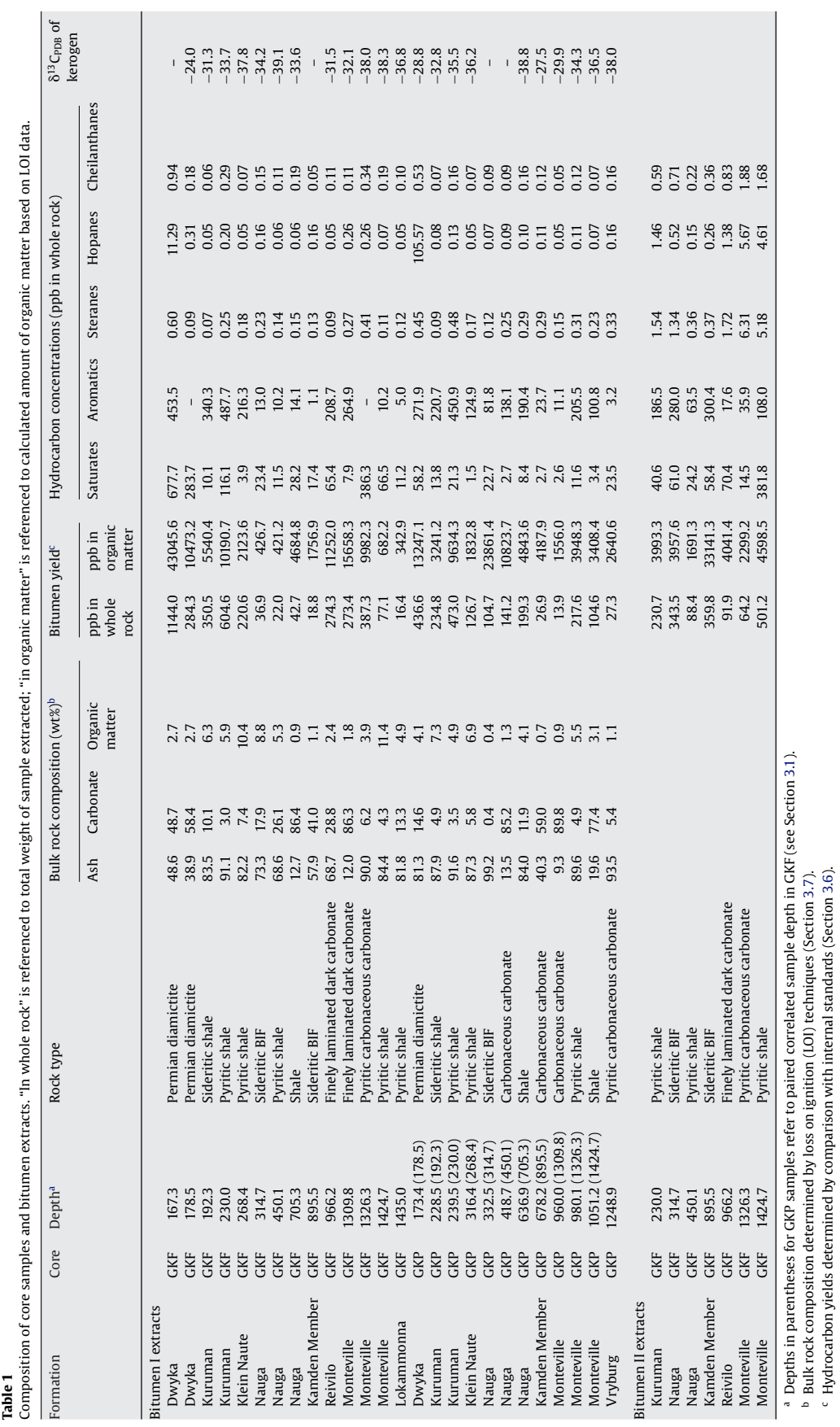




\subsection{Bulk composition by LOI}

Bulk weight percent organic matter, weight percent carbonate, and weight percent ash of each sample was estimated using loss on ignition (LOI) techniques. $300-400 \mathrm{mg}$ of rock powder was weighed into a small ceramic crucible and combusted in a Barnstead/Thermolyne 30400 furnace at $550^{\circ} \mathrm{C}$ for $4 \mathrm{~h}$. Once cool, the powders were weighed, and mass loss at this step taken as the organic matter content. The powders were then re-combusted at $950^{\circ} \mathrm{C}$ for $2 \mathrm{~h}$, and the further mass loss taken as weight percent carbonate. Material remaining after the final combustion (primarily silicate, oxide and sulfide minerals) was considered ash.

\subsection{EA-IRMS analyses}

Rock powders for kerogen isotope measurements were first demineralized using $6 \mathrm{~N} \mathrm{HCl}$ and $48 \% \mathrm{HF}$ as described above. After this treatment, $1 \mathrm{~N} \mathrm{HNO}_{3}$ was added to the samples to dissolve sulfide minerals. The largely demineralized powders were then dried and weighed in triplicate into tin cups $(0.5-0.8 \mathrm{mg}$ each). Carbon isotopic compositions were determined using a Fisons (Carlo Erba) NA 1500 elemental analyzer fitted with a Costech Zero Blank Autosampler and coupled to a Thermo Finnigan Delta Plus XP isotope ratio mass spectrometer.

\section{Composition of Archean organic matter}

The overall composition of the organic matter present in the Griqualand cores is shown schematically in Fig. 1. The Griqualand rocks preserve abundant organic matter - exceeding $10 \mathrm{wt} \%$ in some samples (Table 1) - but very little of that organic matter is present as extractable bitumen. In all cases, in excess of $99.99 \%$ of the organic matter is in the form of insoluble, macromolecular kerogen (Fig. 1) closely associated with the mineral matrix. These characteristics are a result of the extensive diagenetic and thermal histories of the host rocks. The carbon isotope composition of the kerogen isolated from the samples analyzed for biomarkers closely follows the organic carbon isotope stratigraphy for the cores determined by Fischer et al. (this volume) (Fig. 2E).

\subsection{Composition of extractable bitumens}

While solvent-extractable bitumen comprises a tiny fraction of the organic matter in these late Archean rocks, it is the portion most amenable to detailed molecular analyses that might shed paleobiological and paleoenvironmental light on the organisms and ecosystems that produced the preserved organic material. The bulk of the results presented herein are from detailed characterizations of the molecular compositions of bitumen I (extracts of whole-rock powders) from cores GKF and GKP, and bitumen II (extracts of demineralized kerogen) from a subset of samples from core GKF. Figs. 2, 5, 6 and 8 show selected indices of the molecular composition of these three bitumens through the two cores; details of these downcore variations are presented in the following sections. Samples correlated between cores GKF and GKP on sedimentological and stratigraphic grounds (i.e., chosen $a$ priori before organic analysis) are plotted in those figures at equivalent depths on a representative stratigraphy, indicated at the left of each figure. Results from analysis of three samples of the PermoCarboniferous Dwyka formation diamictite, which unconformably overlies the late Archean strata in both cores, are also plotted. Analysis of the Dwyka diamictite, while clearly not informative as to late Archean biogeochemistry, proved very useful as a test of the integrity and syngeneity of molecular fossils in the underlying strata; these results are discussed below in Section 5.5.

Overall, molecular indices of bitumen composition did not significantly correlate with quantitative measures of host rock composition including organic carbon, carbonate, iron and sulfur

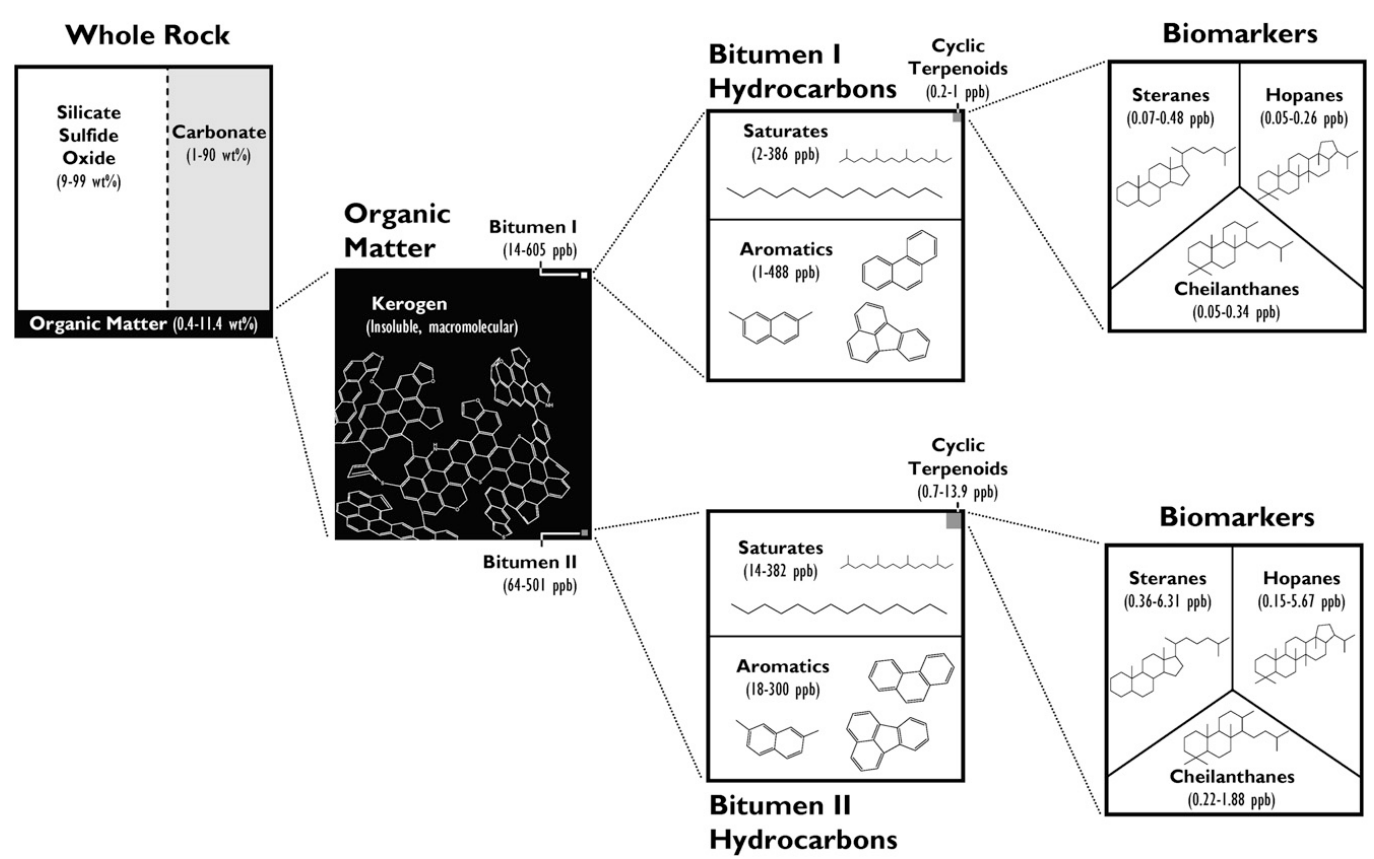

Fig. 1. Schematic of organic matter composition in late Archean core samples. Areas of subdivisions within boxes are proportional to the abundance of different pools of constituents. 
Saturated \& Aromatic Hydrocarbons

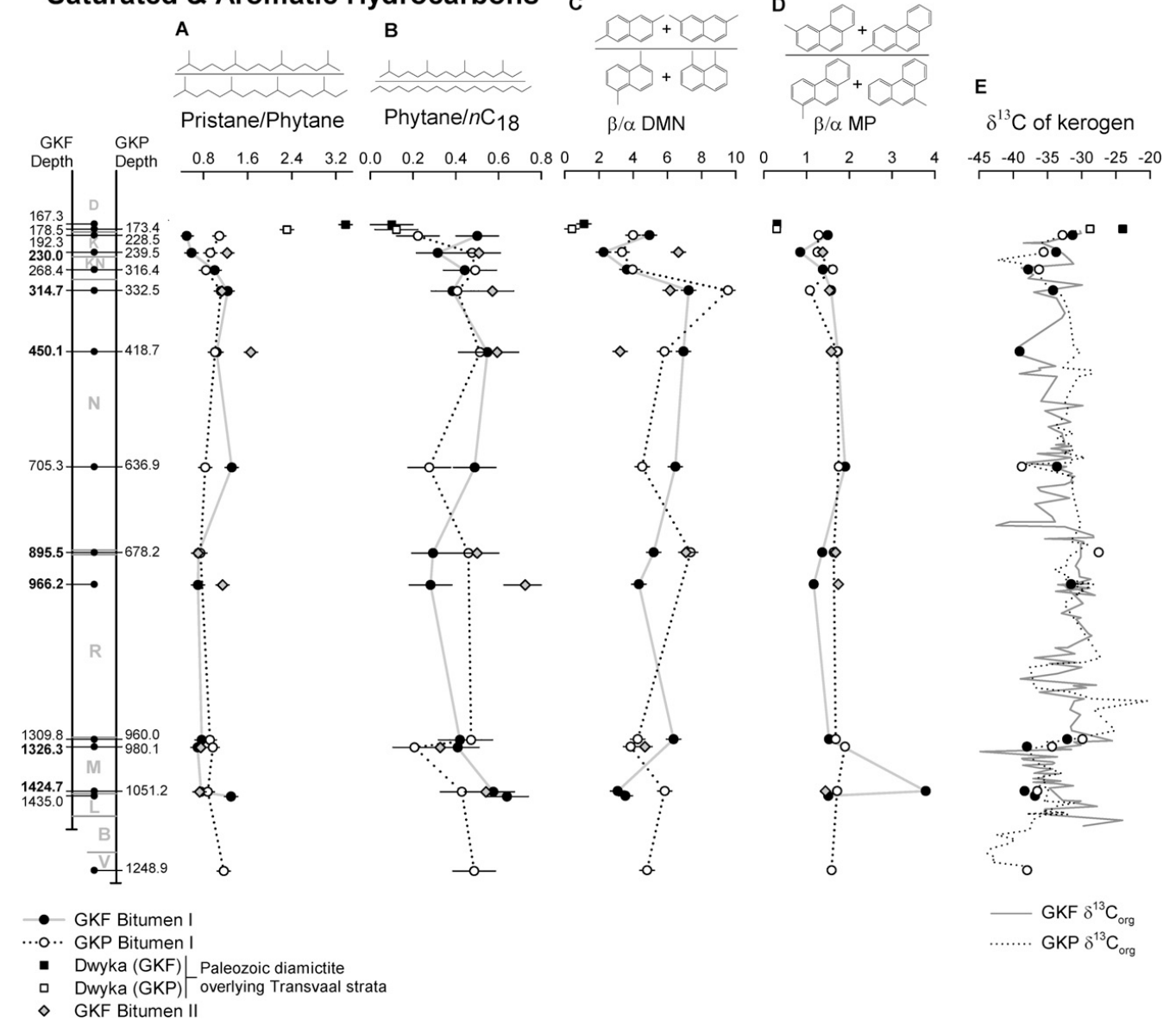

Fig. 2. (A-E) Selected organic matter composition parameters for samples from cores GKF and GKP. Sample depths are indicated at left on a representative stratigraphy and samples correlated between the two cores are plotted at equivalent vertical positions. GKF samples indicated in bold were analyzed for bitumen II. Errors in composition parameters $(2 \sigma)$ are plotted; where bars do not appear, they are smaller than the symbol size. Uncertainties in correlations were generally smaller than symbol size in the vertical dimension. Compound abbreviations: DMN - dimethylnaphthalene, MP - methylphenanthrene. (E) Carbon isotope composition of kerogen isolated from core samples. Lines are the $\delta^{13} \mathrm{C}$ values for total organic carbon determined by Fischer et al. (this volume). Formation abbreviations at left: D - Dwyka, K - Kuruman, KN - Klein Naute, N - Nauga, R - Reivilo, M - Monteville, L - Lokamonna, B - Boomplaas, V - Vryburg.

contents and $\delta^{13} \mathrm{C}$ values. While these quantities are an incomplete description of the complex rock matrix, such correlations have been observed in some other studies of Precambrian strata (Eigenbrode, 2008; Eigenbrode et al., 2008) and interpreted as a paleoenvironmental signal of coupling of organic matter source and depositional environment. The absence of these correlations in the Transvaal dataset may reflect a weaker coupling of the source of the organic matter (microbial activity) and the mode of its incorporation into the sediments with the inputs of clastic and inorganic chemical components. As elemental and isotopic compositions of the Transvaal strata show significant variation on centimeter to meter scales (Ono et al., this volume; Sumner et al., unpublished), this coupling would have to have been quite strong for bulk-molecular correlations to hold over hundreds of meters of stratigraphy. The correspondence in bitumen composition between samples correlated on stratigraphic grounds (Section 5.3), however, suggests that longer-term trends in organic matter sourcing and/or depositional environment are reflected in the cores. It is also possible that more detailed characterization of the rock mineral and organic matrix compositions, including quantification of specific mineral phases and examination of microscale organic-mineral associations, will further reveal depositional controls of bitumen composition.

\subsubsection{Saturated and aromatic hydrocarbons}

Bitumens from the Agouron cores are composed almost exclusively of saturated and aromatic hydrocarbons; more polar oxygenand nitrogen-containing functionalized compounds (e.g., carboxylic acids, porphyrins, phenols, etc.) were below detection limits. Partially unsaturated compounds (e.g., alkenes) were also not detected. Most bitumens contained more aromatic than saturated hydrocarbons, consistent with the highly aromatic character of the kerogen (Table 1). All of these characteristics are expected of highly thermally mature bitumen-kerogen associations where diagenetic and catagenetic reactions have proceeded for long periods and where the disproportionation of the original organic matter 
into disordered, aromatised carbon and light hydrocarbons is nearing completion.

Saturated hydrocarbon fractions extracted from these sediments are dominated by straight-chain $n$-alkanes, with a condensate-like carbon-number distribution peaking between $C_{11}$ and $\mathrm{C}_{20}$. The acyclic isoprenoids pristane and phytane, generally considered to be the products of diagenesis of the side-chain of chlorophyll, were detected in all samples. The pristane/phytane ratios of the late Archean bitumens are low (0.4-1.5; Fig. 2A), which is consistent with (though not necessarily indicative of)
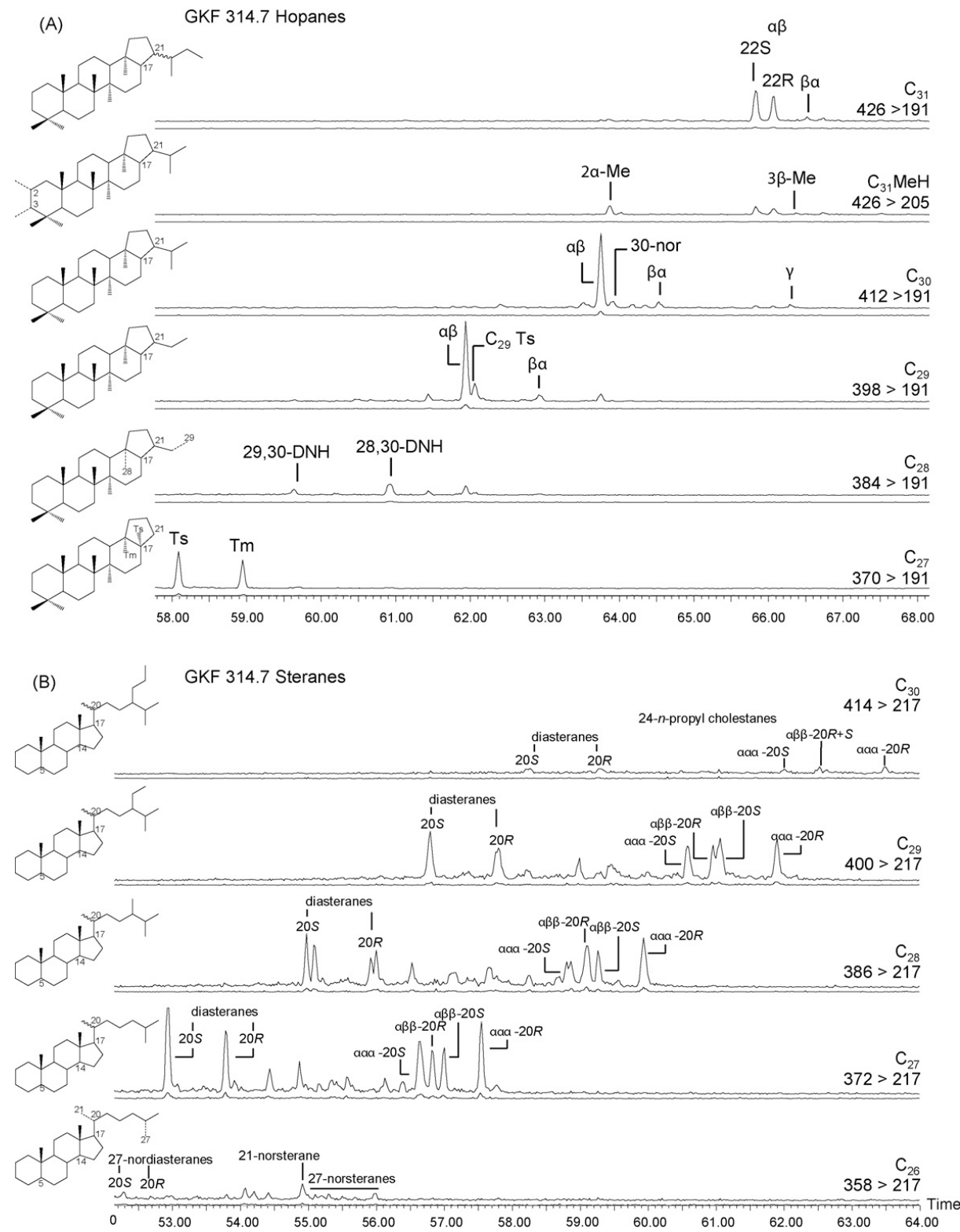

Fig. 3. (A) Typical results of MRM GC-MS-MS analysis for hopane biomarkers. $C_{27}$ through $C_{31}$ and A-ring methylated $C_{31}$ hopanes are shown; homohopanes to $C_{35}$ were routinely detected as well, in lower abundances. MRM transitions are indicated at right, and all chromatograms are shown to the same absolute scaling. Beneath each sample chromatogram is the equivalent trace from analysis of the parallel procedural blank. Compound abbreviations: Ts - 18 $\alpha-22,29,30$-trisnorneohopane, Tm - 17 $\alpha-22,29,30-$ trisnorhopane, DNH - dinorhopane, $\alpha \beta-17 \alpha(\mathrm{H}), 21 \beta(\mathrm{H})$ hopane, $\mathrm{C}_{29}$ Ts - 18 $\alpha$-30-norneohopane, $\beta \alpha-17 \beta(\mathrm{H}), 21 \alpha(\mathrm{H})$ hopane (moretane), 30 -nor - $\mathrm{C}_{30} 30$-norhomohopane, $2 \alpha$-Me - $2 \alpha$-methylhopane, $3 \beta$-Me - 3 $\beta$-methylhopane, $\gamma$ - gammacerane. (B) Typical results from sterane biomarker analysis, showing $\mathrm{C}_{26}$ through $\mathrm{C}_{30}$ regular steranes and diasteranes. Regular sterane structures shown at left; diasteranes have methyl rearrangements to $\mathrm{C} 5$ and $\mathrm{C} 14$. Compound abbreviations: $\alpha \alpha \alpha-5 \alpha(\mathrm{H}), 14 \alpha(\mathrm{H}), 17 \alpha(\mathrm{H})$ sterane, $\alpha \beta \beta-5 \alpha(\mathrm{H}), 14 \beta(\mathrm{H}), 17 \beta(\mathrm{H})$ sterane 
Table 2

Sample-to-blank (S/B) ratios for biomarker compounds, shown as averages across analyses of bitumen I extracts from Transvaal Supergroup samples $(n=23)$. Where a peak corresponding a given isomer was not identified in the analysis of a procedural blank, a baseline noise peak of equivalent retention time was integrated to reflect the detection limit of the mass spectrometer during that analytical session.

\begin{tabular}{|c|c|c|c|c|c|}
\hline Hopanes & Average S/B & Cheilanthanes/terpanes & Average S/B & Steranes & Average S/B \\
\hline $\mathrm{C}_{27}$ hopane Ts & 7.3 & $\mathrm{C}_{19} \alpha \beta$ tricyclic & 17.7 & $C_{26} 27$-nordiasterane $\beta \alpha 20 S$ & 7.3 \\
\hline $\mathrm{C}_{27}$ hopane $\mathrm{Tm}$ & 16.0 & $C_{20} \alpha \beta$ tricyclic & 13.4 & $C_{26} 27$-nordiasterane $\beta \alpha 20 R$ & 6.3 \\
\hline$C_{28}$ 29,30-dinorhopane & 5.6 & $C_{21} \alpha \beta$ tricyclic & 4.9 & $\mathrm{C}_{26}$ 21-norsterane & 16.4 \\
\hline$C_{28}$ 28,30-dinorhopane & 22.9 & $C_{22} \alpha \beta$ tricyclic & 2.9 & $C_{26} 27$-norsterane $\alpha \alpha \alpha 20 S$ & 4.9 \\
\hline$C_{29} \alpha \beta$ hopane & 9.3 & $\mathrm{C}_{23} \alpha \beta$ tricyclic & 4.6 & $C_{26} 27$-norsterane $\alpha \beta \beta 20 R$ & 7.3 \\
\hline $\mathrm{C}_{29} \mathrm{Ts}$ & 35.2 & $\mathrm{C}_{24} \alpha \beta$ tricyclic & 5.0 & $\mathrm{C}_{26} 27$-norsterane $\alpha \beta \beta 20 \mathrm{~S}$ & 3.8 \\
\hline$C_{29} \beta \alpha$ hopane (moretane) & 27.3 & $C_{25} \alpha \beta$ tricyclic $22 R$ & 6.2 & $\mathrm{C}_{26} 27$-norsterane $\alpha \alpha \alpha 20 R$ & 8.8 \\
\hline$C_{30} \alpha \beta$ hopane & 13.5 & $C_{25} \alpha \beta$ tricyclic $22 S$ & 5.7 & $C_{27}$ diasterane $\beta \alpha 20 S$ & 36.8 \\
\hline $\mathrm{C}_{30}$ 30-norhopane & 11.2 & $C_{26} \alpha \beta$ tricyclic $22 R$ & 18.0 & $C_{27}$ diasterane $\beta \alpha 20 R$ & 45.8 \\
\hline$C_{30} \beta \alpha$ hopane & 18.7 & $C_{26} \alpha \beta$ tricyclic $22 S$ & 35.1 & $\mathrm{C}_{27}$ sterane $\alpha \alpha \alpha 20 \mathrm{~S}$ & 21.4 \\
\hline$C_{31} 2 \alpha$-methylhopane & 25.9 & $\mathrm{C}_{24}$ tetracyclic & 9.8 & $C_{27}$ sterane $\alpha \beta \beta 20 R$ & 26.4 \\
\hline$C_{31} \alpha \beta$ hopane $22 S$ & 64.4 & $\mathrm{C}_{30}$ gammacerane & 5.3 & $\mathrm{C}_{27}$ sterane $\alpha \beta \beta$ 20S & 32.0 \\
\hline$C_{31} \alpha \beta$ hopane $22 R$ & 59.2 & & & $C_{27}$ sterane $\alpha \alpha \alpha 20 R$ & 21.0 \\
\hline$C_{32} \alpha \beta$ hopane $22 S$ & 27.2 & & & $C_{28}$ diasterane $\beta \alpha 20 S$ & 29.3 \\
\hline$C_{32} \alpha \beta$ hopane $22 R$ & 14.2 & & & $C_{28}$ diasterane $\beta \alpha 20 R$ & 31.8 \\
\hline$C_{33} \alpha \beta$ hopane $22 S$ & 19.6 & & & $C_{28}$ sterane $\alpha \alpha \alpha$ 20S & 14.9 \\
\hline$C_{33} \alpha \beta$ hopane $22 R$ & 25.5 & & & $C_{28}$ sterane $\alpha \beta \beta 20 R$ & 18.8 \\
\hline$C_{34} \alpha \beta$ hopane $22 S$ & 14.6 & & & $C_{28}$ sterane $\alpha \beta \beta$ 20S & 10.6 \\
\hline$C_{34} \alpha \beta$ hopane $22 R$ & 7.6 & & & $C_{28}$ sterane $\alpha \alpha \alpha 20 R$ & 18.5 \\
\hline$C_{35} \alpha \beta$ hopane $22 S$ & 7.5 & & & $C_{29}$ diasterane $\beta \alpha 20 S$ & 23.4 \\
\hline \multirow{10}{*}{$C_{35} \alpha \beta$ hopane $22 R$} & 5.6 & & & $C_{29}$ diasterane $\beta \alpha 20 R$ & 29.6 \\
\hline & & & & $C_{29}$ sterane $\alpha \alpha \alpha 20 S$ & 34.5 \\
\hline & & & & $C_{29}$ sterane $\alpha \beta \beta 20 R$ & 21.3 \\
\hline & & & & $C_{29}$ sterane $\alpha \beta \beta 20 S$ & 18.1 \\
\hline & & & & $\mathrm{C}_{29}$ sterane $\alpha \alpha \alpha 20 R$ & 15.7 \\
\hline & & & & $C_{30}$ diasterane $\beta \alpha 20 S$ & 8.3 \\
\hline & & & & $C_{30}$ diasterane $\beta \alpha 20 R$ & 5.6 \\
\hline & & & & $C_{30} 24$-n-propyl sterane $\alpha \alpha \alpha 20 S$ & 7.6 \\
\hline & & & & $C_{30} 24-n$-propyl sterane $\alpha \beta \beta 20 R+20 S$ & 9.9 \\
\hline & & & & $C_{30} 24$-n-propyl sterane $\alpha \alpha \alpha 20 R$ & 6.0 \\
\hline
\end{tabular}

deposition in a saline, anoxic environment. The aromatic fractions (as analyzed by SIM-GC-MS) are composed primarily of low-molecular weight polycyclic aromatic hydrocarbons (PAH; naphthalene and phenanthrene) and their alkylated homologues. Larger PAHs, including fluoranthene, pyrene and (in some samples) perylene were detected at much lower abundances. The substitution patterns of dimethylnaphthalenes and methylphenanthrenes showed dominance of the thermodynamically favored $\beta$-isomers over the more sterically hindered $\alpha$-isomers (Fig. 2C and D).

The preponderance of low-molecular weight compounds in the Transvaal Supergroup bitumens also means that that they are very susceptible to evaporation during extraction and analysis. This applies particularly to $n$-alkanes $<C_{14}$ and naphthalenes and may have affected the apparent bitumen yields, which can be considered lower limits for in situ concentrations. With the possible exception of chlorophyll-derived pristane and phytane, little can be said about the specific biological sources of these simpler hydrocarbons or their precursors.

\subsection{Biomarkers}

Though they represent only a small proportion of the bitumen extracts (Fig. 1), the cyclic terpenoids - hopanes, steranes and cheilanthanes - are the most information-rich molecules with regard to interpretations of the paleobiological source of the organic matter and its diagenetic history. These molecules are unambiguously biogenic; the hopanoids and steroids, in particular, are well-characterized as the biosynthetic products of the enzymatic cyclization of squalene and oxidosqualene, respectively (Rohmer et al., 1984; Ourisson et al., 1987; Summons et al., 2006; Fischer and Pearson, 2007), which enables a more informed interpretation of these compounds as molecular fossils. Typical results of MRM GC-MS-MS biomarker analysis of a saturated hydrocarbon frac- tion from a bitumen extract is shown in Fig. 3. These classes of cyclic terpenoids were present at ppb to sub-ppb levels (Table 1 ) with concentrations of individual compounds at the parts per trillion level by weight. Despite these extremely low yields, a broad diversity of biomarker structures could be consistently detected in bitumen extracts over the full depth of Archean strata intersected by both cores. Analytical sample-to-blank ratios (i.e., amount of a given compound detected in a core sample compared to that in the parallel procedural blank) for biomarker compounds are listed in Table 2. For many compounds, the 'blank' reflects the detection limit of the mass spectrometer (baseline noise above which a peak must rise) rather than the identified presence of a compound in the procedural sand blank. The relative contributions of hopanes, steranes and cheilanthanes to the total biomarker content are shown in Fig. 4 . The three types are present in roughly equal abundance, with a slight preference for steranes that may or may not be significant with regard to the source and/or diagenesis of the organic matter (see Section 6.2)

\subsubsection{Hopanes}

Several series of hopanes with 27-35 carbon atoms were detected in all bitumens (Fig. 3A). These include: $17 \alpha(\mathrm{H}), 21 \beta(\mathrm{H})$ hopanes; $2 \alpha$ - and $3 \beta$-methylhopanes; $17 \beta(\mathrm{H}), 21 \alpha(\mathrm{H})$-hopanes (moretanes); and several rearranged and norhopanes (Fig. 3A). Average sample-to-blank ratios generally exceeded 10 for the principal $C_{27}-C_{31}$ isomers (Table 2). The stratigraphic distributions of selected hopane isomers are shown in Fig. 5. The isomer distributions of the hopanes show predominance of the more thermodynamically stable forms, consistent with the high thermal maturity of the Transvaal host rocks. In particular, all the late Archean bitumens show high Ts/Tm ratios (>0.5; Fig. $5 \mathrm{~A}$ ) and low moretane/hopane ratios - approaching the thermodynamic endpoint of 0.05 - in the $C_{29}$ and $C_{30}$ homologues (Fig. 5C and E). These indices of high maturity are consistent between bitumens I and I 


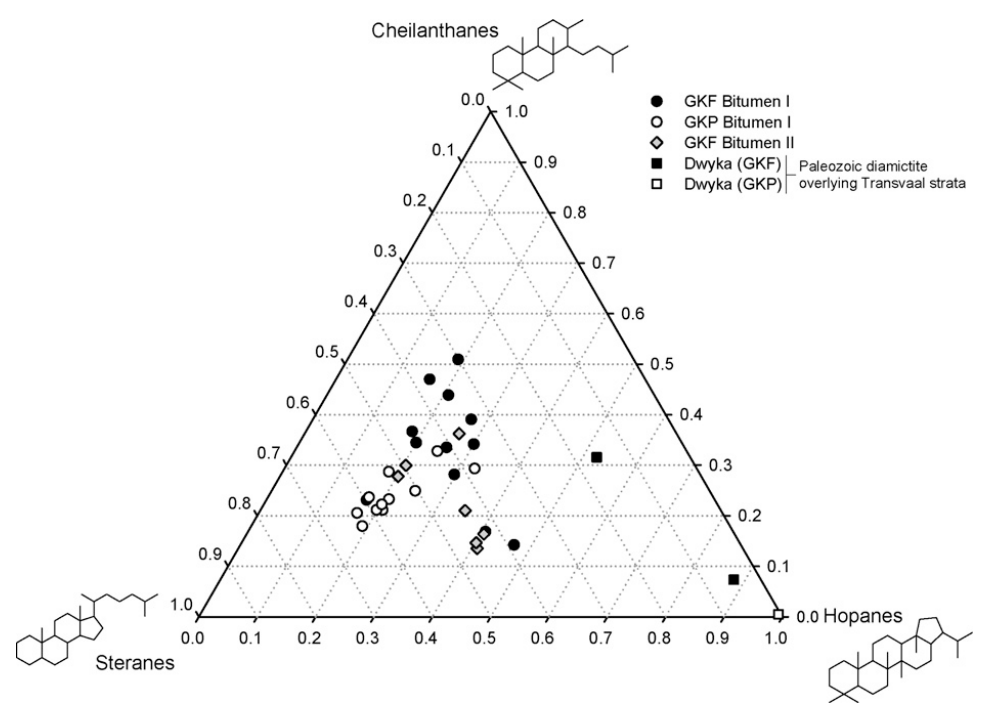

Fig. 4. Ternary diagram showing the composition of the cyclic terpenoid biomarker fraction of the bitumen extracts. Sterane, hopane and cheilanthane contents are calculated as the sum of all detected pseudohomologues and isomers.

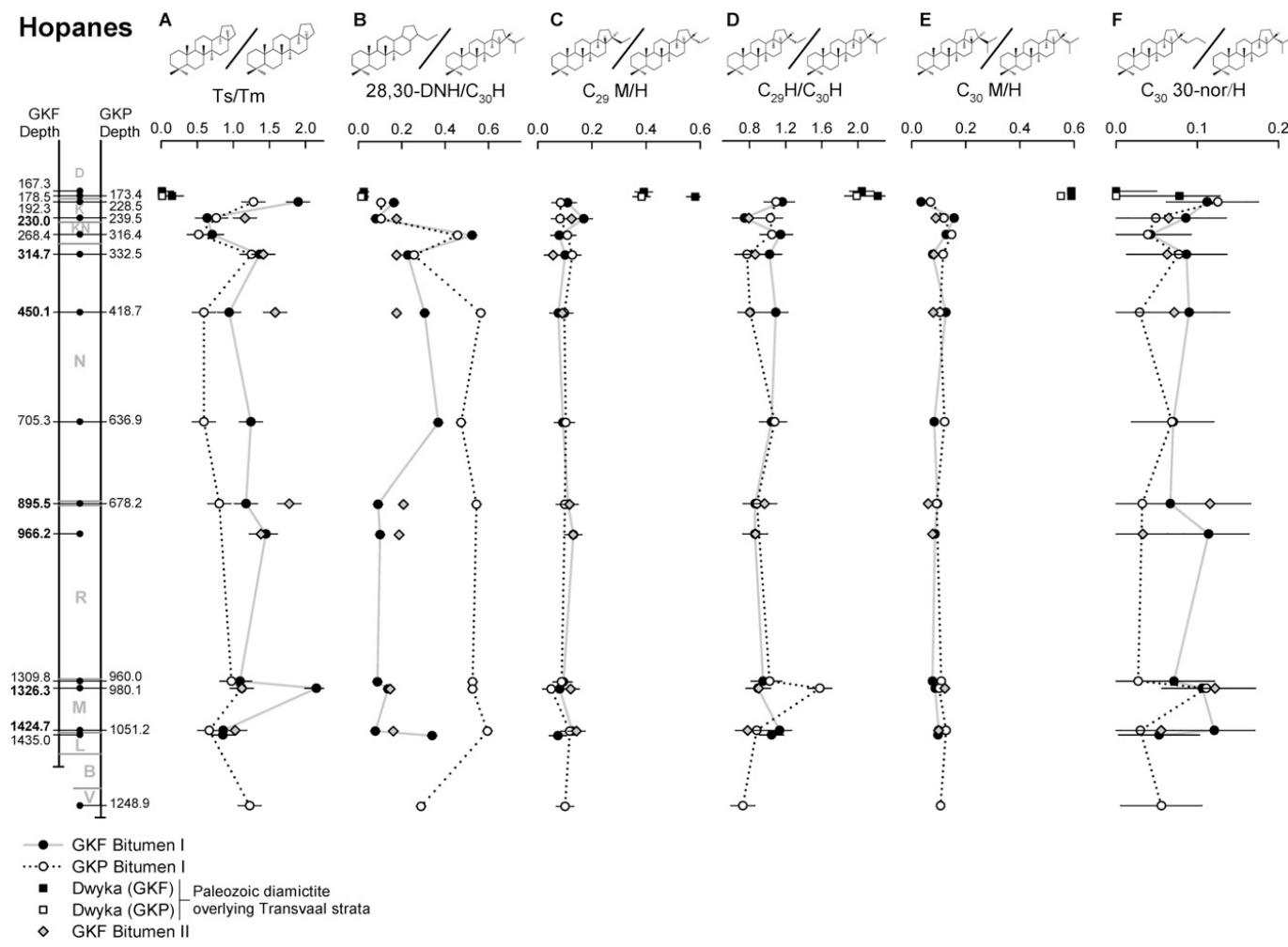

Fig. 5. Selected hopane biomarker parameters for samples from cores GKF and GKP, plotted down-core as in Fig. 2. Compound abbreviations as in Fig. 3A, with $\mathrm{H}=$ hopane and $\mathrm{M}=$ moretane 
(Fig. 5A, C and E), suggesting that the two bitumen pools have the same thermal history.

$\mathrm{C}_{28}$ dinorhopanes, including 28,30- and 29,30-dinor pseudohomologues, are present to varying degrees in all the late Archean bitumens (Fig. 3A). 28,30-Dinorhopane contents also varied between the cores, being higher in GKP than GKF below the uppermost Nauga formation (Fig. 5B); this difference is discussed further in Section 5.3. The 30-norhopane series, commonly observed in Phanerozoic oils and sediments, is also present in the Transvaal Supergroup bitumens. Fig. 3A identifies the elution position of the peak identified as 30-norhomohopane while Fig. 5 shows GKF and GKP down-core variations in the abundances of the $\mathrm{C}_{29}$ (column D) and $C_{30}$ (column $F$ ) members of the series relative to $C_{30}$ $\alpha \beta$-hopane. This series of hopanoids has been identified as particularly abundant in sediments with carbonate lithologies (Subroto et al., 1991, 1992) including those of Precambrian age (Brocks and Summons, 2003) and therefore it is not surprising to find it present in GKF and GKP. The $\mathrm{C}_{29} \mathrm{H} / \mathrm{C}_{30} \mathrm{H}$ ratio varies between 0.8 and 1.2 for the Transvaal Supergroup sediments (Fig. 5D) which is the range commonly found in Phanerozoic oils from carbonate source rocks but above the range (0.3-0.7) observed for oils derived from shales (Knoll et al., 2007). However, within the error limits, no definitive relationship to carbonate content could be discerned in the $\mathrm{C}_{29} \mathrm{H} / \mathrm{C}_{30} \mathrm{H}$ ratios of GKP and GKF.

In all of these indices of hopane content, the Permian Dwyka Formation in the uppermost portion of the cores shows a distinct and less mature composition. The relevance of this contrast in assessment of the syngeneity of the biomarkers from the Agouron cores is discussed in detail in Section 5.5 .

A-ring methylated hopanes were also detected in the Transvaal Supergroup bitumens (Fig. 3A). 2 $\alpha$-Methylhopanes with 31-35 carbon atoms were found, while $3 \beta$-methylhopanes were less abundant and could usually be detected only at $C_{31}$. 3Methylhopanoids, precursors of $3 \beta$-methylhopane, are known principally from aerobic methanotrophic bacteria, though they have been found in Acetobacter sp. as well (Zundel and Rohmer, 1985a, 1985b). While their biological source could in principle be elucidated through isotopic analysis, the extremely small quantities extractable from the core samples preclude this at present. The presence of 3 $\beta$-methylhopanes in the Transvaal bitumens is consistent with proposals for active methane cycling in late Archean marine ecosystems (Hayes, 1983; Eigenbrode and Freeman, 2006). Strong stratigraphic patterns are not apparent (Fig. 6B), though the portion of the Transvaal Supergroup sampled by the Agouron cores does not include intervals with strongly ${ }^{13} \mathrm{C}$-depleted organic carbon (few points are below -40\%; Fig. 2E) that would indicate a large contribution of recycled methane to sedimentary organic matter.

High relative abundances of $2 \alpha$-methylhopanes $\left(\mathrm{C}_{31} 2\right.$ $\mathrm{MeH} / \mathrm{C}_{30} \mathrm{H}>0.05$ ) are typical of Precambrian bitumens (Knoll et al., 2007), and most of the Transvaal samples are in this range (Fig. 6A). Summons et al. (1999) suggested that $2 \alpha$-methylhopanes are markers for oxygenic cyanobacteria. This interpretation is certainly consistent with the presence of steranes, whose precursor steroids require $\mathrm{O}_{2}$ for their biosynthesis (see Section 6.2). Rashby et al. (2007), however, have recently reported the biosynthesis of 2 -methylhopanoids by the $\alpha$-proteobacterium Rhodopseudomonas palustris, including during photosynthetic growth under anaerobic conditions. Whatever its identity, the biological source of the 2-methylhopanoids to the Transvaal sediments must have been ecologically widespread and persistent in the environment, as these biomarkers are present throughout the $>200 \mathrm{Myr}$ period recorded in the Agouron cores. The higher abundance of $2 \alpha-$ methylhopane in core GKF relative to GKP (Fig. 6A) - particularly through the deposition of the Reivilo and Nauga formations where facies in GKF represent significantly shallower depositional environments than those in GKP - suggests that the 2-methylhopanoid producing organisms may have been most abundant in shallowerwater, platform environments. Notably, these are the same kinds of environments where 2-methylhopanoid producing cyanobacteria have left especially strong biomarker (and microfossil) signatures through the Phanerozoic (Summons et al., 1999; Knoll et al., 2007).

\subsubsection{Cheilanthanes and other terpanes}

Tricyclic biomarkers of the cheilanthane type were found in all the Archean bitumens, typically at abundances similar to those of hopanes and steranes (Fig. 4). $13 \beta(\mathrm{H}), 14 \alpha(\mathrm{H})$ homologues from $\mathrm{C}_{19}$ to $\mathrm{C}_{26}$ were detected, with the $\mathrm{C}_{23}$ being consistently the most abundant member of the series (Fig. 6C). As expected for the regular isoprenoid side-chain, $C_{22}$ was the least abundant homologue. $C_{24}$ tetracyclic terpane was also detected in many samples. As the biological source(s) of cheilanthanes and $\mathrm{C}_{24}$ tetracyclic terpane are not known (Brocks et al., 2003b), little paleobiological interpretation can be made of their presence. To the extent that they are syngenetic, however, the tricyclic biomarkers in the Griqualand cores confirm that the biosynthetic pathways leading to the cheilanthane and $\mathrm{C}_{24}$ tetracyclic terpane precursors were operative by the late Archean (Brocks, 2001; Eigenbrode, 2004).

Gammacerane was also detected at low abundance in many of the Transvaal bitumens (Fig. 6D). The biological precursor of gammacerane is tetrahymanol, a lipid with both bacterial (Kleemann et al., 1990; Bravo et al., 2001) and eukaryotic (Harvey and McManus, 1991; Sinninghe Damsté et al., 1995) sources. Some bacteriovorous ciliates produce tetrahymanol as a steroid substitute when feeding below the oxic-anoxic transition in stratified waters, so gammacerane is commonly seen as a marker of water-column redox stratification. While such stratification is certainly a plausible scenario for an Archean marine environment, the biological source of tetrahymanol was not necessarily predatory protozoa. The presence of tetrahymanol in a number of proteobacteria, notably anoxygenic phototrophs (Kleemann et al., 1990; Rashby et al., 2007), makes a prokaryotic source more likely. Gammacerane, unlike the $C_{31} 2 \alpha-$ methylhopane, does not show higher abundance in GKF relative to GKP (compare Fig. 6A and D), suggesting some biological and/or geographical separation of the sources of those two biomarkers.

\subsubsection{Steranes}

Series of steranes with 26-30 carbon atoms were also detected in all bitumens analyzed (Fig. 3B). The predominant isomers of all homologues were $5 \alpha(\mathrm{H}), 14 \alpha(\mathrm{H}), 17 \alpha(\mathrm{H})$ and $5 \alpha(\mathrm{H}), 14 \beta(\mathrm{H}), 17 \beta(\mathrm{H})$ regular steranes (both $20 \mathrm{~S}$ and $20 \mathrm{R}$ epimers) and rearranged $13 \beta(\mathrm{H}), 17 \alpha(\mathrm{H})$-diasteranes. $\mathrm{C}_{27}$ to $\mathrm{C}_{29}$ homologues are dominant, with $\mathrm{C}_{26}$ and $\mathrm{C}_{30}$ steranes comprising only $1.9-5.4 \%$ and $1.5-6.2 \%$ of total steranes, respectively. Average sample-to-blank ratios for compounds of the $\mathrm{C}_{27}$ to $\mathrm{C}_{29}$ series range from 10.6 to 45.8 (Table 2 ), and certain isomers in individual samples were present at up to 248 times their abundance in the parallel procedural blank. Among the $\mathrm{C}_{27}-\mathrm{C}_{29}$ steranes, the three carbon numbers show similar abundances, with a slight preference for $C_{27}$ homologues (Fig. 7). The carbon-number distribution of steranes is very consistent between cores GKF and GKP and between bitumens I and II in GKF. The sterane carbon-number distributions in the late Archean bitumens fall within the range of compositions of Phanerozoic petroleum (gray line in Fig. 7), suggesting that the sources of steroids to the late Archean sediment were not radically different from that of later periods and involved multiple protistan taxa. Identified $C_{26}$ steranes included the 21-nor and 27-norcholestanes. $C_{30}$ steranes included 24- $n$-propyl regular and diasteranes. Steranes bearing additional methylation in the ring system (including 4-methyl and 4,4-dimethyl steranes) were not detected in any of the Griqualand core samples, despite use of sensitive MRM techniques to search for them specifically. While such methylsteranes cannot truly be said to be absent from the rocks - only to be below the detection limit of 


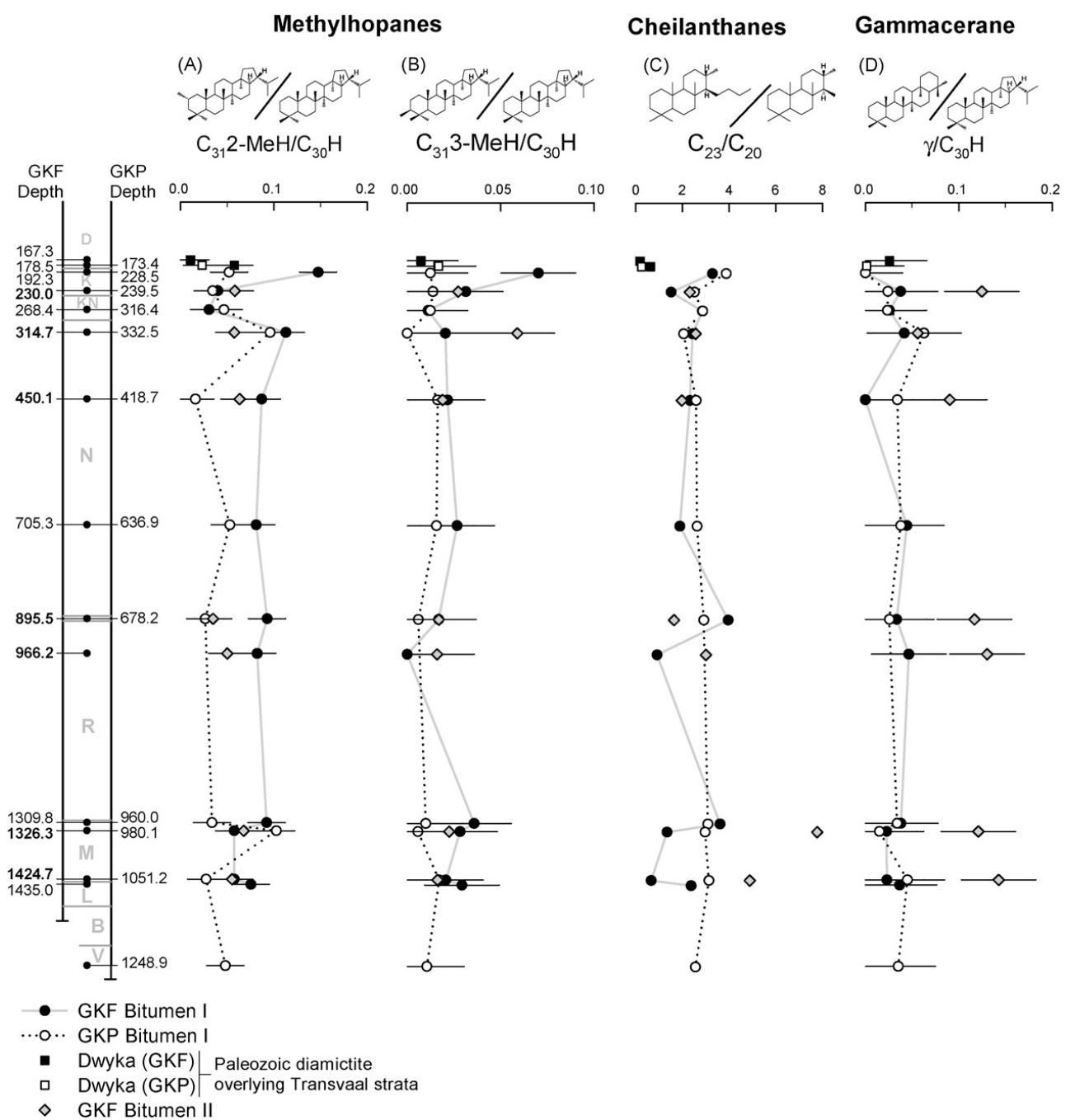

Fig. 6. (A) and (B) Ratios of A-ring methylated hopanes to desmethyl homologues. (C) and (D) Selected terpane ratios of cheilanthanes and gammacerane. Down-core plots as in Fig. 2.

the methods employed - it is clear that the precursors of desmethyl steranes were a much larger proportion of the organic matter input to these sediments than were methylsteroids.

Fig. 8 shows the stratigraphic variation in selected sterane isomer distributions down the two cores. As with the hopanes, the steranes show isomer distributions indicative of high thermal maturity. Specifically, the sterane isomers are distributed towards thermally favored configurations around $\mathrm{C} 14$ and $\mathrm{C} 17$ ( $\alpha \alpha \alpha$ to $\alpha \beta \beta$; Fig. 8D) and $C 20$ ( $R$ to $S$; Fig. $8 \mathrm{E}$ ). Diasteranes, which arise from sterene intermediates that have undergone an acidor clay-catalyzed backbone rearrangement during early diagenesis (Rubinstein et al., 1975), are abundant in the late Archean bitumens: diasterane/regular sterane ratios range from 0.4 up to 2.3. Notably, the down-core variation in diasterane/regular sterane ratios track exceptionally closely among all the pseudohomologues in bitumen I extracts from both cores and in bitumen II (Fig. 8A-C). This correspondence among the $C_{27}$ to $C_{29}$ steranes in their degrees of rearrangement is highly suggestive of a single source and a common diagenetic history for the steranes in the Transvaal Supergroup bitumens. A detailed discussion of the paleobiological interpretation of the steranes from the Agouron cores is presented in Section 6.2 .

\section{Syngeneity of molecular fossils}

The determination of the source of biomarker molecules is of paramount importance in their interpretation. Without confidence that biomarkers extracted from a sedimentary rock sample actually derive from organic matter that was a constituent of the original sediment, the antiquity of these molecules and their utility as molecular fossils is in question. The Agouron Griqualand Basin cores have provided a unique opportunity to test the syngeneity of Archean molecular fossils. Since the cores were drilled without hydrocarbon drilling fluids, were handled and curated with 


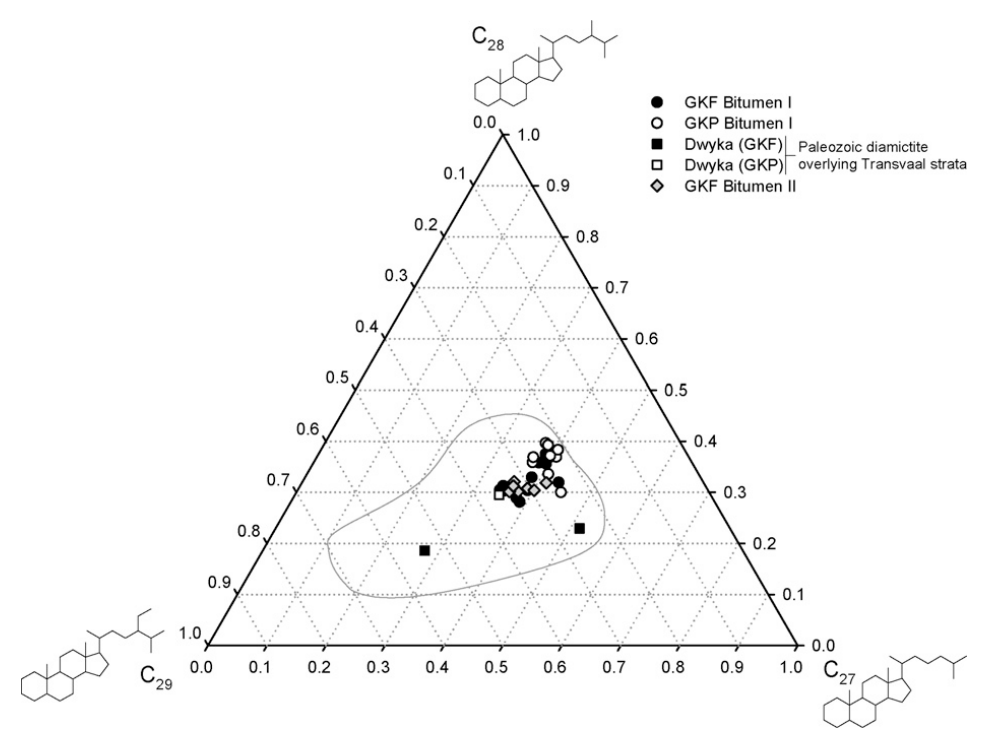

Fig. 7. Ternary composition diagram showing sterane carbon-number distributions in bitumen extracts. The gray line indicates the range of compositions observed in Phanerozoic petroleum systems, from the GeoMark Reservoir Fluid Database (http://www.geomarkresearch.com).

exceptional care and were analyzed shortly after recovery, the potential for contamination of the core material was minimized. The very small quantities of extractable organic matter remaining in these rocks after their long history makes it very easy to overprint syngenetic biomarker contents with exogenous hydrocarbons, so attention to clean drilling, handling, storage and analysis is essential. The low procedural blank achieved using the methods described above (Fig. 3; Table 2) demonstrates that laboratory contamination during analysis was also minimal. Nevertheless, even when laboratory blanks are well below sample hydrocarbon contents, the data resulting from analysis of bitumen extracts must still be scrutinized for evidence of contamination or characteristics incompatible with syngenetic organic matter. We did not, for instance, assume that cutting off the outer portions of the core samples necessarily removed contaminant hydrocarbons (contra Brocks et al., 2008). We present here five lines of evidence indicating that the molecular fossils reported here from the Agouron cores are syngenetic with their host rocks and are late Archean in age. It should be noted that no single one of these criteria constitutes a sufficient condition for syngeneity, and we do not take satisfaction of one criterion (e.g., thermal maturity; cf. Brocks et al., 2008) as prima facie evidence for indigenous molecular fossils. Rather, it is the simultaneous occurrence of all these characteristics that demonstrates syngeneity.

\subsection{Thermal maturity}

All the bitumens extracted from late Archean strata in the Griqualand cores have molecular compositions indicative of very high thermal maturity. Syngenetic organic matter must have the same burial and thermal history as its host rock, so high thermal maturity is to be expected for bitumens from host rocks that have experienced prehnite-pumpellyite facies metamorphism, indicating extended periods at temperatures $>200^{\circ} \mathrm{C}$. In the part of the Griqualand West Basin sampled by cores GKF and GKP, metamorphic alteration temperatures likely peaked ca. $2 \mathrm{Ga}$ (Duane et al., 2004). Overall, the molecular composition of the late Archean bitumens is consistent with thermal maturities in the wet-gas zone, as found in the Hamersley Basin (Brocks, 2001; Brocks et al., 2003a; Eigenbrode, 2004). The condensate-like distributions of $n$-alkanes, strong preference for $\beta$-substitution of polycyclic aromatics and overall dominance of low-molecular-weight hydrocarbons are indicative of high degrees of thermal cracking and maturation. The trace quantities of biomarkers detected in the bitumens all show thermodynamically controlled isomerization and stereochemical rearrangement patterns (see Section 4.2), consistent with high thermal maturity. As noted by Brocks et al. (2003a), it is difficult to use these molecular parameters to accurately gauge relative thermal maturity in rocks of this age, since so few organic geochemical studies of Archean and Paleoproterozoic rocks have been performed and little is known about the physicochemical behavior of these hypermature bitumens over their exceptionally long histories.

While quantitative assessments of the thermal maturity of Archean bitumens remain challenging, it is clear that the hydrocarbon biomarkers detected in the Griqualand core samples reflect the time-temperature histories of their host rocks. Notably, their high thermal maturity constrains contributions to the bitumens to before the time of peak heating of the host strata, ca. $2 \mathrm{Ga}$. The biomarkers in these bitumens are molecular fossils, not functionalized biochemicals, and certainly do not derive from modern, or even geologically recent, microbial activity in the host rocks.

\subsection{Absence of petrochemical and Phanerozoic signatures}

Petrochemicals - anthropogenic products of refined petroleum - are ubiquitous in industrialized settings, including drill sites and research laboratories. As petroleum-derived hydrocarbons are a principal source of potential contamination, detection of synthetic petrochemical compounds in bitumen extracts would sharply compromise their interpretation as molecular fossils. Grosjean and Logan (2007) and Brocks et al. (2008) have highlighted the utility of branched alkanes with quaternary carbons (BAQCs) as tracers of polyethylene contamination in drill cores. Using selected ion and difference chromatograms, the Agouron core extracts were examined for several series of BAQCs, including 2,2- through 9,9- 


\section{Steranes}

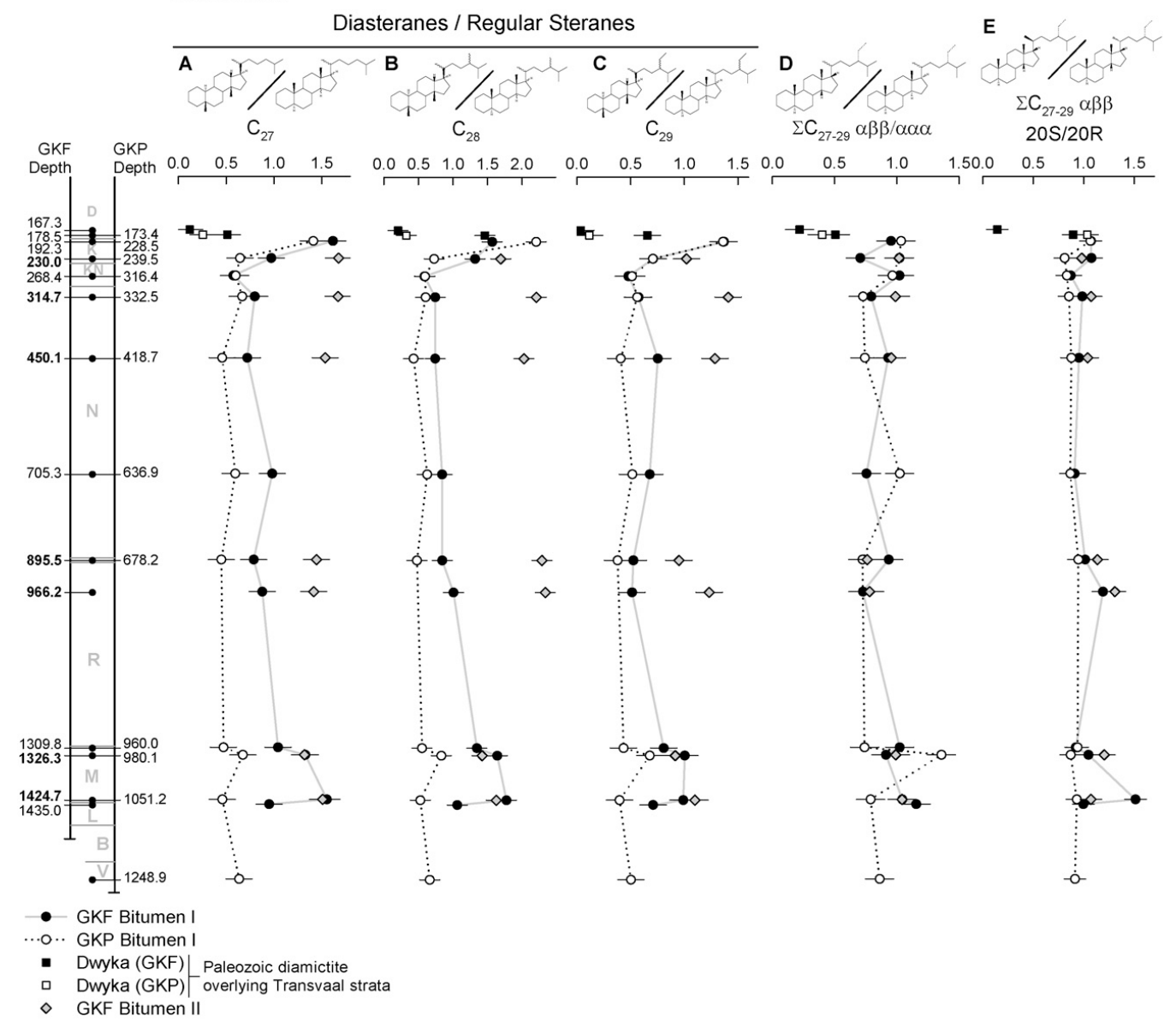

Fig. 8. Selected sterane biomarker parameters for samples from cores GKF and GKP, plotted down-core as in Fig. 2. Compound abbreviations as in Fig. 3B.

dimethyl, -diethyl and mixed alkyl (e.g., ethyl-, methyl-; butyl-, ethyl-) alkanes. No signals indicative of the presence of BAQCs were detected. The absence of this one class of contaminants does not exclude the possibility of other forms of contamination, but lends some confidence that core storage and handling conditions did not introduce high levels of exogenous petrochemicals into the samples.

Syngenetic organic matter from Precambrian rocks should also be devoid of biomarkers known only from later-evolving organisms. The largest class of these characteristically Phanerozoic biomarkers are the wide variety of polyterpenoid compounds synthesized by vascular plants. No fossils of plant terpenoids could be identified in the late Archean bitumens, despite specific investigation when potential signals of their presence were detected. In one instance, this investigation led to detection of an additional series of biomarkers.

MRM-GC-MS-MS analysis of a number of core extracts showed a small, partly resolved doublet peak in the $m / z 412>191$ reaction chromatogram for a compound eluting just before $C_{30}$ hopane at the time expected for oleanane, a diagnostic marker for angiosperms (indicated with an asterisk in Fig. 9A). Oleanane is abundant in the sedimentary record only from the late Cretaceous onwards
(Moldowan et al., 1994) so its presence in the Transvaal Supergroup bitumens would be clear evidence of contamination by younger hydrocarbons. Identification based solely on the $m / z 412>191$ transition is insufficient, however, since oleanane is known to co-elute with other triterpenoids including members of the 25-norhopane series, as shown by Dzou et al. (1999). 25-norhopanes are generally considered to be the products of microbial degradation of hopanes (Volkman et al., 1983; Peters et al., 1996), and, owing to the removal of the C10 methyl group, give a strong $m / z 177$ fragment ion.

We thus re-examined the samples with the potential oleanane peak by measuring a series of $\mathrm{M}^{+}>177$ transitions, and compared the results with analysis of a biodegraded crude oil from the Llanos Basin of Colombia that contains abundant 25-norhopanes (Fig. 9A). Inspection of the trace for the $412>177$ transition revealed the presence of two peaks at the retention times of the $C_{30}$ 25 -norhomohopanes $(22 S+R)$. Note the inversion of the relative contributions of the $22 S$ and $22 R$ epimers between the $412>191$ and $412>177$ traces, observed in both the oil and core samples. Examination of other MRM transitions indicated the presence of $\mathrm{C}_{29}$ 25-norhopane and $\mathrm{C}_{28}$ 25,30-dinorhopane as well (Fig. 9B) confirming the presence of a pseudohomologous series of these biomarkers. In fact, the series of 25-norhopanes appeared to be 
(A)

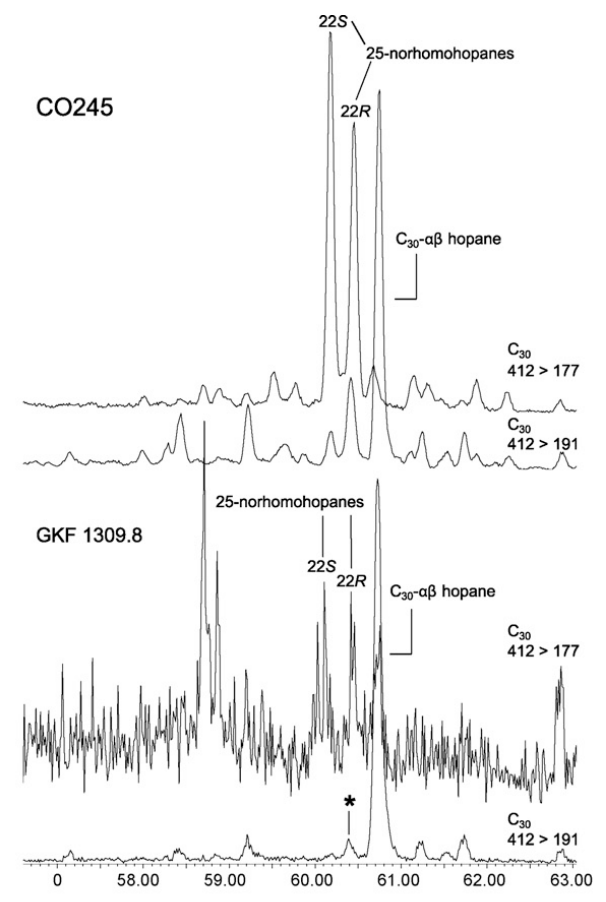

(B)

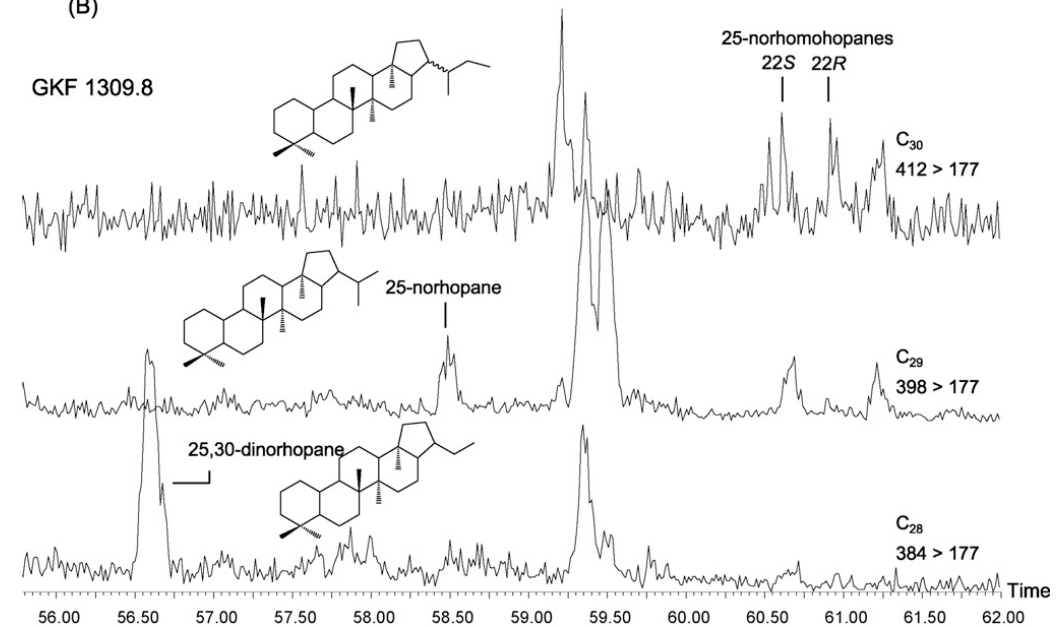

Fig. 9. (A) MRM GC-MS-MS traces from analyses of an Archean core sample (lower) and a biodegraded oil sample from the Cretaceous-Miocene Llanos Basin, Colombia (upper). The peak indicated with a star, observed in a number of the late Archean bitumens, prompted further investigation (see Section 5.2). (B) MRM GC-MS-MS chromatograms of $\mathrm{M}^{+}>177$ transitions showing the presence of a 25-norhopane series.

present at a low level in all samples analyzed. We conclude that the small peak preceding the $C_{30} \alpha \beta$ hopane in the $412>191$ trace arises largely from the presence of 25 -nor- $\alpha \beta$-homohopane- $22 R$, rather than from oleanane. While the observation of the $\mathrm{M}^{+}>177$ series makes a 25-norhomohopane the favored candidate for the source of at least part of this signal, other terpenoids (including ring E-methylated 28-norhopanes and the $C$ ring-enlarged $C_{31}-22 S 14 a-$ homo-26-nor-17 $\alpha(\mathrm{H})$-hopane) are known to elute in this region under certain chomatographic conditions (Nytoft et al., 2002). At present, there is insufficient extract from our samples to conduct analyses that would definitively identify the compound or compounds constituting the peak in question. Besides this peak, no other potentially plant-derived signals have been identified in the late Archean bitumens.

The presence of 25-norhopanes superficially suggests that the Transvaal bitumens were subjected to some degree of biodegradation earlier in their geological history. Alternatively, the series of 25-norhopanes could reflect an original microbial source (Blanc and Connan, 1992; Chosson et al., 1992) with the series becoming enriched relative to the more common hopane series over time. 
In the present case, the origins of the 25-norhopanes cannot be ascribed; they may derive from original microbial input or from earlier in situ biodegradation or, possibly, they are somewhat more thermodynamically stable and resistant to cracking.

Beyond the absence of diagnostic markers of later-evolving organisms, the biomarker composition of the late Archean bitumens would be very unusual if detected in a Phanerozoic setting. For example, the Transvaal Supergroup bitumens have relatively large amounts of $\mathrm{C}_{28}$ steranes (30-40\% of total $\mathrm{C}_{27-29}$; Fig. 7), which are typically $<25 \%$ of the total in Paleozoic oils (Grantham and Wakefield, 1988; Knoll et al., 2007). Mesozoic and younger bitumens contain more $\mathrm{C}_{28}$ steranes, owing to the rise to ecological prominence of the chlorophyll c-containing phytoplankton (Knoll et al., 2007), but also usually contain plant markers (especially oleanane in Cretaceous and younger systems) and/or abundant dinosteranes, none of which were detected here. The high relative abundance 2-methylhopane, exceeding $10 \%$ of $C_{30}$ hopane in a number samples of various lithologies (Fig. 6A), is also a signature more typical of Precambrian deposits than Phanerozoic ones (Knoll et al., 2007). Taken together, the constellation of biomarkers seen in the Transvaal Supergroup bitumens is inconsistent with a source of Phanerozoic age. It is thus difficult - merely on compositional grounds - to construct a scenario by which the bitumen in the Transvaal strata could have migrated from a much younger source.

\subsection{Correlation between cores GKF and GKP}

An important aspect of the Agouron Griqualand drilling project, unique among Precambian Earth-history drilling efforts, is the recovery of long intervals of correlated stratigraphy from geographically distant parts of a single depositional system. Cores GKF and GKP, drilled $24 \mathrm{~km}$ apart, are well correlated over much of their lengths, representing ca. 200 million years of deposition of the Transvaal Supergroup. The basis for these correlations and detailed discussion of the depositional environment are presented elsewhere (Schröder et al., 2006; Sumner et al., unpublished). These correlated sections provide a singular opportunity to look for chemostratigraphic patterns in biomarker contents. The late Archean bitumens, while of a uniformly high maturity, do exhibit enough compositional heterogeneity that variations along the stratigraphy are potentially informative.

When the molecular composition of bitumens from GKF and GKP are compared down-core, the stratigraphic correspondence in biomarker variation is striking. The values of many biomarker parameters from samples correlated on sedimentological grounds plot nearly on top of one another, even as they vary between strata by amounts significantly greater than the analytical precision (Figs. 2, 5 and 8). The correspondence is particularly notable in the upper sections of the core over the Kuruman, Klein Naute, and uppermost Nauga formations, where $\beta / \alpha$ dimethylnaphthalene (Fig. 2C), Ts/Tm (Fig. 5A), 28,30-dinorhopane/ $C_{30}$ hopane (Fig. 5B), $\mathrm{C}_{30}$ 30-norhopane/hopane (Fig. 5F) and each of $\mathrm{C}_{27}-\mathrm{C}_{29}$ diasterane/regular sterane (Fig. $8 \mathrm{~A}-\mathrm{C}$ ) ratios all track exceptionally closely over more than $100 \mathrm{~m}$ of stratigraphy. The two cores sample similar deepwater depositional environments through this interval, and so likely had similar organic matter inputs, and the lithologies of the host rocks are similar, creating similar physicochemical environments for diagenesis. While one expects similar biomarker signatures from similar depositional settings with similar organicmatter sources, finding such correspondence preserved over 2.5 billion years in trace quantities of molecular fossils is remarkable.

In the lower portion of the cores, from the Nauga Formation down to the lowermost correlated samples in the Monteville, there is more inter-core variation and the bitumen compositions of the two cores diverge in certain respects. The lower parts of the cores represent less similar depositional settings, with GKF comprising relatively more marginal and GKP more basinal facies. However, nowhere do we detect gross compositional differences that suggest radically different sources of organic matter to the sites of the two cores, and a number of biomarker ratios (e.g., Fig. 5C-E, Fig. 8E) do correlate, albeit within a small range of variation comparable to the analytical precision. Some parameters (e.g., Figs. 2C and D, 5F, and 8D and F) show apparently uncorrelated variation around a constant mean. A few consistent differences, however, can be discerned between the lower sections of GKF and GKP, and these may be related to their relative positions on the platform margin.

Below the upper Nauga, GKP has lower diasterane/regular sterane ratios (Fig. $8 \mathrm{~A}-\mathrm{C}$ ), higher relative amounts of 28,30 dinorhopane (Fig. 5B) and lower Ts/Tm ratios (Fig. 5A) than GKF. $C_{29}$ Ts/hopane ratios, analogous to Ts/Tm, show similar behavior (data not shown). This is in spite of similar patterns of other hopanes $\left(C_{29}\right.$ and $\mathrm{C}_{30}$ moretane/hopane; Fig. $5 \mathrm{C}$ and $\left.\mathrm{E}\right)$ and steranes $(\alpha \beta \beta / \alpha \alpha \alpha$ and 20S/20R; Fig. 8D and E) in a majority of samples. Several compositional differences are also apparent: GKP has relatively more steranes (at the expense of cheilanthanes; Fig. 4), higher relative $\mathrm{C}_{28}$ sterane content (Fig. 7) and less $2 \alpha$-methylhopane (Fig. $6 \mathrm{~A}$ ) than GKF and less variable ratios of some cheilanthanes such as $\mathrm{C}_{23} / \mathrm{C}_{20}$ (Fig. $6 \mathrm{C}$ ). These differences, while not large, suggest that there may have been a cross-platform gradient in microbial community composition and, more tentatively, that sedimentary redox conditions at the location of GKP may have been more reducing, up until partway through the deposition of the Nauga Formation.

The consistency down-core of the differences in $\mathrm{C}_{27}-\mathrm{C}_{29}$ diasterane and 28,30-DNH (Fig. 5B) contents between GKF and GKP are particularly striking and, perhaps, record a subtle sedimentary or environmental distinction that decreased over the time of platform deposition. The biomarker $28,30-\mathrm{DNH}$ is typically abundant in highly reducing environments (Grantham et al., 1980; Peters et al., 2005). However, in the case of immature Miocene California Borderland sediments, where the whole sedimentary section records a reducing paleoenvironment, the abundance of 28,30 -DNH relative to the total hopane content is inversely correlated with the content of clay minerals (Brincat and Abbott, 2001) whose presence should promote the formation of diasteranes. In accord with this pattern, below the uppermost Nauga Formation the relative contents of diasteranes and Ts in GKF increase compared to the correlated intervals in GKP while $28,30-\mathrm{DNH}$ is relatively more abundant in GKP.

The observed chemostratigraphic relationships between correlated samples in GKF and GKP provide compelling evidence for syngeneity. The bed-to-bed correlation is especially close in the deepwater facies in the upper $\sim 350 \mathrm{~m}$ of each core, and the lower portions show compositional differences that may reflect their relative positions on the Campbellrand Platform margin. These relationships can only arise when the organic contents of rocks are depositionally controlled and not subsequently overprinted by hydrocarbon migration or anthropogenic contamination.

\subsection{Composition of bitumens I and II}

To further test the syngeneity of the Archean bitumens, seven samples from core GKF (which had already been solvent extracted to prepare bitumen $\mathrm{I}$ ) were treated with acids ( $\mathrm{HCl}$ and $\mathrm{HF}$ ) to dissolve and disaggregate much of the mineral matter and were then re-extracted to yield a fraction termed bitumen II (see Section 3.5). In contrast to bitumen I (the bitumen extractable from whole-rock powder), bitumen II represents material that was not solvent-accessible in situ or even after mechanical crushing. While its precise microscale distribution is unclear, bitumen II is certainly more closely associated with the kerogen and mineral matrices of the rock than is bitumen I. Additionally, since contaminants 
(anthropogenic or migrated) are necessarily mobile, they are more likely to be found in the more solvent-accessible bitumen I. Hence, where bitumen I and II extracts from the same rock show similar compositions, they are likely derived from syn-depositional organic matter, with the caveat that bitumen II may be enriched in compounds produced by mineral-surface-catalyzed diagenetic reactions and derived from maturing kerogen.

The compositions of bitumens I and II from the GKF core samples appear similar in many respects. Yields of the two bitumens are in the same tens-to-hundreds of ppb range (Table 1); there is not a very much larger quantity of solvent-extractable bitumen liberated by acid demineralization. The gross molecular composition of the two bitumens is the same, dominated by low-molecularweight saturated and aromatic hydrocarbons (Fig. 1). Bitumen II contains cyclic terpenoid biomarkers at slightly higher concentrations than bitumen I (Table 1). The carbon-number distributions of steranes (Fig. 7), hopanes (Fig. 5D) and cheilanthanes (Fig. 6C) fully overlap between bitumens I and II, and moretanes and norhopanes show very similar relative abundances (Fig. 5B-D and F), consistent with the biomarkers in the two pools of organic matter deriving from the same source. Crucially, the thermal maturities of the two bitumens inferred from the biomarker isomer distributions are equivalent-an essential and characteristic quality of syngenetic organic matter. Evidence for equivalent thermal maturities includes similar values in bitumen I and II extracts for: hopanoid Ts/Tm ratios (Fig. 5A) and extent of $\beta \alpha$-to- $\alpha \beta$ isomerization around C17 and C21 for $\mathrm{C}_{29}$ and $\mathrm{C}_{30}$ homologues (moretane/hopane; Fig. 5C and $\mathrm{E}$ ), as well as ratios of $\mathrm{C}_{27-29} \alpha \beta \beta$ to $\alpha \alpha \alpha$ steranes (Fig. 8D) and epimerizaton around $\mathrm{C} 20$ (Fig. 8E). The down-core variability for several of these indices tracks closely between bitumens I and II as well; this is good evidence that organic matter source and depositional environment, rather than later overprinting by contaminants, controls differences in bitumen composition between strata. Taken together, the consistent similarities in molecular composition, thermal maturity and patterns of stratigraphic variation between bitumens I and II are strong evidence for syngeneity.

Furthermore, the compositional differences between the two bitumen extracts are in fact of the kind one might expect in comparing pools of organic matter that have the same source, but are more- versus less-closely matrix-associated in the same rock. One example is the higher diasterane/regular sterane ratios in bitumen II than bitumen I, especially in the upper $\sim 1000 \mathrm{~m}$ of core GKF, above the Monteville formation (Fig. 8A-C). Diasteranes are thought to be generated from sterenes by concerted methyl-rearrangement reactions that are catalyzed by acidic sites on clay minerals such as montmorillonite and illite (Rubinstein et al., 1975; Sieskind et al., 1979; van Kaam-Peters et al., 1998). Hence, the more closely mineral-associated bitumen II, having greater access to clays in the rock matrix, would plausibly be enriched in diasteranes relative to bitumen I. The two samples from the Monteville formation, however, show nearly identical diasterane/regular sterane ratios. This may be related to a distinct clay mineralogy or style of organic sedimentation in the Monteville; in any event, it points toward depositional control of organic matter composition and diagenesis. As noted above in comparing between GKF and GKP, these downcore patterns are consistent in bitumens I and II between $C_{27}, C_{28}$ and $\mathrm{C}_{29}$ (dia)steranes. Both similarities and contrasts in molecular composition between bitumens I and II indicate the hydrocarbons' derivation from syn-depositional organic matter.

\subsection{Contrasting composition of overlying Dwyka diamictite}

One unintended - but unexpectedly useful - aspect of the Griqualand drilling was the intersection (in both the GKF and GKP boreholes) of the Dwyka Formation, a Permo-Carboniferous diamictite that was deposited over a large portion of southern
Africa (Visser, 1989). This Phanerozoic glacial deposit overlies the late Archean Transvaal sedimentary sequence above a 2-billionyear unconformity with the Kuruman Formation. While not the target of the Agouron drilling project, the Dwyka was intersected in the two cores to depths approaching $200 \mathrm{~m}$, well below the modern weathering horizon. The recovery of unweathered Phanerozoic sedimentary rock in the same borehole provides another opportunity to test the syngeneity of the Archean bitumens, since the organic matter in the Dwyka has very different sources and thermal history from the Transvaal sediments, yet the core samples were all exposed to the same drilling equipment, handling and storage conditions, and chemical analyses. Pervasive contamination during drilling and analysis would obliterate the contrast that should be apparent across the Dwyka/Kuruman unconformity. On the other hand, if the very different character of the Dwyka organic matter were observed in analyses of the Griqualand cores, this would be strong evidence that anthropogenic contamination did not overprint syngenetic organic signatures in the core samples.

By nearly every metric of molecular composition, the Dwyka samples from the Griqualand cores are, in fact, distinct from the late Archean rocks directly below. Both saturate and aromatic hydrocarbon fractions show differences, including higher pristane/phytane and lower phytane $/ n-\mathrm{C}_{18}$ ratios (Fig. $2 \mathrm{~A}$ and $\mathrm{B}$ ), strong even-overodd alkane preferences in the GKF Dwyka samples, and much higher contents of thermodynamically disfavored $\alpha$-substituted dimethylnaphthalenes and methylphenanthrenes (Fig. 2C and D). Bitumen from the Dwyka also contains less thermally stable molecules, such as anthracene (Smith et al., 1995), that are not detected in the Transvaal.

The contrast is especially clear in cyclic biomarker contents, which uniformly indicate a significant difference in source and thermal history across the unconformity. The biomarkers in two Dwyka samples are strongly hopane-dominated, while the third is less so but still outside the range of the Archean samples (Fig. 4). Organic matter from the Dwyka appears rather heterogeneous, which is expected in a clastic glaciogenic deposit. Consistent with this depositional setting, the studied core samples contained numerous large, irregularly shaped clasts with dissimilar lithologies, indicating multiple sedimentary sources. The sterane carbon-number distributions of the Archean samples define a small field in Fig. 7, while those of the Dwyka vary much more widely, with the GKP sample plotting just on the edge of the Archean field and the two GKF samples completely distinct from it. Their relatively low content of $\mathrm{C}_{28}$ steranes is also a typically Paleozoic signature. Even more striking are the sharp contrasts in biomarker maturity parameters across as little as $15 \mathrm{~m}$ of stratigraphy, shown in Figs. 5 and 8. By every measure, the organic matter in the Dwyka is far less thermally mature than the Archean sedimentary rocks just a few meters below. Evidence includes: much lower Ts/Tm and higher $\beta \alpha / \alpha \beta$ hopane ratios (Fig. $5 \mathrm{~A}, \mathrm{C}$ and D), lower diasterane/regular sterane and lower sterane $\alpha \beta \beta / \alpha \alpha \alpha$ ratios (Fig. $8 \mathrm{~A}-\mathrm{D}$ ), and the detection in the Dwyka of thermodynamically less-stable isomers, such as $17 \beta$-hopanes, which are absent from the Transvaal rocks. Carbon-number distributions of hopanes (Fig. 5D) and cheilanthanes (Fig. 6C) are also distinct.

Clearly, the sharp compositional and maturity contrasts between the Dwyka and the underlying late Archean rocks are preserved in both cores. These contrasts constitute an original, unconformity-related relationship and the process of recovering and analyzing the core material has not compromised this signal. The distinct and immature composition of the Dwyka organic matter also precludes it from being the source of the bitumens in the Transvaal strata. The detection of this demonstrably syngenetic characteristic of the organic matter in both GKF and GKP cores provides additional strong evidence that the biomarkers in the late Archean bitumens are not contaminants. 


\section{Discussion}

6.1. Late Archean molecular fossils and the antiquity of microbial diversity

Taken together, we find the evidence for the syngenetic nature of the Transvaal bitumens (Section 5) to be compelling. As they are syngenetic, and thus late Archean in age, the bitumens constitute a molecular fossil record of microbial activity during the deposition of the Transvaal Supergroup. The Transvaal sediments, like many Precambrian sequences, bear a number of signs of microbial involvement in their deposition. Numerous sedimentary textures can be attributed to microbial activity (Schröder et al., this volume) and large amounts of organic matter with textures and isotopic compositions consistent with a biogenic origin are present (Fischer et al., this volume), so there is no doubt that vigorous marine ecosystems were functioning widely by the late Archean, if not much earlier. A molecular fossil record aids our understanding of this period by contributing information about the specific identities and physiologies of the microorganisms active in late Archean oceans.

The late Archean molecular fossil record from this study and others of early Precambrian rocks (Brocks et al., 2003b; Eigenbrode, 2004; Dutkiewicz et al., 2006) is the most specific evidence at present for the antiquity of the three principal Domains of cellular life: the Bacteria, Eukarya and Archaea. The hopanoid fossils detected throughout the Griqualand cores are bacterial in origin; in extant organisms extended $C_{35}$ hopanoids are exclusive to bacteria (Rohmer et al., 1984), and it is virtually certain that the bulk of other hopanoids in late Archean bitumens are bacterial (rather than eukaryotic) as well. The fossil steroids detected in late Archean rocks have been more controversial, and their interpretation as molecular fossils is discussed in detail below (Section 6.2). We consider the suite of steranes present in the Transvaal bitumens from these cores to be strong evidence for the presence of eukaryotes in late Archean coastal oceans. In contrast to another report (Ventura et al., 2007), molecular fossils specifically diagnostic for the Archaea, such as mid-chain cyclized or head-to-head-linked > $\mathrm{C}_{20}$ isoprenoids, were not detected. These extended isoprenoids, even if they were initially present, are unlikely to have survived the long thermal and diagenetic history that the Transvaal Supergroup has experienced. The finding of characteristic fossils of the other two Domains, however, makes the presence of at least stem-group archaea by the late Archean a likely scenario. Indirect geochemical evidence for widespread methanogenesis even earlier in Earth history (Hayes, 1983) is further support for Archean archaea. The consensus emerging from multiple, independent molecular fossil studies of the late Archean rock record supports the presence of members of all three Domains by at least 2.7 billion years ago.

Below Domain level, few molecular fossils have been detected that carry taxonomic specificity. Possible exceptions include the A-ring methylated hopanes where $2 \alpha$-methylhopanes are provisionally indicative of cyanobacterial primary productivity while the $3 \beta$-methylhopanes are likely proteobacterial and possibly from methanotrophic $\gamma$-proteobacteria. Gammacerane (fossil tetrahymanol), while known to originate from some ciliates, is also probably proteobacterial.

\subsection{Interpretation of late Archean steranes}

Perhaps the most contentious claim to arise from molecular fossil analysis of late Archean organic matter has been the interpretation of steranes as indicative of the presence of eukaryotes in marine environments as far back as $2.7 \mathrm{Ga}$ (Brocks et al., 1999, 2003b). These molecular fossils are almost a billion years older than the earliest convincingly eukaryotic body fossils (Knoll et al., 2007) although a recent report offers evidence for an earlier appearance (Zang, 2007). The presence of steranes in Archean sediments suggests (though does not require) that cardinal events in eukaryotic evolution took place very early in Earth history. Furthermore, since steroid biosynthesis requires molecular oxygen, the presence of fossil steranes in late Archean rocks implies that $\mathrm{O}_{2}$ was available in at least some aquatic ecosystems several hundred million years before the first geological and geochemical evidence for persistent atmospheric $\mathrm{O}_{2}$, ca. $2.4 \mathrm{Ga}$ (Holland, 2006; Summons et al., 2006). Since the only environmentally significant source of $\mathrm{O}_{2}$ is oxygenic photosynthesis, fossil steranes also imply that oxygenic photosynthesis arose long before the Paleoproterozoic appearance of atmospheric $\mathrm{O}_{2}$.

This set of interpretations of Archean steranes has been questioned on several grounds. First, the syngeneity of the sterane fossils has been doubted. The evidence presented herein (Section 5) for the syngeneity of Archean molecular fossils, including steranes, is, in our opinion, the strongest yet. We have demonstrated stratigraphic and compositional relationships in cleanly recovered drill-core samples that are inconsistent with anthropogenic or geologically recent contamination. Second, some (Raymond and Blankenship, 2004; Kopp et al., 2005) have proposed that steroid biosynthesis might have been conducted by an ancestral, anaerobic pathway that was subsequently "replaced" by the modern, $\mathrm{O}_{2}$-dependent route. These proposals have been addressed in detail by Summons et al. (2006); we will not repeat that discussion here, except to say that we do not consider the arguments for a hypothetical anaerobic steroid biosynthetic pathway to be convincing. Evidence for the antiquity of the known aerobic pathway is robust, and its operation during the late Archean remains the most probable and parsimonious explanation for the presence of steranes in rocks of that age.

A third objection has been that a very few bacteria do produce steroids de novo (Bird et al., 1971; Bode et al., 2003; Pearson et al., 2003) and so the steranes in Archean rocks could have a prokaryotic source (Cavalier-Smith, 2006). Aspects of the phylogenetic distribution of steroid biosynthesis among the bacteria and the genes involved are discussed by Summons et al. (2006). It has been noted (Brocks and Summons, 2003; Volkman, 2005) that no prokaryote is known to be able to produce the $\mathrm{C}_{28}$ and $\mathrm{C}_{29}$ 24-alkyl steroid structures detected in abundance in the late Archean rocks. Additionally, the majority of prokaryotic steroids are 4-methyl and 4,4-dimethyl, resulting from the typically abbreviated steroid biosynthetic pathways of these few bacteria. A dominance of 4-methyl steroids was found in the Mesoproterozic $(1.64 \mathrm{Ga})$ Barney Creek Formation, likely deposited under strongly euxinic conditions and reflecting minimal eukaryotic activity in highly sulfidic waters (Brocks et al., 2005). As noted above, 4-methyl steranes were not observed in the Griqualand core material, despite efforts taken specifically to detect them, suggesting that 4-desmethyl (i.e., more typically eukaryotic) steroids were a much larger component of the sedimentary organic matter.

To these biosynthetic considerations, analysis of the two Agouron cores adds an ecological dimension: steranes with all the hallmarks of syngenetic molecular fossils are found consistently over more than $2500 \mathrm{~m}$ of core that stratigraphically represent depositional environments from shallow marine to deep slope. The source organisms for the fossil steranes must therefore have had a broad ecological distribution in the coastal ocean and have contributed substantially to organic matter input to sediments over such a range of conditions. The source organisms must also have maintained that ecological distribution and organic matter production throughout the $\sim 200$ million years of geological history represented in the cores. None of the known bacterial steroid producers has such an ecological distribution or prominence in the fossil record. Arguments for a prokaryotic origin for fossil steranes 
must account for both biosynthetic and ecological discrepancies between the established biology of steroid-producing bacteria and the fossil record, or else postulate extinct biochemistries and ecologies for putative marine steroid producing bacteria for which all other evidence has been lost.

The range of sterane skeletons observed suggests that a number of different steroid synthesis pathways were operative in this coastal marine environment, at the very least with regard to sidechain alkylation. The carbon-number distributions $\left(\mathrm{C}_{27} / \mathrm{C}_{28} / \mathrm{C}_{29}\right.$; Fig. 7) of steranes in the late Archean bitumens are suggestive of the simultaneous presence of multiple protistan sources. In the absence of a more continuous Precambrian body and molecular fossil record, it is premature to interpret these carbon-number patterns in terms of the predominance of particular protistan groups, as has been done successfully over much of the Phanerozoic (Grantham and Wakefield, 1988; McCaffrey et al., 1994; Holba et al., 1998; Knoll et al., 2007). We cannot say definitively whether these eukaryotes were photo- or heterotrophic - which would put a constraint on the timing of primary plastid endosymbiosis - as steroid synthesis appears to have been present in the last common ancestor of all extant eukaryotes (Summons et al., 2006). Whatever the metabolic modes of their producers, the finding of steranes throughout the Transvaal Supergroup strata attests to a broad ecological distribution and persistence of the source organisms. No sterane fossils are found that cannot be attributed to recognized extant biosynthetic pathways, nor are there 'orphan' molecules indicative of extinct steroid biochemistry. Remarkably, much of steroid biosynthesis - at least with regard to ring-system formation and side-chain modification - appears to have been operative by at least $2.6 \mathrm{Ga}$.

It seems unlikely that the relative amounts of biomarker classes - such as sterane/hopane ratios - could be used to infer relative contributions to sedimentary organic matter or community abundance of groups of organisms, such as eukaryotes versus bacteria, in these ancient and overmature bitumens. On organic geochemical grounds, not enough is known about the relative stabilities of different biomarker structures once such high degrees of thermal maturity and extreme ages are reached, and small differences will become magnified over very long alteration histories. On biosynthetic grounds, while the vast majority of free-living eukaryotes produce steroids, only an unquantified minority of bacteria make hopanoids, and this proportion is almost certainly not constant over different environmental conditions or Earth history. The steranes detected in the Transvaal Supergroup bitumens indicate the presence of eukaryotes, but do not necessarily assign to them any specific ecological prominence.

In summation, the steranes detected throughout the Griqualand cores have all the hallmarks of syngenetic molecular fossils of eukaryotes. Their isomeric distributions are fully consistent with the high thermal maturity expected of this ancient organic matter. Their stratigraphic distribution, particularly their correlation between two separate boreholes and sharp maturity contrast with overlying rocks, is a geochemical signature difficult to reconcile with scenarios of contamination by younger migrated hydrocarbons or during drilling and analysis. The sterane structures detected in the Archean bitumens are typical of those produced by eukaryotes, unknown (or at best highly atypical) among prokaryotes, and occur over a range of paleoenvironments inconsistent with present knowledge of the ecology of steroid-producing bacteria. Current understanding of the chemistry of steroid biosynthesis suggests that molecular oxygen is absolutely required, especially for production of the 4,14-desmethyl steranes that dominate the late Archean (and most younger) bitumens (Summons et al., 2006). Hence, this molecular fossil record provides simultaneous evidence for the three Domains of life, oxygenic photosynthesis and the anabolic use of $\mathrm{O}_{2}$.

\section{Conclusions}

The Agouron Griqualand drilling project has recovered relatively well-preserved organic-rich strata from the Transvaal Supergroup, and has done so without overprinting syngenetic molecular fossil signatures with contaminant hydrocarbons. These drill cores have enabled stratigraphic comparisons of biomarker contents that support their syngenetic nature. The suite of molecular fossils identified in the late Archean bitumens includes hopanes attributable to bacteria, potentially including cyanobacteria and methanotrophs, and steranes almost certainly of eukaryotic origin. This work is in accord with other reports (Brocks, 2001; Brocks et al., 2003b; Eigenbrode, 2004; Dutkiewicz et al., 2006) of early Precambrian molecular fossils and extends those findings to the Kaapvaal craton.

What, we might ask, would constitute yet stronger evidence for microbial (including eukaryotic) diversity in the Archean? There is always the hope that some sort of Lagerstätte might yet be found, a trove of spectacularly well preserved fossils - morphological or molecular - of Archean microbes. Hope of such finds rests on continued exploration of the Archean rock record. The Agouron Griqualand drilling project and other Earth-history drilling efforts have highlighted the value of extending such exploration to the subsurface. Beyond the perhaps faint hope of finding a pocket of lightly altered, thermally immature Archean sediment, drilling projects recover long stretches of continuous stratigraphy, unavailable in outcrop, that allow multiproxy geochemical records such as the ones presented in this volume to be constructed from material whose provenance is controlled.

The Archean paleobiological record, incomplete and problematic as it is, is beginning to yield a picture of the early phases of evolutionary history. An emerging finding is that much of cellular biochemistry, including diverse metabolic pathways of electron transport, photosynthesis, carbon fixation, nutrient assimilation and lipid biosynthesis, appears to have arisen very early in Earth history. The Archean might be viewed as the cardinal epoch of biochemical innovation, resulting in most of the molecular toolkit on which a wide spectrum of biological form and function would eventually be based. This possibility makes understanding of the Archean surface environment - the physicochemical context in which this innovation took place - all the more essential.

\section{Acknowledgements}

We thank the Agouron Institute and members of the Griqualand Drilling Project team for provision of the cores. Alex Birch oversaw the clean drilling operation and Francis McDonald provided field support. We are especially grateful to Nic Beukes and Joe Kirschvink for discussions of geologic context and for sampling logistics, and to Gordon Love for discussions of biomarker analyses. Funding support for this work came from the NASA Exobiology Program (Grants NNG05GN62G and NNG04GJ13G), NSF (EAR0418619) and the Agouron Institute. JRW receives support through an NDSEG Fellowship from the Office of Naval Research and a Graduate Research Fellowship from the National Science Foundation. John Zumberge of GeoMark Research provided crude oil samples used as reference materials. Carolyn Colonero provided laboratory assistance, particularly in the maintenance and operation of the mass spectrometers. Thanks to Andrew Knoll for helpful comments, and to reviewers Simon George and Roger Buick for suggestions that improved the manuscript.

\section{References}

Allwood, A.C., Walter, M.R., Kamber, B.S., Marshall, C.P., Burch, I.W., 2006. Stromatolite reef from the Early Archaean era of Australia. Nature 441, 714-718. 
Altermann, W., Kazmierczak, J., Oren, A., Wright, D.T., 2006. Cyanobacterial calcification and its rock-building potential during 3.5 billion years of Earth history. Geobiology 4, 147-166.

Armstrong, R.A., Compston, W., Retief, E.A., Williams, I.S., Welke, H.J., 1991. Zircon ion microprobe studies bearing on the age and evolution of the Witwatersrand triad. Precambrian Research 53, 243-266.

Barton, E.S., Altermann, W., Williams, I.S., Smith, C.B., 1994. U-Pb zircon age for a tuff in the Campbell Group, Griqualand West Sequence, South Africa: implications for Early Proterozoic rock accumulation rates. Geology 22, 343-346.

Beukes, N.J., 1987. Facies relations, depositional environments and diagenesis in a major Early Proterozoic stromatolitic carbonate platform to basin sequence, Campbellrand Subgroup, Transvaal Supergroup, Southern Africa. Sedimentary Geology 54, 1-46.

Beukes, N.J., Smit, C.A., 1987. New evidence for thrusting in Griqualand West, South Africa: Implications for stratigraphy and the age of red beds. South African Journal of Geology 90, 378-394.

Beukes, N.J., Evans, D.A.D., Grotzinger, J.P., Kirschvink, J.L., Knoll, A.H., Sumner, D.Y., 2004. Multidisciplinary study of the precambrian biosphere and surficial oxygenation, Kaapvaal Craton, South Africa: the Agouron Cores. International Journal of Astrobiology 3 (Suppl. 1), 15.

Bird, C.W., Lynch, J.M., Pirt, F.J., et al., 1971. Steroids and squalene in Methylococcus capsulatus grown on methane. Nature 230, 473-474.

Blanc, P., Connan, J., 1992. Origin and occurrence of 25-norhopanes: a statistical study. Organic Geochemistry $18,813-828$.

Bode, H.B., Zeggel, B., Silakowski, B., Wenzel, S.C., Reichenbach, H., Muller, R., 2003. Steroid biosynthesis in prokaryotes: identification of myxobacterial steroids and cloning of the first bacterial 2,3(S)-oxidosqualene cyclase from the myxobacterium Stigmatella aurantiaca. Molecular Microbiology 47, 471-481.

Bosak, T., Greene, S.E., Newman, D.K., 2007. A likely role for anoxygenic photosynthetic microbes in the formation of ancient stromatolites Geobiology 5, 119-126.

Bravo, J.M., Perzl, M., Hartner, T., Kannenberg, E.L., Rohmer, M., 2001. Novel methylated triterpenoids of the gammacerane series from the nitrogen-fixing bacterium Bradyrhizobium japonicum USDA 110. European Journal of Biochemistry 268, 1323-1331.

Brincat, D., Abbott, G.D., 2001. Some aspects of the molecular biogeochemistry of laminated and massive rocks from the Naples Beach Section (Santa BarbaraVentura Basin). In: Isaacs, C.M., Rullkotter, J. (Eds.), The Monterey Formation: From Rocks to Molecules. Columbia University Press, New York, pp. 140-149.

Brocks, J.J., 2001. Molecular fossils in Archean rocks. Ph.D. Thesis. Sydney University, Sydney, Australia.

Brocks, J.J., Summons, R.E., 2003. Sedimentary hydrocarbons, biomarkers for early life. In: Holland, H., Turekian, K.K. (Eds.), Treatise on Geochemistry. Pergamon, Oxford, pp. 63-115.

Brocks, J.J., Logan, G.A., Buick, R., Summons, R.E., 1999. Archean molecular fossils and the early rise of Eukaryotes. Science 285, 1033-1036.

Brocks, J.J., Buick, R., Logan, G.A., Summons, R.E., 2003a. Composition and syngeneity of molecular fossils from the 2.78 to 2.45 billion-year-old Mount Bruce Supergroup, Pilbara Craton, Western Australia Ceochimica et Cosmochimica Acta 67, 4289-4319.

Brocks, J.J., Buick, R., Summons, R.E., Logan, G.A., 2003b. A reconstruction of Archean biological diversity based on molecular fossils from the 2.78 to 2.45 billion-yearold Mount Bruce Supergroup, Hamersley Basin, Western Australia. Geochimica ol Mount Bruce Supergroup, Hameshimica Acta 67, 4321-4335.

Brocks, J.J., Love, G.D., Summons, R.E., Knoll, A.H., Logan, G.A., Bowden, S.A., 2005. Biomarker evidence for green and purple sulphur bacteria in a stratified Palaeoproterozoic sea. Nature 437, 866-870.

Brocks, J.J., Grosjean, E., Logan, G.A., 2008. Assessing biomarker syngeneity using branched alkanes with quaternary carbon (BAQCs) and other plastic contaminants. Geochimica et Cosmochimica Acta 72, 871-888.

uick, R., 1992. The antiquity of oxygenic photosynthesis: evidence from stromatolites in sulphate-deficient Archaean lakes. Science 255, 74-77.

Button, A., 1973. The stratigraphic history of the Malmani dolomite in the eastern and north-eastern Transvaal. Transactions of the Geological Society of South Africa $76,229-247$

Cavalier-Smith, T., 2006. Rooting the tree of life by transition analyses. Biology Direct 1, doi:10.1186/1745-6150-1-19.

Chosson, P., Connan, J., Dessort, D., Lanau, C., 1992. In vitro biodegradation of steranes and terpanes: A clue to understanding geological situations. In: Moldowan, J.M., Albrecht, P., Philp, R.P. (Eds.), Biological Markers in Sediments and Petroleum. Prentice Hall, Englewood Cliffs, NJ, pp. 320-349.

Clay, A.N., 1986. The stratigraphy of the Malmani Dolomite Subgroup in the Carletonville area, Transvaal: genetic implications for lead-zinc mineralization. In: Anhaeusser, C.R., Maske, S.(Eds.), Mineral Deposits of Southern Africa. Geological Society of South Africa, Johannesburg, pp. 853-860.

Cloud, P., 1973. Paleoecological significance of the banded iron-formation. Economic Geology 68, 1135-1143.

Cloud, P., Licari, G.R., 1968. Microbiotas of the banded iron formations. PNAS 61, 779-786.

Duane, M.J., Kruger, F.J., 1991. Geochronological evidence for tectonically driven brine migration during the early Proterozoic Kheis Orogeny of southern Africa. Geophysical Research Letters 18, 975-978.

Duane, M.J., Kruger, F.J., Roberts, P.J., Smith, G.B., 1991. Pb and Sr isotope and origin of Proterozoic base metal (fluorite) and gold deposits, Transvaal Sequence, South Africa. Economic Geology 86, 1491-1505.

Duane, M.J., Kruger, F.J., Turner, A.M., Whitelaw, H.T., Coetzee, H., Verhagen, B.T., 2004. The timing and isotopic character of regional hydrothermal alteration and asso- ciated epigenetic mineralization in the western sector of the Kaapvaal Craton (South Africa). Journal of African Earth Sciences 38, 461-476.

Dutkiewicz, A., Ridley, J., Buick, R., 2003a. Oil-bearing $\mathrm{CO}_{2}-\mathrm{CH}_{4}-\mathrm{H}_{2} \mathrm{O}$ fluid inclusions: oil survival since the Palaeoproterozoic after high temperature entrapment. Chemical Geology 194, 51-79.

Dutkiewicz, A., Volk, H., Ridley, J., George, S.C., 2003b. Biomarkers, brines, and oil in the Mesoproterozoic, Roper Superbasin, Australia. Geology 31 (11), 981-984.

Dutkiewicz, A., Volk, H., Ridley, J., George, S.C., 2004. Geochemistry of oil in fluid

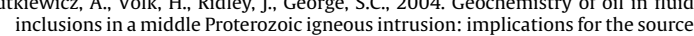
inclusions in a middle Proterozoic igneous intrusion: implications for the source
of hydrocarbons in crystalline rocks. Organic Geochemistry 35 (8), 937-957.

Dutkiewicz, A., Volk, H., George, S.C., Ridley, J., Buick, R., 2006. Biomarkers from Huronian oil-bearing fluid inclusions: an uncontaminated record of life before the Great Oxidation Event. Geology 34, 437-440.

Dutkiewicz, A., George, S.C., Mossman, D.J., Ridley, J., Volk, H., 2007. Oil and its biomarkers associated with the Palaeoproterozoic Oklo natural fission reactors, Gabon. Chemical Geology 244, 130-154.

Dzou, L., Holba, A.G., Ramon, J., Moldowan, J.M., Zinniker, D., 1999. Application of new diterpane biomarkers to source, biodegradation and mixing effects on Central Llanos Basin oils, Colombia. Organic Geochemistry 30, 515-534.

Eglinton, G., Scott, P.M., Besky, T., Burlingame, A.L., Calvin, M., 1964. Hydrocarbons from a one-billion-year-old sediment. Science 145, 263-264.

Eigenbrode, J.L., 2004. Late Archean Microbial Ecology: an integration of molecular isotopic and lithological studies. Ph.D Thesis. The Pennsylvania State University, State College, $\mathrm{PA}$.

Eigenbrode, J.L., 2008. Fossil lipids for life-detection: a case study from the early earth record. Space Science Reviews, doi:10.1007/s11214-007-9252-9.

Eigenbrode, J.L., Freeman, K.H., 2006. Late Archean rise of aerobic microbial ecosystems. PNAS 103 (43), 15759-15764.

Eigenbrode, J.L., Freeman, K.H., Summons, R.E., 2008. Methylhopane biomarker hydrocarbons in Hamersley Province sediments provide evidence for Neoarchean aerobiosis. Earth and Planetary Science Letters 273, 323-331.

Fischer, W.W., Pearson, A., 2007. Hypotheses for the origin and early evolution of triterpenoid cyclases. Geobiology 5, 19-34.

George, S.C., Volk, H., Dutkiewicz, A., Ridley, J., Buick, R., 2008. Preservation of hydrocarbons and biomarkers in oil trapped inside fluid inclusions for $>2$ billion years. Geochimica et Cosmochimica Acta 72 , 844-870.

Grantham, P.J., Wakefield, L.L., 1988. Variations in the sterane carbon number distributions of marine source rock derived crude oils through geological time. Organic Geochemistry 12, 61-73.

Grantham, P.J., Posthuma, J., DeGroot, K., 1980. Variation and significance of the $C_{27}$ and $C_{28}$ triterpane content of a North Sea core and various North Sea crude oils. In: Douglas, A.G., Maxwell, J.R. (Eds.), Advances in Organic Geochemistry 1979. Pergamon Press, New York, pp. 29-38.

Grosjean, E., Logan, G.A., 2007. Incorporation of organic contaminants into geochemical samples and an assessment of potential sources: examples from Geoscience Australia marine survey S282. Organic Geochemistry 38, 853-869.

Grotzinger, J.P., Rothman, D.H., 1996. An abiotic model for stromatolite morphogenesis. Nature $383,423-425$

Harvey, H.R., McManus, G.B., 1991. Marine ciliates as a widespread source of tetrahymanol and hopan- $3 \beta$-ol in sediments. Geochimica et Cosmochimica Acta 55 , manol and 3390

Hayes, J.M., 1983. Geochemical evidence bearing on the origin of aerobiosis, a speculative hypothesis. In: Schopf, J.W. (Ed.), Earth's Earliest Biosphere: Its Origin and lative hypothesis. In: Schopf, J.W. (Ed.), Earth's Earliest Biosphere:
Evolution. Princeton University Press, Princeton, NJ, pp. 291-301.

Hofmann, H., Grey, K., Hickman, A., Thorpe, R., 1999. Origin of 3.45 Ga coniform stromatolites in Warrawoona Group, Western Australia. Geological Society of America Bulletin 111, 1256-1262.

Holba, A.G., Dzou, L.I.P., Masterson, W.D., Hughes, W.B., Huizinga, B.J., Singletary, M.S., Moldowan, J.M., Mello, M.R., Tegelaar, E., 1998. Application of 24-norcholestanes for constraining source age of petroleum. Organic Geochemistry 29, 1269-1283. Holland, H.D., 2006. The oxygenation of the atmosphere and oceans. Philosophical Transactions of the Royal Society B 361, 903-915.

House, C.H., Schopf, J.W., McKeegan, K.D., Coath, C.D., Harrison, T.M., Stetter, K.O. 2000. Carbon isotopic composition of individual Precambrian microfossils. Geology $28,707-710$

Kleemann, G., Poralla, K., Englert, G., Kjøsen, H., Liaaen-Jensen, S., Neunlist, S., Rohmer, M., 1990. Tetrahymanol from the phototrophic bacteriaum Rhodopseudomonas palustris: first report of a gammacerane triterpene from a prokaryote. Journal of General Microbiology 136, 2551-2553.

Knoll, A.H., 2003. Life on a young planet. Princeton University Press, Princeton, NJ.

Knoll, A.H., Summons, R.E., Waldbauer, J.R., Zumberge, J., 2007. The geological succession of primary producers in the oceans. In: Falkowski, P., Knoll, A.H. (Eds.), The Evolution of Primary Producers in the Sea. Academic Press, Boston, pp. 133163.

Kopp, R.E., Kirschvink, J.L., Hilburn, I.A., Nash, C.Z., 2005. The Paleoproterozoic snowball Earth: a climate disaster triggered by the evolution of oxygenic photoSnowball Earth: a climate disaster
synthesis. PNAS 102, 11131-11136.

Martini,J.E.J., 1976. The fluorite deposits in the Dolomite Series of the Marico District, Transvaal, South Africa. Economic Geology 71, 625-635.

McCaffrey, M.A., Moldowan, J.M., Lipton, P.A., Summons, R.E., Peters, K.E., Jeganathan, A., Watt, D.S., 1994. Paleoenvironmental implications of novel C 30 steranes in PreA., Watt, D.S., 1994. Paleoenvironmental implications of novel $C_{30}$ steranes in Pre-
cambrian to Cenozoic Age petroleum and bitumen. Geochimica et Cosmochimica Acta 58, 529-532.

Miyano, T., Beukes, N.J., 1984. Phase relations of stilpnomelane, ferri-annite, and riebeckite in very low-grade metamorphosed iron-formations. Transactions of the Geological Society of South Africa 87, 111-124. 
Moldowan, J.M., Dahl, J., Huizinga, B.J., Fago, F.J., Hickey, L.J., Peakman, T.M., Taylor, D.W., 1994. The molecular fossil record of oleanane and its relation to angiosperms. Science 265, 768-771.

Nytoft, H.P., Bojesen-Koefoed, J.A., Christiansen, F.G., Fowler, M.G., 2002. Oleanane or lupane? Reappraisal of the presence of oleanane in Cretaceous-Tertiary oils and sediments. Organic Geochemistry 33, 1225-1240.

Ourisson, G., Albrecht, P., 1992. Geohopanoids: the most abundant natural products on Earth? Accounts of Chemical Research 25, 298-402.

Ourisson, G., Rohmer, M., Poralla, K., 1987. Prokaryotic Hopanoids and other Polyterpenoid Sterol Surrogates. Annual Review of Microbiology 41, 301-333.

Pearson, A., Budin, M., Brocks, J.J., 2003. Phylogenetic and biochemical evidence for sterol synthesis in the bacterium Gemmata obscuriglobus. PNAS 100, $15352-15357$.

Peters, K.E., Moldowan, J.M., McCaffrey, M.A., Fago, F.J., 1996. Selective biodegradation of extended hopanes to 25-norhopanes in petroleum reservoirs: insights from molecular mechanics. Organic Geochemistry 24, 765-783.

Peters, K.E., Walters, C.C., Moldowan, J.M., 2005. The Biomarker Guide, Second Edition. Cambridge University Press, Cambridge, UK.

Rashby, S.E., Sessions, A.L., Summons, R.E., Newman, D.K., 2007. Biosynthesis of 2-methylbacteriohopanepolyols by an anoxygenic phototroph. PNAS 104, 15099-15104.

Raymond, J., Blankenship, R.E., 2004. Biosynthetic pathways, gene replacement and the antiquity of life. Geobiology 2, 199-203.

Rohmer, M., Bouvier-Nave, P., Ourisson, G., 1984. Distribution of Hopanoid Triterpenes in Prokaryotes. Journal of General Microbiology 130, 1137-1150.

Rubinstein, I. Sieskind, O. Albrecht, P. 1975. Rearranged steranes in a shale: occurrence and simulated formation. Journal of the Chemical Society, Perkin Transaction I, 1833-1836.

Schopf, J.W., 2006. Fossil evidence of Archaean life. Philosophical Transactions of the Royal Society B 361, 869-885.

Schopf, J.W., Walter, M.R., 1983. Archean microfossils-new evidence of ancient microbes. In: Schopf, J.W. (Ed.), Earth's earliest biosphere: Its origin and evomicrobes. In: Schopf, J.W. (Ed.), Earth's earliest biosphere: Its
lution. Princeton University Press, Princeton, NJ, pp. 214-239.

Schröder. S. Lacassi, U.P. Beukes, N.J. 2006. Stratigraphic and geochemical framework of the Agouron drill cores, Transvaal Supergroup (Neoarchean-Paleoproterozoic, South Africa). South African Journal of Geology 109, 23-54.

Shen, Y., Buick, R., 2004. The antiquity of microbial sulfate reduction. Earth Science Reviews 64, 243-272.

Shen, Y., Buick, R., Canfield, D.E., 2001. Isotopic evidence for microbial sulphate reduction in the early Archaean era. Nature 410, 77-81.

Sherman, L.S., Walbauer, J.R., Summons, R.E., 2007. Improved methods for isolating and validating indigenous biomarkers in Precambrian rocks. Organic Geochemistry $38,1987-2000$

Sieskind, O., Joly, G., Albrecht, P., 1979. Simulation of the geochemical transformation of sterols: superacid effects of clay minerals. Geochimica et Cosmochimica Acta 43, 1675-1679.

Sinninghe Damsté, J.S., Kenig, F., Koopmans, M.P., Koster, J., Schouten, S., Hayes, J.M., de Leeuw, J.W., 1995. Evidence for gammacerane as an indicator of water column de Leeuw, J.W., 1995. Evidence for gammacerane as an indicator of wa

Smith, J.W., George, S.C., Batts, B.D., 1995. The geosynthesis of alkylaromatics. Organic Geochemistry 23, 71-80.

Subroto, E.A., Alexander, R., Kagi, R.I., 1991. 30-Norhopanes: their occurrence in sediments and crude oils. Chemical Geology 93, 179-192.
Subroto, E.A., Alexander, R., Pranjoto, U., Kagi, R.I., 1992. The use of 30-norhopane series, a novel carbonate marker, in source rock to crude oil correlation in the North Sumatra basin. In: Proceedings of the Indonesian Petroleum Association 21st Annual Convention, pp. 145-163.

Summons, R.E., Jahnke, L.L., Hope, J.M., Logan, G.A., 1999. 2-Methylhopanoids as biomarkers for cyanobacterial oxygenic photosynthesis. Nature 400, 554-556.

Summons, R.E., Bradley, A.S., Jahnke, L.L., Waldbauer, J.R., 2006. Steroids, triterpenoids and molecular oxygen. Philosophical Transactions of the Royal Society B 361, 951-968.

Sumner, D.Y., Beukes, N.J., 2006. Sequence stratigraphic development of the Neoarchean Transvaal carbonate platform, Kaapvaal Craton, South Africa. South African Journal of Geology 109, 11-22.

Sumner, D.Y., Bowring, S.A., 1996. U-Pb geochronologic constraints on deposition of the Campbellrand Subgroup, Transvaal Supergroup, South Africa. Precambrian Research 79, 25-35.

Tyler, R., Tyler, N., 1996. Stratigraphic and structural controls on gold mineralization in the Pilgrim's Rest goldfield, eastern Transvaal, South Africa. Precambrian Research 79, 141-169.

van Kaam-Peters, H.M.E., Koster, J., va der Gaast, S.J., Dekker, M., de Leeuw, J.W., Sinninghe Damsté JS 1998. The effect of clay minerals on diasterane/sterane ratios. Geochimica et Cosmochimica Acta 62, 2923-2929.

Ventura, G.T., Kenig, F., Reddy, C.M., Schieber, J., Frysinger, G.S., Nelson, R.K., Dinel, E., Gaines, R.B., Schaeffer, P., 2007. Molecular evidence of Late Archean archaea and
the presence of a subsurface hydrothermal biosphere. PNAS 104, 14260-14265.

Visser. J.N.J.. 1989. The Permo-Carboniferous Dwyka Formation of Southern Africa: deposition by a predominantly subpolar marine ice sheet. Palaeogeography, deposition by a predominantly subpolar marine
Palaeoclimatology, Palaeoecology 70, 377-391.

Volk, H., Dutkiewicz, A., George, S.C., Ridley, J., 2003. Oil migration in the Middle Proterozoic Roper Superbasin, Australia: evidence from oil inclusions and their geochemistries. Journal of Geochemical Exploration 78-79, 437-441.

Volkman. J.K. 2005. Sterols and other triterpenoids: source specificity and evolution of biosynthetic pathways. Organic Geochemistry 36, 139-159.

Volkman, J.K., Alexander, R., Kagi, R.I., Woodhouse, G.W., 1983. Demethylated hopanes in crude oils and their applications in petroleum geochemistry. Geochimica et Cosmochimica Acta 47, 785-794.

Walraven, F., Martini, J., 1995. Zircon Pb-evaporation age determinations of the Oak Tree Formation, Chuniespoort Group, Transvaal Sequence: implications for Transvaal-Griqualand West basin correlations. South African Journal of Geology 98, 58-67.

Walter, M.R., 1983. Archean stromatolites: evidence of Earth's earliest benthos. In: Schopf, J.W. (Ed.), Earth's earliest biosphere. Princeton University Press, Princeton, NJ, pp. 187-213.

Walter, M.R., Buick, R., Dunlop, J.S.R., 1980. Stromatolites 3,400-3,500 Myr old from the North Pole area, Western Australia. Nature 284, 443-445.

Zang, W.-L., 2007. Deposition and deformation of late Archaean sediments and preservation of microfossils in the Harris Greenstone Domain, Gawler Craton, South Australia. Precambrian Research 156, 107-124.

Zundel, M., Rohmer, M., 1985a. Hopanoids of the methylotrophic bacteria Methylococcus capsulatus and Methylomonas sp. as possible presursors of $\mathrm{C}_{29}$ and $\mathrm{C}_{30}$ hopanoid chemical fossils. FEMS Microbiology Letters 28, 61-64.

Zundel, M., Rohmer, M., 1985b. Prokaryotic triterpenoids. 1. 33-Methylhopanoids from Acetobacter sp. and Methylococcus capsulatus. European Journal of Biochemistry $150,23-27$. 


\section{Chapter Three}

Microaerobic steroid biosynthesis and the molecular fossil record of Archean life

Jacob R. Waldbauer, Dianne K. Newman and Roger E. Summons 


\title{
Microaerobic steroid biosynthesis and the molecular fossil record of Archean life
}

\author{
Jacob R. Waldbauer, Dianne K. Newman, and Roger E. Summons
}

The power of molecular oxygen to drive many crucial biogeochemical reactions, from respiration to weathering, makes reconstructing the history of its production and accumulation a first-order question for understanding earth's evolution. Among the various geochemical proxies for the presence of oxygen in the environment, molecular fossils present a record of $\mathrm{O}_{2}$ where it was first produced and consumed by biology, in sunlit aquatic habitats. As steroid biosynthesis requires molecular oxygen, fossil steranes have been key to inferences about aerobiosis in the early Precambrian, but better quantitative constraints on the oxygen requirement of this biochemistry would clarify these molecular fossils' implications for environmental conditions at the time of their production. Here we demonstrate that steroid biosynthesis is a microaerobic process, enabled by dissolved oxygen concentrations in the nanomolar range. We also present evidence that microaerobic marine environments were likely to have been widespread and persistent for long periods of time prior to the earliest geologic evidence for atmospheric oxygen.

\section{GEOCHEMICAL RECORDS OF OXIDATION, OXYGENATION AND EVOLUTION}

Over the course of earth's history, life has left one particularly indelible mark: the surface of the planet is far more oxidized than it would be without biological activity. Life has done this by transferring electrons from a number of donors (notably oxygen, sulfur and iron) to carbon, for the purpose of producing the organic carbon that is the stuff of biomass. In doing so, life has effected a net reduction of carbon and caused oxidized waste products, including molecular oxygen, sulfate, and ferric iron, to accumulate in the atmosphere, oceans and upper crust to much higher levels than would be present otherwise. This net export of oxidizing power by the carbon cycle has gone on more or less continuously since at least the mid-Archean (ca. $3.5 \mathrm{Ga} ; \mathrm{Ga}=$ billion years ago), and represents a biologically-driven, unidirectional change in the state of the earth system.

In quantitative terms, molecular oxygen is actually the smallest of these pools of accumulated oxidants. At present, the oxygen in the atmosphere and dissolved in the 
oceans amounts to only about $10 \%$ of the oxidizing power represented by sulfate, and only $2-3 \%$ of that represented by the excess ferric iron in the crust over the proportion in the mantle (Hayes and Waldbauer, 2006). Of course, since oxygen is the most powerful of those three oxidants, sulfate and ferric iron can be produced (either biotically or abiotically) from sulfide and ferrous iron, using $\mathrm{O}_{2}$ as the oxidant. Hence much of the $\mathrm{SO}_{4}{ }^{2-}$ and $\mathrm{Fe}(\mathrm{III})$ in the modern world also stems from oxygenic primary production. But the relatively small size of the molecular oxygen pool highlights the distinction between oxidation - the net transfer of electrons from inorganic species to carbon - and oxygenation - the accumulation of $\mathrm{O}_{2}$ in the atmosphere-ocean system. The two are undoubtedly closely linked, but have not necessarily proceeded apace. In particular, secular oxidation is a global process involving redox transformations between a wide range of species, where reservoir effects and geophysical processes come into play and a number of important fluxes are poorly constrained. Oxygenation, on the other hand, is (at least conceptually) simpler: the abundance of $\mathrm{O}_{2}$ at a given time represents the integration of a long-term source-sink balance, of which the source is biological and the sink is geochemical.

\subsection{The emergence of oxygenic photosynthesis: wildfire or slow burn?}

Because molecular oxygen is a biological product, its influence on biogeochemistry has a start date: the evolution of water-splitting, oxygenic photosynthesis. This biochemistry was apparently invented exactly once, by an ancestor of the cyanobacteria, when type I and type II photosystems were combined with a unique cofactor, the oxygen evolving center. The specifics of how oxygenic photosynthesis came to be are not fully clear and remain the subject of active investigation and debate (Allen and Martin, 2007). In geochemical terms, our ability to infer the history of the influence of biological activity on the redox state of the earth's surface would be greatly aided by knowledge of this start date. 
The geologic record, helpfully, does provide some evidence of a minimum age for oxygenic photosynthesis. An ensemble of geological and geochemical evidence for atmospheric oxygenation, which has come to be known as the "Great Oxidation Event", points to the period ca. $2.40-2.45 \mathrm{Ga}$ as the time when $\mathrm{O}_{2}$ became a persistent and geochemically significant component of the atmosphere, which it has remained ever since. The suite of observed geochemical signals and their prevailing interpretations have recently been reviewed (Catling and Claire, 2005; Buick, 2008; Sessions et al., 2009); suffice it here to say that ca. $2.45 \mathrm{Ga}$ stands as a minimum age by which oxygenic photosynthesis must have arisen. The basic question, then, is: does the Great Oxidation Event (GOE) actually document the evolution of oxygenic photosynthesis, or is the process significantly older? In other words, was the spread of oxygenic photoautotrophs a wildfire or a slow burn?

The appearance of the GOE ensemble in the geologic record could be taken as prima facie evidence of the dawn of oxygenic photosynthesis, an interpretation to which, with amendments, some authors hold (Kopp et al., 2005; Kirschvink and Kopp, 2008). It is important to keep in mind, however, the distinction between the accumulation of $\mathrm{O}_{2}$ in the atmosphere and its production by microorganisms in aquatic environments. The latter is necessary but not sufficient for the former; the oxygenation of the atmosphere depends on the integrated imbalance between sources and sinks of oxygen. The interpretation of the GOE as marking the origin of oxygenesis requires that this balance have been upset rapidly by vigorous $\mathrm{O}_{2}$ production, overwhelming the capacity of existing sinks.

The source term for atmospheric oxygen in the early Precambrian is relatively simple: net primary production (photosynthesis in excess of local aerobic respiration) drives supersaturation of $\mathrm{O}_{2}$ in surface waters, which results in a sea-to-air flux of oxygen. (Throughout, we will refer to oxygen production in marine environments, since it is marine sedimentary sequences that bear geochemical records of oxygenation. This does 
not, however, exclude the possibility that lacustrine or other freshwater environments contributed to the oxygen flux - there is simply very little geological record of such environments from the early Precambrian.) The sink or loss term, on the other hand, is rather complicated: a wide variety of geochemical pathways exist for oxygen reduction, involving many atmospheric and mineral reaction partners. Fortunately, the loss of molecular oxygen from the Archean atmosphere has been the subject of several recent, detailed modeling studies (Pavlov and Kasting, 2002; Claire et al., 2006; Goldblatt et al., 2006; Zahnle et al., 2006), which provide valuable constraints on how the composition of the atmosphere might evolve given a biogenic $\mathrm{O}_{2}$ flux.

The scenario of rapid spread of newly-evolved oxygenic photoautotrophs triggering the $\mathrm{GOE}$ is predicated on the idea that, prior to ca. $2.45 \mathrm{Ga}$, marine primary producers were strongly and globally limited by the availability of reductant. If iron- and sulfide- and hydrogen-oxidizing autotrophs were limited only by supplies of their respective reductants, and not by any other factor, then the cyanobacteria's newly-developed ability to use water as a reductant for carbon fixation would have given them a profound ecological advantage. This assumption, however, is quite a strong one. It requires that all other growth substrates (including phosphate, fixed nitrogen, trace metals and light) be in plentiful supply, and be underexploited by anoxygenic primary producers, presumably because of their reductant limitation. This notion contradicts the tendency of ecosystems towards co-limitation, where overabundance of one substrate relative to another drives its preferential exploitation until multiple forms of limitation occur simultaneously. Anoxygenic autotrophs are not necessarily less efficient at nutrient or light harvesting than cyanobacteria, and were well-adapted to their respective environments. Cyanobacteria had to 'shoulder aside' and gradually outcompete these prior inhabitants in order to occupy their sunlit habitats. In doing so, they had the distinct advantage of never facing reductant limitation and producing a metabolic waste product that was deleterious to their competitors. But it is a strong and, in our view, unsupported assumption to posit that this global-scale process of ecological succession occurred in a geologic eyeblink. 
The concept of a rapid takeover of global primary production by oxygenic photoautotrophs at $2.4 \mathrm{Ga}$ is also at odds with longer-term geologic records. For all the geologic evidence of the appearance of atmospheric oxygenation at that time, there is relatively little evidence of large-scale oxidation. The period is not marked by unusually large deposits of organic carbon, and the only perturbations to the isotopic compositions of sedimentary carbon reservoirs come later, closer to $2.1 \mathrm{Ga}$, and are more plausibly related to changes in global patterns in diagenesis than to enhanced organic carbon burial (Hayes and Waldbauer, 2006). These patterns are inconsistent with the notion that there were large excesses of nutrients just waiting to be unlocked and rapidly consumed by water-splitting primary producers. The persistence of deep-water anoxia and coexistence of oxygenic and anoxygenic primary producers in marine settings for another nearly two billion years (Johnston et al., 2009), until the latest Neoproterozoic, is further evidence of the gradual nature of the succession in oceanic primary production.

And there is also another good reason to doubt that the GOE indicators immediately recorded the emergence of oxygenic photosynthesis: other proxies for molecular oxygen document its production and consumption long before its accumulation in the atmosphere. The next section discusses the distinctions between what is recorded by the various geochemical proxies for oxygenation.

\subsection{Proxies for oxygenation}

Most of what we know about the history of the oxygenation of the surface environment comes from geologic and geochemical proxies that are sensitive to the involvement of $\mathrm{O}_{2}$ in weathering processes and/or atmospheric chemistry. The ensemble of 'classic' GOE indicators fall into this category: oxidized paleosols record oxic chemical weathering directly, detrital redox-sensitive minerals such as pyrite, siderite and uraninite record erosion and anoxic fluvial transport, and the sharp termination of mass-independent 
fractionation of $\mathrm{S}$ isotopes that cemented the GOE paradigm documents a change in atmospheric chemistry brought on by oxygenation. A number of other oxygen proxies track the mobility, speciation and/or isotopic fractionation of redox-sensitive metals, including Mo, Re (Anbar et al., 2007), U (Coogan, 2009), Cr (Frei et al., 2009) and Fe (Reinhard et al., 2009), and also test the involvement of oxygen in weathering, transport and sedimentation processes.

For signals to be recorded in these geologic and geochemical proxies, oxygen must have already accumulated in the atmosphere to some extent and persisted there for a geologically-significant period of time. As noted in the previous section, there are reasons to question the assumption that signals would necessarily appear in these proxies promptly after the evolution of oxygenic photosynthesis. They are at somewhat of a remove - separated by considerations of transport and source-sink balances - from the biology of oxygen production. Ideally, we could find a direct proxy for the oxygenic biochemistry itself; unfortunately, the molecular components of the water-splitting apparatus do not leave identifiable fossil remains. The body fossil record of early Precambrian microbes is also very poor, and even those microstructures accepted as biogenic do not reliably inform taxonomic or physiological identifications.

A remarkable recent finding suggests what may be some of the most immediate fossil evidence yet for oxygenic photosynthetic microbes. Bosak et al. (2009) examined the conical structures built by some filamentous cyanobacteria, which are plausible analogues for types of stromatolites seen in the Archean rock record. They observed that oxygenic photosynthesis by these organisms resulted in the formation of oxygen bubbles, which accumulated near the tips of the cones. Spherical features similar to bubbles are also seen enclosed by mat laminae in the crests of some late Archean stromatolites, beginning about 2.7Ga, and Bosak et al. (2009) detail why these features are likely signatures of oxygenic photosynthesis. If so, they are very nearly "fossil oxygen". 
Hydrocarbon molecular fossils (biomarkers) have provided some of the earliest evidence for the production and biological utilization of molecular oxygen (Brocks et al., 1999; Brocks et al., 2003; Dutkiewicz et al., 2006; George et al., 2007; Eigenbrode et al., 2008). Chapter 2 of this thesis presents a molecular fossil record from late Archean sedimentary rocks of Transvaal Supergroup, dating from ca. 2.67-2.46Ga. As diagenetically-altered, but still identifiable biochemicals, biomarkers offer a proxy for biological activity that was once an actual functional component of living cells. A few specific types of molecular fossils are specific proxies for either oxygenic organisms or aerobic biochemistry. 2-methylhopanoids are markers for at least the preexistence of cyanobacteria, if not their direct input to sedimentary organic matter (Summons et al., 1999; Sessions et al., 2009). 3-methylhopanoids, particularly when found strongly depleted in ${ }^{13} \mathrm{C}$, are markers for aerobic Type 1 methanotrophs, whose preferred habitat at the chemocline between oxygenic and methanogenic habitats was likely widespread in the late Archean (Blumenberg et al., 2007). This chapter focuses on the third class of biomarker proxies for oxygen, the steranes. All of these molecules have been found in sedimentary deposits dating as far back as $2.72 \mathrm{Ga}$, including, as documented in Chapter 2 , in the samples with the strongest contamination controls. While the challenges involved in their analysis and interpretation are considerable (Sherman et al., 2007), molecular fossils from sedimentary rocks provide a unique record of $\mathrm{O}_{2}$ where it was first produced, accumulated and utilized: sunlit aquatic ecosystems.

Fossils of steroids have been especially significant as proxies for oxygenation, since steroid biosynthesis specifically requires molecular oxygen. A summary view of the biosynthetic pathway of steroids is shown in Figure 1. As indicated, there are at least four steps that require $\mathrm{O}_{2}$. The first oxygen-dependent step is also the first committed step in steroid synthesis: the epoxidation of the isoprenoid squalene. The enzyme that cyclizes oxidosqualene to form the characteristic $6,6,6,5$ - steroid ring structure cannot act on squalene itself, but requires the (3S) 2,3-epoxide (Summons et al., 2006). Subsequently, to produce the 4,14-desmethyl sterols that make up the vast majority of 


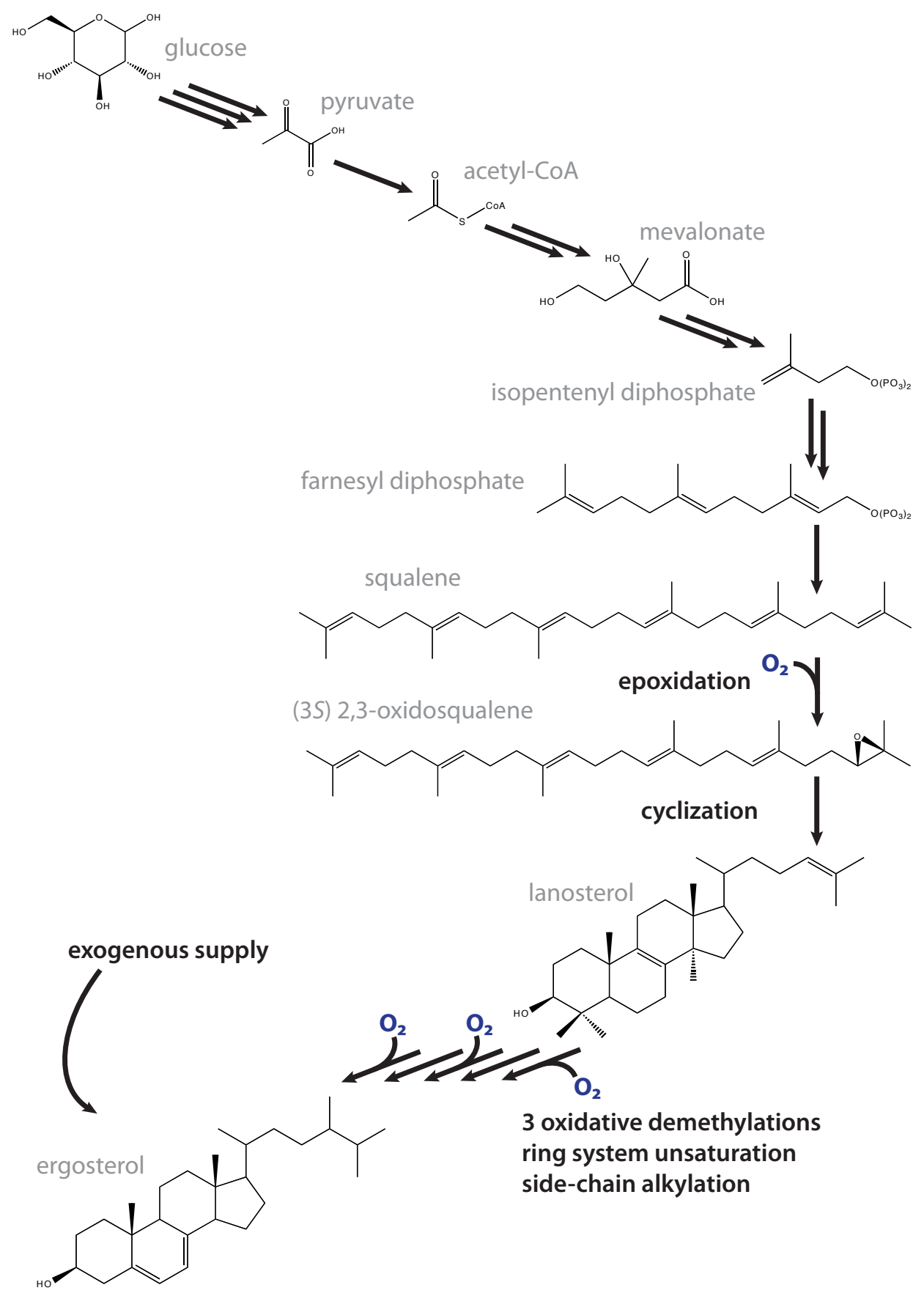

Figure 1. An abbreviated summary of the biosynthesis of sterols in yeast, showing the flow of carbon from glucose to ergosterol. The first oxygen-requiring step is the epoxidation of squalene; subsequently, $\mathrm{O}_{2}$ is also required for the removal of three methyl groups from the sterol ring system to produce the functional membrane lipid. In these experiments, cells can either synthesize sterols de novo (and hence with a ${ }^{13} \mathrm{C}$ label) or take up unlabeled ergosterol from the medium. 
both membrane lipids and molecular fossils, nine more molecules of $\mathrm{O}_{2}$ are necessary to effect three oxidative demethylation reactions. The synthesis of some steroids (e.g., cholesterol) requires yet more oxygen to introduce unsaturations into the carbon skeleton. Steroid biosynthesis is highly evolutionarily conserved, and was present in the last common ancestral lineage of all extant eukaryotes (Summons et al., 2006; Desmond and Gribaldo 2009).

Steranes - defunctionalized, saturated, diagenetically altered fossil steroids - have been detected in sedimentary rocks as old as $2.72 \mathrm{Ga}$, roughly 300 million years before the GOE. The presence of steranes in rocks of this age implies that some molecular oxygen was available in the marine environments that produced the organic matter preserved in late Archean sediments. The implication is that the integrated source-sink imbalance for atmospheric oxygen remained very small for hundreds of millions of years after the evolutionary origin of oxygenic photosynthesis, keeping atmospheric $\mathrm{O}_{2}$ somewhere below $10^{-6}$ of its modern level. At present, however, constraints on the environmental oxygen concentration that enables steroid biosynthesis are limited: Jahnke and Klein (1983) reported an apparent oxygen $\mathrm{K}_{\mathrm{m}}$ (half-saturation content) for squalene epoxidase of $4.3 \mu \mathrm{M}$, and Rogers and Stewart reported apparent $\mathrm{O}_{2} \mathrm{~K}_{\mathrm{m}}$ values for ergosterol contents in yeast of $0.5 \mu \mathrm{M}$ (Rogers and Stewart, 1973b) and " $0.3 \mu \mathrm{M}$ or less" (Rogers and Stewart, 1973a). The former is an in vitro measure of a crude preparation of a single part of the pathway, while the latter reflects a physiological response (cellular sterol levels) rather than biosynthesis itself; nor have these experiments been repeated in the years since. Hence it is difficult to assess the consistency between interpretations of the biomarker record and reconstructions of Archean biogeochemistry and atmospheric evolution. The experiments presented in this chapter seek to provide constraints on the oxygen requirement of sterol biosynthesis that more directly apply to the interpretation of the molecular fossil record. 
To directly assay the oxygen requirements of steroid biosynthesis, we adopted an isotopic labeling strategy. Yeast (S. cerevisiae) was chosen as a test organism because it is a facultatively anaerobic eukaryote, able to grow at any oxygen concentration. Yeast cells were grown in a defined minimal medium containing ${ }^{13} \mathrm{C}$-glucose and unlabeled $\left({ }^{12} \mathrm{C}\right.$ - $)$ ergosterol. Since steroid biosynthesis requires molecular oxygen, the cells are obligately auxotrophic for steroids under anaerobic conditions, and take up the supplied (unlabeled) ergosterol. Under aerobic conditions, however, steroids are made de novo from carbon substrates, and so acquire the ${ }^{13} \mathrm{C}$ label from glucose. By analyzing the cells' lipid complements using mass spectrometry, we could examine the incorporation of ${ }^{13} \mathrm{C}$ into specific compounds, including sterols. Our goal was to determine the lowest dissolved oxygen concentration that would enable de novo steroid synthesis.

\section{EXPERIMENTAL METHODS}

\subsection{Microaerobic growth experiments}

\subsubsection{Culture conditions and sampling}

Saccharomyces cerevisiae (strain D273-10B, ATCC) cells were grown in a defined minimal medium containing $4 \mathrm{~g} / \mathrm{l}$ uniformly-labeled ${ }^{13} \mathrm{C}_{6}$-glucose (99\%, Cambridge Isotope), supplemented with $10 \mathrm{mg} / 1$ ergosterol (Alfa Aesar) and $0.5 \mathrm{ml} / 1$ Tween 80 (polyoxyethylene sorbitan monooleate). The Tween 80 provided a source of unsaturated fatty acids, which yeast also require molecular oxygen to produce. The medium was also amended with 1ml/1 FG-10 (Dow Chemical), a polydimethylsiloxane-based surfactant, as an antifoaming agent. Cultures were maintained in an anaerobic chamber (Coy) in an atmosphere of 5\% hydrogen, $15 \% \mathrm{CO} 2$ and $<1 \mathrm{ppm} \mathrm{O}_{2}$. For microaerobic growth, $6 \mathrm{ml}$ of late log-phase culture $\left(\mathrm{OD}_{600} \sim 0.8\right)$ was inoculated into $300 \mathrm{ml}$ of media in a bubbler bottle with the headspace exhausted to external vacuum. Dissolved oxygen concentration in the media was controlled by vigorous bubbling with $\mathrm{O}_{2}: \mathrm{N}_{2}$ mixtures (5-5000ppm $\mathrm{O}_{2}$, Airgas). Cells were grown with bubbling and stirring for 12-16h, until an $\mathrm{OD}_{600}$ of 0.2 
was reached. The cells were then harvested by vacuum filtration in the anaerobic chamber onto a precombusted GF/F filter (Whatman). Filters were placed into glass centrifuge tubes filled with $19 \mathrm{ml}$ of Bligh-Dyer extraction solvent (10:5:4 chloroform:methanol:water; Bligh and Dyer, 1959) that had been pre-equilibrated with the anaerobic atmosphere, and incubated in the anaerobic chamber overnight to ensure complete enzyme deactivation prior to oxygen exposure.

\subsubsection{Dissolved $\mathrm{O}_{2}$ measurements}

Oxygen concentration in the cultures during microaerobic growth was measured by two methods: a colorimetric assay based on Rhodazine-D (Chemetrics) and a novel type of microelectrode with a polarizable front guard (Unisense). The Rhodazine-D test, based on a proprietary redox-sensitive chromophore, is convenient and inexpensive, but is limited in sensitivity to dissolved oxygen concentrations above approximately $300 \mathrm{nM}$. It is also susceptible to interferences from $\mathrm{Fe}(\mathrm{III})$ and $\mathrm{Cu}(\mathrm{II})$, and higher concentrations of suspended particulates (such as cells) can require prefiltration, potentially limiting accuracy. The recently-developed STOX (Switchable Trace Oxygen) electrode (Revsbech et al., 2009) allows in situ electrochemical detection of $\mathrm{O}_{2}$ down to concentrations as low as $5-10 \mathrm{nM}$ (equivalent to equlibrium at $25^{\circ} \mathrm{C}$ and $1 \mathrm{~atm}$ with an atmosphere containing 4-8ppmv $\mathrm{O}_{2}$ ).

\subsection{Lipid analysis}

\subsubsection{Lipid extraction/derivitisation}

Tubes containing filters and solvent were removed from the anaerobic chamber and ultrasonicated for 20 minutes. Tubes were then centrifuged for 5 minutes at $1000 \times g$, the supernatant decanted (first extract) and 19ml Bligh-Dyer solvent added again. After a second ultrasonication and centrifugation, the second extract was pooled with the first, and $10 \mathrm{ml}$ water and $10 \mathrm{ml}$ chloroform added to induce phase separation. Phase separation continued overnight at $-20^{\circ} \mathrm{C}$, after which the upper phase and interfacial debris were 
removed and the lower phase concentrated under $\mathrm{N}_{2}$. Extracts were filtered over $1 \mathrm{~cm}$ of silica gel in pipette columns, eluting lipids with 8:2 dichloromethane:ethyl acetate. Lipid extracts were evaporated to dryness to determine yield and $1 \mathrm{mg}$ of lipid reacted with $40 \mu 1$ BSTFA $+1 \%$ TMCS (Alfa Aesar) and $40 \mu 1$ pyridine (Acros) at $70^{\circ} \mathrm{C}$ for 60 minutes to produce trimethylsilyl derivatives.

\subsubsection{Gas chromatography/Mass spectrometry}

Derivatized lipid extracts were analyzed by gas chromatography-mass spectrometry on a 7980A GC / 5975C MSD system (Agilent). $1 \mu$ l of extract was injected onto a DB-1 or DB-5 column $(0.250 \mathrm{~mm}$ ID, $0.25 \mu \mathrm{m}$ film, $30 \mathrm{~m}$ length; Agilent). Following the solvent delay, the oven ramped from $70^{\circ} \mathrm{C}$ to $230^{\circ} \mathrm{C}$ at $20^{\circ} \mathrm{C} / \mathrm{min}$; carrier helium flow was $1 \mathrm{ml} / \mathrm{min}$. Analytes were ionized by electron impact at $70 \mathrm{eV}$.

\subsection{Modeling of Archean oxygen fluxes}

To link our experimental determination of the oxygen requirements of steroid biosynthesis with models of Archean atmospheric evolution, we constructed a simple model for the $\mathrm{O}_{2}$ outgassing flux from a partially-oxygenated surface ocean. Under an anaerobic atmosphere, the saturation level of oxygen in the ocean is very small, ingassing is negligible, and the net sea-to-air gas flux can be expressed as:

$$
J_{O_{2}}=\alpha A_{E} v_{p}\left[O_{2}\right]_{\alpha}
$$

where $J_{O 2}$ is the sea-to-air oxygen flux, $\alpha$ is the proportion of the surface area of the earth $\left(A_{E}\right)$ contributing to that oxygen flux, $v_{p}$ is the globally-averaged gas exchange piston velocity, and $\left[\mathrm{O}_{2}\right]_{\alpha}$ is the average oxygen concentration of surface waters in the area represented by $\alpha . A_{E}$ is taken as the present value, 510 million $\mathrm{km}^{2}$. Air-sea gasexchange, while strongly dependent on climatic variables such as wind speed, temperature and sea state that are poorly constrained for the Archean ocean, is an 
A
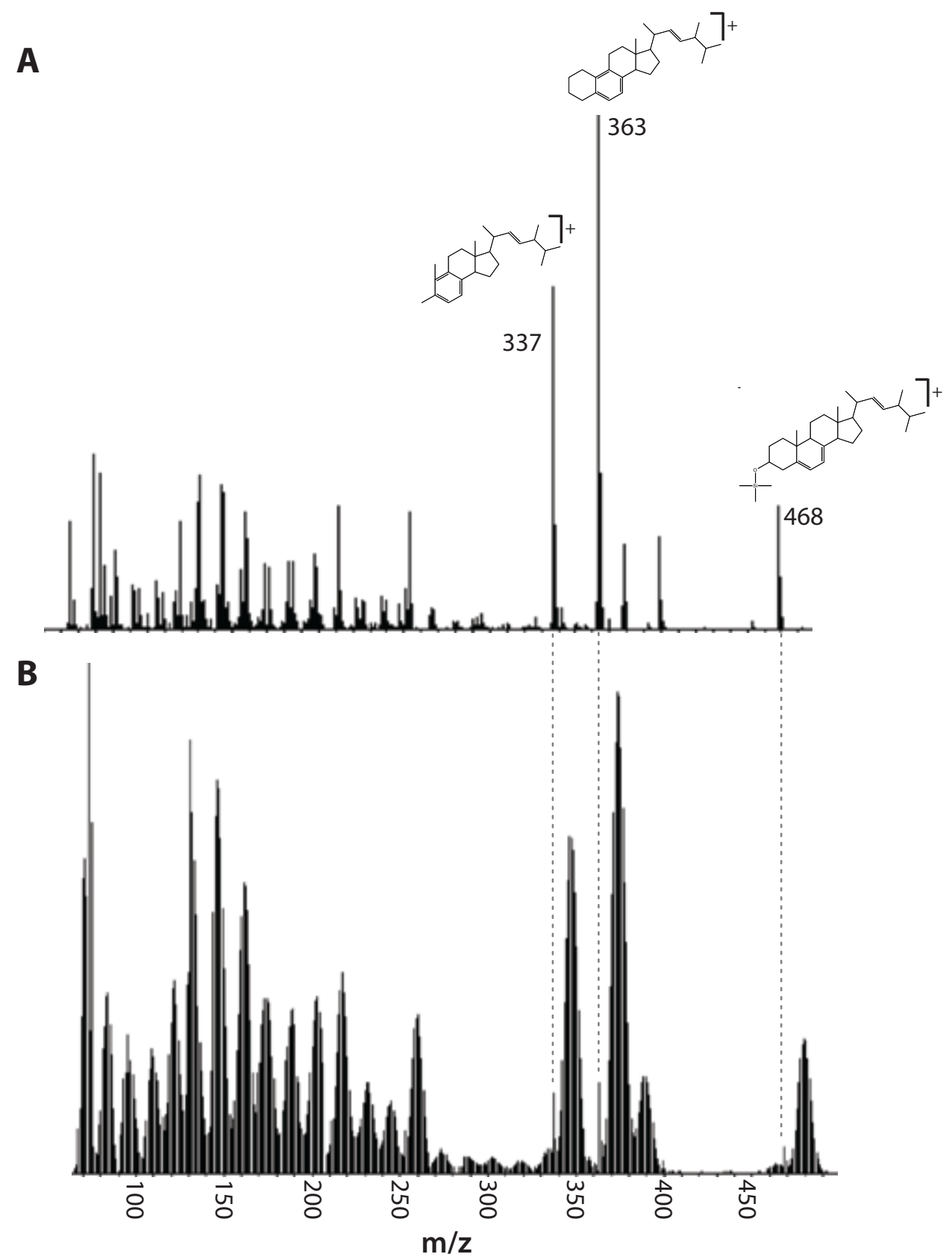

Figure 2. Mass spectra of unlabeled $(\mathbf{A})$ and ${ }^{13} \mathrm{C}$-labeled $(\mathbf{B})$ trimethylsilyl derivatives of ergosterol. Incorporation of ${ }^{13} \mathrm{C}_{6}$-glucose results in broad peaks in the mass spectrum, shifted to higher masses than the unlabeled compound. Fragment ion assignments are after Brooks et al. (1968). 
essentially physical process likely to have been more or less constant over time. Here we utilize the modern, long-term global average value of $16 \mathrm{~cm} / \mathrm{h}$ of Glover et al. (2007). We note that $v_{p}$ is unlikely to vary by more than an order of magnitude, while the modeled range of interest of $\mathrm{J}_{\mathrm{O} 2}$ and $\left[\mathrm{O}_{2}\right]_{\alpha}$ span roughly four orders of magnitude, so overall the model is not expected to be highly sensitive to the precise physics of gas exchange.

\section{RESULTS: STEROID BIOSYNTHESIS IS A MICROAEROBIC PROCESS}

\subsection{The oxygen requirements of steroid biosynthesis}

The incorporation of the ${ }^{13} \mathrm{C}$ label from glucose produced a distinct shift in the appearance of lipid mass spectra (Fig. 2). ${ }^{13} \mathrm{C}$-labeled compounds showed large peaks in the mass spectra, shifted up the mass scale relative to peaks in the spectra of unlabeled compounds. These distinct spectra provide an unambiguous signal of de novo lipid biosynthesis.

The carbon-13 label was incorporated into squalene - the last steroid synthesis intermediate reachable in the absence of $\mathrm{O}_{2}-$ in all conditions (Fig. 3). Under anaerobic conditions, no lanosterol was detected, and only unlabeled ergosterol (taken up from the medium) was present. This is the pattern expected if steroid synthesis is not operative, and is entirely consistent with the oxygen dependence of sterol biosynthesis. Once oxygen was provided to the culture by bubbling, however, labeled steroids were produced de novo. In the presence of oxygen, lanosterol is synthesized and bears the ${ }^{13} \mathrm{C}$ label, and labeled ergosterol is also present.

Surprisingly, even the lowest oxygen concentration tested to date - estimated at $7 \mathrm{nM}-$ appears to support steroid biosynthesis. This is a remarkably low oxygen level to enable what has been thought of as a classically aerobic biochemistry. It should be noted, however, that this result has yet to be duplicated in a replicate experiment, and that the 


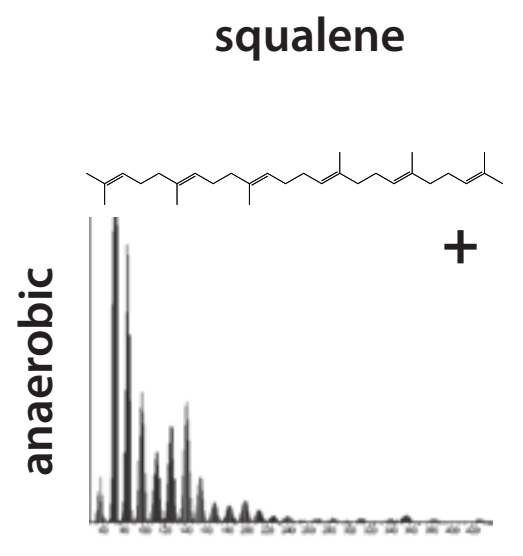

lanosterol

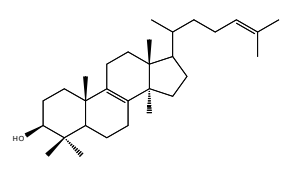

n.d. ergosterol

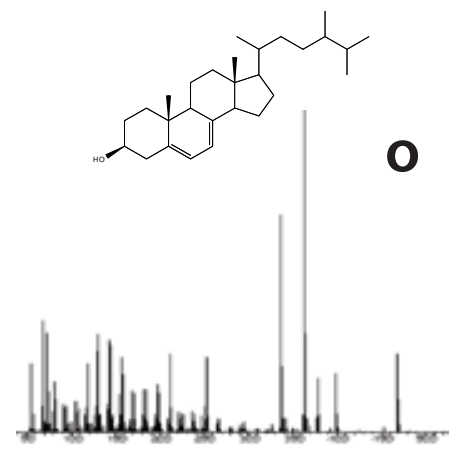

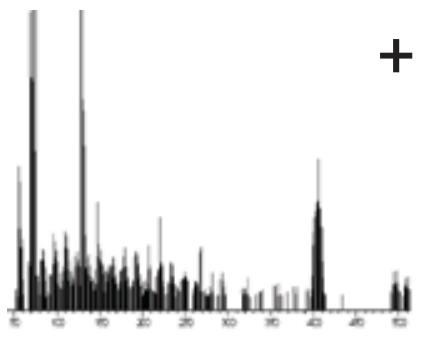
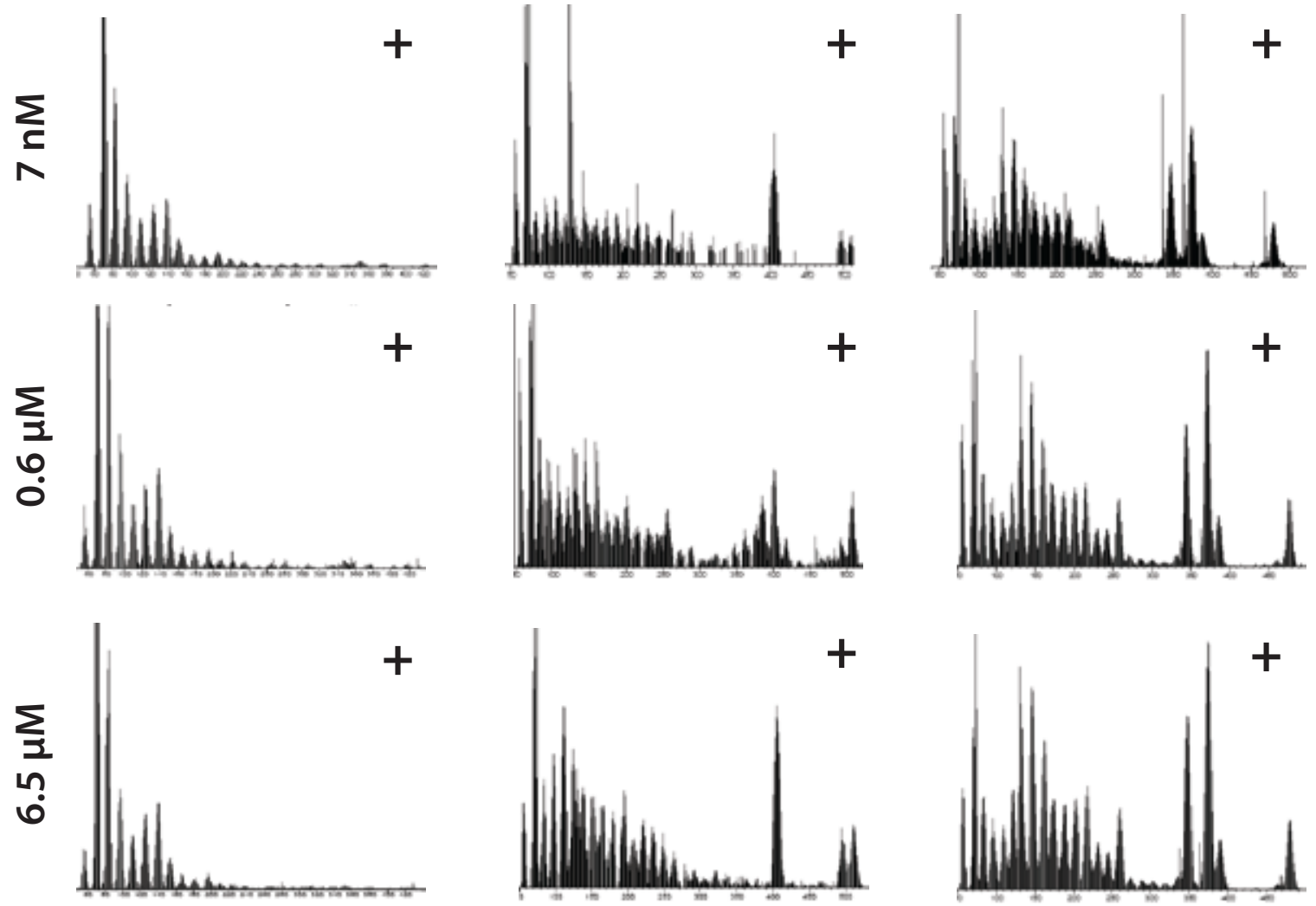

Figure 3. Mass spectra of lipids from microaerobic growth experiments. Compounds are indicated across the top; dissolved oxygen concentrations in the various growth experiments are indicated at left. $\mathrm{A}^{\text {" }}+$ " indicates that the ${ }^{13} \mathrm{C}$ labeled compound was detected, a "o" indicates that only the unlabeled compound was detected; n.d., not detected. 
oxygen concentration is only an estimate, based on the calculated solubility and the composition of the gas mixture as certified by the manufacturer. At the time this experiment was performed, we did not have access to the new STOX electrode, and so had no way of directly measuring such low $\mathrm{O}_{2}$ concentrations. This experiment will be repeated in the very near future, with the dissolved oxygen monitored electrochemically.

With those caveats in mind, we provisionally interpret these results as a useful, perhaps even conservative, lower bound for the oxygen requirement for steroid synthesis. While yeast is certainly an incomplete model for the unknown diversity of eukaryotic organisms in the Archean ocean, the fact that a modern organism can perform this biochemistry at such low oxygen concentrations suggests that early eukaryotes, adapted to chronic $\mathrm{O}_{2}$ scarcity, likely could as well. Notably, steroid biosynthesis is possible well below the Pasteur point of $1 \%$ PAL $\left(\sim 2 \mu \mathrm{M} \mathrm{O}_{2}\right)$, above which fermentation is inhibited. The Pasteur point has sometimes been misinterpreted as a switching point between aerobic and anaerobic metabolism (Rutten, 1970; Goldblatt et al., 2006). Along with recent results suggesting microaerobic respiration in E. coli (Stolper et al., 2009), this experiment shows that there is a geochemically-significant range of dissolved $\mathrm{O}_{2}$ concentrations below the Pasteur point that enable aerobic biochemistry.

The exceptionally low oxygen levels enabling steroid synthesis also highlight the close connection between steroid biochemistry and oxygen sensing, metabolism and defense (Galea and Brown, 2009). Studies of industrial fermentations have documented that, during aeration pulses, the first aerobic biochemistry to become active is steroid synthesis, not respiration (Rosenfeld et al., 2003). In our experiment, de novo steroid synthesis occurred despite an abundant supply of exogenous sterols in the growth medium. It would appear that sterol homeostasis is one of the most sensitive oxygendetecting systems in biology, which is consistent with its emergence in microaerobic aquatic settings, well before the oxygenation of the atmosphere. 


\subsection{Oxygenation of the Archean surface ocean}

Our results demonstrate that steroid biosynthesis is possible in microaerobic environments, which has significant implications for the biogeochemical interpretation of steranes in the fossil record. The fossil steranes in late Archean sedimentary rocks can be interpreted as evidence for microaerobic conditions in a portion of the marine water column in an otherwise anaerobic world. Is this interpretation of the molecular fossil record consistent with other geochemical evidence for the evolution of atmospheric oxygen levels? In particular, could microaerobic regions of the surface ocean have supplied the $\mathrm{O}_{2}$ fluxes called for by current models of Precambrian atmospheric evolution? To answer this question, we constructed a simple model of global air-sea gas exchange and used it to predict the mixed-layer supersaturation of dissolved oxygen implied by given values of the $\mathrm{O}_{2}$ flux to the atmosphere (Sec. 2.3).

The results of this model, shown in Figure 4, indicate that microaerobic ocean surface environments capable of supporting steroid biosynthesis are entirely consistent with even required by - models of Archean atmospheric evolution. To provide the oxygen outgassing fluxes called for in the models of Pavlov and Kasting (2002) and Zahnle et al. (2006), that the average supersaturation in ocean regions contributing to sea-to-air oxygen outgassing would be in the range of $0.1-1 \mu \mathrm{M}$. This is $10-100$ times our experimental determination of the concentration required for sterol biosynthesis. These models show how such an oxygen flux can persist for hundreds of millions of years without causing oxygenation of the atmosphere to the extent that would 'trip' the geologic and geochemical proxies whose signals appear at or near the GOE. Even if the total flux were an order of magnitude less than the lower limit considered by Zahnle et al. (2006), if it were concentrated in high-productivity hotspots ("oxygen oases"), the local oxygen concentration was likely to have been high enough to allow steroids to be made. It is important to recognize that no threshold value of sea-to-air $\mathrm{O}_{2}$ flux requires that the atmosphere become oxygenated; so long as the oxygen is accompanied by inputs of 


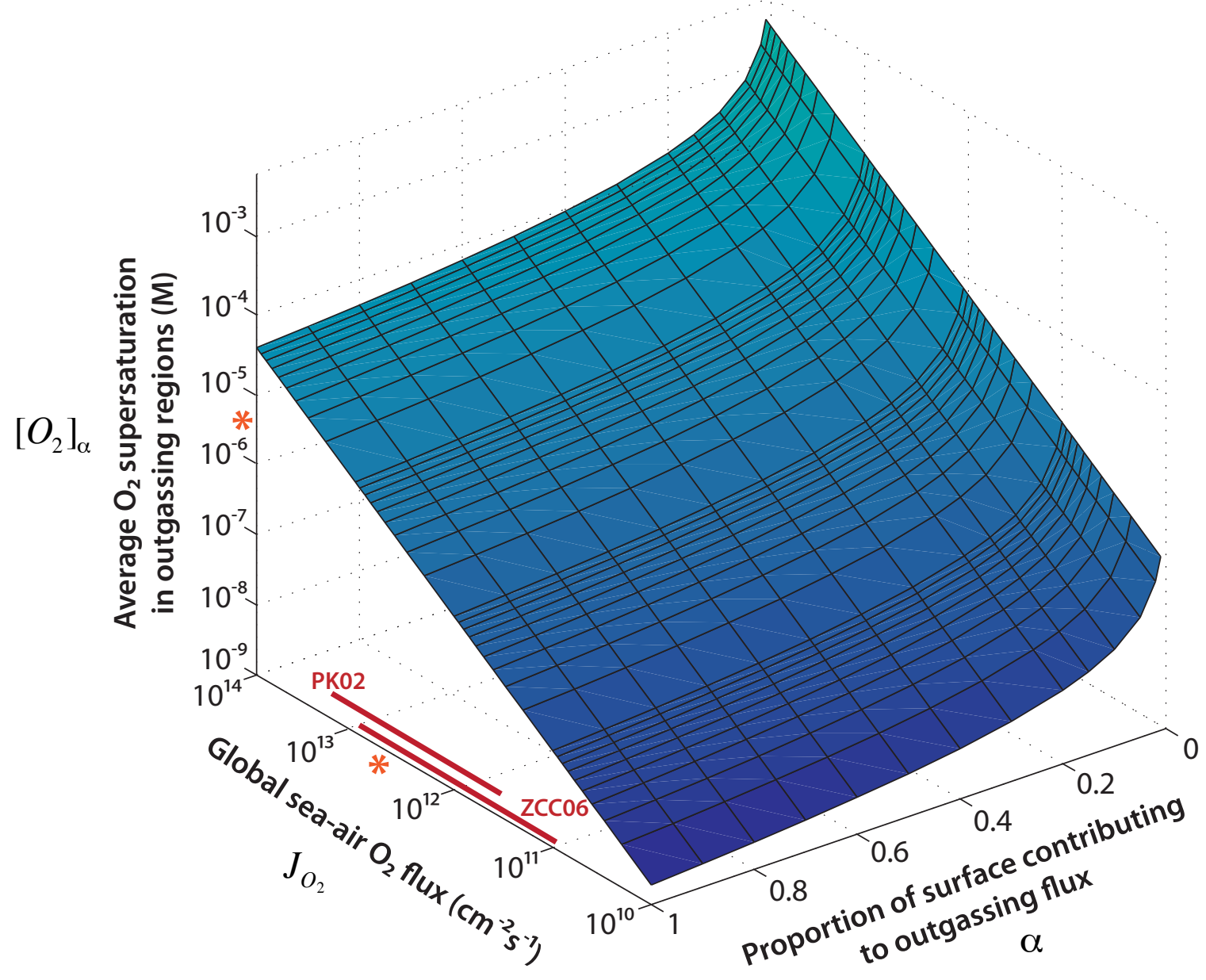

Figure 4. The modeled average dissolved oxygen concentration (i.e., supersaturation) in oxygenated regions of the Archean ocean, as a function of the sea-to-air $\mathrm{O}_{2}$ flux and the proportion of the earth's surface contributing to that flux. The larger the outgassing flux, and the smaller the fraction of the earth's surface it comes from, the more oxygenated surface waters in that region must be. Red bars indicate the range of oxygen fluxes in the Archean atmospheric evolution models of Pavlov \& Kasting (2002) (PK02) and Zahnle et al. (2006) (ZCC06). Orange stars indicate approximate modern values for annual net biogenic marine oxygen outgassing and associated surface-water supersaturations (Najjar \& Keeling 2000). 
sufficient reductant, even the modern $\mathrm{O}_{2}$ flux could coexist with an anaerobic atmosphere (Claire et al., 2006; Goldblatt et al., 2006; Zahnle et al., 2006).

The biogeochemical distribution of oxygen in the Archean ocean likely resembled (in general terms) that of biogenic trace gases in the modern ocean, such as dimethylsulfide (DMS). In fact, the parallels between Archean oxygen and modern DMS are striking: both have biological sources in the upper water column, vanishingly small atmospheric concentrations and atmospheric lifetimes of hours to days (Kettle et al., 1999; Kloster et al., 2007). Oxygen in the Archean ocean probably had rather heterogeneous geographic and seasonal distributions, as DMS does today. Moreover, in both cases, some degree of supersaturation of the surface ocean is persistent over long time scales (a phenomenon also seen with some abiogenic gases, such as ${ }^{222} \mathrm{Rn}$ ) and biologically relevant.

The past $\mathrm{O}_{2}$ content of the surface ocean cannot be inferred accurately from atmospheric $\mathrm{O}_{2}$ levels alone, nor should its concentration be assumed to have been homogeneous. This is especially true when considering biochemical processes, since, as steroid biosynthesis exemplifies, micromolar-scale deviations from saturation are highly significant. Hence, the geologic record of atmospheric $\mathrm{O}_{2}$ does not directly constrain the evolutionary history of aerobic biochemistries, as has sometimes been assumed (Goldblatt et al., 2006; de Duve, 2007). While the oxygenation of the ocean may seem trivial compared to that of the atmosphere (the ocean contains only about $0.5 \%$ of the $\mathrm{O}_{2}$ in the modern world), the ocean has played a pivotal role as a source region for oxygen production.

\section{A bRIEF PORTRAit OF LIFE \& GEOCHEMISTRY IN THE LATE ARCHEAN}

The support from molecular (e.g., Chapter 2), morphological (Bosak et al., 2009) and newer geochemical (Frei et al., 2009) proxies for microaerobic surface ocean environments as far back as $2.7 \mathrm{Ga}$ adds new features to the picture of Archean 
biogeochemistry. A cartoon representation of a late Archean coastal ocean environment and some of the biogeochemical processes that went on there is shown in Figure 5. A wide variety of microbial metabolisms were probably present, in an ecosystem every bit as spatially structured as those of the Phanerozoic. Microaerobic regions where cyanobacteria were burgeoning primary producers provided an outgassing flux of $\mathrm{O}_{2}$ to the atmosphere. While most gross primary production was likely locally aerobically respired (as it is today), any net export of organic from microaerobic zones was, in a low$\mathrm{Fe}(\mathrm{III})$, low-sulfate ocean, most likely subject to methanogenic diagenesis. Where the methane so produced met the fringes of the oxygenated zones, aerobic methanotrophy would have been active and formed another sink for $\mathrm{O}_{2}$. The biogenic gases that escaped the photosynthesis-respiration-methanogenesis-methanotrophy loop in the water column and evaded to the atmosphere would have provided a stoichiometric 2:1 flux of oxygen:methane that, in the absence of an ozone shield, would have undergone rapid photochemical recombination (Claire et al., 2006). Catling et al. (2001) have noted that the small, steady leak of methane out of this atmospheric process - due to hydrogen escape to space - may have been essential to the eventual oxygenation of the atmosphere. Meanwhile, hydrothermal supplies of reductants (including $\mathrm{H}_{2}$ produced by serpentinization of basaltic crust (Hayes and Waldbauer, 2006)) fueled various phototrophic and chemotrophic metabolisms, both at the periphery of microaerobic zones and distant from them. A fruitful direction for future research will be to examine how and when shifts from dominantly anoxygenic to dominantly oxygenic primary productivity occurred, a process that, as discussed above, was probably gradual and spatially heterogeneous.

The biodiversity implied by this range of biogeochemical processes is considerable, and implies that much of microbial metabolism and that many, if not most, of the major taxonomic lineages had appeared by the late Archean. The molecular fossil record provides specific evidence for bacteria and eukaryotes (Chapter 2), methanogenesis implies the presence of archaea, and the active biogeochemical cycles evident in the 


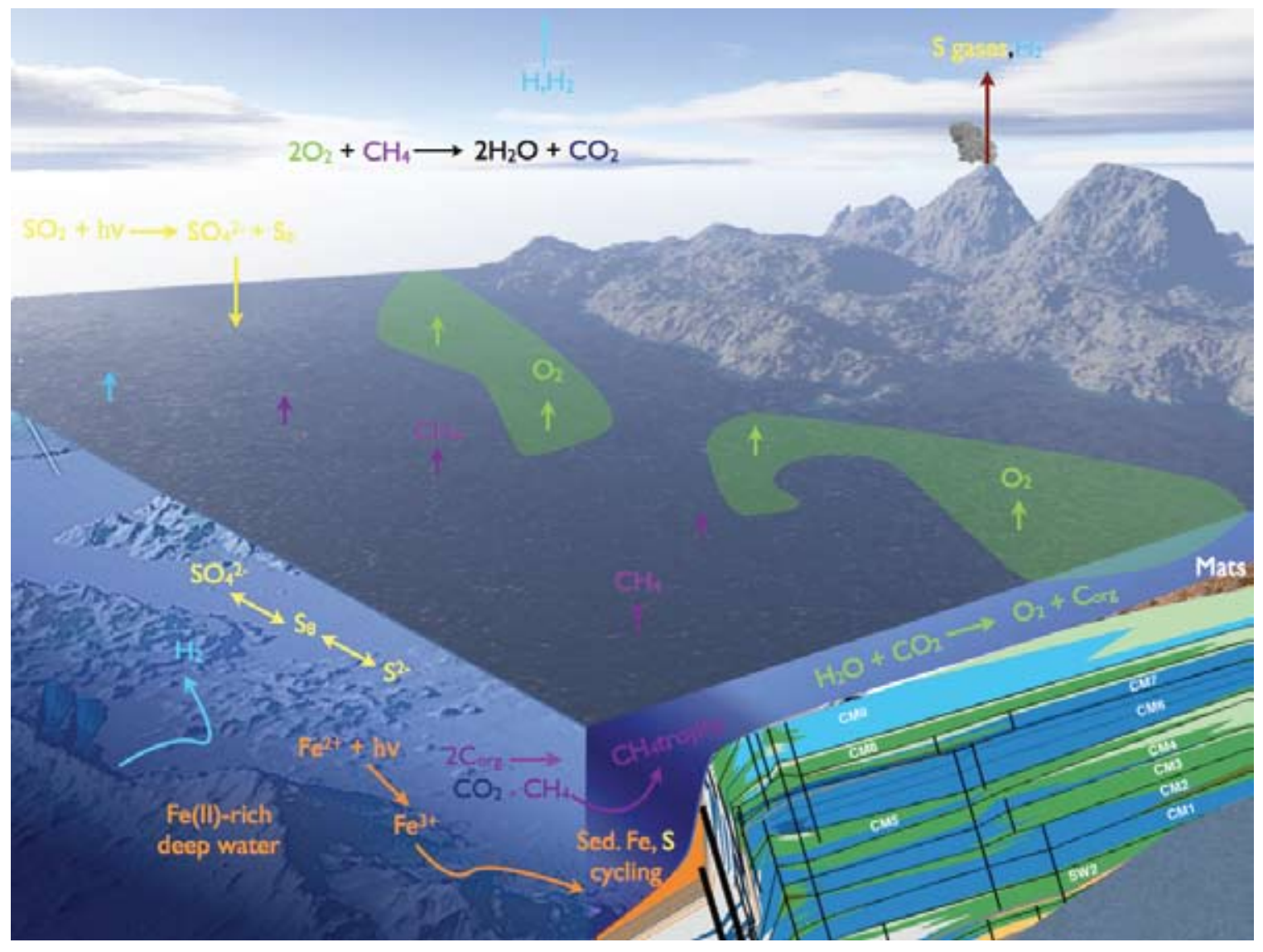

Figure 5. Cartoon representation of biogeochemistry in the late Archean ocean. In this depiction, localized oxygenic photosynthesis in coastal waters drives a sea-air $\mathrm{O}_{2}$ flux, which is balanced by a methane flux deriving from methanogenic diagenesis of aerobic net primary production. As a result, atmospheric chemistry with the same net reaction as aerobic methanotrophy acts to consume the biogenic gas flux. The activity of oxygenic and microaerobic marine microbial communities is recorded in coastal sedimentary deposits, such as the Transvaal Supergroup (sequence stratigraphy at lower right). Other indicated processes include: hydrothermal inputs of ferrous iron and molecular hydrogen to deep waters, iron-oxidizing photoautotrophy and sedimentary iron reduction/cycling, atmospheric sulfur photochemistry (the source of mass-independently fractionated $\mathrm{S}$ pools) and aquatic/sedimentary $\mathrm{S}$ cycling, volcanic $\mathrm{S}$ and $\mathrm{H}_{2}$ gas fluxes, and hydrogen escape to space. 
geologic record suggest that life was widely diversified. It seems that the majority of key biochemical networks, including energy-generating and biosynthetic pathways, were operative by the late Archean. Studies such as this one are showing that some classically aerobic biochemical pathways, including some characteristic of eukaryotic biology, are operative under the very low dissolved oxygen concentrations that may have been widespread and persistent during the late Archean.

It should be noted that the production of sterols does not strictly entail an oxygenated water column. It could be argued that the oxygen used to synthesize sterols in the Archean ocean was actually produced by the eukaryotes themselves, and that their presence in sedimentary organic matter reflects only the intracellular availability of $\mathrm{O}_{2}$. A remarkable example of eukaryotes maintaining intracellular $\mathrm{O}_{2}$ while living in anoxic environments was recently described by Esteban et al. (2009), who found that certain freshwater ciliates living just below an oxic-anoxic transition zone sequester still-active chloroplasts from algal prey in order to provide an oxygen supply for aerobic biochemistry. But while solely intracellular $\mathrm{O}_{2}$ is a conceivable explanation for sterol production in isolation, all eukaryotic photosynthesis is ultimately 'borrowed' from cyanobacteria, through either endosymbiosis or kleptoplasty as described by Esteban et al. (2009). Hence the presence of photosynthetic eukaryotes of any sort would mean that oxygenic cyanobacteria had already emerged, and thus more probable that oxygenation of the water column was underway to some extent.

Accurate reconstruction of the history of oxygen in the atmosphere and oceans and evolution of the organisms and biochemistry responsible for its production are central to understanding the reciprocal interactions between life and environments. The demonstration here that steroid biosynthesis is possible under microaerobic (but not anaerobic) conditions provides new context for the interpretation of the biomarker record and inferences about ocean biogeochemistry. The assumption that an anaerobic atmosphere means an anaerobic ocean is misleading, as biologically-sustained deviations 
from physical equilibria are common and important today and were in the distant past as well. Reconstructions of microbial evolution, often framed in the context of global, longterm trends, should keep in mind the heterogeneity of natural environments.

\section{ACKNOWLEDGEMENTS}

We are grateful to Alexa Price-Whelan and Paula Welander for guidance with anaerobic culturing techniques, Lars Dietrich for help with microelectrode measurements, and Maureen Coleman for discussions. This work was supported by grants from the Agouron Institute and the NASA Astrobiology Institute to RES, and by an Office of Naval Research NDSEG Fellowship and an NSF Graduate Research Fellowship to JRW.

\section{REFERENCES}

Allen, JF, and W Martin. 2007. Evolutionary biology: out of thin air. Nature 445, no. 7128: 610-612.

Anbar, AD, Y Duan, TW Lyons, GL Arnold, B Kendall, RA Creaser, AJ Kaufman, GW Gordon, C Scott, J Garvin, and R Buick. 2007. A whiff of oxygen before the great oxidation event? Science 317, no. 5846: 1903-1906.

Bligh, EG, and WJ Dyer. 1959. A rapid method of total lipid extraction and purification. Canadian Journal of Physiology and Pharmacology 37, 911-917.

Blumenberg, M, R Seifert, and W Michaelis. 2007. Aerobic methanotrophy in the oxic-anoxic transition zone of the Black Sea water column. Organic Geochemistry 38, no. 1: 84-91.

Bosak, T, B Liang, MS Sim, and AP Petroff. 2009. Morphological record of oxygenic photosynthesis in conical stromatolites. Proc Natl Acad Sci USA 106, no. 27: 10939-10943.

Brocks, JJ, GA Logan, R Buick, and RE Summons. 1999. Archean molecular fossils and the early rise of eukaryotes. Science 285, no. 5430: 1033-1036.

Brocks, JJ, R Buick, RE Summons, and GA Logan. 2003. A reconstruction of Archean biological diversity based on molecular fossils from the 2.78 to 2.45 billion-yearold Mount Bruce Supergroup, Hamersley Basin, Western Australia. Geochimica et Cosmochimica Acta 67, no. 22: 4321-4335.

Brooks, CJW, ES Horning and JS Young. 1968. Characterization of Sterols by Gas Chromatography-Mass Spectrometry of the Trimethylsilyl Ethers. Lipids 3, no. 5: 391-402.

Buick, R. 2008. When did oxygenic photosynthesis evolve? Philosophical Transactions of the Royal Society B: Biological Sciences 363, no. 1504: 2731.

Catling, DC, KJ Zahnle, and C McKay. 2001. Biogenic methane, hydrogen escape, and the irreversible oxidation of early Earth. Science 293, no. 5531: 839-843. 
Catling, DC, and MW Claire. 2005. How Earth's atmosphere evolved to an oxic state: a status report. Earth and Planetary Science Letters 237, no. 1-2: 1-20.

Claire, MW, DC Catling, and KJ Zahnle. 2006. Biogeochemical modelling of the rise in atmospheric oxygen. Geobiology 4, no. 4: 239.

Coogan, L. 2009. Did natural reactors form as a consequence of the emergence of oxygenic photosynthesis during the Archean? GSAT 19, no. 10: 4-10.

de Duve, C. 2007. The origin of eukaryotes: a reappraisal. Nature Reviews Genetics 8, no. 5: 395-403.

Desmond, E and S Gribaldo. 2009. Phylogenomics of Sterol Synthesis: Insights into the Origin, Evolution, and Diversity of a Key Eukaryotic Feature. Genome Biology and Evolution 1, no. 1: 364-381.

Dutkiewicz, A, H Volk, SC George, J Ridley, and R Buick. 2006. Biomarkers from Huronian oil-bearing fluid inclusions: an uncontaminated record of life before the Great Oxidation Event. Geology 34, no. 6: 437.

Eigenbrode, JL, KH Freeman, and RE Summons. 2008. Methylhopane biomarker hydrocarbons in Hamersley Province sediments provide evidence for Neoarchean aerobiosis. Earth and Planetary Science Letters 273, no. 3-4: 323-331.

Esteban, GF, BJ Finlay, and KJ Clarke. 2009. Sequestered organelles sustain aerobic microbial life in anoxic environments. Environmental Microbiology 11, no. 2: 544-550.

Frei, R, C Gaucher, SW Poulton, and DE Canfield. 2009. Fluctuations in Precambrian atmospheric oxygenation recorded by chromium isotopes. Nature 461, no. 7261: 250-253.

Galea, AM, and AJ Brown. 2009. Special relationship between sterols and oxygen: Were sterols an adaptation to aerobic life? Free Radical Biology and Medicine 47, no. 6: 880-889.

George, SC, H Volk, A Dutkiewicz, J Ridley, and R Buick. 2007. Preservation of hydrocarbons and biomarkers in oil trapped inside fluid inclusions for $>2$ billion years. Geochimica et Cosmochimica Acta

Glover, DM, NM Frew, and SJ McCue. 2007. Air--sea gas transfer velocity estimates from the Jason-1 and TOPEX altimeters: prospects for a long-term global time series. Journal of Marine Systems 66, no. 1-4: 173-181.

Goldblatt, C, TM Lenton, and AJ Watson. 2006. Bistability of atmospheric oxygen and the Great Oxidation. Nature 443, no. 7112: 683-686.

Hayes, JM, and JR Waldbauer. 2006. The carbon cycle and associated redox processes through time. Philos Trans $R$ Soc Lond, B, Biol Sci 361, no. 1470: 931-950.

Jahnke, L, and HP Klein. 1983. Oxygen requirements for formation and activity of the squalene epoxidase in Saccharomyces cerevisiae. J Bacteriol 155, no. 2: 488-492.

Johnston, D, F Wolfe-Simon, A Pearson, and AH Knoll. 2009. Anoxygenic photosynthesis modulated Proterozoic oxygen and sustained Earth's middle age. Proceedings of the National Academy of Sciences

Kettle, AJ, MO Andreae, D Amouroux, TW Andreae, TS Bates, H Berresheim, H Bingemer, R Boniforti, MAJ Curran, and GR DiTullio. 1999. A global database of sea surface dimethylsulfide (DMS) measurements and a procedure to predict 
sea surface DMS as a function of latitude, longitude, and month. Global Biogeochemical Cycles 13, no. 2:

Kirschvink, JL, and RE Kopp. 2008. Palaeoproterozoic ice houses and the evolution of oxygen-mediating enzymes: the case for a late origin of photosystem II.

Philosophical Transactions of the Royal Society B: Biological Sciences 363, no. 1504: 2755.

Kloster, S, KD Six, J Feichter, E Maier-Reimer, E Roeckner, P Wetzel, P Stier, and M Esch. 2007. Response of dimethylsulfide (DMS) in the ocean and atmosphere to global warming. Journal of Geophysical Research-Biogeosciences 112, no. G3: G03005.

Kopp, RE, JL Kirschvink, IA Hilburn, and CZ Nash. 2005. The Paleoproterozoic snowball Earth: a climate disaster triggered by the evolution of oxygenic photosynthesis. Proc Natl Acad Sci USA 102, no. 32: 11131-11136.

Najjar, RG, and RF Keeling. 2000. Mean annual cycle of the air-sea oxygen flux: A global view. Glob Biogeochem Cycles 14, no. 2: 573-584.

Pavlov, AA, and JF Kasting. 2002. Mass-independent fractionation of sulfur isotopes in Archean sediments: strong evidence for an anoxic Archean atmosphere. Astrobiology 2, no. 1: 27-41.

Reinhard, CT, R Raiswell, C Scott, AD Anbar, and TW Lyons. 2009. A Late Archean Sulfidic Sea Stimulated by Early Oxidative Weathering of the Continents. Science 326, no. 5953: 713-716.

Revsbech, NP, LH Larsen, J Gundersen, T Dalsgaard, O Ulloa, and B Thamdrup. 2009. Determination of ultra-low oxygen concentrations in oxygen minimum zones by the STOX sensor. Limnol. Oceanogr. Methods 7, 371-381.

Rogers, PJ, and P Stewart. 1973a. Mitochondrial and peroxisomal contributions to the energy metabolism of Saccharomyces cervisiae in continuous culture. Journal of General Microbiology 79, 205-217.

Rogers, PJ, and PR Stewart. 1973b. Respiratory development in Saccharomyces cerevisiae grown at controlled oxygen tension. J Bacteriol 115, no. 1: 88-97.

Rosenfeld, E, B Beauvoit, B Blondin, and J-M Salmon. 2003. Oxygen consumption by anaerobic Saccharomyces cerevisiae under enological conditions: effect on fermentation kinetics. Appl Environ Microbiol 69, no. 1: 113-121.

Rutten, MG. 1970. The history of atmospheric oxygen. Space Life Sciences 2, 5-17.

Sessions, AL, DM Doughty, PV Welander, RE Summons, and DK Newman. 2009. The continuing puzzle of the great oxidation event. Curr Biol 19, no. 14: R567-74.

Sherman, LS, JR Waldbauer, and RE Summons. 2007. Improved methods for isolating and validating indigenous biomarkers in Precambrian rocks. Organic Geochemistry 38, no. 12: 1987-2000.

Stolper, D, N Revsbech, and DE Canfield. 2009. Growth of E. coli at nanomolar concentrations of oxygen. American Geophysical Union Annual Meeting Abstracts.

Summons, RE, LL Jahnke, JM Hope, and GA Logan. 1999. 2-Methylhopanoids as biomarkers for cyanobacterial oxygenic photosynthesis. Nature 400, no. 6744: 554-557. 
Summons, RE, AS Bradley, LL Jahnke, and JR Waldbauer. 2006. Steroids, triterpenoids and molecular oxygen. Philosophical Transactions B 361, no. 1470: 951.

Zahnle, K, M Claire, and D Catling. 2006. The loss of mass-independent fractionation in sulfur due to a Palaeoproterozoic collapse of atmospheric methane. Geobiology 4, no. 4: 271-283. 


\section{Chapter Four}

A multilevel view of gene expression and regulation in the Prochlorococcus diel cell cycle

Jacob R. Waldbauer, Sébastien Rodrigue, Maureen L. Coleman, and Sallie W. Chisholm 


\section{A multilevel view of gene expression and regulation in the diel cell cycle of Prochlorococcus}

Jacob R. Waldbauer, Sébastien Rodrigue, Maureen L. Coleman and Sallie W. Chisholm

The cell division cycle of Prochlorococcus populations is tightly coupled to the diel cycle of light and darkness, with chromosome replication in the late afternoon being followed by cellular fission after sunset. Experiments in culture have shown that the mRNA-level expression profiles of most genes also have a diel rhythm. Here we quantify the abundance and periodicity of the Prochlorococcus proteome over a diel cycle and compare transcriptlevel and protein-level expression patterns. Strong diel oscillations in transcript abundance are broadly damped at the protein level, and temporal offsets between the two suggest that posttranslational regulatory mechanisms are important in determining the abundance dynamics of a number of proteins. The overall composition of the proteome is quite stable over the diel cycle, with some proteins consistently among the most abundant in the cell at all times of day. The small changes in protein abundance that accompany significant changes in central metabolic activities imply that Prochlorococcus biochemical networks may be poised near balance points that allow for redirection of metabolic fluxes with relatively small changes in the abundance of their components.

\section{INTRODUCTION: THE IMPORTANCE OF THE DIEL CYCLE TO LIFE IN THE}

\section{SUBTROPICAL EPIPELAGIC}

The twenty-four hour alternation of light and darkness caused by the earth's rotation is one of the strongest periodicities imposed on natural systems. Since the sun is the ultimate source of nearly all energy input to ecosystems, its diurnal motion has dramatic consequences for the activity of surface-dwelling organisms ranging from bacteria to humans. This is especially true for phototrophs, whose physiology is directly tied to solar energy utilization. In the ocean, the great majority of photosynthesis is carried out by microbes, whose small body sizes require them to closely balance metabolic demands of growth with the availability of light energy. In the low-latitude epipelagic where Prochlorococcus is a dominant primary producer, the diel cycle is one of the main timevarying properties of the ecosystem. 
As the most abundant photosynthetic organism on earth and the base of the food web in vast areas of the open ocean, Prochlorococcus is a central player in marine biogeochemistry. Shortly after its discovery and initial characterization in the late 1980s, a remarkable property of Prochlorococcus populations was observed: their growth is clearly synchronized to the diel cycle. Fortuitously, the tool used to discover and identify Prochlorococcus in the ocean, flow cytometry, is also ideal for assessing cell cycling, and utilization of DNA-binding fluorescent dyes allowed the cell cycle progression of natural communities to be tracked quantitatively. A well-conserved pattern emerged: cells replicate their DNA in the afternoon and divide from around sunset into the evening (Vaulot et al., 1995). With generally one cell division per day (though see Shalapyonok et al., 1998), this synchronization results in well-defined B-, C- and D-phases equivalent to the $\mathrm{G}_{1}, \mathrm{~S}$ and $\mathrm{G}_{2}+\mathrm{M}$ phases, respectively, of eukaryotic cells (Wang and Levin, 2009).

The diel cell cycle of Prochlorococcus has one additional characteristic that has enabled its detailed investigation: it can be reproduced in laboratory culture. Principal cell cycle parameters of axenic cultures maintained on a light-dark cycle, including growth rate and the length of DNA synthesis and cell division phases, are similar to those observed in field populations - suggesting that the biology occurring in the laboratory is at least a first-order representation of what occurs in nature. A number of studies have explored aspects of the Prochlorococcus diel cell cycle using a variety of experimental arrangements, and have added insights into changes in photophysiology (Bruyant et al., 2005) and optical properties (Claustre et al., 2002) as well as in the expression of selected photosynthesis- (Garczarek et al., 2001) and cell cycle-related (Holtzendorff et al., 2001) genes.

A further intriguing aspect of diel cell cycling in Prochlorococcus is the absence of the kind of true circadian clock that has been documented in other cyanobacteria. Typical cyanobacterial circadian clock systems are comprised of three proteins, KaiA, KaiB and KaiC. The molecular basis of the clock is a 24-hour oscillation in the phosphorylation 
state of KaiC, with KaiA promoting phosphorylation and KaiB promoting dephosphorylation. All sequenced Prochlorococcus strains, however, lack a homologue of KaiA. Holtzendorff et al. (2008) have demonstrated that the diel coordination of growth and associated expression oscillations damp rapidly (at least in terms of their population averages) when cultures are shifted to continuous light, implying that the Prochlorococcus timing system relies on external cues during each photoperiod. Recently, Axmann et al. (2009) showed that the KaiC of Prochlorococcus MED4 is hyperphosphorylated by default, apparently enabling the KaiBC-only system to oscillate. It may be that a streamlined, less robust timing mechanism is sufficient for Prochlorococcus because it inhabits the low-latitude open ocean, and therefore experiences smaller seasonal changes in day length than phototrophs at higher latitudes or those in freshwater settings where shading by vegetation or sediment is more likely (Axmann et al., 2009; Mullineaux and Stanewsky, 2009).

The most comprehensive molecular picture of the Prochlorococcus diel cell cycle to date has come from the work of Zinser et al. (2009), who used microarrays to track gene expression genome-wide with 2-hour resolution over two consecutive diel periods in axenic cultures of Prochlorococcus MED4. This study found that $82 \%$ of transcripts of protein-coding genes had a detectable circadian oscillation of expression. The high proportion of transcripts cycling with 24 hour periodicity suggests that the diel cycle could be seen as the 'master regulator' of Prochlorococcus gene expression - though how this regulation is effected is unclear. The MED4 genome encodes only a small number of the complement of regulatory systems typically found in bacteria (Mary and Vaulot, 2003; Vogel et al., 2003; Kielbasa et al., 2007). Small RNAs, including antisense transcripts, likely play important regulatory roles (Steglich et al., 2008; Richter et al., 2010).

In light of these previous findings, the experiment described here was designed to address two key aspects of the diel cell cycle in Prochlorococcus. One is the regulatory 
underpinnings of the observed transcriptional program. The small complement of regulatory systems in MED4 makes a genome-wide analysis of the dynamics of the transcriptional regulatory network feasible. Using a combination of 5'- and 3'-RACE (Rapid Amplification of cDNA Ends) and ChIP-Seq (Chromatin Immunoprecipitation and Sequencing) techniques, the binding sites and DNA association patterns of transcription factors can be mapped over the diel cycle. This work is in progress and will be presented elsewhere (Rodrigue et al., in prep.). This chapter presents the second focus of the diel growth experiment: the relationship between gene expression at the transcript and protein levels.

The central dogma of molecular biology holds that gene sequence information in cells generally flows from DNA to RNA to protein. While this specifies the topology of the chemical network involved in gene expression, it leaves open the question of the relative abundances of gene products. It is clear that the relative abundance of a gene at the RNA level is not readily predictable from its abundance at the DNA level: two genes each present at a single copy in the genome can be transcribed at very different rates. The same is true for the translation of RNA into protein. Transcript abundance, while undoubtedly a major factor influencing protein level, does not fully determine, predict or explain it. The observed abundance of a gene product reflects the dynamic balance between production (by transcription or translation) and degradation, hence the motivation to complement the mRNA-level picture of diel cyclic gene expression in Prochlorococcus with a protein-level one.

In biogeochemical terms, to understand how organisms effect molecular transformations in the environment, protein quantification is essential. Proteins are the catalysts for many biogeochemically-significant reactions, and themselves represent a large proportion of the nutrient requirements of microbial cells. Large-scale gene expression measurements are coming to the fore as assays of the activities and physiological states of microbial communities, including investigations of the diel cycle in marine surface waters 
(Poretsky et al., 2009). If protein and transcript levels diverge, however, the mRNA-level view of biological activity may be misleading. It is therefore increasingly important to quantify the relationships between gene products at multiple biological levels, so that accurate inferences about community function can be drawn from biochemical measurements.

We also sought to address the relationship between the proportional cellular abundances of mRNAs and proteins. Previous microarray analyses (Zinser et al., 2009) have accurately resolved relative changes in mRNA-level gene expression (that is, changes in the abundance of a given transcript over time), but, due to widely varying hybridization efficiencies and the potential for saturation of the microarray signal (Levicky and Horgan, 2005), it has been difficult to compare the abundance of different gene products at a given time. The use of parallel RNA-sequencing and mass spectrometry to measure transcript and protein abundance, respectively, allow comparison of abundances between gene products. Hence we obtain a first view of the rank-abundance structure of Prochlorococcus gene products at the transcript and protein levels and how it changes over the diel cell cycle. The relationships between transcript and protein abundance are an important component of systems-level understanding of cellular functions and the biogeochemistry they participate in.

\section{EXPERIMENTAL METHODS \& TECHNICAL DISCUSSION}

\subsection{Diel growth experiment}

\subsubsection{Culture conditions}

Axenic Prochlorococcus MED4 was grown in duplicate batches in 30L acid-cleaned polycarbonate carboys (Nalgene) in a modified I-66LL illuminated incubator (Percival Scientific), depicted in Figure 1A. The illumination in the incubator was controlled by custom PID-controlled dimmer circuitry and programmed to match a diel irradiance 

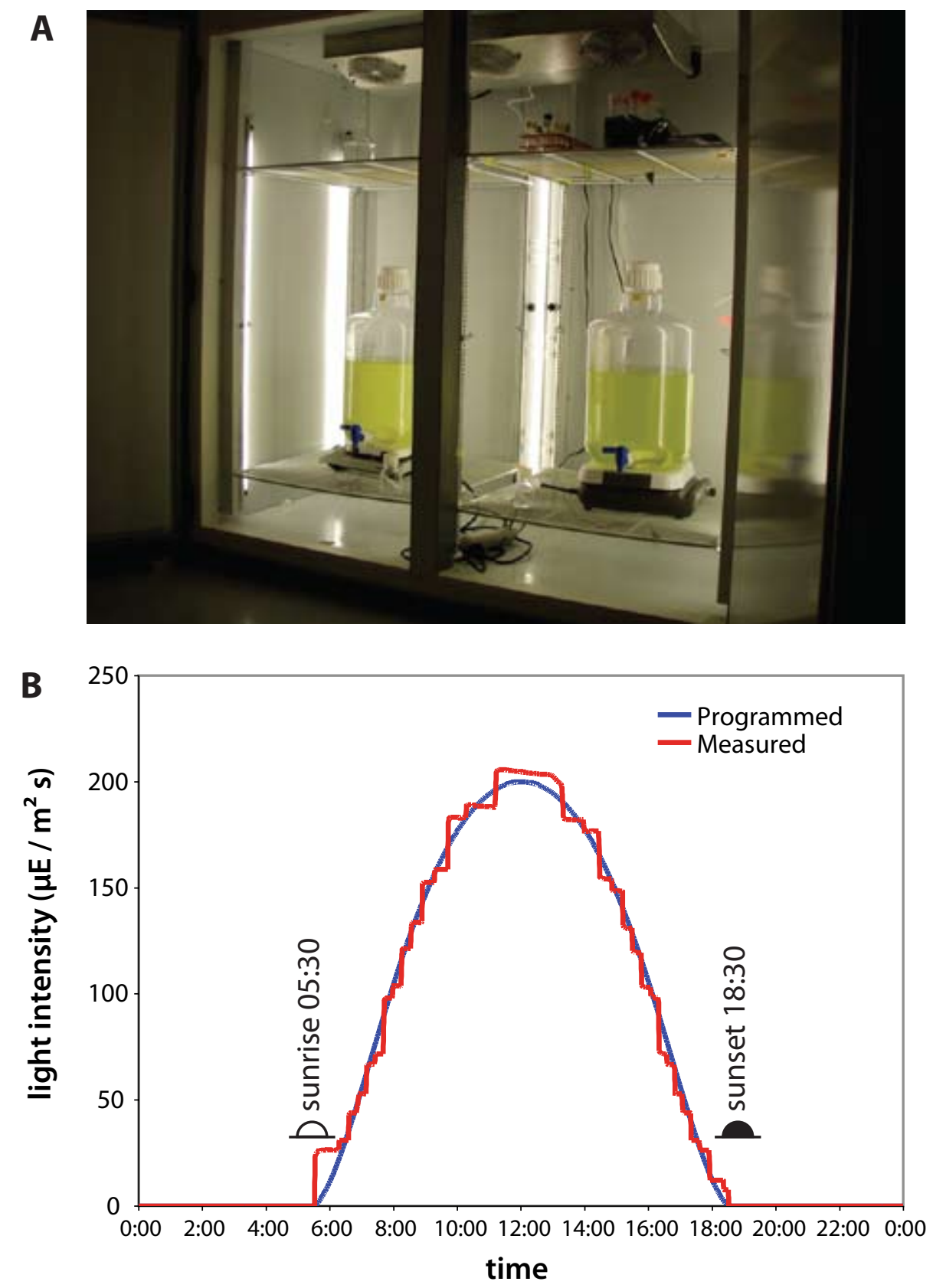

Figure 1. A Photograph of Prochlorococcus MED4 batch cultures in sunbox incubator. B Programmed and measured irradiance curves for the sunbox. Programmed curve derived from data from the Hawaii Ocean Time-series (HOT) station ALOHA. 
curve from the Hawaii Ocean Time-series station ALOHA, as shown in Figure 1B. Temperature in the incubator was maintained at $24^{\circ} \mathrm{C}$. Each culture was stirred continuously by a large teflon-coated magnetic stir bar. Prior to inoculation of the largevolume batch cultures for this experiment, the culture had been maintained for several months in the same incubator to ensure synchronization to the light-dark cycle. The culture medium was a modified version of Pro99 (Moore et al., 2007) based on Vineyard Sound seawater (collected at Woods Hole, MA) and pH-buffered with 10mM HEPES and $6 \mathrm{mM}$ sodium bicarbonate. Starting culture volume for the diel growth experiment was 20L. Separately, Prochlorococcus MED4 labeled with ${ }^{15} \mathrm{~N}$ were prepared by growing cells from the same axenic strain used in the diel growth experiment on Pro99 medium in continuous light, where the ammonium chloride that is the sole fixed $\mathrm{N}$ source in the medium was $>99 \%{ }^{15} \mathrm{NH}_{4} \mathrm{Cl}$ (Cambridge Isotope). A 1L culture was grown to lateexponential phase and harvested by centrifugation as described below, with samples preserved for flow cytometry to provide accurate cell counts of the pellets.

\subsubsection{Sampling}

During the diel growth experiment, samples were taken every two hours over a 26 hour span, beginning at local midnight, resulting in 14 total timepoints. At each timepoint, $500 \mathrm{ml}$ samples of each culture were withdrawn using spigots mounted at the bottom of the carboys and dispensed in to $2 \times 250 \mathrm{ml}$ centrifuge bottles (Nalgene). For sampling during dark and low-light periods, a low-power green lamp was used to provide indirect work light. Additionally, five $1 \mathrm{ml}$ samples of the culture were preserved with $0.125 \%$ glutaraldehyde (Tousimis) in cryovials (Nunc) for flow cytometric determination of cell density and growth cycling. Large-volume samples were centrifuged at $16,000 \times g$ for 10 minutes in an Avanti J-10 centrifuge (Beckman Coulter). After pipetting off the supernatant, the two pellets from each culture were resuspended, combined in a $15 \mathrm{ml}$ conical tube (VWR), and the volume brought to $5 \mathrm{ml}$ with Pro99 media. Duplicate $10 \mu 1$ aliquots of the resuspended concentrate were diluted $1: 100$ with $0.125 \%$ glutaraldehyde in Pro99 in for flow cytometric analysis to ensure precise and accurate determination of 
cell counts in the sample pellets. The remainder of the concentrate was then split into $2 \times 2 \mathrm{ml}$ and $4 \times 0.25 \mathrm{ml}$ aliquots in $2 \mathrm{ml}$ microcentrifuge tubes (Sarstedt), the former for RNA-sequencing and the latter for proteomics. These aliquots were then centrifuged for 6 minutes at 14,000 $\times g$ in a 5418 centrifuge (Eppendorf). The supernatant was then removed and preserved with $5 \mu 125 \%$ glutaraldehyde in cryovials for flow cytometric counting to assess pelleting efficiency. Cell pellets and flow cytometry samples were kept frozen at $-80^{\circ} \mathrm{C}$ until analysis.

\subsubsection{Flow cytometry}

Cell counts and cell cycle were analyzed using an InFlux flow cytometer (Cytopeia). Glutaraldehyde-fixed samples were diluted in filtered sterile seawater to appropriate concentrations. Light scatter and fluorescence signals were detected using a $488 \mathrm{~nm}$ excitation beam, triggering on forward (small-angle) light scatter and counting cells on the basis of scatter characteristics and chlorophyll fluorescence. Cell cycle analysis was performed using SYBR Green (Invitrogen) to stain DNA. Flow cytometry data were analyzed using FlowJo (TreeStar).

\subsection{Proteomics sample preparation}

\subsubsection{Protein extraction}

Prochlorococcus cell pellets were resuspended in $2 \times$ LDS buffer (Invitrogen) with $10 \mathrm{mM}$ dithiothreitol (DTT), vortexed, and incubated in a heat block at $95^{\circ} \mathrm{C}$ for 20 minutes. They were subsequently vortexed again and incubated at $37^{\circ} \mathrm{C}$ for 60 minutes. After cooling to room temperature, iodoacetamide $\left(0.5 \mathrm{M}\right.$ in $\left.100 \mathrm{mM} \mathrm{NH}_{4} \mathrm{HCO}_{3}\right)$ was added to a concentration of $45 \mathrm{mM}$ and incubated at room temperature in the dark for 60 minutes to alkylate cysteine residues. DTT (2M) was then added to a concentration of $45 \mathrm{mM}$ (assumed to be $10 \mathrm{mM}$ after quenching of residual iodoacetamide). Volumes of extraction buffer and reduction/alkylation reagents were chosen to result in a final concentration of $1 \times 10^{9}$ extracted cells $/ 50 \mu 1$. Extract from sample pellets was then mixed 
1:1 by cell numbers (established previously by flow cytometry) with extract from an identically and simultaneously processed ${ }^{15} \mathrm{~N}$-labeled cell pellet.

\subsubsection{SDS-PAGE}

Extract from up to $1 \times 10^{9}$ total cells was loaded per lane into 10 -lane, $10 \mathrm{~cm} \times 1.5 \mathrm{~mm} 10 \%$ SDS-PAGE gels (NuPAGE, Invitrogen) with MOPS running buffer (Invitrogen). Gels were run at $50 \mathrm{~mA}$ per gel with water cooling to $25^{\circ} \mathrm{C}$ in a vertical format (SE260, Hoefer) for 90 minutes. Benchmark protein ladder (Invitrogen) was used as a molecular weight standard. After electrophoresis, gels were removed from their cases and fixed in $50 \%$ ethanol $/ 7.5 \%$ acetic acid overnight on an orbital shaker. They were then rehydrated in Milli-Q water for 30 minutes before staining with SimplyBlue coomassie stain (Invitrogen). Gels were imaged on a flatbed scanner prior to slicing with a razor blade into $\sim 1 \mathrm{~mm}$ cubes. Eight separate molecular weight fractions were prepared for each timepoint. The fastest-running component of the extract (the bottom-most band, rich in chlorophyll as evidenced by its green color during electrophoresis) was excised and not included in further processing. The gel slices were transferred to $1.5 \mathrm{ml}$ microcentrifuge tubes (Axygen) and destained by shaking for 4 hours in 50\% ethanol/ $7.5 \%$ acetic acid at $37^{\circ} \mathrm{C}$. The destain solution was then changed and the gel pieces returned to the incubator/shaker overnight.

\subsubsection{Trypsin digestion}

The destained gel pieces were washed five times: twice alternating acetonitrile $/ 50 \mathrm{mM}$ ammonium bicarbonate for 20 minutes each, then a final acetonitrile wash until fully dehydrated. The acetonitrile was then removed and the dry pieces chilled on ice. Sequencing grade modified porcine trypsin (Promega) was diluted in 10\% acetonitrile in $50 \mathrm{mM}$ ammonium bicarbonate and chilled on ice. Sufficient trypsin solution was added to the sample tubes to cover the gel pieces $(\sim 100-200 \mu 1 /$ tube $)$, and the incubation continued on ice until the gel pieces were clear. The tubes were then incubated for 24 
hours at $37^{\circ} \mathrm{C}$, at which point $200 \mu \mathrm{l}$ of $10 \%$ acetonitrile in $50 \mathrm{mM}$ ammonium bicarbonate was added and incubated for a further 24 hours at $37^{\circ} \mathrm{C}$.

\subsubsection{Peptide extraction}

After incubation with trypsin for 48 hours, the liquid from the digestion tubes was transferred to a new set of $1.5 \mathrm{ml}$ microcentrifuge tubes, which were kept at $4{ }^{\circ} \mathrm{C} .200 \mu 1$ of $10 \%$ acetonitrile in $50 \mathrm{mM}$ ammonium bicarbonate was added to the pieces and incubated

for 1 hour at $37^{\circ} \mathrm{C}$ before being pooled with the initial extract. This process was repeated once more with $10 \%$ acetonitrile in $50 \mathrm{mM}$ ammonium bicarbonate and then twice with $50 \%$ acetonitrile in $0.1 \%$ formic acid; total extract volume was $\sim 1 \mathrm{ml}$. Extracts were frozen solid at $-80^{\circ} \mathrm{C}$ before being concentrated in a vacuum centrifuge for 4-5 hours at $30^{\circ} \mathrm{C}$. The dried extracts were then resuspended in $500 \mu 1$ of $5 \%$ acetonitrile in $0.25 \%$ formic acid, vortexed vigorously, and centrifuged for 10 minutes at $14,000 \times \mathrm{g}$ to pellet residual gel fragements and debris. The supernatant was removed, frozen solid at $-80^{\circ} \mathrm{C}$ and finally concentrated by vacuum centrifuge for $\sim 2$ hours at $30^{\circ} \mathrm{C}$.

\subsection{LC-MS/MS analysis}

\subsubsection{Liquid chromatography/nanoelectrospray ionization}

Frozen, dried peptide fractions ( 8 per timepoint) were resuspended in $10 \mu 1$ of $5 \%$ acetonitrile in $0.25 \%$ formic acid and transferred to a 384-well plate (Nunc). $6 \mu 1$ of each sample was injected via qualitative $\mu 1$ pickup with a MicroAS autosampler (Thermo/Spark Holland) fitted with a $10 \mu 1$ sample loop. Mobile phase was delivered by a Surveyor LC pump (Thermo) fitted with a backpressure regulator (P-880, Upchurch) and a fixed-T flow splitter (ratio $90: 1$ ). Peptides were loaded onto a reversed-phase capillary LC column (Hypersil Gold $\mathrm{C}_{18}, 0.18 \times 100 \mathrm{~mm}, 3 \mu \mathrm{m} \times 175 \AA$ particles, Thermo). The mobile phase system for the LC consisted of $0.1 \%$ formic acid in water (buffer A) and $0.1 \%$ formic acid in acteonitrile (B). Solvents for LC-MS were HPLC grade (Burdick \& Jackson). Peptides were eluted with a gradient of 5\% to $37.5 \%$ B over 105 
minutes, followed by a ramp to $100 \% \mathrm{~B}$ and washing for 25 minutes, then 40 minutes reequilibration with $5 \% \mathrm{~B}$. Column flow rate was $1.3 \mu \mathrm{l} / \mathrm{min}$, and total $\mathrm{LC}$ cycle time was 180 minutes. The LC system was interfaced to the mass spectrometer through an TriVersa Nanomate nanospray source/fraction collector (Advion). Post-column flow split between the nanospray chip and the collector mandrel was 1:2, resulting in a flow of $\sim 400 \mathrm{nl} / \mathrm{min}$ to the chip. The spray chip was operated at a voltage of $1.6-1.8 \mathrm{kV}$, and spray current was monitored to ensure ionization stability.

\subsubsection{Mass spectrometry}

Mass spectral data was acquired on a LTQ-FT Ultra (Thermo) in a data-dependent manner. Each full scan in the ICR cell (profile mode, m/z 300-1600, resolution 100,000) was followed by 4 CID MS/MS scans on selected precursors in the linear ion trap. Dynamic exclusion was enabled, with repeat count set to 2 and exclusion duration 30 seconds. Singly charged ions, ions whose charge state could not be assigned, and common contaminant ions were excluded from MS/MS precursor selection. The LTQFT ion optics were tuned on a mixture of angiotensin (Calbiochem) and bradykinin (Genscript) for best sensitivity and transmission of peptide ions.

\subsubsection{Peptide/protein identification}

Mass spectral data acquired by Xcalibur (v. 2.0, Thermo .RAW format) was converted to mzXML format with ReAdW (v. 4.3.1). Peptide-spectrum matching was done against a database consisting of the Prochlorococcus MED4 genome (Rocap et al., 2003), its reversed complement, and a set of common contaminant proteins including porcine trypsin and human keratins. Three MS/MS database search algoriths were employed: X!Tandem (v. TORNADO, Craig and Beavis, 2004; with the k-score plugin, MacLean et al., 2006), MyriMatch (v. 060509, Tabb et al., 2007) and OMSSA (v. 2.1.7, Geer et al., 2004). For all search engines, semi-tryptic searches were conducted with two missed cleavages allowed. Amino acid modfications included static carbamidomethylated cysteine, variably oxidized methionine, and variable formation of pyro-glutamine, - 
glutamate or -carbamidomethylcysteine on the N-termini of peptides. For OMSSA searching, a concatenated DTA file (.odta format) was first produced from each mzXML file using mzXML2Search, and spectra were then searched on the Darwin computing cluster at MIT. Output from the 3 search engines was converted (if necessary) to pepXML format and a Perl script used to unify their formats. Each pepXML file was then processed with PeptideProphet (Keller et al., 2002) to assign probabilities to each peptide identification; peptides longer than 6 amino acids were considered, and accurate mass information was used. The peptide identifications in the 24 pepXML files from each timepoint ( 8 gel slices $\times 3$ search engines) were merged using iProphet (Shteynberg et al., in prep; all models enabled). A Perl script was used to reindex the merged pepXML file, to ensure that each peptide ID had a unique index value. Peptide identifications were then assigned to proteins by ProteinProphet (Nesvizhskii et al., 2003) using Occam's razor logic, and requiring a minimum PeptideProphet score of 0.05 to filter out the weakest spectrum IDs. Further filtering based on an arbitrary ProteinProphet score cutoff was not necessary at this point (except to exclude those with scores of 0.00 ), as the false discovery rate (FDR) was already acceptably low: 33 decoy

proteins were identified among 1021 MED4 proteins, for a nominal dataset-wide proteinidentification FDR of 3.2\% (Supplementary Fig. 2). If only proteins identified at 2 or more timepoints are considered, the FDR was 1.6\% (14 decoys among 873 MED4 proteins). As discussed below (Sec. 2.4.1), the process of constructing expression timecourses eliminated all decoy data, resulting in a protein-ID FDR in the time-series data of $<0.2 \%$.

\subsection{Protein timecourse quantification}

\subsubsection{Isotope-labeling-based quantification}

The method of quantification used here to construct timecourses of protein abundance over the diel sampling period is based on the use of isotopically-labeled internal standards. Metabolic isotope labeling, here using ${ }^{15} \mathrm{~N}$, affords an internal standard for 
virtually every peptide observed in the sample. Mixing the samples taken at each diel sampling timepoint with aliquots of identical ${ }^{15} \mathrm{~N}$-labeled cells at the earliest stage of protein extraction minimizes the effect of subsequent experimental biases (protein extraction yields, peptide ionization efficiencies, etc.) on the observed abundance ratio. Accurate cell counts ensure that the ${ }^{14} \mathrm{~N} /{ }^{15} \mathrm{~N}$ mixing ratio was near 1:1. Analysis of quantitative proteomics data using ${ }^{15} \mathrm{~N}$-labeling does present challenges, however, not the least of which is the variable mass difference between sample and standard. Details of the method used to construct timecourses of protein abundance over the diel cycle is described below.

MS ${ }^{1}$ peaks corresponding to ${ }^{15} \mathrm{~N}$-labeled versions of identified MED4 peptides were matched to their unlabeled, coeluting partners by ASAPRatio (Li et al., 2003), and the abundance ratios of the ${ }^{15} \mathrm{~N}$ - versus ${ }^{14} \mathrm{~N}$-peaks were computed. A window of $0.05 \mathrm{~m} / \mathrm{z}$ was used for integration, background correction was set to zero, multiple charge states were used for quantification, and peakgroup pairs were constrained to the same elution time range. ASAPRatio estimates an integrated intensity error for each peak by the difference between the integrated raw intensity and the integrated area under a fitted peak generated by a Savitzky-Golay smoothing filter; this error was used to calculate a coefficient of variation (CV) for each individual peakgroup ratio. The set of quantified peakgroup ratios for the entire dataset was extracted from the protXML files for each timepoint, and a data matrix constructed and subsequent analyses performed in MATLAB (MathWorks). Redundant values for the same physical LC-MS feature were removed by requiring a $5 \%$ integrated intensity difference for duplicate, identical ratio values for a given peptide at a given timepoint. The total dataset comprised isotope ratios for 95,542 unique peakgroup pairs - here referred to as "peaks" for brevity, but actually representing in excess of 500,000 distinct LC-MS features, including both ${ }^{14} \mathrm{~N}$ - and ${ }^{15} \mathrm{~N}$ partners and ${ }^{13} \mathrm{C}$-isotopologues for each peptide across multiple charge states. 
The set of unique peak ratios was then filtered to remove peaks with a ratio $\mathrm{CV}$ greater than $41 \%$, corresponding to the $80^{\text {th }}$ percentile in $\mathrm{CV}$ ranking. These high-CV peaks are generally of low intensity and/or poor peak shape, making them more prone to quantification errors. The remaining data were then $\log _{2}$-transformed, and the peak ratios were adjusted to compensate for slight variations away from 1:1 in the mixing ratio of ${ }^{14} \mathrm{~N}$ - and ${ }^{15} \mathrm{~N}$-protein at different timepoints. This was done by finding the median of the $\log _{2}$-peak ratios between -1 and 1 for each timepoint, and subtracting that value from each peak ratio at that timepoint. This normalization procedure ensures that the peak ratio distributions for each timepoint have a common median (i.e., 0 on a logarithmic scale) and that timecourse variations for indivdual proteins are not due to systematic biases in the overall dataset.

Protein-level expression timecourses were constructed from the peak-level data in the following manner: for a given protein, if four or more peaks were found at eight or more timepoints, those timepoints were included in the timecourse. If a timecourse could not be constructed with those criteria, the threshold number of peaks required for that protein was sequentially lowered (to 3,2 , or 1 ) until 8 or more timepoints were included. This approach was chosen to maximize the quality of the extracted timecourses for data-rich proteins, but to also allow timecourses to be constructed for proteins consistently detected at low levels. For timepoints with at least 4 peaks, the set of peak ratios was tested for outliers using a variation of the integrated inconsistent rate (IIR) method (Hsiao et al., 2009), with an IIR cutoff value of 1.81 .

Protein expression ratios were calculated from the peak-level data at each timepoint by maximum likelihood estimation of the parameters of a lognormal fit to the data, taking the mean of the lognormal distribution as the protein ratio. The uncertainty in the ratio was taken as the upper and lower 95\% confidence limits calculated from an unbiased estimate of the standard error of the mean (Gurland and Tripathi, 1971). Thus the protein-level uncertainty is given by: $\pm\left(1.96 c_{\mathrm{N}} s\right) /\left(N^{0.5}\right)$, where $s$ is the standard deviation 
of the lognormal fit to the peak-level data, $N$ is the number of (filtered) peaks observed for a given protein at a given time, and the factor $c_{\mathrm{N}}$ is taken from Table 2 of Gurland and Tripathi (1971).

Finally, 70 outlier timepoints (1.1\% of the 6157 total) were excluded from protein expression timecourses by measuring the ratio difference between each point in a timecourse and its nearest neighbors in time. Timepoints whose summed nearestneighbor distances were greater than an empirically-determined threshold value of 4.15 times greater than the mean value for that timecourse were identified as outliers. This timepoint outlier detection method does not assume any underlying model for temporal structure in the data. The effect of the various filtering steps on dataset size is summarized in Table 1. The filtered dataset used for timecourse construction included 66,186 peak ratios, or $69.2 \%$ of the full dataset before filtering. Ultimately, expression timecourses over the diel cycle were constructed for 548 proteins, which are shown in Supplementary Figure 1.

\begin{tabular}{|c|c|c|c|c|}
\hline Filtering Step & $\begin{array}{c}\text { Unique LC-MS } \\
\text { peakgroup } \\
\text { pairs }\end{array}$ & $\begin{array}{l}\text { Protein-by- } \\
\text { time } \\
\text { datapoints }\end{array}$ & $\begin{array}{c}\text { Detected/ } \\
\text { quantified } \\
\text { proteins }\end{array}$ & $\begin{array}{c}\text { Proteins } \\
@ 14 \\
\text { timepoints }\end{array}$ \\
\hline Full diel dataset & $\begin{array}{c}95542 \\
(147 / 0.15 \%)\end{array}$ & $\begin{array}{c}8812 \\
(85 / 0.96 \%)\end{array}$ & $\begin{array}{c}1021 \\
(33 / 3.2 \%)\end{array}$ & $\begin{array}{c}360 \\
(0 / 0 \%)\end{array}$ \\
\hline Peak CV & $\begin{array}{c}76434 \\
(72 / 0.09 \%)\end{array}$ & $\begin{array}{c}8181 \\
(43 / 0.53 \%)\end{array}$ & $\begin{array}{c}967 \\
(25 / 2.6 \%)\end{array}$ & $\begin{array}{c}307 \\
(0 / 0 \%)\end{array}$ \\
\hline Peaks/timepoint & 73185 & 6157 & $\begin{array}{c}548 \\
(0 /<0.18 \%)\end{array}$ & 170 \\
\hline IIR peak outliers & 66661 & 6157 & 548 & 170 \\
\hline Timepoint outliers & 66186 & 6087 & 548 & 154 \\
\hline
\end{tabular}


Table 1 also shows the effect of data filtering on the false discovery rate (FDR). As noted above, the FDR for protein identification in the full dataset was 3.2\% (33/1021), but decoys amounted to only $<1 \%$ of the protein-by-time datapoints, and even less $(0.15 \%)$ of the total number of peaks. In the unfiltered dataset, the greatest number of timepoints at which any one decoy protein was identified was 9, and the majority (19/33) were found at only one timepoint (Supplementary Fig. 2). Filtering on the basis of ${ }^{14} \mathrm{~N} /{ }^{15} \mathrm{~N}$ ratio $\mathrm{CV}$ eliminated more than half of the decoy peaks, as expected if most of them are spurious identifications of features without a true isotopic partner of appropriate mass difference. After peak CV filtering, no decoy protein was found at more than 5 timepoints; hence no decoys passed the 8-timepoint requirement for timecourse construction. Thus, at least on the basis of decoy hits, the estimated protein-ID FDR for the timecourse data is bounded at $<0.18 \%(<1 / 548)$.

\subsubsection{Analysis of diel expression cycling}

Analysis of the temporal cycling of protein expression followed an approach based on that outlined by Futschik and Herzel (2008). For the 548 proteins quantified over the diel timecourse, any missing timepoint values were imputed using $k$-nearest neighbors (Troyanskaya et al., 2001), with the missing data inferred as the weighted average of the two closest timepoints by Euclidean distance in expression profile. The imputed data were used solely for the purpose of assessing the significance of expression cycling. The protein timecourses were then standardized to mean $=0$ and standard deviation $=1$. Two tests were then applied to identify proteins whose expression cycled over the diel.

First, Fourier scores were caluclated for all timecourses on the basis of a 24-hour cell cycle, as in Zinser et al. (2009). To assess the significance of a given Fourier score value, a background distribution of scores from 1000 simulated first-order autoregressive (AR(1)) timecourses was generated for each protein, using autoregression coefficients and white noise distributions derived from the protein timecourse. Futschik and Herzel (2008) have shown that an AR(1) background model is a more stringent and specific test 
of cyclic expression than Gaussian or randomized backgrounds. From comparison with these background distributions, a $p$-value (designated $p_{\mathrm{f}}$ ) was derived for each protein for the null hypothesis of no significant 24-hour cycling. Second, the highest autoregression coefficient of each protein timecourse (allowing a maximum lag of 2 timepoints) was compared with a distribution of autoregression coefficients from 1000 random whitenoise timecourses of equal variance. We implemented this procedure as an complementary check for time-coherent signals because the relative noisiness of the proteomics data resulted in occasional artifacts in the Fourier score calculation. This test directly addressed the autoregressive nature of the timecourse data, generating a $p$-value (designated $p_{\text {a }}$ ) for the null hypothesis of mutual independence of the timepoints.

The $p$-values for the two tests were combined using the truncated product method (Zaykin et al., 2002), with a cutoff of $p_{\mathrm{a}}<0.05$ (no truncation was applied to the $p_{\mathrm{f}}$ values). This cutoff was chosen to reflect the expectation of significant autocorrelation in timecourses of cyclic expression; since the two tests are not independent, the combined test score had to be corrected for the covariance of the two $p$-values (Brown, 1975). The combined test statistic $X_{k}^{2}$ is then given by

$$
X_{k}^{2}= \begin{cases}\frac{-2 \ln \prod_{i=f, a} p_{i}}{1+\frac{\operatorname{Cov}\left(-2 \ln p_{f},-2 \ln p_{a}\right)}{2 k}}, k=2 & p_{a}<0.05 \\ -2 \ln p_{a}, k=1 & p_{a} \geq 0.05\end{cases}
$$

Combined $p$-values $p_{\mathrm{X}}$ were derived from the combined test statistic assuming it follows a $\chi^{2}$ distribution with $2 k$ degrees of freedom when the joint null hypothesis is true. A false discovery rate approach was then used for multiple hypothesis testing with the program QVALUE (v. 1.1, Storey and Tibshirani, 2003) in the R statistical environment (v. 2.10.0, R Development Core Team). From the distribution of $548 p_{\mathrm{X}}$ values, a $q$ value was computed for each timecourse; QVALUE parameters included smoother method for $\pi_{0}$ with 5 degrees of freedom and robust $q$-value method. 
The time of peak abundance for each protein timecourse was found by fitting a series of shifted cosine curves to the standardized expression timecourses (Zinser et al., 2009). The abundance peak was identified as the phase shift of the best-fit cosine curve by minimizing the residual between the cosine and the data:

$$
R(\phi)=\sum_{t=1}^{14}\left(P_{s}(t)-\frac{\cos \left(\frac{\pi(t-1)}{6}-\frac{\pi \phi}{12}\right)}{\sigma_{\cos }}\right)
$$

where $t$ is the timepoint, $P_{s}(t)$ is the standardized protein timecourse, $\sigma_{\mathrm{cos}}$ is the standard deviation of the cosine over the range 0 to $2 \pi$, and $\phi$ is the phase (an integer between 0 and 23, inclusive). The value of $\phi$ that produced the minimum value of $R$ was considered the phase (time of peak abundance) of the timecourse. This procedure yielded estimates of the phasing of protein expression with hourly resolution.

\subsection{Quantification of fractional cellular protein \& transcript abundance}

The foregoing analysis (Sec. 2.4) was designed to provide a high-resolution picture of the timecourse expression of individual genes over the diel cycle. The quantification results based on isotope labeling enable comparison of the abundance of a given gene between timepoints, and the presence of a consistent internal standard (the ${ }^{15} \mathrm{~N}$-labeled peptides) allows relatively small changes in expression to be resolved, even for proteins that are not expressed at high levels. It is also informative, however, to compare the abundances of different proteins to each other, both within and across samples. This allows, for instance, one to see that protein $\mathrm{X}$ is present at $n$ times as many copies per cell as protein $\mathrm{Y}$ in a given sample. The approach adopted here to assess the cellular abundances of different proteins is based primarily on spectral counting. Spectral counting quantification stems from the empirical correlation between the abundance of a protein in a sample and number of times MS-MS fragmentation spectra of peptides of that protein are observed in a given dataset. The strength of this correlation is highly dependent on a host of chemical characteristics of the protein and its peptides as well as on the 
particulars of the analytical setup used to collect the mass spectral data. The utility of spectral counting as a mode of quantification is greatly enhanced by computational modeling of, and correction for, these experimental biases (Mallick et al., 2007). In this work, we used a computational approach to spectral counting termed APEX (Lu et al., 2007; Vogel and Marcotte, 2008) for Absolute Protein Expression.

The term "absolute abundance" is somewhat problematic. What spectral-counting techniques such as APEX calculate is in fact the fraction of the total amount of protein detected in a sample comprised by any one gene product. Thus it is still a form of relative quantification, though it is protein $\mathrm{X}$ relative to protein $\mathrm{Y}$ in sample $\mathrm{A}$, as opposed to protein $\mathrm{X}$ in sample A relative to protein $\mathrm{X}$ in sample $\mathrm{B}$. The latter is the kind of quantification obtained by isotope labeling methods. Spectral counting methods can be (and often are) used successfully for intersample comparisons of protein abundance, particularly for the more abundant components of the proteome (Hendrickson et al., 2006). Here we use spectral counts for protein-to-protein relative abundance measurements. These can be converted to nominally "absolute" units if the total amount of protein in the cell is known; for example, if the APEX score of a protein is 0.005 , meaning it comprises $0.5 \%$ of all (detected) protein, and there are 500,000 protein molecules in the cell (estimated from cellular $\mathrm{N}$ content, amino acid analysis, or colorimetric assays such as the Bradford, Lowry or BCA), we could say that there are 2500 copies of that protein in the average cell in that sample. However, APEX is blind to all the non-detected proteins, which are generally numerous and, as noted in Section 3.5.1, not necessarily of low cellular abundance. This is what makes spectral-countbased "absolute" quantification qualitatively different from the use of synthetic, absolutely-quantified peptide standards: the latter affords a measurement of the molar abundance of one molecular species irrespective of the presence of others, and is thus truly absolute. Since APEX has an inherently limited analytical window, we chose not to convert its fractional abundance results to quasi-absolute, copies-per-cell values, as those will inevitably be overestimates of the true cellular abundance. 
With those caveats in mind, however, protein-to-protein relative comparisons remain valuable. To perform this analysis, the set of $\mathrm{MS}^{2}$ spectra at each of the 14 diel timepoints identified as belonging to peptides of Prochlorococcus MED4 proteins were analyzed using the APEX Quantitative Proteomics Tool (v. 1.0.0, Braisted et al., 2008). The Random Forest classifier was trained on a merged set of data from the 06:00, 12:00, 18:00 and 24:00 timepoints, using a set of 50 proteins observed in all 14 timepoints and the same protein database used for peptide identification. All peptide properties were used by the classifier, with a minimum peptide length of 6 amino acids and 2 missed cleavages allowed. After training and generation of $\mathrm{O}_{\mathrm{i}}$ values, APEX scores were generated for each timepoint. All data were normalized such that the APEX scores for proteins observed at a given timepoint sum to one. Hence the APEX score for each protein is equivalent to its fraction of the total (detected) cellular protein at a given timepoint.

We also compared the results of the APEX quantification to the simple, MS ${ }^{1}$-based, label-free "absolute" quantification method of Silva et al. (2006). The Silva et al. (2006) method (hereinafter referred to as "Top3") is based on an empirical observation of the correlation between protein abundance and the average of the $\mathrm{MS}^{1}$ intensities of the three peptides belonging to that protein giving the largest $\mathrm{MS}^{1}$ peaks. Here we limited the application of the Top3 method to proteins where 10 or more unique peptides were detected, as was the case for the results of Silva et al. (2006). The Top3 abundance values were normalized in the same way as the APEX scores, i.e., as a proportion of the total at a given timepoint. Top3-based abundances were thus generated for 1799 (20\%) of the 8812 protein-by-time datapoints in the full diel dataset. A comparison between APEX and Top3 abundance values is shown in Supplementary Figure 3. The correlation coefficient between the results of the two quantification methods is 0.59 over two orders of magnitude in predicted abundance, indicating broad agreement. Similar results were obtained by Malmström et al. (2009) in their quantification of the Leptospira interrogans 
proteome. It should be noted that the predicted abundances of some proteins vary by over 50-fold between APEX and Top3 (Supplementary Figure 3). Combined with the biases inherent to a finite analytical window, these limitations suggest that fractional abundance values are perhaps best interpreted in terms of the distribution of the cellular proteome among different gene products, rather than as precise measures of the abundances of individual proteins.

Finally, while the comparison between the transcript and protein levels of the cyclic expression of individual genes in the following sections utilizes the published diel mRNA timecourses of Zinser et al. (2009), we sought to incorporate preliminary results from our in-progress RNA-sequencing transcriptomics dataset in order to compare the celluar abundances of gene products. Since RNA-sequencing is, to first order, unbiased across templates, the fractional abundance of reads in an RNA-Seq dataset belonging to a given transcript equals the proportional abundance of that transcript in the sample. To enable comparison with the protein abundance values, we calculated an equivalent transcript abundance score, termed AMEX (Absolute Message Expression), and given by:

$$
A M E X_{i}=\frac{\frac{\text { bp of sequence from transcript } i}{\text { length of transcript } i}}{\frac{\text { bp of sequence from protein - coding regions }}{\text { total length of protein - coding genes }}}
$$

At the time of this writing, RNA-Seq results have been obtained for only two timepoints, 08:00 and 18:00. AMEX analysis of those datasets is presented in Section 3.5.

It should be noted that, with the exceptions of Xcalibur (used for primary mass spectral data acquisition on the LTQ-FT) and MATLAB, all software used in the analysis of this proteomics dataset is freely distributed and open source. 


\section{Results: Protein And transCRIPT EXPRESSiOn OVER The Prochlorococcus DIEL CELL CYCLE}

\subsection{Cell growth \& diel cycling}

The cells were well-entrained to the diel cycle in the sunbox incubator. Figure 2A shows the density of cells in the culture over the period of diel sampling. Cell density was steady throughout most of the day, increasing sharply after sunset to close to double the value at the start of the experiment. Cell cycle analysis (Figure 2B) confirms that the culture was synchronized to the diel cycle. Cells spent the hours from early morning until noon in $\mathrm{B} / \mathrm{G} 1$ phase. DNA synthesis ( $\mathrm{C} / \mathrm{S}$ phase) began in the afternoon and was largely complete by dusk. The peak of $\mathrm{D} / \mathrm{G} 2+\mathrm{M}$ phase was at sunset and cells began to divide then, but, as shown by the slope of the density curve, cytokinesis continued until midnight or shortly thereafter. The observed progression and timing of the cell cycle is entirely consistent with previous observations in culture and in the field (Vaulot et al., 1995; Jacquet et al., 2001), and further demonstrates the consistency and reproducibility of the Prochlorococcus diel cell cycle. In particular, the diel growth in this experiment is very similar to that observed by Zinser et al. (2009) using the same strain under similar (though not identical) conditions, enabling comparison of results between the two studies. Differences between the culture conditions of Zinser et al. (2009) and those used here include the length of the light period (14 hours vs. 13 hours) and the maximum midday light intensity (232 vs. $205 \mu \mathrm{mol}$ quanta $\mathrm{m}^{-2} \mathrm{~s}^{-1}$ ).

\subsection{Quantitative proteomic analysis of expression cycling}

A total of 1021 different Prochlorococcus proteins were observed over the course of the diel experiment - more than half of the annotated MED4 genome. Between 524 and 764 proteins were detected at each sampling timepoint, out of total of 1984 putative open reading frames (ORFs) in the current annotation of the MED4 genome (Figure 3A). The 

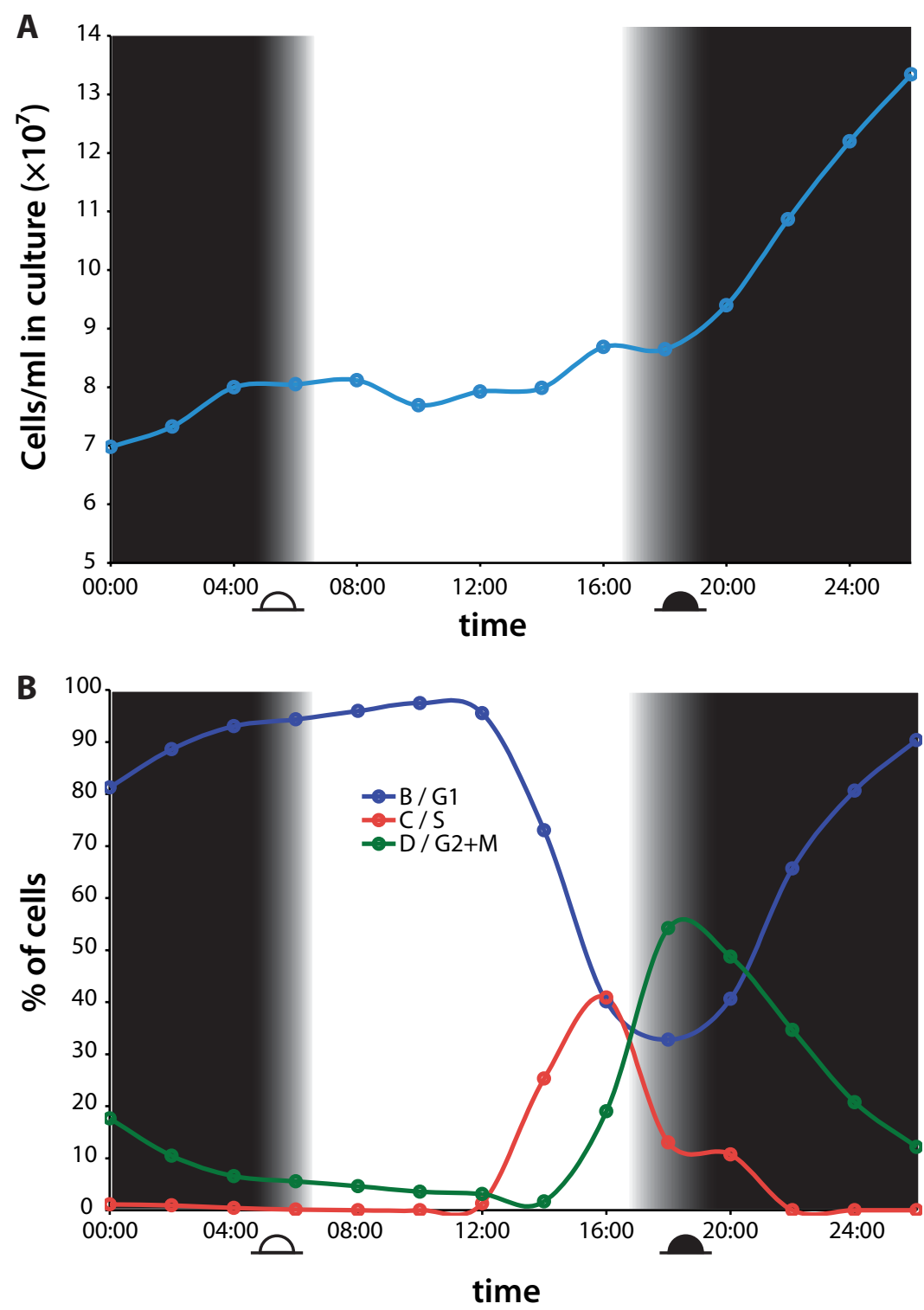

Figure 2. A Cell density in the culture over the timecourse of the experiment, as counted by flow cytometry. Density was essentially constant between sunrise and sunset, and increased after dark as cells began cytokinesis. Cell density doubled over the course of the experiment, meaning $>90 \%$ of cells divided. B Proportion of cells in the 3 phases of the cell cycle, measured by DNA staining and flow cytometry. DNA replication began in the afternoon and was largely complete by dusk, when cell division began. The times of experimental sunrise and sunset (05:30 and 18:30, respectively), are indicated by symbols. 
average coverage (i.e., proportion of amino acid positions represented in observed peptides) of detected proteins was $25.1 \%$, with a mean of 6.8 unique peptides per protein. Since a primary goal of this study is to quantify protein expression over time, consistent observation of a set of proteins across all (or at least a majority of) timepoints is critical. Figure 3B shows the number of timepoints at which each observed protein was detected. Of the 1021 total detected proteins, 584 were found in at least 8 timepoints, and 360 were detected in all 14 timepoints. The regularly-observed proteins actually constitute the majority of the dataset, since they exhibit the greatest number of detected peptides and LC-MS peaks. These consistently-observed proteins are the group we will focus on in examining expression patterns over the diel. The proteins observed at only a few timepoints are very likely genuine observations (having passed through the same identification and validation process), but little can be said about their expression timecourses; there are not, for example, clear-cut instances of catching only the peak of a protein's expression in just a few successive timepoints. The great majority of these infrequently-observed proteins are detected sporadically over the diel, consistent with them being expressed at relatively low levels and hovering on the edge of the analytical window.

In the current MED4 genome annotation, there are 963 ORFs for which no peptides were detected at any of the diel timepoints. Some of these genes are essential to critical cell functions and were undoubtedly expressed during this experiment; their absence reflects the widely varying detectability of different proteins in LC-MS-based proteomics.

Examples of such genes include subunits of photosystems I and II and the cytochrome $b_{6} f$ complex, and several nucleotide biosynthesis enzymes. A majority the undetected proteins, however, are short and/or hypothetical ORFs that may or may not actually produce proteins. Of the 963 undetected ORFs, 53\% (512) are annotated as "hypothetical" or "conserved hypothetical", which is significantly higher than the $25 \%$ of detected proteins bearing those annotations. Peptides from 252 hypothetical ORFs were 

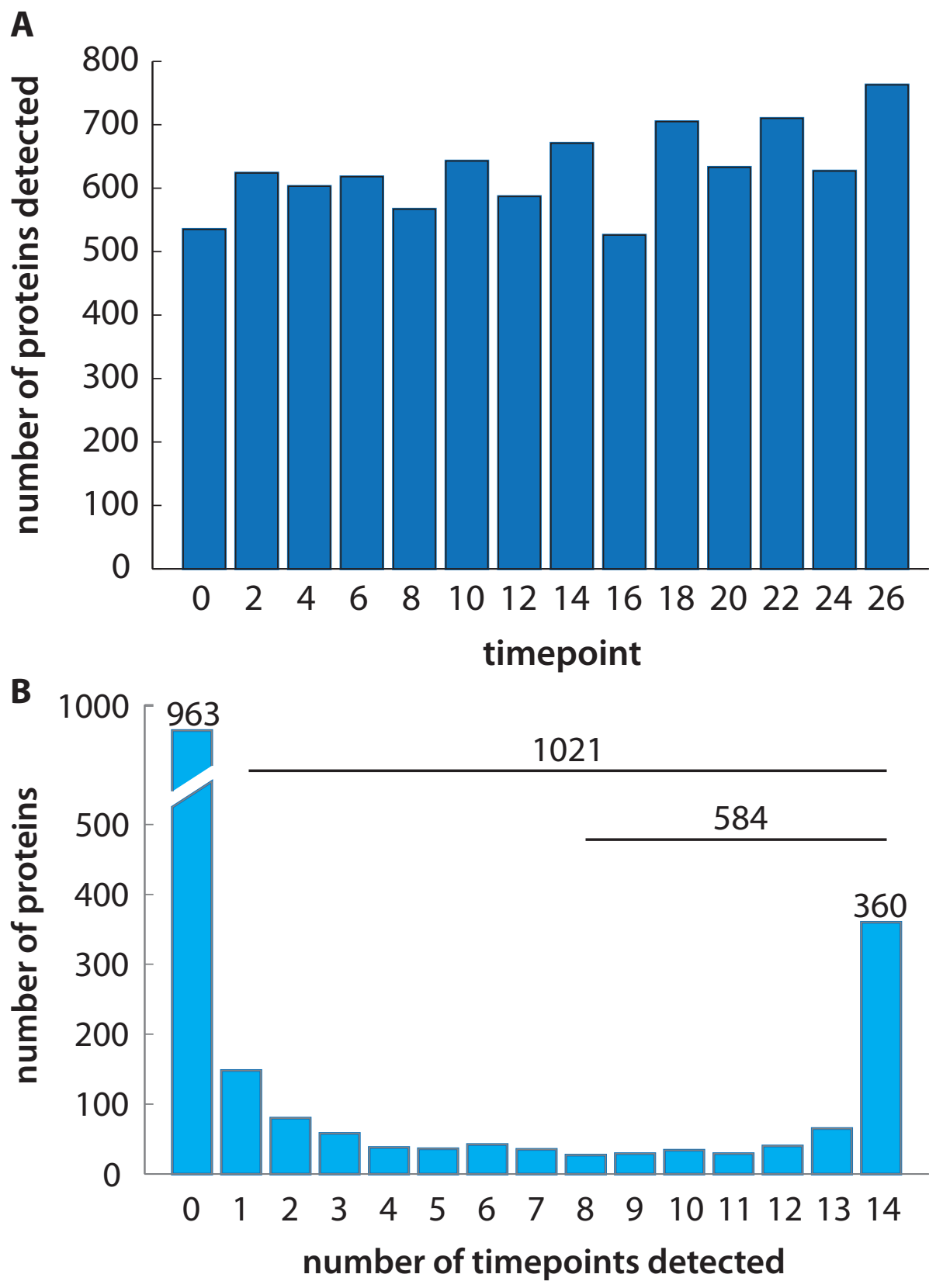

Figure 3. A Number of Prochlorococcus MED4 proteins detected at each of the 14 timepoints over the 28-hour diel sampling period. B Number of timepoints at which each MED4 protein was detected. In the total (unfiltered) dataset, 1021 proteins were detected at at least one timepoint, 584 at eight or more timepoints, and 360 proteins were found in all of the 14 timepoints. No peptides were detected from 963 ORFs in the current MED4 genome annotation; these genes include a substantial number of small and/or hypothetical proteins. 
detected in this dataset, however, confirming their translation into an actual protein product.

Using the criteria and procedures described in Section 2.4, the data from the 584 proteins observed in the majority of timepoints was filtered to produce protein expression timecourses for 548 genes. These timecourses are plotted in Supplementary Figure 1. To assess how many of these timecourses showed evidence of cyclic expression over the diel, we performed an analysis (described in Sec. 2.4.2) that produced a $q$-value for each timecourse for the null hypothesis of no significant cycling with 24-hour periodicity. The $q$-value is a measure of the false discovery rate, i.e., the proportion of false positives expected if cycling in a given timecourse is considered significant. Zinser et al. (2009) used a $q$-value cutoff of 0.1 and found that $82 \%$ of expressed protein-coding genes had significantly cyclic expression at the mRNA level. As shown by the dotted line in Figure 4A, applying the same cutoff to the diel proteomics dataset results in only 92 of 548 timecourses exhibiting significant diel periodicity. Taken at face value, this result would imply that the protein-level expression of only 15\% of MED4 genes cycles over the diel.

From inspection of the timecourses, however, it is clear that this quantitative proteomics data is substantially sparser than the microarray data of Zinser et al. (2009), and it is likely that the cycling of a substantial number of genes is masked by analytical noise. The $15 \%$ of timecourses at $q \leq 0.1$, then, is probably a minimum estimate for the proportion of cycling proteins, and this number would increase with dataset richness. An alternative approach to estimating the proportion of cycling timecourses is to calculate the proportion of expected true positives as a function of the $q$-value cutoff. As the $q$ value threshold is raised, the proportion of expected true positives (i.e., cycling proteins) reaches a plateau where $q=0.5$, since at that point each protein added to the dataset is equally likely to be cycling as non-cycling. As shown in Figure 4B, this plateau is reached at a value of roughly $43 \%$ cycling proteins. So while specific evidence of cycling can be identified in only 92 timecourses, the shape of the $q$-value distribution 

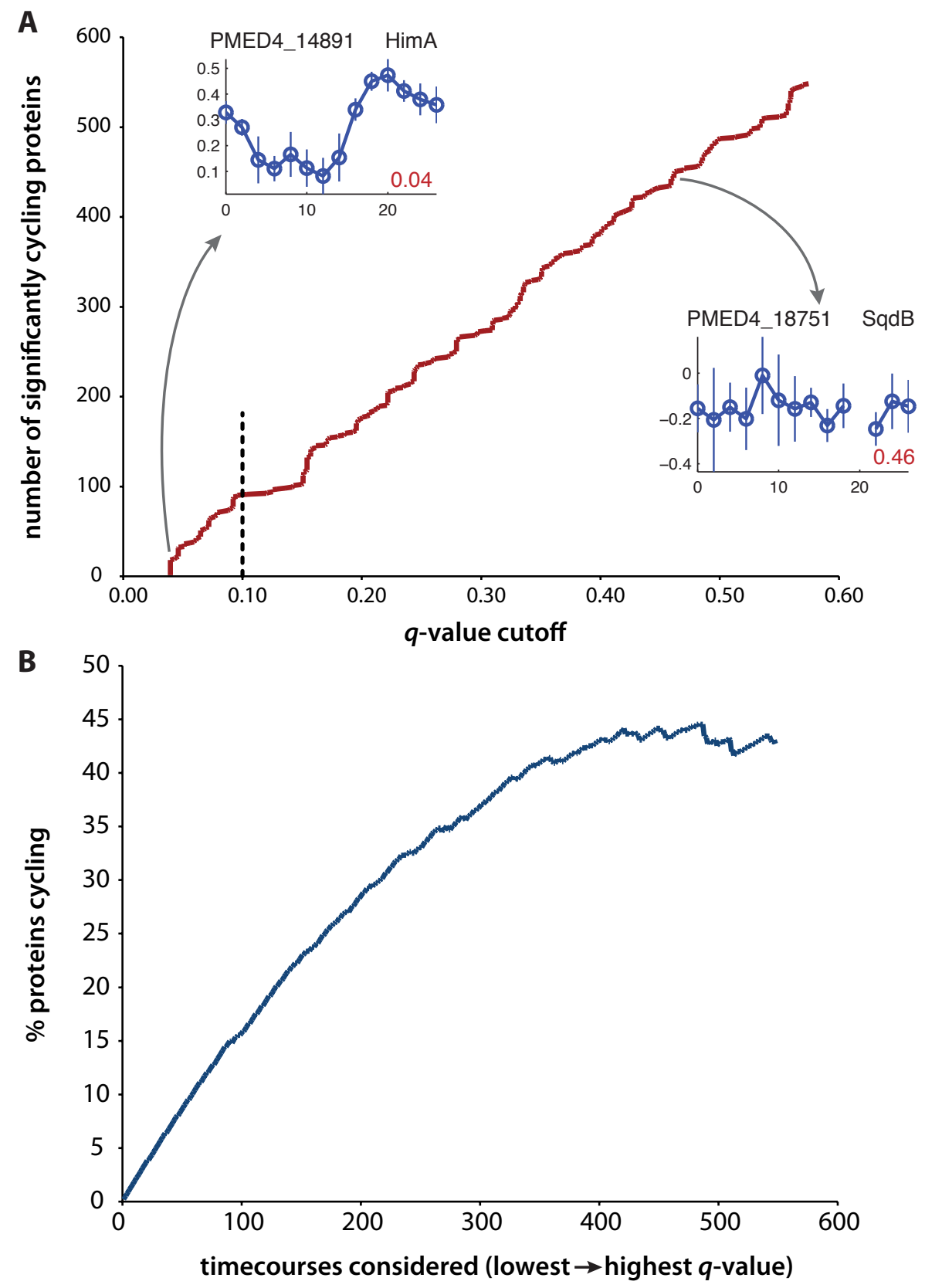

Figure 4. Results of analysis of diel cycling of protein timecourses. A Number of proteins considered to be significantly cycling with 24-hour periodicity, as a function of $q$-value, a measure of the false discovery rate. Insets show examples of protein timecourses with low (HimA, $q=0.04)$ and high (SqdB, $q=0.46) q$-values. At the chosen cutoff of 0.1 (shown by the dotted line and equivalent to that used by Zinser et al. (2009)), 92 proteins show significant cycling. Note that the number of cycling proteins is not very sensitive to $q$-value in the range 0.09-0.15. B An estimate of the proportion of expected cycling proteins, calculated from proportion of expected true positives (i.e., cycling proteins). As more timecourses are considered, the expected proportion of cycling proteins plateaus near $43 \%$, once the $q$-value reaches 0.5 . So while clear evidence of cycling can be seen in only $15 \%$ of individual protein timecourses, the shape of the $q$-value distribution suggests that up to $43 \%$ may have some diel periodicity. 
suggests that almost half of proteins could be cycling - though it does not specify the members of that group, nor the parameters (e.g., phase, amplitude) of their cycling. This result, while hardly robust in itself, is generally consistent with an estimate of the proportion of cycling genes derived from the temporal distribution of peak protein abundances (see Sec. 3.3.3).

It should be noted that mass spectrometry-based proteomics data in general is much sparser in terms of coverage and redundant observations of gene product abundance than microarray or massively parallel RNA-sequencing is. Large-scale comparisons of gene expression patterns at different biological levels using different methodologies must be mindful of the relative richness and biases of various datasets. In the next section, we focus on the relationship between mRNA and protein expression for genes where the cycling (or lack thereof) is well-resolved at both levels.

\subsection{Relationships of mRNA and protein expression cycles}

Beyond simply characterizing the Prochlorococcus proteome, a key aim of this study is to illuminate the dynamic relationship between gene product abundances at the transcript and protein levels. Here we compare protein expression timecourses with the mRNAlevel results of Zinser et al. (2009). It is important to recognize that certain aspects of the two experiments limit how precisely they can be intercompared. In particular, the photoperiod in the work of Zinser et al. (2009) was 14 hours, an hour longer than in this experiment, and the sampling points in the two studies were shifted relative to their respective dusk and dawn. While there may be some quantitative differences in detail, we expect the mRNA-protein relationships outlined below to remain qualitatively the same.

In the near future, a complete diel transcriptome from this experiment will be available (Rodrigue et al., in prep), allowing direct and precise comparisons of simultaneous transcript and protein abundance measurements. Analysis of the transcriptome samples is 
currently underway; to date, RNA-Seq libraries have been sequenced for two timepoints from this experiment, 08:00 and 18:00. While data from just two timepoints is insufficient to assess transcript expression cycling, analysis of this data is presented in Sec. 3.4 in the context of cellular gene product abundances.

\subsubsection{Temporal patterns of transcript \& protein abundance}

Three examples of the types of mRNA-protein cycling relationships observed in the Prochlorococcus diel cell cycle are shown in Figure 5. The first is exemplified by $n r d J$, encoding the ribonucleotide reductase that converts ribonucleotides to deoxyribonucleotides by removing the 2'-hydroxyl of ribose, which is an essential step in DNA synthesis. This gene shows what might be considered the 'expected' relationship between transcript and protein abundance dynamics: the protein abundance tracks the transcript abundance quite closely, showing the same amplitude of variation and perhaps a slight temporal lag. Both mRNA and protein vary in abundance by about 10 -fold over the diel cycle. The timing of peak abundance also makes intuitive sense: the abundance of $n r d J$ gene products increases through $\mathrm{C} / \mathrm{S}$ phase, when cells are replicating their chromosomes and thus require deoxyribonucleotides, and declines thereafter. $n r d J$, then, is likely an example of gene whose expression is dominated by transcriptional regulation. It turns out, however, that this apparently straightforward relationship between transcript and protein is the exception rather than the rule.

A more widely observed pattern is exemplified by $r b c L$, the large subunit of the principal carbon fixation enzyme Rubisco (Figure 5B and E). In this case, the abundance oscillation over the diel is 35 times stronger at the transcript level (46-fold change) than at the protein level (1.3-fold). We hypothesize that, in this case, the mRNA-level variability in expression is strongly damped by posttranscriptional processes.

Nevertheless, the small-amplitude diel cycle in RbcL protein abundance was wellresolved (Fig. 5B, inset), and it shows a peak in the early afternoon - a time when rbcL transcript abundance is declining from its peak near sunrise. So in this case, mRNA and 


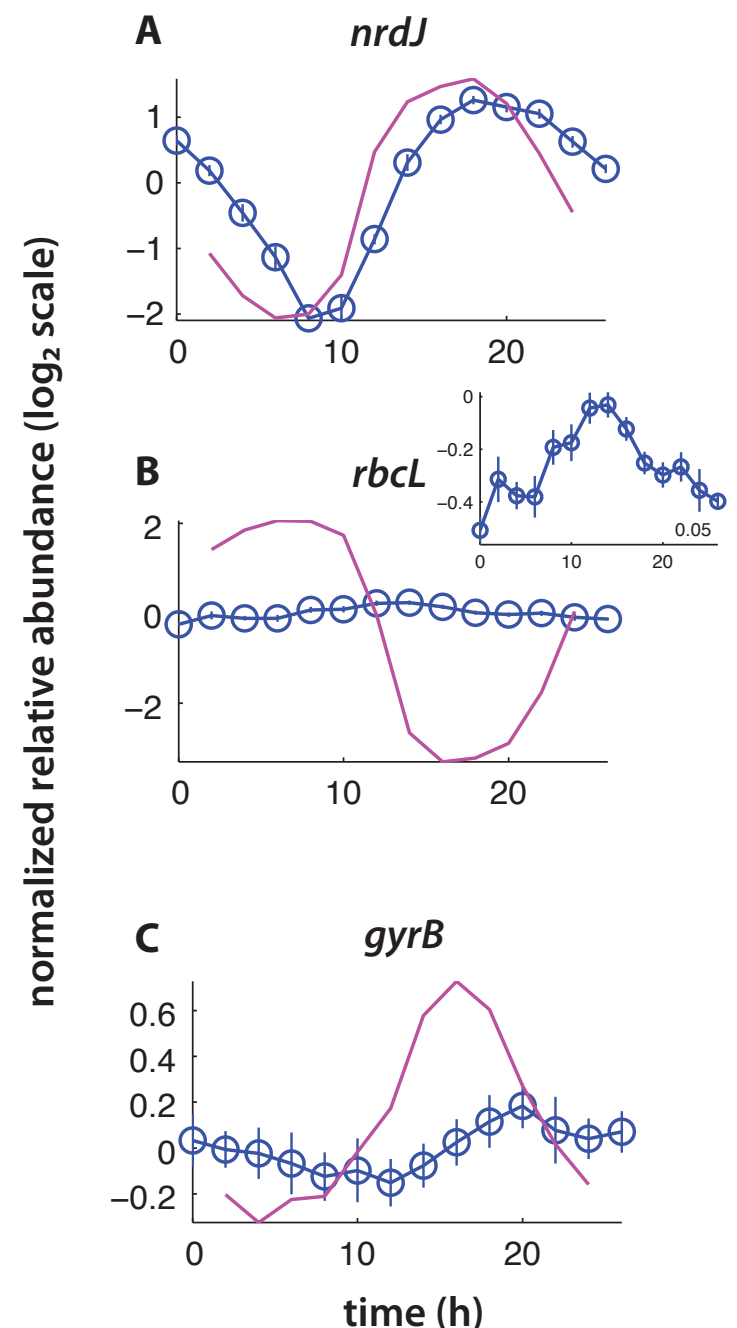

time (h)
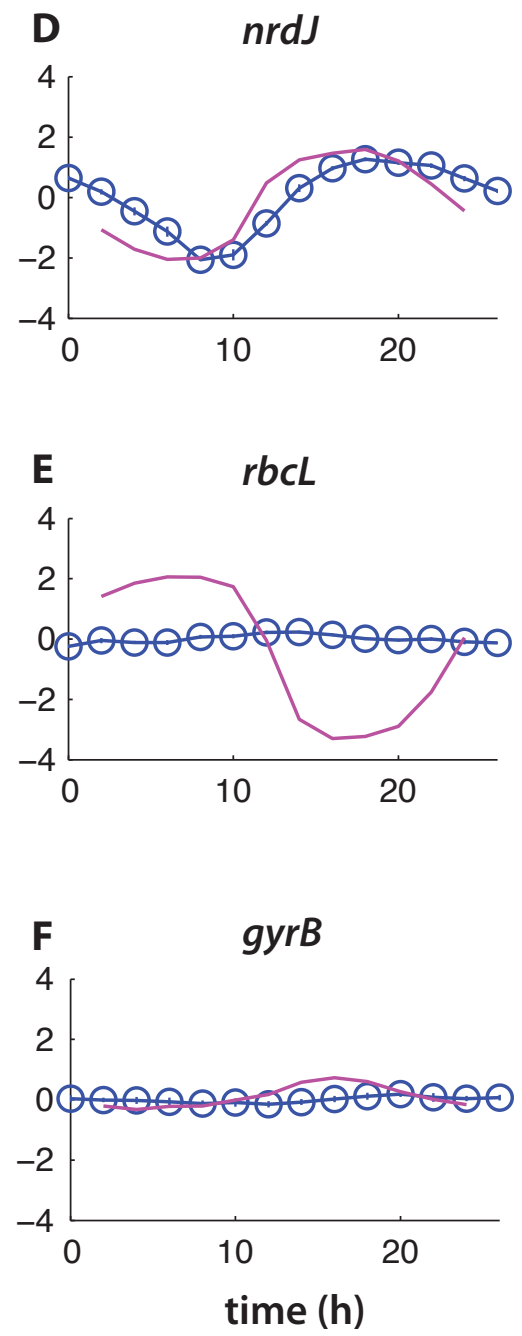

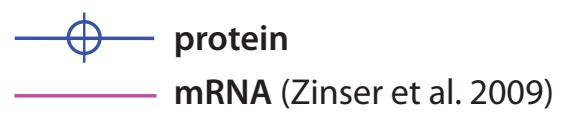

Figure 5. Examples of relationships between expression cycling at the mRNA and protein levels. Protein (blue) and mRNA (pink) timecourses for three genes are shown: $n r d J$ (ribonucleotide reductase), $r b c L$ (Rubisco large subunit), and gyrB (subunit B of DNA gyrase). All 3 genes have $q$-values for cycling $\leq 0.1$ for both mRNA and protein. For comparison, expression timecourses for each gene are shown scaled to their own amplitude range $(\mathbf{A}-\mathbf{C})$ and on a common scale of \pm 4 $\log _{2}$-units (D-F), reflecting the range of fold changes in Prochlorococcus genes over the diel cycle $\left(2^{8}=256\right.$-fold). Transcript and protein abundances for $n r d J$ are well-correlated, tracking closely in both amplitude and phase, and varying 10-fold over the diel. $r b c L$, on the other hand, oscillates much more strongly at the mRNA level (46-fold) than at the protein level (1.3-fold); the protein timecourse is shown inset. gyrB, while cycling at both levels, shows a small amplitude of variation $(<2$-fold), and is damped and somewhat phase-shifted at the protein level compared to the mRNA. 
protein dynamics are decoupled in both amplitude and phase. As documented in Section 3.4, this pattern is observed repeatedly in central metabolic pathways in Prochlorococcus.

The third example is less extreme than the first two. For the gene gyrB, which codes for a subunit of the DNA gyrase that causes negative supercoiling of the bacterial chromosome, neither transcript nor protein show more than 2-fold abundance changes over the diel. On the scale of the nrdJ and rbcL oscillations, the abundance of these gene products hardly appears to change at all (Figure 5F). The low-amplitude cycles of gyrB and GyrB are fairly well resolved, however (Figure 5D), and show that the protein cycle is again somewhat damped and phase-shifted relative to the mRNA. As discussed below, this is a common pattern seen between transcript and protein levels over the Prochlorococcus diel cycle.

\subsubsection{Transcript-protein dynamics: amplitude}

The examples in the previous section show that the relationship between gene product abundances at the protein and mRNA levels can be highly variable from gene to gene. For 253 genes whose proteins were observed at 12 or more timepoints, we examined the relationship between the fold change (the ratio of highest to lowest abundance) of protein and transcript (Fig. 6). Considering first just those with protein cycling $q$-values less than 0.1 (the blue points in Fig. 6), it is clear that the great majority (50 of 55) are below the 1:1 line. That is to say, for $91 \%$ of these genes, the amplitude of variation seen in the mRNA abundance cycle is diminished at the protein level. When all genes plotted in Figure 6 are considered, the proportion below the 1:1 line drops to $79 \%$, though many of those above the line are noisier protein timecourses whose amplitudes would likely decrease with more data. This relationship also highlights how unusual the case of $n r d J$ is: it supports by far the strongest correlation between transcript and protein levels. $r b c L$, while towards an extreme, is part of a general trend of strong-transcript, weak-protein cycles. $g y r B$ is more typical of the most densely-populated region of the amplitude 


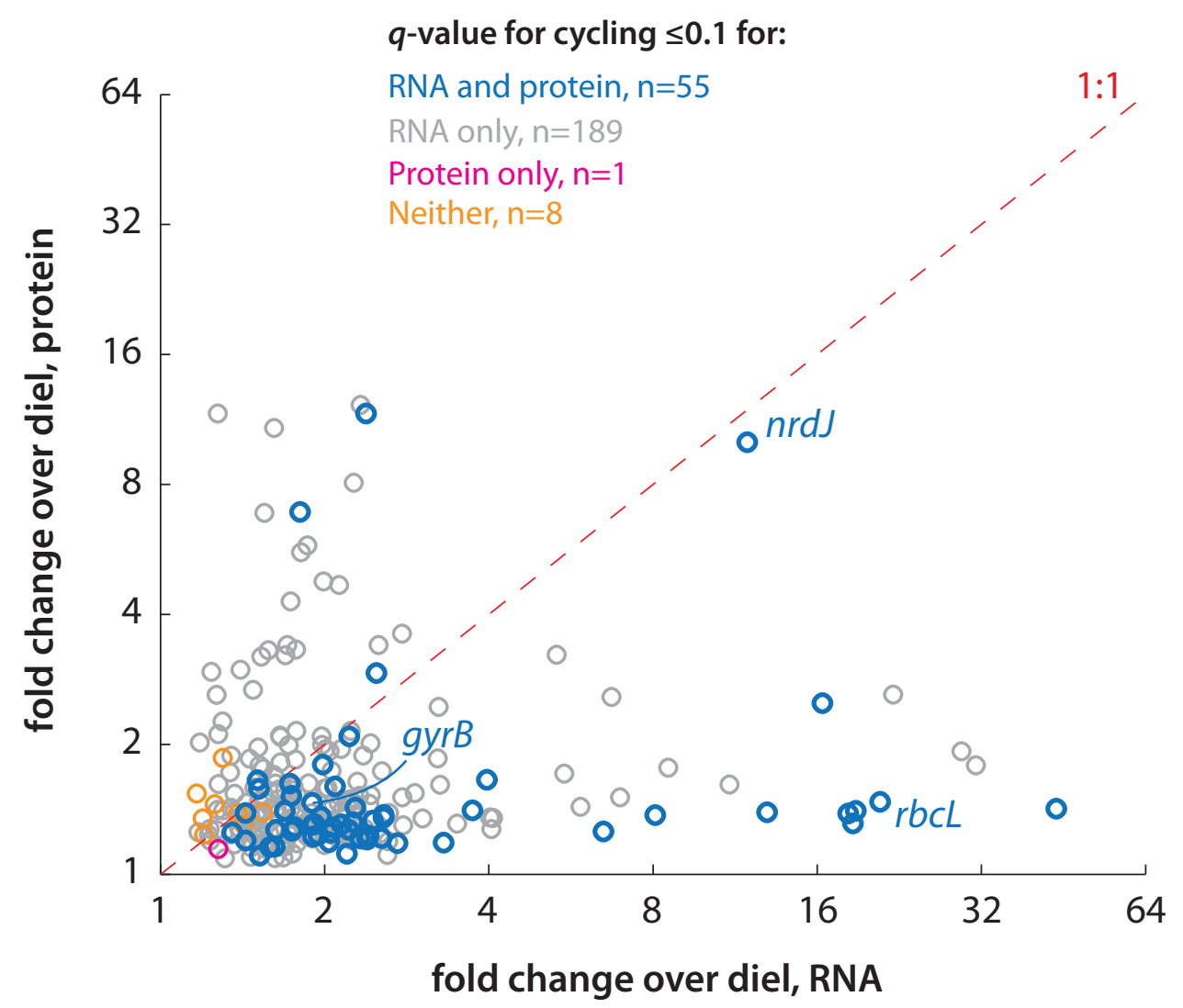

Figure 6. Comparison of the amplitudes of expression cycling at the mRNA and protein levels. If protein-level expression tracked transcript-level variation quantitatively, points would lie on or near the 1:1 line shown. Instead, most genes show quite different amplitudes of variation between the protein and mRNA levels, with the majority being damped at the protein level compared to the transcript. Genes are colored by their $q$-values for diel cycling; most of the cases of protein fold change appearing greater than that of mRNA are attributable to noisy, non-cycling protein timecourses (gray points above 1:1 line). For clarity, only genes whose protein timecourses included at least 12 timepoints ( 253 total) are shown. Comparisons of the mRNA and protein timecourses for genes indicated by name are shown in Figure 5. mRNA-level data from Zinser et al. (2009). 
space. Overall, the comparison of expression fold-changes suggests that mRNA-level variations are broadly damped at the protein level.

The methods used here to detect cyclic protein expression (metabolic isotope labeling, Fourier scoring with false discovery rates; see Sec. 2.4) can confidently detect protein abundance oscillations even when the amplitude is less than 2-fold - as it is for most observed proteins (Figure 6). When protein abundances are tracked over time with sufficient temporal resolution, small variations that would be impossible to resolve in 2sample comparisons become apparent and interpretable. If we had simply contrasted protein abundances at, say, sunrise and sunset or midday and midnight, few differences would be significant. It would appear that the protein abundance variations that accompany (and underlie) the light-dark entrained cell cycle of Prochlorococcus are generally of a smaller magnitude than those observed in typical laboratory gene expression experiments involving strong, systemic perturbations such as acute nutrient starvation, temperature shock or toxin exposure.

\subsubsection{Transcript-protein dynamics: phase}

Besides the amplitude or magnitude of abundance changes, the phase of expression cycles (i.e., the timing of the peaks and troughs in abundance) can also vary between the mRNA and protein levels. If transcriptional control were dominant, we might expect to see a small, more or less consistent lag between the peaks of transcript and protein abundance, simply due to the finite speed of translation - much as observed for $n r d J$ (Figure 5A). As shown in Figure 7, however, the protein abundance cycles observed in this experiment show a wide range of phase relationships with their respective transcripts. A substantial number do plot slightly above and to the left of the 1:1 "in phase" diagonal, which is where proteins lagging their transcripts would fall, though the range of lags in that group spans 1-8 hours. This is many times longer than the half-life of Prochlorococcus mRNA molecules (which averages 2.4 minutes; Steglich et al., in prep) and, even given the slow translation rates in Prochlorococcus (see Chapter 5), it is 

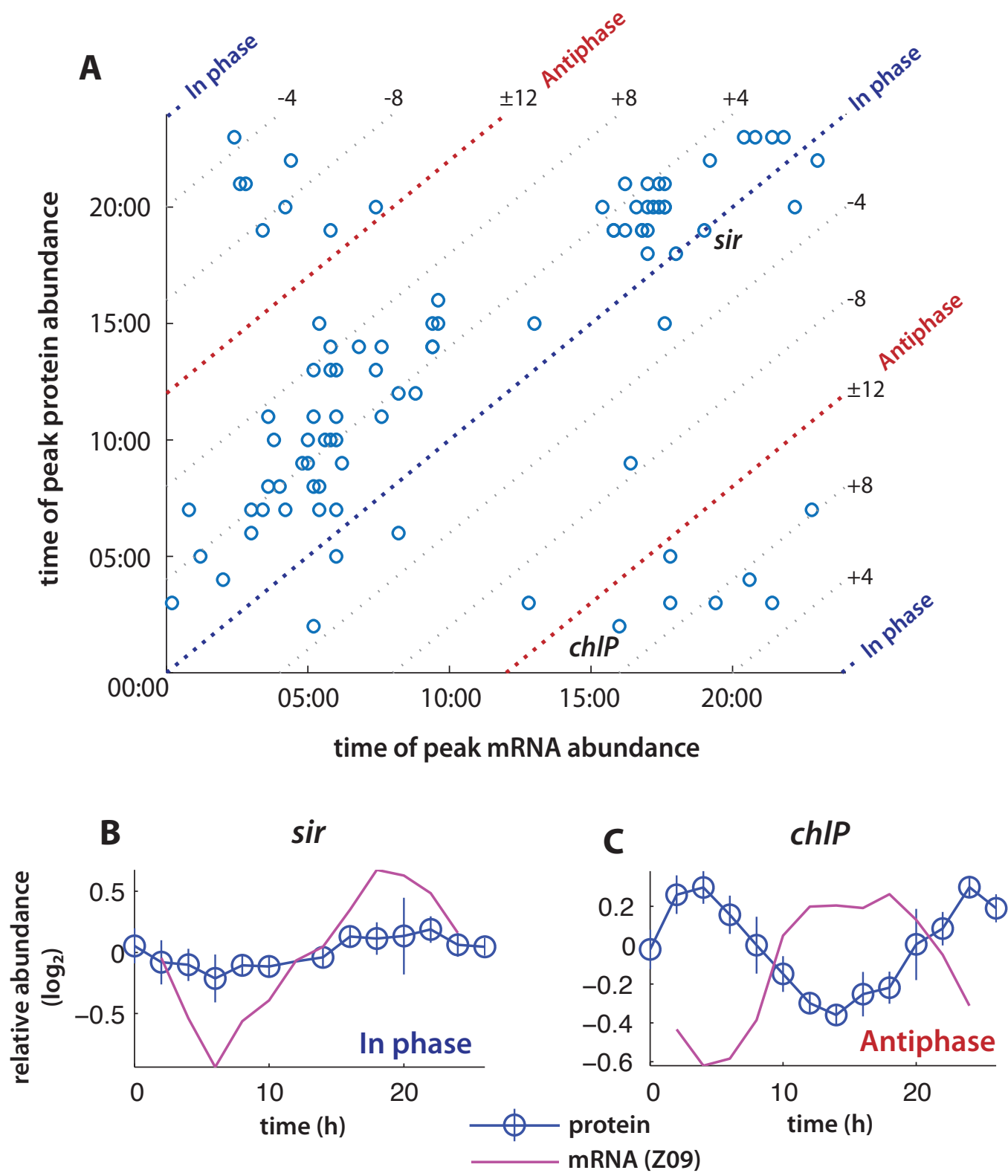

Figure 7. Relationship between phasing of protein- and mRNA-level expression, based on data from the current experiment and Zinser et al. (2009), respectively. A Peak abundance times at the protein and transcript levels for genes with protein cycling $q$-values $\leq 0.1(n=92)$. Genes plotting along the central blue diagonal (or in the upper left or lower right corners) have their peaks of protein and transcript abundance at the same time during the diel cycle, i.e., the protein and mRNA are in phase. An example is sir (ferredoxin-sulfite reductase), whose expression timecourses are shown in B. Genes plotting near either of the two red diagonals have their protein and mRNA peaks offset by 12 hours (i.e., antiphase). C shows an example of antiphase expression, chlP (geranylgeranyl diphosphate reductase). 
difficult to see how transcript abundance variations would take, say, 6 hours (a quarter of the cell cycle) to be transmitted to the protein level. Assessment of the significance of these smaller phase offsets will await the transcriptome timecourses from the current experiment.

There are, however, a number of genes (e.g., chlP, Fig. 7C) for which the phasing of mRNA and protein abundance are clearly divergent and involve posttranscriptional, and probably posttranslational, processes. It is conceivable that for these genes, protein degradation is also strongly cyclic, and this imposes another time-varying driver on protein abundance. If protein degradation and transcript abundance both cycle with sufficient amplitude and with different phases, the observed timecourse of protein abundance would represent the sum of these two oscillating source and sink terms. Why certain proteins would be subject to this mode of regulation remains a question for future investigation.

Zinser et al. (2009) found that a majority of Prochlorococcus transcripts peak in abundance near sunrise and sunset. A two-peaked distribution of peak abundance times was also found in the protein timecourses from this experiment (Figure 8). Consistent with the peaks in the abundance of transcripts, the peaks in the protein phase distribution follow shortly after sunrise and sunset. The histograms in Figure 8 also provide an way to estimate the proportion of non-cycling genes. If no genes were significantly cycling, the distribution of phases would be random, and the histogram should look flat, with the same number of genes 'peaking' at each timepoint. Viewed in this way, the sunrise and sunset peaks sit on top of a flat, 'background' distribution of roughly 12 proteins per timepoint. If those 288 ( 12 proteins $\times 24$ timepoints) are considered non-cycling, then the predicted proportion of cycling proteins is $47 \%$, similar to the $43 \%$ estimated from the shape of the $q$-value distribution, above. 

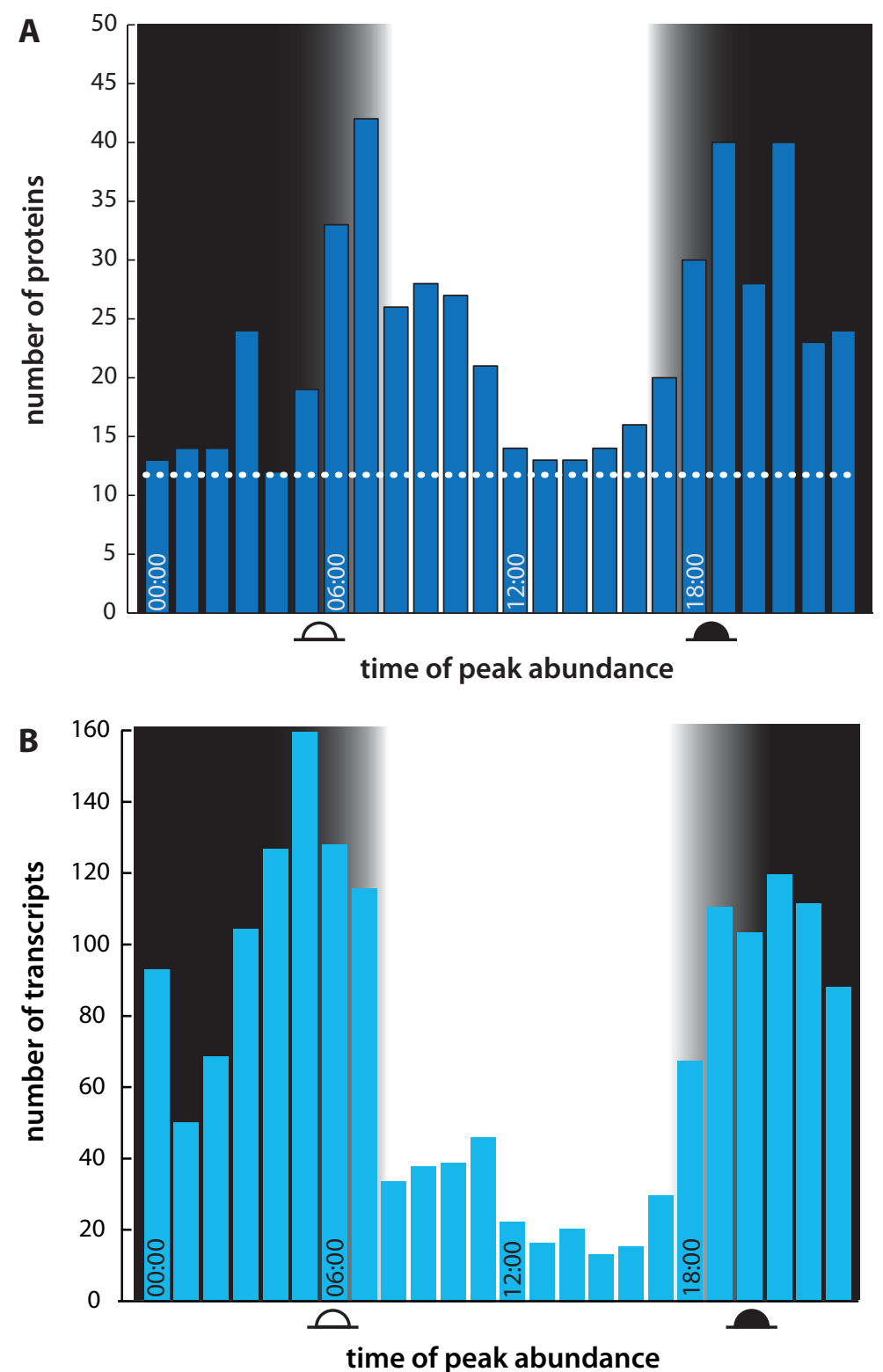

Figure 8. Histograms of times of peak expression with hourly resolution for (A) proteins in the current experiment and (B) transcripts in the diel microarray experiment of Zinser et al. (2009). Both distributions show peaks near sunrise and sunset, whose times during the two experiments are indicated by symbols. Note that the photoperiod is one hour longer in $\mathbf{B}$ than in $\mathbf{A}$. The white dotted line in the upper panel represents a hypothetical null distribution of non-cycling proteins, approximately 12 per timepoint. 


\subsection{Diel balance of carbon metabolism in Prochlorococcus}

One of the most important cellular functions in Prochlorococcus, and arguably the most important with regard to its role in ocean biogeochemistry, is carbon fixation. The immediate incorporation of $\mathrm{CO}_{2}$ into organic matter is catalyzed by Rubisco, but this is just one reaction in a central carbon metabolic network that involves both reductive $(\mathrm{C}$ fixing) and oxidative (C-respiring) pathways. Two components of this metabolic network, the Calvin cycle and the pentose phosphate pathway, can be viewed as a superpathway of two intersecting cycles working in opposite directions (Fig. 9A). In essence, the reductive portion (the Calvin cycle) trades energy (ATP) and reducing power (NADPH) for fixed carbon, while the oxidative portion (the pentose phosphate pathway) trades fixed carbon for reducing power. During the day, photosynthesis can replenish the ATP and NADPH consumed by the Calvin cycle, allowing net fixation of carbon. At night, reserves of fixed carbon stored as glycogen are consumed and NADPH is regenerated.

Proper regulation of the intersection of these two cycles is essential. To illustrate why, consider what happens if the metabolic flux is allowed to run around the outside of the superpathway: chemistry accomplished in one part of the cycle is undone in another, with no net result except the waste of 3 molecules of ATP. It is the balance of fluxes through the intersection of the reductive and oxidative portions that determines whether net carbon fixation or respiration occurs.

The expression of the genes coding for enzymes of central carbon metabolism was discussed in detail by Zinser et al. (2009). They found that transcripts for components of the reductive and shared portions of the network were most abundant near sunrise, while those for the oxidative section were highest near sunset. The amplitude of expression variation was particularly strong for the initial C-fixing enzymes of the Calvin cycle, Rubisco and phosphoglycerate kinase. 
Figure 9. (Facing page) Central carbon metabolism in Prochlorococcus. A The network of central carbon metabolism, showing the intersection of the reductive (Calvin cycle) and oxidative (pentose phosphate pathway) components. The Calvin cycle trades reducing power for fixed carbon, while the oxidative pentose phosphate pathway does the reverse. The two share a set of enzymes, shown in the central portion of the diagram. The direction of metabolic flux -- and hence whether net $C$ fixation or respiration occurs -- depends on regulation of the activity of these shared enzymes. OpcA is a posttranslational regulator of Zwf (see text). B Expression of carbon metabolism genes at the protein (blue) and transcript (pink; Zinser et al. 2009) levels. Most genes, especially those of the Calvin cycle, show strong oscillations in mRNA abundance that are damped and phase-shifted at the protein level. Of these enzymes, only transaldolase (Tal) changes in abundance more than 2 -fold over the diel cycle. $\mathbf{A}$ is redrawn after a figure by L.R. Thompson and used with kind permission.

compound abbreviations:

RuBP ribulose-1,5-bisphosphate R5P ribose-5-phosphate

PGA 3-phosphoglyceric acid E4P erythrose-4-phosphate

BPG 1,3-bisphosphoglycerate FBP fructose-1,6-bisphosphate

GAP glyceraldehyde 3-phosphate F6P fructose-6-phosphate

DHAP dihydroxyacetone phosphate G6P glucose-6-phosphate

SBP sedoheptulose-1,7-bisphosphate 6PGL 6-phosphonoglucone- $\delta$-lactone

S7P sedoheptulose-7-phosphate 6PG 6-phosphogluconate

X5P xylulose-5-phosphate Ru5P ribulose-5-phosphate

\section{enzyme names:}

rbcL/rbcS ribulose-1,5-bisphosphate carboxylase/oxygenase (Rubisco)

pgk phosphoglycerate kinase

gap2 glyceraldehyde-3-phosphate dehydrogenase

tpi triosephosphate isomerase

cbbA fructose-1,6-bisphosphate/sedoheptulose-1,7-bisphosphate aldolase

glpX fructose-1,6-bisphosphatase/sedoheptulose-1,7-bisphosphatase

tktA transketolase

rpiA phosphopentose isomerase

rpe phosphopentose epimerase

prkB phosphoribulokinase

tal transaldolase

pgi phosphoglucose isomerase

zwf glucose-6-phosphate dehydrogenase

pgl 6-phosphogluconolactonase

gnd 6-phosphogluconate dehydrogenase 


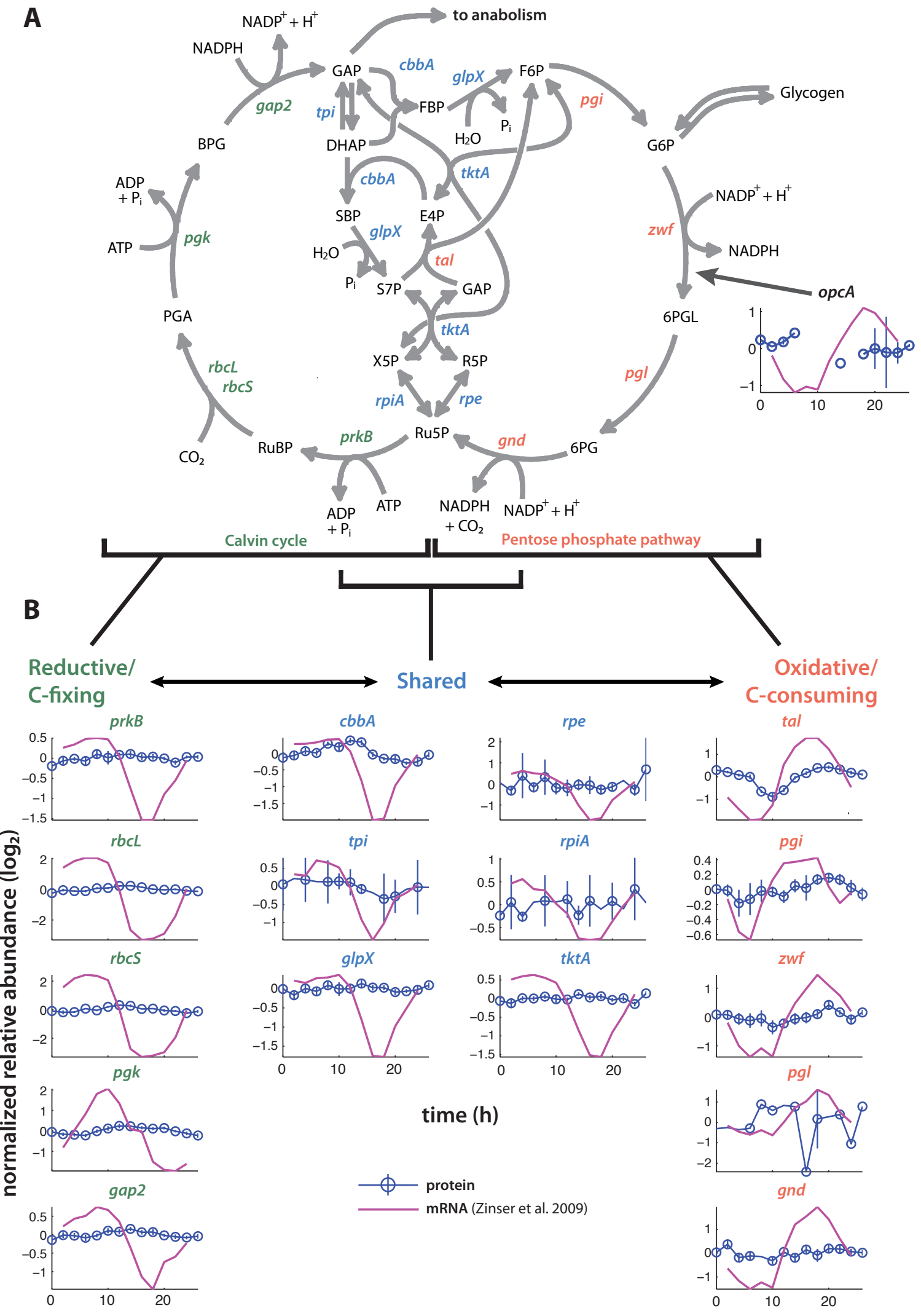


The results shown in Figure 9B add new insights to our understanding of carbon metabolism and fixation by Prochlorococcus. The large-amplitude oscillation of expression at the mRNA level, especially for genes in the reductive/Calvin cycle portion of the pathway, is strongly damped at the protein level. The abundances of central carbon metabolism enzymes show only small variations over the diel cycle. If these small changes are sufficient to alter the balance of fluxes through the central intersection - as they apparently are - this implies that this metabolic network is poised near a kind of balancing point. The many-fold changes in transcript levels observed by Zinser et al. (2009) do not result in wholesale redistribution of protein abundances between the oxidative and reductive portions of the cycle, but rather nudge the network to one side or the other of the flux balance point.

Notably, the only one of these central carbon metabolism enzymes to show more than 2fold changes in abundance is transaldolase (tal), which is also the only component of the central intersection to act solely in the oxidative direction. These data suggest that the abundance of the transaldolase protein is a key parameter regulating the direction of flux through central carbon metabolism. The lower abundance of transaldolase during the morning hours may be just enough to constrict flux through the oxidative pentose phosphate pathway, promoting carbon fixation. The importance of transaldolase levels has previously been inferred from the presence of genes encoding transaldolase in cyanophage genomes and the production of proteins from those genes during phage infection (L.R. Thompson et al., in prep).

Zinser et al. (2009) also raised the possibility of posttranslational regulation of carbon metabolism. In particular, the PrkB/Gap2-binding inhibitor CP12 and the allosteric Zwf effector OpcA were suggested to promote flux through the oxidative pathway at nighttime. CP12 was not observed in our proteomics dataset; it is quite small (74aa) and would not remain bound to its regulatory targets under the denaturing conditions used to 
prepare the samples. OpcA, though, was observed, and almost exclusively during the dark timepoints (Figure 9A). While it is difficult to quantify its cycling amplitude since it dropped out of the analytical window for much of the day, OpcA is clearly significantly more abundant at night, when it likely promotes flux through the oxidative portion of the pathway. The observed constancy of the abundances of enzymes such as Zwf reinforces the importance of the role of these regulatory proteins in modulating fluxes through metabolic pathways.

The relatively constant abundance of Calvin cycle enzymes also helps explain a somewhat puzzling observation in the experiment of Zinser et al. (2009). They noted that, at night, the maximal light-saturated rate of carbon fixation drops to only 2- to 3fold below its daytime peak (c.f. Figure 3B of Zinser et al., 2009). This is surprising given that Rubisco shows a more than 40-fold change in transcript abundance over the diel, and other enzymes in the C-reduction pathway cycle by 4-10 fold at the mRNA level (Fig. 9B). It is now clear that the troughs in transcript abundance for Calvin cycle genes at the sunset do not result in low levels of their respective proteins during the night. Nighttime abundances of Calvin cycle proteins are only $40 \%$ below their daytime peaks; the remaining $10-25 \%$ drop in $\mathrm{C}$ fixation capacity between day and night is likely due to the more oxidized state of the cellular metabolite pool in the dark (L.R. Thompson et al., in prep). Central carbon metabolism in Prochlorococcus is clearly both a biogeochemically important pathway and one that needs to be understood at multiple levels of gene expression and regulation.

\subsection{Cellular gene product abundances over the diel cycle}

\subsubsection{Correlation of fractional transcript and protein abundance}

The use of the APEX (Absolute Protein Expression) technique (Lu et al., 2007), and an equivalent measure of transcript abundance, here termed AMEX (Absolute Message Expression), allows us to express gene product abundances in fractional terms - that is, as 
a percent of the total amount of protein detected at any one timepoint. The basis for these metrics and the caveats to their interpretation are discussed in more detail in Sec. 2.5; suffice it here to note that the APEX/AMEX abundances represent fractions of detected gene products - since the amount of detected protein or mRNA is always less than the total, such measurements will overestimate true cellular abundances to some degree. This problem is significantly more acute at the protein level, since many fewer gene products are represented in the proteomics datasets (27-39\%) than in the RNA-Seq libraries $(89-92 \%)$. With these experimental limitations in mind, however, we can gain biological insights from comparisons of fractional gene product abundances.

We analyzed the relationship between fractional abundances at the protein and transcript levels, for the two timepoints (08:00 and 18:00) for which mRNA sequencing has been performed to date (Fig. 10). The correlation between the two is quite low: mRNA abundance explains only $8-18 \%$ of the variance in protein abundance. While it is perhaps surprising that transcript and protein abundances should be so decoupled, similar results have been obtained from protein-transcript comparisons in a variety of organisms, including yeast (Gygi et al., 1999; Foss et al., 2007; Garcia-Martinez and GonzalezCandelas, 2007; Tuller et al., 2007; Ingolia et al., 2009), E. coli (Ishihama et al., 2008), Streptomyces (Jayapal et al., 2008), Desulfovibrio (Nie et al., 2006), mouse (Huttlin et al., 2009) and human (Gry et al., 2009). The loose connection between mRNA and protein abundances is a theme emerging from multiple studies, and an important one if we are to quantify molecular interactions between microbes and their environments.

It should also be noted that the genes whose protein products were not detected were not necessarily rare at the transcript level. The gray points along the $\mathrm{x}$-axes in Figure 10 show the fractional abundances of transcripts for which no peptides were detected, some of which rank among the most abundant transcripts in the cell at a given timepoint. While some of these are transcripts of hypothetical ORFs that may actually not be translated, some of them are genes that encode important cellular functions and their 

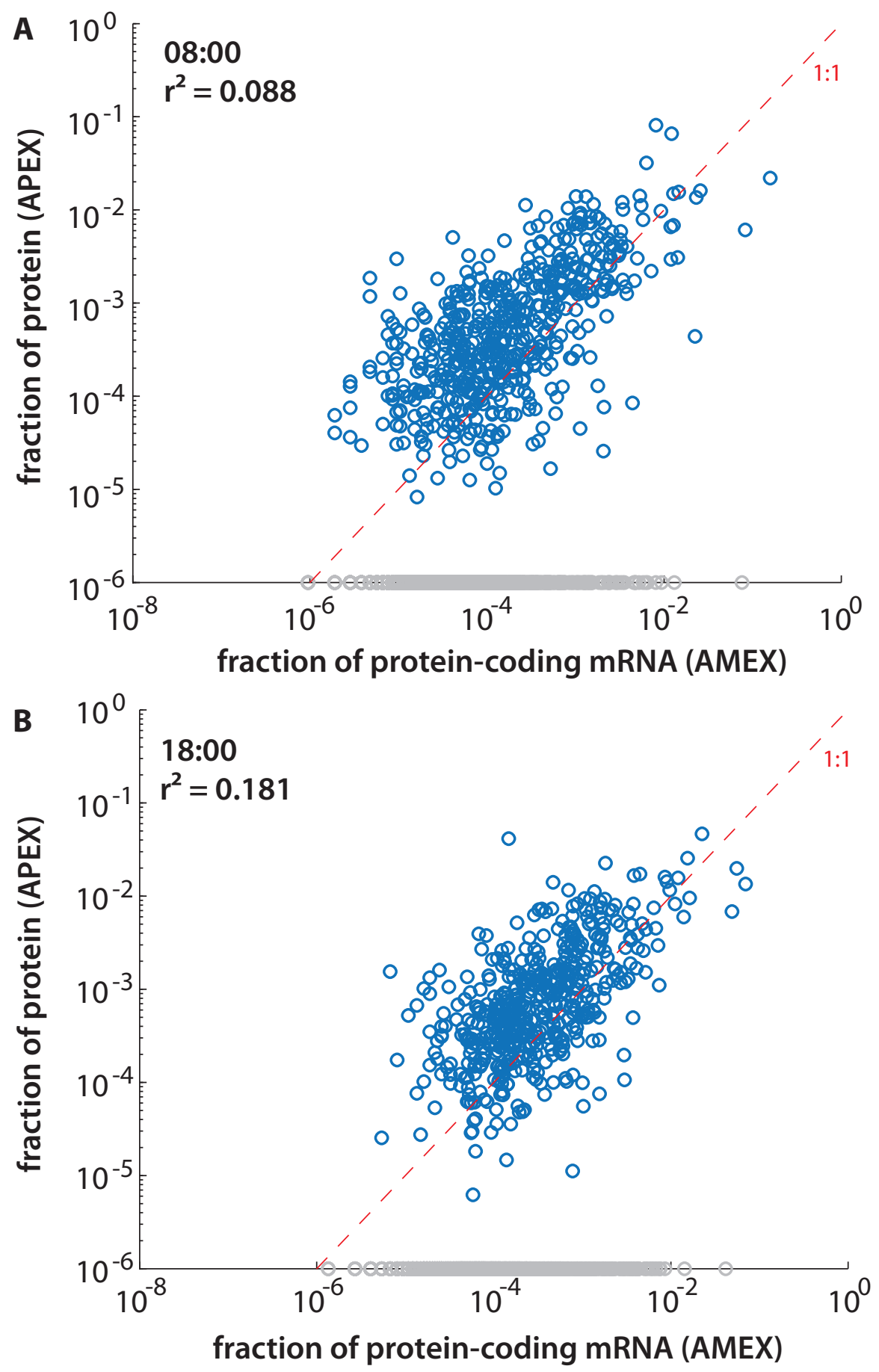

Figure 10. Correlation between mRNA and protein abundances (expressed as fraction of the cellular total, based on AMEX/APEX scores) at (A) 08:00 and (B) 18:00. Blue points are genes detected as both transcript and protein, while gray points along the $x$-axes are genes detected only as mRNA. The correlation between abundance at the mRNA level and abundance at the protein level, while positive, is not strong: it explains only $8-18 \%$ of the variance that spans $>4$ orders of magnitude. 
absence is due to the limited size and experimental biases of the proteomic dataset. The possibility that a number of relatively abundant protein products are outside the analytical window reinforces the approximate nature of fractional abundance measurements.

\subsubsection{Stability of proteome composition over the diel cycle}

One very useful question that gene product fractional abundance measurements allow us to address is to what extent cellular resources are redistributed among different parts of metabolism over the diel cycle. Are, for example, photosynthesis proteins a major portion of the cellular proteome during the day, but then degraded and hardly present in the cell at night? Does the diel cycle involve large-scale sifts of amino acids among proteins involved in different cellular processes? As documented in Section 3.4, enzymes of the Calvin cycle are still present in the cell at night, at more than half of their daytime abundance, even though Prochlorococcus respires carbon at night rather than fixing it. A proteome-wide view of fractional abundance changes over the diel is shown in Figure 11A, as the correlations between abundance profiles at three timepoints: 06:00 (just after sunrise), 08:00, and 18:00 (just before sunset). If a large proportion of the proteome were being redistributed among different sets of proteins over time, we would expect the contrast between sunrise and sunset to be significantly greater than that between successive timepoints. This is not borne out by the observations: the fractional abundance of proteins at 06:00 correlates equally well with abundances at 08:00 as with those at 18:00. The correlation between 06:00 and 18:00 is actually slightly higher than that between 06:00 and 08:00, due primarily to closer correspondence in a few highabundance proteins. There does not appear to be evidence of major remodeling of the proteome over the diel cycle.

A yet broader picture of proteome stability over the diel is revealed by the matrix of pairwise correlations between fractional abundances at all timepoints (Fig. 11B). Each set of APEX values was compared to every other, with the aim of gauging whether protein abundances correlate better between nearby timepoints than between distant ones. 

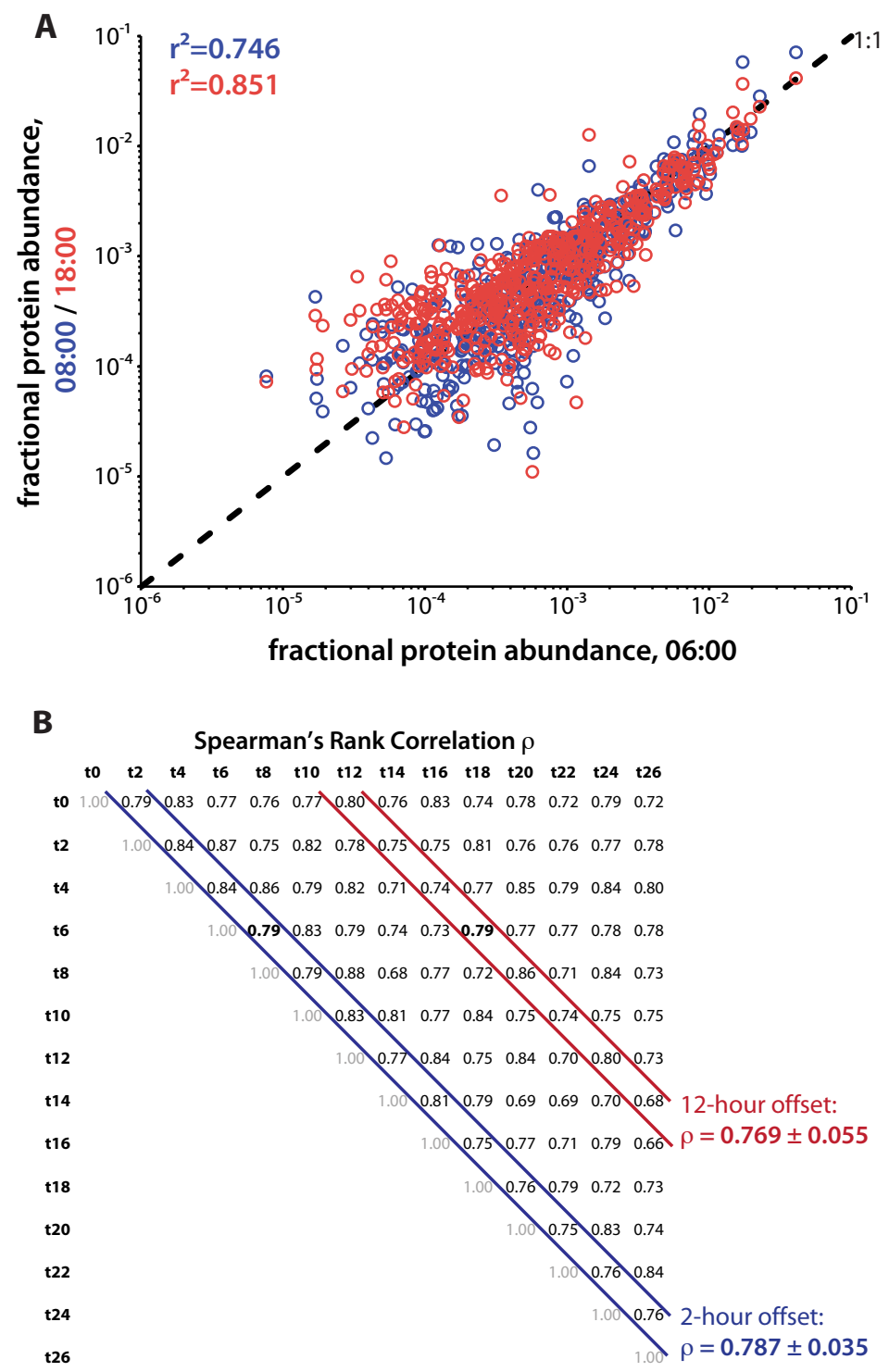

Figure 11. Stability of the MED4 proteome over the diel cycle. A Correlation of fractional protein abundances (APEX values) across timepoints. Protein abundance values at 06:00 (just after sunrise) are compared with values two and twelve hours later. If the rank-abundance structure of the proteome varied strongly over the diel, we would expect protein abundances to correlate better between nearby timepoints (e.g., 06:00 and 08:00) than between widely separated ones (e.g., 06:00 and 18:00). However, this is not observed; in this case, the correlation between timepoints separated by 12 hours is actually slightly better than that between successive samples. B Matrix of Spearman's rank correlation coefficient $(\rho)$ values for protein abundances between all diel timepoints. If proteome abundance structure changed with time, the $\rho$ values would be expected to decrease moving away from the diagonal, presumably to a minimum at an offset of 12 hours. Instead, no significant difference is seen in $\rho$ values between the smallest ( 2 hours) and greatest (12 hours) temporal offsets. The two correlations plotted in $\mathbf{A}$ are shown in bold. 
Spearman's rank correlation ( $\rho$; simply the standard Pearson's $r$ calculated on ranks) was used here to avoid weighting the highest-abundance proteins too heavily and maintain a proteome-wide measure of correspondence. Using $\rho$, the two correlations shown in Figure 11A give the same coefficient. This matrix of correlations allows us to assess the similarity in proteome composition as a function of temporal offsets between timepoints. If proteome composition varied systematically over the diel cycle, the $\rho$ values should decrease away from the main diagonal to a minimum where the offset is 12 hours. In fact, however, the $\rho$ values at 12 -hour offsets are not significantly lower than those at 2hour offsets (one-tailed $t$-test, $p=0.19$ ). This data strongly suggests that the overall composition of the Prochlorococcus proteome remains stable over the diel cycle.

\subsubsection{Cellular functions of abundant proteins}

The stability of proteome composition means that a particular set of proteins are consistently the most abundant in the cell. One enumeration of this set is listed in Table 2, where all 51 proteins with average fractional abundance over the diel cycle of 0.005 or greater are grouped by function. To illustrate the consistency of protein abundance, consider that these 51 proteins occupy $92 \%(656 / 714)$ of the spots in the combined top-51 abundance rankings of the 14 timepoints. The biochemical functions of many of the abundant proteins are ones expected from Prochlorococcus: carbon fixation, photosynthesis, ATP synthesis and nutrient uptake. Necessary cellular functions such as

protein folding (chaperonins), nucleotide metabolism, fatty acid biosynthesis, cell division (FtsZ) and translation (TufA) are also represented. Notably, the most abundant central carbon metabolism proteins are predominantly those involved in the Calvin cycle; none of the oxidative pentose phosphate pathway-specific proteins appear (the most abundant of these, transaldolase, has an average APEX score of 0.0024).

A few biochemical systems appear in the list of abundant proteins that have not to date been the subject of detailed investigation in Prochlorococcus. Two components of the thioredoxin system, TrxA and AhpC, are highly abundant. Thioredoxin (TrxA) is a 


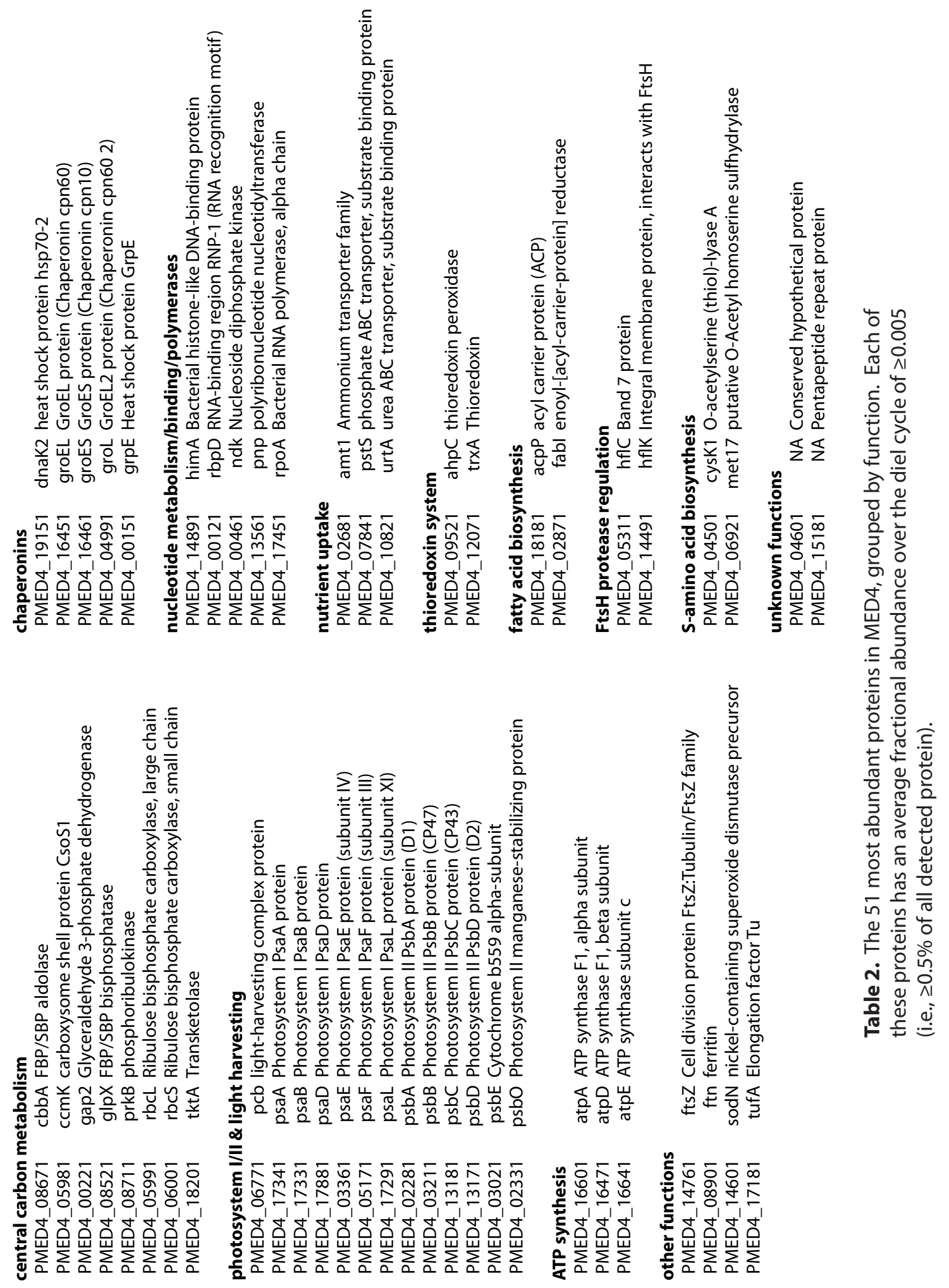


redox-active protein that interacts with a wide range of enzymes involved in photosynthesis (Schurmann and Buchanan, 2008). Thioredoxin peroxidase (AhpC) is an inorganic and alkylperoxide detoxifying enzyme (Parsonage et al., 2008) that uses thioredoxin as an electron donor; since Prochlorococcus lacks genes for catalase and heme peroxidases, it likely relies on this thioredoxin-based system to deal with peroxides that are an inevitable byproduct of its oxygenic metabolism. This may be especially true in axenic culture conditions (such as the current experiment), as it has been shown that one of the key "helper" roles of heterotrophic bacteria in co-culture with Prochlorococcus is as peroxide scavengers (Morris et al., 2008). Nickel-containing superoxide dismutase was also one of the most abundant proteins, consistent with the notion that these dense $\left(\sim 10^{8}\right.$ cells $\left./ \mathrm{ml}\right)$, axenic cultures of Prochlorococcus are oxidatively stressful environments for the cells.

Another surprise is the abundance of two enzymes, CysK1 and Met17, involved in the biosynthesis of the sulfur-containing amino acids, cysteine and methionine. These two enzymes catalyze the same step in their respective pathways: the insertion of sulfide (from assimilatory sulfate reduction) into $O$-acetyl(homo)serine. This is unexpected because Cys and Met are two of the least-common amino acids in the MED4 proteome $\left(20^{\text {th }}\right.$ - and $17^{\text {th }}$-most abundant, respectively; see Chapter 5). Moreover, conditions known to promote high expression of these genes, such as sulfur limitation (Wirtz et al., 2004), seem unlikely in a sulfate-rich medium like seawater. The high abundance of these proteins is therefore puzzling. It may be that the MED4 alleles of these two enzymes are catalytically slow, necessitating high abundances even to provide the small required supply of Cys and Met. It is also possible that Prochlorococcus is especially sensitive to sulfide, and the abundance of these enzymes ensures that $\mathrm{H}_{2} \mathrm{~S}$ produced by assimilatory sulfate reduction is quickly utilized for its intended biosynthetic purpose and does not build up in the cytosol. 


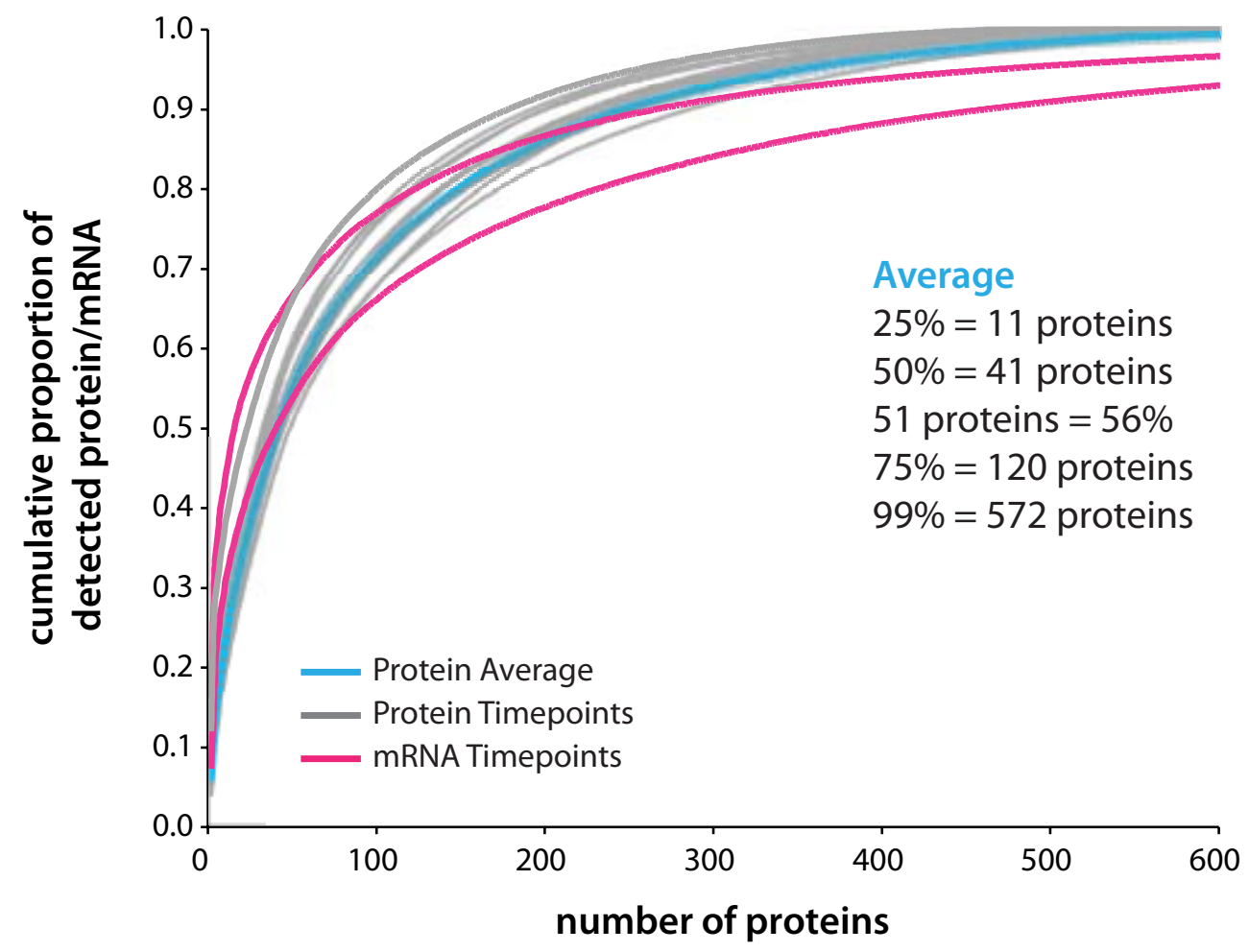

Figure 12. Rank-abundance structure of the MED4 proteome. Plotted is the proportion of cellular protein represented by the $n$ most abundant proteins, based on APEX scores. Each of the 14 diel sampling timepoints is represented by a gray curve, and the average across the diel dataset is in blue. For comparison, the mRNA abundance curves for the two timepoints sequenced to date are shown in pink. The shape of the cumulative protein abundance curve shows relatively little variation across the diel, suggesting that amino acids are not more evenly distributed among proteins at one time of day than another. The inset lists the number of proteins accounting for the given percentages of the cellular proteome (e.g., on average, $75 \%$ of protein is in 120 different gene products). With significant caveats concerning experimental sensitivity and bias, this suggests that a few hundred proteins account for the bulk of the proteome, while the rest ( $\geq 1000$ in MED4) are quite rare or nonexpressed. 


\subsubsection{Rank-abundance structure of the proteome}

Finally, fractional abundance measurements can provide an estimate of how concentrated a cell's total complement of proteins are in the most abundant gene products. Figure 12 shows the rank-cumulative abundance structure for the MED4 proteome as measured over the diel cycle. The 51 most abundant proteins discussed in the previous section make up, on average, $56 \%$ of the total amount of detected protein. The shapes of the rank-abundance curves for the various timepoints (gray lines) do not vary dramatically, further evidence that the overall composition of the proteome is generally stable over the diel cycle. $99 \%$ of the detected protein is accounted for by roughly 572 proteins; taken at face value, this implies that the products of all other gene (a total of 1356 ORFs) sum to $\leq 1 \%$ of cellular protein. As discussed above, however, fractional abundance estimates are blind to undetected proteins, which are not necessarily rare. By comparison, the higher-coverage transcriptomics datasets (pink lines in Figure 12) suggest that the top 600 gene products make up $\sim 90 \%$ of the total. While the estimates are uncertain at this point, these data do point towards a "long tail" of gene product abundance, with over 1000 gene products accounting for perhaps $\sim 10-20 \%$ of the cellular total. Similar "long tails" have been observed in other biological systems, including human plasma (Anderson and Anderson, 2002). These distributions illustrate the enormous expansion in the dynamic range of gene product abundance between DNA and protein.

\section{CONCLuSions}

A principal finding of this study is that the strong diel periodicity in the expression of many Prochlorococcus genes at the mRNA level (Zinser et al., 2009) is substantially damped at the protein level. While Zinser et al. (2009) found that $82 \%$ of transcripts of protein-coding genes exhibit a diel abundance cycle, we estimate that only $15-47 \%$ of proteins cycle significantly over the diel. While mass-spectrometry based proteomics data is not as rich as microarrays or RNA-sequencing as a probe of gene expression, it is clear that, for $80-90 \%$ of genes, the amplitude of oscillation in gene product abundance is 
lower at the protein level than at the mRNA level. Additionally, for many proteins the timing of peak abundance is quite different from - or even opposite to - the maximum in transcript abundance, and these offsets are highly variable from gene to gene. Hence the temporal dynamics of protein expression cannot be directly extrapolated from transcript abundance timecourses. Our results show that substantial decoupling of transcript and protein abundances occurs even in a small, 'simple' organism like Prochlorococcus that has streamlined metabolism and regulatory systems.

We have also established that the overall composition of the proteome, in terms of proportional protein abundance, is quite stable over the diel cycle. Proteins that are highly abundant in the cell remain so around the clock, not being degraded down to low levels and then re-synthesized. Thus in proteomic terms, Prochlorococcus is not a "phototroph by day, heterotroph by night"; the protein machinery for its photoautotrophic lifestyle is present in the cells in the dark, and the enzymes used for respiration at night are present during the day as well. The fact that Prochlorococcus actually does perform net $\mathrm{C}$ fixation during the day and net respiration at night suggests that its metabolic networks are poised near a flux balance point. Relatively small changes in the abundance of enzymes at key points in the network, likely combined with other forms of posttranslational regulation, can redirect metabolic fluxes to match cellular demands and environmental conditions. This picture of metabolism reflects the evolutionary refinement of microbial physiology, whereby fluxes through a complex biochemical network are modulated by small, targeted shifts rather than system-wide remodeling.

Since most proteins change so little in abundance over the diel cycle, the few that do oscillate strongly stand out. In particular, ribonucleotide reductase (NrdJ) changes more than 10-fold in abundance between morning and late afternoon. This variation is certainly coherent with NrdJ's role in DNA synthesis, which occurs between noon and sunset. But other cell-cycle-specific proteins do not oscillate nearly so strongly; FtsZ and MreB, for example, vary only 1.3 -fold. Why the large cycle in NrdJ? It may be that the 
presence of ribonucleotide reductase in the cytosol outside of $\mathrm{C} / \mathrm{S}$ phase is deleterious; at other times, the main goal of nucleotide synthesis is RNA, and having material diverted to DNA may be substantially counterproductive. But the presence of $n r d J$ in phage genomes (L.R. Thompson et al., in prep) offers another hypothesis: the low abundance of NrdJ outside of the DNA synthesis may be a mode of defense against phage infection. When a bacteriophage infects a host cell, it shuts down translation of the host genome and begins expressing its own using the host's machinery. Thus, to make progeny, the phage is dependent on the presence of DNA-synthesis enzymes (notably NrdJ) in the host cell at the time of infection. If infection occurs when NrdJ protein levels are low (as they are in the morning), phage infection may stall for want of deoxyribonucleotides to copy the phage genome. In this scenario, the presence of ribonucleotide reductase genes in the majority of sequenced cyanophage genomes (Sullivan et al., 2005; Sullivan et al., in press) is a strategy adopted by phage to circumvent dependence on host NrdJ by encoding their own copy and expressing it during infection.

The results of this experiment also have implications for how inferences can be drawn between lab- and field-based datasets, and what sorts of techniques might be most useful as assays of conditions and microbial activities in natural environments. First, it appears that growth in dense, axenic culture imposes particular oxidative stress on Prochlorococcus cells. Assessing the true biochemistry and regulation of the response of Prochlorococcus to reactive oxygen species under oceanic conditions is therefore likely to be challenging, since the cell densities currently required to produce sufficient material for some kinds of molecular analyses (as well as the absence of exogenous reductants produced by other members of the community) are a source of oxidative stress. Second, if measurements of gene product abundances are to be used as reporters of the physiological activities of, and environmental stresses felt by, organisms in natural settings, then a multilevel, systems view of gene expression and regulation is warranted. This experiment exemplifies how the dynamics of protein abundance can be very different in both timing and magnitude from those of transcripts, yet for a variety of 
methodological reasons (such as the ability to selectively amplify a gene of interest), it is often desirable to assay gene expression at the transcript level. Given the findings presented here, and similar results emerging from systems biology more generally (Beyer et al., 2004; Nie et al., 2007), we suggest that complementing transcript-level measurements with information from other biological metrics (be they proteins, metabolites, activity assays, etc.) will often be valuable for drawing accurate biogeochemical inferences.

The quantitative concordance between levels of biological organization (transcriptome, proteome, metabolism) is likely to be greatest in concerted responses to acute stresses (Halbeisen and Gerber, 2009). The majority of laboratory gene expression studies test such responses, as the contrast between stress and control conditions is distinct and produces strong experimental signals. With the exception of studies of specific perturbation events, however, observations of natural populations are generally not studies of acute stress responses. When we look at a microbial community in its natural habitat, we are seeing a group of organisms that have necessarily adapted to that environment over many generations. When we contrast two environments that differ in, for example, availability of a particular nutrient, the adaptation involved is to a chronic stressor, and is not the same as the kind of acute nutrient limitation that is often imposed in the laboratory. The longest-running experimental evolution study, the E. coli Longterm Evolution Experiment (Barrick et al., 2009), has tracked populations over 40,000 generations, and most steady-state culture studies (e.g., in chemostats) are much shorter. Prochlorococcus has undergone roughly 40,000 generations just since the year 1900, while living in an ocean undergoing both natural and anthropogenic change on a continuum of timescales ranging from decadal to geologic. Experimental nutrient limitation in culture can help identify important genes and functions (e.g., Martiny and Coleman et al., 2006), but the quantitative dynamics of that response are distinct from those involved in long-term adaptation. The laboratory study presented here attempts to approximate, however imperfectly, the quotidian activities of Prochlorococcus over its 
natural, unstressed diel growth cycle. In taking a multilevel view of gene expression and metabolic regulation, we hope to contribute to bridging the gap between laboratory and environmental characterizations of microbial roles in biogeochemistry.

\section{ACKNOWLEDGEMENTS}

We are grateful to Luke Thompson for discussions of carbon metabolism and permission to reproduce Figure 9A, Anne Thompson for assistance with sampling during the diel growth experiment, Bryan Krastins for advice on proteomics sample preparation, and to David Shteynberg and Matt Chambers for help with proteomics data analysis. LC-MS data were collected at the Fourier-Transform Mass Spectrometry Facility at the Woods Hole Oceanographic Institution; we thank Facility Manager Melissa Soule for guidance and technical support. This work was supported by grants from the Department of Energy Genomics:GtL program, the NSF Center for Microbial Oceanography: Research and Education, and the Gordon and Betty Moore Foundation to SWC, and by an Office of Naval Research NDSEG Fellowship and an NSF Graduate Research Fellowship to JRW.

\section{REFERENCES}

Anderson, NL, and NG Anderson. 2002. The Human Plasma Proteome: History, Character, and Diagnostic Prospects. Mol Cell Proteomics 1: 845-867.

Axmann, IM, U Duhring, L Seeliger, A Arnold, JT Vanselow, A Kramer, and A Wilde. 2009. Biochemical Evidence for a Timing Mechanism in Prochlorococcus. $J$ Bacteriol 191, no. 17: 5342-5347.

Barrick, JE, DS Yu, SH Yoon, H Jeong, TK Oh, D Schneider, RE Lenski, and JF Kim. 2009. Genome evolution and adaptation in a long-term experiment with Escherichia coli. Nature 461:1243-1247.

Beyer, A, J Hollunder, H-P Nasheuer and T Wilhelm. 2004. Post-transcriptional Expression Regulation in the Yeast Saccharomyces cerevisiae on a Genomic Scale. Mol Cell Proteomics 3: 1083-1092.

Braisted, JC, S Kuntumalla, C Vogel, EM Marcotte, AR Rodrigues, R Wang, S-T Huang, ES Ferlanti, AI Saeed, RD Fleischmann, SN Peterson, and R Pieper. 2008. The APEX Quantitative Proteomics Tool: generating protein quantitation estimates from LC-MS/MS proteomics results. BMC Bioinformatics 9, 529.

Brown, MB. 1975. 400: A Method for Combining Non-Independent, One-Sided Tests of Significance. Biometrics 31, no. 4: 987-992.

Bruyant, F, M Babin, B Genty, O Prasil, MJ Behrenfeld, H Claustre, A Bricaud, L Garczarek, J Holtzendorff, and M Koblizek. 2005. Diel variations in the photosynthetic parameters of Prochlorococcus strain PCC 9511: Combined effects of light and cell cycle. Limnology and Oceanography 50, no. 3: 850-863. 
Claustre, H, A Bricaud, M Babin, F Bruyant, L Guillou, FL Gall, D Marie, and F Partensky. 2002. Diel variations in Prochlorococcus optical properties. Limnology and Oceanography 1637-1647.

Craig, R, and RC Beavis. 2004. TANDEM: matching proteins with tandem mass spectra. Bioinformatics 20, no. 9: 1466-1467.

Foss, EJ, D Radulovic, SA Shaffer, DM Ruderfer, A Bedalov, DR Goodlett, and L Kruglyak. 2007. Genetic basis of proteome variation in yeast. Nature Genetics 39, no. 11: 1369-1375.

Futschik, ME, and H Herzel. 2008. Are we overestimating the number of cell-cycling genes? The impact of background models on time-series analysis. Bioinformatics 24, no. 8: 1063.

Garcia-Martinez, J, and F Gonzalez-Candelas. 2007. Common gene expression strategies revealed by genome-wide analysis in yeast. Genome Biology

Garczarek, L, F Partensky, H Irlbacher, J Holtzendorff, M Babin, I Mary, JC Thomas, and WR Hess. 2001. Differential expression of antenna and core genes in Prochlorococcus PCC 9511 (Oxyphotobacteria) grown under a modulated lightdark cycle. Environmental Microbiology 3, no. 3: 168-175.

Geer, LY, SP Markey, JA Kowalak, L Wagner, M Xu, DM Maynard, X Yang, W Shi, and SH Bryant. 2004. Open mass spectrometry search algorithm. J Proteome Res 3, no. 5: 958-964.

Gry, M, R Rimini, S Stromberg, A Asplund, F Ponten, M Uhlen, and P Nilsson. 2009. Correlations between RNA and protein expression profiles in 23 human cell lines. BMC Genomics 10, 365.

Gurland, J, and RC Tripathi. 1971. A simple approximation for unbiased estimation of the standard deviation. American Statistician 30-32.

Gygi, SP, Y Rochon, BR Franza, and R Aebersold. 1999. Correlation between protein and mRNA abundance in yeast. Mol Cell Biol 19, no. 3: 1720-1730.

Halbeisen, R, and A Gerber. 2009. Stress-Dependent Coordination of Transcriptome and Translatome in Yeast. PLoS Biol 7, no. 5: e105.

Hendrickson, EL, Q Xia, T Wang, JA Leigh, and M Hackett. 2006. Comparison of spectral counting and metabolic stable isotope labeling for use with quantitative microbial proteomics. The Analyst 131, no. 12: 1335-1341.

Holtzendorff, J, F Partensky, S Jacquet, F Bruyant, D Marie, L Garczarek, I Mary, D Vaulot, and WR Hess. 2001. Diel expression of cell cycle-related genes in synchronized cultures of Prochlorococcus sp. strain PCC 9511. J Bacteriol 183, no. 3: 915-920.

Holtzendorff, J, F Partensky, D Mella, J-F Lennon, H WR, and L Garczarek. 2008. Genome streamlining results in loss of robustness of the circadian clock in the marine cyanobacterium Prochlorococcus marinus PCC9511. Journal of Biological Rhythms 23, 187-199.

Hsiao, C, H Chen, K Furuse, and N Ohbo. 2009. Figure and Ground: A Complete Approach to Outlier Detection. AIP Conference Proceedings

Huttlin, EL, X Chen, GA Barrett-Wilt, AD Hegeman, RB Halberg, AC Harms, MA Newton, WF Dove, and MR Sussman. 2009. Discovery and validation of colonic 
tumor-associated proteins via metabolic labeling and stable isotopic dilution. Proc Natl Acad Sci USA 106, no. 40: 17235-17240.

Ingolia, NT, S Ghaemmaghami, JRS Newman, and JS Weissman. 2009. Genome-Wide Analysis in Vivo of Translation with Nucleotide Resolution Using Ribosome Profiling. Science 324, no. 5924: 218-223.

Ishihama, Y, T Schmidt, J Rappsilber, M Mann, FU Hartl, MJ Kerner, and D Frishman. 2008. Protein abundance profiling of the Escherichia coli cytosol. BMC Genomics 9, 102.

Jacquet, S, F Partensky, D Marie, R Casotti, and D Vaulot. 2001. Cell cycle regulation by light in Prochlorococcus strains. Appl Environ Microbiol 67, no. 2: 782-790.

Jayapal, KP, RJ Philp, Y-J Kok, MGS Yap, DH Sherman, TJ Griffin, and W-S Hu. 2008. Uncovering genes with divergent mRNA-protein dynamics in Streptomyces coelicolor. PLoS ONE 3, no. 5: e2097.

Keller, A, AI Nesvizhskii, E Kolker, and R Aebersold. 2002. Empirical statistical model to estimate the accuracy of peptide identifications made by MS/MS and database search. Anal Chem 74, no. 20: 5383-5392.

Kielbasa, SM, H Herzel, and IM Axmann. 2007. Regulatory elements of marine cyanobacteria. Genome Informatics 18, 1-11.

Levicky, R and A Horgan. 2005. Physicochemical perspectives on DNA microarray and biosensor technologies. Trends Biotechnol 23, no. 3: 143-149.

Li, X-J, H Zhang, JA Ranish, and R Aebersold. 2003. Automated statistical analysis of protein abundance ratios from data generated by stable-isotope dilution and tandem mass spectrometry. Anal Chem 75, no. 23: 6648-6657.

Lu, P, C Vogel, R Wang, X Yao, and EM Marcotte. 2007. Absolute protein expression profiling estimates the relative contributions of transcriptional and translational regulation. Nat Biotechnol 25, no. 1: 117-124.

MacLean, B, JK Eng, RC Beavis, and M McIntosh. 2006. General framework for developing and evaluating database scoring algorithms using the TANDEM search engine. Bioinformatics 22, no. 22: 2830-2832.

Mallick, P, M Schirle, SS Chen, MR Flory, H Lee, D Martin, J Ranish, B Raught, R Schmitt, T Werner, B Kuster, and R Aebersold. 2007. Computational prediction of proteotypic peptides for quantitative proteomics. Nat Biotechnol 25, no. 1: $125-131$.

Malmström, J, M Beck, A Schmidt, V Lange, EW Deutsch and R Aebersold. 2009. Proteome-wide cellular protein concentrations of the human pathogen Leptospira interrogans. Nature 460: 762-765.

Martiny, AC, ML Coleman, and SW Chisholm. 2006. Phosphate acquisition genes in Prochlorococcus ecotypes: evidence for genome-wide adaptation. PNAS 103, no. 33: 12552-12557.

Mary, I, and D Vaulot. 2003. Two-component systems in Prochlorococcus MED4: genomic analysis and differential expression under stress. FEMS Microbiol Lett 226, no. 1: 135-144. 
Moore, LR, A Coe, ER Zinser, MA Saito, MB Sullivan, D Lindell, K Frois-Moniz, J Waterbury, and SW Chisholm. 2007. Culturing the marine cyanobacterium Prochlorococcus. Limnology and Oceanography: Methods 5, 353-362.

Morris, JJ, R Kirkegaard, MJ Szul, ZI Johnson, and ER Zinser. 2008. Facilitation of Robust Growth of Prochlorococcus Colonies and Dilute Liquid Cultures by "Helper" Heterotrophic Bacteria. Appl Environ Microbiol 74, no. 14: 4530-4534.

Mullineaux, CW, and R Stanewsky. 2009. The Rolex and the Hourglass: a Simplified Circadian Clock in Prochlorococcus? J Bacteriol 191, no. 17: 5333-5335.

Nesvizhskii, AI, A Keller, E Kolker, and R Aebersold. 2003. A statistical model for identifying proteins by tandem mass spectrometry. Anal Chem 75, no. 17: 46464658.

Nie, L, G Wu and W Zhang. 2006. Correlation between mRNA and protein abundance in Desulfovibrio vulgaris: A multiple regression to identify sources of variations. Biochem Biophys Res Comm 339: 603-610.

Nie, L, G Wu, DE Culley, JCM Scholten and W Zhang. 2007. Integrative Analysis of Transcriptomic and Proteomic Data: Challenges, Solutions and Applications. Crit Rev Biotechnol 27:63-75.

Parsonage, D, PA Karplus, and LB Poole. 2008. Substrate specificity and redox potential of AhpC, a bacterial peroxiredoxin. Proc Natl Acad Sci USA 105, no. 24: 82098214.

Poretsky, RS, I Hewson, S Sun, AE Allen, JP Zehr, and MA Moran. 2009. Comparative day/night metatranscriptomic analysis of microbial communities in the North Pacific subtropical gyre. Environmental Microbiology 11, no. 6: 1358-1375.

Richter, AS, C Schleberger, R Backofen and C Steglich. 2010. Seed-based INTARNA prediction combined with GFP-reporter system identifies mRNA targets of the small RNA Yfr1. Bioinformatics 26, no. 1: 1-5.

Rocap, G, FW Larimer, J Lamerdin, S Malfatti, P Chain, NA Ahlgren, A Arellano, ML Coleman, L Hauser, WR Hess, ZI Johnson, M Land, D Lindell, AF Post, W Regala, M Shah, SL Shaw, C Steglich, MB Sullivan, CS Ting, AC Tolonen, EA Webb, ER Zinser, and SW Chisholm. 2003. Genome divergence in two Prochlorococcus ecotypes reflects oceanic niche differentiation. Nature 424, no. 6952: 1042-1047.

Schurmann, P, and BB Buchanan. 2008. The ferredoxin thioredoxin system of oxygenic photosynthesis. Antioxidants \& Redox Signaling 10, no. 7: 1235-1274.

Silva, JC, MV Gorenstein, G-Z Li, JPC Vissers, and SJ Geromanos. 2006. Absolute quantification of proteins by LCMSE: a virtue of parallel MS acquisition. Mol Cell Proteomics 5, no. 1: 144-156.

Shalapyonok, A, RJ Olson and LS Shalapyonok. 1998. Ultradian Growth in Prochlorococcus spp. Appl Env Micr 64, no. 3: 1066-1069.

Steglich, C, ME Futschik, D Lindell, B Voss, SW Chisholm and WR Hess. 2008. The Challenge of Regulation in a Minimal Photoautotroph: Non-Coding RNAs in Prochlorococcus. PLoS Genet 4, no. 8: e1000173.

Storey, JD, and R Tibshirani. 2003. Statistical significance for genomewide studies. Proc Natl Acad Sci USA 100, no. 16: 9440-9445. 
Sullivan, MB, ML Coleman, P Weigele, F Rohwer, and SW Chisholm. 2005. Three Prochlorococcus cyanophage genomes: signature features and ecological interpretations. PLoS Biol 3, no. 5: e144.

Sullivan, MB, KH Huang, JC Ignacio-Espinoza, A Berlin, L Kelly, PR Weigele, AS DeFrancesco, SE Kern, LR Thompson, S Young, C Yandava, R Fu, B Krastins, M Chase, D Sarracino, MS Osburne, MR Henn and SW Chisholm. In press. Genomic analysis of oceanic cyanobacterial myoviruses compared to T4-like myoviruses from diverse hosts and environments. Env Microbiol.

Tabb, DL, CG Fernando, and MC Chambers. 2007. MyriMatch: highly accurate tandem mass spectral peptide identification by multivariate hypergeometric analysis. $J$ Proteome Res 6, no. 2: 654-661.

Troyanskaya, O, M Cantor, G Sherlock, P Brown, T Hastie, R Tibshirani, D Botstein, and RB Altman. 2001. Missing value estimation methods for DNA microarrays. Bioinformatics 17, no. 6: 520-525.

Tuller, T, M Kupiec, and E Ruppin. 2007. Determinants of protein abundance and translation efficiency in S. cerevisiae. PLoS Comput Biol 3, no. 12: e248.

Vaulot, D, D Marie, R Olson, and S Chisholm. 1995. Growth of Prochlorococcus, a Photosynthetic Prokaryote, in the Equatorial Pacific Ocean. Science 268, no. 5216: 1480-1482.

Vogel, C, and EM Marcotte. 2008. Calculating absolute and relative protein abundance from mass spectrometry-based protein expression data. Nat Protoc 3, no. 9: 14441451.

Vogel, J, IM Axmann, H Herzel, and WR Hess. 2003. Experimental and computational analysis of transcriptional start sites in the cyanobacterium Prochlorococcus MED4. Nucleic Acids Res 31, no. 11: 2890-2899.

Wang, JD, and PA Levin. 2009. Metabolism, cell growth and the bacterial cell cycle. Nat Rev Microbiol 7, no. 11: 822-827.

Wirtz, M, M Droux, and R Hell. 2004. O-acetylserine (thiol) lyase: an enigmatic enzyme of plant cysteine biosynthesis revisited in Arabidopsis thaliana. J Exp Bot 55, no. 404: 1785-1798.

Zaykin, DV, LA Zhivotovsky, PH Westfall, and BS Weir. 2002. Truncated product method for combining P-values. Genet Epidemiol 22, no. 2: 170-185.

Zinser, ER, D Lindell, ZI Johnson, ME Futschik, C Steglich, ML Coleman, MA Wright, T Rector, R Steen, N McNulty, LR Thompson, and SW Chisholm. 2009. Choreography of the transcriptome, photophysiology, and cell cycle of a minimal photoautotroph, Prochlorococcus. PLoS ONE 4, no. 4: e5135. 
Supplementary Figure 1. Timecourses for 548 proteins quantified over the diel cycle in Prochlorococcus MED4. For each protein, relative abundance is shown on a $\log _{2}$ scale. Proteinlevel abundance at each timepoint was calculated as the mean of a lognormal fit to the filtered peak-level ${ }^{14} \mathrm{~N} /{ }^{15} \mathrm{~N}$ abundance ratios, and error bars indicate $95 \%$ confidence intervals based on an unbiased estimate of the standard error of that mean (Sec. 2.4.1). Titles indicate the gene locus and name, where applicable. In the lower right corner of each timecourse panel is the $q$-value calculated for the significance of diel cycling (Sec. 2.4.2). 

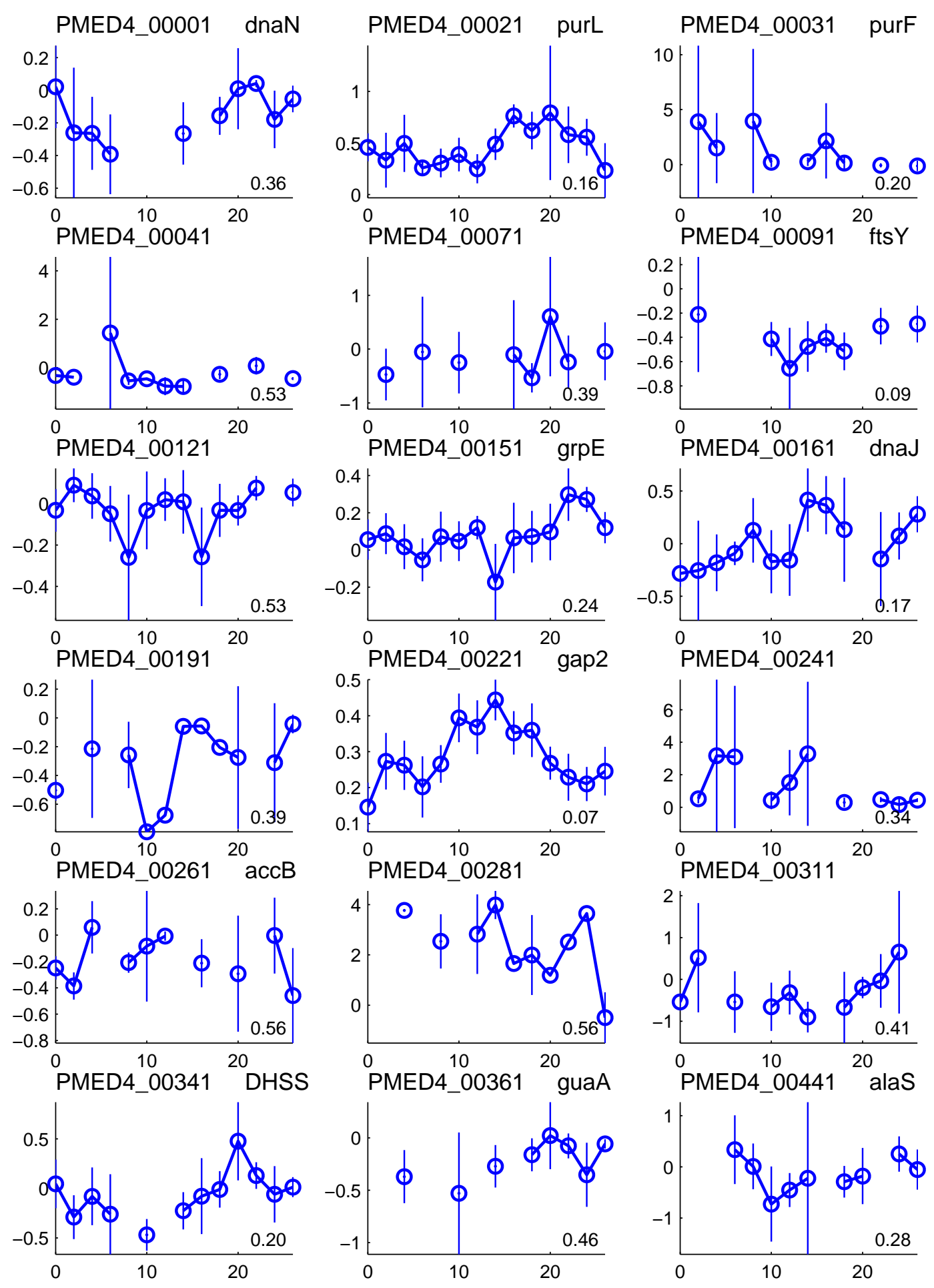

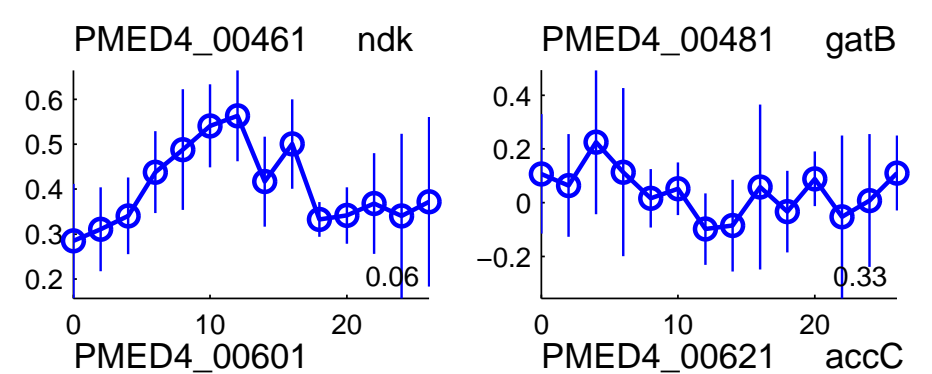

PMED4_00551
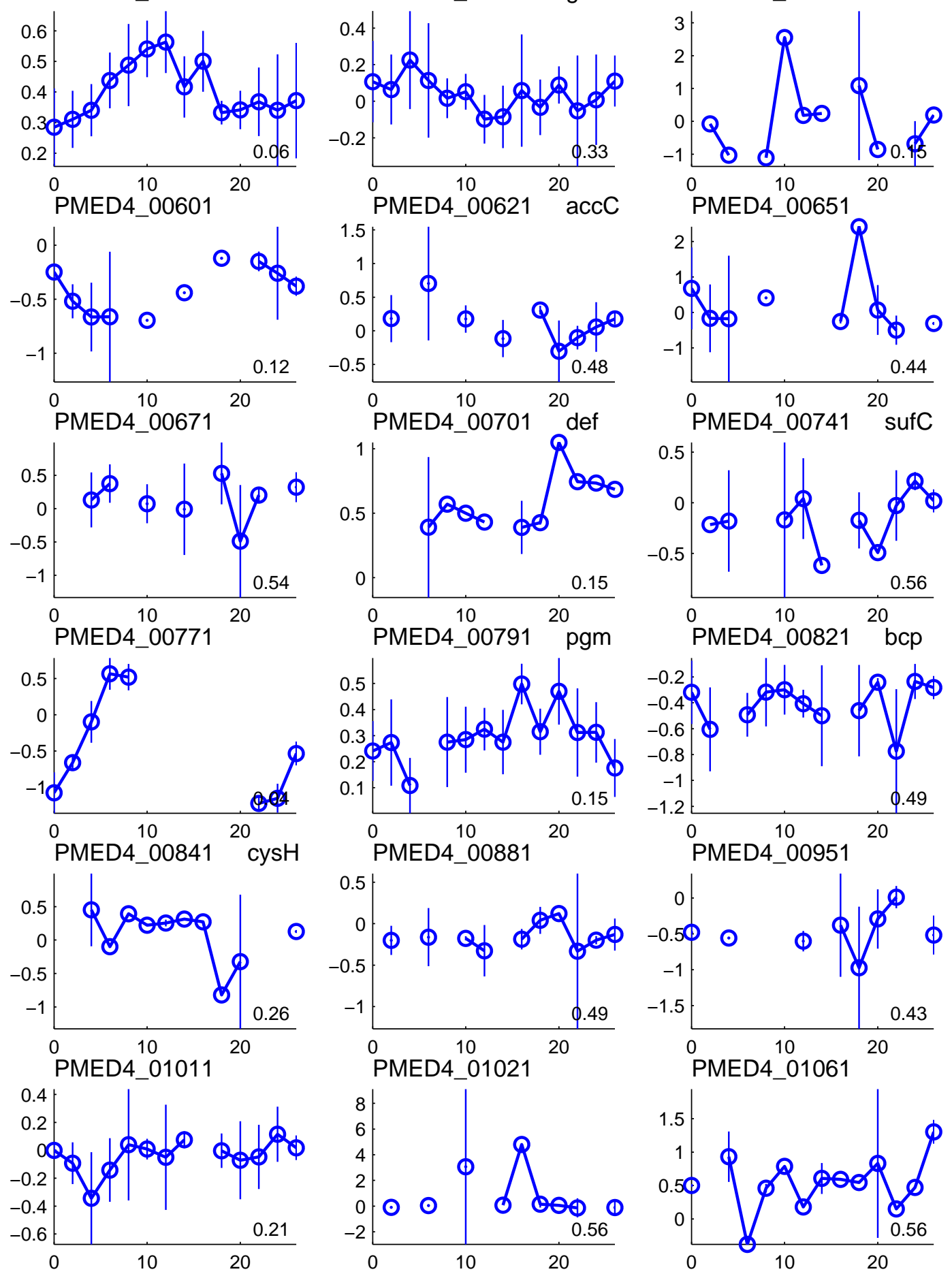

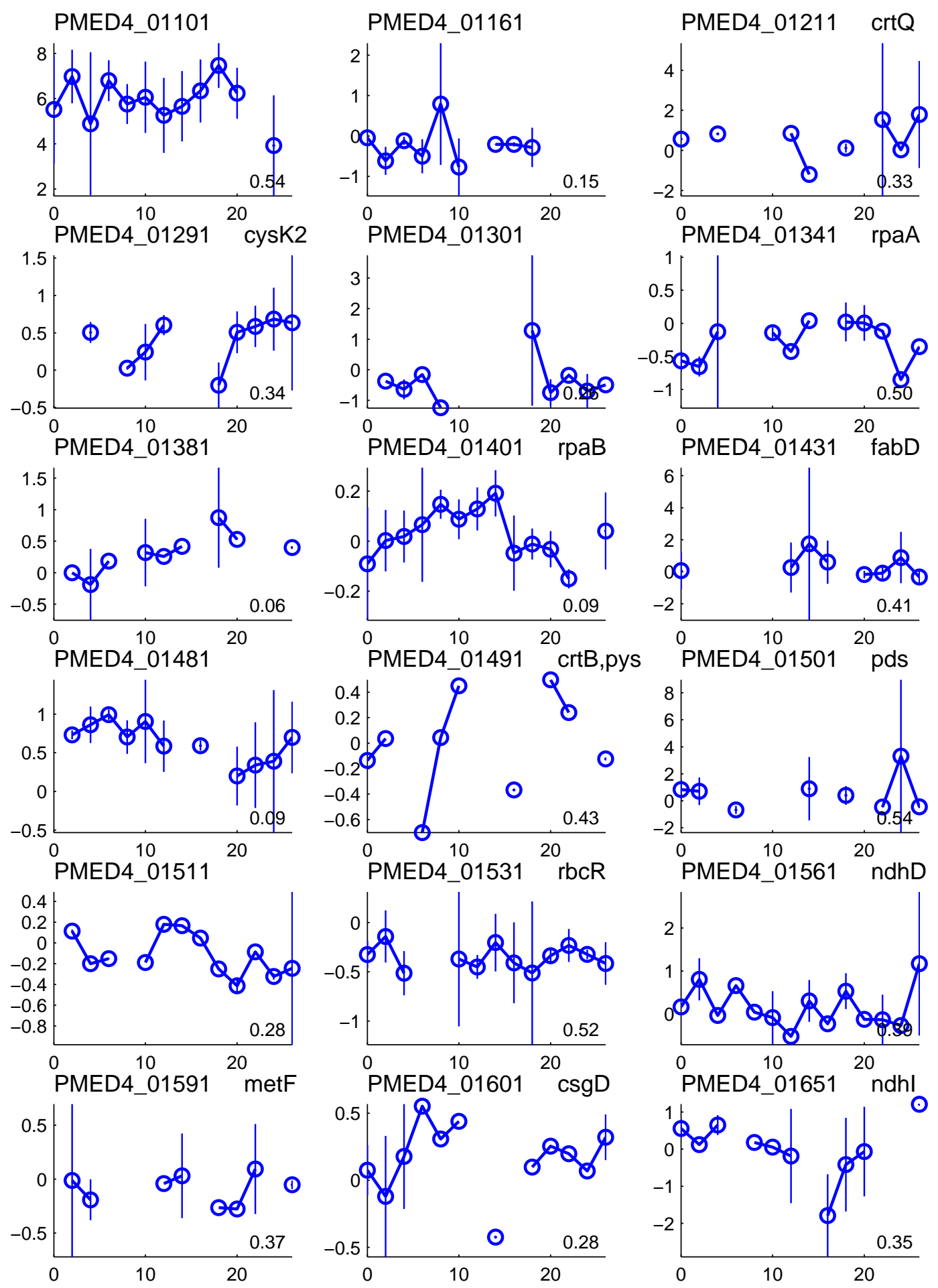

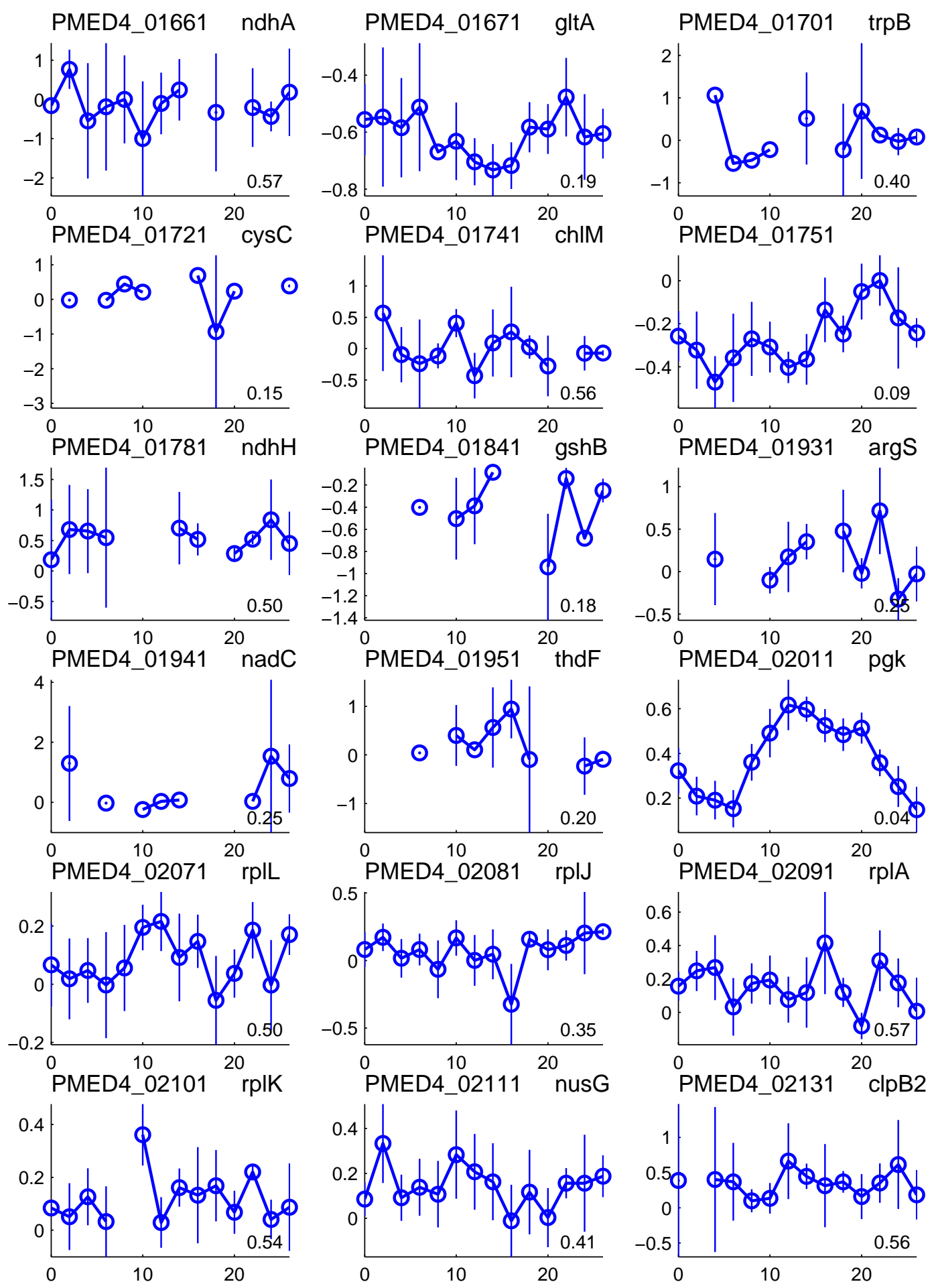

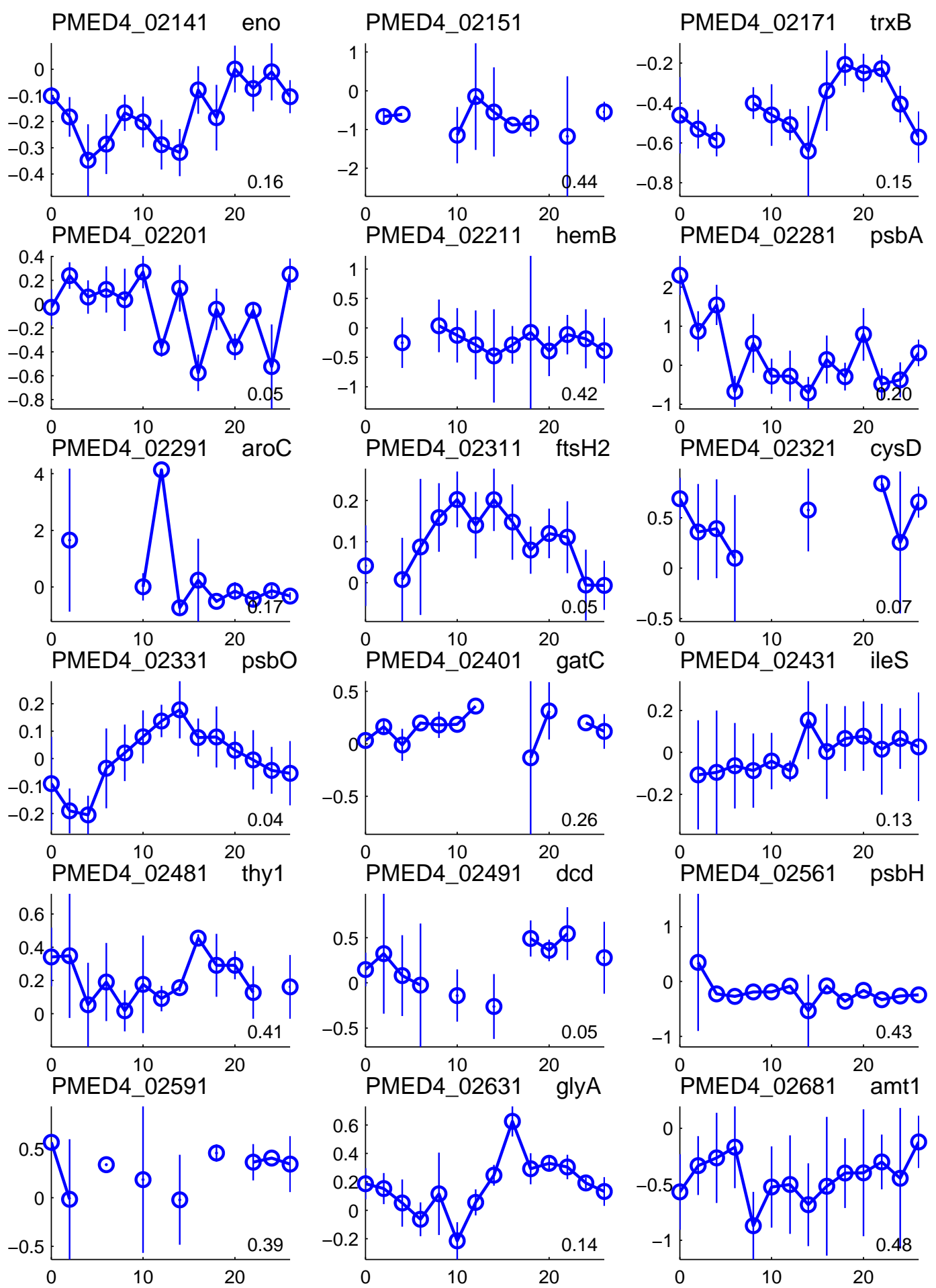
PMED4_02701
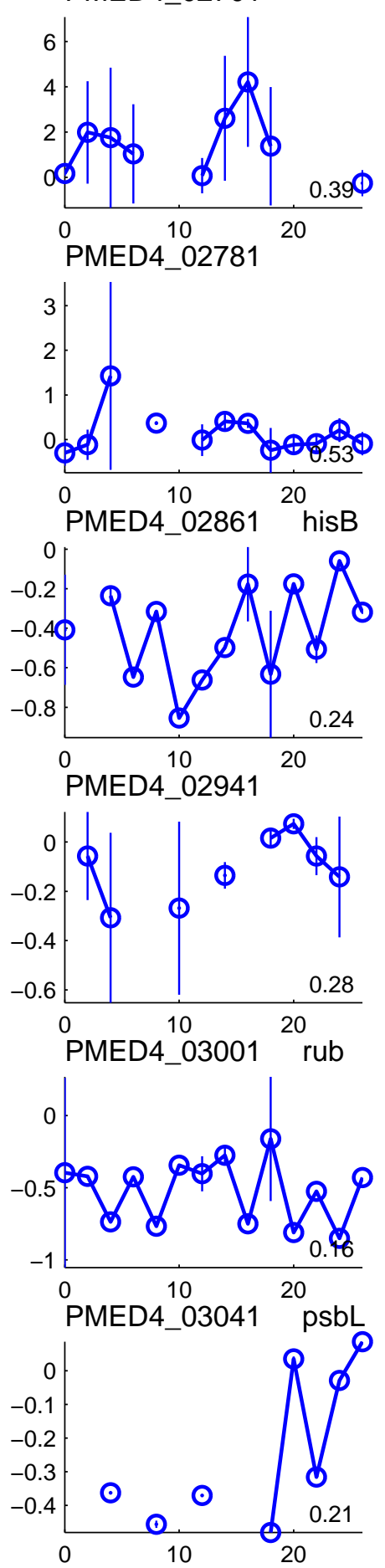

PMED4_02711 purH PMED4_02721
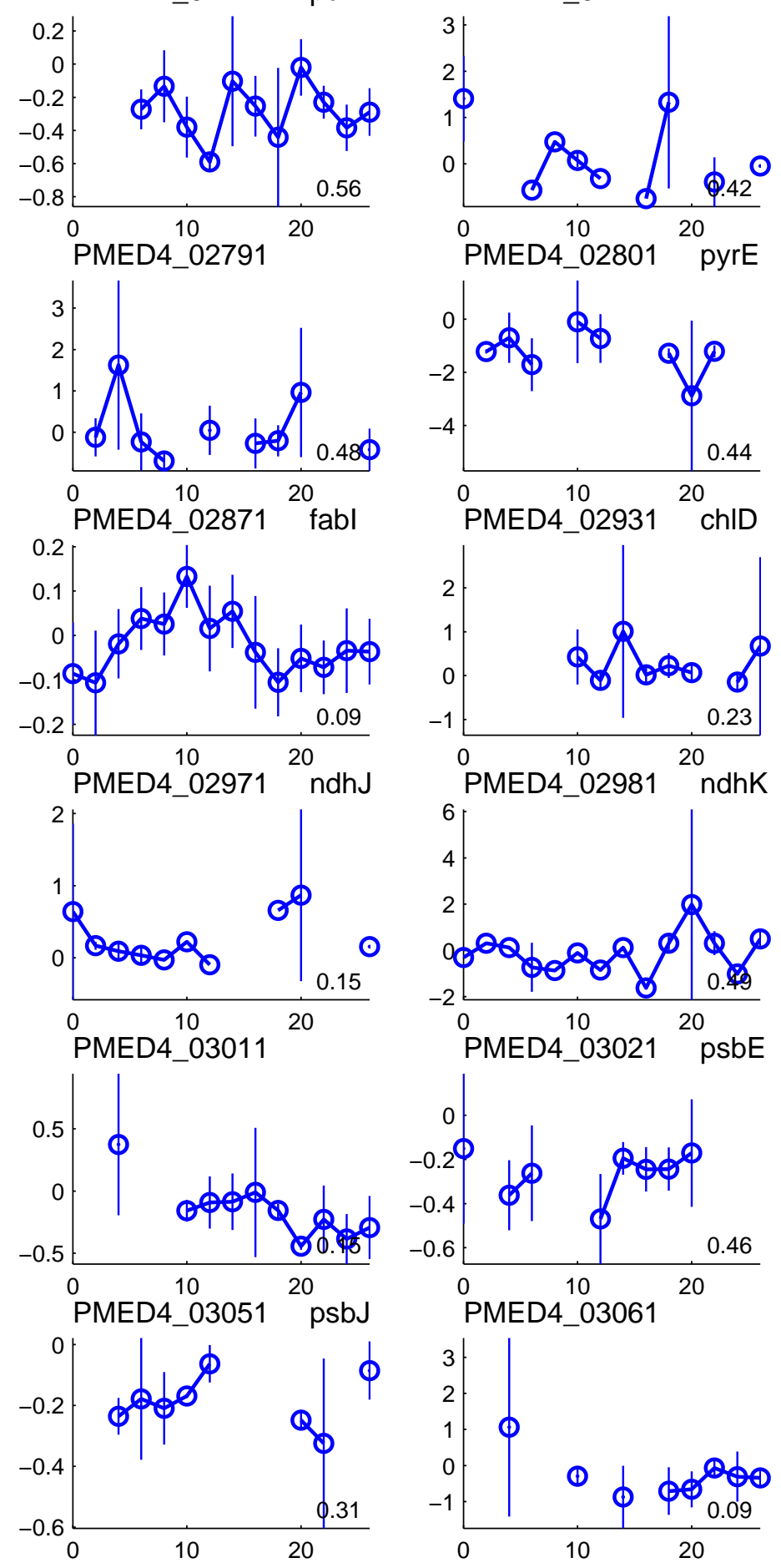

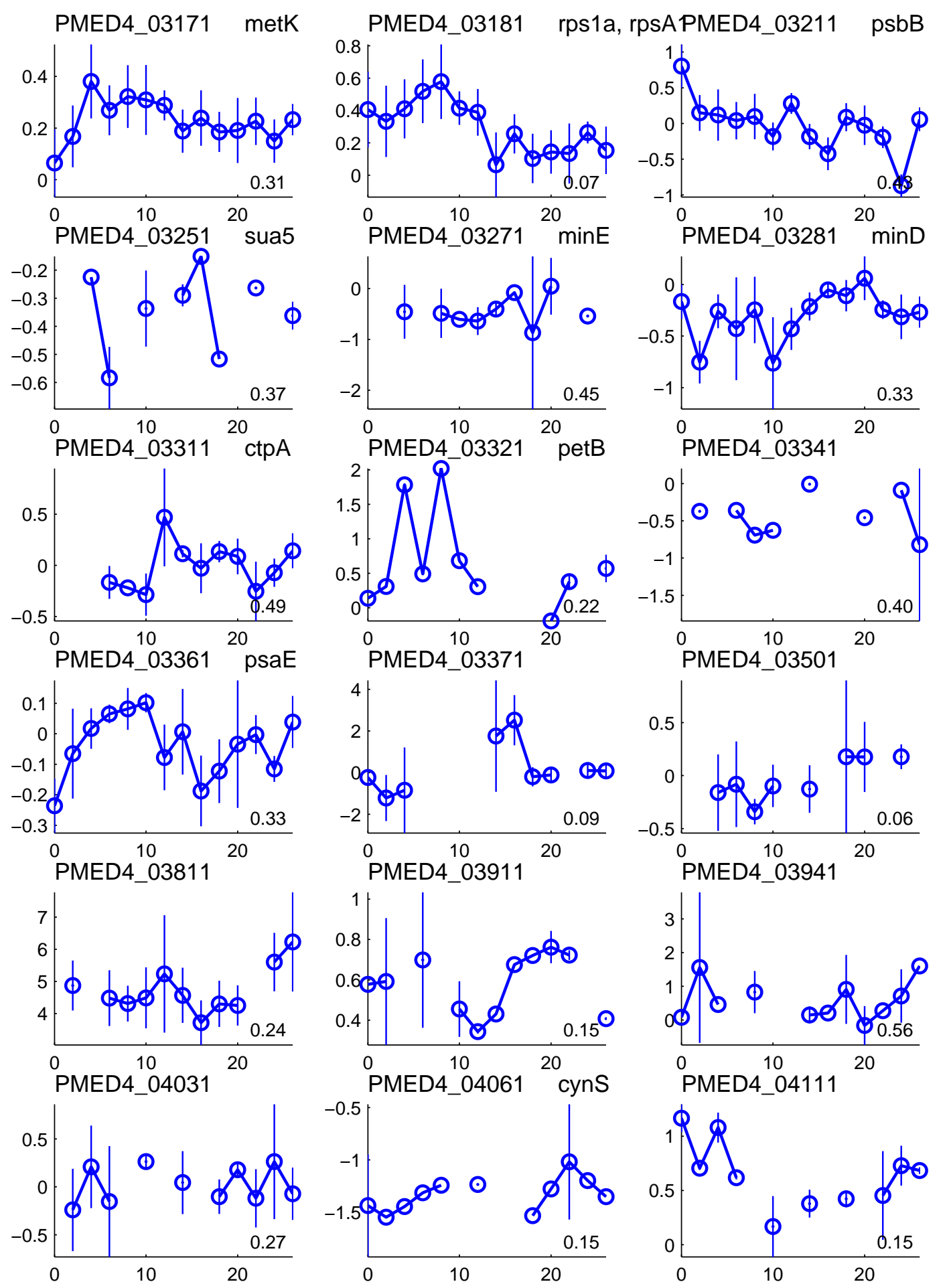
PMED4_04231 spnll-interruPAtteD\&_04411 IrtA PMED4_04441
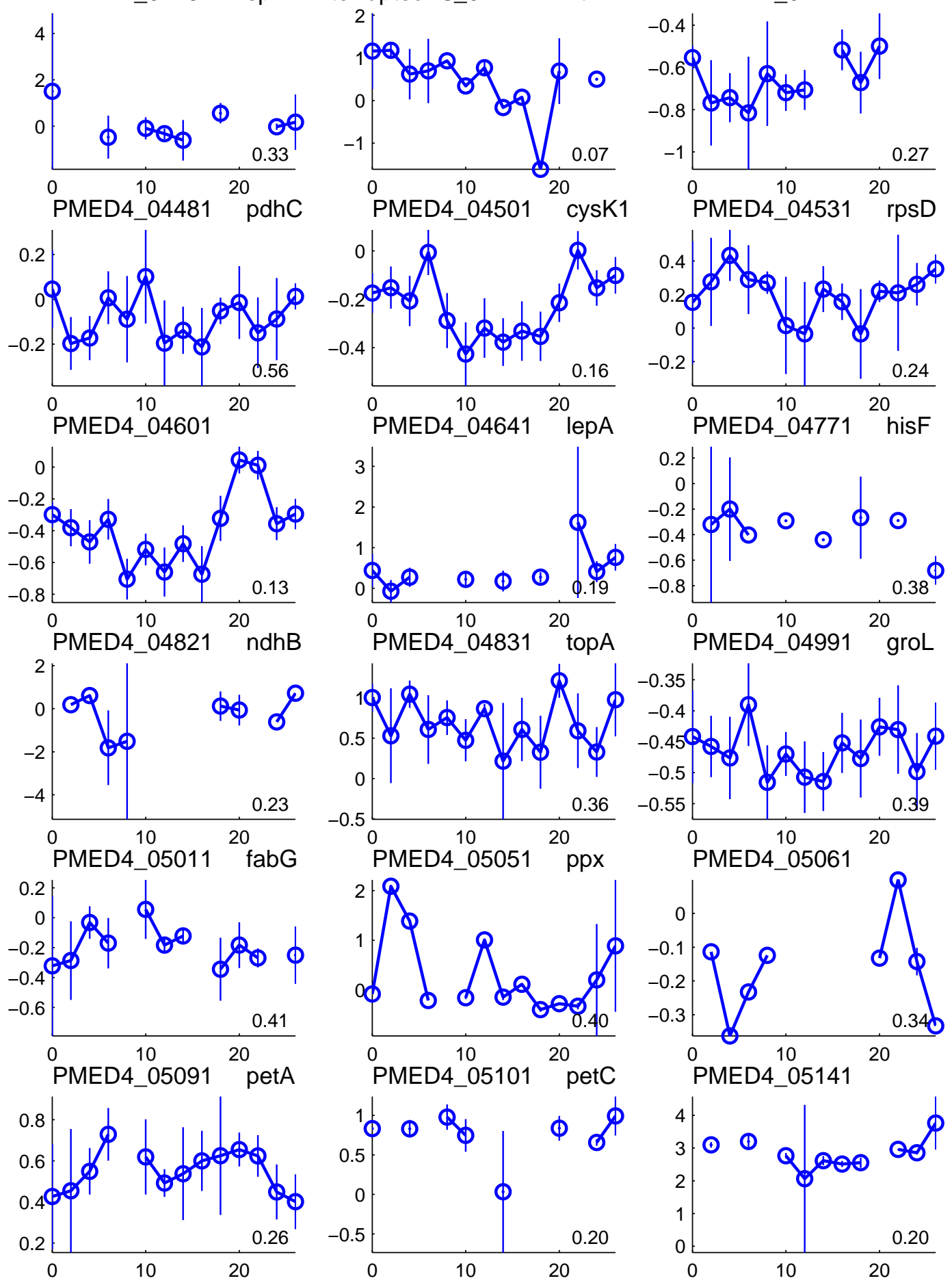

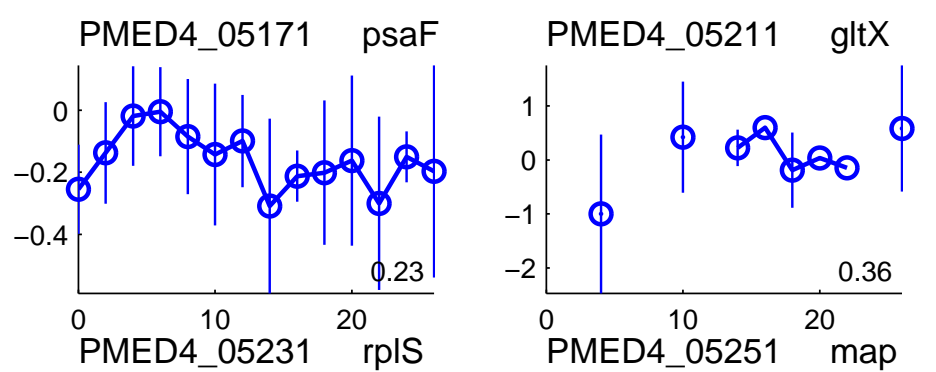

PMED4_05221
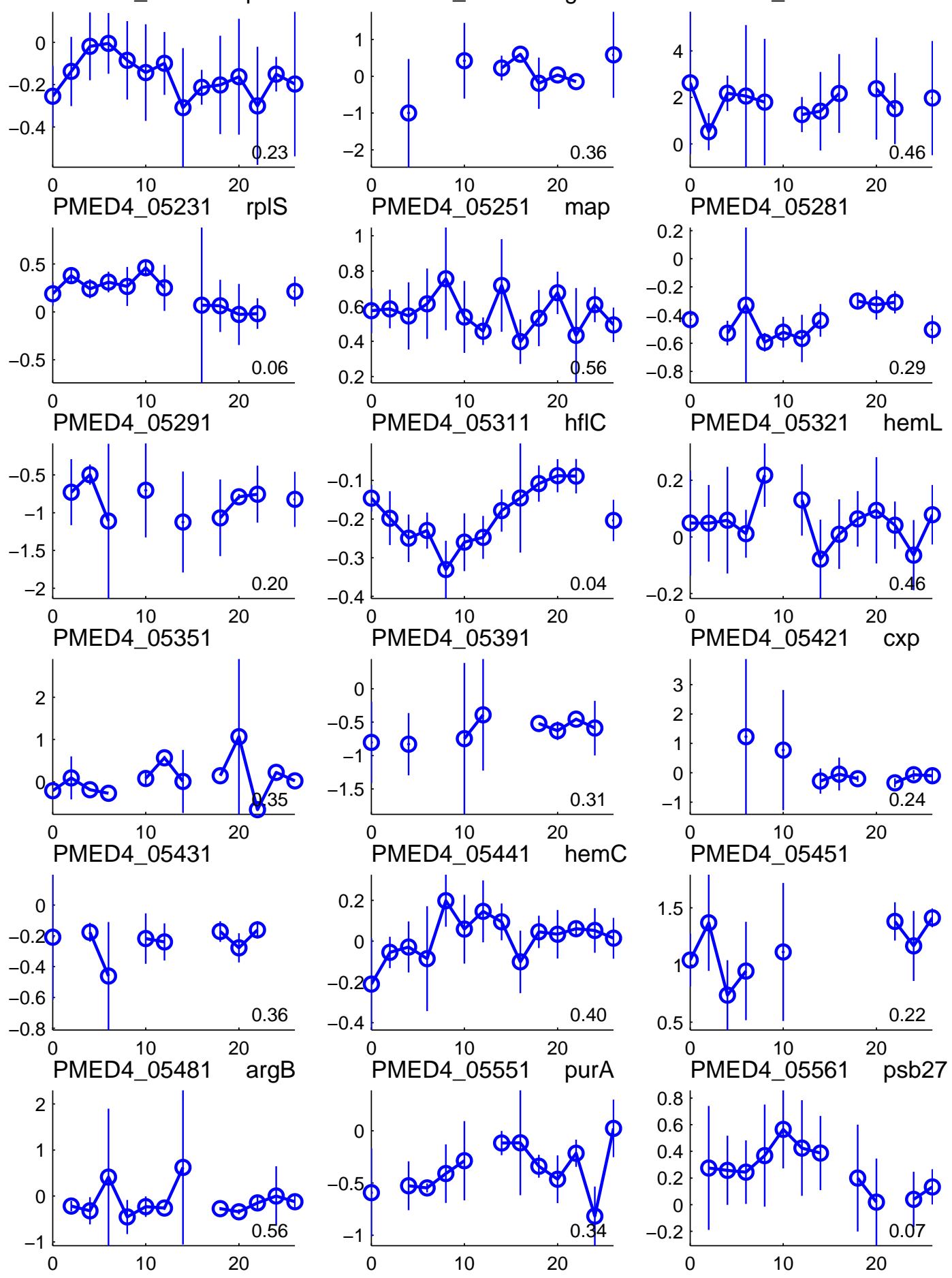

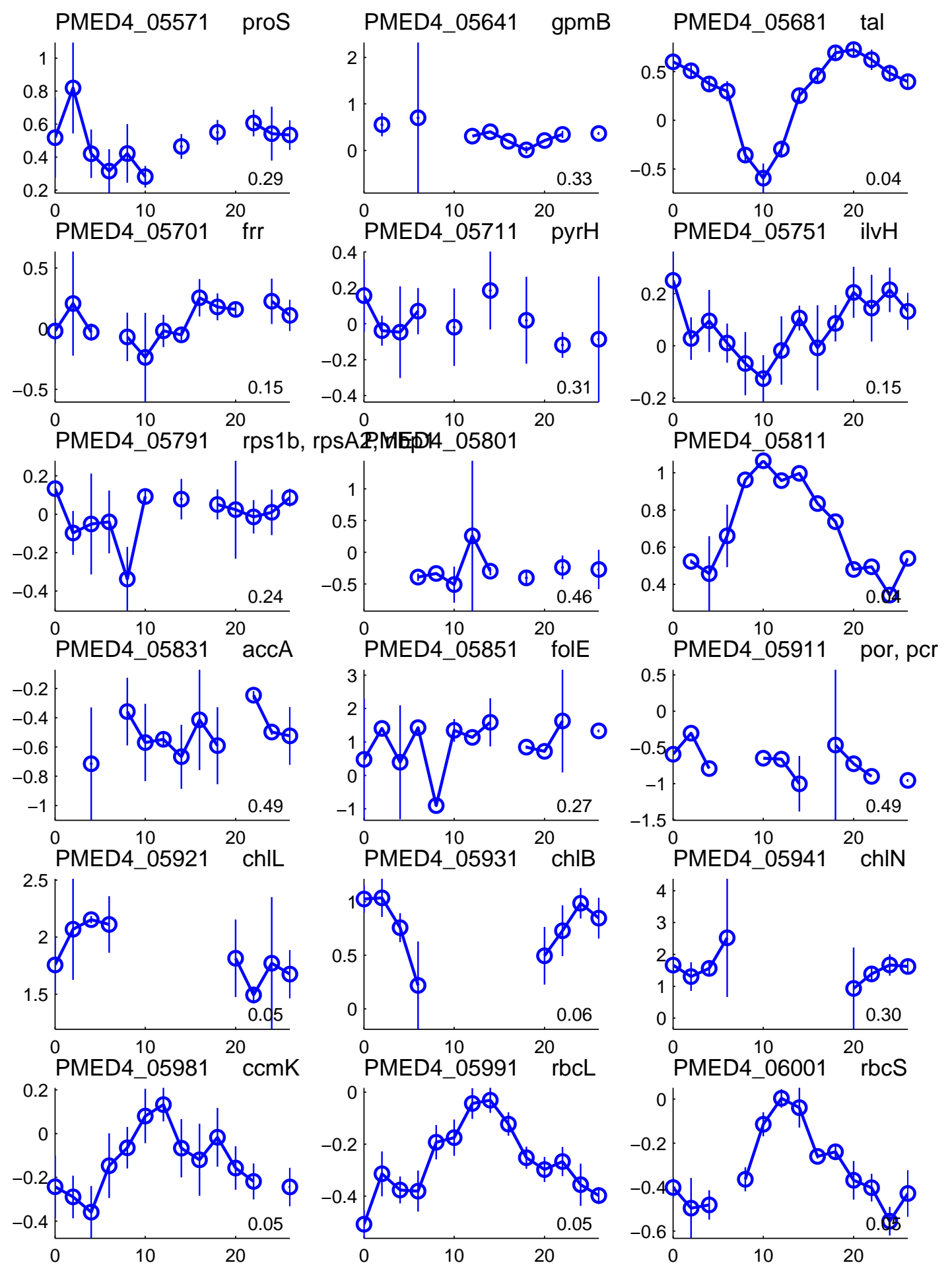

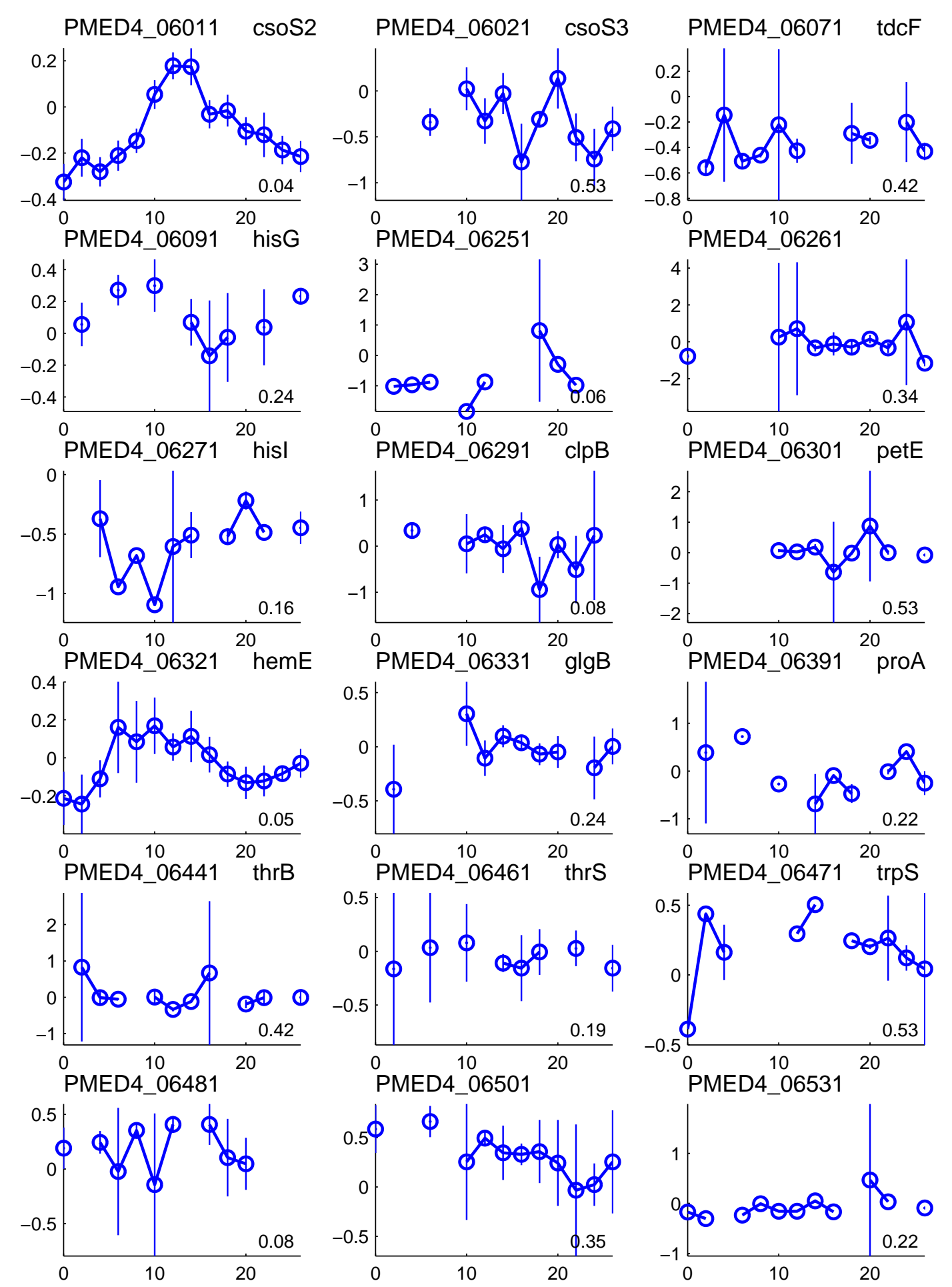

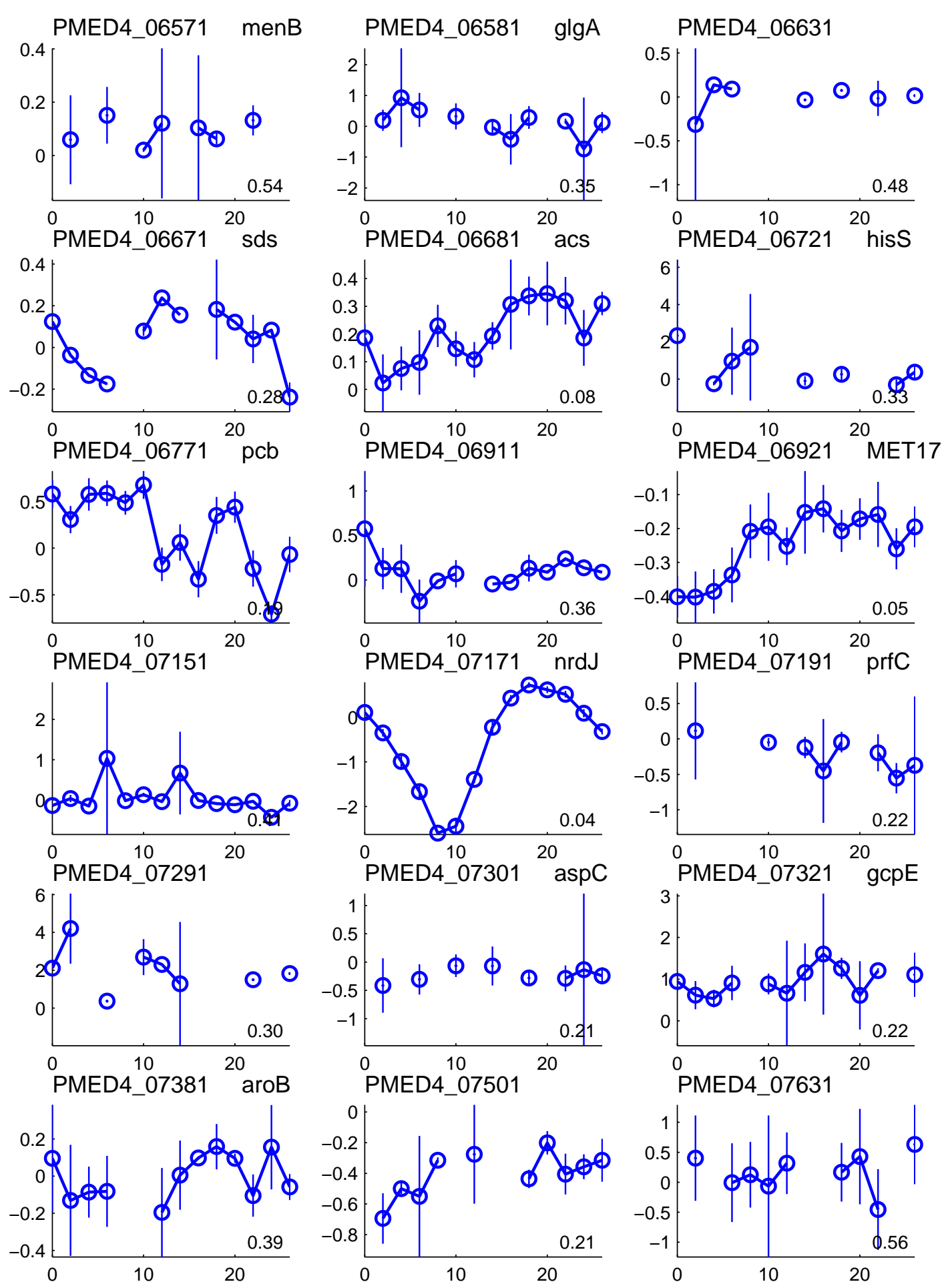

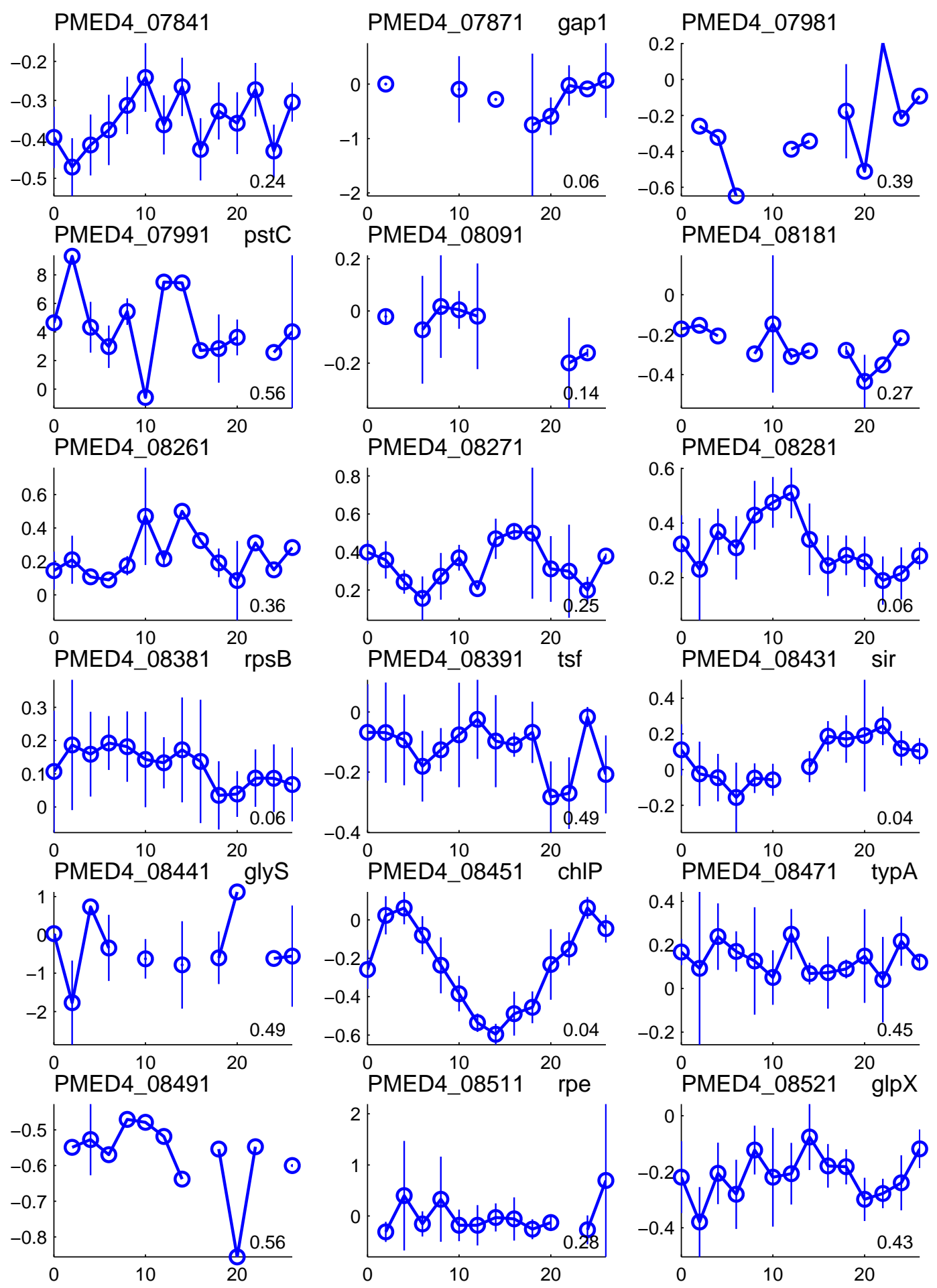

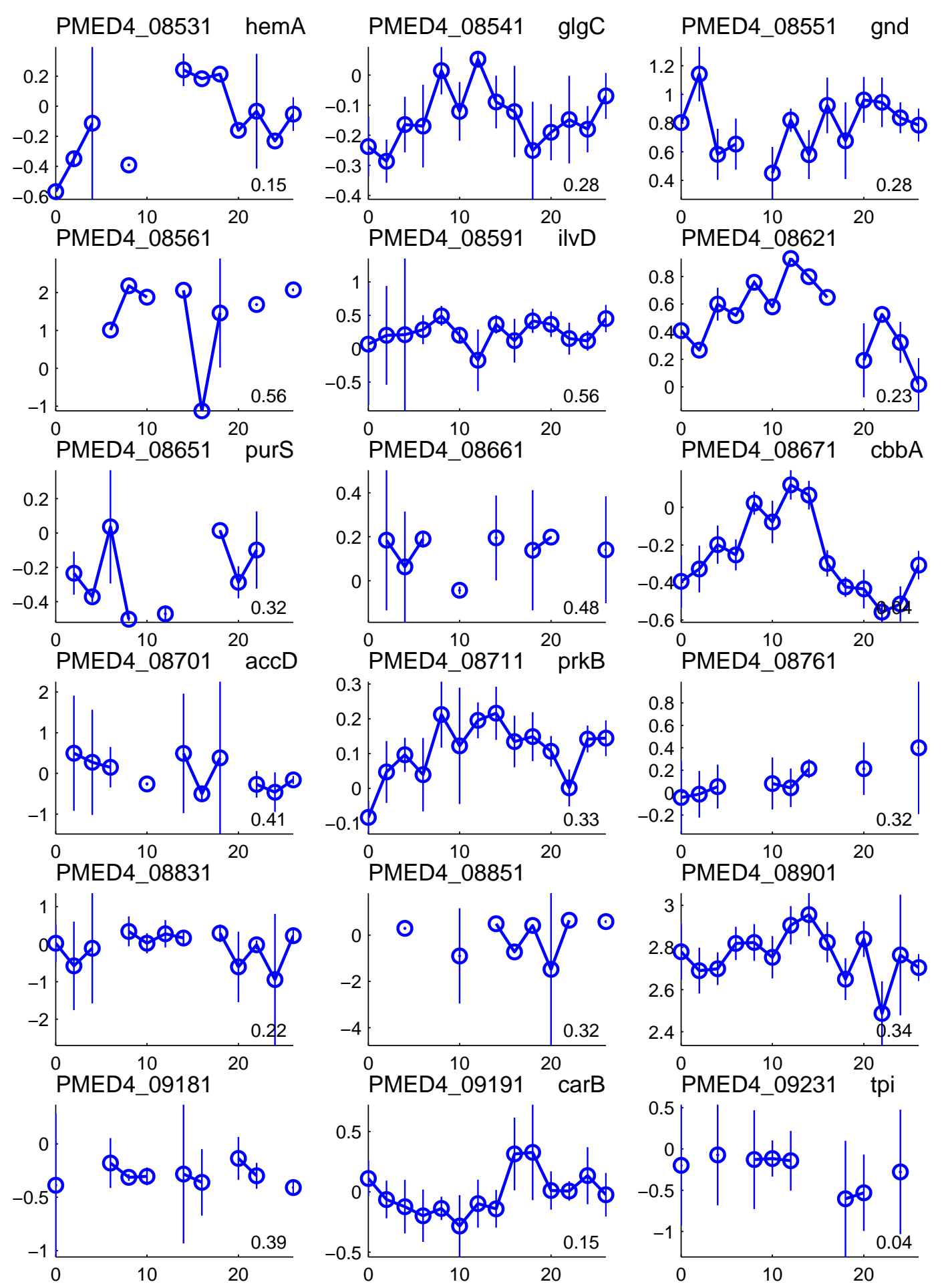
PMED4_09251 chlH PMED4_09261 dapB PMED4_09341 mfd
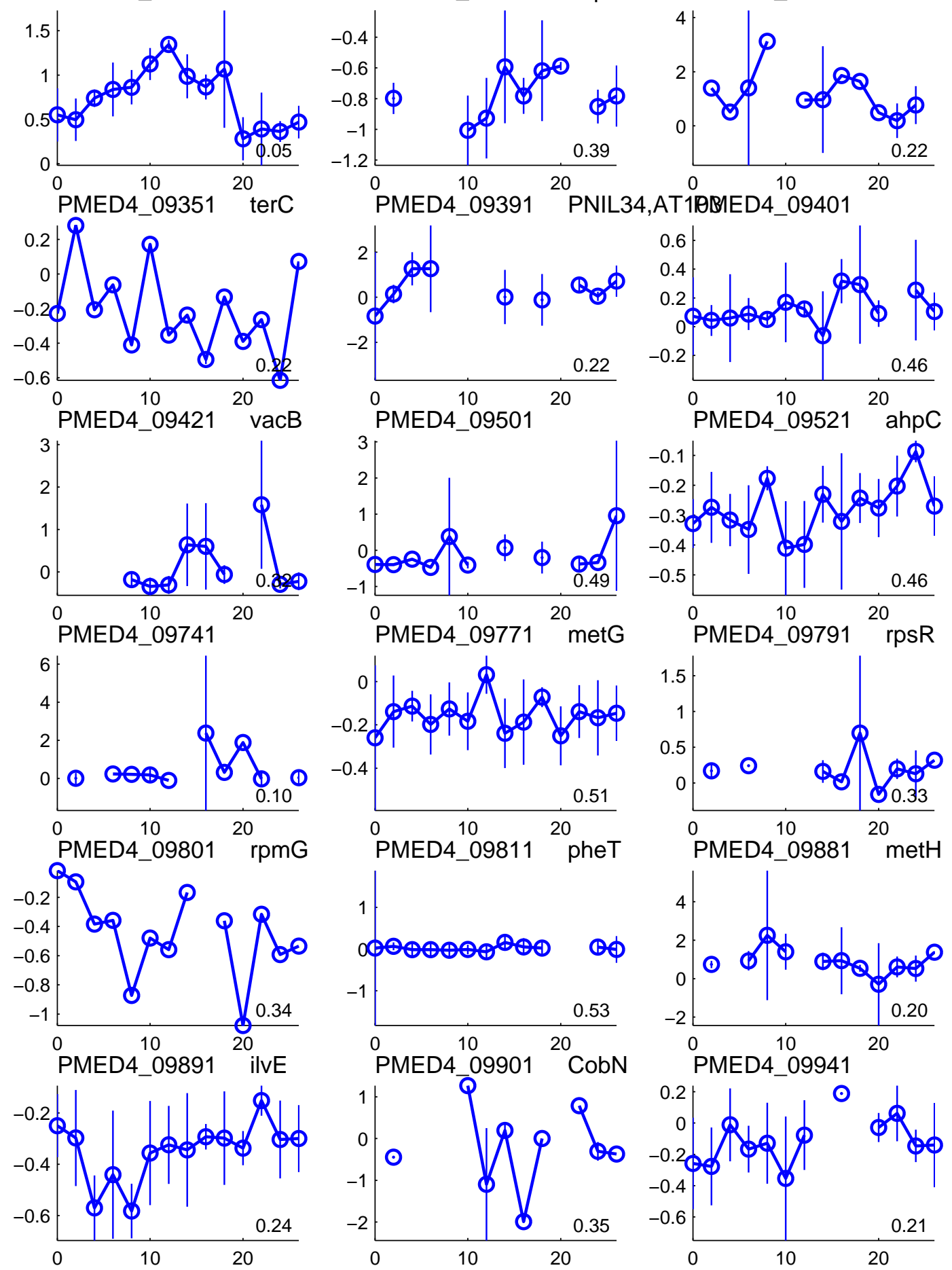

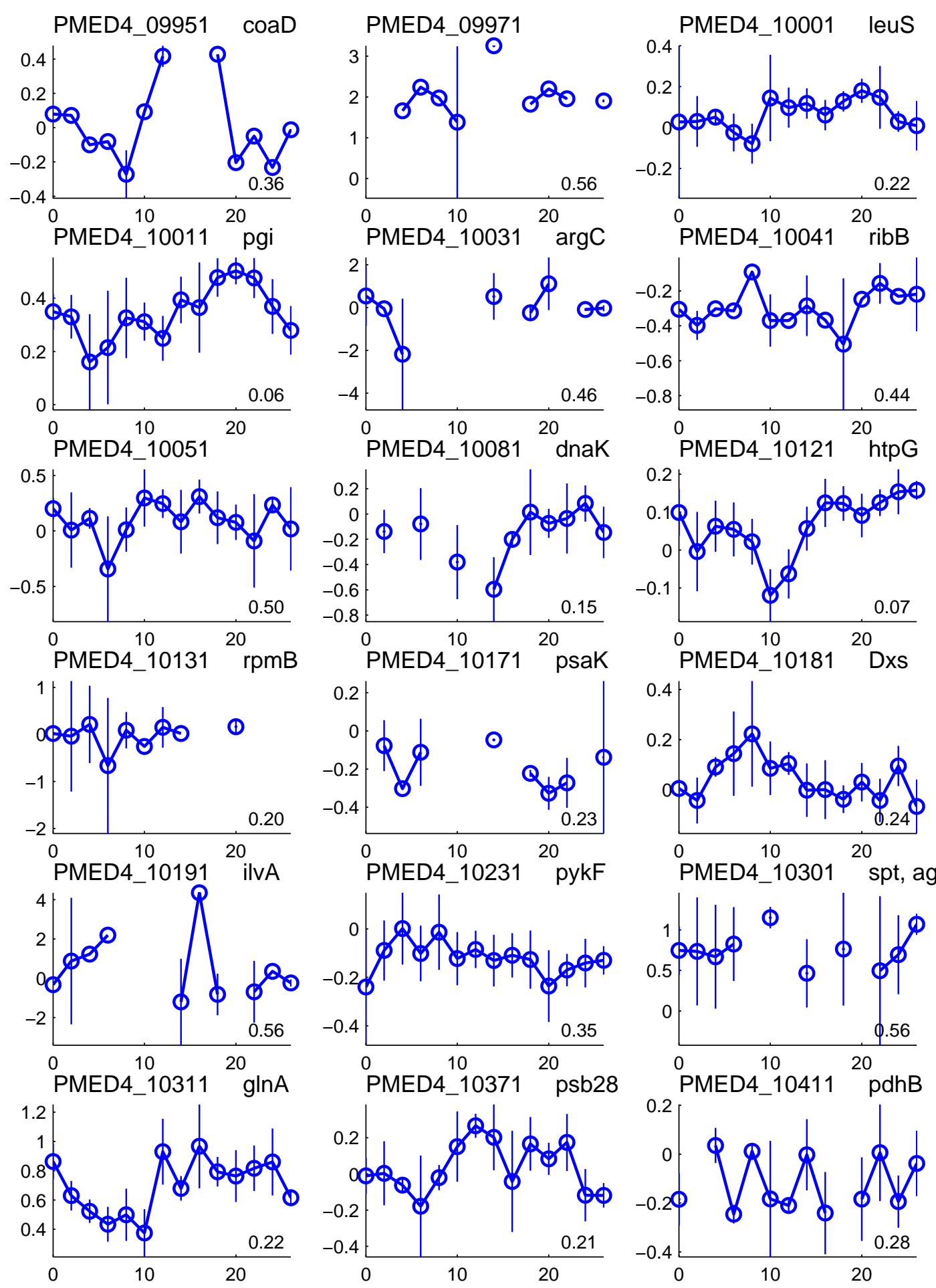

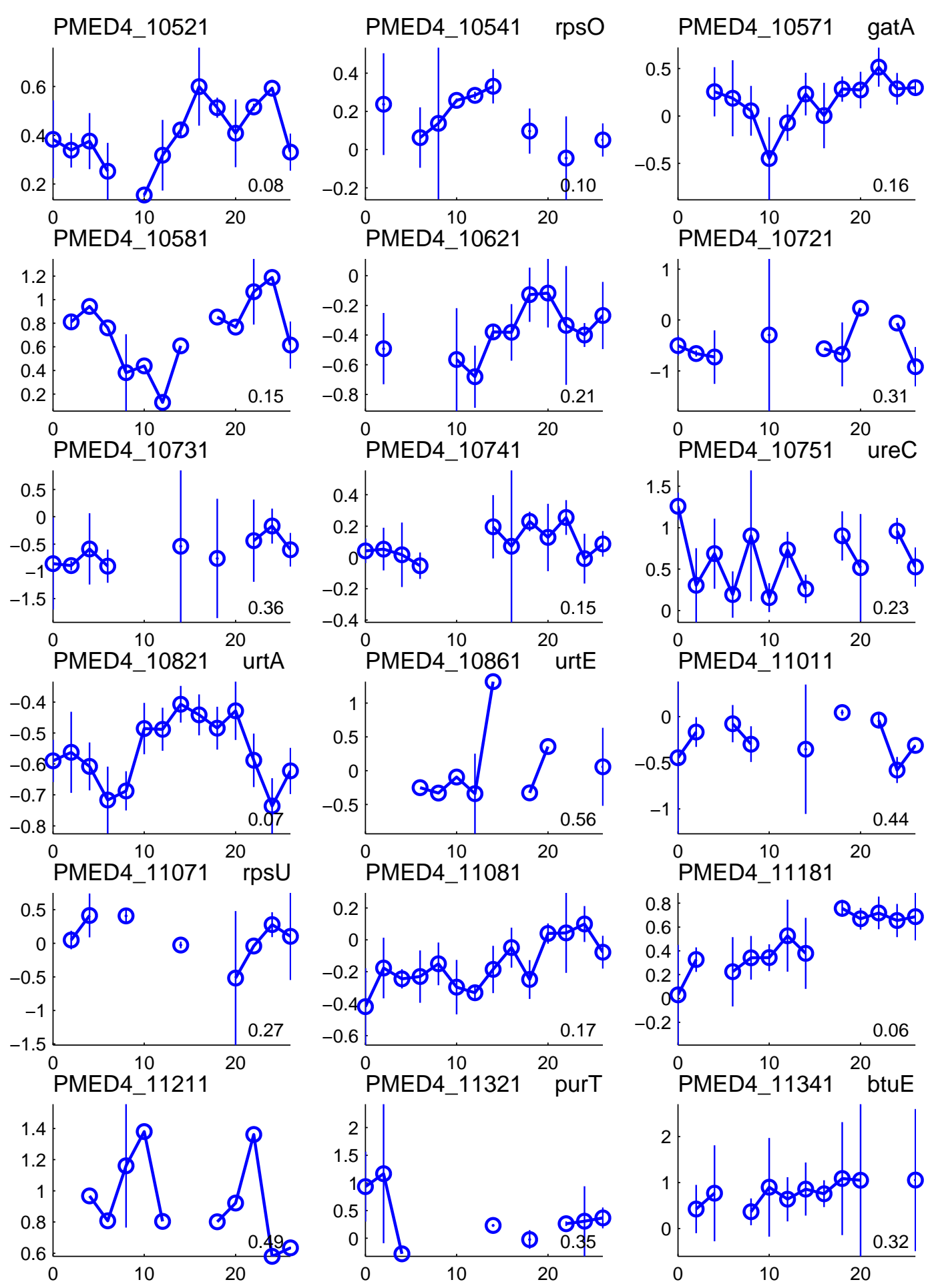

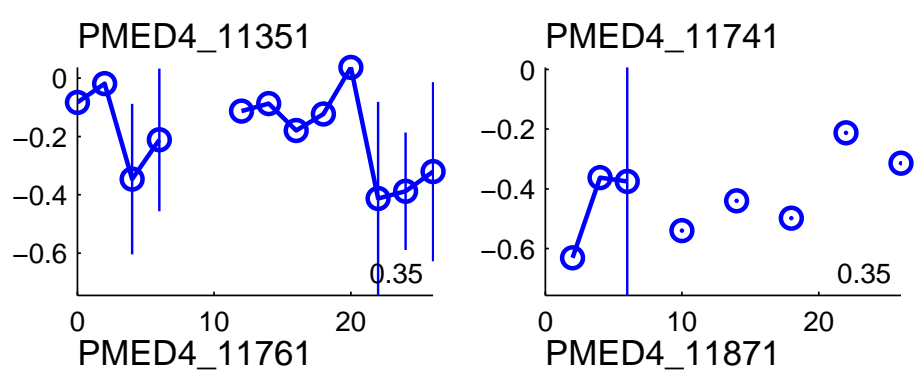

PMED4_11751 Iral
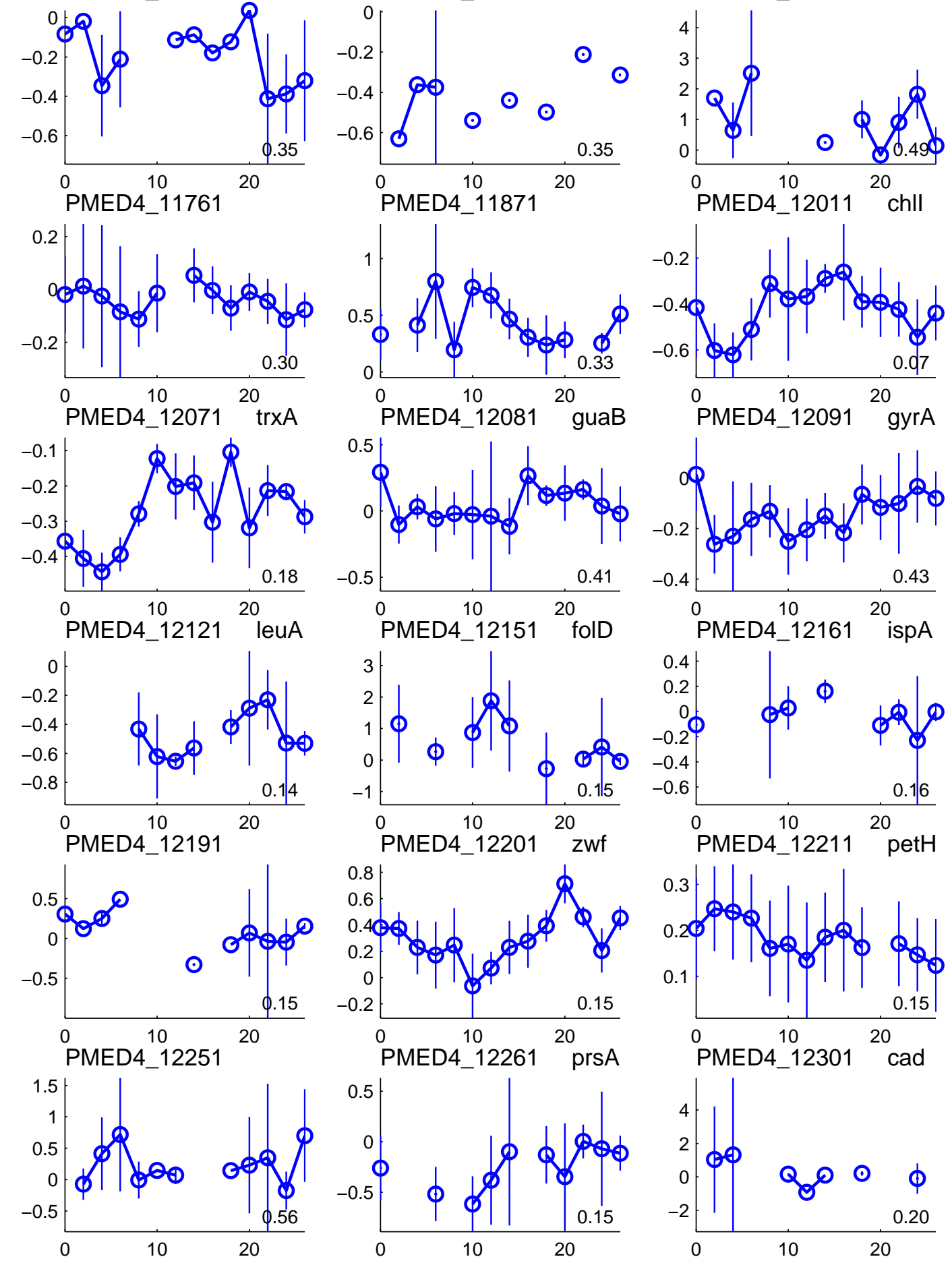

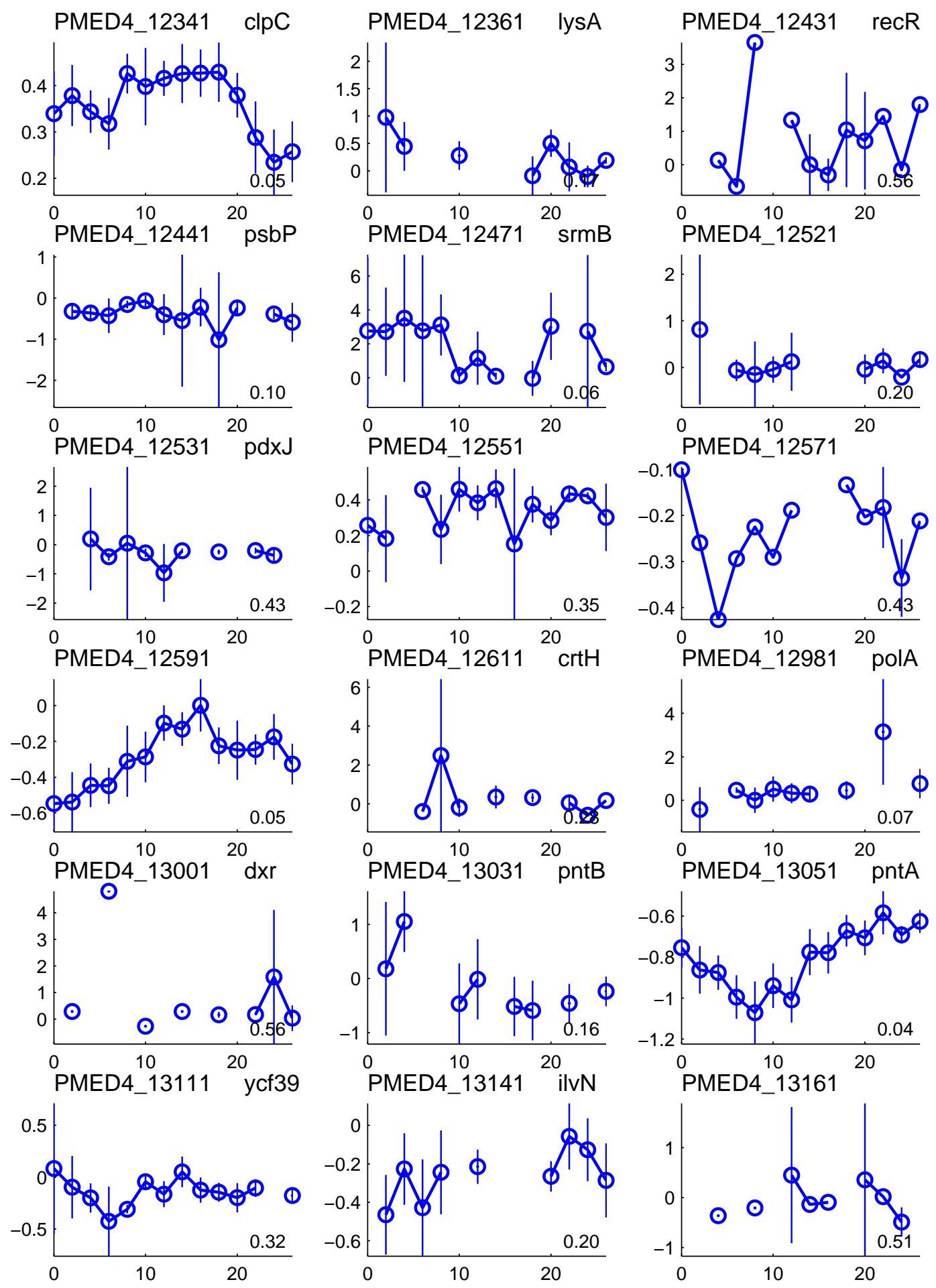

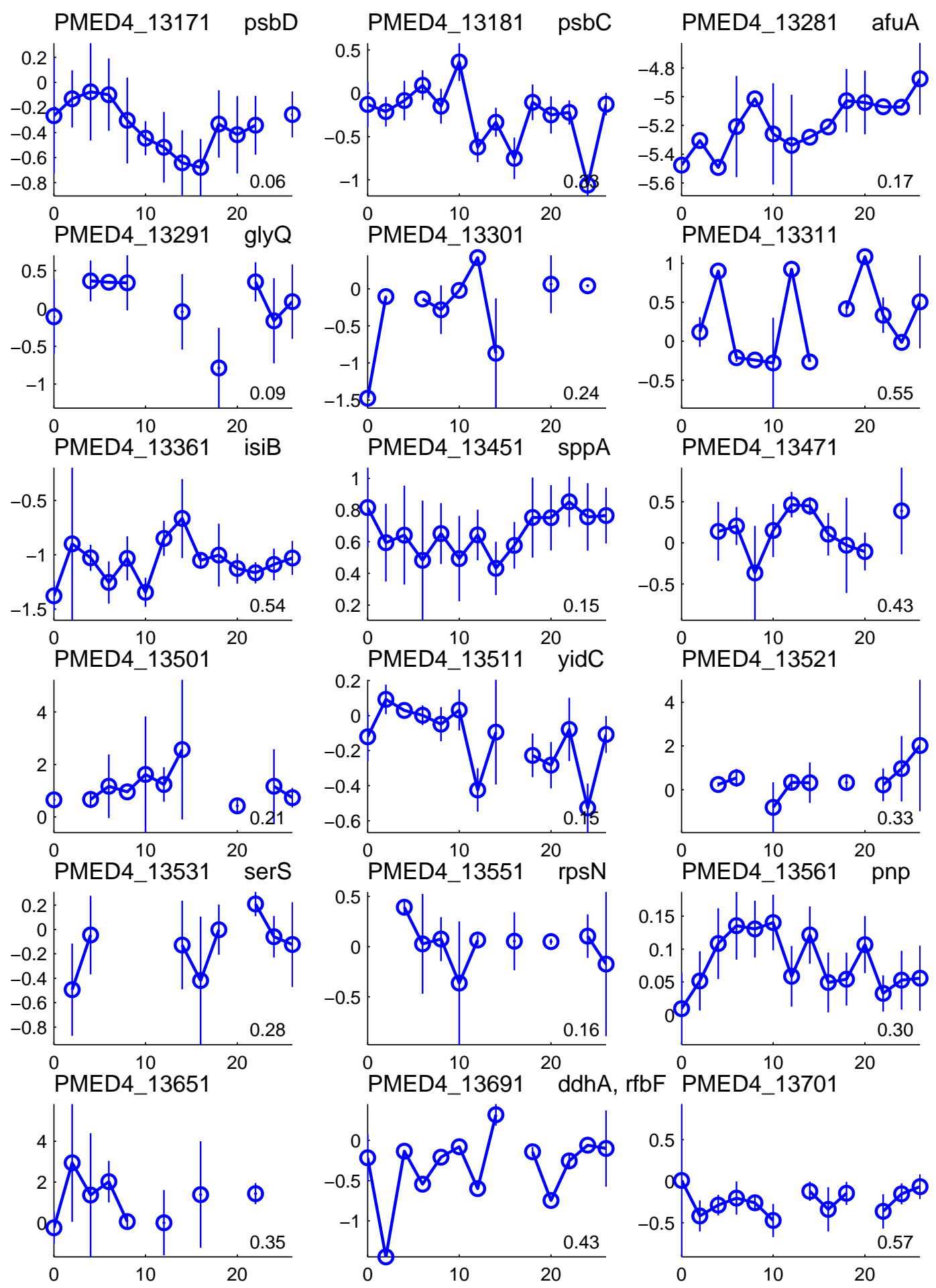

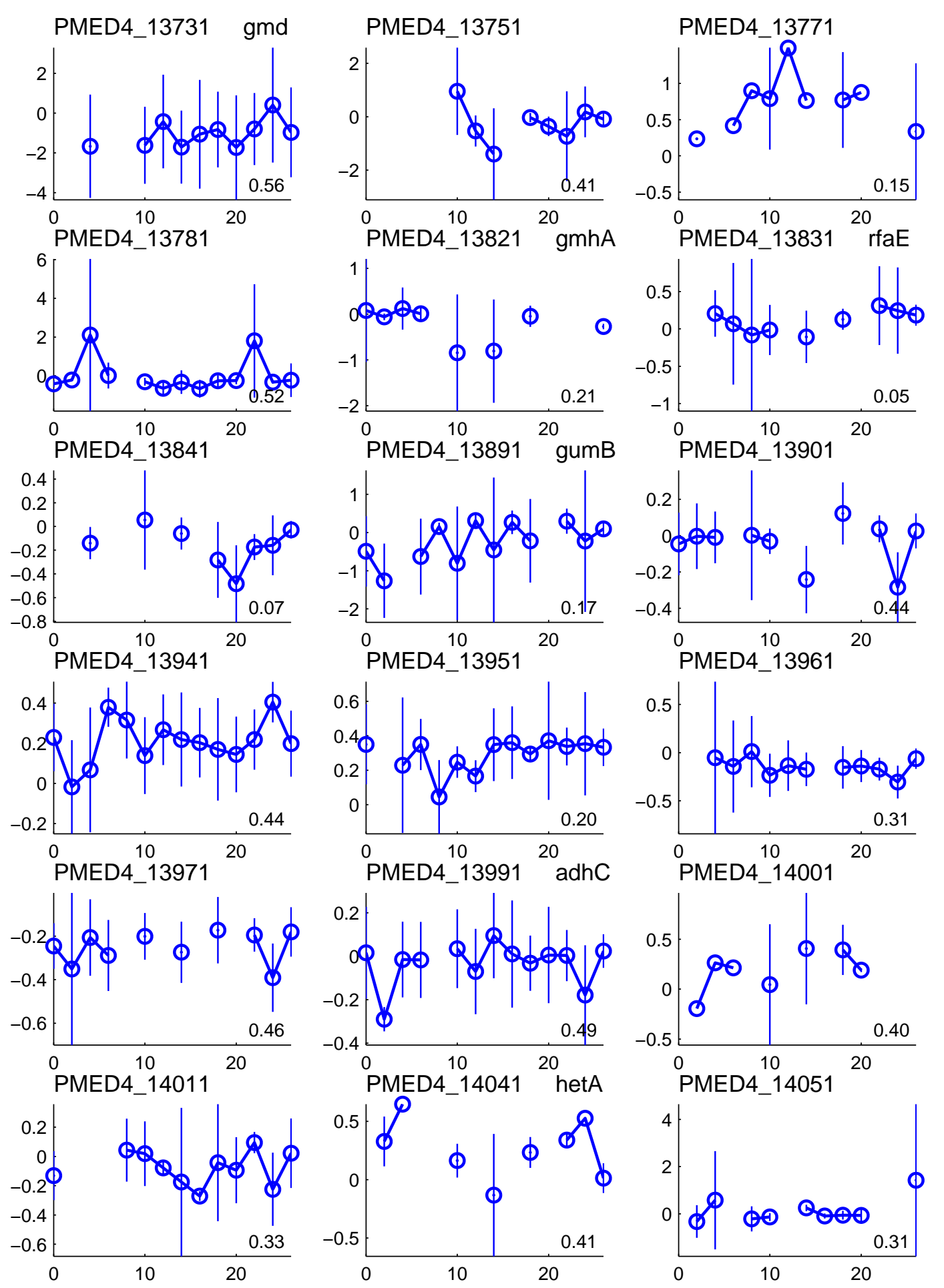

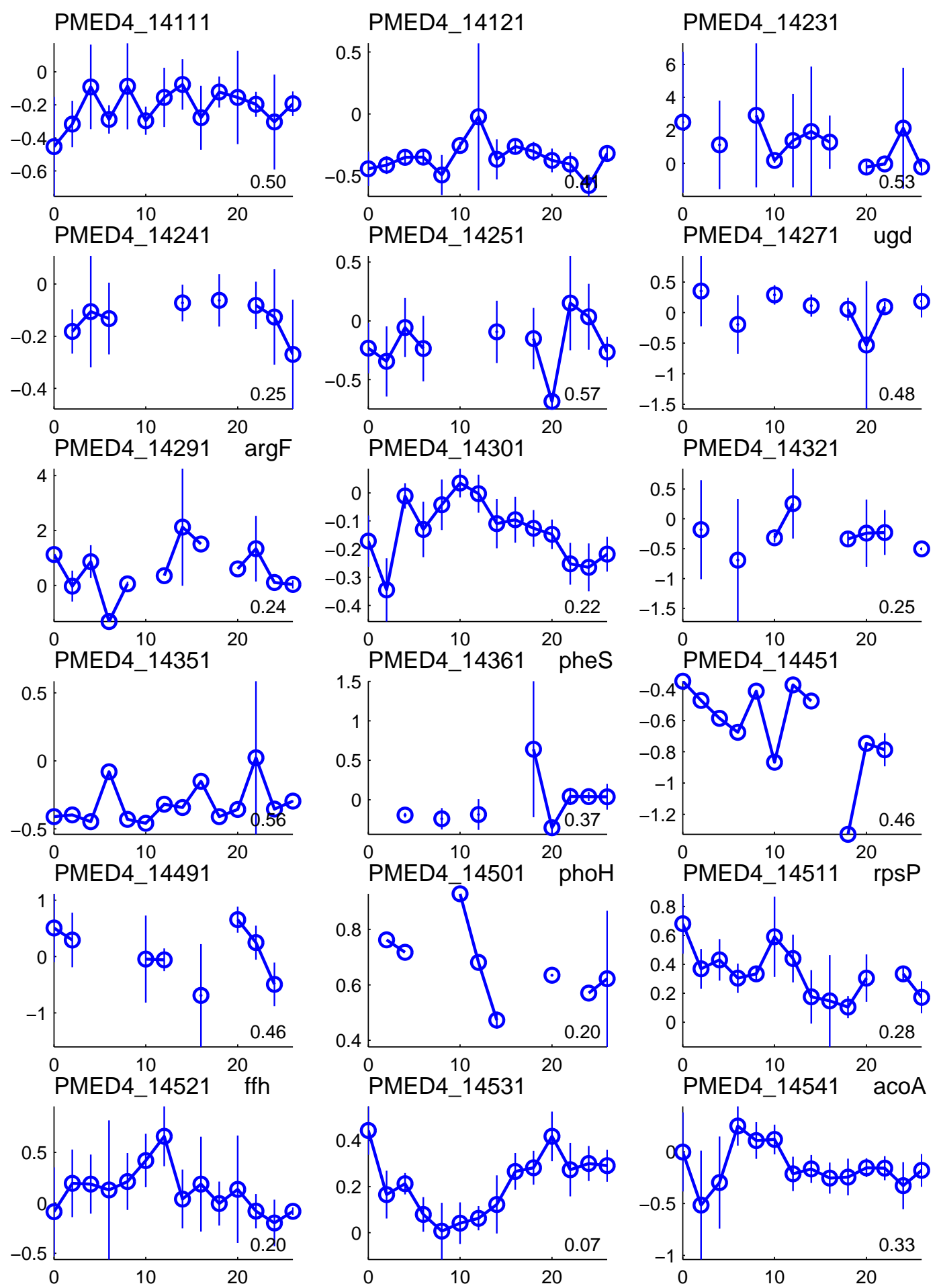

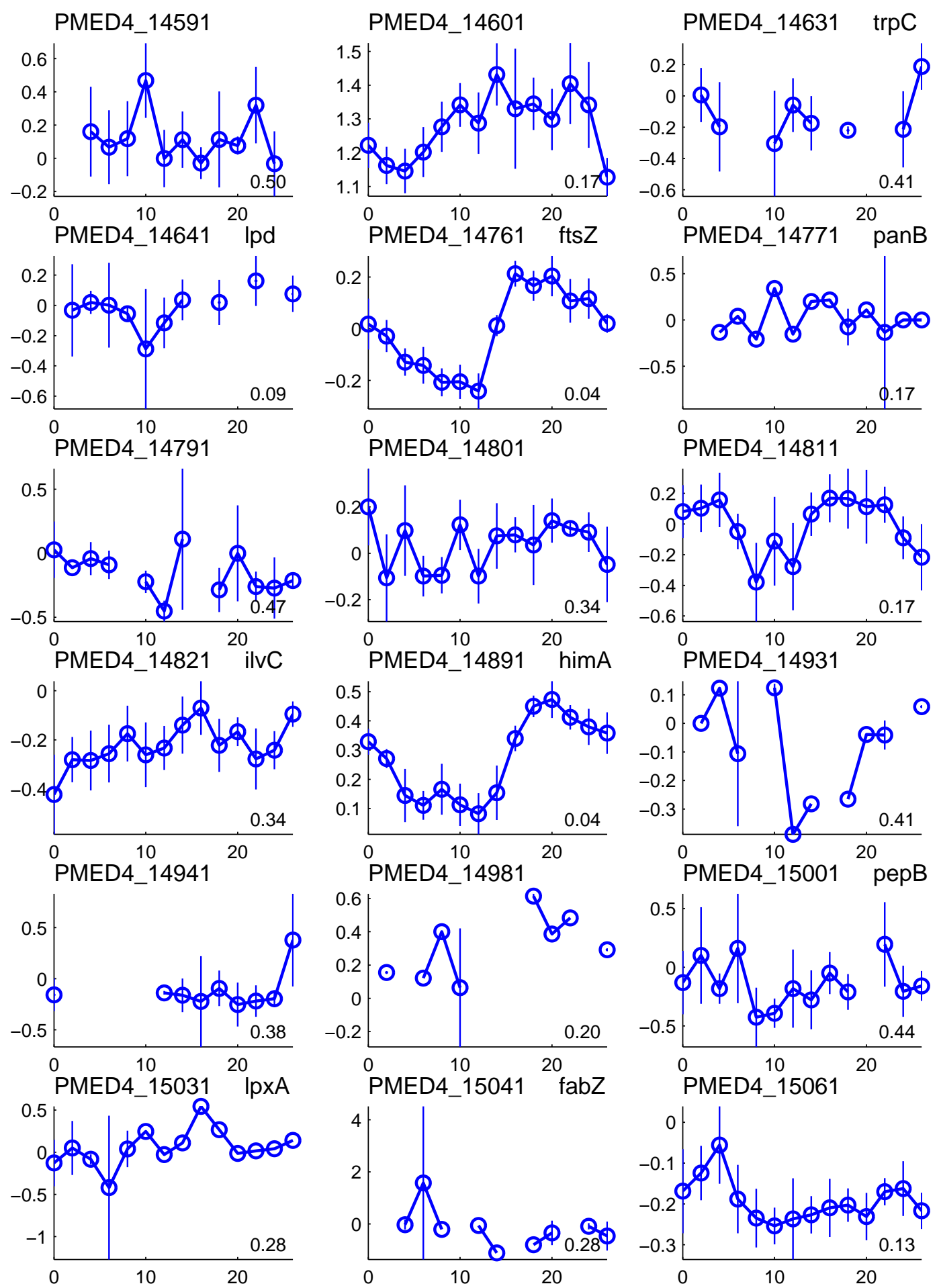

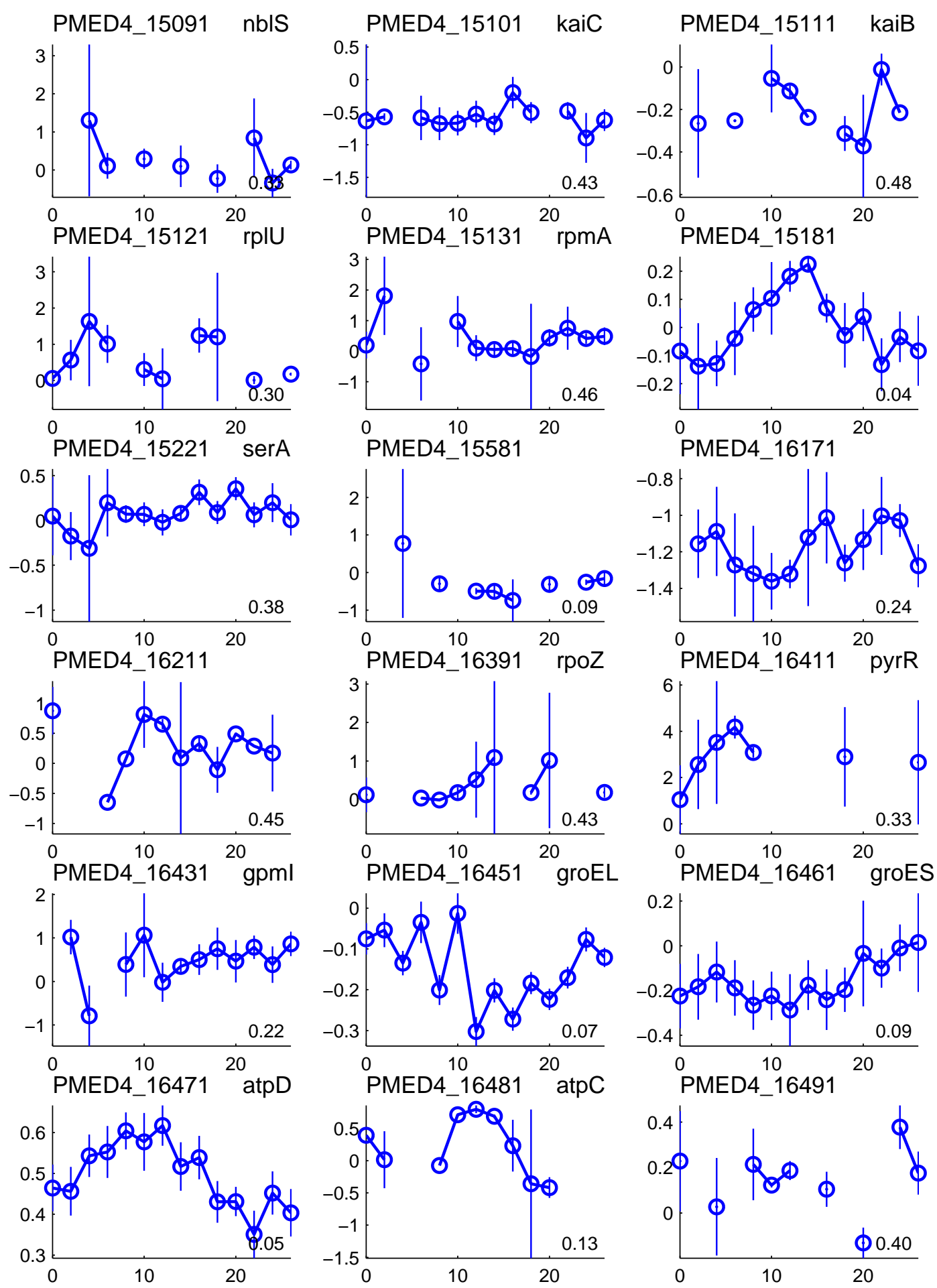
PMED4_16591 atpC PMED4_16601 atpA PMED4_16611 atpH
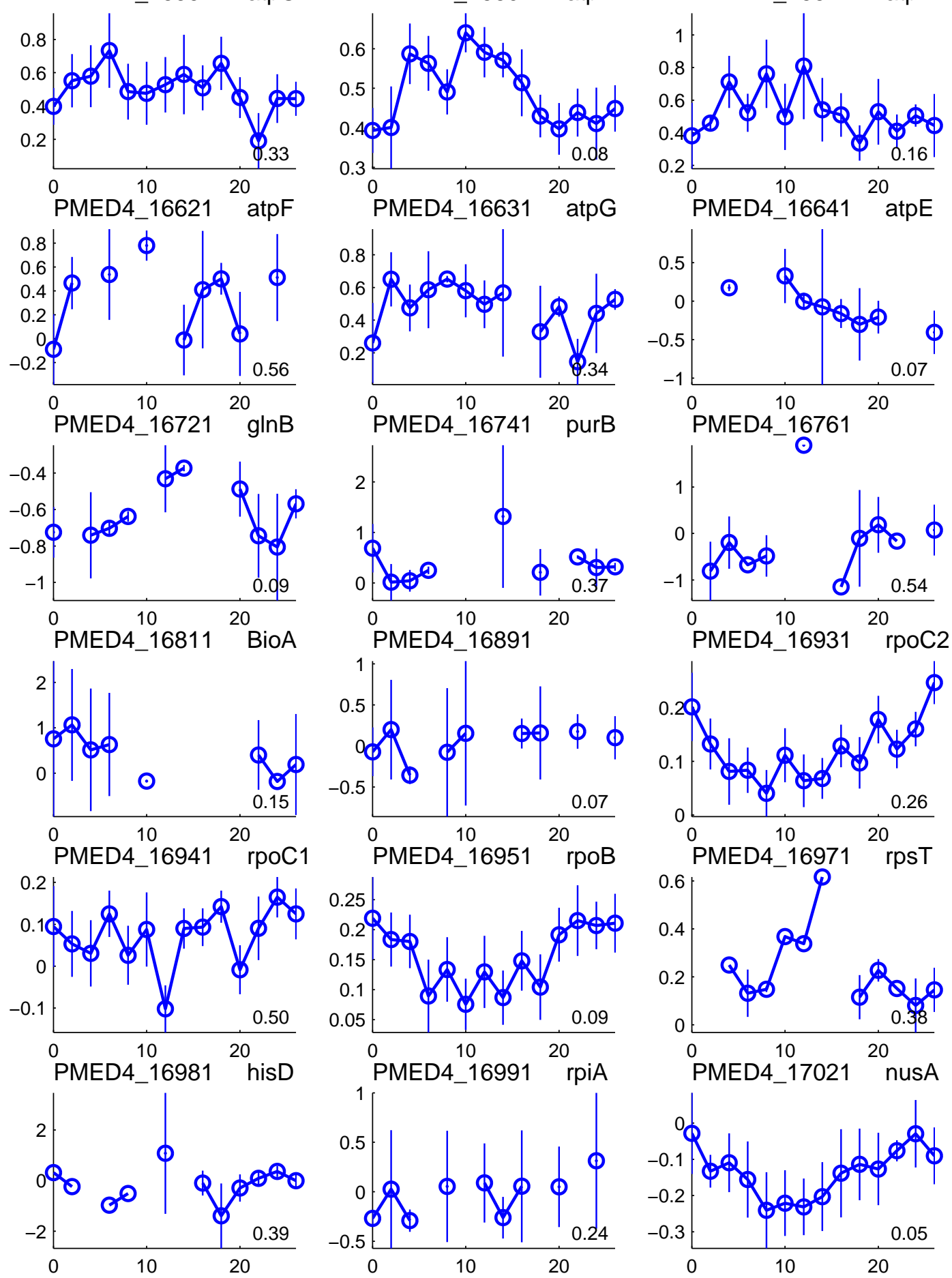

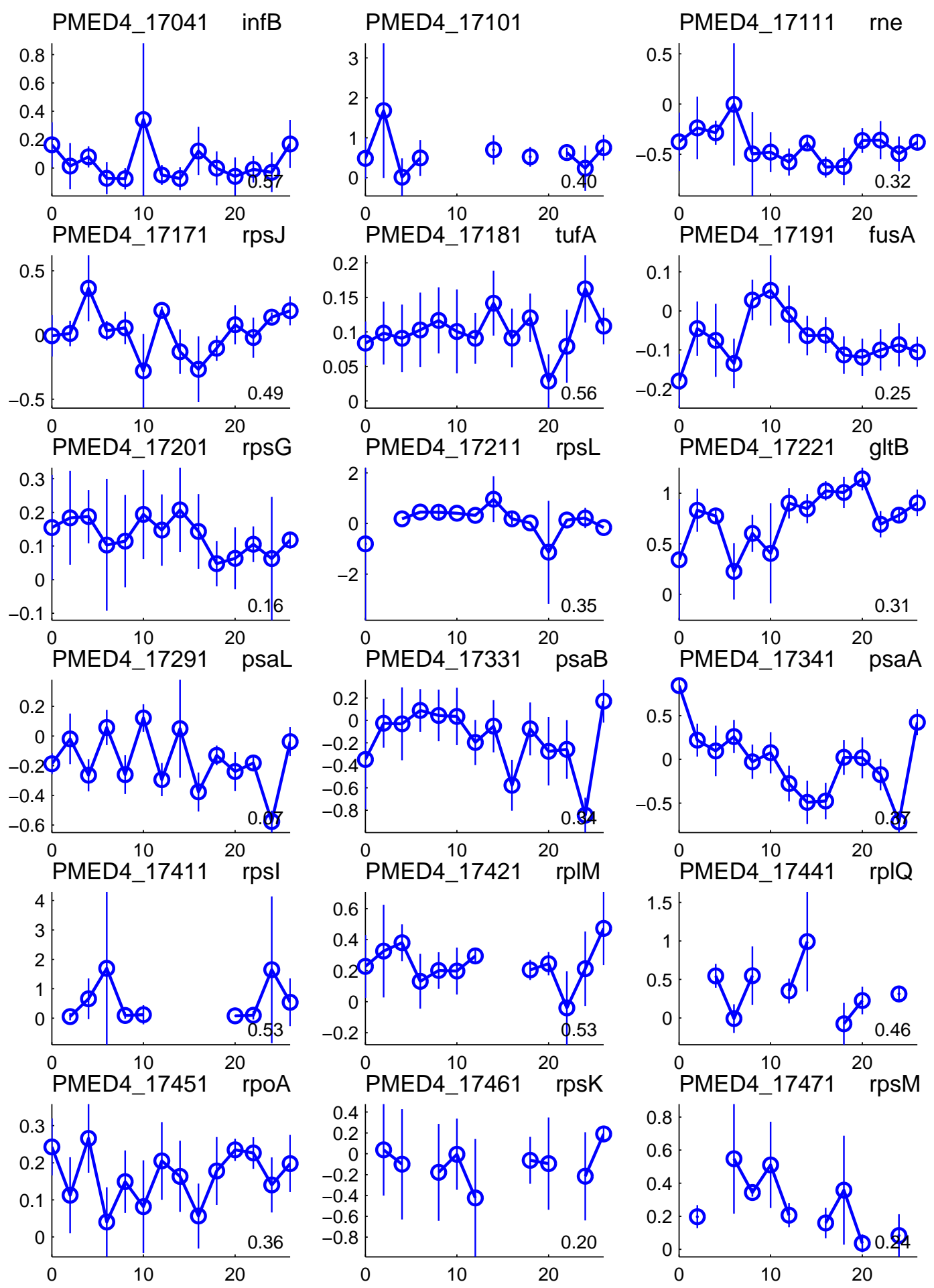

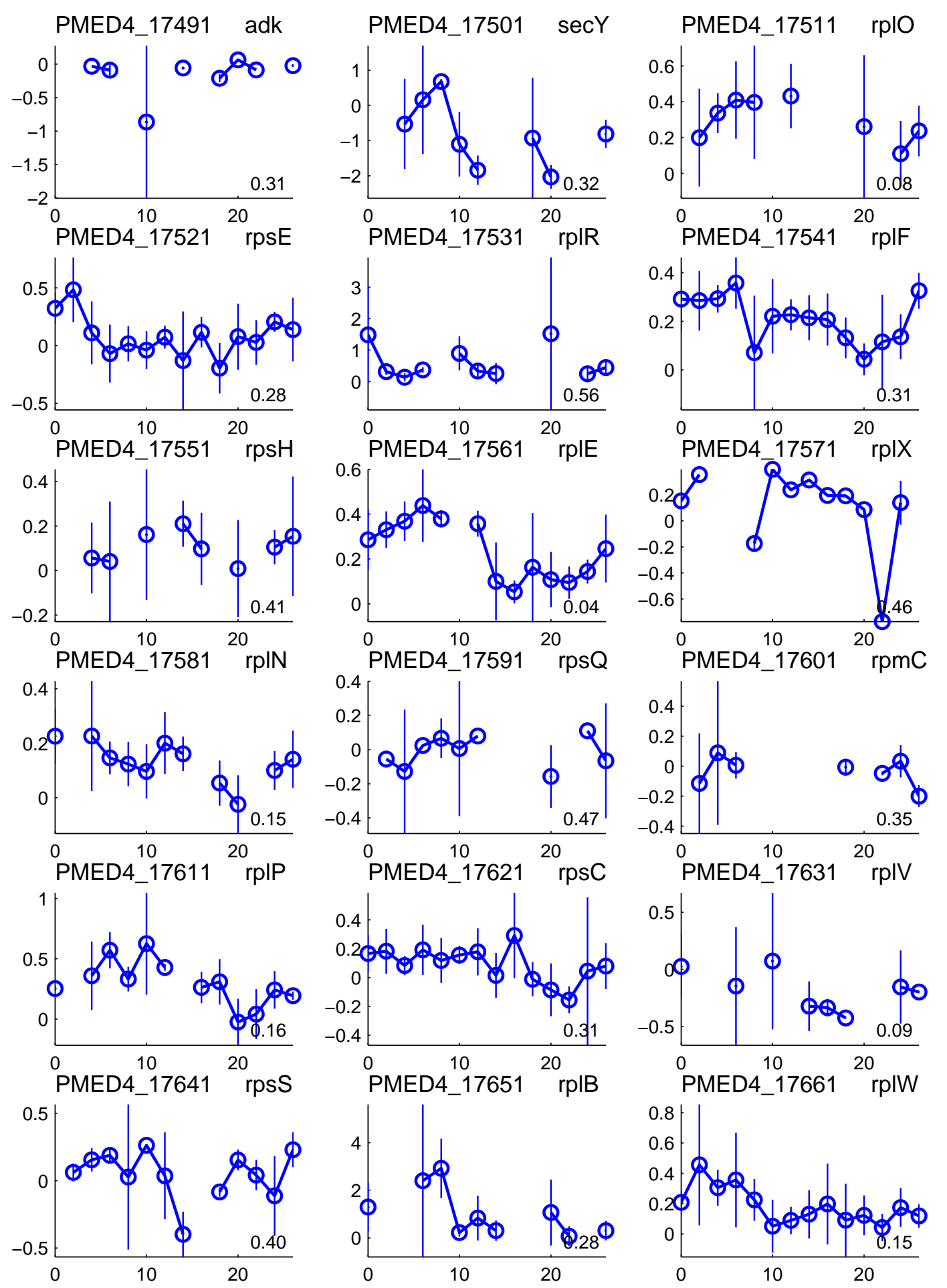

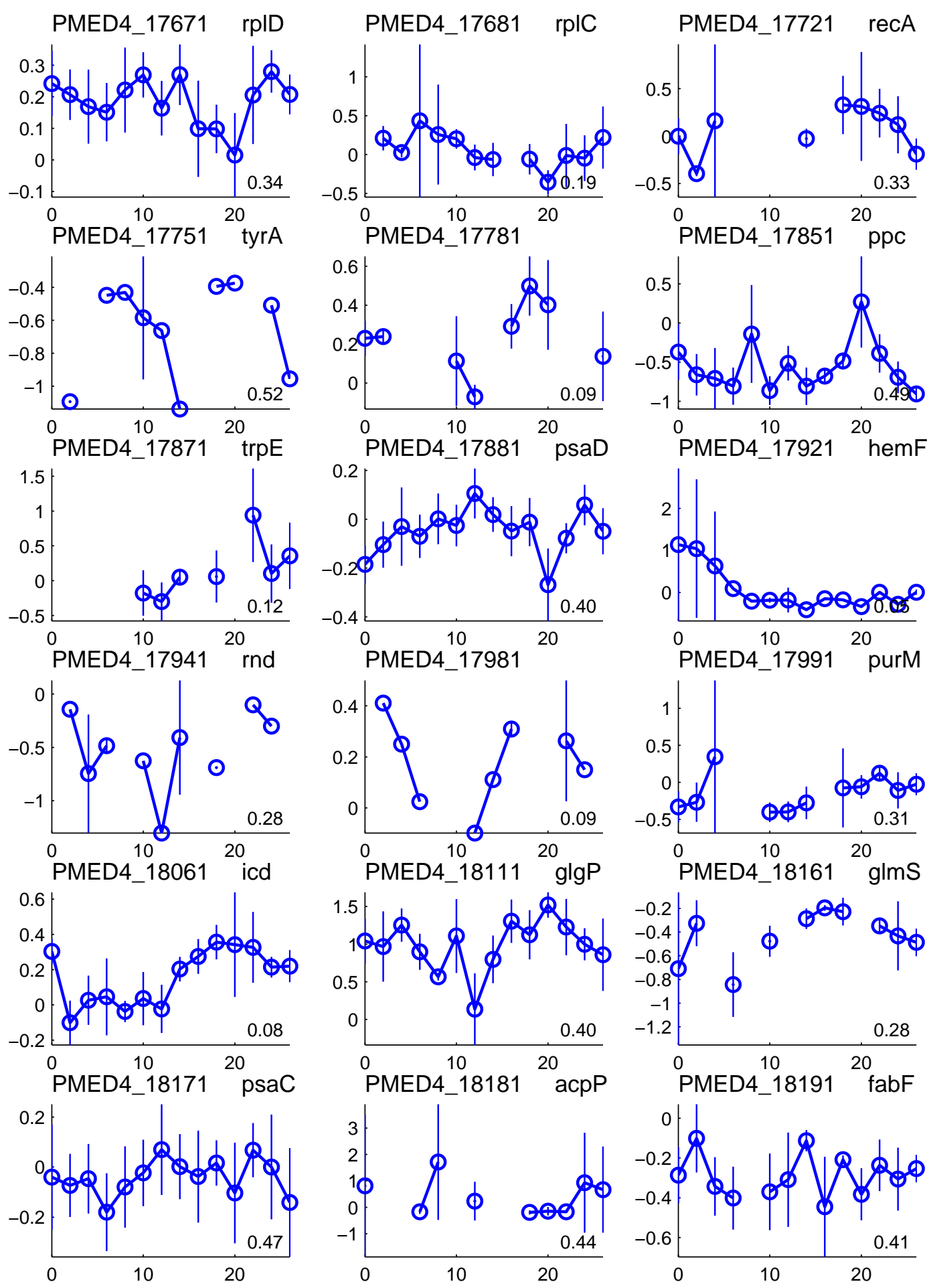

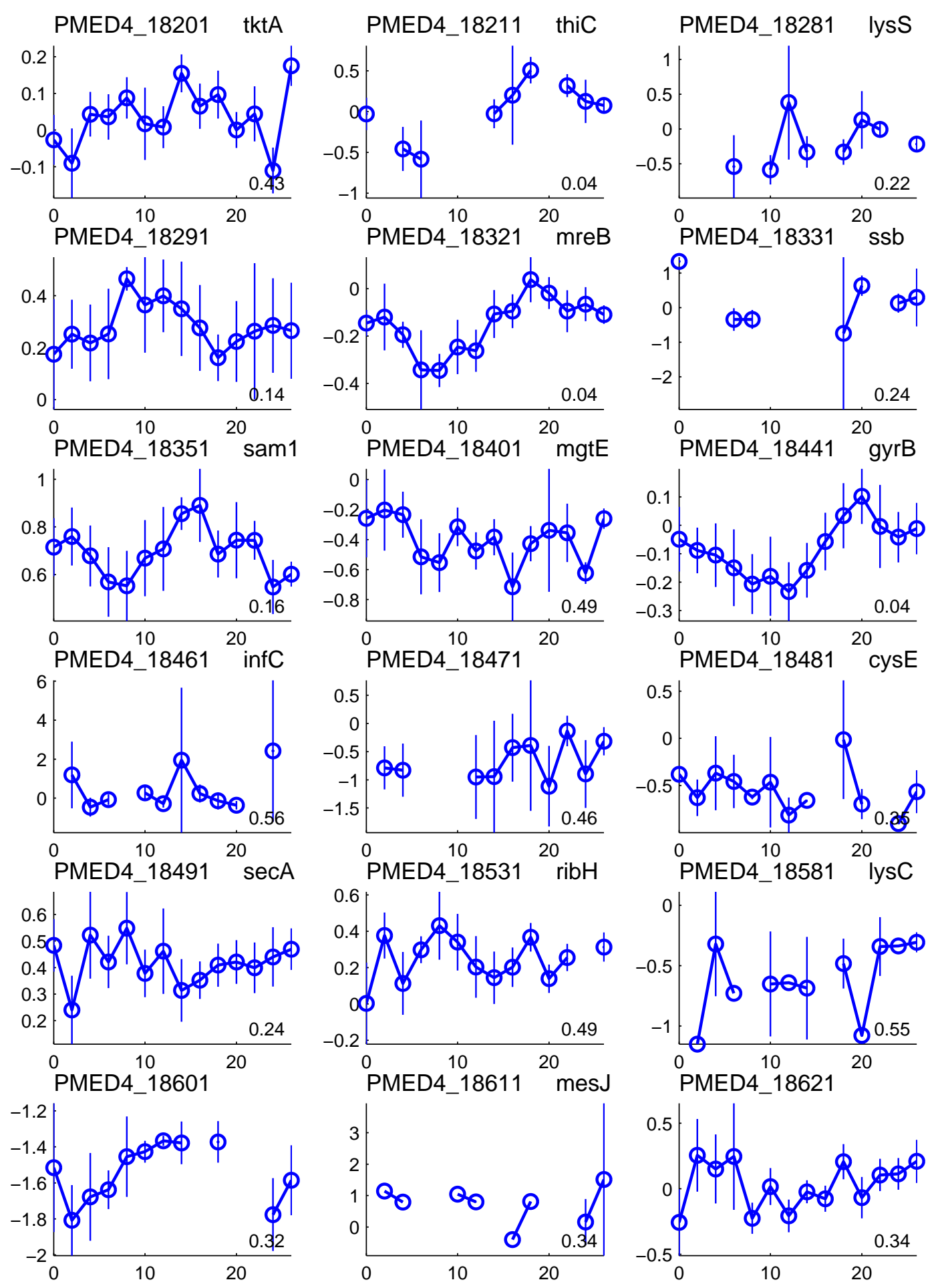


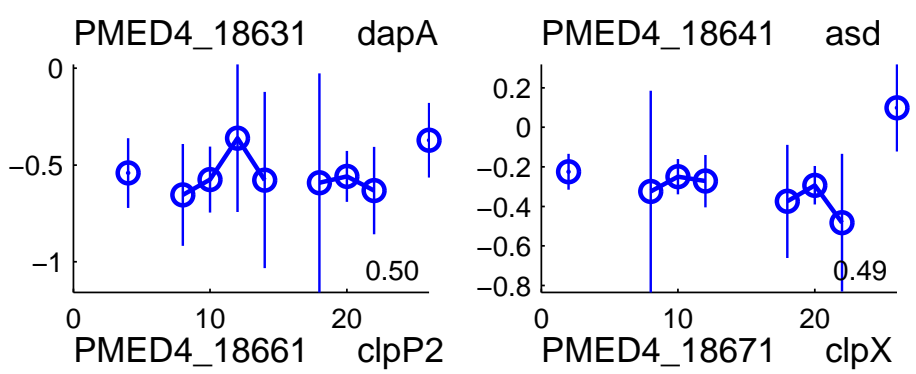

PMED4_18651 tig
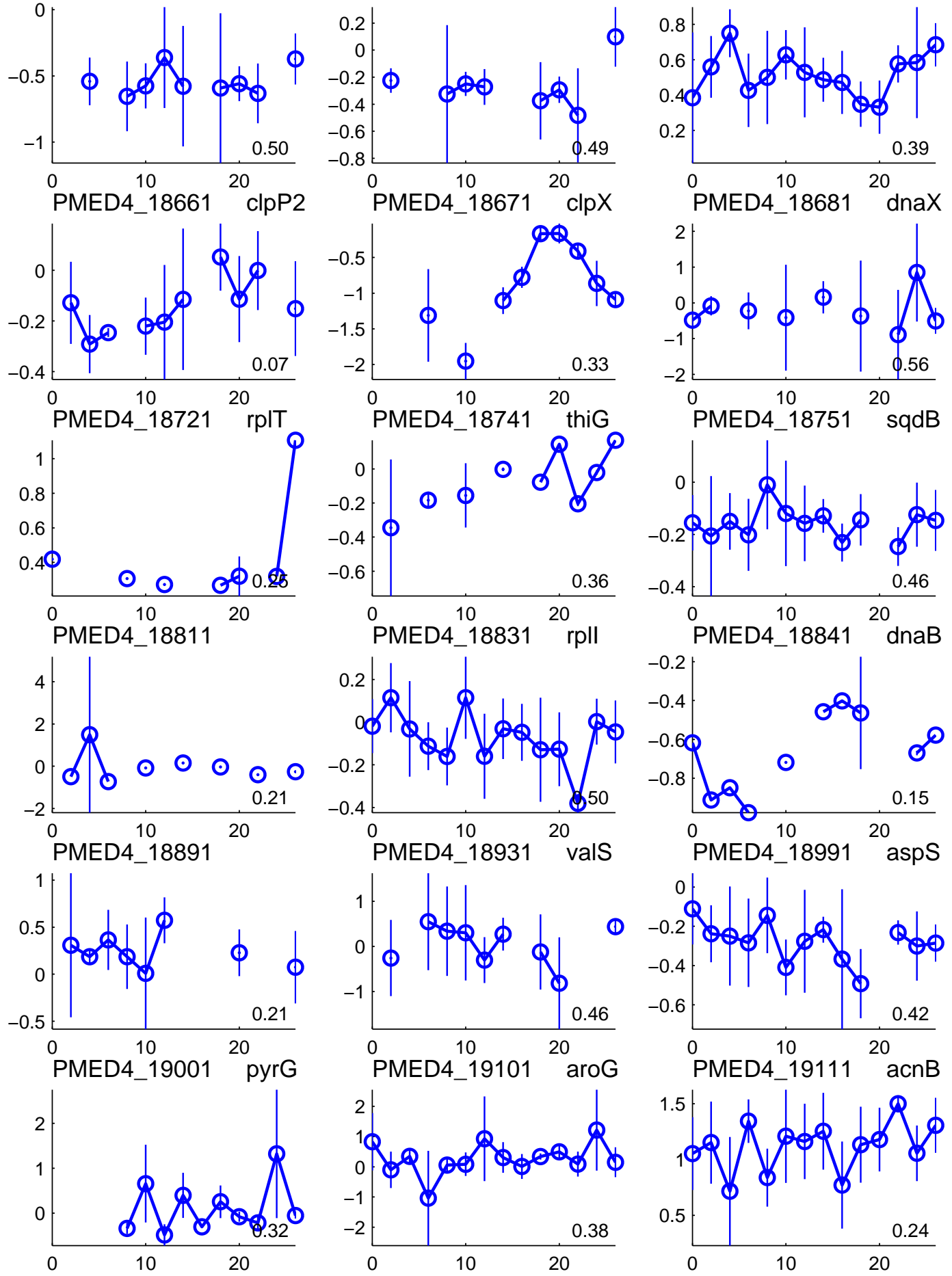

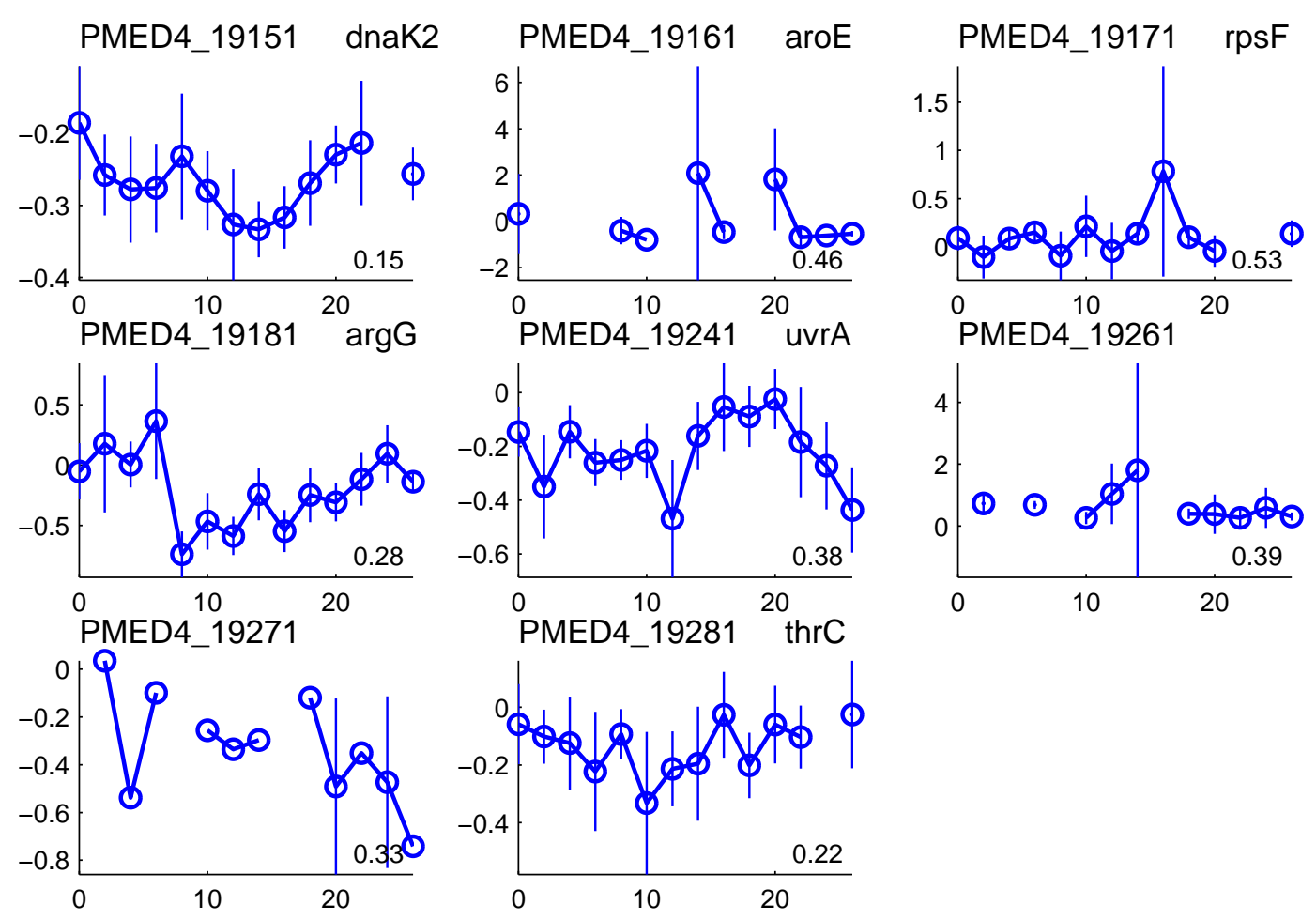


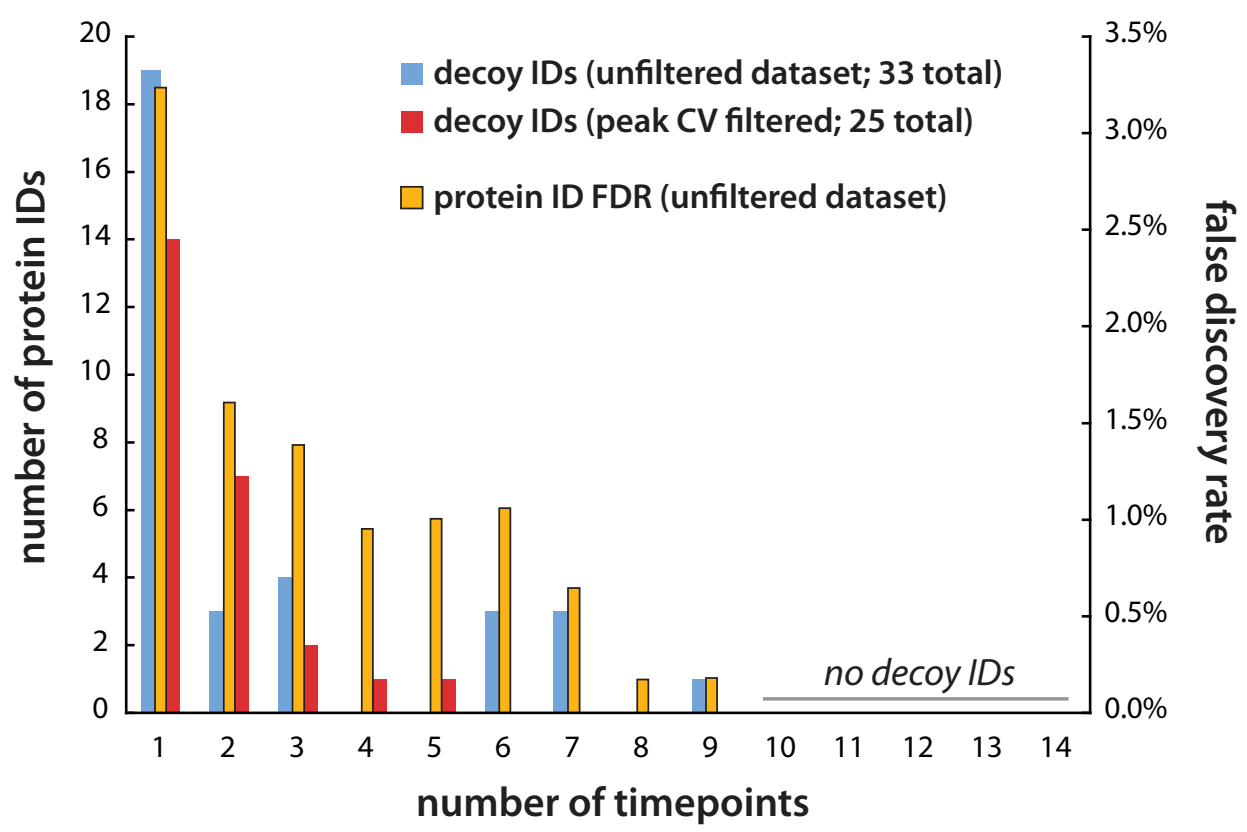

Supplementary Figure 2. Identifications of decoy proteins (i.e., reversed MED4 sequences) in the full diel dataset (blue bars) and after filtering on the basis of peak ratio $\mathrm{CV}$ (see Sec. 2.4.1), as a function of the number of timepoints at which each decoy sequence was found. The false discovery rate (FDR), calculated from the ratio of decoy IDs to MED4 protein IDs, is also shown, as a function of the minimum number of timepoints required for an ID. In the unfiltered dataset, with only one timepoint required, the FDR is $3.2 \%$; if two are required, the FDR drops to $1.6 \%$. Filtering based on peak ratio CV reduces the maximum number of timepoints at which any one decoy protein is found from 9 to 5 ; futher filtering for expression timecourses eliminated decoy data entirely, indicating a protein-ID FDR in the diel timeseries dataset of $<0.2 \%$. 


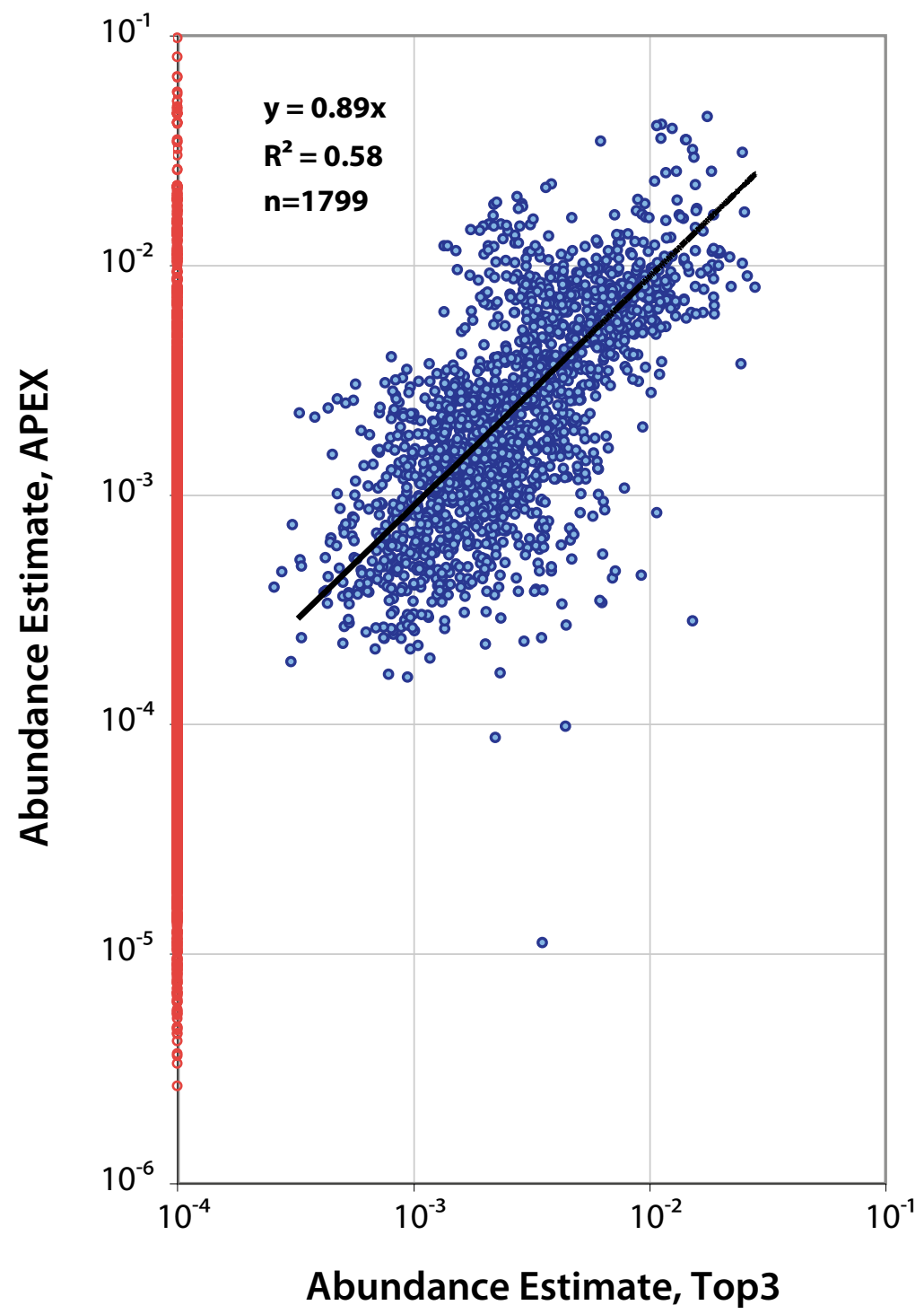

Supplementary Figure 3. Comparison of fractional abundance estimates using normalized spectral counting-based (APEX; Lu et al. 2007) and label-free, MS'-based (Top3; Silva et al. 2006) techniques. Blue points $(n=1799)$ are protein timeponts for which both APEX and Top3 abundance estimates were obtained; for the red points $(n=7013)$, only APEX values were calculated. Note that, in this case, detection of a minimum of 10 peptides was required for calculation of a Top3 score. 


\section{Chapter Five}

Biogeochemical insights from Prochlorococcus systems biology

Jacob R. Waldbauer and Sallie W. Chisholm 


\title{
Biogeochemical insights from Prochlorococcus systems biology
}

\author{
Jacob R. Waldbauer and Sallie W. Chisholm
}

In order to see cells as biogeochemical agents and incorporate cellular metabolism into a systems-level view of natural environments, an understanding of organisms' molecular composition is fundamental. Specifying molecular composition - albeit largely in a laboratory context - has emerged as a basic goal of systems biology en route to an integrated view of cellular function. The tools developed for systems biology with mainly biomedical or biotechnological goals in mind have been increasingly turned to address questions in biogeochemistry and environmental science. Chapter 4 presented an example of using such tools, including microarrays, proteomics and RNA-sequencing, to assess the inventories and dynamics of gene products in Prochlorococcus. In this chapter, we take a holistic view of the molecular composition of Prochlorococcus cells, first modeling how elemental budgets are apportioned among major pools of biochemicals, and then addressing how cellular molecular composition informs hypotheses concerning genome evolution and stochastic gene expression effects. We propose that genome streamlining in Prochlorococcus is unlikely to be driven primarily by adaptation to oligotrophic conditions, and that noise in gene expression may play an ecological role in limiting the growth rates of very small cells.

\section{ELEMENTAL AND BIOCHEMICAL BUDGETS FOR PROCHLOROCOCCUS CELLS}

We consider first the major elemental (carbon, nitrogen, phosphorus) budgets of a 'hypothetical' high-light Prochlorococcus cell. Our goal is to provide a first-order accounting of biochemical components, on the basis of available experimental and genomic data. We focus on the B/G1 period of the cell cycle, when the cell has a single copy of its chromosome (Wang and Levin, 2009); for Prochlorococcus, this period extends from approximately midnight to mid-afternoon each day. Populations will naturally show variation around the values presented, and that variation is itself likely to be interesting and ecologically relevant. The calculations and assumptions involved in deriving these values are described in the following section. 


\subsection{Budgeting calculations}

\subsubsection{Elemental stoichiometry}

Several studies have presented measurements of the elemental composition of

Prochlorococcus cells (Bertilsson et al., 2003; Heldal et al., 2003; Fu et al., 2007) and provide a basis for major-element budgets. These papers report a range of elemental contents and $\mathrm{C}: \mathrm{N}: \mathrm{P}$ stoichiometries for Prochlorococcus cells. Here we treat the C:N ratio as an independent variable, and allow it to range over the span reported in the literature (5.7-9.9). We further take the cellular P content to be 13 amol, which, as discussed further below, represents the majority of results reported in all three studies. At a given $\mathrm{C}: \mathrm{N}$ ratio, then, the $\mathrm{C}: \mathrm{P}$ ratio is chosen so that the cellular carbon budget (detailed below) closes (i.e., such that the cellular $\mathrm{C}$ content equals the sum of the constituent biochemicals). The resultant modeled elemental contents and $\mathrm{C}: \mathrm{N}: \mathrm{P}$ ratios are shown in Figures 1 and 2, plotted along with the literature values. The elemental budgets for three cases (I, II and III; indicated in Figs. 1 and 2) that span the range of modeled stoichiometries are included in Table 1; the assumptions and calculations behind these budgets are detailed in Sections 1.1.2-1.1.4, and the results are discussed in Section 1.2.

The method for constructing cellular elemental budgets used here was designed to produce self-consistent, closed budgets, so that the distribution of $\mathrm{C}, \mathrm{N}$ and $\mathrm{P}$ among biochemical pools can be meaningfully interpreted. As shown in Figures 1 and 2, no single set of elemental contents or ratios can encompass all of the literature data, which in any case represent differing strains, media, culture conditions and growth rates. It is also not generally possible to close budgets based on a fixed stoichiometry without including 'mystery pool' of biochemicals of arbitrary composition and size, chosen solely to account for material stipulated by the stoichiometry. Including such a chemically illdefined component of the cells renders the proportional demands of major constituents such as protein and RNA much less interpretable. The modeled cellular $\mathrm{C}$ and $\mathrm{N}$ contents do match the experimental values quite well (Figure 1A). The choice was made to fix the 

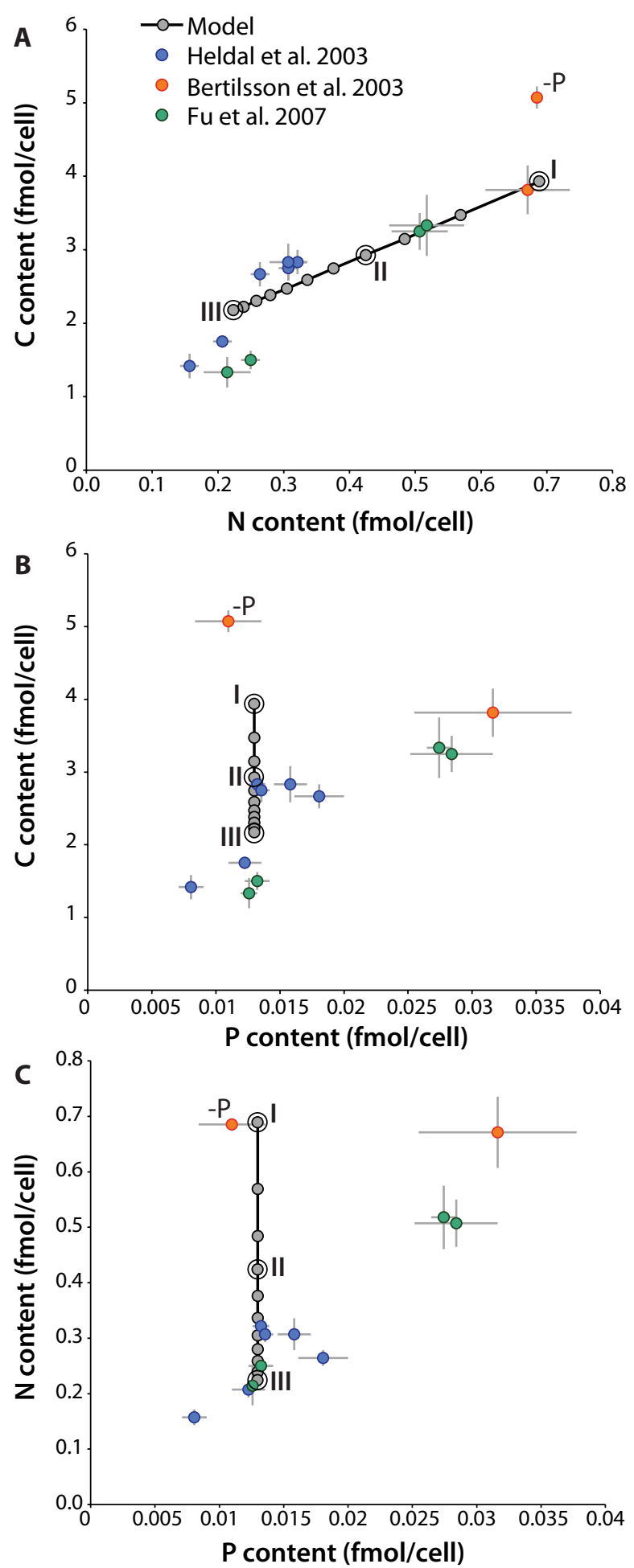

Figure 1. Cellular contents of carbon, nitrogen and phosphorus, from literature reports and as modeled here. The phosphorus-limited measurement by Bertilsson et al. (2003) (the only data from nutrient-limited culture) is indicated by the -P. The content values for the three model cases summarized in Table $1(\mathrm{I}, \mathrm{II}, \mathrm{II})$ are indicated by circled symbols. 

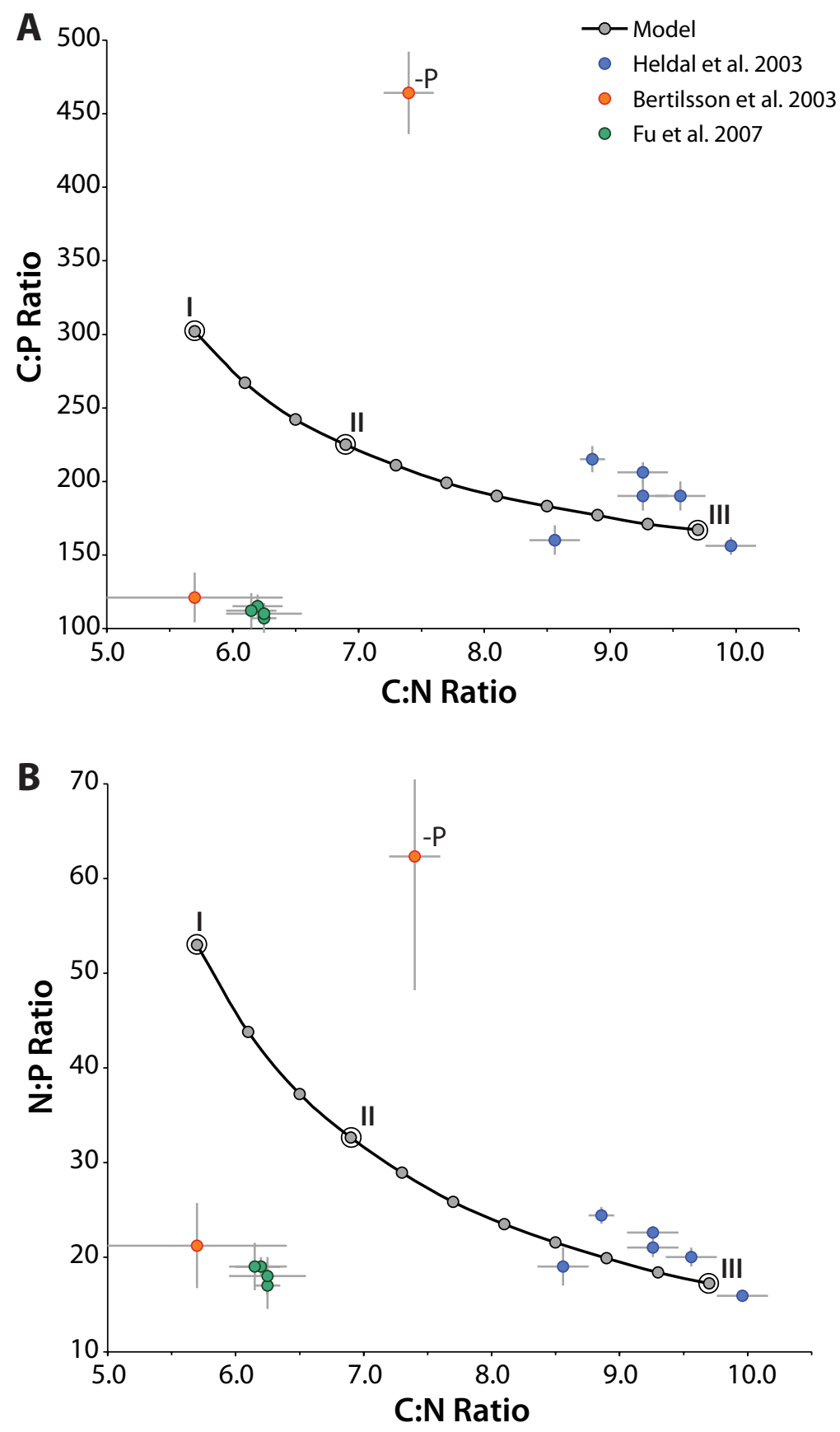

Figure 2. Cellular C:N:P stoichiometries, from literature reports and as modeled here. The phosphorus-limited measurement by Bertilsson et al. (2003) (the only data from nutrient-limited culture) is indicated by the -P. The ratio values for the three model cases summarized in Table 1 (I, II, III) are indicated by circled symbols. 


\begin{tabular}{|c|c|c|c|c|c|c|c|}
\hline \multirow[b]{2}{*}{ Model Case } & \multirow[t]{2}{*}{ Phosphorus } & \multicolumn{3}{|c|}{ Nitrogen } & \multicolumn{3}{|c|}{ Carbon } \\
\hline & & $\mathbf{I}$ & II & III & $\mathbf{I}$ & II & III \\
\hline Ratio to Phosphorus & 1 & 53 & 33 & 17 & 302 & 225 & 167 \\
\hline Cell Content (fmol) & 0.013 & 0.69 & 0.42 & 0.22 & 3.93 & 2.93 & 2.17 \\
\hline $\begin{array}{l}\text { Chromosome } \\
\text { RNA }\end{array}$ & $42.4 \%$ & $2.9 \%$ & $4.8 \%$ & $9.0 \%$ & $1.4 \%$ & $1.9 \%$ & $2.5 \%$ \\
\hline rRNA & $40.0 \%$ & $3.0 \%$ & $4.8 \%$ & $9.1 \%$ & $1.3 \%$ & $1.7 \%$ & $2.3 \%$ \\
\hline tRNA & $7.5 \%$ & $0.6 \%$ & $0.9 \%$ & $1.7 \%$ & $0.2 \%$ & $0.3 \%$ & $0.4 \%$ \\
\hline mRNA & $2.5 \%$ & $0.2 \%$ & $0.3 \%$ & $0.6 \%$ & $0.1 \%$ & $0.1 \%$ & $0.1 \%$ \\
\hline $\begin{array}{l}\text { Protein } \\
\text { Membrane Lipids }\end{array}$ & $5.0 \%$ & $91.2 \%$ & $85.6 \%$ & $72.8 \%$ & $60.5 \%$ & $46.9 \%$ & $28.4 \%$ \\
\hline $\begin{array}{l}\text { Miembrane LIpIas } \\
\text { LPS }\end{array}$ & $5.0 \%$ & $0.5 \%$ & $0.9 \%$ & $1.7 \%$ & $5.2 \%$ & $7.0 \%$ & $9.4 \%$ \\
\hline Cell Envelope & & & & & $8.3 \%$ & $11.1 \%$ & $15.0 \%$ \\
\hline Thylakoids & & & & & $15.4 \%$ & $20.6 \%$ & $27.8 \%$ \\
\hline $\begin{array}{l}\text { Peptidoglycan } \\
\text { Pigments }\end{array}$ & & $1.0 \%$ & $1.6 \%$ & $2.9 \%$ & $0.9 \%$ & $1.3 \%$ & $1.7 \%$ \\
\hline Chlorophyll & & $0.6 \%$ & $1.0 \%$ & $2.0 \%$ & $1.5 \%$ & $2.1 \%$ & $2.8 \%$ \\
\hline Zeaxanthin & & & & & $2.2 \%$ & $3.0 \%$ & $4.0 \%$ \\
\hline Metabolites & $2.5 \%$ & $0.10 \%$ & $0.16 \%$ & $0.31 \%$ & $3.0 \%$ & $4.0 \%$ & $5.4 \%$ \\
\hline
\end{tabular}

Table 1. The distributions of carbon, nitrogen and phosphorus contents among major biochemical constituents, for three model cases (I, II and III) with cellular stoichiometries as indicated. Note that the cellular phosphorus content is the same (13 amol) for all cases. 
cellular P content at 13 amol because values in that range appear in all three studies and it appears to approximate a lower limit of P content in Prochlorococcus (Figure 1B and C). We are less interested here in precisely modeling exponential growth under optimum, nutrient-replete conditions than in assessing how a cell can be built with the small nutrient complements likely to prevail in oligotrophic habitats. In our judgment, the sole measurement of Prochlorococcus cellular elemental stoichiometry under nutrient-limited conditions (that of Bertilsson et al., 2003, indicated in Figures 1 and 2), is quite relevant to real oceanic conditions and should be given due consideration. The C:N:P stoichiometries that result from this model approach those found by Heldal et al. (2003) at higher $\mathrm{C}: \mathrm{N}$ ratios, and $\mathrm{C}: \mathrm{P}$ and $\mathrm{N}: \mathrm{P}$ ratios rise as $\mathrm{C}: \mathrm{N}$ drops (Figure 2).

These broad uncertainties in cellular elemental composition mean that the biochemical budgets outlined here should be considered flexible. Certainly, a more precisely constrained composition would be required for detailed systems modeling, such as flux balance analysis. Nevertheless, the kinds of budgeting calculations described here can readily accommodate a range of stoichiometries, and hopefully future data (especially from field samples) will better constrain the $\mathrm{C}: \mathrm{N}: \mathrm{P}$ ranges most relevant to oceanic conditions.

\subsubsection{Phosphorus budget}

Phosphorus is present in Prochlorococcus as a component of several types of biochemicals: nucleic acid polymers (DNA and RNA), phospholipids, and small molecule metabolites. The amount in the chromosome can be calculated exactly from the genome size (1.66Mbp). Phospholipids are rather rare in Prochlorococcus compared to most other microbes likely as an adaptation to chronic P scarcity (Van Mooy et al., 2006). We calculate the total number of lipid molecules in the cell by assuming a typical geometry for Prochlorococcus: the cell envelope (outer membrane, murein layer and inner membrane) is set at $0.6 \mu \mathrm{m}$ in diameter. Within the cell are two complete, spherical thylakoid membranes averaging $0.5 \mu \mathrm{m}$ in diameter (compare Fig. 1A-D of Ting et al., 
2007) - for geometric simplicity, we neglect the small 'hairpin' region where each membrane wraps around itself to enclose the lumen. Thus the inner and outer cell membranes comprise 4 leaflets ( 2 bilayers), while the thylakoids comprise 8 leaflets ( 4 bilayers), and we assume a membrane area of $0.55 \mathrm{~nm}^{2}$ per lipid molecule (Lopez Cascales et al., 1996) and that lipids constitute $80 \%$ of total membrane area (Nikaido, 1996). Hence our hypothetical cell contains about 14 million lipid molecules, $2 \%$ of which are assumed to be phosphatidylglycerol (Van Mooy et al., 2006). Little data exists on metabolites or their concentrations in Prochlorococcus; here we assume a cytosolic concentration of $1 \mathrm{mM}$ for nucleotide triphosphates (e.g., ATP, GTP), probably the most abundant P-containing metabolites (Bennett et al., 2009). Finally, all of the 13 amol P complement not accounted for by DNA, lipids and metabolites is assumed to be RNA, thereby closing the cellular phosphorus budget. Total RNA is assumed to be $80 \%$ rRNA, 15\% tRNA and 5\% mRNA (Bremer and Dennis, 2008).

\subsubsection{Nitrogen budget}

Nitrogen in DNA is again calculated exactly from the genome size and base composition $(30.8 \% \mathrm{G}+\mathrm{C})$. Nitrogen in RNA is calculated from the RNA P content, with an RNA $\mathrm{N}: \mathrm{P}$ ratio of 3.8:1 derived from MED4 RNA sequences. To calculate the $\mathrm{N}$ in metabolites, we assume that the metabolite pool is dominated by compatible solutes at a concentration of $0.1 \mathrm{M}$ (Bennett et al., 2009). The compatible solute pool is taken to be comprised of sucrose / glucosylglycerate / glutamate in a 10:5:1 stoichiometric ratio (Klähn et al., 2009); hence metabolite N reflects cytosolic glutamate. Nitrogen in chlorophyll is based on a chlorophyll content of $1 \mathrm{fg} / \mathrm{cell}$, an average of literature values that range from 0.3-5 fg/cell (Morel et al., 1993; Moore and Chisholm, 1999; Claustre et al., 2002). The amount in peptidoglycan is again calculated from the cell geometry, assuming a cell-wall area of $2 \mathrm{~nm}^{2}$ per disaccharide unit (Vollmer and Holtje, 2004), and an $\mathrm{N}$ content of 7 atoms/unit. Nitrogen in lipopolysaccharide (LPS) is also calculated from membrane area, assuming a membrane area of $0.202 \mathrm{~nm}^{2}$ per fatty acid chain in LPS. LPS structure was assumed to be the same as that reported by Snyder et al. (2009) 
for strains of marine Synechococcus, with two nitrogen atoms and four fatty acid chains per unit, resulting in roughly 1.1 million LPS units per cell. Finally, all remaining nitrogen is assumed to be in protein, thereby closing the $\mathrm{N}$ budget. The number of amino acids in the cell can be calculated from the average nitrogen content of $1.310 \mathrm{~N}$ per residue, derived from the composition of the MED4 proteome as measured over the diel cycle (see Sec. 2).

\subsubsection{Carbon budget}

Carbon in DNA is calculated from genome size and base composition. Carbon in RNA is calculated from P content, with an RNA C:P ratio of 9.5:1. Metabolite C is calculated based on the same assumptions about compatible osmolytes made in the nitrogen budget. Chlorophyll $\mathrm{C}$ is again based on $1 \mathrm{fg} \mathrm{Chl/cell,} \mathrm{and} \mathrm{zeaxanthin} \mathrm{C}$ is based on a Zea:Chl ratio of 1.25 (wt/wt; Claustre et al., 2002). Protein $C$ is calculated from the cellular amino acid content, with an average residue composition of 5.075 $\mathrm{C}$ per amino acid, based on protein sequences. Lipid carbon is based on the measurements of Van Mooy et al. (2006), which suggest an average $C$ content of 39.7 atoms per lipid molecule. Based on the cell geometry calculations described above, there are approximately 20 million lipid molecules in our hypothetical Prochlorococcus cell. This is, however, probably the most uncertain component of the cellular carbon budget, as membrane geometry and lipid composition are likely to vary strongly with growth rate, light intensity and temperature.

\subsection{Budgeting results}

Three scenarios of C:N:P stoichiometry span the modeled range: cases I (302:53:1), II (225:33:1), and III (167:17:1). Case III is likely most analogous to exponential growth in culture under nutrient-replete conditions. Case I reflects extreme phosphorus limitation, akin to the results of Bertilsson et al. (2003), and possibly applicable to exceptionally Ppoor oceanic regions such as the Sargasso Sea (Ammerman et al., 2003) or eastern Mediterranean (Thingstad et al., 2005), though whether the high cell contents of $\mathrm{C}$ and $\mathrm{N}$ 
suggested by the model would actually obtain in such situations is uncertain. Case II is probably the most relevant for the majority of conditions encountered by

Prochlorococcus in oligotrophic marine habitats. The range presented here should not be construed to necessarily encompass the whole range of possible compositions of Prochlorococcus cells in the world's oceans. In particular, should a cell operate with a phosphorus quota substantially larger or smaller than 13 amol, a number of budgetary aspects could be qualitatively different. However, as discussed in the next section, the P quota can hardly be much smaller, and given that most of the elemental stoichiometry data is from P-replete cultures, it seems unlikely to be very much larger. Just how 'hardwired' the phosphorus quota is into a given strain of Prochlorococcus remains an intriguing and important question.

\subsubsection{Phosphorus economy}

The P budget of our hypothetical Prochlorococcus cell illustrates the "phosphorus economy" (Coleman and Chisholm, 2007) of small cells adapted to nutrient-poor environments. Note that the phosphorus budget is the same for cases I, II and III, since the cellular P content is fixed in this model. More than $40 \%$ of the cellular P quota is devoted to the 'fixed cost' of the chromosome. This makes the fact that most Prochlorococcus cells contain a substantial number of rare flexible genes (i.e., genes present in some but not all Prochlorococcus strains; Kettler et al., 2007) in their genomes - likely recently acquired and only occasionally with adaptive value - all the more telling of the importance of continuous gene exchange to microbial ecology and evolution (Coleman and Chisholm, submitted). We discuss the relationship between genome size, composition and nutrient budgets in detail in Section 2, below.

The amount of $\mathrm{P}$ in rRNA (5.2 amol) is sufficient to build 598 ribosomes. This is quite a small number; by comparison, log-phase $E$. coli cells have between 8,000 and 73,000 ribosomes, depending on growth rate (Bremer and Dennis, 2008). Similarly, the number of Prochlorococcus tRNA molecules predicted from the budget $(7,866)$, is 10 - to 100 - 
fold lower than E. coli (74,000-680,000; Bremer and Dennis, 2008). The predicted amount of mRNA is also quite small: just $200 \mathrm{~kb}$ (compared to $1.0-4.3 \mathrm{Mb}$ in E. coli; Bremer and Dennis, 2008), which allows only about $12 \%$ of the genome to be transcribed at any given time. Together, the DNA to encode genes and the RNA to express them account for over $90 \%$ of the cellular phosphorus budget. To a first approximation, Prochlorococcus uses any acquired P for nucleic acids - about half for DNA, half for RNA.

The other two phosphorus-containing classes of biochemicals, phospholipids and metabolites, are relatively minor in the cellular $\mathrm{P}$ budget. The low phospholipid content of picocyanobacteria, especially Prochlorococcus ( $\sim 2 \mathrm{~mol} \%$ ), has recently been explored in detail by Van Mooy et al. (2006; 2009). Interestingly, Prochlorococcus does not seem to upregulate phospholipid biosynthesis even when grown in P-replete conditions (Van Mooy et al., 2006). This is in stark contrast to other bacteria: marine heterotrophs contain $>80 \%$ phospholipids (Van Mooy et al., 2006), while E. coli uses phospholipids almost exclusively (Cronan and Rock, 2008). The amount of phosphorus in metabolites, on the other hand, is more uncertain. Little metabolome-wide quantitative data exists for bacteria, and none in Prochlorococcus; the estimates here are based on the recent comprehensive study by Bennett et al. (2009) in E. coli. It has recently been shown that sucrose is the major compatible solute in Prochlorococcus cells, with glucosylglycerate taking the place of glutamate as the second-most abundant metabolite under N-limiting conditions (Klähn et al., 2009). While glucosylglycerate contains neither P nor N, it is synthesized from relatively P-rich precursors (ADP-glucose and 3-phosphoglycerate), suggesting that the glycosylglycerate / glutamate exchange may also be a P/N substitution.

\subsubsection{Nitrogen}

The great majority of cellular nitrogen - 80 to $90 \%$ - is in protein (Figure 3A), in accord with findings for other phytoplankton and bacteria generally (Falkowski and Raven, 

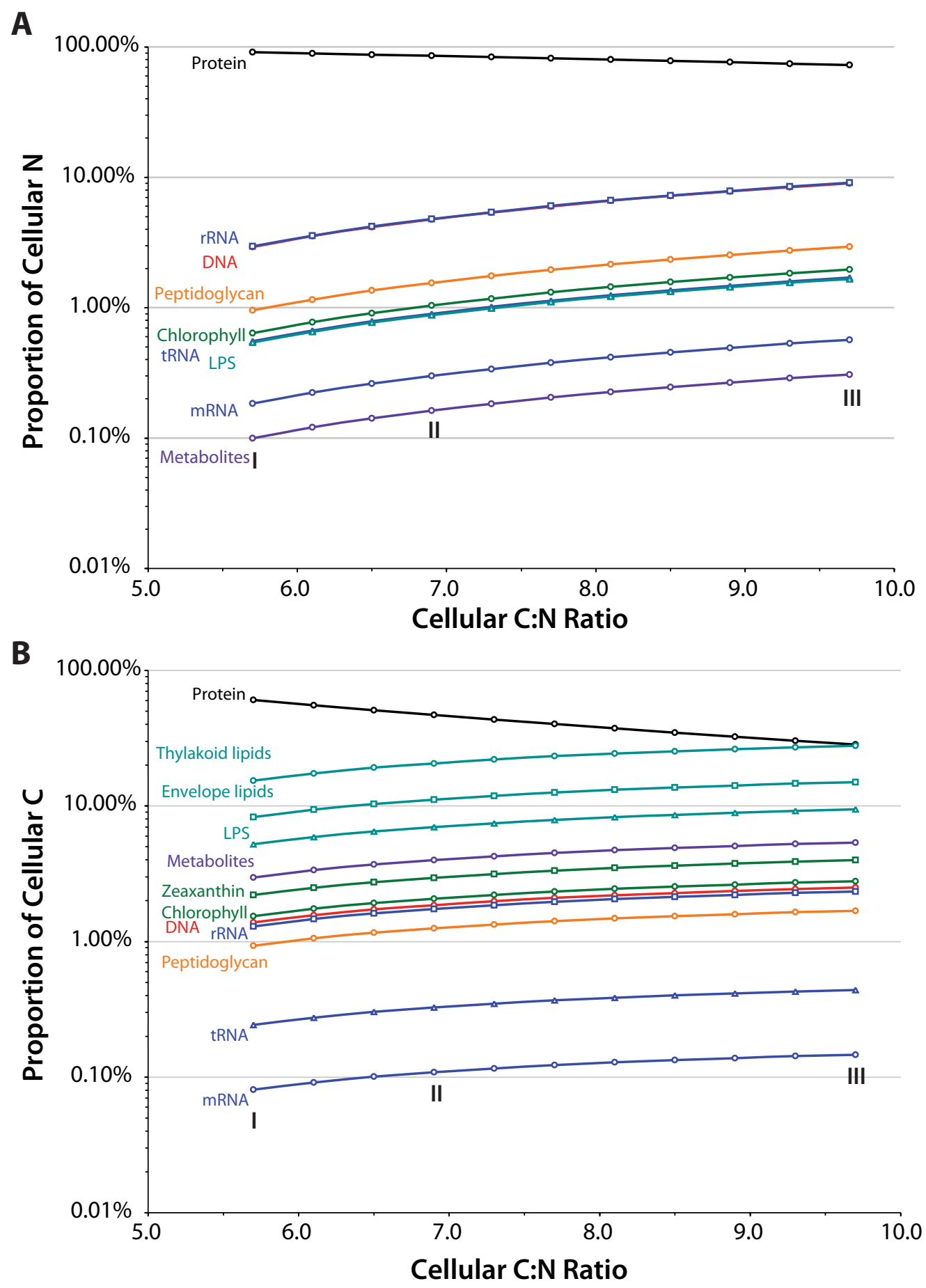

Figure 3. The distributions of cellular nitrogen (A) and carbon (B) among pools of biochemicals, as a function of modeled C: $\mathrm{N}$ ratio. The C:N ratios of the three model cases summarized in Table $1(I=5.7 ;\|=6.9 ;\| I=9.7)$ are indicated. 
2007). Even at the highest $\mathrm{C}: \mathrm{N}$ ratios considered (case III), the next largest pool of $\mathrm{N}$, RNA, is less than $10 \%$ of the total. It is worth noting, however, that the amount of $\mathrm{N}$ in peptidoglycan (up to 3\%) was calculated for a monolayer. If there are conditions under

which Prochlorococcus produces a much thicker cell wall, peptidoglycan could become a more significant part of its nitrogen requirements. Metabolite $\mathrm{N}$ is quite small (0.1$0.3 \%$ ), since Prochlorococcus uses carbohydrates as its primary compatible solutes. As noted above, Prochlorococcus seems to engage in a glucosylglycerol-for-glutamate substitution under $\mathrm{N}$ limitation, which could have a marginal impact on the overall nitrogen budget.

Over the C:N range considered, the number of amino acids in the cell varies almost fourfold, from $0.75 \times 10^{8}$ to $2.9 \times 10^{8}$, comprising between 290,000 and 1.12 million protein molecules. For comparison, exponentially-growing E. coli contains $7.6 \times 10^{8}$ to $23.7 \times 10^{8}$ amino acids per cell (Bremer and Dennis, 2008). Based on the results in Chapter 4, more than half of the amino acids are concentrated in the 50 most abundant proteins. Having an estimate of the amount of cellular protein also allows further interpretation of the fractional abundance values (APEX scores), though, as noted in Chapter 4, these will generally be overestimates of the true proportional abundance of a given protein. In particular, we can see what the range of fractional abundances implies in copy-number terms. The most abundant proteins have fractional abundances up to $\sim 0.05$, implying a range of 15,000-50,000 copies per cell. The lowest observed APEX scores from the diel experiment are $\sim 10^{-5}$, which would equate to between 3 and 11 copies per cell. As the low end of the calculated fractional abundance range is of order single-copy, it appears that the dynamic range of protein abundances in Prochlorococcus MED4 spans roughly four orders of magnitude.

\subsubsection{Carbon}

Carbon allocation to biochemicals is shown in Figure 3B for our range of modeled C:N ratios. The two major components of the cellular carbon budget are protein and lipids, 
and the largest effect of shifting elemental stoichiometry is on the relative amounts of $\mathrm{C}$ in the two pools. In case I, there is twice as much carbon in protein as in the lipids, while in case III, the proportions are reversed (nearly twice as much in lipids as in protein). Most cells probably divide net photosynthate approximately equally between the two, which is typical for phytoplankton (Falkowski and Raven, 2007). This budget does not include a specific amount of stored carbon, likely to be in the form of glycogen or other polysaccharides, which is produced during the day and catabolized at night (Chapter 4 and Zinser et al., 2009) and could constitute a substantial proportion of the cellular C budget early in the dark period. Other components of the carbon budget are small, amounting to no more than 5\% individually and 15\% collectively, but should not be disregarded. Metabolites, in particular, might comprise only 3-5\% of cellular carbon, but this is probably the pool of cellular biochemicals that is turning over at the highest rate, as well as that most likely to exchange with the extracellular medium and thereby impact water-column chemistry.

These elemental and biochemical budgets provide a useful baseline for considering how gene expression and nutrient utilization can influence one another. In the following sections, we explore two aspects of this interplay:

- To what extent adaptation to oligotrophic conditions might drive genome streamlining in Prochlorococcus

- How these small cells with limited pools of biochemicals cope with stochastic effects in gene expression

\section{IMPACT OF GENOME STREAMLINING ON CELLULAR NUTRIENT BUDGETS}

\subsection{Genome streamlining: occurrence and hypotheses}

The cellular biochemical budgets in Section 1 enable us to assess the effect of genome streamlining in Prochlorococcus on its nutrient requirements. It has been suggested that 
the loss of genes and decrease in genomic $\mathrm{G}+\mathrm{C}$ content evident along the evolutionary grade from Synechococcus through low-light Prochlorococcus to high-light

Prochlorococcus (Figure 4) reflects specialization for growth in oligotrophic habitats (Dufresne et al., 2005; Partensky and Garczarek, 2010). Other small marine bacteria, notably the abundant Pelagibacter/SAR11 $\alpha$-proteobacteria, also have small, A+T-rich genomes, and a similar nutrient-economy rationale has been proposed to explain this (Giovannoni et al., 2005), though the evolutionary context is not well characterized in these cases. One hypothesis (Wilhelm et al., 2007) holds that nutrient limitation places these bacteria are under strong selective pressure to excise any 'extraneous' DNA whose expression is not essential or immediately advantageous.

Small, A+T-rich genomes are not, however, limited to bacteria from nutrient-poor environments (nor are all oligotroph genomes compact and $\mathrm{G}+\mathrm{C}$-poor). Instead, the majority of such genomes are found in parasites (Dagan et al., 2006), for whom the explanation for genome streamlining is quite different. For parasites, the stable, nutrientrich environment of the host renders many cellular functions (especially biosynthetic ones) superfluous, thereby relaxing selective pressure to maintain those functions and allowing drift to eventually eliminate genes from the genome. So in one case, genome streamlining is proposed to be the result of strong selective pressures from the environment, while in the other, streamlining results from weakening of environmental selective pressure. These scenarios are not necessarily contradictory and both could be true; it would, however, be an unusual example of convergent evolution for these opposing causes to produce the same effect.

Using the cellular elemental and biochemical budgets developed above, we can quantify the relative impact of genome size reduction and base composition changes on nutrient requirements. We consider the changes in genome composition between Prochlorococcus MIT9313 and MED4, namely a reduction in genome size by $750 \mathrm{kbp}$ and a drop in $\mathrm{G}+\mathrm{C}$ content from $50.7 \%$ to $30.8 \%$ (Fig. 4). The effects on nutrient budgets 


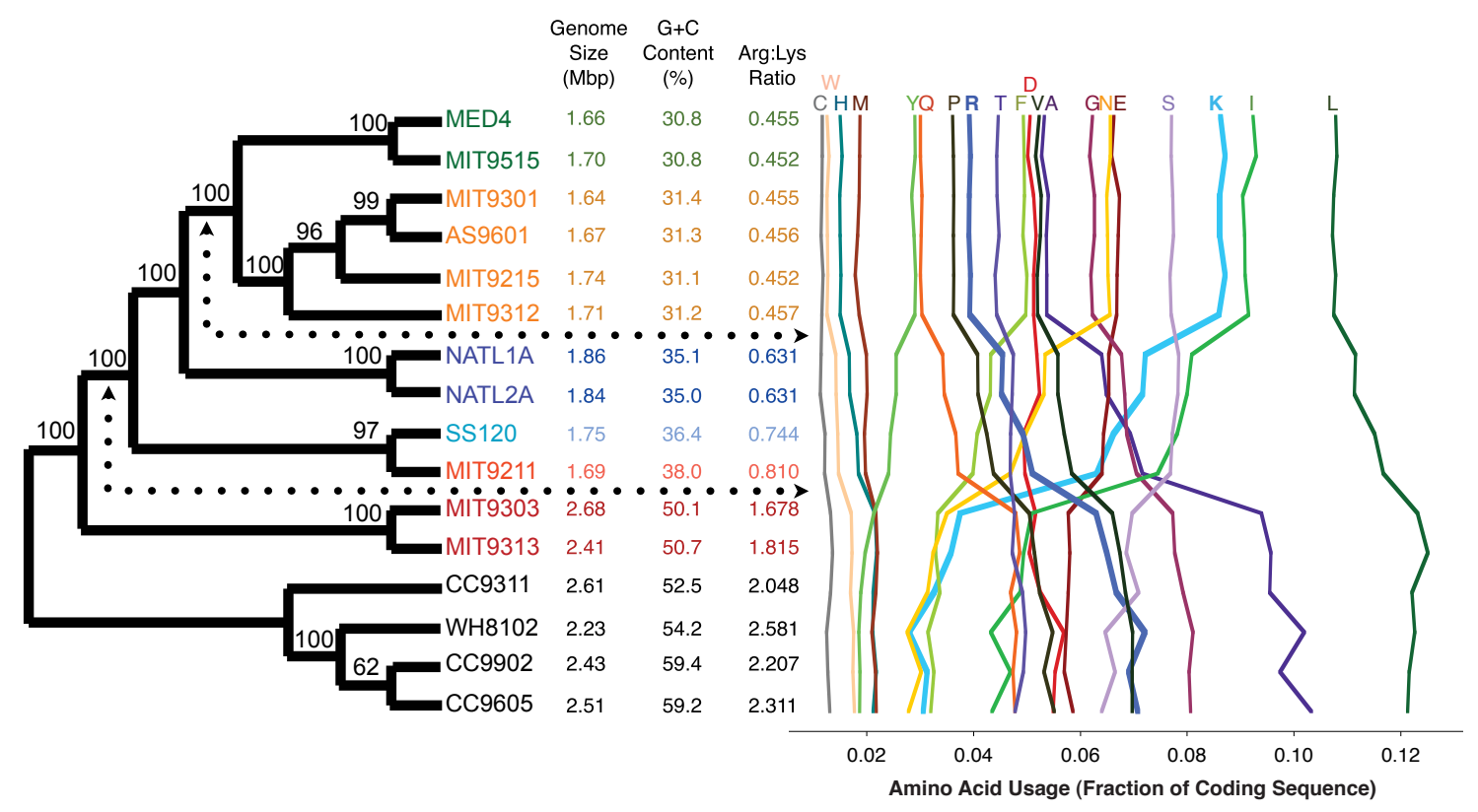

Figure 4. Genome characteristics of marine picocyanobacteria. At left, cladogram of sequenced Prochlorococcus and marine Synechococcus strains (from Kettler et al. 2007). At right, plot of proportional amino acid usage in protein-coding sequences. Changes are largely tied to the trend of decreasing genomic $\mathrm{G}+\mathrm{C}$ content, exemplified by the 5 -fold drop in the Arginine:Lysine ratio (right-hand column and bold lines in plot). Dotted arrows indicate branches on the tree where the largest shifts in amino acid usage appear to have occurred. 
are calculated relative to the cellular compositions in Section 1, which is for a high-light adapted, MED4-like cell. The intent here is not to compare nutrient requirements of MIT9313- and MED4-type cells, but rather to gauge the additional burden that carrying a MIT9313-like genome would have imposed on a MED4-like cell.

\subsection{Consequences for nutrient requirements}

The larger genome, of course, requires more phosphorus to duplicate. An additional $750 \mathrm{kbp}$ would increase the size of the MED4 genome by $45 \%$. The net increase in the cellular P requirement for just the extra DNA would be 19.1\%. Assuming these additional genes were expressed at the same average level as the rest of the genome, the additional mRNA would add $1.1 \%$ to the phosphorus quota. Hence the total P savings from the smaller, high-light genome amounts to roughly $20.2 \%$ of the cellular phosphorus budget - in total, an amount likely visible to selection under P-limiting conditions. However, DNA is lost from bacterial genomes in fragments much smaller than $750 \mathrm{kbp}-$ perhaps only a few tens of bases at a time - and the incremental effect of each loss is much smaller and less likely to be selected for. Bragg and Wagner (2009) have shown how single-atom changes in gene composition can be adaptive under nutrient limitation, if the selective pressure is strong enough. Quantifying this selective pressure would require knowledge of the effective population size of Prochlorococcus (i.e., the size of a subpopulation bearing the same degree of neutral genetic varation as the total population), which is difficult to ascertain for bacteria generally (Fraser et al., 2007). The

global census population is of order $10^{27}$ cells (Partensky et al., 1999), though the effective population size could be many orders of magnitude smaller. The potential exists for a huge effective population size to select for even small chromosomal changes in Prochlorococcus. As discussed below, however, there are multiple lines of evidence from high-light isolate genomes that suggest that 'extraneous' DNA is not always rapidly excised from cells. 
The effects of genome streamlining on the cellular nitrogen budget are more equivocal. 750,000 base pairs of DNA amounts to $1.2-3.7 \%$ of the cellular $\mathrm{N}$ budget. The effect of the decrease in $\mathrm{G}+\mathrm{C}$ content is even smaller - if the MED4 genome were $50.7 \% \mathrm{G}+\mathrm{C}$, the cellular $\mathrm{N}$ requirement would increase by only $0.1-0.2 \%$. Because of a bias in the genetic code that associates $\mathrm{G}+\mathrm{C}$-rich codons with $\mathrm{N}$-rich amino acids, a decrease in genomic $\mathrm{G}+\mathrm{C}$ will also produce a lower-N proteome. This can be clearly seen in the trend in the Arg:Lys ratio along the evolutionary grade, which decreases fivefold from 2.3 in Synechococcus to 0.45 in high-light Prochlorococcus (Fig. 4). Arginine and lysine are both basic amino acids, and can often substitute for one another in proteins. Since arginine has 4 nitrogens to lysine's two, and Arg is encoded by more G+C-rich codons (CGx, AGA, AGG) than Lys (AAA, AAG), a single transition mutation could save the cell 3 nitrogen atoms. If the MED4 genome coded for amino acids at the same relative frequency as MIT9313, its proteome would be predicted to be richer in nitrogen by $0.031 \mathrm{~N}$ per amino acid. For the cell compositions outlined in Section 1, this would increase the $\mathrm{N}$ budget by $1.8-2.1 \%$. Hence the total potential nitrogen savings associated with genome streamlining in MED4 amount to 3.4-5.7\% of the cellular $\mathrm{N}$ requirement.

As in the case of phosphorus, each small, incremental change in genome composition would have to be visible to selection - which could be plausible, given the potentially huge effective population size of Prochlorococcus, and would be influenced by the expression level of each protein. Nitrogen savings in highly-expressed proteins will have a proportionally larger impact on the cellular $\mathrm{N}$ budget, and therefore be more likely to be selected under $\mathrm{N}$ limitation. The calculation of fractional protein abundances based on the data presented in Chapter 4 equips us to address this question. Figure 5 shows three views of the amino acid composition of the proteome: the average of coding sequences in the genome (also plotted in Figure 4), the proteome composition suggested by the fractional abundance measurements (i.e., the average of sequences of detected proteins, weighted by APEX scores), and the average of sequences of the 51 most abundant proteins in MED4 (i.e., those with highest average APEX scores over the diel cycle; see 


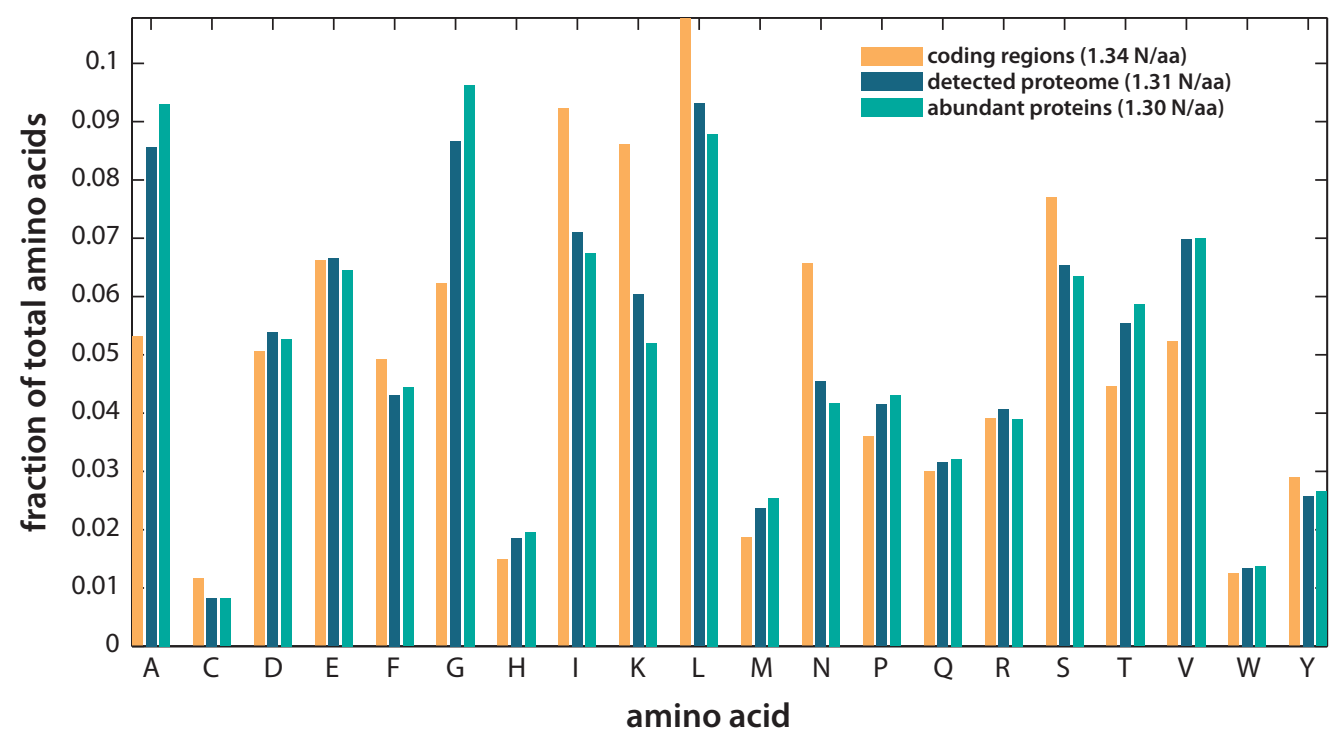

Figure 5. Amino acid usage in the MED4 proteome, contrasting the proteome-as-coded (orange) with the proteome-as-expressed (light \& dark green). Orange bars are the aa usage in protein-coding regions of the genome (as also plotted in Fig. 4). Dark green bars are the composition of the proteome as detected over the diel cell-division cycle (see Chapter 4; based on weighting by APEX scores). Light green bars are the average amino acid composition of the 51 most abundant MED4 proteins (Chap. 4, Table 2). The number of nitrogen atoms per amino acid each set of sequences is also indicated. 
Chapter 4). The proteome-as-expressed is indeed lower in nitrogen than the proteome-ascoded, to the tune of $0.03 \mathrm{~N}$ per amino acid (1.34 vs. 1.31$)$, or $2.4 \%$. The most abundant proteins are even slightly lower in $\mathrm{N}$ content. This suggests that the sequences of highlyexpressed proteins have in fact been selected to limit $\mathrm{N}$ demand.

Does the genome streamlining process explain the relative nitrogen efficiency of highlyexpressed proteins? Surprisingly, the answer seems to be no. The abundant proteins in MED4 are not endmembers or extreme examples of the evolutionary compositional trends illustrated in Figure 4. Rather, the low $\mathrm{N}$ content of abundant proteins stems primarily from less usage of two N-rich amino acids (Lys and Asp), whose genomic frequency increases towards the high-light clade (Fig. 4), in favor of more usage of three $\mathrm{N}$-poor residues (Ala, Gly and Val) whose frequency decreases along the trend. Since A, $\mathrm{G}$ and $\mathrm{V}$ are encoded by $\mathrm{G}+\mathrm{C}$-rich codons (GCx, GGx and GTx, respectively), the $\mathrm{G}+\mathrm{C}$ content of the 50 most highly-expressed genes is actually higher than the genome as a whole (38.9\% vs. 30.8\%). In other words, highly-expressed proteins show economy of $\mathrm{N}$ usage despite the overall effect of genome streamlining, not because of it. Whatever the evolutionary process driving genome streamlining, it does not appear to be closely linked to reducing cellular demand for nitrogen.

\subsection{Streamlining in the light of evolution}

Viewed in a broader evolutionary context, the case for genome streamlining in Prochlorococcus as being driven primarily by adaptation to nutrient scarcity is tenuous. The two kinds of genomic changes - size reduction and $\mathrm{G}+\mathrm{C}$ loss - are uncoupled in the

evolutionary history of Prochlorococcus. From the phylogenetic tree in Figure 4 it would at first appear that, rather than being a gradual, continuous process, there was a single episode of genome size reduction in Prochlorococcus, along the branch between the MIT9313/MIT9303 divergence and all other strains. With more careful consideration of the histories of individual gene families, a different picture emerges: the Synechococcus- 
like size of the MIT9313/MIT9303 genomes is not due to retention of nearly all genes from a Prochlorococcus/Synechococcus ancestor, but rather substantial gene gain within that lineage (Kettler et al., 2007) - implying that genome size reduction actually occurred in the Prochlorococcus common ancestor. The slightly enlarged genomes of the NATL strains - bigger than either the more basal or distal groups in the tree - also reflects lineage-specific gene acquisition. Moreover, none of these patterns correlates at all with the distributions of phosphorus-stress related genes among Prochlorococcus strains. As shown by Martiny and Coleman et al. (2006), closely-related isolates with similar genome sizes often bear radically different complements of $\mathrm{P}$ acquisition and utilization genes. As noted by Coleman and Chisholm (2007), adaptation to phosphorus availability appears to occur on a very different evolutionary scale from changes in genome size.

Drops in $\mathrm{G}+\mathrm{C}$ content, and the associated changes in amino acid usage, also have a phylogenetic distribution distinct from the pattern of genome size reduction. These appear to be concentrated in two places on the evolutionary tree: between MIT9313/MIT9303 and other Prochlorococcus, and between the NATL strains and the ancestor of the high-light adapted group (Figure 4). If $\mathrm{G}+\mathrm{C}$ content changes were primarily influenced by nitrogen availability while genome size reduction is a phosphorus-saving measure, it is unclear how the two should become so decoupled over evolutionary timescales.

Most significantly, there is increasing evidence that, even under the strongest forms of Plimitation, cells bear a significant number of genes that have little or no immediate adaptive value. In high-light Prochlorococcus isolates, roughly $10 \%$ of the genome consists of hypervariable regions, and the size of these regions is uncorrelated with the distribution of phosphate-adaptation genes. If genome size were linked to adaptation to $\mathrm{P}$ limitation, we would expect strains with larger complements of P-acquisition genes to have smaller genomes, but no such correlation is observed among strains sequenced to date. The hypervariable $10 \%$ of the genome represents approximately $4.5 \%$ of the 
cellular P budget that could be considered the cell's 'investment' in gene exchange. Metagenomic sequence data from the HOT and BATS ocean time-series stations also provides evidence that adaptation to strong P limitation at BATS has not produced a decrease in genome size. If genome size reduction were a strategy to adapt to nutrient limitation, we would expect many genes to be less abundant at the more strongly Plimited site. In fact, the opposite pattern is observed: core genes (i.e., those shared by all sequenced Prochlorococcus strains; Kettler et al., 2007) show indistinguishable per-cell abundance distributions at HOT and BATS, and of the 29 flexible genes that are differentially abundant, 26 are actually more abundant at BATS than HOT (Coleman and Chisholm, submitted).

If nutrient-utilization efficiency fails to account for the evolution of small, A+T-rich genomes in Prochlorococcus, what processes offer a more satisfactory explanation? Partensky and Garczarek (2010) discuss the loss of several DNA-repair genes within the Prochlorococcus lineage, and note that the inactivation of these systems is likely to increase the rate of G:C-to-A:T mutations. These authors also point out, however, that gene losses are not clearly linked to high mutation rates, as that should result in the presence of numerous inactivated pseudogenes, which are nearly absent in Prochlorococcus genomes. Quantification of mutation rates in various Prochlorococcus ecotypes would be highly valuable in assessing the role of DNA repair in genome streamlining.

With regard to gene loss, Lynch (2006) has suggested that the presence of nonfunctional DNA in bacterial genomes is opposed by selection because of the mutational burden it introduces. For example, a mutation in a 5' untranslated region could introduce an alternative start site for its associated ORF, with deleterious consequences. The strength of the selection that opposes the maintenance of such sequences is proportional to the effective population size, which as noted above, is difficult to discern at present. In organisms such as Prochlorococcus, it would appear that not only nonfunctional DNA, 
but also coding regions have been excised. This may be tied to reductions in cell size and metabolic simplification: as overall expression levels drop, some genes enter a 'gray zone' between functional and nonfunctional DNA. A gene that is no longer (or not yet) expressed may be a waste of a couple of thousand phosphorus atoms, but its much greater burden is as a neighbor to genes that do need to be expressed efficiently and need a wellordered chromosomal neighborhood to do so. This 'use it or lose it' notion of genome streamlining is also more consistent with the clustering of recently-acquired genes in hypervariable islands (Coleman et al., 2006), as islands could serve as genomic 'holding pens' that allow active gene exchange without undue disruption of expression of the rest of the chromosome. Ultimately, genome streamlining in Prochlorococcus (and other marine bacteria) may well be tied to oligotrophic conditions - but through overall reductions in gene expression (as noted in Section 1, the major nutrient demand on the cell) rather than selection on the composition of DNA per se. Furthermore, this scenario unifies the root causes of genome streamlining in parasitic and oligotrophic bacteria, as both could stem from genetic disuse. Much remains to be explored regarding the evolutionary trajectories of Prochlorococcus genomes, and multiple driving forces are likely to be at work. Nevertheless, the ability of population-genomic processes to explain genome characteristics of a wide variety of organisms inhabiting many different habitats (Lynch, 2006) offers promise for a deeper understanding of microbial evolution.

\section{STOCHASTIC GENE EXPRESSION EFFECTS AND THE ECOLOGICAL CONSEQUENCES OF NOISE}

\subsection{The nature of noise}

The expression of genes by cells is a stochastic phenomenon. Gene expression is stochastic in that fluctuations in gene product abundances, both in a particular cell over time and between nominally identical cells at a given time, are governed by processes, such as macromolecular diffusion, that are random and can only be described statistically 
(Kærn et al., 2005; Larson et al., 2009). Note that this does not mean that expression levels as typically considered - as population or steady-state averages - are random or non-deterministic. Regulatory mechanisms clearly control the averages and central tendencies; it is in the variance and high-frequency dynamics that stochastic effects become significant. For individual cells, however, long-term or population averages are irrelevant, and stochasticity can have a major impact on how a cell goes about accomplishing a physiological programme such as cell division.

To illustrate this idea, consider the abundance of a given gene's transcript in a hypothetical Prochlorococcus cell. As noted above (Section 1.2.1), only about $12 \%$ of the cell's genome is transcribed at any given time, so the mean transcript abundance (averaged across all genes and cells) is 0.12 copies/cell. Of course, transcripts only exist in integer copy numbers, so this result is more meaningfully interpreted along the lines of, "A given cell contains a copy of a given transcript about $12 \%$ of the time," or, "A given transcript is present in about $12 \%$ of cells in a population at any given time." In reality, there is a wide range of average transcript abundances (spanning four orders of magnitude, according to the AMEX results in Chapter 4), so some transcripts are probably found in most cells most of the time (average abundances at or above 1), while others are very rare ( $\sim 0.001$ copies/cell). Nevertheless, the fact that the average transcript abundance is significantly less than 1 copy/cell means that each individual transcription event (i.e., each mRNA molecule produced) usually causes a huge proportional change in the abundance of that gene product in the cell. Since transcription is a stochastic process, the low average abundance of transcripts in Prochlorococcus cells mean that the actual number of copies of a particular mRNA molecule in a given cell will be subject to strong and unpredictable fluctuations over time. As a cell tries to perform more or less of a certain metabolic process (e.g., in response to changing light availability over the diel cycle) by changing the expression of a related gene, it must cope with the random noise imposed by stochastic effects on the gene product abundance is it attempting to regulate. 
The small size, limited chemical complements and low absolute gene expression levels of Prochlorococcus cells could have significant implications for their physiological functioning. In particular, small cells might 'sample' the range of physiological states (phenotypes) available to them in a more discrete fashion than larger cells do, because the pools of biochemicals that enable those different states are smaller and thus integer changes in copy numbers of gene products are proportionally more significant. This 'granularity' in gene product abundance makes small cells especially susceptible to stochastic effects in gene expression. Stochasticity can give rise to phenotypic variability even within an isogenic population, with various aspects of cell physiology and metabolism varying around a population mean (Longo and Hasty, 2006). In large populations, then, rare phenotypes are more likely to arise and have an ecological, and potentially evolutionary, impact. Nutrient scarcity can also exacerbate stochastic effects, particularly for autotrophic organisms, since gene products (mRNA and proteins) and gene expression machinery (especially ribosomes) are the most nutrient-intensive biochemicals in the cell. Prochlorococcus, as one of the smallest and most abundant free-living bacteria and an inhabitant of exceptionally nutrient-poor habitats, appears to be in the 'perfect storm' of stochastic gene expression effects. Here we develop a hypothesis for how Prochlorococcus - and small cells in general - might cope with expression noise, which suggests that modulating these effects could be an important constraint on these organisms' physiology and ecology.

Stochastic effects in gene expression (also termed 'noise') have been of interest for a number of years, and a variety of experimental and theoretical studies have been published. The significance of stochastic effects in processes such as determination of cell fate (Losick and Desplan, 2008) and responses to environmental perturbations (Acar et al., 2008) been demonstrated. The exploration of stochastic effects in Prochlorococcus, however, is hindered by the lack of genetic tools that would enable the kind of single-cell studies that have elucidated similar phenomena in other microbes. 
Fluorescent protein fusions have been especially powerful tools for visualizing stochastic effects, and allow detection by flow cytometry (Newman et al., 2006). Transfer of a GFP-containing plasmid into (and its expression by) one Prochlorococcus strain has been acheived (Tolonen et al., 2006), but expressed fusions to native proteins have yet to be demonstrated. While direct experimental investigation of noisiness in Prochlorococcus gene expression awaits further developments, the cellular biochemical budgets outlined in the first section allow us to bound the problem and generate some useful hypotheses for how such effects might be manifested.

\subsection{Bounding the noisiness of Prochlorococcus}

We consider two different scenarios for gene expression noise, depending on the noise source: a "Poisson" scenario, where expression noise is dominated by the random births and deaths of mRNA molecules, and a "Telegraph" scenario, where noise is dominated by random promoter binding events (Kaufmann and van Oudenaarden, 2007). The Poisson scenario is a one-state model in that the probability of transcription is constant with time. The Telegraph scenario is a two-state model, since transcription is dependent on promoter binding, which is itself stochastic. While the Poisson model has generally been considered to sufficiently explain observations of expression fluctuations in bacteria (Raj and van Oudenaarden, 2008), there is some evidence for the transcriptional bursting implied by the Telegraph model (Golding and Cox, 2006), so here we consider both possibilities. The equations describing mRNA and protein noise under the two scenarios are given in Table 2 (Kaufmann and van Oudenaarden, 2007), and we introduce the ratio of the noise at the mRNA and protein levels, designated $H^{2}$.

A description of each of the noise parameters, and their values for Prochlorococcus near the upper and lower limits of environmentally-observed growth rates (Veldhuis et al., 2005), are given in Table 3. Where required, values from the budgets in Section 1 of this chapter were taken, using case II. Note that because of the way gene product abundances 


$$
\begin{array}{ccc} 
& \text { Poisson } & \text { Telegraph } \\
\text { mRNA noise } & \eta_{m}^{2}=\frac{1}{\langle m\rangle} & \eta_{m}^{2}=\frac{\lambda_{m} \tau_{m}}{\langle m\rangle\left(1+b \tau_{m}\right)} \\
\text { protein noise } & \eta_{p}^{2}=\frac{\lambda_{p} \tau_{m}}{\langle p\rangle} \quad \eta_{p}^{2}=\frac{\lambda_{p} \lambda_{m} \tau_{m}}{\langle p\rangle b} \\
\text { noise ratio } & & H^{2}=\frac{\eta_{m}^{2}}{\eta_{p}^{2}}
\end{array}
$$

Table 2. Equations for noise in gene expression at the mRNA and protein levels, from Kaufman \& van Oudenaarden (2007). In the Poisson scenario, noise is dominated by the random births and deaths of low-copy transcripts. In the Telegraph scenario, transcriptional bursts result from random promoter binding events. See Table 3 for parameter values.

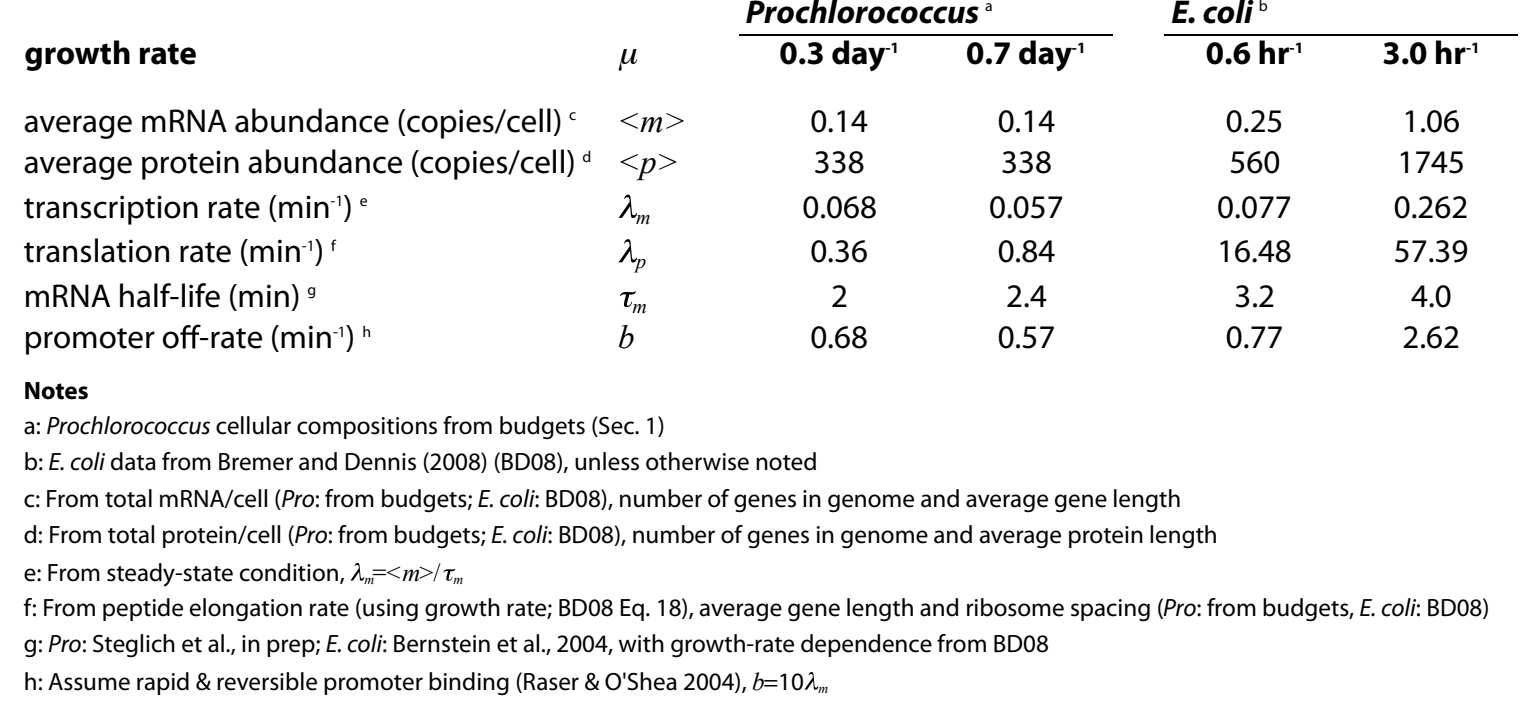

Table 3. Values of cellular parameters used in calculations of expression noise. 
and rates are calculated here, stochastic effects for Prochlorococcus are independent of cell stoichiometry at a given growth rate. This is certainly a simplification, and in real settings there are likely tradeoffs between growth, noise and budgets that are important to environmental adaptation. To help put the Prochlorococcus results in context, values of the same parameters for E. coli growing at two different rates are also shown (Table 3). As expected from its slower growth and lower ribosome content, the translation rate in Prochlorococcus is substantially lower than in E. coli.

Under both the Poisson and Telegraph scenarios, gene expression is noisier in Prochlorococcus than E. coli at the mRNA level, but less noisy at the protein level (Table 4). For both organisms, noise scales inversely with growth rate, principally due to the larger pools of gene products and expression components in the faster-growing cells. Stochastic effects due to mRNA fluctuations (the Poisson scenario) are larger than those predicted for promoter noise (the Telegraph scenario), though this is contingent on promoter kinetics that likely vary widely between individual genes.

A key prediction of this model is that gene expression in Prochlorococcus is much noisier at the mRNA level - probably by a factor of over a thousand - than at the protein level. Values of the mRNA:protein noise ratio ( $\mathrm{H}^{2}$ value) in Prochlorococcus range from near 700 in the Telegraph promoter-noise scenario at fast growth rates to over 3000 in the Poisson scenario at slow growth rates. By comparison, the noise ratios in E. coli range from $\sim 6$ up to only 43 . The main factors contributing to the high noise ratio in Prochlorococcus are the small numbers of transcripts and slow translation rate. This results in fewer proteins being produced per transcript, limiting the effects of translational bursting. In fact, the model presented here implies that many transcripts in Prochlorococcus may go untranslated, essentially for want of an available ribosome. Since transcription (and therefore transcript degradation) is not slowed to nearly the same degree as translation, a sizeable proportion of transcripts might be recycled before a ribosome binds to them. While 'wasteful' to a certain extent, Prochlorococcus invests 


\section{growth rate}

$$
\mu
$$

Poisson scenario

mRNA noise

protein noise

Noise ratio

\section{Telegraph scenario}

mRNA noise

protein noise

Noise ratio

$\eta_{m}{ }^{2}$

$\eta_{p}^{2}$

$H^{2}$
Prochlorococcus

\section{3 day $^{-1}$}

0.7 day $^{-1}$

7.35

0.0021

3453

7.35

0.0060

1233

$\eta_{m}{ }^{2}$
$\eta_{p}{ }^{2}$
$H^{2}$

0.424
0.0002
1990

0.424
0.0006
711

0.289

0.087

0.0094

0.0133

30.7

6.5

Table 4. Results of expression noise modeling, using equations and values from Tables 2 and 3. Under both noise scenarios and over the full range of growth rates, gene expression in Prochlorococcus is noisier at the mRNA level but less noisy at the protein level than in E. coli. Protein noise is effectively damped by the slow translation rate in Prochlorococcus. 
only a relatively small proportion of nutrients in mRNA. High mRNA turnover could be energy-intensive, but if the phenotypic consequences of protein-level noise are severe enough, it may be worthwhile to express genes in a manner that damps stochastic fluctuations in protein abundances.

If protein-level expression noise is in fact a constraint on cellular metabolism, the equations in Table 2 outline several potential strategies for dealing with it. Perhaps most straightforward is to increase $<p>$-- that is, to make larger pools of proteins. This, of course, requires greater nutrient supplies and larger cells, and would be ecologically disadvantageous in an oligotrophic marine habitat. Another strategy is to decrease mRNA half-life (smaller $\boldsymbol{\tau}_{m}$ ). Prochlorococcus has quite short mRNA half-lives (Steglich et al., submitted), but it seems likely that this strategy can only be taken so far, as half-lives would become too short for mRNAs to be translated at all. The third option is to slow down protein synthesis (smaller $\lambda_{p}$ ) - which can be taken as far as the ecological consequences of the concomitant slowdown in cell growth can be tolerated. The damping of protein noise by slow translation (in the sense of low protein yield per transcript molecule, not necessarily due to "slow ribosomes") may be a substantial factor in the low, fixed cell division rate of Prochlorococcus.

It is interesting, then, that Prochlorococcus makes relatively little use the first noisedamping option - making more protein and bigger cells - even when nutrients are abundant. It is clear that some "facultatively copiotrophic" (or conversely, "facultatively oligotrophic") microbes can tune cellular protein abundances, sizes and growth rates to the prevailing nutrient regimes, making smaller, slow-growing cells under oligotrophic conditions and larger, faster-growing ones when nutrients are replete. It may be that nutrient enrichments to epipelagic waters are infrequent enough that Prochlorococcus lost the genetic machinery to speed growth under meso- to eutrophic conditions through disuse, as described in the previous section. Not all open-ocean microbes have lost these abilities, however (Martin and Macleod, 1984), suggesting that the putatively large 
effective population size of Prochlorococcus may have driven it further down the path of genome streamlining.

The relative lifetimes of proteins and transcripts may also be crucial to expression noise. Steglich et al. (submitted) have shown that mRNA half-lives in Prochlorococcus are relatively short, and the timecourse expression results presented in Chapter 4 suggest (though do not require) that protein lifetimes are relatively long. This $\tau_{p} / \tau_{m}$ ratio figures prominently in several more recent models of stochastic gene expression. In the models of Pedraza and Paulsson (2008) and Shahrezaei and Swain (2008), which treat bursting effects explicitly in a temporal framework, the contribution of mRNA noise to stochasticity at the protein level is modulated by $\tau_{p} / \tau_{m}$. In fact (cf. Pedraza and Paulsson, 2008, Eq. 1 and 2; Shahrezaei and Swain, 2008, Eq. 21), in the case where $\tau_{p}>>\tau_{m}$, as may well be the case for Prochlorococcus, protein noise in these models simplifies to $1 /<p>$, the inherent small-number lower limit. Temporally-resolved proteomics measurements, akin to those presented in Chapter 4, should allow gene-product lifetime experiments analogous to that of Steglich et al. to be performed at the protein level, providing a global view of $\tau_{p} / \tau_{m}$ ratios for Prochlorococcus genes. This combination of transcript and protein half-life measurements would test whether the combination of long-lived proteins and rapid mRNA turnover contributes to noise reduction, and how this effect varies among different genes.

\subsection{The growth costs of noise, or, You can't just shrink a Ferrari}

The low translation rate that contributes to limiting protein-level expression noise in Prochlorococcus goes hand-in-hand with relatively slow cell growth. Slow growth may be a general feature of very small cells, if low translation rates are a common strategy to

damp protein noise and stabilize metabolism. To apply an automotive metaphor, in small cells, the 'engine' of transcription is running fast, producing (and degrading) mRNA at rates comparable to faster-growing cells, but the 'transmission' of translation is in low 
gear, producing the 'torque' required to control the cell division program at the expense of slowing protein production. In this scenario, small cells grow slowly for the same reason a moped can't go $60 \mathrm{mph}$ up a steep hill: the small engine can only generate so much torque with the largest gear that will fit on the frame. As with motor vehicles, there may be a size-speed tradeoff in cells: initial increases in size produce speed (growth rate) gains, as a more powerful drivetrain (expression machinery) can be accommodated by the frame. But eventually, an optimum power:weight ratio is reached, maximizing speed, as exemplified by racecars or E. coli. Above this optimum size, top speed/maximum growth rate declines (for bigger 'vehicles', e.g., cargo trucks, or large protists such as dinoflagellates) as the chassis accommodates heavier and heavier loads.

There is biological evidence for the growth rate-cell mass relationship on both sides of this proposed optimum. In E. coli, growth rate increases proportionally to cell mass up to a maximum of a 20 minute doubling time at a mass of $1.02 \mathrm{pg}$ (Bremer and Dennis, 2008). Interestingly, this positive correlation between cell size and growth rate is the opposite of the relationship proposed from some compilations of phytoplankton growth rates, which have been fit with a (mass) $)^{-1 / 4}$ trend (Finkel et al., 2009); it should be noted that positive correlations between cell size and growth rate have been also observed for some phytoplankton (Costello and Chisholm, 1981). A negative correlation between growth and size in larger organisms has been justified theoretically in the context of the metabolic theory of ecology (Brown et al., 2004). So one hypothesis, shown diagrammatically in Figure 6, might be that rapidly-growing bacteria such as E. coli are perched at an optimum at the border between metabolically-dominated and stochastically-dominated growth regimes. In this view, the increase in growth rate gained by alleviating metabolic burdens in smaller cells is counterbalanced by the challenge of speeding up a transcriptional program whose smaller pools of gene products are more susceptible to stochastic effects. Testing this hypothesis would require intercalibration of growth rate-cell size relationships across a broad range of taxa (especially small, slow- 


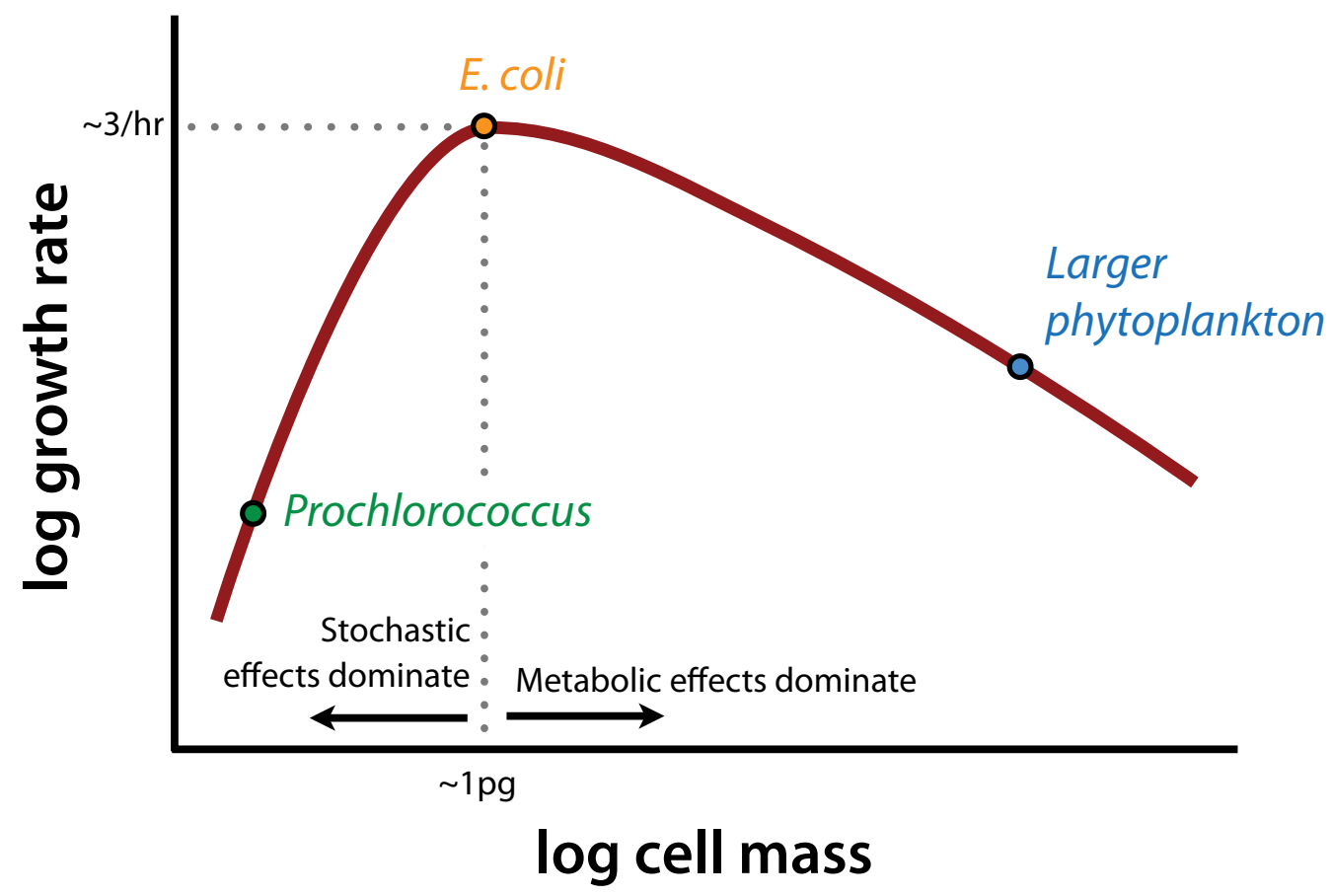

Figure 6. Schematic of hypothesized relationship between cell mass and growth rate. At low cell mass, small pools of gene products result in large stochastic effects in gene expression; slow growth is suggested as one way to damp these effects at the protein level and thereby maintain metabolic/ phenotypic stability. For larger cells, stochastic effects are small and growth rates are limited primarily by metabolic considerations. Fast-growing bacteria such as E. coli are proposed to live at the border between the two growth-limitation regimes. 
growing bacteria and archaea), which is challenging (Finkel et al., 2009). But one can't simply shrink down a Ferrari and expect it to still go as fast.

While stochastic gene expression effects might be observable in the laboratory (though not yet in Prochlorococcus) and at least partially predictable on theoretical grounds, it is less clear how consequential they are for organismal physiology and even less clear for ecology. Experimental studies to date have shown that increased stochasticity can provide an advantage when environmental conditions fluctuate. With the notable exception of the diel cycle (Chapter 4), the tropical/subtropical open-ocean habitat of Prochlorococcus is generally considered one of the more stable environments on earth. Physical and chemical conditions in the upper water column of subtropical gyres change relatively slowly, and seasonality is weak. In recent years, however, increasing attention has been paid to smaller-scale variability that may be highly relevant for microbial physiology. Even in the well-mixed upper open ocean, eddies and aeolian deposition events produce substantial changes in nutrient availability over scales of $10^{2}-10^{5} \mathrm{~m}$ and days to weeks. Perhaps more importantly over longer time scales, even conditions that appear stable using conventional, 'bulk' oceanographic measurements are increasingly appreciated as highly heterogeneous on the spatial scales of individual microbial cells (Seymour et al., 2004; Mitchell et al., 2008). Microscale events such as the formation and dissipation of nutrient patches or the aggregation and sinking of rare, large particles are occurring constantly and represent environmental fluctuations from the perspective of individual cells. The ecological (and ultimately evolutionary) consequences of stochastic gene expression in planktonic marine microbes may well be tied to the exploitation of microscale, transient features in the water column.

\section{CONCLUSIONS AND FUTURE DIRECTIONS}

This chapter presents a simple model for the molecular composition of hypothetical highlight Prochlorococcus cells, based on available experimental and genomic data. 
Budgeting of cellular contents of carbon, nitrogen and phosphorus reveals that almost half of cellular $\mathrm{P}$ is contained in the chromosome, leaving enough for only about 600 ribosomes and the transcription of $10-15 \%$ of the genome. $70-90 \%$ of cellular $\mathrm{N}$ is in protein, and copy numbers of individual proteins range up to perhaps 50,000 copies per cell. We suggest that genome streamlining is unlikely to be driven primarily by adaptation to oligotrophic conditions, and hypothesize that population-level evolutionary processes are more likely to explain genome evolution in Prochlorococcus.

Consideration of cell composition in light of stochastic effects in gene expression yields a prediction that transcript levels in Prochlorococcus cells are likely to be very noisy, but that noise is strongly damped at the protein level due to slow translation. Slow translation is concomitant with slow growth, and we propose a hypothesis for growth limitation of small cells by the need to limit the metabolic and phenotypic influence of stochastic gene expression.

There is a large gulf in microbial biogeochemistry between the rapidly-expanding inventory of microbial diversity and knowledge of the molecular composition of microbial communities. A very few organisms, notably E. coli and S. cerevisiae, have been the subject of extensive quantitative investigation. While these lab models are of limited environmental relevance in themselves, they have provided essential data on the molecular organization of universal biological structures, such as ribosomes and membranes. Armed with such parameters, and with some basic information about cell size and elemental composition - and a sequenced genome - one can actually constrain the molecular makeup of any microorganism to a reasonably informative degree. For a cultivable organism like Prochlorococcus, more physiological and biochemical data are available, enabling greater refinement of a cell composition model. Any natural microbial population is comprised of a wide variety of organisms, ranging in characterization from undiscovered to cultivated, and in-depth investigation of the properties of each and every relevant microbial type is unfeasible. A more viable goal is to build conceptual framework to 'bootstrap' the information about the composition of 
better-characterized organisms into inferences about less well-known members of the community.

Ultimately, we would like to be able to use data from high-throughput, cultureindependent techniques as inputs to biogeochemical models of microbial communities. Such techniques are already available to measure some aspects of cellular composition: flow cytometry, for example, can count cells and detect a variety of pigments, and with the right sort of dye or probe can assay for a huge range of properties, and can examine thousands of cells per second. Nucleic acid sequence information, of course, is now a readily observed property of any microbial system, and can even be obtained from single, uncultivated cells (Rodrigue and Malmstrom et al., 2009). Chemical imaging methods such as SIMS (secondary ion mass spectrometry) and X-ray microanalysis are increasingly used to examine the makeup of microbial cells, and high-speed cell sorting and microfluidic devices promise new ways of preparing environmental samples for molecular analysis. The real benefit of a compositional view of cells will come in integrating information from these disparate technologies, identifying key unknowns for further investigation, and feeding into process models of microbial activity and its biogeochemical consequences. Biogeochemistry that is informed by systems biology will undoubtedly afford deeper insights into the structure, function and coevolution of earth's life and environments.

\section{ACKNOWLEDGEMENTS}

We are grateful to Maureen Coleman for discussions of genome evolution and expression dynamics and for analysis of amino acid utilization patterns, and to Jason Bragg and Chris Kempes for discussions of interactions between organismal stoichiometry, metabolism and growth. This work was supported by grants from the Department of Energy Genomics:GtL program, the NSF Center for Microbial Oceanography: Research and Education, and the Gordon and Betty Moore Foundation to SWC, and by an Office of Naval Research NDSEG Fellowship and an NSF Graduate Research Fellowship to JRW. 


\section{REFERENCES}

Acar, M, JT Mettetal, and A van Oudenaarden. 2008. Stochastic switching as a survival strategy in fluctuating environments. Nat Genet 40, no. 4: 471-475.

Ammerman, JW, RR Hood, DA Case, and JB Cotner. 2003. Phosphorus deficiency in the Atlantic: an emerging paradigm in oceanography. Eos, Transactions American Geophysical Union 84, no. 18: 165.

Bennett, BD, EH Kimball, M Gao, R Osterhout, SJV Dien, and JD Rabinowitz. 2009. Absolute metabolite concentrations and implied enzyme active site occupancy in Escherichia coli. Nat Chem Biol 5, no. 8: 593-599.

Bertilsson, S, O Berglund, DM Karl, and SW Chisholm. 2003. Elemental Composition of Marine Prochlorococcus and Synechococcus: Lmplications for the Ecological Stoichiometry of the Sea. Limnology and Oceanography 1721-1731.

Bragg, JG, and A Wagner. 2009. Protein material costs: single atoms can make an evolutionary difference. Trends Genet 25, no. 1: 5-8.

Bremer, H., and P.P. Dennis. 2008. Modulation of chemical composition and other parameters of the cell at different exponential growth rates. In EcoSal -Escherichia coli and Salmonella typhimurium: Cellular and Molecular Biology, eds. A. Böck, R. Curtiss III, J. B. Kaper, P. D. Karp, F. C. Neidhardt, T. Nyström, J. M. Slauch, C. L. Squires, and D. Ussery, Washington, D.C.: ASM Press.

Brown, JH, JF Gillooly, AP Allen, VM Savage, and GB West. 2004. Toward a metabolic theory of ecology. Ecology 85, no. 7: 1771-1789.

Claustre, H, A Bricaud, M Babin, F Bruyant, L Guillou, FL Gall, D Marie, and F Partensky. 2002. Diel variations in Prochlorococcus optical properties. Limnology and Oceanography 1637-1647.

Coleman, ML, and SW Chisholm. 2007. Code and context: Prochlorococcus as a model for cross-scale biology. Trends Microbiol 15, no. 9: 398-407.

Coleman, ML, MB Sullivan, AC Martiny, C Steglich, K Barry, EF Delong, and SW Chisholm. 2006. Genomic islands and the ecology and evolution of Prochlorococcus. Science 311, no. 5768: 1768-1770.

Costello, JC and SW Chisholm. 1981. The influence of cell size on the growth rate of Thalassiosira weissflogii. Journal of Plankton Research 3, 415-419.

Cronan, J.E., and C.O. Rock. 2008. Biosynthesis of membrane lipids. In EcoSal -Escherichia coli and Salmonella typhimurium: Cellular and Molecular Biology, eds. A. Böck, R. Curtiss III, J. B. Kaper, P. D. Karp, F. C. Neidhardt, T. Nyström, J. M. Slauch, C. L. Squires, and D. Ussery, Washington, D.C.: ASM Press.

Dagan, T, R Blekhman, and D Graur. 2006. The" domino theory" of gene death: gradual and mass gene extinction events in three lineages of obligate symbiotic bacterial pathogens. Molecular biology and evolution 23, no. 2: 310.

Dufresne, A, L Garczarek, and F Partensky. 2005. Accelerated evolution associated with genome reduction in a free-living prokaryote. Genome Biol 6, no. 2:

Falkowski, P.G., and J.A. Raven. 2007. Aquatic Photosynthesis. Princeton, NJ: Princeton University Press. 
Finkel, ZV, J Beardall, KJ Flynn, A Quigg, TAV Rees, and JA Raven. 2009.

Phytoplankton in a changing world: cell size and elemental stoichiometry. Journal of Plankton Research 1-19.

Fraser, C, WP Hanage, and BG Spratt. 2007. Recombination and the nature of bacterial speciation. Science 315, no. 5811: 476.

Fu, FX, ME Warner, Y Zhang, Y Feng, and DA Hutchins. 2007. Effects of increased temperature and $\mathrm{CO} 2$ on photosynthesis, growth, and elemental ratios in marine Synechococcus and Prochlorococcus (cyanobacteria). Journal of Phycology 43, no. 3: 485-496.

Giovannoni, SJ, HJ Tripp, S Givan, M Podar, KL Vergin, D Baptista, L Bibbs, J Eads, TH Richardson, M Noordewier, MS Rappe, JM Short, JC Carrington, and EJ Mathur. 2005. Genome streamlining in a cosmopolitan oceanic bacterium. Science 309, no. 5738: 1242-1245.

Golding, I, and EC Cox. 2006. Physical nature of bacterial cytoplasm. Physical review letters 96, no. 9: 98102.

Heldal, M, DJ Scanlan, S Norl, TF Thingstad, and NH Mann. 2003. Elemental composition of single cells of various strains of marine Prochlorococcus and Synechococcus using X-ray microanalysis. Limnol Oceanogr 48, no. 5: 1732 1743.

Kærn, M, TC Elston, WJ Blake, and JJ Collins. 2005. Stochasticity in gene expression: from theories to phenotypes. Nature Reviews Genetics 6, no. 6: 451-464.

Kaufmann, BB, and A van Oudenaarden. 2007. Stochastic gene expression: from single molecules to the proteome. Curr Opin Genet Dev 17, no. 2: 107-112.

Kettler, G, AC Martiny, K Huang, J Zucker, ML Coleman, S Rodrigue, F Chen, A Lapidus, S Ferriera, J Johnson, C Steglich, GM Church, PM Richardson, and SW Chisholm. 2007. Patterns and Implications of Gene Gain and Loss in the Evolution of Prochlorococcus. PLoS Genet 3, no. 12: e231.

Klähn, S, C Steglich, WR Hess, and M Hagemann. 2009. Glucosylglycerate: a secondary compatible solute common to marine cyanobacteria from nitrogen-poor environments. Environmental Microbiology

Larson, D, R Singer, and D Zenklusen. 2009. A single molecule view of gene expression. Trends in Cell Biology

Longo, D, and J Hasty. 2006. Dynamics of single-cell gene expression. Mol Syst Biol 2, 64.

Lopez Cascales, JJ, JGdl Torre, SJ Marrink, and HJC Berendsen. 1996. Molecular dynamics simulation of a charged biological membrane. J. Chem. Phys 104, no. 7:

Losick, R, and C Desplan. 2008. Stochasticity and cell fate. Science 320, no. 5872: 6568.

Lynch, M. 2006. Streamlining and simplification of microbial genome architecture. Annu Rev Microbiol 60, 327-349.

Martin, P, and R Macleod. 1984. Observations on the Distinction Between Oligotrophic and Eutrophic Marine Bacteria. Appl Environ Microbiol 47, no. 5: 1017-1022. 
Martiny, AC, ML Coleman, and SW Chisholm. 2006. Phosphate acquisition genes in Prochlorococcus ecotypes: evidence for genome-wide adaptation. PNAS 103, no. 33: 12552-12557.

Mitchell, JG, H Yamazaki, L Seuront, F Wolk, and H Li. 2008. Phytoplankton patch patterns: Seascape anatomy in a turbulent ocean. Journal of Marine Systems 69, no. 3-4: 247-253.

Moore, LR, and SW Chisholm. 1999. Photophysiology of the marine cyanobacterium Prochlorococcus: ecotypic differences among cultured isolates. Limnology and Oceanography 628-638.

Morel, A, YH Ahn, F Partensky, D Vaulot, and H Claustre. 1993. Prochlorococcus and Synechococcus: A comparative study of their optical properties in relation to their size and pigmentation. Journal of Marine Research 51, no. 3: 617-649.

Newman, JRS, S Ghaemmaghami, J Ihmels, DK Breslow, M Noble, JL DeRisi and JS Weissman. 2006. Single-cell proteomic analysis of $S$. cerevisiae reveals the architecture of biological noise. Nature 441: 840-846.

Nikaido, H. 1996. Outer Membrane. In Escherichia coli and Salmonella typhimurium: Cellular and Molecular Biology, ed. F. C. Neidhardt, Washington, D.C.: ASM Press.

Partensky, F, and L Garczarek. 2010. Prochlorococcus: Advantages and Limits of Minimalism. Annual Review of Marine Science 2, 211-237.

Partensky, F, WR Hess, and D Vaulot. 1999. Prochlorococcus, a marine photosynthetic prokaryote of global significance. Microbiol Mol Biol Rev 63, no. 1: 106-127.

Pedraza, JM, and J Paulsson. 2008. Effects of molecular memory and bursting on fluctuations in gene expression. Science 319, no. 5861: 339-343.

Raj, A, and A van Oudenaarden. 2008. Nature, nurture, or chance: stochastic gene expression and its consequences. Cell 135, no. 2: 216-226.

Rodrigue, S, RR Malmstrom, AM Berlin, BW Birren, MR Henn, and SW Chisholm. 2009. Whole genome amplification and de novo assembly of single bacterial cells. PLoS ONE 4, no. 9: e6864.

Seymour, JR, JG Mitchell, and L Seuront. 2004. Microscale heterogeneity in the activity of coastal bacterioplankton communities. Aquatic Microbial Ecology 35, no. 1: 116.

Shahrezaei, V, and PS Swain. 2008. Analytical distributions for stochastic gene expression. Proc Natl Acad Sci USA 105, no. 45: 17256-17261.

Snyder, DS, B Brahamsha, P Azadi, and B Palenik. 2009. Structure of Compositionally Simple Lipopolysaccharide from Marine Synechococcus. J Bacteriol 191, no. 17: 5499-5509.

Thingstad, TF, MD Krom, RFC Mantoura, GAF Flaten, S Groom, B Herut, N Kress, CS Law, A Pasternak, P Pitta, S Psarra, F Rassoulzadegan, T Tanaka, A Tselepides, P Wassmann, EMS Woodward, CW Riser, G Zodiatis, and T Zohary. 2005. Nature of phosphorus limitation in the ultraoligotrophic eastern Mediterranean. Science 309, no. 5737: 1068-1071.

Ting, CS, C Hsieh, S Sundararaman, C Mannella, and M Marko. 2007. Cryo-Electron Tomography Reveals the Comparative Three-Dimensional Architecture of 
Prochlorococcus, a Globally Important Marine Cyanobacterium. J Bacteriol 189, no. 12: 4485-4493.

Tolonen, AC, GB Liszt and WR Hess. 2006. Genetic Manipulation of Prochlorococcus Strain MIT9313: Green Fluorescent Protein Expression from an RSF1010 Plasmid and Tn5 Transposition. Appl Env Microbiol 72, no. 12: 7607-7613.

Van Mooy, BAS, G Rocap, HF Fredricks, CT Evans, and AH Devol. 2006. Sulfolipids dramatically decrease phosphorus demand by picocyanobacteria in oligotrophic marine environments. PNAS 103, no. 23: 8607-8612.

Van Mooy, BAS, H Fredricks, B Pedler, S Dyhrman, D Karl, M Koblizek, M Lomas, T Mincer, L Moore, T Moutin, M Rappe, and E Webb. 2009. Phytoplankton in the ocean use non-phosphorus lipids in response to phosphorus scarcity. Nature

Veldhuis, MJW, KR Timmermans, P Croot, and Bvd Wagt. 2005. Picophytoplankton; a comparative study of their biochemical composition and photosynthetic properties. Journal of Sea Research 53, no. 1-2: 7-24.

Vollmer, W, and J-V Holtje. 2004. The architecture of the murein (peptidoglycan) in gram-negative bacteria: vertical scaffold or horizontal layer(s)? J Bacteriol 186, no. 18: 5978-5987.

Wang, JD, and PA Levin. 2009. Metabolism, cell growth and the bacterial cell cycle. Nat Rev Microbiol 7, no. 11: 822-827.

Wilhelm, LJ, HJ Tripp, S Givan, D Smith, and SJ Giovannoni. 2007. Natural variation in SAR11 marine bacterioplankton genomes inferred from metagenomic data. Biol Direct 2, no. 1: 27.

Zinser, ER, D Lindell, ZI Johnson, ME Futschik, C Steglich, ML Coleman, MA Wright, T Rector, R Steen, N McNulty, LR Thompson, and SW Chisholm. 2009. Choreography of the transcriptome, photophysiology, and cell cycle of a minimal photoautotroph, Prochlorococcus. PLoS ONE 4, no. 4: e5135. 


\section{Chapter Six}

Conclusions and Future Directions 
The work presented in this thesis has sought to contribute to understanding of how, when and why biological activity, especially primary production by oxygenic photoautotrophs, has driven the secular oxidation of earth's surface environment. Using a variety of molecular tools and examining processes at timescales spanning hours to billions of years, the goal of these studies has been to provide new insights into the biogeochemistry of marine microbes, both as documented in the rock record from the distant past and visible in the activities of living organisms. The principal findings include:

- The molecular fossil record bears clear evidence of a diverse microbial community in the oceans by at least 2.7 billion years ago, including members of all three domains of life.

- The presence of biomarkers for aerobic biochemistry long before the oxygenation of the atmosphere is fully consistent with their production in persistently microaerobic regions of the surface ocean.

- The diel cell cycle of Prochlorococcus and associated shifts in metabolic fluxes are underlain by small variations in a proteome whose composition is generally stable, despite strong oscillations in expression at the transcript level.

- The relatively simple cellular and biochemical architecture of Prochlorococcus enables a compositional budget to be constructed of the sort that can inform biogeochemical models, as well as guide inferences about the ecological consequences of genome streamlining and stochastic gene expression in small cells. 


\section{FUTURE DIRECTIONS: THE MOLECULAR RECORD OF PRIMARY PRODUCTION AND OXIDATION}

The work presented here has helped to place the late Archean biomarker record on firmer footing, but much remains to be learned about the molecular fossils contained in the vast and still-growing archive of material recovered by the Agouron drilling project and other scientific drilling initiatives. In particular, it would be helpful to characterize and identify the controls on finer spatial-scale variability in biomarker compositions, and map correlations with other geochemical proxies in greater detail. While the low extractable hydrocarbon contents of these overmature rocks make high-resolution measurements quite challenging, an understanding of such distributions would both lend greater confidence to the molecular fossils' syngeneity and likely provide new insights into the ecological and biogeochemical structure of the microbial community.

The late Archean Hamersley and Transvaal basins, on which much Precambrian biomarker work has focused, are essentially the end of the line: no older sedimentary rocks of equivalently low metamorphic grade (such that biogeochemically-informative molecular fossils are likely to have been preserved) have yet been found. Pushing the molecular fossil record deeper into the Archean will be a considerable challenge, and likely contingent on both methodological advances and new subsurface sampling efforts. But, having jumped as far back as possible, it may be fruitful to turn back towards the Proterozoic, during which time both the redox structure of the atmosphere and oceans and the ecology of primary producers underwent repeated reorganizations. Establishing a more global, continuous molecular fossil record for the Paleo- and Mesoproterozoic will help to clarify the biogeochemical and evolutionary transitions involved in the long journey from the late Archean to the dawn of the modern Phanerozoic earth system. 


\section{FUTURE DIRECTIONS: MOLECULAR NETWORKS IN BIOGEOCHEMISTRY}

It is clear that the tools of systems biology can open new windows on biogeochemistry; the two fields share a desire to unpack the interlocking, nested, overlapping molecular interactions in complex networks while keeping sight of how those networks produce first-order emergent phenomena like cell growth and net primary production. Likewise, biogeochemistry can offer systems biology a framework for understanding the real environmental conditions that biological systems respond to, where all variables are continuous rather than binary. Much fruitful cross-fertilization seems possible between the two fields. For instance, an integrative transcriptomic/proteomic/metabolomic/ biochemical/regulatory investigation of central carbon metabolism in Prochlorococcus would illustrate the systems-level control of a key physiological process. A notinsubstantial portion of marine primary production flows through this network, so a more in-depth understanding of its functioning would also yield biogeochemical and oceanographic insights.

The development of novel assays for biogeochemically-significant processes that can be applied to environmental samples is one of the most challenging yet exciting prospects of this interaction. Taking the tools of systems biology outside would enable a far more direct view of both the molecular bases of biogeochemistry and of the full range of adaptive strategies employed by life's diversity in the face of myriad environmental pressures that cannot be simulated in the laboratory. The challenges lie in the molecular complexity of natural materials, where analytes of interest are often a small minority of a vast number of potentially confounding molecular species. Such technological obstacles are, to one degree or another, surmountable. The promise of a deeper, richer understanding of the functioning of earth system, its evolution and our impacts on it, is too valuable to pass up. 


\title{
Appendix A
}

\section{Use of stable isotope labeled cells to identify active grazers of picocyanobacteria in ocean surface waters}

\author{
Jorge Frias-Lopez, Anne W. Thompson, Jacob R. Waldbauer and
} Sallie W. Chisholm

Reprinted with permission from Environmental Microbiology

(C) 2008 The authors

Frias-Lopez, J., Thompson, A.W., Waldbauer, J.R. and Chisholm, S.W. (2009) Use of stable isotope labeled cells to identify active grazers of picocyanobacteria in ocean surface waters. Environmental Microbiology 11: 512-525. 


\title{
Use of stable isotope-labelled cells to identify active grazers of picocyanobacteria in ocean surface waters
}

\author{
OnlineOpen: This article is available free online at www.blackwell-synergy.com
}

Jorge Frias-Lopez, ${ }^{1 *}$ Anne Thompson, ${ }^{1,2}$ Jacob Waldbauer ${ }^{1,3}$ and Sallie W. Chisholm ${ }^{1}$

${ }^{1}$ Department of Civil and Environmental Engineering,

Massachusetts Institute of Technology, 15 Vassar

Street, Cambridge, MA 02139, USA.

${ }^{2}$ MIT/Woods Hole Joint Program in Biological

Oceanography, Cambridge, MA 02139, USA.

${ }^{3}$ MIT/Woods Hole Joint Program in Chemical

Oceanography, Cambridge, MA 02139, USA.

\section{Summary}

Prochlorococcus and Synechococcus are the two most abundant marine cyanobacteria. They represent a significant fraction of the total primary production of the world oceans and comprise a major fraction of the prey biomass available to phagotrophic protists. Despite relatively rapid growth rates, picocyanobacterial cell densities in open-ocean surface waters remain fairly constant, implying steady mortality due to viral infection and consumption by predators. There have been several studies on grazing by specific protists on Prochlorococcus and Synechococcus in culture, and of cell loss rates due to overall grazing in the field. However, the specific sources of mortality of these primary producers in the wild remain unknown. Here, we use a modification of the RNA stable isotope probing technique (RNA-SIP), which involves adding labelled cells to natural seawater, to identify active predators that are specifically consuming Prochlorococcus and Synechococcus in the surface waters of the Pacific Ocean. Four major groups were identified as having their 18S rRNA highly labelled: Prymnesiophyceae (Haptophyta), Dictyochophyceae (Stramenopiles), Bolidomonas (Stramenopiles) and Dinoflagellata (Alveolata). For the first three of these, the closest relative of the sequences identified was a photosynthetic organism, indicating the presence of mixotrophs among picocyanobacterial predators. We conclude that the use of RNA-SIP is

Received 7 May, 2008; accepted 13 September, 2008. *For correspondence. E-mail jfrias@mit.edu; Tel. (+1) 617253 1771; Fax (+1) 6173240336 .

Re-use of this article is permitted in accordance with the Creative Commons Deed, Attribution 2.5, which does not permit commercial exploitation. a useful method to identity specific predators for picocyanobacteria in situ, and that the method could possibly be used to identify other bacterial predators important in the microbial food-web.

\section{Introduction}

The mechanisms that regulate microbial communities are a central issue in ocean ecology. Phagotrophic protists and viruses are the main sources of mortality for these microbes in oligotrophic environments (Fuhrman and Campbell, 1998; Partensky et al., 1999a) and play an important role in shaping microbial communities in the ocean (so-called 'top-down' regulation) (Sherr and Sherr, 2002; Pernthaler, 2005). One of the outstanding questions is precisely how the food-web is structured: which protists eat which microbes?

Grazing activity by eukaryotes is a major factor of bacterial mortality in the ocean and a major force for shaping microbial communities in those environments (Jurgens and Matz, 2002). Heterotrophic nanoflagellates and ciliates are considered to be the primary grazers on planktonic marine bacteria (Sherr et al., 1989; Simek and Chrzanowski, 1992; Cho et al., 2000; Sherr and Sherr, 2002). In general, grazing by bacterivorous protists upon suspended bacteria is size selective (Chrzanowski and Símek, 1990; Gonzalez et al., 1990; Simek and Chrzanowski, 1992; Jürgens and Güde, 1994; Anderson and Rivkin, 2001) with most protists grazing preferentially on medium-sized bacterial cells.

Because Prochlorococcus and Synechococcus numerically dominate the oxygenic phototrophs in ocean waters (Chisholm et al., 1988; Partensky et al., 1999a,b), understanding their sources of mortality is central to understanding the structure of the microbial food-web, and the regulation of marine productivity and nutrient cycling in the ocean. Laboratory studies using cultured heterotrophic flagellates and ciliates have shown that they can survive when fed Prochlorococcus and Synechococcus (Christaki et al., 1999; Guillou et al., 2001) and that some feed preferentially on one or the other (Christaki et al., 1999; Guillou et al., 2001). Studies using natural nanoflagellate populations show that the nanoflagellate community composition shapes the picoautotrophic community structure and, vice versa, the picoautotrophic

(C) 2008 The Authors

Journal compilation @ 2008 Society for Applied Microbiology and Blackwell Publishing Ltd 
community structure favours or inhibits the growth of some nanoflagellates groups (Christaki et al., 2005). However, these studies do not address the question of the identity of the grazers feeding on bacteria.

While rates of grazing-induced mortality of picocyanobacteria have been measured in situ (Sherr et al., 1987; Hall et al., 1993; Ishii et al., 2002; Massana et al., 2002; Worden and Binder, 2003; An-Yi et al., 2007), the specific identity of the grazers feeding on these cells has not been studied. In the present work, we have used a modification of a RNA stable isotope probing technique (RNA-SIP) (Radajewski et al., 2000; Manefield et al., 2002; Lueders et al., 2004) to identify eukaryotic cells that consume Prochlorococcus and Synechococcus in surface waters at the Hawaii Ocean Time Series (HOT) station ALOHA. A similar approach had been previously used to identify micropredators of Escherichia coli in a sample of agricultural soil (Lueders et al., 2006). The use of this method avoids problems associated with using non-active bacteria (González et al., 1990; Landry et al., 1991; del Giorgio et al., 1996; Ishii et al., 2002; Koton-Czaarnecka and Chrost, 2003), and enables molecular taxonomic resolution.

\section{Results and discussion}

\section{Characterization of the indigenous eukaryotic protist} community

We first characterized the diversity of protists in our sample, collected from the study site, Station ALOHA (Hawaii Ocean Time Series) through the analysis of the indigenous 18S rDNA sequences (Figs $1 \mathrm{~A}$ and 2 and Fig. S1). The community was similar to those reported for other oligotrophic surface ocean waters (Countway et al., 2005; 2007; Not et al., 2007), in terms of firstand second-rank marine protistan and Super-group taxa defined by Adl and colleagues (2005). Alveolates, and specifically Dinozoa, including novel Alveolate groups I and II (NAI and NAII), are among the most abundant sequences found. Stramenopiles, including novel Marine Stramenopiles (MAST), are also well represented (Figs $1 \mathrm{~A}$ and 2 and Fig. S1).

\section{Incubation experiments with labelled cultures}

To determine which protists from this community most actively grazed on Prochlorococcus and Synechococcus, ${ }^{13} \mathrm{C}$ - and ${ }^{15} \mathrm{~N}$-labelled cultures of these cyanobacteria were added to seawater samples and incubated for 1 day, allowing the indigenous community to consume the labelled cells (see Experimental procedures for details). After $24 \mathrm{~h}$, the microbial community was collected by filtration, RNA was extracted, and 'heavy' (labelled) and 'light' (unlabelled) RNA was separated by density gradient ultracentrifugation. Density-resolved 18S rRNA sequences were amplified, sequenced and analysed. Sequences from the labelled subfraction (which are enriched in a subset of sequences as they are physically separated from the bulk community before sequencing) are interpreted as being derived from eukaryotic cells that consumed high numbers of labelled Prochlorococcus or Synechococcus cells during the incubation. Sequences in the unlabelled RNA fraction represent protists that did not graze on the labelled cells during the incubation. Because different levels of RNA labelling are likely to occur depending on what fraction of the diet of a particular grazer consists of Prochlorococcus and Synechococcus, we analysed only the most highly labelled fractions (Fig. 3).

We recognize that there are, theoretically, a number of possible indirect routes for the heavy isotopes to end up in the 18S rRNA. We analysed these possibilities in detail in a separate section below, and conclude that direct grazing on Prochlorococcus and Synechococcus is the most consistent explanation for the incorporation of label into $18 \mathrm{~S}$ rRNA in our experiments.

Community structure analysis using terminal restriction fragment length polymorphism (T-RFLP)

Before analysing the sequences of rRNA from the labelled and unlabelled fractions in detail, we assessed the quality of the biological replicates and general differences and similarities among the treatments, using terminal restriction length polymorphism (T-RFLP) and cluster analysis (GEPAS, http://www.gepas.org) (Dollhopf et al., 2001). The eukaryotic cells at the onset of the experiment (time 0), as well as those that remained unlabelled after a $24 \mathrm{~h}$ incubation (i.e. those that did not prey on either Prochlorococcus or Synechococcus), cluster together in both replicates (Fig. 4). The similarity of these two groups indicates that there were no significant changes in the food-web structure in the incubation bottles during the $24 \mathrm{~h}$ incubation. More importantly, the 18S rRNA sequences containing the Prochlorococcusderived label and Synechococcus-derived label clustered separately from the time 0 and unlabelled rRNA samples, indicating that we are identifying a specific subset of the community that is preying upon these cyanobacteria. Furthermore, the predator sequences originating from addition of Prochlorococcus and Synechococcus did not cluster together, suggesting distinct predators for these two types of cyanobacteria, consistent with observations from laboratory studies (Guillou et al., 2001; Pernthaler, 2005).

(C) 2008 The Authors

Journal compilation (C) 2008 Society for Applied Microbiology and Blackwell Publishing Ltd, Environmental Microbiology, 11, 512-525 


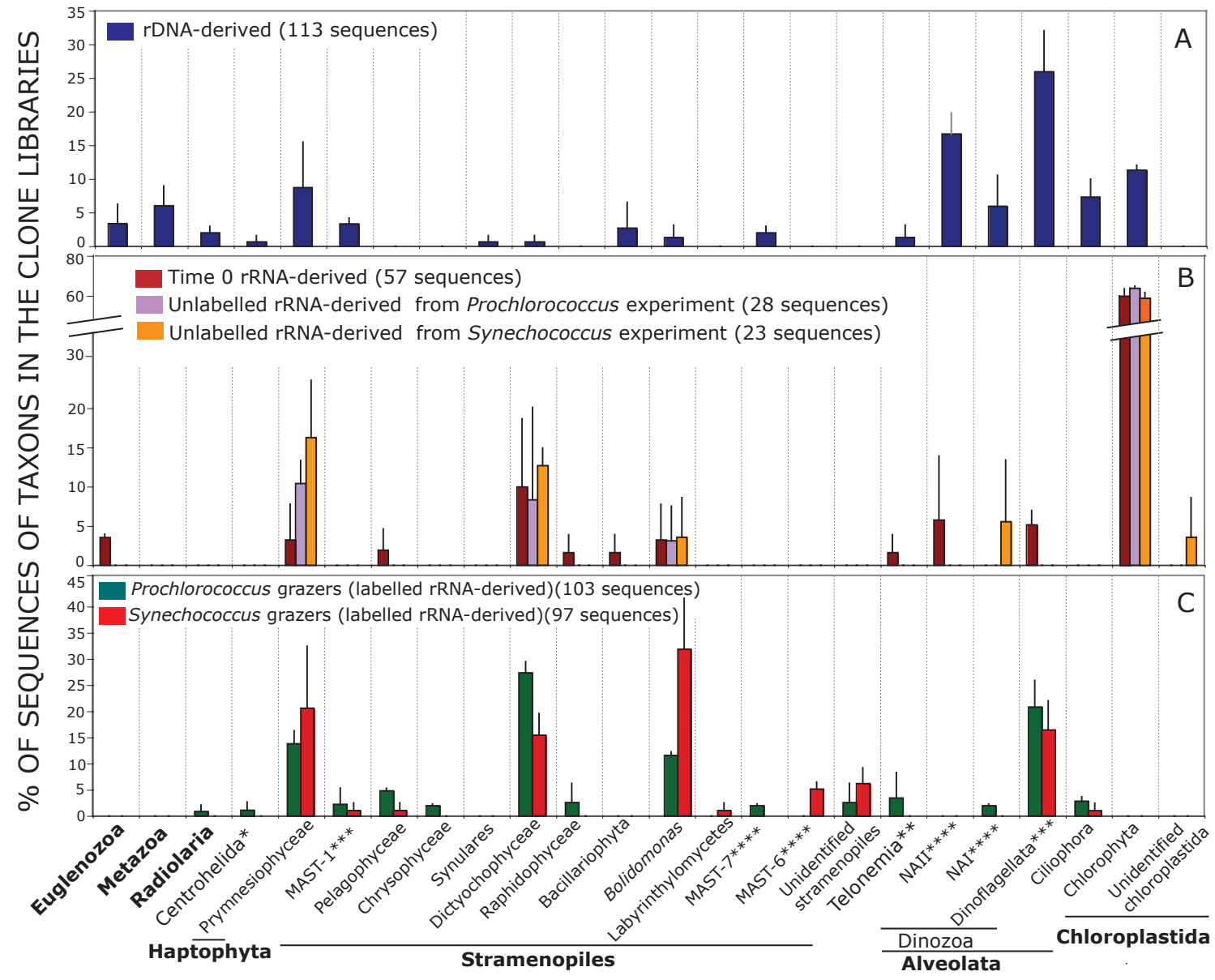

Fig. 1. Phylogenetic assignments and relative frequencies of the rDNA sequences from indigenous eukaryotic community, and the labelled and unlabelled rRNA fractions in the experimental treatments.

A. rDNA extracted from the total community.

B. Unlabelled fractions from the density gradient separations and time 0 samples.

C. Samples with label originating from Prochlorococcus or Synechococcus added to the experimental bottles.

Error bars represent the standard deviation of the values obtained for the biological duplicates of the libraries. Phylogenetic assignment

follows Adl and colleagues (2005) with classification at the first- (in bold) and second-rank taxonomic level except when indicated as follows: *Super-groups, ${ }^{* *}$ Phylum, ${ }^{* * *}$ third-rank taxonomic level and ${ }^{* * * *}$ novel Alveolate groups I and II (NAI and NAll), or the novel MAST following

Not and colleagues (2007).

Fig. 2. Unrooted phylogenetic tree inferred by maximum likelihood (ML) analysis of the reference sequences used in the phylogenetic analysis of the clone libraries presented in this work (see Supporting information). Selected representative clones and colour circles indicate the phylogenetic adscription of the sequences obtained in the different clone libraries. Blue clones and circles: sequences originating from the DNA-derived libraries. Purple clones and circles: sequences originating in the unlabelled fractions from the density gradient separations and time 0 samples. Green clones and circles: sequences originating from the labelled fraction of the Prochlorococcus inoculation experiment. Red clones and circles: sequences originating from the labelled fraction from the Synechococcus inoculation experiment. Partial sequences ranging from a minimum of $604 \mathrm{bp}$ up to $827 \mathrm{bp}$ were used in the alignment. Bootstrap values over $50 \%$ are indicated on the internal branches obtained from Bootstrap values $<50 \%$, which have been omitted. The proportion of invariant sites $(I)$ was 0.214 . The scale bar indicates $5 \%$ divergence. Classification is based on Adl and colleagues (2005) and Not and colleagues (2007). All groups correspond to first and second rank according to Adl and colleagues (2005) except *Super-group and **Phylum (Shalchian-Tabrizi et al., 2006), ***third-rank taxonomic level and ${ }^{* * * *}$ novel Alveolate groups I and II (NAI and NAII), or the novel MAST following Not and colleagues (2007). 


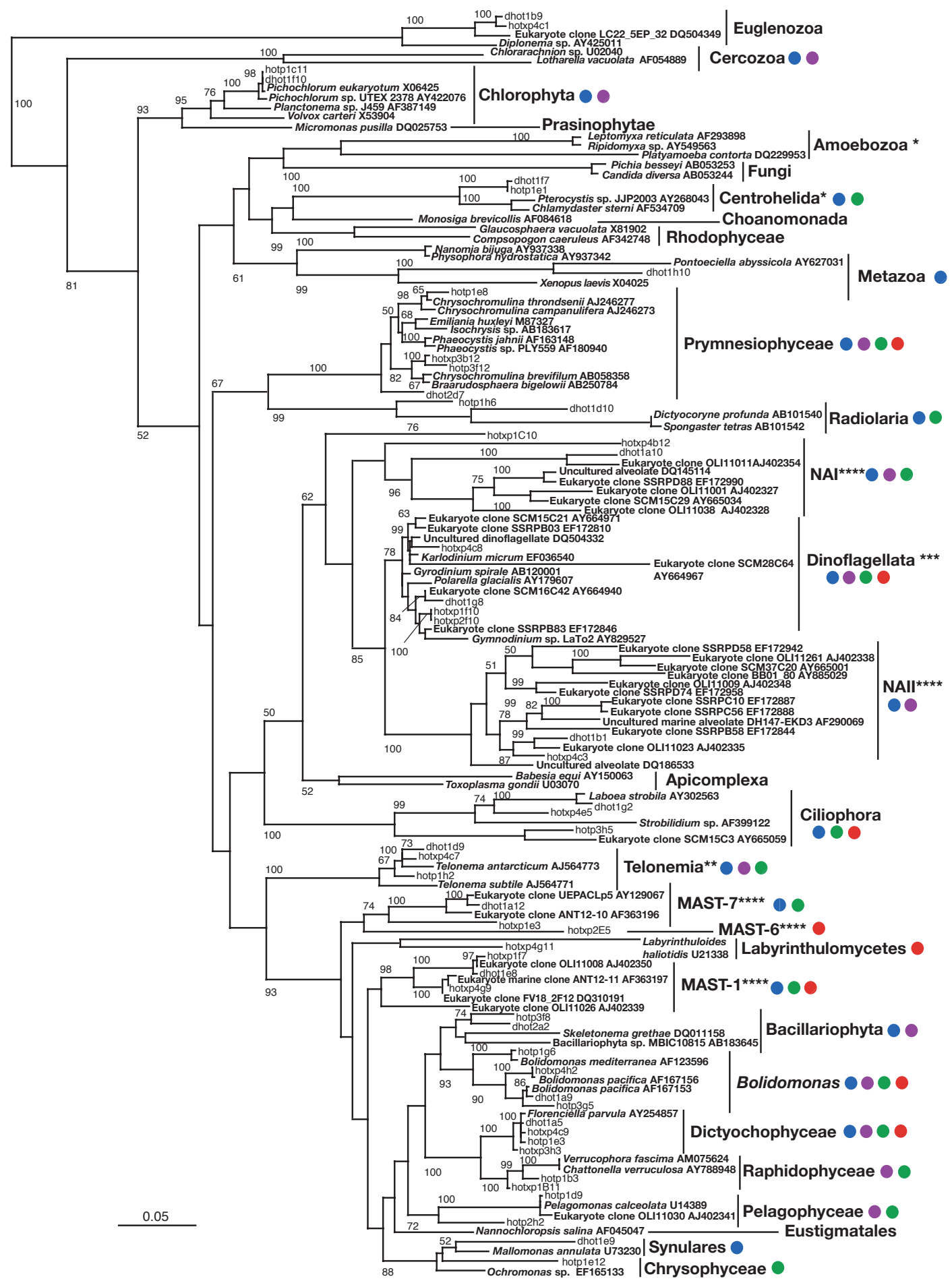

(c) 2008 The Authors

Journal compilation @ 2008 Society for Applied Microbiology and Blackwell Publishing Ltd, Environmental Microbiology, 11, 512-525 


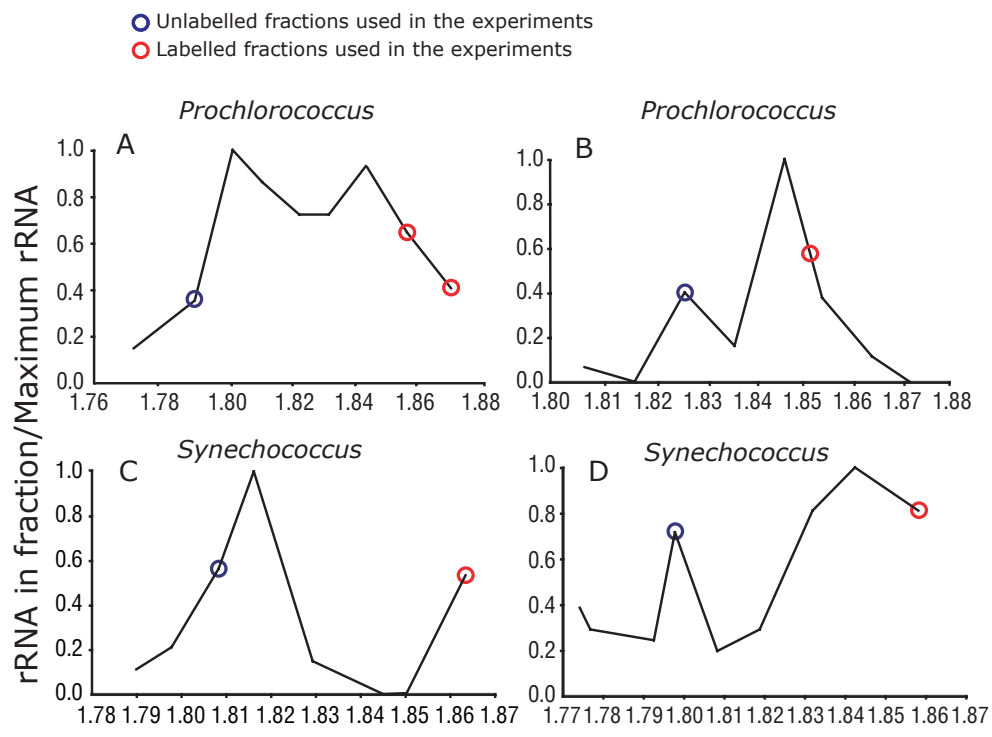

CsTFA buoyant density $\left(\mathrm{g} \mathrm{ml}^{-1}\right)$

Fig. 3. Relative amount of rRNA in different fractions separated by density gradient centrifugation of $18 \mathrm{~S}$ rRNA analysed in this study. Two peaks of RNA were detected in each sample, the lighter containing sequences that did not incorporate the isotopic label $\left({ }^{15} \mathrm{~N}\right.$ and $\left.{ }^{13} \mathrm{C}\right)$ during the $24 \mathrm{~h}$ incubation, and the heavier to the RNA greatly enriched in the heavy isotope, i.e. from cells that incorporated label from the

Prochlorococcus and Synechococcus that were added to the samples. The particular samples indicated in a blue circle were analysed as the 'unlabelled fraction' from the experimental bottles, and those in a red circle the 'heavily labelled' fraction. These particular samples were chosen to maximize the sample size while at the same time avoiding cross-contamination of light and heavy RNA in the subsequent analyses. (A) and (B) represent sequences from biological replicates of samples amended with labelled Prochlorococcus (in A two heavy fractions were used to increase the amount of total RNA used for constructing the clone libraries) and (C) and (D) those amended with Synechococcus. Total RNA was detected fluorometrically using Ribogreen (see Experimental procedures).

A

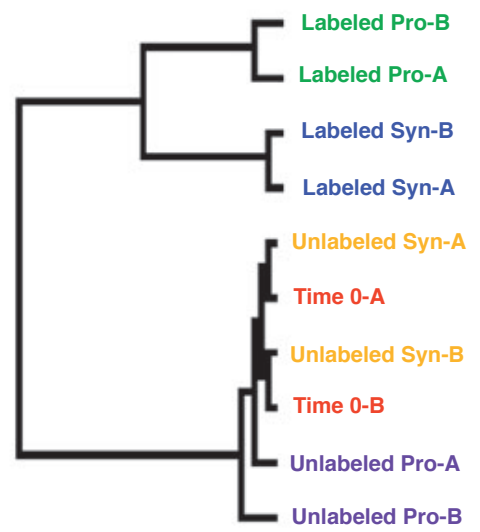

Hhal digestion

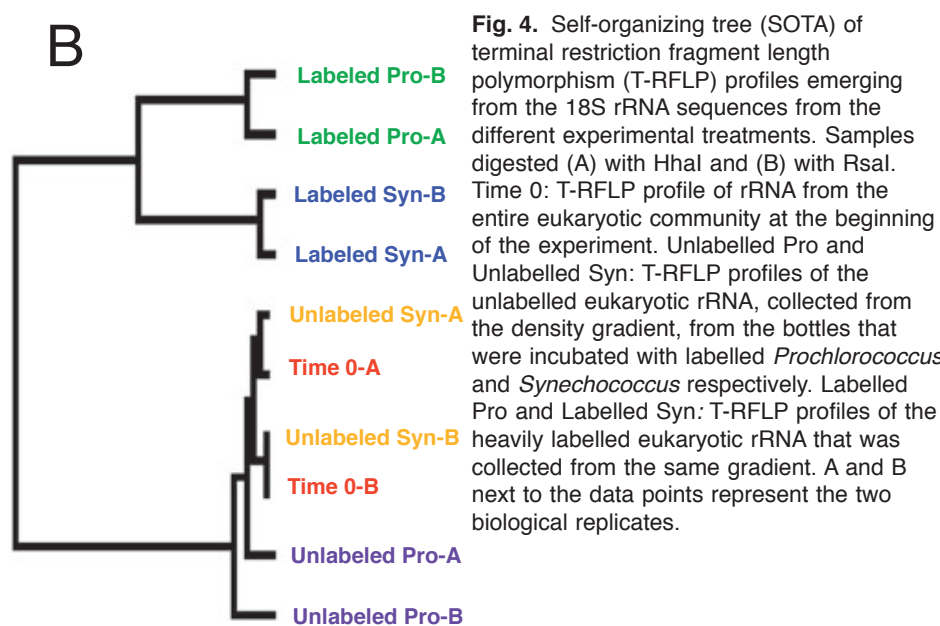

Rsal digestion 
Analysis of the unlabelled and labelled 185 rRNA sequences

We next analysed the identity of the unlabelled and labelled eukaryotes by cloning and sequencing the $18 \mathrm{~S}$ rRNA fragments from the heavily labelled and unlabelled fractions isolated from the density gradient separation (Fig. 3). Heavily labelled fractions represent eukaryotes that have eaten either Prochlorococcus or Synechococcus. The unlabelled fractions represent eukaryotes in the community with relatively high levels of rRNA that did not assimilate label from the cyanobacteria. As has been reported previously (Stoeck et al., 2007) the sequences in the unlabelled rRNA-derived library are substantially different from those in the rDNA library (Figs $1 \mathrm{~A}$ and $\mathrm{B}$ and 2; Figs S1 and S2), showing that there are some members of the community that are much more 'active' (as measured by rRNA levels) than others.

Most sequences obtained in the rRNA-derived library from the time 0 samples and unlabelled fractions represented members of the Chlorophyta, principally close relatives of the genus Picochlorum (Figs $1 \mathrm{~B}$ and 2 and Fig. S2). Other taxonomic groups identified in these libraries included the Dictyochophyceae (Stramenopiles) and Prymnesiophyceae (Haptophyta), and in smaller numbers relatives of members of Raphidophyceae, Bolidomonas, Bacillariophyta, Pelagophyceae (all Stramenopiles), Euglenozoa and Dinozoa (Alveolota) (Figs 1B and 2 and Fig. S2).

The sequences that appeared in the labelled fractions (Figs 1C and 2, Figs S3 and S4) - i.e. from cells grazing on Prochlorococcus and Synechococcus - belonged primarily to four groups: the Prymnesiophyceae, Dictyochophyceae, Bolidomonas and Dinoflagellata. Dictyochophyceae dominated the 18S rRNA sequences that had incorporated label from Prochlorococcus, while Bolidomonas dominated those that had incorporated the label from Synechococcus (Fig. 1C), but it appears that the four dominant grazers consume both types of cells. Novel MAST also appeared in both labelled rRNA-derived libraries and they have been identified as non-pigmented heterotrophic flagellates with bacterivory activity (Massana et al., 2002). Some taxonomic groups appear to be specific to either Prochlorococcus or Synechococcus (Figs $1 \mathrm{C}$ and 2, Figs S3 and S4), but this could be simply due to the small library sample size. Certain groups that were present in the labelled rRNA-derived clone libraries but were absent in the unlabelled rRNA-derived clone libraries could have been simply masked by the high dominance of Chlorophyta in the rRNA-derived clone libraries in relation to the rest of identified phylogenetic groups.

Ciliates, which are considered important grazers in some aquatic environments (Sherr and Sherr, 2002; Pern- thaler, 2005), represent a small fraction of the labelled sequences, which is consistent with recent work showing that subtropical marine ciliates exhibit almost no grazing activity on bacterium-sized particles (An-Yi et al., 2007) and with the experimental results by Christaki and colleagues (1999) showing that Prochlorococcus and Synechococcus proved to be poor food sources for ciliate growth.

The most striking observation in these results is that three of the four most abundant sequences in the labelled 18S rRNA fraction belong to the taxa Prymnesiophyceae, Dictyochophyceae and Bolidomonas, whose characterized members are photosynthetic. Two groups present in the labelled 18S rRNA fraction, the Pelagophyceae and Bolidomonas, have not previously been found to consume bacteria. Some Pelagophyceae feed heterotrophically on dissolved organic matter (Lomas et al., 2001) but this group has previously been described as nonphagotrophic (Cavalier-Smith and Chao, 2006). Characterized members of the Bolidomonas, the most frequently detected labelled group in the Synechococcus-fed samples (Figs 1C and 2 and Fig. S4), are all photosynthetic. While some members of the Dictyochophyceae, which dominate the clone libraries from the labelled Prochlorococcus-fed samples (Figs 1C and 2 and Fig. S3), are heterotrophs, the closest relative to the sequences we have identified is Florenciella parvula, which is photosynthetic. Also among the identified predators of Prochlorococcus and Synechococcus are representatives of groups known to be capable of mixotrophy, including the Chrysophyceae (Nygaard and Tobiesen, 1993), Prymnesiophyceae (Nygaard and Tobiesen, 1993; Hansen and Hjorth, 2002) and Dinoflagellata (Hansen and Nielsen, 1997). Almost all of the sequences from the labelled clone libraries belong to plastid-containing lineages; only sequences identified as relatives of Telonema (phylum Telonemia) (Shalchian-Tabrizi et al., 2006) and Centrohelida come from groups not known to contain autotrophic members.

Previous work had already presented evidence that mixotrophic nanoflagellates are important predators in surface waters and may make up more than $50 \%$ of the bacterivory in them, and that they are more abundant near ocean surface waters than in the deeper euphotic zone (Arenovski et al., 1995; Caron, 2000). Moreover, previous studies have demonstrated that pigmented and nonpigmented nanoflagellates had similar grazing rates on heterotrophic bacteria (Hall et al., 1993).

\section{Detection of label in plastid $16 S$ rRNA}

To further test the mixotrophy hypothesis we examined whether the labelled fraction contained plastid DNA using primers designed specifically for the $16 \mathrm{~S}$ rRNA sequence

(C) 2008 The Authors

Journal compilation (c) 2008 Society for Applied Microbiology and Blackwell Publishing Ltd, Environmental Microbiology, 11, 512-525 


\section{J. Frias-Lopez, A. Thompson, J. Waldbauer and S. W. Chisholm}

in chloroplast DNA (Table S1). We designed these primers specifically to amplify plastid 16S rRNA genes, but not Prochlorococcus and Synechococcus 16S rRNA, since the latter would have dominated our signal. This meant we did not recover as many plastid sequences as we might have if we had used published plastid 16S rRNA primers (Fuller et al., 2006), but this was an unavoidable limitation, given the experimental design.

Two of the primer sets for plastid 16S rRNA (primers sets 6 and 15, Table S1) yielded PCR products of the expected size, and in the case of primer set 15 the product was long enough (approximately $650 \mathrm{bp}$ ) to be sequenced and analysed. Although it is difficult to determine the exact affiliation of these chloroplast sequences given the short length of the amplified PCR product, and the limited coverage of chloroplast sequences from different plastid-containing phylogenetic groups in the database, the phylogenetic analysis showed that the amplified sequences from the labelled fraction were indeed from chloroplasts (Fig. S5). Furthermore, the phylogenetic analysis showed that the closest relatives of the chloroplasts identified in our labelled fraction were related to Bolidomonas mediterranea and diatom chloroplasts (Fig. S5). As there are a limited number of chloroplast sequences representing other groups of Stramenopiles in the databases, and given the short size of the analysed product, the exact phylogenetic affiliation of these sequences is not entirely clear. The key finding, however, is that all of the sequences obtained cluster with chloroplasts indicating that the heavy label ended up in eukaryotic cells capable of photosynthesis.

\section{Analysis of alternative routes for label incorporation}

In this and other types of labelling experiments with natural populations, the possibility that the isotopic label might have been acquired by protists via a route other than phagotrophic predation must be considered. For example, it is conceivable that the label might have passed through a dissolved phase, either organic or inorganic, and was acquired through non-phagotrophic nutrient uptake. Alternatively, the label could have been initially acquired by bacterial heterotrophs that were subsequently grazed by phagotrophs. Below we consider each of these possibilities in turn, and present evidence that they do not appear to be playing a role in these experiments.

The labelled cyanobacterial biomass could have been transformed to dissolved inorganic carbon (DIC) through respiration, either by the picocyanobacteria themselves or by other heterotrophs. Had a substantial amount of the added biomass been respired, that labelled carbon would have become broadly available for fixation by all of the autotrophs in the sample, which would then appear in the labelled fraction. In fact, the most abundant sequences in the unlabelled rRNA-derived clone libraries - the photoautotrophic Chlorophyta - were not represented in the labelled fraction (Figs $1 \mathrm{~B}$ and $\mathrm{C}$ and 2, Figs S2-S4). This demonstrates that no significant quantity of labelled DIC was available for photosynthetic fixation, and passage of the label through the dissolved carbonate pool can be excluded.

Another possibility would be that the initially supplied, isotopically labelled biomass might have entered the dissolved organic carbon (DOC) pool by exudation, lysis or 'sloppy feeding' by zooplankton. The latter two mechanisms would result in substantial declines in the picocyanobacterial population during the experiment; however, the concentration of picocyanobacteria did not change dramatically over the $24 \mathrm{~h}$ of incubation. In all cases the initial and final concentration, after $24 \mathrm{~h}$ of incubation, of both Prochlorococcus and Synechococcus was of $10^{5}$ cells $\mathrm{ml}^{-1}$, suggesting that mechanisms involving cell death (including lysis and sloppy feeding) did not release large amounts of biomass into the dissolved phase. To consider exudation, we can use the Prochlorococcus addition experiment as an example. Prochlorococcus MED4 cells were added to the seawater sample at a concentration of $1.7 ¥ 10^{5}$ per $\mathrm{ml}$ and typically contain about $60 \mathrm{fg}$ of carbon per cell (Bertilsson et al., 2003). If we imagine that the added Prochlorococcus could somehow exude all of their initial labelled carbon as DOC - while suffering no great decline in cell numbers - this is equivalent to the addition of $0.9 \mathrm{mM}$ of ${ }^{13} \mathrm{C}$-DOC, clearly an upper limit for the potential contribution of the isotopically labelled Prochlorococcus to the DOC pool. Typical surface total DOC concentrations at station ALOHA, where the samples for this study were taken, are around $75 \mathrm{mM}$, of which $40 \mathrm{mM}$ is likely refractory organic matter that turns over very slowly (Carlson, 2002). Hence there is roughly $35 \mathrm{mM}$ of labile DOC available for rapid heterotrophic consumption. Addition of Prochlorococcusderived ${ }^{13} \mathrm{C}-\mathrm{DOC}$ to this could result in a $36 \mathrm{mM}$ pool of labile DOC with maximum ${ }^{13} \mathrm{C}$ content of 3.5 atom\%, which is in turn the upper limit for labelling by DOC consumption. Similar considerations limit the ${ }^{13} \mathrm{C}$ content of DOC in the Synechococcus addition experiments to 10.6 atom\%.

Next, we consider the extent of labelling of the heavy RNA fractions in our incubation experiments. The difference in buoyant density between heavy and light RNA fractions in these experiments ranged from 0.034 to $0.078 \mathrm{~g} \mathrm{ml}^{-1}$ (Fig. 3), equal to or exceeding the buoyancy differences $\left(0.035-0.04 \mathrm{~g} \mathrm{ml}^{-1}\right)$ observed by Lueders and colleagues (2004) for $100 \%{ }^{13} \mathrm{C}$-labelled SSU rRNA. This large difference in buoyant density suggests that the heavy fractions analysed in this experiment were highly labelled, likely in excess of 90 atom $\%{ }^{13} \mathrm{C}$. This is far 
greater than the $3-11 \%$ possible from DOC consumption, even under the assumption of maximally rapid exudation by the added cyanobacteria. The buoyancy differences observed here in excess of the $\sim 0.4 \mathrm{~g} \mathrm{ml}^{-1}$ reported by Lueders and colleagues (2004) may reflect ${ }^{15} \mathrm{~N}$ incorporation and/or differences in centrifugation conditions. In any event, the heavy RNA in these experiments is much too highly labelled to derive from heterotrophic consumption of DOC.

A third, even more mechanistically complicated possibility is the direct and specific consumption of picocyanobacteria by heterotrophic bacteria or the consumption of labelled DOC exuded by, or otherwise released from, the picocyanobacteria by those heterotrophs. Protistan predators can then graze on these labelled heterotrophs. If this occurred, the $18 \mathrm{~S}$ sequences observed in the heavy fraction would reflect grazing activity, though not specifically on Prochlorococcus or Synechococcus. Under this scenario, a subset of heterotrophic bacteria would become highly labelled, and their RNA should be found in the heavy fraction. To address this possibility, we constructed 16S rRNA clone libraries as described in Experimental procedures. If there had been transfer of labelled organic matter through heterotrophic bacteria at the level needed to fractionate differentially in a CsTFA gradient we would expect to find 16S rRNA sequences from heterotrophic bacteria. Forty-three clones from the labelled fractions were sequenced. Seventeen clones came from the fraction obtained from the bottles inoculated with labelled Prochlorococcus MED4 and in all cases the best BLASTN match for those sequences corresponded to Prochlorococcus marinus. Similarly, 26 clones coming from the fraction obtained from the bottles inoculated with labelled Synechococcus WH8102 and in all cases the best BLASTN match corresponded to Synechococcus. Additionally, 11 clones coming from the unlabelled fraction from the Prochlorococcus experiment were sequenced and $18 \%$ of those corresponded to P. marinus, while the rest were sequences from heterotrophic bacteria. These results demonstrate that 16S rRNA compositions of the labelled and unlabelled fractions were indeed distinct, and that heterotrophic bacteria did not appear to become highly labelled over the course of the incubation. We thus conclude that the labelled eukaryotes did not obtain their label indirectly via predation of heterotrophic bacteria.

\section{Conclusions and implications}

The reproducibility and internal consistency of the results obtained in the study indicate that the use of RNA-SIP for studying the marine microbial food-webs in situ has tremendous potential. There are a multitude of variations on this experimental design that could yield many insights into the specific pathways of the flow of carbon and energy in the marine food-web. These particular results also reveal that a significant fraction of the eukaryotes that we identified as grazing specifically on Prochlorococcus and Synechococcus were likely mixotrophs - i.e. cells that utilize both phototrophy and phagotrophic heterotrophy as a way of obtaining nutrients and energy (Raven, 1997; Jones, 2000). While a few studies have provided evidence of the importance of mixotrophy in marine aquatic environments (Arenovski et al., 1995; An-Yi et al., 2007; Unrein et al., 2007), this is the first study to identify marine mixotrophs through their grazing activity on specific prey.

The adoption of mixotrophy as a survival strategy under oligotrophic oceanic conditions might confer a fitness advantage for a number of reasons (Raven, 1997). First, phagotrophy may be a way for relatively large eukaryotic cells to acquire inorganic nutrients such as $\mathrm{N}, \mathrm{P}$ and $\mathrm{Fe}$ in oligotrophic waters. Arenovski and colleagues (1995) presented experimental evidence of a decrease in the abundance of mixotrophic phototrophs under nutrient enrichment conditions, suggesting that phagotrophy is used under low dissolved nutrient concentrations, conditions that are normal in surface oligotrophic water. With their larger surface to volume ratio, picocyanobacteria like Prochlorococcus and Synechococcus likely have an advantage over larger eukaryotic cells in acquiring dissolved nutrients. Consuming cyanobacteria may also be a way for the larger cells to increase their relative fitness by reducing the abundance of their competitors for nutrients. Mixotrophy has been linked to survival of nanoflagellates under nutrient limitation (Unrein et al., 2007) and it has been shown that algal flagellates increase bacterivory under phosphate limitation (Nygaard and Tobiesen, 1993). Moreover, the metabolic costs of adding phagotrophic machinery to an otherwise photosynthetic metabolism may be rather low in comparison with the potential benefits (Raven, 1997).

Predation by mixotrophs also has implications for our understanding of the population dynamics of marine picocyanobacteria. While picocyanobacteria are generally the numerically dominant phytoplankton in stratified oligotrophic open-ocean waters, they usually do not bloom (i.e. increase markedly in cell concentrations) in response to episodic nutrient supplies (Mann and Chisholm, 2000). This behaviour has been explained by concomitant increases in grazing rates, implying that these grazers are able to respond very quickly to shifts in prey growth and quality. Our identification of mixotrophic predators may shed further light on this dynamic: eukaryotic mixotrophs directly exploit the same episodic supplies of dissolved nutrients as their picocyanobacterial prey, and thus could grow faster, through stimulated autotrophy, as nutrients become more abundant. As their populations grow and consume the available nutrients, they may shift towards

(C) 2008 The Authors

Journal compilation (c) 2008 Society for Applied Microbiology and Blackwell Publishing Ltd, Environmental Microbiology, 11, 512-525 
phagotrophy, increasing the mortality rate of cyanobacteria, preventing bloom formation even in the face of rapid growth rates. This hypothesis is directly testable using the approach we have described.

As evidence increasingly points towards the mixotrophic capabilities of both nominally photo- and heterotrophic organisms it is becoming clear that a sharp distinction between photosynthetic and predatory lifestyles is a false dichotomy. It is likely that marine protists utilize a spectrum of trophic strategies, ranging between obligate photoautotrophic and strictly phagotrophic end members and occupying nearly all gradations in between (Sanchez-Puerta et al., 2007). Further investigations regarding other ocean sites and different depths are needed to confirm the potential importance of mixotrophy as a common metabolic strategy for grazes feeding on picocyanobacteria.

\section{Experimental procedures}

\section{Sampling and incubation conditions}

Prochlorococcus MED4 and Synechococcus WH8102 were grown for 4 days at $19^{\circ} \mathrm{C}$ under continuous cool white light (16.6 $\mathrm{mmol} \mathrm{Q} \mathrm{m}^{-2} \mathrm{~s}^{-1}$ ) in artificial seawater medium (Rippka et al., 2000) amended with $6 \mathrm{mM}{ }^{13} \mathrm{C}$-sodium bicarbonate and $800 \mathrm{mM}{ }^{15} \mathrm{~N}$-ammonium chloride. Cells were harvested by centrifugation at $8000 \mathrm{~g}$ for $15 \mathrm{~min}$ and washed twice in unlabelled artificial seawater medium and re-suspended in the same medium. Cells were counted by flow cytometry to have an estimate of the volume of inoculum to be used in the experiment, in order to have a final concentration of picocyanobacteria similar to the concentration found in natural samples (approximately $10^{5} \mathrm{cells} \mathrm{ml}^{-1}$ ). Final isotopic enrichment of the cultures was measured by mass spectrometry at UC Davis Stable Isotope Facility using on-line combustion (Europa Integra): atom $\%{ }^{13} \mathrm{C}$ for Prochlorococcus MED4 was $98.86 \%$ and for Synechococcus WH8102 $84.20 \%$ and atom\% ${ }^{15} \mathrm{~N}$ for Prochlorococcus MED4 was $61.13 \%$ and for Synechococcus WH8102 39.83\%. These cultures were then transported overnight in the dark to the field site for use in the grazing experiments.

Samples of ocean surface water (3-5 m depth) were collected in $500 \mathrm{ml}$ acid cleaned bottles during the month of March 2006 as a part of HOT cruise 179, and inoculated with either labelled Prochlorococcus MED4 or Synechococcus WH8102 at a final concentration of $10^{5}$ cells $\mathrm{ml}^{-1}$. All shipboard incubations were performed in duplicate and analysed independently. The incubations were set in an on-deck incubator, which was constantly re-circulated with surface seawater to maintain temperature. Two samples of $200 \mathrm{ml}$ were collected at the beginning of the experiment as a control to identify the initial eukaryotic community. Samples of $250 \mathrm{ml}$ were collected from the bottles with added labelled Prochlorococcus and Synechococcus after $24 \mathrm{~h}$ of incubation. The $24 \mathrm{~h}$ period allowed enough time for the labelled isotopes to be incorporated into the nucleic acids of the grazers yet prevented both significant changes in the eukaryotic community, and potential indirect incorporation of labelled isotopes that could occur during an extended incubation. All water samples were filtered through 0.2 -mm-pore-size membranes and preserved in RNAlater at $-80^{\circ} \mathrm{C}$ until analysis.

\section{DNA and RNA extraction, gradient fractionation and $c D N A$ synthesis}

RNAlater was removed by washing the filters with cold $70 \%$ ethanol. DNA was extracted following Coffroth and colleagues (1992) protocol. Filters were placed in $0.5 \mathrm{ml}$ of CTAB (hexadecyltrimethyl ammonium bromide) buffer (1.4 M $\mathrm{NaCl}, 20 \mathrm{mM}$ EDTA, $100 \mathrm{mM}$ Tris- $\mathrm{HCl}$ pH 8.0, 0.2\% CTAB and $0.2 \% 2$-mercapthoethanol) and the tubes were placed in a mini-bead beater (BioSpec Products, Bartlesville, OK, USA) and vortexed for 2 min at the maximum speed (4800 r.p.m.) to re-suspend the cells. Proteinase $\mathrm{K}$ was added to a final concentration of $0.1 \mathrm{mg} \mathrm{ml}^{-1}$ and samples were incubated at $65^{\circ} \mathrm{C}$ for $1 \mathrm{~h}$. An equal volume of chloroform was added, mixed and spun at $14000 \mathrm{~g}$ for $10 \mathrm{~min}$. The aqueous layer was transferred to a new tube and DNA was extracted with an equal volume of phenol:chloroform: isoamyl alcohol (25:24:1). Finally, DNA was precipitated by addition of 2 vols of cold $95 \%$ ethanol without addition of additional salt. Pellet was washed twice with $70 \%$ cold ethanol dried and re-suspended in water.

For RNA extraction filters were placed in $100 \mathrm{ml}$ of $10 \mathrm{mM}$ Tris- $\mathrm{HCl} \mathrm{pH} \mathrm{8.0,} 4 \mathrm{ml}$ of RNase inhibitor (Ambion, Austin, TX, USA) and $2 \mathrm{ml}$ lysozyme $\left(50 \mathrm{mg} \mathrm{ml}^{-1}\right)$. Samples were incubated for $30 \mathrm{~min}$ at $37^{\circ} \mathrm{C}$. An additional $2 \mathrm{ml}$ of the $50 \mathrm{mg} \mathrm{ml}^{-1}$ lysozyme solution was added and the samples were incubated again for $30 \mathrm{~min}$ at $37^{\circ} \mathrm{C}$. Total RNA was immediately extracted by a mirVana RNA isolation kit (Ambion, Austin, TX, USA).

Labelled and unlabelled RNA were separated by density gradient centrifugation, performed according to the protocol of Lueders and colleagues (2004). Centrifugation media were prepared by mixing $4.5 \mathrm{ml}$ of a $2 \mathrm{~g} \mathrm{ml}^{-1}$ CsTFA stock solution (Amersham Pharmacia Biotech), up to $1 \mathrm{ml}$ of gradient buffer (GB; $0.1 \mathrm{M}$ Tris- $\mathrm{HCl} \mathrm{pH}$ 8; $0.1 \mathrm{M} \mathrm{KCl} ; 1 \mathrm{mM}$ EDTA) and RNA extracts (up to $500 \mathrm{ng}$ ). Additionally, $175 \mathrm{ml}$ of formamide was added to centrifugation media to guarantee that RNA was denatured. The average density of all prepared gradients was checked with an AR200 digital refractometer (Leica Microsystems), and adjusted by adding small volumes of Cs salt solution or gradient buffer, if necessary. 18S rRNA was resolved in CsTFA gradients with an average density of $1.8316 \mathrm{~g} \mathrm{ml}^{-1}$ at $20^{\circ} \mathrm{C}$. Quick-Seal Polyallomer tubes, $3.9 \mathrm{ml}$ (Beckmann Instruments), were filled up with centrifugation media plus sample, and centrifuged in an Optima TLX ultracentrifuge using a TLN100 vertical rotor (Beckmann Instruments). Centrifugation conditions were $>60 \mathrm{~h}$ at 61000 r.p.m. (131000 g).

Centrifuged gradients were fractionated from bottom to top into 12 equal fractions $(\sim 400 \mathrm{ml})$. A precisely controlled flow rate was achieved by displacing the gradient medium with water at the top of the tube using a syringe pump (Harvard Apparatus). The density of $15 \mathrm{ml}$ from each collected fraction was determined using an AR200 digital refractometer (Leica Microsystems). Total RNA was precipitated with 1 vol. of isopropanol. Precipitates from gradient fractions were washed once with $70 \%$ ethanol and re-suspended in $25 \mathrm{ml}$ of EB for Journal compilation (c) 2008 Society for Applied Microbiology and Blackwell Publishing Ltd, Environmental Microbiology, 11, 512-525 
subsequent determination of total RNA using RiboGreen (Molecular Probes, Invitrogen, Carlsbad, CA, USA) assays.

Primers for 18S rRNA eukaryotic genes were designed using the Design Probes tool from the ARB software (Ludwig et al., 2004): EukF (5'-GGGTTCGATTCCGGAGAG-3') EukR (5'-CCGTGTTGAGTCAAATT-3') (Integrated DNA Technologies Coralville, IA, USA). The database used contained 27887 complete sequences, all of eukaryotic origin. EukF primer matched 19378 sequences with 0 mismatches and 23459 sequences with one mismatch. EukR primer matched 25739 sequences with 0 mismatches and 27447 sequences with one mismatch. They were tested in two cultures of Cafeteria, two cultures of Paraphysomonas and one culture of Dullaniella, given in all cases the expected-size PCR product of approximately $830 \mathrm{bp}$.

Total RNA $(0.5-5 \mathrm{ng})$ from fractions containing highly labelled and unlabelled RNA was reverse transcribed with the specific primers using the ThermoScript RT-PCR system (Invitrogen, Carlsbad, CA, USA). Reverse transcription was performed for $2 \mathrm{~h}$ at $50^{\circ} \mathrm{C}$.

PCR reactions were performed using Taq DNA polymerase from NEB and primers at $2 \mathrm{mM}$ concentration. After $5 \mathrm{~min}$ at $95^{\circ} \mathrm{C}, 35$ cycles of denaturation $\left(95^{\circ} \mathrm{C}, 45 \mathrm{~s}\right)$, annealing $\left(52^{\circ} \mathrm{C}, 1 \mathrm{~min}\right)$, elongation $\left(72^{\circ} \mathrm{C}, 1 \mathrm{~min}\right)$ and a final elongation step $\left(72^{\circ} \mathrm{C}, 10 \mathrm{~min}\right)$ were run in a MJ Research PTC 100 Thermal Cycler. PCR products were cleaned up using a QIAquick PCR purification kit (Qiagen, Valencia, CA, USA) and cloned into either TOPO TA cloning vector (Invitrogen, Carlsbad, CA, USA) or pGEM-T cloning vector (Promega, Madison, WI, USA). Inserts were sequenced either at Genaissance Pharmaceuticals (New Haven, CT; now Cogenics, MA, USA) using primers for the T7 promoter region or in house using the same primer and the BigDye sequencing kit (Applied Biosystems, Foster City, CA, USA) at 1 min denaturation and 25 cycles of $95^{\circ} \mathrm{C}-30 \mathrm{~s}, 50^{\circ} \mathrm{C}-20 \mathrm{~s}, 60^{\circ} \mathrm{C}-4 \mathrm{~min}$, and finally held at $4^{\circ} \mathrm{C}$. The reactions were then purified by ethanol precipitation and run on an ABI PRISM 3730 (Applied Biosystems) capillary DNA sequencer.

16S rRNA genes from bacteria present in the heavy fractions were cloned and sequenced using universal primers $9 \mathrm{~F}$ (5'-GAGTTTGATYMTGGCTC) and 1509R (5'-GYTACCTT GTTACGACTT) (Integrated DNA Technologies Coralville, IA, USA). PCR and cloning were performed as described above but elongation at $72^{\circ} \mathrm{C}$ was extended to $2 \mathrm{~min}$. Fragments were sequenced using the ABI PRISM BigDye Terminator v3.1 Cycle Sequencing Kit (Applied Biosystems, Warrington, UK) and primers for the T7 promoter region.

\section{Taxonomic affiliation and phylogenetic analysis}

Vector contamination was assessed using VecScreen (http:// www.ncbi.nlm.nih.gov/VecScreen/VecScreen.html). On the basis of the evaluation by the CHECK_CHIMERA program of the Ribosomal Database Project (Maidak et al., 2001) only sequences that showed no evidence for potential chimeric gene artefacts were analysed.

Preliminary taxonomic affiliation of the sequences was determined using BLASTN against the GenBank nr database (March 2005). Phylogenetic analysis was based on partial sequences trimmed to the shortest common denominator. A first analysis to confirm the taxonomic affiliation of the sequences, and have a raw picture of the overall phylogenetic tree, was performed using ARB software. Sequences were aligned against the eukaryotic database (SSRef release 90 12.05.2007, SILVA database project http://www.arbsilva.de/ with 27887 pre-aligned sequences) (Pruesse et al., 2007) in the ARB software version 07.02.20 (Ludwig et al., 2004) and performed using the Fast Alignment tool. Alignments were edited manually and sequences were added to the backbone tree using ARB's 'Parsimony insertion' feature.

For maximum likelihood (ML), neighbour joining (NJ)distance and maximum parsimony (MP) analyses, alignments were generated using MAFFT (Katoh et al., 2002; 2005) and edited manually using Sequence Alignment Editor v2.0 (http://tree.bio.ed.ac.uk/software/seal/). Maximum parsimony analysis was performed using the 'fast' stepwiseaddition algorithm in PAUP $4.0 \mathrm{~b} 10$ (Altivec) with 1000 bootstraps replicates. For each alignment the best DNA substitution model was evaluated using MrModeltest 2.2 (Nylander, 2004), which ranked General Time Reversiblegamma-Proportion invariant $(\mathrm{GTR}+\mathrm{g}+\mathrm{l})$ best model in all cases. Maximum likelihood analysis was performed using the software PHYML_v2.4.4 (Guindon and Gascuel, 2003) and GTR as a substitution model with 100 bootstraps replicates. Neighbour joining-distance analysis was performed using PAUP 4.0b10 (Altivec) using the also GTR as a substitution model, with 1000 bootstraps replicates, and the values of Gamma-shape and proportion of invariable sites estimated by PHYML. Trees were visualized and plotted using NJPlot v2.1 (Perriere and Gouy, 1996).

\section{T-RFLP analysis}

Fluorescently labelled PCR products for the T-RFLP analysis were generated by the PCR protocol described above, using a FAM-labelled forward primer. PCR products were digested with the restriction endonucleases Hhal and Rsal (New England Biolabs, Ipswich, MA, USA). The resulting fluorescent terminal fragments were resolved and analysed at the Roy J. Carver Biotechnology Center (University of Illinois at UrbanaChampaign) using an ABI Prism 3730xI Analyser automated sequencer, and GeneMapper version 3.7 software.

Clustering of the different T-RFLP profiles was performed using the Self-Organizing Tree Algorithm (SOTA) from the GEPAS 4.0 (GEPAS website http://www.gepas.org).

\section{Chloroplast 16S rRNA analysis}

Labelled fractions from both Prochlorococcus and Synechococcus grazers were tested for the presence of $16 \mathrm{~S}$ rRNA chloroplast sequences. Specific oligonucleotides against chloroplast sequences (SSRef release 90 12.05. 2007, SILVA database project http://www.arb-silva.de/) were design using the Design Probes tool from the ARB software (Ludwig et al., 2004). Although a total of 16 sets of primers were used in the experiment (Table S1), only the set of primers $15 \mathrm{~F}\left(5^{\prime}\right.$ TTAACTCAAGTG GCGGACGG) and 15R (AGTGTTAG TAATAGCCCAGTA) gave a PCR product long enough to be sequenced. PCR reactions were performed using Taq DNA polymerase from NEB and primers at $2 \mathrm{mM}$ concentration. After $5 \mathrm{~min}$ at $95^{\circ} \mathrm{C}, 40$ cycles of denaturation $\left(95^{\circ} \mathrm{C}, 45 \mathrm{~s}\right)$,

(C) 2008 The Authors

Journal compilation (c) 2008 Society for Applied Microbiology and Blackwell Publishing Ltd, Environmental Microbiology, 11, 512-525 
annealing $\left(56^{\circ} \mathrm{C}, 1 \mathrm{~min}\right)$, elongation $\left(72^{\circ} \mathrm{C}, 1 \mathrm{~min}\right)$ and a final elongation step $\left(72^{\circ} \mathrm{C}, 10 \mathrm{~min}\right)$ were run in a MJ Research PTC 100 Thermal Cycler. PCR products were clean up using a QIAquick PCR purification kit (Qiagen, Valencia, CA, USA) and cloned into TOPO TA cloning vector (Invitrogen, Carlsbad, CA, USA) and sequenced as described above.

\section{Nucleotide sequence accession numbers}

Ribosomal RNA sequences have been deposited at GenBank/EMBL under Accession Nos EF695076-EF695247 and EU499951-EU500232.

\section{Acknowledgements}

This research was supported by grants from the National Science Foundation Biological Oceanography Program, the Gordon and Betty Moore Foundation Marine Microbiology Program and the Department of Energy GTL Program. Jacob Waldbauer was supported in part by an NSF Graduate Fellowship. We thank Marcia Osborne for helpful discussions and comments, which improved the manuscript. We also thank Edward F. DeLong for access to the ABI PRISM 3730 (Applied Biosystems) capillary DNA sequencer in his laboratory.

\section{References}

Adl, S.M., Simpson, A.G.B., Farmer, M.A., Andersen, R.A., Anderson, O.R., Barta, J.R., et al. (2005) The new higher level classification of eukaryotes with emphasis on the taxonomy of protists. J Eukaryot Microbiol 52: 399-451.

Anderson, M.R., and Rivkin, R.B. (2001) Seasonal patterns in grazing mortality of bacterioplankton in polar oceans: a bipolar comparison. Aquat Microb Ecol 25: 195-206.

An-Yi, T., Chiang, K.-P., Chan, Y.-F., Lin, Y.-C., and Chang, J. (2007) Pigmented nanoflagellates in the coastal western subtropical Pacific are important grazers on Synechococcus populations. J Plankton Res 29: 71-77.

Arenovski, A.L., Lim, E.L., and Caron, D.A. (1995) Mixotrophic nanoplankton in oligotrophic surface waters of the Sargasso Sea may employ phagotrophy to obtain major nutrients. J Plankton Res 17: 801-820.

Bertilsson, S., Berglund, O., Karl, D.M., and Chisholm, S.W. (2003) Elemental composition of marine Prochlorococcus and Synechococcus: implications for the ecological stoichiometry of the sea. Limnol Oceanogr 48: 1721-1731.

Carlson, C.A. (2002) Production and removal processes. In Biogeochemistry of Marine Dissolved Organic Matter. Hansell, D.A., and Carlson, C.A. (eds). San Diego: Academic Press, pp. 91-151.

Caron, D.A. (2000) Symbiosis and Mixotrophic among Pelagic Microorganism. New York, USA: Wiley-Liss.

Cavalier-Smith, T., and Chao, E.E.Y. (2006) Phylogeny and megasystematics of phagotrophic heterokonts (kingdom Chromista). J Mol Evol 62: 388-420.

Chisholm, S.W., Olson, R.J., Zettler, E.R., Waterbury, J.B., Goericke, R., and Welschmeyer, N. (1988) A novel freeliving prochlorophyte occurs at high cell concentrations in the oceanic euphotic zone. Nature 334: 340-343.
Cho, B.C., Na, S.C., and Choi, D.H. (2000) Active ingestion of fluorescently labeled bacteria by mesopelagic heterotrophic nanoflagellates in the East Sea, Korea. Mar Ecol Prog Ser 206: 23-32.

Christaki, U., Jacquet, S., Dolan, J.R., Vaulot, D., and Rassoulzadegan, F. (1999) Growth and grazing on Prochlorococcus and Synechococcus by two marine ciliates. Limnol Oceanogr 44: 52-61.

Christaki, U., Vazquez-Dominguez, E., Courties, C., and Lebaron, P. (2005) Grazing impact of different heterotrophic nanoflagellates on eukaryotic (Ostreococcus tauri) and prokaryotic picoautotrophs (Prochlorococcus and Synechococcus). Environ Microbiol 7: 1200-1210.

Chrzanowski, T.H., and Símek, K. (1990) Prey-size selection by freshwater fagellated protozoa. Limnol Oceanogr 35: 1424-1436.

Coffroth, M.A., Lasker, H.R., Diamond, M.E., Bruenn, J.A., and Bermingham, E. (1992) DNA fingerprints of a gorgonian coral: a method for detecting clonal structure in a vegetative species. Mar Biol 114: 317-325.

Countway, P.D., Gast, R.J., Savai, P., and Caron, D.A. (2005) Protistan diversity estimates based on 18S rDNA from seawater incubations in the western North Atlantic. $J$ Eukaryot Microbiol 52: 95-106.

Countway, P.D., Gast, R.J., Dennett, M.R., Savai, P., Rose, J.M., and Caron, D.A. (2007) Distinct protistan assemblages characerize the euphotic zone and deep sea $(2500$ $\mathrm{m}$ ) of the western North Atlantic (Sargasso Sea and Gulf Stream). Environ Microbiol 9: 1219-1232.

Dollhopf, S.L., Hashsham, S.A., and Tiedje, J.M. (2001) Interpreting 16S rDNA T-RFLP data: application of selforganizing maps and principal component analysis to describe community dynamics and convergence. Microb Ecol 42: 495-505.

Fuhrman, J.A., and Campbell, L. (1998) Marine ecology microbial microdiversity. Nature 393: 410-411.

Fuller, N.J., Campbell, C., Allen, D.J., Pitt, F.D., Zwirglmaierl, K., Le Gall, F., et al. (2006) Analysis of photosynthetic picoeukaryote diversity at open ocean sites in the Arabian Sea using a PCR biased towards marine algal plastids. Aquat Microb Ecol 43: 79-93.

del Giorgio, P.A., Gasol, J.M., Vaque, D., Mura, P., Agusti, S., and Duarte, C.M. (1996) Bacterioplankton community structure: protists control net production and the proportion of active bacteria in a coastal marine community. Limnol Oceanogr 41: 1169-1179.

Gonzalez, J.M., Sherr, E.B., and Sherr, B.F. (1990) Sizeselective grazing on bacteria by natural assemblages of estuarine flagellates and ciliates. Appl Environ Microbiol 56: 583-589.

González, J.M., Iriberri, J., Egea, L., and Barcina, I. (1990) Differential rates of digestion of bacteria by freshwater and marine phagotrophic protozoa. Appl Environ Microbiol 56: 1851-1857.

Guillou, L., Jacquet, S., Chretiennot-Dinet, M.J., and Vaulot, D. (2001) Grazing impact of two small heterotrophic flagellates on Prochlorococcus and Synechococcus. Aquat Microb Ecol 26: 201-207.

Guindon, S., and Gascuel, O. (2003) A simple, fast, and accurate algorithm to estimate large phylogenies by maximum likelihood. Syst Biol 52: 696-704. 
Hall, J.A., Barret, D.P., and James, M.R. (1993) The importance of phytoflagellates, heterotrophic flagellate and ciliate grazing on bacteria and picophytoplankton sized prey in a coastal marine environment. J Plankton Res 15: 1075-1086.

Hansen, P.J., and Hjorth, M. (2002) Growth and grazing responses of Chrysochromulina ericina (Prymnesiophyceae): the role of irradiance, prey concentration and pH. Mar Biol 141: 975-983.

Hansen, P.J., and Nielsen, T.G. (1997) Mixotrophic feeding of Fragilidium subglobosum (Dinophyceae) on three species of Ceratium: effects of prey concentration, prey species and light intensity. Mar Ecol Prog Ser 147: 187196.

Ishii, N., Takeda, H., Doi, M., Fuma, S., Miyamoto, K., Yanagisawa, K., and Kawabata, Z. (2002) A new method using enhanced green fluorescent protein (EGFP) to determine grazing rate on live bacterial cells by protists. Limnology 3 : $47-50$.

Jones, R.I. (2000) Mixotrophy in planktonic protists: an overview. Freshw Biol 45: 219-226.

Jurgens, K., and Matz, C. (2002) Predation as a shaping force for the phenotypic and genotypic composition of planktonic bacteria. Antonie Van Leeuwenhoek 81: 413434.

Jürgens, K., and Güde, H. (1994) The potential importance of grazing-resistant bacteria in planktonic systems. Mar Ecol Prog Ser 112: 169-188.

Katoh, K., Misawa, K., Kuma, K., and Miyata, T. (2002) MAFFT: a novel method for rapid multiple sequence alignment based on fast Fourier transform. Nucleic Acids Res 30: 3059-3066.

Katoh, K., Kuma, K., Toh, H., and Miyata, T. (2005) MAFFT version 5: improvement in accuracy of multiple sequence alignment. Nucleic Acids Res 33: 511-518.

Koton-Czaarnecka, M., and Chrost, R.J. (2003) Protozoans prefer large and metabolically active bacteria. Polish $J$ Environ Studies 12: 325-334.

Landry, M.R., Lehner-Fournier, J.M., Sundstrom, J.A., Fagerness, V.L., and Selph, K.E. (1991) Discrimination between living and heat-killed prey by a marine zooflagellate, Paraphysomonas vestita (Stokes). J Exp Mar Biol Ecol 146: 139.

Lomas, M.W., Glibert, P.M., Clougherty, D.A., Huber, D.R., Jones, J., Alexander, J., and Haramoto, E. (2001) Elevated organic nutrient ratios associated with brown tide algal blooms of Aureococcus anophagefferens (Pelagophyceae). J Plankton Res 23: 1339-1344.

Ludwig, W., Strunk, O., Westram, R., Richter, L., Meier, H., Yadhukumar, et al. (2004) ARB: a software environment for sequence data. Nucleic Acids Res 32: 1363-1371.

Lueders, T., Manefield, M., and Friedrich, M.W. (2004) Enhanced sensitivity of DNA- and rRNA-based stable isotope probing by fractionation and quantitative analysis of isopycnic centrifugation gradients. Environ Microbiol 6: 73-78.

Lueders, T., Kindler, R., Miltner, A., Friedrich, M.W., and Kaestner, M. (2006) Identification of bacterial micropredators distinctively active in a soil microbial food web. Appl Environ Microbiol 72: 5342-5348.

Maidak, B.L., Cole, J.R., Lilburn, T.G., Parker, C.T., Jr,
Saxman, P.R., Farris, R.J., et al. (2001) The RDP-I (Ribosomal Database Project). Nucleic Acids Res 29: 173174.

Manefield, M., Whiteley, A.S., Griffiths, R.I., and Bailey, M.J. (2002) RNA stable isotope probing, a novel means of linking microbial community function to phylogeny. Appl Environ Microbiol 68: 5367-5373.

Mann, E.L., and Chisholm, S.W. (2000) Iron limits the cell division rate of Prochlorococcus in the eastern equatoria Pacific. Limnol Oceanogr 45: 1067-1076.

Massana, R., Guillou, L., Diez, B., and Pedros-Alio, C. (2002) Unveiling the organisms behind novel eukaryotic ribosomal DNA sequences from the ocean. Appl Environ Microbiol 68: 4554-4558.

Not, F., Gausling, R., Azam, F., Heidelberg, J.F., and Worden, A.Z. (2007) Vertical distribution of picoeukaryotic diversity in the Sargasso Sea. Environ Microbiol 9: 1233 1252.

Nylander, J. (2004) MrModeltest v2. Program distributed by the author. Evolutionary Biology Centre, Upssala University.

Nygaard, K., and Tobiesen, A. (1993) Bacterivory in algae a survival strategy during nutrient limitation. Limnol Oceanogr 38: 273-279.

Partensky, F., Hess, W.R., and Vaulot, D. (1999a) Prochlorococcus, a marine photosynthetic prokaryote of global significance. Microbiol Mol Biol Rev 63: 106-127.

Partensky, F., Blanchot, J., and Vaulot, D. (1999b) Differential distribution and ecology of Prochlorococcus and Synechococcus in oceanic waters: a review. Bull Inst Océanogr Monaco Special No. 19: 457-475.

Pernthaler, J. (2005) Predation on prokaryotes in the water column and its ecological implications. Nat Rev Microbiol 3: 537-546.

Perriere, G., and Gouy, M. (1996) WWW-Query: an on-line retrieval system for biological sequence banks. Biochimie 78: 364-369.

Pruesse, E., Quast, C., Knittel, K., Fuchs, B.M., Ludwig, W. Peplies, J., and Glockner, F.O. (2007) SILVA: a comprehensive online resource for quality checked and aligned ribosomal RNA sequence data compatible with ARB. Nucleic Acids Res 35: 7188-7196.

Radajewski, S., Ineson, P., Parekh, N.R., and Murrell, J.C. (2000) Stable-isotope probing as a tool in microbial ecology. Nature 403: 646-649.

Raven, J.A. (1997) Phagotrophy in phototrophs. Limnol Oceanogr 42: 198-205.

Rippka, R., Coursin, T., Hess, W., Lichtle, C., Scanlan, D.J. et al. (2000) Prochlorococcus marinus Chisholm et al. 1992 subsp. pastoris subsp. nov. strain PCC 9511, the first axenic chlorophyll a2/b2-containing cyanobacterium (Oxyphotobacteria). Int J Syst Evol Microbiol 50 (Part 5): 18331847.

Sanchez-Puerta, M.V., Lippmeier, J.C., Apt, K.E., and Delwiche, C.F. (2007) Plastid genes in a non-photosynthetic dinoflagellate. Protist 158: 105-117.

Shalchian-Tabrizi, K., Eikrem, W., Klaveness, D., Vaulot, D. Minge, M.A., Le Gall, F., et al. (2006) Telonemia, a new protist phylum with affinity to chromist lineages. Proc Biol Sci 273: 1833-1842.

Sherr, B.F., Sherr, E.B., and Fallon, R.D. (1987) Use of 
monodispersed, fluorescently labeled bacteria to estimate in situ protozoan bacterivory. Appl Environ Microbiol 53: 958-965.

Sherr, E.B., and Sherr, B.F. (2002) Significance of predation by protists in aquatic microbial food webs. Antonie Van Leeuwenhoek (Int J Gen Mol Microbiol) 81: 293-308.

Sherr, E.B., Rassouladegan, F., and Sherr, B.F. (1989) Bacterivory by pelagic choreotrichous ciliates in coastal waters of the NW Mediterranean Sea. Mar Ecol Prog Ser 55: 235-240.

Simek, K., and Chrzanowski, T.H. (1992) Direct and indirect evidence of size-selective grazing on pelagic bacteria by freshwater nanoflagellates. Appl Environ Microbiol 58: 3715-3720.

Stoeck, T., Zuendorf, A., Breiner, H.W., and Behnke, A (2007) A molecular approach to identify active microbes in environmental eukaryote clone libraries. Microb Ecol 53: 328-339.

Unrein, F., Massana, R., Alonso-Saez, L., and Gasol, J.M. (2007) Significant year-round effect of small mixotrophic flagellates on bacterioplankton in an oligotrophic coastal system. Limnol Oceanogr 52: 456-469.

Worden, A.Z., and Binder, B.J. (2003) Application of dilution experiments for measuring growth and mortality rates among Prochlorococcus and Synechococcus populations in oligotrophic environments. Aquat Microb Ecol 30: 159174.

\section{Supporting information}

Additional Supporting Information may be found in the online version of this article:

Fig. S1. Phylogenetic analysis of the sequences derived from the 18S rDNA sequences in the indigenous microbial community. Unrooted phylogenetic tree inferred by maximum likelihood (ML) analysis. A total of 1090 positions used, including gaps (sequences ranging from a minimum of $585 \mathrm{bp}$ up to $893 \mathrm{bp}$ ), from an alignment of 203 partial sequences were used. Bootstrap values over $50 \%$ are indicated on the internal branches obtained from both $\mathrm{ML}$, neighbour joining-distance methods (NJ-Dis) and using maximum parsimony (MP) (in the order ML/NJ-Dist/MP). Bootstrap values $<50 \%$, or not supported at least in two of the analyses, have been omitted. The gamma distribution parameter (a) was estimated at 0.520 ; and the proportion of invariant sites $(I)$ was 0.015 . The scale bar indicates $10 \%$ divergence. The sequences from the duplicate biological samples are indicated as (dhot1) and (dhot2). Classification is based on Adl and colleagues (2005) and Not and colleagues (2007). All groups correspond to first and second rank according to Adl and colleagues (2005) except *Super-group and **Phylum (Shalchian-Tabrizi et al., 2006).

Fig. S2. Phylogenetic analysis of the sequences derived from the 18S rDNA sequences in the unlabelled fractions from the experiments (time 0 and blue circles in Fig. 2). Unrooted SSU rRNA-derived library phylogenetic tree of eukaryotes inferred by maximum likelihood (ML) analysis. A total of 1058 positions used, including gaps (sequences ranging from a minimum of $512 \mathrm{bp}$ up to $886 \mathrm{bp}$ ), from an alignment of 200 partial sequences were used. Bootstrap values over $50 \%$ are indicated on the internal branches obtained from both $\mathrm{ML}$, neighbour joining-distance methods (NJ-Dis) and using maximum parsimony (MP) (in the order $\mathrm{ML} / \mathrm{NJ}$-Dist/MP). Bootstrap values $<50 \%$, or not supported at least in two of the analyses, have been omitted. The gamma distribution parameter (a) was estimated at 0.594; and the proportion of invariant sites (I) was 0.000 . The scale bar indicates $10 \%$ divergence. The sequences coming from the duplicate biological samples is indicated as (A) and (B). Clones colour code: dark red: sequences from the time 0 sample, representing the metabolically active initial eukaryotic microbial community; purple: sequences from the unlabelled eukaryotic RNA obtained from the samples incubated with Prochlorococcus; orange: sequences from the unlabelled fraction from the samples incubated with Synechococcus. Classification is based on Adl and colleagues (2005) and Not and colleagues (2007). All groups correspond to first and second rank according to Adl and colleagues (2005) except when noted as follows: ${ }^{*}$ Super-group and ${ }^{* *}$ Phylum (Shalchian-Tabrizi et al., 2006). ${ }^{* * *}$ Unidentified chloroplastida, BLAST results gave no clear match and the sequences did not cluster clearly with any of the second-rank groups used in the tree that could indicate the exact affiliation of the sequence.

Fig. S3. Phylogenetic analysis of the sequences derived from the labelled $18 \mathrm{~S}$ rDNA sequences (red circles in Fig. 2) from the experimental bottles amended with labelled Prochlorococcus cells. Unrooted $18 \mathrm{~S}$ rRNA-derived library phylogenetic tree of eukaryotes inferred by maximum likelihood (ML) analysis. A total of 1145 positions used, including gaps (sequences ranging from a minimum of $545 \mathrm{bp}$ up to $980 \mathrm{bp}$ ), from an alignment of 192 partial sequences were used. Bootstrap values over $50 \%$ are indicated on the internal branches obtained from both $\mathrm{ML}$, neighbour joining-distance methods (NJ-Dis) and using maximum parsimony (MP) (in the order $\mathrm{ML} / \mathrm{NJ}$-Dist/MP). Bootstrap values $<50 \%$, or not supported at least in two of the analyses, have been omitted. The gamma distribution parameter (a) was estimated at 0.512; and the proportion of invariant sites (I) was 0.000 . The scale bar indicates $10 \%$ divergence. The sequences coming from the duplicate biological samples is indicated as (A) and (B). Classification is based on Adl and colleagues (2005) and Not and colleagues (2007). All groups correspond to first and second rank according to Adl and colleagues (2005) except when noted as follows: *Super-group and ${ }^{* *}$ Phylum (ShalchianTabrizi et al., 2006). ${ }^{* *}$ Unidentified stramenopiles, BLAST results gave no clear match and the sequences did not cluster clearly with any of the second-rank groups used in the tree that could indicate the exact affiliation of the sequence. Fig. S4. Phylogenetic analysis of the sequences derived from the labelled 18S rDNA sequences (red circles in Fig. 2) from the experimental bottles amended with labelled Synechococcus cells. Unrooted 18S rRNA-derived library phylogenetic tree of eukaryotes inferred by maximum likelihood (ML) analysis. A total of 1156 positions used, including gaps (sequences ranging from a minimum of $507 \mathrm{bp}$ up to $977 \mathrm{bp}$ ), from an alignment of 188 partial sequences were used. Bootstrap values over $50 \%$ are indicated on the internal branches obtained from both $\mathrm{ML}$, neighbour joining-distance methods (NJ-Dis) and using maximum parsimony (MP) (in the order ML/NJ-Dist/MP). Bootstrap values $<50 \%$, or not supported at least in two of the analyses, have been omitted. The gamma 
distribution parameter (a) was estimated at 0.543 ; and the proportion of invariant sites (I) was 0.036 . The scale bar indicates $10 \%$ divergence. The sequences coming from the duplicate biological samples is indicated as (A) and (B). Classification is based on Adl and colleagues (2005) and Not and colleagues (2007). All groups correspond to first and second rank according to Adl and colleagues (2005) except when noted as follows: *Super-group and ${ }^{* *}$ Phylum (ShalchianTabrizi et al., 2006). ${ }^{* \star *}$ Unidentified stramenopiles, BLAST results gave no clear match and the sequences did not cluster clearly with any of the second-rank groups used in the tree that could indicate the exact affiliation of the sequence. Fig. S5. Phylogenetic tree 16S rRNA sequences from chloroplasts and bacteria inferred by maximum likelihood (ML) analysis. Blue: cyanobacterial 16S rRNA sequences. Green: sequences originating from the labelled fraction of the Prochlorococcus inoculation experiment. Orange: sequences originated from the labelled fraction from the Synechococcus inoculation experiment. (A) and (B) represent the two biological replicates in the experiments. A total of 724 positions were used, including gaps (sequences ranging from a minimum of $358 \mathrm{bp}$ up to $668 \mathrm{bp}$ ), from an alignment of 103 partial sequences. Bootstrap values over $50 \%$ are indicated on the internal branches obtained from ML, neighbour joiningdistance methods (NJ-Dist) and using maximum parsimony (MP) (in the order ML/NJ-Dist/MP). Bootstrap values $<50 \%$, or not supported at least in two of the analyses, have been omitted. The proportion of invariant sites (I) was 0.241 . The scale bar indicates $10 \%$ divergence. An archaeal sequence was used as out-group (Sulfolobus acidocaldarius).

Table S1. Oligonucleotides used for the amplification of $16 S$ rRNA chloroplast genes from different groups defined based on the ARB tree (SSRef release 90 12.05.2007) for these group of sequences. F, forward primer. R, reverse primer.

Please note: Wiley-Blackwell are not responsible for the content or functionality of any supporting materials supplied by the authors. Any queries (other than missing material) should be directed to the corresponding author for the article.

(C) 2008 The Authors

Journal compilation (c) 2008 Society for Applied Microbiology and Blackwell Publishing Ltd, Environmental Microbiology, 11, 512-525 


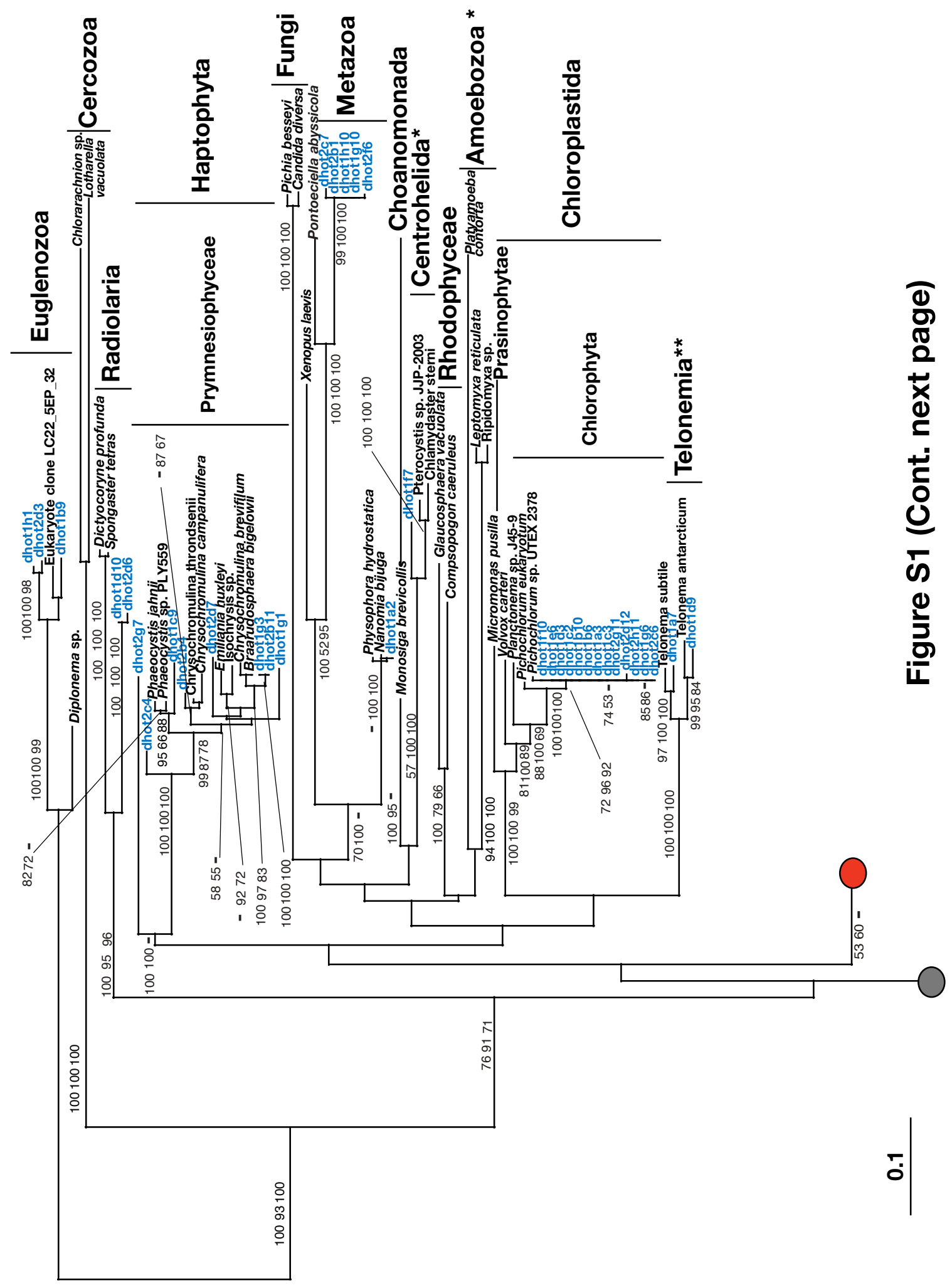




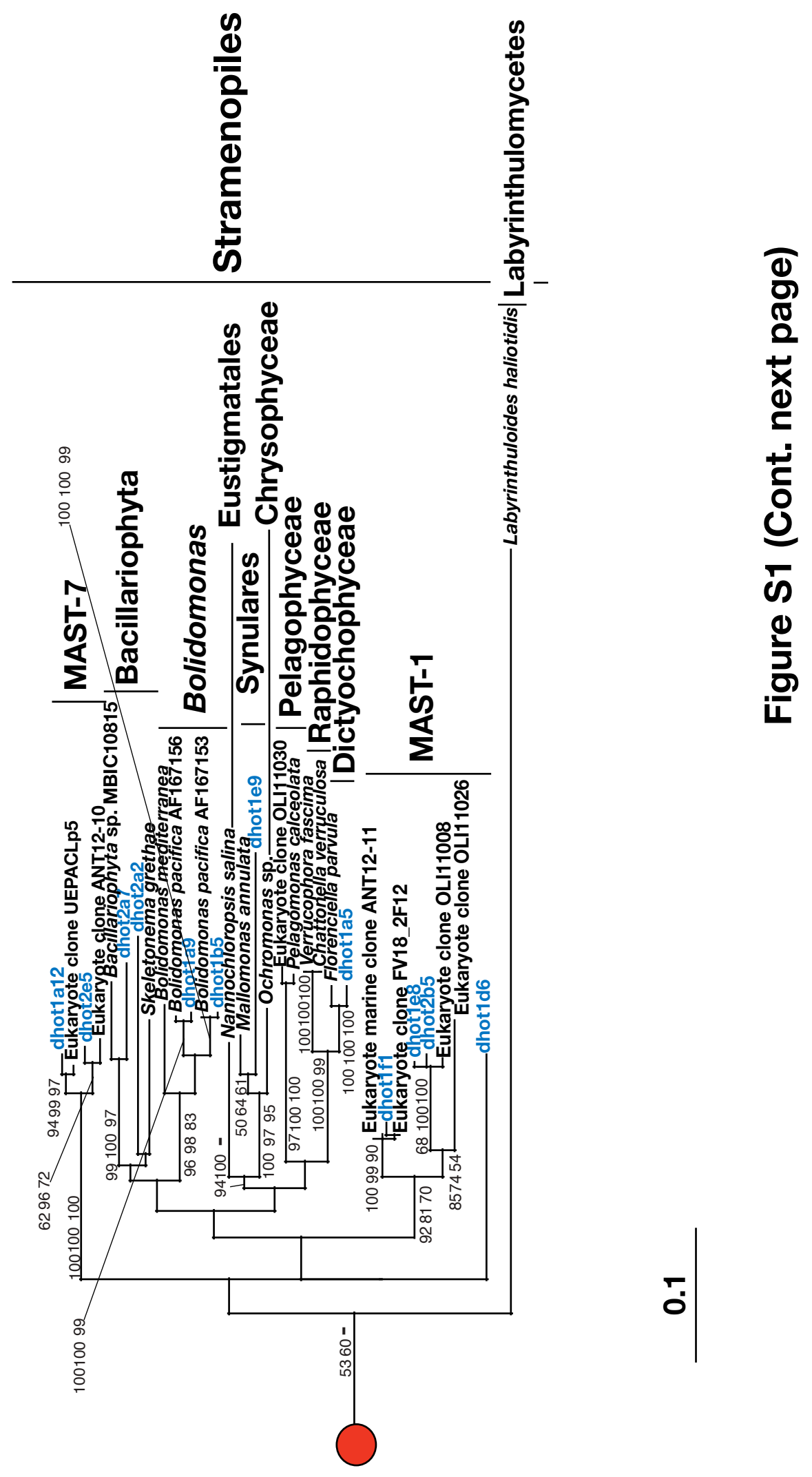




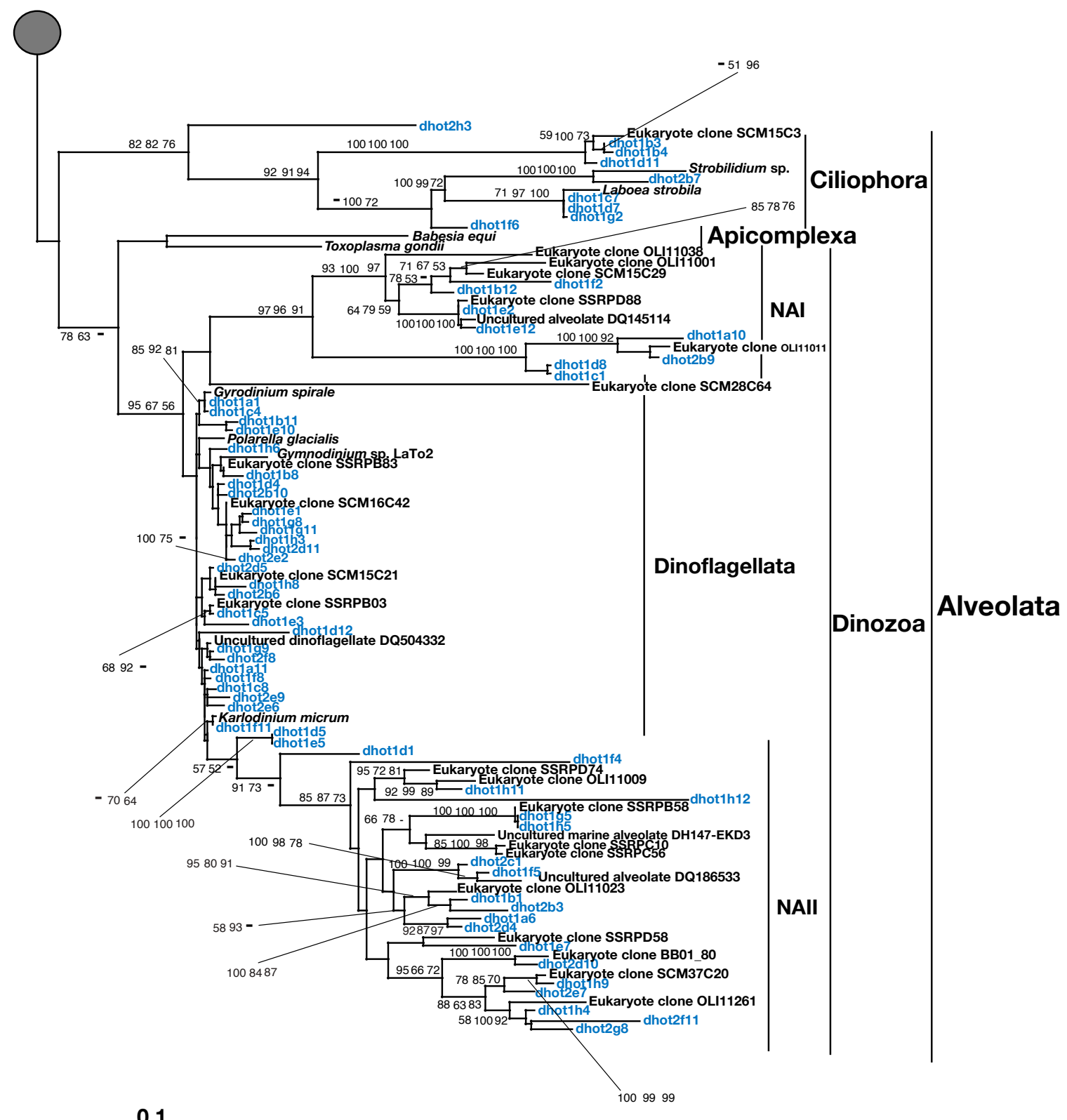

Figure S1 


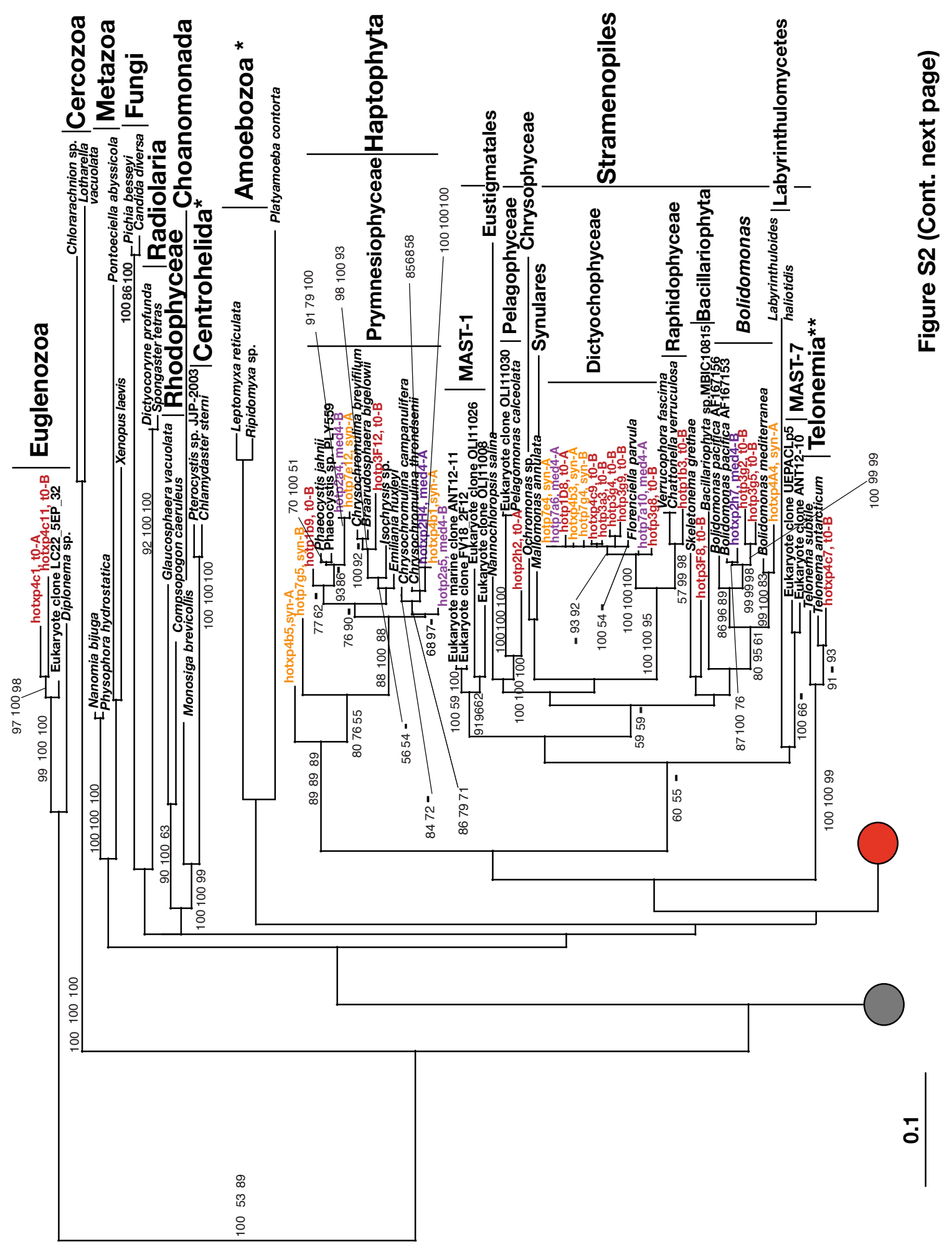




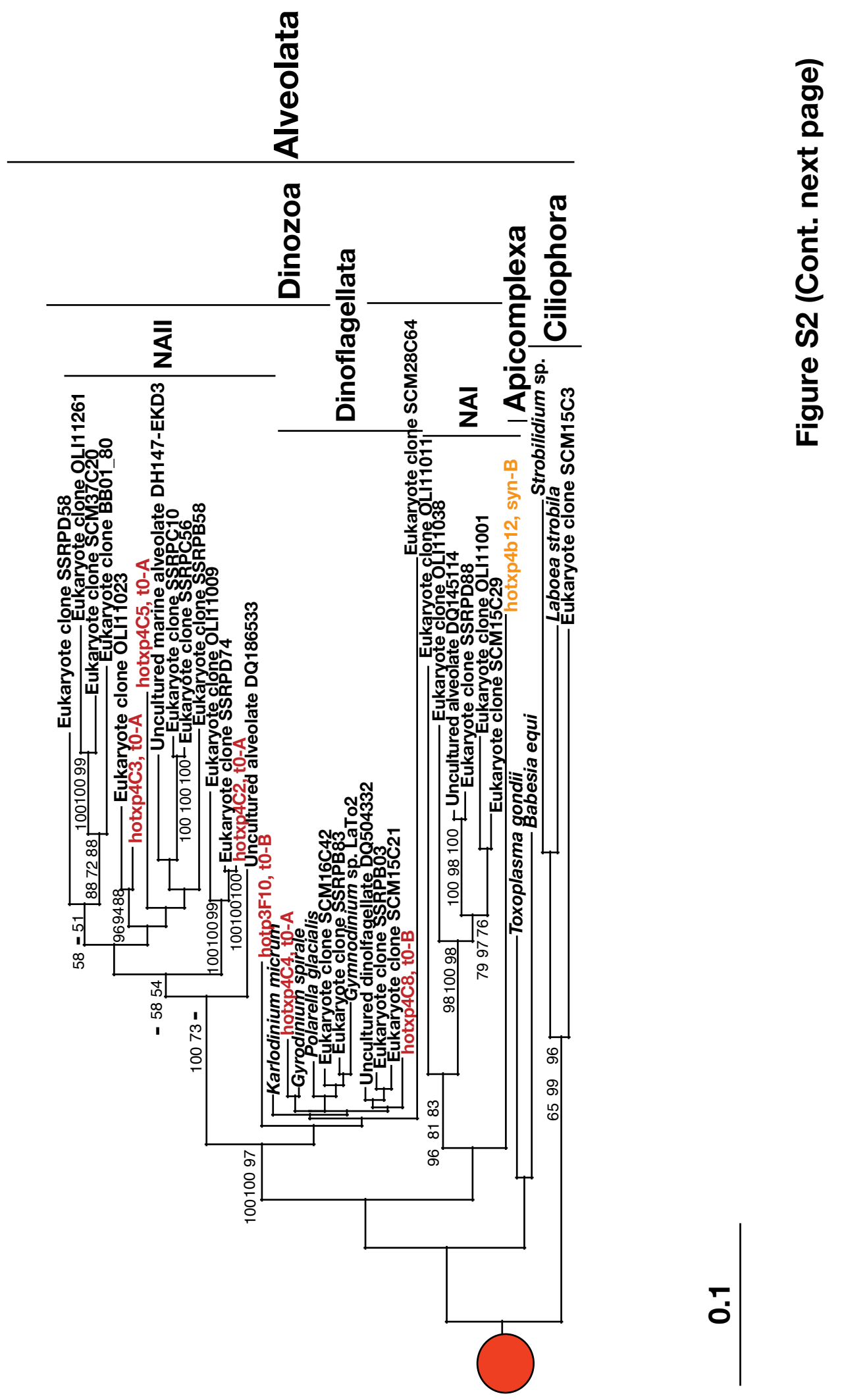




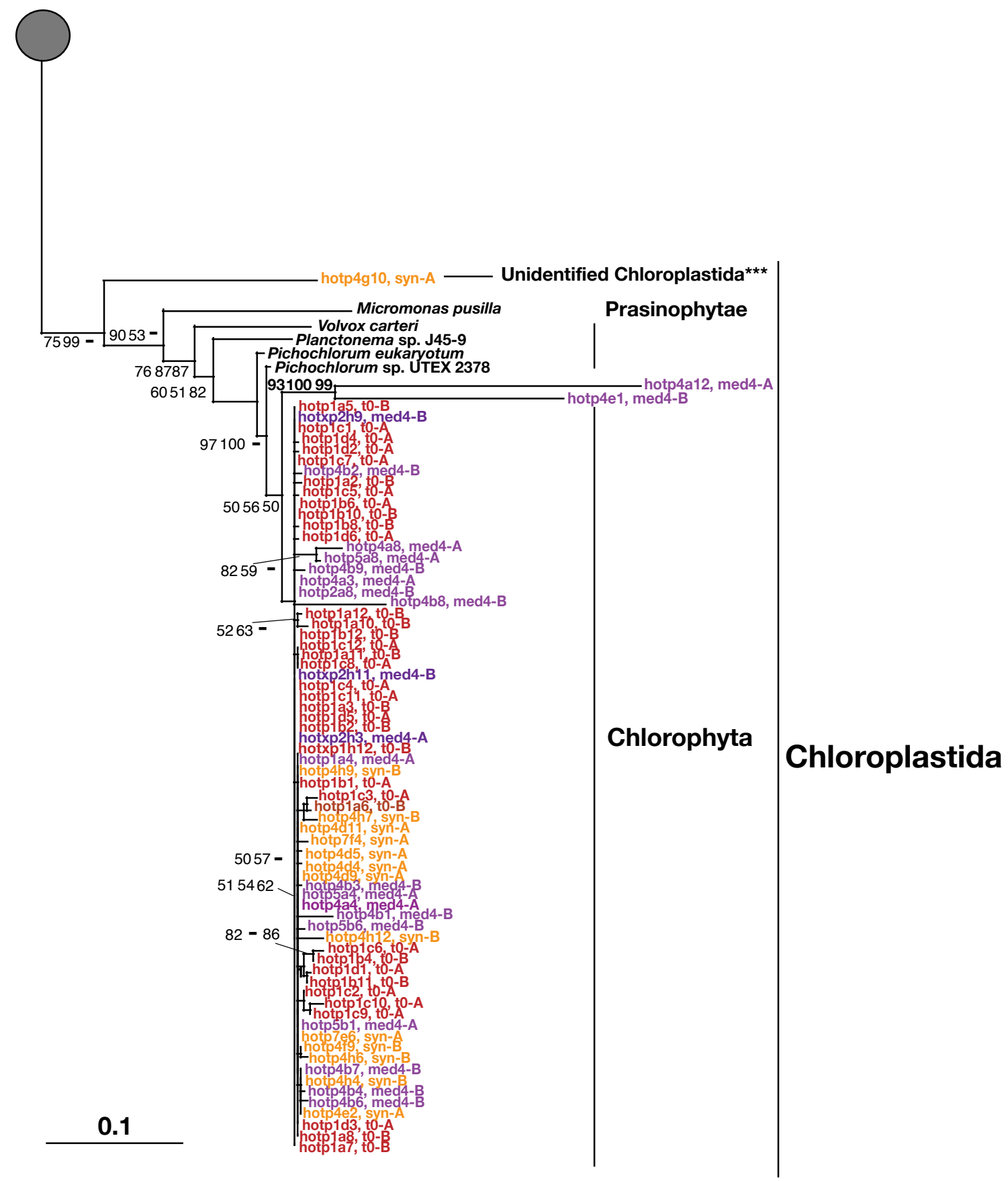

Figure S2 


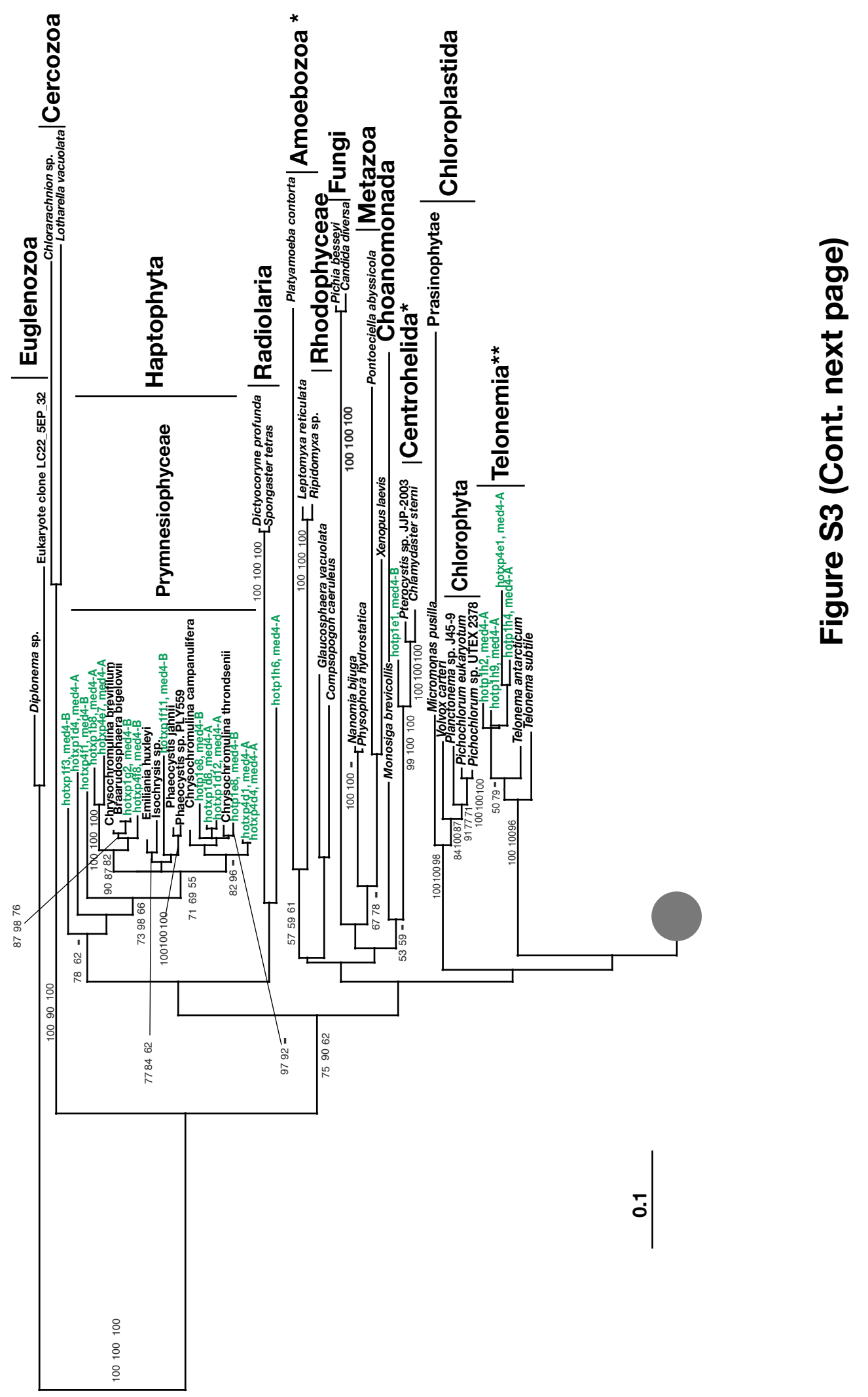




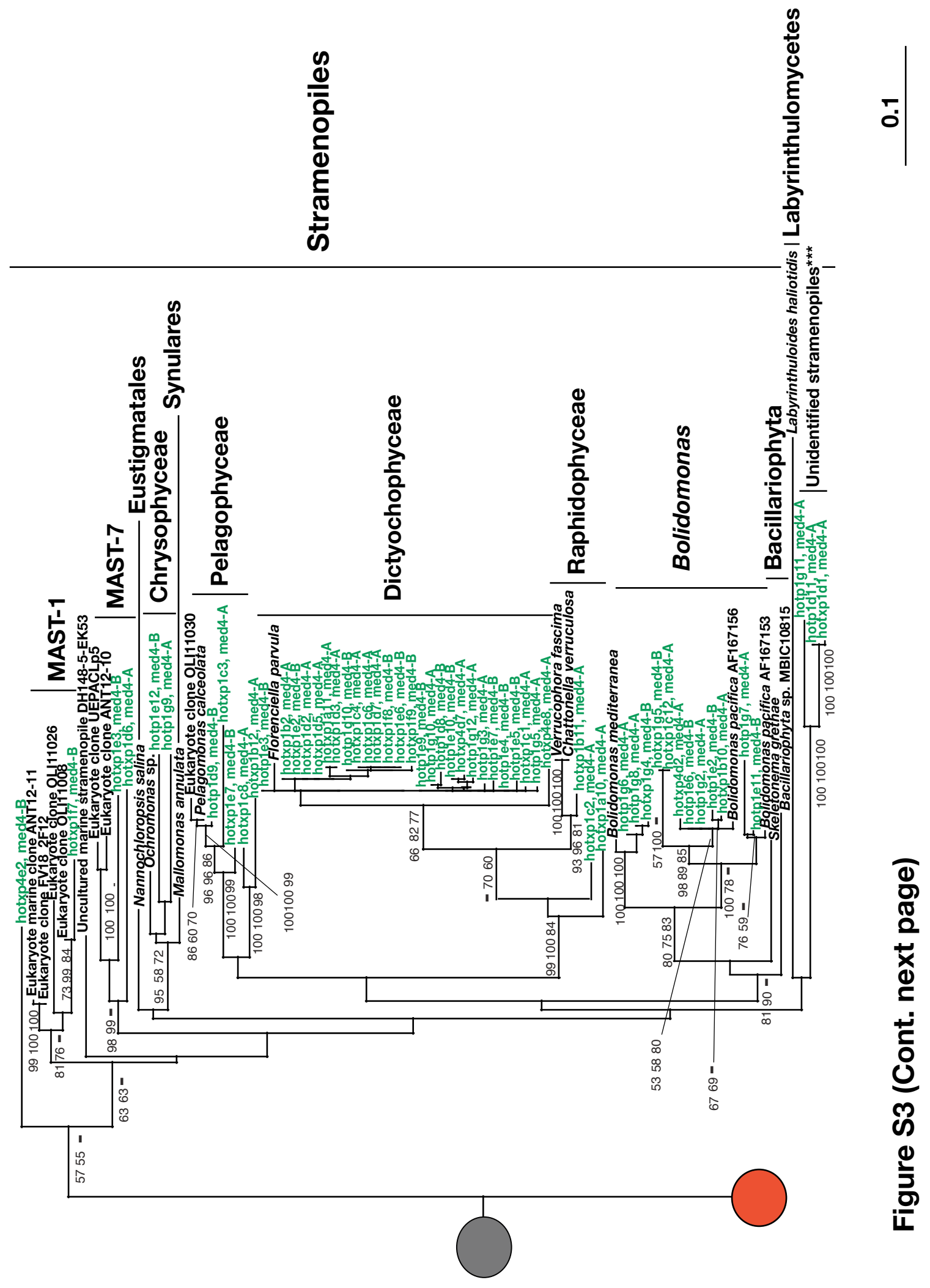




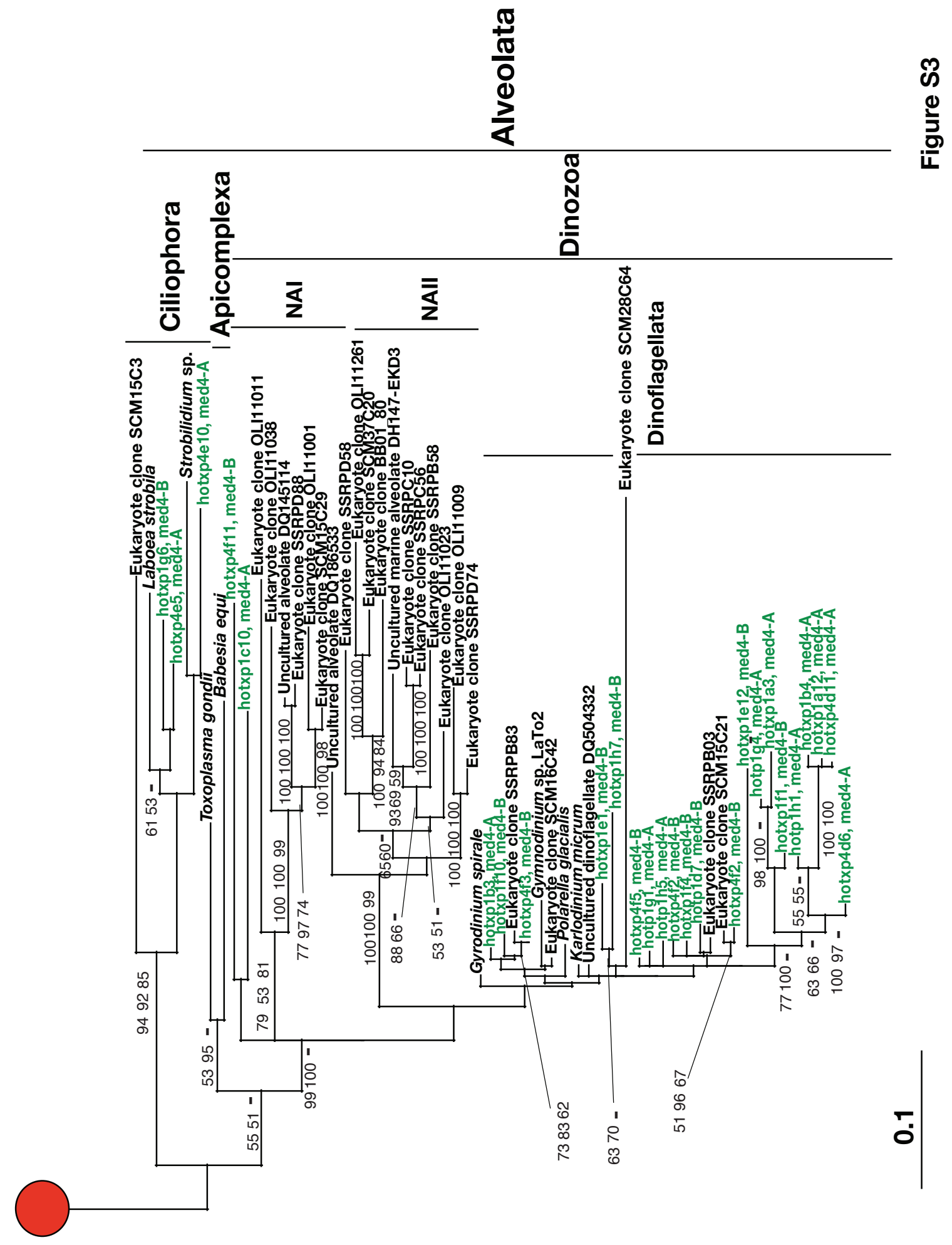




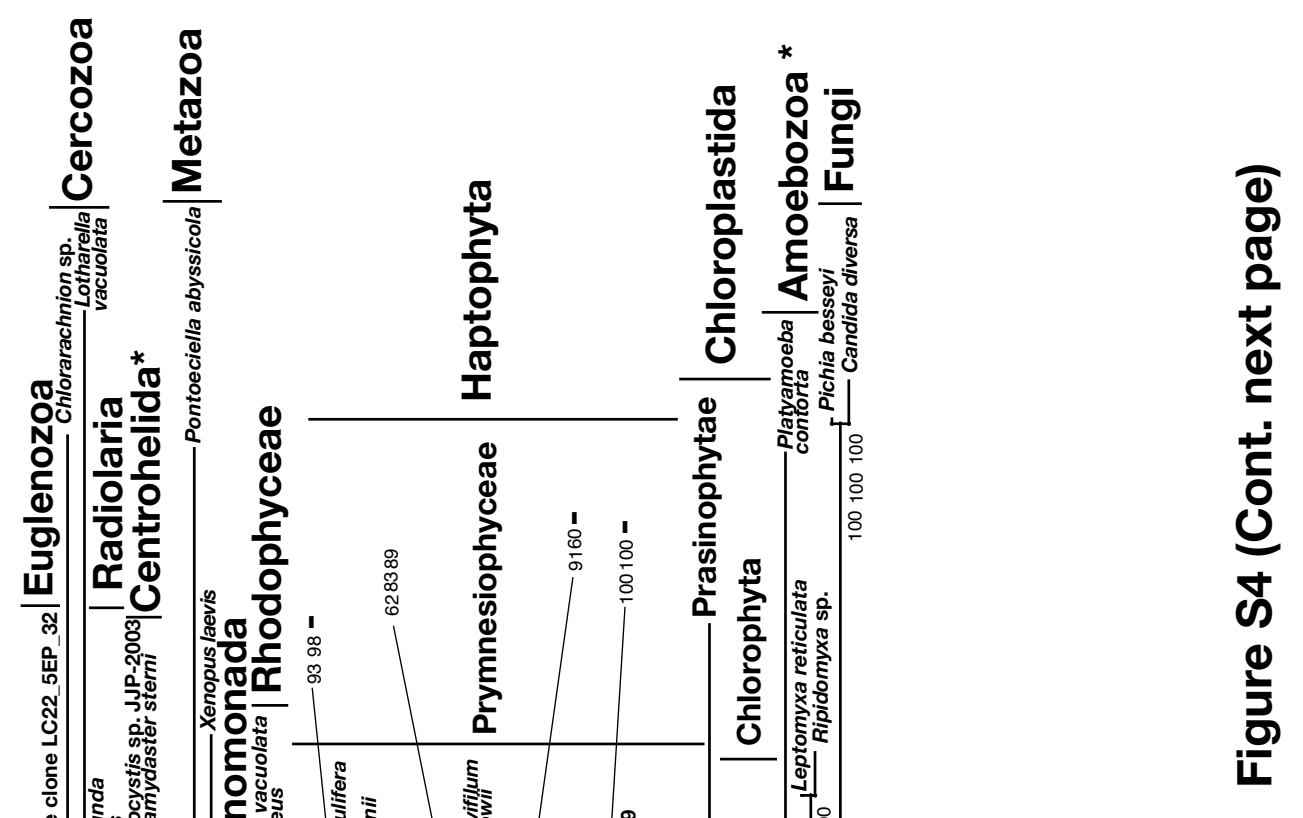




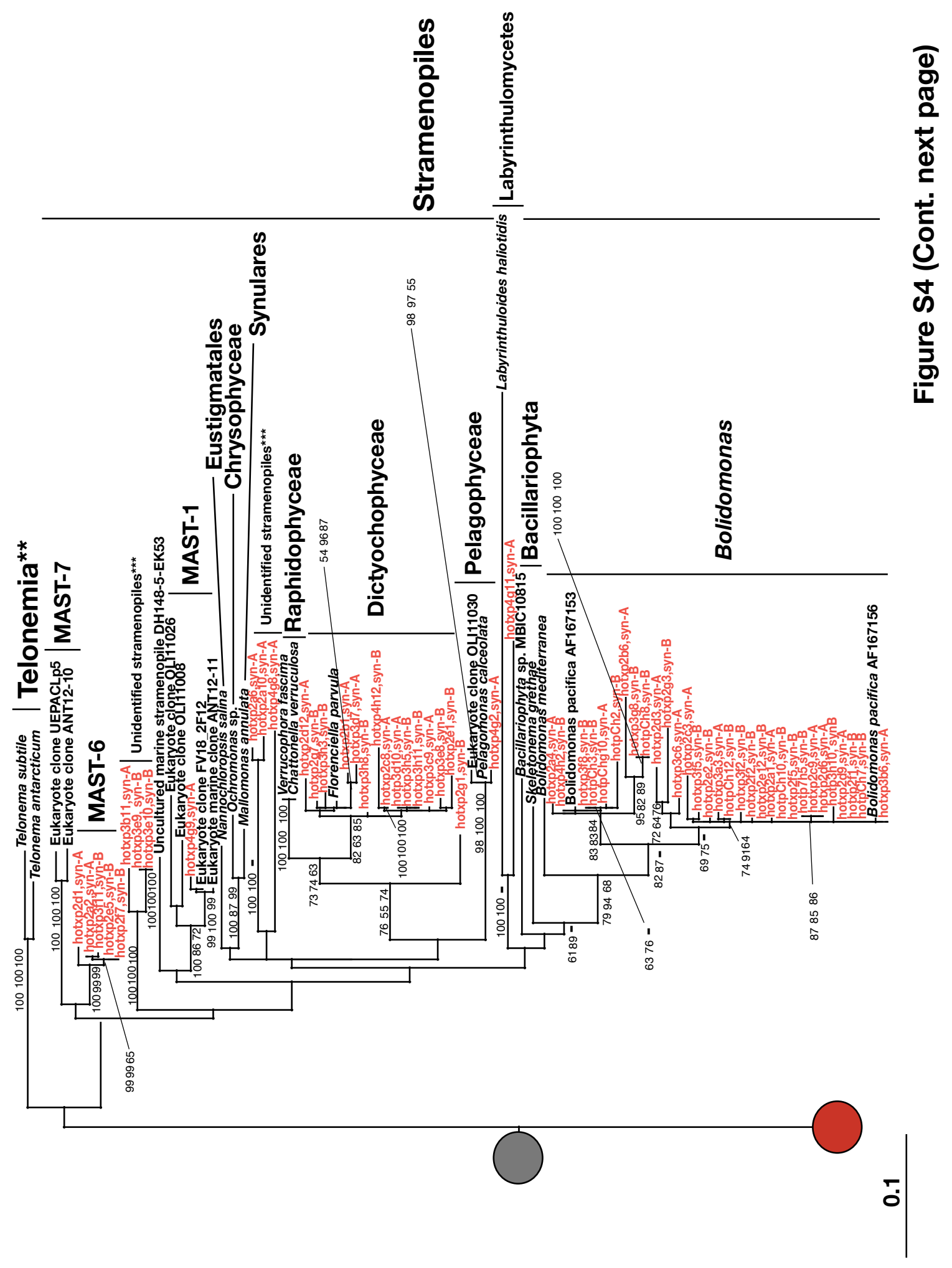




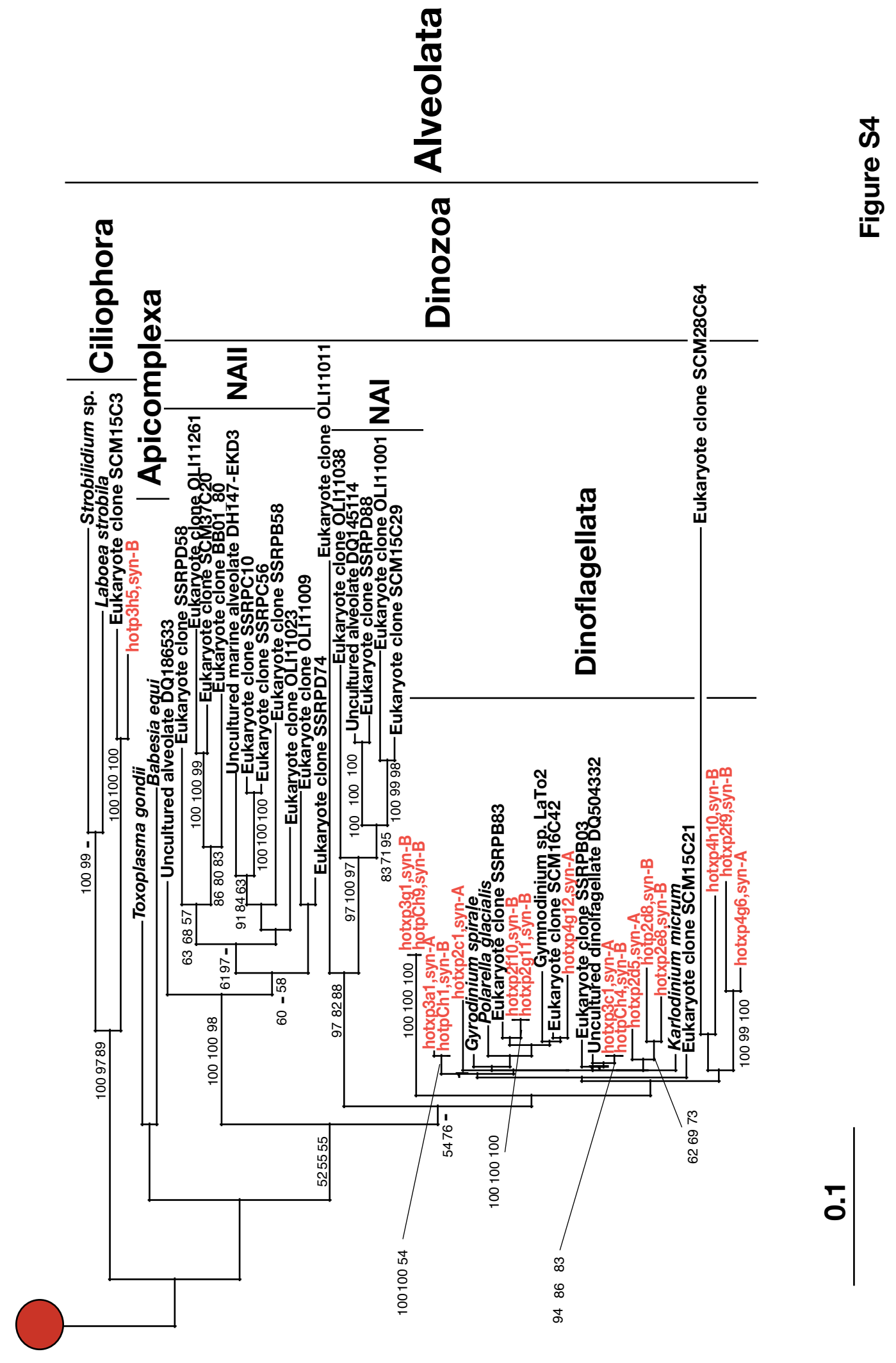




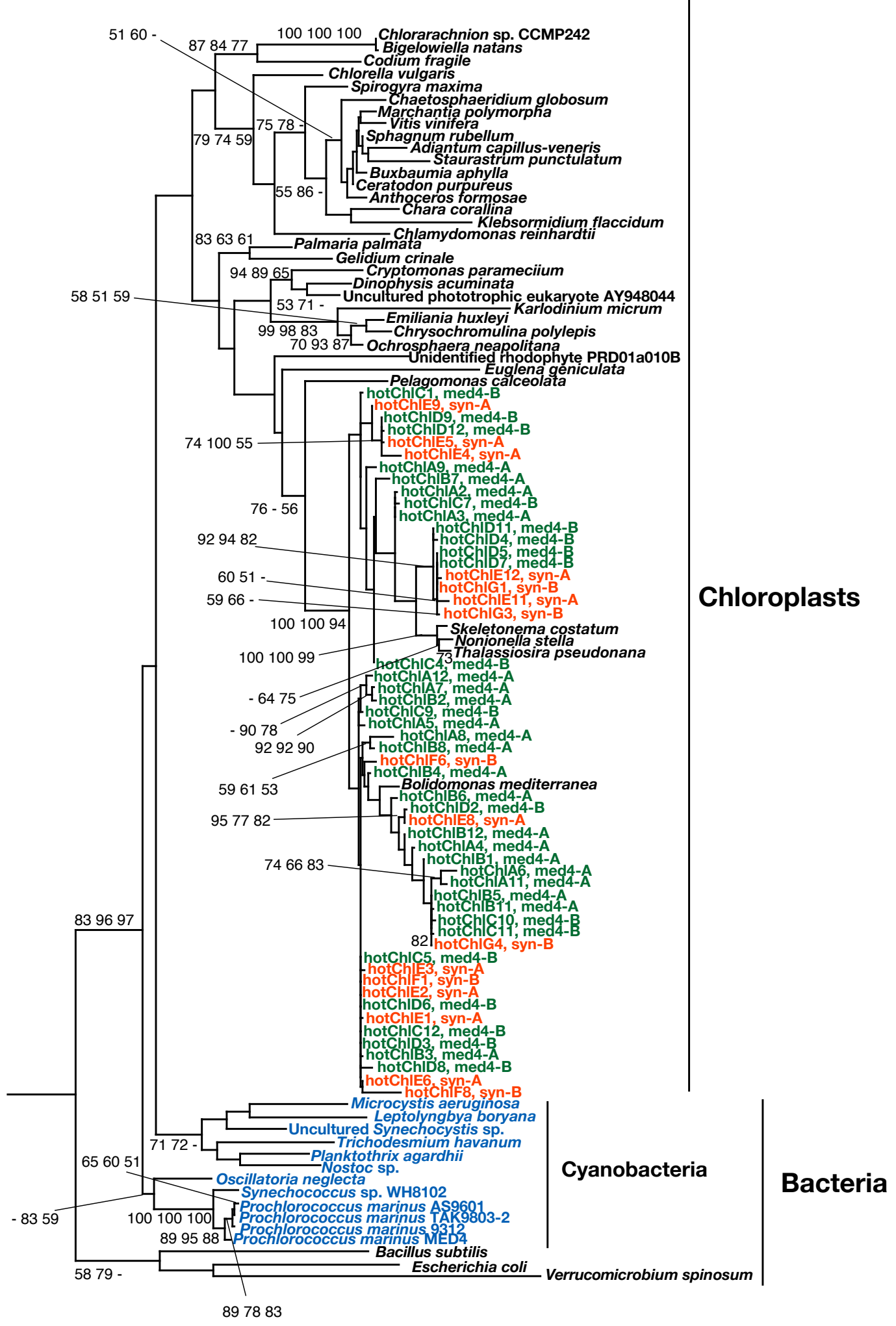

Figure S5 
Table S1. Oligonucleotides used for the amplification of 16S rRNA chloroplast genes from different groups defined based on the ARB tree (SSRef release 90 12.05.2007) for these group of sequences. $F$, forward primer. $R$, reverse primer.

\begin{tabular}{|c|c|c|c|}
\hline $\begin{array}{l}\text { Primers' } \\
\text { set } \\
\text { number }\end{array}$ & Group targeted & Sequence & $\begin{array}{l}\text { Expected size } \\
\text { of amplified } \\
\text { product in bp }\end{array}$ \\
\hline \multirow[t]{2}{*}{1} & Zea and rel.-F & GAA GTG GTG TTT CCA GTG GC & 351 \\
\hline & Zea and rel.-R & AAA AGA AGT TCA CGA CCC GT & \\
\hline \multirow[t]{2}{*}{2} & Chlamydomonas and rel.-F & ACA CGT CAA CGC ACG AGC TG & 821 \\
\hline & Chlamydomonas and rel.-R & TAG CTA GTT GGT GGG GGT AA & \\
\hline \multirow[t]{2}{*}{3} & Anthocerus and rel.-F & TAA GGA GGG GCT TGC GTT TG & 218 \\
\hline & Anthocerus and rel.-R & GTC ATT GCT TCT TCT CTA AG & \\
\hline \multirow[t]{2}{*}{4} & Marchantia and rel.-F & СCT TTT CTC AGA GAA GAT GC & 813 \\
\hline & Marchantia and rel.- $R$ & GCG AGG TCG CGA CCC TTT GT & \\
\hline \multirow[t]{2}{*}{5} & Spirogyra and rel.-F & TAG TCT CCA CCG CCT GGC CA & 485 \\
\hline & Spirogyra and rel.- $R$ & GGC GGG GGA CCA CCA CTG GA & \\
\hline \multirow[t]{2}{*}{6} & Palmaria_1 and rel.-F & CGC CTT AGC TAC GAT ACT GC & 89 \\
\hline & Palmaria_1 and rel.- $\mathrm{R}$ & AGA CGA CAG CTA GGG GAG CA & \\
\hline \multirow[t]{2}{*}{7} & Ochromonas and rel.-F & CCA CCT GTG TAA GAG GCC GT & 581 \\
\hline & Ochromonas and rel.- $R$ & GGA AGA TCT GAC GTT ACT TG & \\
\hline \multirow[t]{2}{*}{8} & Palmaria_2 and rel.-F & CTA CGA TAC TGC ACG GAT CG & 136 \\
\hline & Palmaria_2 and rel.-R & TGG GAA GAA CAC CAG AAG CG & \\
\hline \multirow[t]{2}{*}{9} & Chara and rel.-F & GCA CTG AAC GGA TCA AAT CG & 712 \\
\hline & Chara and rel.-R & AAG GAC TTG CCC TTG GGT GG & \\
\hline \multirow[t]{2}{*}{10} & Bryophyta and rel.-F & GGA GCG AAA GGA GGA ATC CA & 31 \\
\hline & Bryophyta and rel.-R & ACG CAA GCC CCT CCT TGG GT & \\
\hline \multirow[t]{2}{*}{11} & Chlorella and rel.-F & CCC CAG GCG GGA TAC TTC ACG & 160 \\
\hline & Chlorella and rel.- $R$ & GGG AGG AAC ACC AAA GGC GA & \\
\hline \multirow[t]{2}{*}{12} & Chlorarachnion and rel.-F & AGC GAG GGG AGA GAA TGG GA & 436 \\
\hline & Chlorarachnion and rel.-R & GCC CAG AAC TTA AGG GGC AT & \\
\hline \multirow[t]{2}{*}{13} & Osmunda and rel.-F & AGC AAA AGG GAG GGA TCC GC & 398 \\
\hline & Osmunda and rel.-R & TTG ACA GCG GAC TTA AGG AG & \\
\hline \multirow[t]{2}{*}{14} & Chaetosphaeridium and rel.-F & CTT GCG TCT GAT TAT GCT AG & 175 \\
\hline & Chaetosphaeridium and rel.- $R$ & ACC CGT AAG CTT TCT TCC T & \\
\hline \multirow[t]{2}{*}{15} & Skeletonema and rel.-F & TTA ACT CAA GTG GCG GAC GG & 650 \\
\hline & Skeletonema and rel.- $R$ & AGT GTT AGT AAT AGC CCA GTA & \\
\hline \multirow[t]{2}{*}{16} & Euglenales and rel.-F & GGG GAG TAC GCT TGC AAA AG & 153 \\
\hline & Euglenales and rel.- $\mathrm{R}$ & CAT GCA CCA CCT GTG TCT AG & \\
\hline
\end{tabular}




\section{Appendix B}

\section{Improved methods for isolating and validating indigenous biomarkers in Precambrian rocks}

Laura S. Sherman, Jacob R. Waldbauer and Roger E. Summons

Reprinted with permission from Organic Geochemistry

(C) 2007 Elsevier Ltd.

Sherman, L.S., Waldbauer, J.R. and Summons, R.E. (2007) Improved methods for isolating and validating indigenous biomarkers in Precambrian rocks. Organic Geochemistry 38: 1987-2000. 


\title{
Improved methods for isolating and validating indigenous biomarkers in Precambrian rocks
}

\author{
Laura S. Sherman ${ }^{\text {a }}$, Jacob R. Waldbauer ${ }^{\text {b }}$, Roger E. Summons ${ }^{\text {a,* }}$ \\ ${ }^{a}$ Department of Earth, Atmospheric and Planetary Sciences, Massachusetts Institute of Technology, Cambridge, MA 02139, USA \\ ${ }^{\mathrm{b}}$ Joint Program in Chemical Oceanography, Massachusetts Institute of Technology and Woods Hole Oceanographic Institution, \\ Cambridge, MA 02139, USA \\ Received 24 July 2007; accepted 31 August 2007 \\ Available online 14 September 2007
}

\begin{abstract}
Hydrocarbon biomarkers in highly mature Precambrian rocks have the potential to provide important information about the diversity ecology, and evolution of early life, but studying them presents special analytical challenges. Extractable hydrocarbons are present in Archean and most Paleoproterozoic sedimentary rocks in such trace concentration that even slight contamination from petroleum-derived materials in situ or during drilling, storage, sampling, handling and laboratory analysis would compromise the results and, thereby, any consequent inferences. Here we report protocols that we have developed for the analysis of cores from several recently completed deep-time scientific drilling initiatives. By paying special attention to cutting, cleaning, crushing and extraction, it is possible to significantly reduce laboratory blanks to acceptable levels. When these methods are utilized, meaningful variations in the patterns of biomarkers over stratigraphic and lithologic boundaries provide compelling evidence for syngeneity.
\end{abstract}

(c) 2007 Elsevier Ltd. All rights reserved.

\section{Introduction}

Molecular fossils of biogenic lipids (i.e., biomarkers) preserved over geologic time in sedimentary rocks of low metamorphic grade can be used to describe ancient microbiota and environmental conditions (Eglinton et al., 1964; Brocks et al., 1999, 2003b). Because biomarkers are the hydrocarbon skeletons of biogenic precursor lipids, those that are created through known biosynthetic and diagenetic pathways convey information about the identity and physiology of their source organisms

\footnotetext{
${ }^{*}$ Corresponding author.

E-mail address: rsummons@mit.edu (R.E. Summons).
}

(Brocks and Summons, 2003; Brocks and Pearson, 2005). Biomarker geochemistry is therefore a potentially powerful tool for establishing the record of ancient life on Earth and for better understanding early microbial evolution. However, the application of biomarker techniques to highly mature Precambrian organic matter presents unique challenges, requires the use of special methods and necessitates extraordinary attention to concerns of contamination.

Although some Precambrian rocks contain abundant amounts of syndepositional organic matter (exceeding $10 \mathrm{wt}^{\%} \%$ in some samples in this study), very little is in the form of extractable bitumen. Over billions of years, even under relatively low 
grade metamorphic conditions, the majority of the organic constituents of a sediment are incorporated into the macromolecular kerogen network (Brocks and Summons, 2003). Because very few hydrocarbons are present as extractable biomarker molecules (sub-ppb quantities), even low level contamination introduced during drilling, sampling, storage, or laboratory processing may overprint the original signatures.

The first purportedly Precambrian biomarkers were amino acids identified by P.H. Abelson in the late 1950s (Barghoorn, 1957); numerous studies in the 1960 s subsequently reported finding fatty acids, amino acids, $n$-alkanes, porphyrins and acyclic isoprenoids in Precambrian rocks (McKirdy, 1974; Hayes et al., 1983; Imbus and McKirdy, 1993). However, these findings were quickly called into question (Hoering, 1966; Leventhal et al., 1975). Hoering (1966) noted that most high maturity Archean rocks had thermal histories inconsistent with the preservation of soluble organic molecules. $\mathrm{He}$ also detailed numerous sources of contamination, including anthropogenic petroleum products, laboratory equipment and sample storage bags (Hoering, 1966; Hoering, 1967). Furthermore, subsequent reports described the potential for younger oils and drill fluids to penetrate and be preserved in Precambrian units (Barghoorn et al., 1965; Meinschein, 1965; Nagy, 1970; Sanyal et al., 1971). These problems overshadowed Precambrian organic geochemistry investigations for almost thirty years.

More recent studies, including those by Brocks (2001), Brocks et al. (1999), Brocks and Summons (2003), Eigenbrode (2004) and Dutkiewicz et al. (2006) have re-visited Precambrian organic geochemistry with extreme care, in order to assess the syngeneity of detected molecules. The current study was undertaken to both gain a better understanding of early microbial evolution and develop a protocol for sampling, handling, and analyzing high maturity Precambrian organic matter.

We examined rocks from two cores (GKP01 and GKF01, hereafter GKP and GKF) drilled as part of the Agouron Griqualand Drilling Project and rocks from one core (ABDP9) drilled as part of the Deep Time Drilling Project of the NASA Astrobiology Drilling Program. These sedimentary successions contain some of the earliest evidence of the accumulation and cycling of molecular oxygen in surface environments (Bekker et al., 2004; Anbar et al., 2007; Kaufman et al., 2007). The Agouron cores were each drilled without the use of hydrocarbon fluids through more than a kilometer of Archean sediments. The cores intersect platform and slope facies of the Transvaal Supergroup and were deposited on the Kaapvaal Craton of South Africa between 2670-2460 Ma (Armstrong et al., 1986; Barton et al., 1994; Walraven and Martini, 1995; Sumner and Bowring, 1996). Stratigraphic correlations between the two Agouron cores, based on the presence of volcanic ash beds, spherule layers, flooding surfaces, sequence boundaries and lithologic marker beds (Schroder et al., 2006), enabled intercomparison of the biomarker contents of correlated beds. Core ABDP9 was drilled through the Pilbara Craton of western Australia, intersecting iron formations, shales, mixed clastics and carbonates. Although drilling difficulties required the use of petroleum-based additives part way through the coring, the top $200 \mathrm{~m}$ were obtained with minimal contamination. These two drilling projects are among the first efforts to recover long stretches of Archean strata with the express purpose of collecting samples for molecular fossil analysis.

\section{Methodology: sample collection and processing}

The methods were developed for the extraction and analysis of Precambrian hydrocarbons with minimal contamination. The methodology is based on techniques described by Brocks (2001), Brocks et al. (2003a,b) and Eigenbrode (2004), with refinements as described. Rationalization of key aspects of the methods can be found in Section 4 .

\subsection{Drilling}

As described above, the data presented in this study are results of analysis of rock samples from the Agouron Griqualand Drilling Project and the Deep Time Drilling Project. Drill cores have several advantages over outcrop samples for the purposes of biomarker analysis. The surface exposures of early Precambrian terranes are generally heavily weathered, meaning they have been exposed to circulating modern surface water and groundwater that carry contaminant lipids and hydrocarbons. Drilling below the modern weathering horizon which can be tens to hundreds of meters deep affords rock samples that have not been in contact with fluids in the recent geologic past. Drill cores also sample long stretches of continuous stratigraphy, generally much longer intervals than can be accessed in often sparse surface exposure. Impor- 
tantly, drill cores represent a group of samples taken at one time that have all been subject to the same handling and storage conditions. This facilitates stratigraphic comparisons of biomarker contents, which can be valuable both for both assessing the syngeneity of the extracted hydrocarbons and establishing records of paleoenvironmental change.

While deep core drilling offers several key advantages in sample recovery for Precambrian biomarker analysis, other potentially complicating factors must be kept in mind. Individual drill cores necessarily represent a finite amount of material deposited at each time interval and cannot be used, as outcrop samples often can, to determine the lateral variability of a stratigraphic unit. Because these projects are costly and time consuming, it is often not practical to drill a series of cores in a small area. A central concern when studying drill cores is that the samples will necessarily have come into contact with drilling equipment and fluids. It is essential that the hydrocarbon-based lubricants typically used in diamond-bit core drilling be eschewed, ideally in favor of pure water as a drilling fluid. Use of water as the sole drilling fluid is difficult, and generally increases rate of equipment fatigue and failure. Nevertheless, the extremely low quantities of extractable bitumen in high maturity Precambrian rocks mean that the syngenetic signal can easily be overprinted by even minimal hydrocarbon content in the drilling fluid. Any additive or substance to be put into the borehole should be pre-screened for hydrocarbon and biomarker content. Some commercial products, such as those based on plant oils, are less likely to be confused with indigenous Precambrian biomarkers because they are composed primarily of polar compounds and display immature isomer distributions. Other products, advertised as 'hydrocarbon free', often contain quantities of petroleum-derived biomarkers that could compromise trace level analysis. Even for cores drilled under the cleanest practicable conditions, we have found that the outer surfaces of core pieces bear signals of low level contamination, necessitating their removal (see Sections 2.3 and 4.1).

From hundreds of meters of drill core, only a small subset of the recovered stratigraphy can be feasibly analyzed. We chose our sub-samples for biomarker work on the basis of several criteria. Originally, visibly organic-rich strata (primarily fine grained black shales, often with abundant pyrite) were sampled because we expected that they would yield the most extractable bitumen. Ultimately, we found little correlation between bitumen yield and whole rock organic matter content, with more carbonate-rich beds sometimes having as much extractable hydrocarbon as shales (Waldbauer et al., 2007). Hence, we consider it useful to sample all lithofacies intersected in a given core, particularly because contrasts between siliciclastic and carbonate-dominated depositional environments are a potentially strong paleoenvironmental signal (Eigenbrode, 2004). Similarly, we sampled across formation and sequence boundaries to explore how changes in sediment sourcing and deposition might be recorded in biomarker content.

\subsection{Materials}

In general, it is important to minimize the number of external surfaces that touch Archean rock samples. Each surface contacted represents an opportunity for contaminant hydrocarbons to be introduced into the samples. Surfaces that samples do contact are either cleaned exhaustively with organic solvents or thoroughly combusted. Additionally, materials used to process high maturity samples are reserved only for these samples and are kept separate from general laboratory supplies.

Laboratory solvents including hexane, dichloromethane (DCM) and methanol $(\mathrm{MeOH})$ are high purity and organic free (OmniSolv, EMD Chemicals). Prior to use, glassware, glass wool, aluminum foil and silica gel are combusted at $550{ }^{\circ} \mathrm{C}(8 \mathrm{~h})$ and quartz sand (Accusand, Unimin Corp.) is combusted at $850{ }^{\circ} \mathrm{C}(12 \mathrm{~h})$. De-ionized (DI) water and hydrochloric acid $(\mathrm{HCl})$ used in sample processing are extracted $(\times 5)$ with DCM. Metal tools used to process samples are rinsed with DI water and cleaned $(\times 5$ each) with $\mathrm{MeOH}, \mathrm{DCM}$ and hexane. Crushing tools (see Section 2.3) are scrubbed with combusted quartz sand, rinsed with DI water and sonicated for $30 \mathrm{~min}$ each in $\mathrm{MeOH}$ and DCM.

\subsection{Sample preparation}

Quarter core samples (1/4 NQ size, $\sim 47.6 \mathrm{~mm}$ whole core diameter) were approximately 50 to $200 \mathrm{~g}$ in weight and 20 to $50 \mathrm{~cm}$ in length. They were processed in batches of six with at least one procedural sand blank per set. Samples were labeled according to core name and downcore depth in meters (e.g., GKF 230). We found that foreign contaminants are present on the outside surfaces of the 
cores and that it is necessary to remove at least the outer 3 to $5 \mathrm{~mm}$ of core material to remove these hydrocarbons (see Section 4.1). To do this, samples are cut using a table saw with a water lubricated, diamond edge blade (UKAM). DCM-extracted DI water is used as the blade lubricant. Between samples the saw is washed in DI water and the blade is washed and sonicated for $20 \mathrm{~min}$ each in $\mathrm{MeOH}$ and DCM. After cutting, to remove potential surficial contaminants, the trimmed core pieces are sonicated for $20 \mathrm{~min}$ each in DCM-extracted DI water, $\mathrm{MeOH}$, and DCM.

After cutting the samples, it is necessary to crush them to a fine powder prior to extraction (see Section 4.1.1). We first crush samples to pieces of ca. 1/4" diameter using a 300 series stainless steel mortar and pestle (MIT Machine Shop design, Fig. 1a). Samples are then crushed to a fine powder (sub140 mesh) using a stainless steel puck mill modeled after a SPEX 8507 steel puck mill (MIT Machine Shop design, Fig. 1b). Between samples, the mortar, pestle and puck mill are scrubbed with fired sand to remove particulate matter, washed with DI water and sonicated for 30 min each in $\mathrm{MeOH}$ and DCM.

\subsection{Extraction and fractionation}

After crushing, the powdered samples (40-90 g) are divided into $60 \mathrm{ml}$ vials (ca. $15 \mathrm{~g}$ powder per vial). DCM is added to each vial (ca. 25-30 ml), the vials are sonicated for $30 \mathrm{~min}$ and the bitumen is collected. More solvent is added and the process is repeated (see Section 4.2). It is then necessary to filter the extracts over a small amount of silica gel (ca. $3 \mathrm{~cm}$ ) in a wide bore glass column to remove particulates. The bitumens are next treated with acid-activated copper shot (Alfa Aesar) to remove elemental sulfur and are loaded on to Pasteur pipette silica columns (ca. $8 \mathrm{~cm}$ of silica). The saturated, aromatic and polar hydrocarbons are separated using hexane (1 column volume), hexane/DCM (4 column volumes, 8/2) and DCM/ $\mathrm{MeOH}$ (4 column volumes, 7/3), respectively.

\subsection{Preparation of bitumen II}

To prepare demineralized powder, ca. $30 \mathrm{~g}$ of extracted rock powder is placed in Teflon tubes which are cleaned of organics first in aqua regia and then with $\mathrm{MeOH}$ and DCM (ca. $15 \mathrm{~g}$ powder per tube). DCM-extracted $\mathrm{HCl}(6 \mathrm{~N})$ is added and, after agitation, the acid is left to react for $24-48 \mathrm{~h}$ to remove carbonates. After pouring off the $\mathrm{HCl}, 48 \%$ hydrofluoric acid (HF) is added for at least $72 \mathrm{~h}$ to dissolve silicates. Finally, after the $\mathrm{HF}$ is decanted, $6 \mathrm{~N}$ DCM-extracted $\mathrm{HCl}$ is again added to remove fluoride precipitates. Throughout this process, to aid acid digestion, the tubes are periodically agitated. After $24 \mathrm{~h}$ in $\mathrm{HCl}$, the samples are washed at least $\times 5$ with DCM-extracted water. Once dry, the powders are extracted using sonication as described above and the resulting bitumen is analyzed.

\subsection{Gas chromatography (GC-MS) and metastable reaction monitoring (MRM) mass spectrometry}

After separation, the fractions are carefully dried to a volume of roughly $80 \mu \mathrm{l}$. Standards are added a

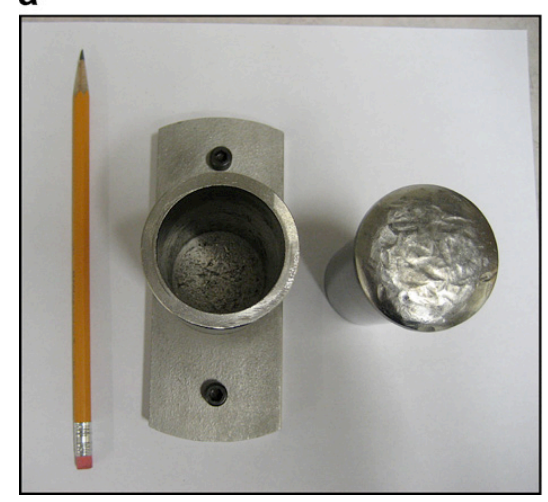

b

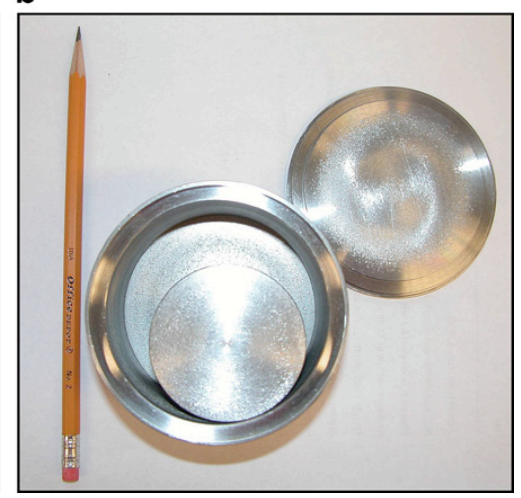

Fig. 1. Photographs of rock crushing equipment. (a) Stainless steel mortar and pestle with pencil for scale. (b) Stainless steel puck mill with pencil for scale. 
as follows: $10 \mathrm{ng} \quad \mathrm{D}_{4} \quad\left(\mathrm{D}_{4}-\alpha \alpha \alpha\right.$-ethylcholestane, Chiron) and $1 \mu \mathrm{g} \quad a i \mathrm{C}_{22}$ (3-methylheneicosane, $99+\%$, ULTRA Scientific) to the saturated fraction and $413 \mathrm{ng} \mathrm{D}_{14}$ ( $\mathrm{D}_{14}$-para-terphenyl, 98 at.\% D, Cambridge Isotope) to the aromatic fraction.

The saturated fractions are analyzed using $\mathrm{GC}-$ MS in full scan mode and metastable reaction monitoring GC-MS (MRM-GC-MS-MS; Table 1). The aromatic fractions are analyzed using GC-MS with selected ion monitoring. All the analyses are conducted with a Micromass AutoSpec Ultima coupled to an Agilent $6890 \mathrm{~N}$ gas chromatograph, fitted with

Table 1

MRM-GC-MS-MS precursor to product transitions

\begin{tabular}{|c|c|c|}
\hline $\begin{array}{l}\text { Precursor mass } \\
(\mathrm{Da})^{\mathrm{a}}\end{array}$ & $\begin{array}{l}\text { Product mass } \\
(\mathrm{Da})^{\mathrm{a}}\end{array}$ & Biomarkers \\
\hline 262.265 & 191.179 & $\mathrm{C}_{19}$ Tricyclic terpane \\
\hline 276.280 & 191.179 & $\mathrm{C}_{20}$ Tricyclic terpane \\
\hline 290.296 & 191.179 & $\mathrm{C}_{21}$ Tricyclic terpane \\
\hline 304.312 & 191.179 & $\mathrm{C}_{22}$ Tricyclic terpane \\
\hline 318.328 & 191.179 & $\mathrm{C}_{23}$ Tricyclic terpane \\
\hline 330.328 & 191.179 & $\begin{array}{l}\mathrm{C}_{24} \text { Tetracyclic } \\
\text { terpane }\end{array}$ \\
\hline 332.344 & 191.179 & $\mathrm{C}_{24}$ Tricyclic terpane \\
\hline 346.359 & 191.179 & $\mathrm{C}_{25}$ Tricyclic terpane \\
\hline 360.374 & 191.179 & $\mathrm{C}_{26}$ Tricyclic terpane \\
\hline 358.353 & 217.196 & $\mathrm{C}_{26}$ Steranes \\
\hline 372.376 & 217.196 & $\mathrm{C}_{27}$ Steranes \\
\hline 386.391 & 217.196 & $\mathrm{C}_{28}$ Steranes \\
\hline 404.432 & 221.221 & $\mathrm{D}_{4} \mathrm{C}_{29}$ Sterane $^{\mathrm{b}}$ \\
\hline 400.407 & 217.196 & $\mathrm{C}_{29}$ Steranes \\
\hline 414.423 & 217.196 & $\mathrm{C}_{30}$ Steranes \\
\hline 426.421 & 205.194 & $\mathrm{Me}-\mathrm{C}_{30}$ Hopanes $^{\mathrm{c}}$ \\
\hline 370.359 & 191.179 & $\mathrm{C}_{27}$ Hopanes \\
\hline 440.437 & 205.194 & $\begin{array}{l}\mathrm{Me}-\mathrm{C}_{31} \\
\text { Homohopanes }\end{array}$ \\
\hline 384.374 & 191.179 & $\mathrm{C}_{28}$ Hopanes \\
\hline 454.452 & 205.194 & $\begin{array}{l}\mathrm{Me}-\mathrm{C}_{32} \\
\text { Homohopanes }\end{array}$ \\
\hline 398.390 & 191.179 & $\mathrm{C}_{29}$ Hopanes \\
\hline 468.467 & 205.194 & $\begin{array}{l}\mathrm{Me}-\mathrm{C}_{33} \\
\text { Homohopanes }\end{array}$ \\
\hline 412.406 & 191.179 & $\mathrm{C}_{30}$ Hopanes \\
\hline 482.482 & 205.194 & $\begin{array}{l}\mathrm{Me}-\mathrm{C}_{34} \\
\text { Homohopanes }\end{array}$ \\
\hline 426.421 & 191.179 & $\mathrm{C}_{31}$ Homohopanes \\
\hline 496.496 & 205.194 & $\begin{array}{l}\mathrm{Me}-\mathrm{C}_{35} \\
\text { Homohopanes }\end{array}$ \\
\hline 440.437 & 191.179 & $\mathrm{C}_{32}$ Homohopanes \\
\hline 454.452 & 191.179 & $\mathrm{C}_{33}$ Homohopanes \\
\hline 468.467 & 191.179 & $\mathrm{C}_{34}$ Homohopanes \\
\hline 482.482 & 191.179 & $\mathrm{C}_{35}$ Homohopanes \\
\hline
\end{tabular}

${ }^{\text {a }}$ Precursor and product mass in Daltons.

b " $\mathrm{D}_{4} \quad \mathrm{C}_{29}$ sterane" is the internal standard, $\mathrm{D}_{4}-\alpha \alpha \alpha-$ ethylcholestane.

c "Me- $\mathrm{C}_{x}$ " steranes and hopanes are methylated versions of the indicated compounds. a DB-1 fused silica column $(60 \mathrm{~m} \times 0.25 \mathrm{~mm}$ i.d., $0.25 \mu \mathrm{m}$ film thickness, J\&W Scientific) and using $\mathrm{He}$ as carrier gas. The mass spectrometer source operates at $250{ }^{\circ} \mathrm{C}$ under electron ionization conditions $(70 \mathrm{eV})$ and an accelerating voltage of $8 \mathrm{kV}$ is used. Full scan analyses are conducted over a range of $m / z 50-600$. Peaks are integrated manually and quantified on the basis of comparison to the internal standards (using $\mathrm{m} / \mathrm{z} 85$ from full scan analyses).

\section{Typical results}

Agouron Archean core samples ranged from 34.1 to $95.6 \mathrm{~g}$ rock powder and yielded between 0.31 and $21.1 \mu \mathrm{g}$ of bitumen (saturated hydrocarbons and cyclic terpenoid biomarkers). By weight, the bitumen content ranges from 13.9 to $604.6 \mathrm{ppb}$ whole rock or 0.34 to $23.9 \mathrm{ppm}$ organic matter. All the Archean bitumens yielded broadly similar types and abundances of hydrocarbons. The compound classes detected, and their overall abundances and relative amounts of rearranged isomers, are characteristic of highly thermally mature organic matter. Typical biomarker and saturated hydrocarbon chromatograms from a representative sample (GKF 705.3) are presented in Fig. 2. The Archean bitumens are comprised almost entirely of saturated and aromatic hydrocarbons; polar and partially unsaturated compounds are below detection limit. The saturated fractions are composed primarily of low molecular weight $n$-alkanes with condensatelike carbon number distributions (Fig. 2b). The aromatic fractions are dominated by low molecular weight polycyclic hydrocarbons and their alkylated homologs.

Cyclic terpenoid biomarker compounds are present in ppb to sub-ppb quantities; cheilanthanes, hopanes and steranes are present at roughly equal abundances. Thermodynamically-favored isomers from each of these terpenoid classes are found in greater relative abundance. Tricyclic biomarkers, including $13 \beta(\mathrm{H}), 14 \alpha(\mathrm{H})$ homologs of $\mathrm{C}_{19}$ to $\mathrm{C}_{26}$ cheilanthanes, and a $\mathrm{C}_{24}$ tetracyclic terpane are present in all of the samples. Gammacerane is also found in low abundance in many of the samples. $\mathrm{C}_{27}$ to $\mathrm{C}_{35}$ hopanes of several series including $17 \alpha(\mathrm{H}), 21 \beta(\mathrm{H})$-hopanes, $17 \beta(\mathrm{H}), 21 \alpha(\mathrm{H})$-hopanes (moretanes), $2 \alpha$ - and $3 \beta$-methylhopanes, 25- and 30-norhopane, and $\mathrm{C}_{28}$ dinorhopanes are present (Fig. 2a). $\mathrm{C}_{26}$ to $\mathrm{C}_{30}$ steranes are also found in every sample, those with 27, 28 and 29 carbons being the most abundant (Fig. 2a). Among these, $5 \alpha(\mathrm{H})$, 

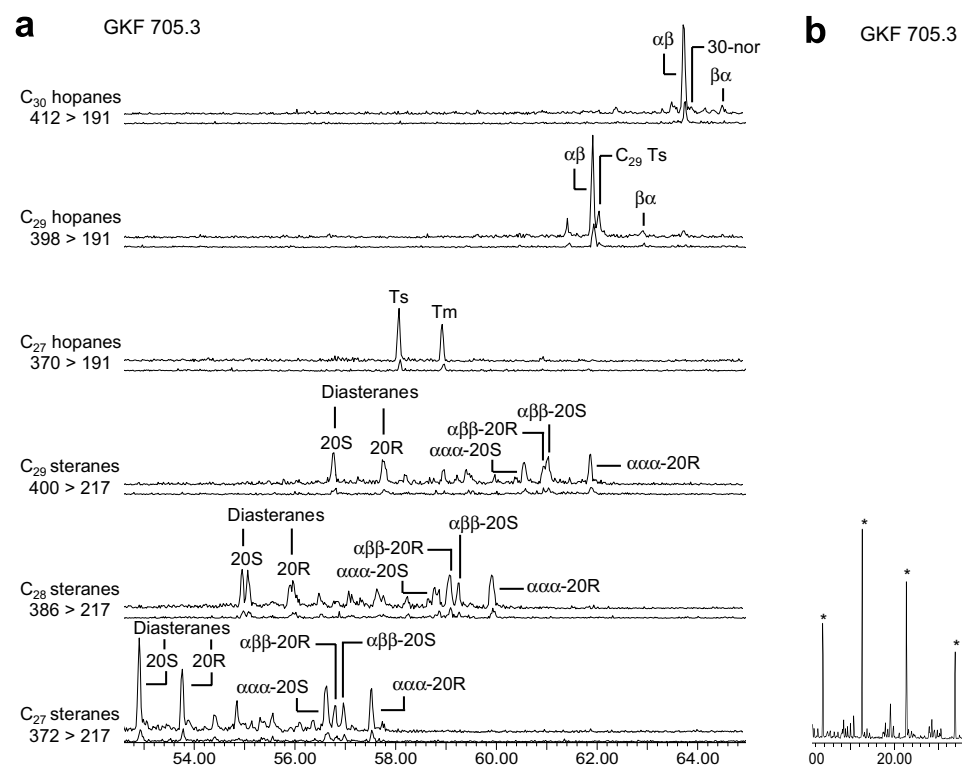

Fig. 2. Typical results (from sample GKF 705.3). (a) Typical Archean biomarker traces. $\mathrm{C}_{27}$ to $\mathrm{C}_{29}$ steranes and $\mathrm{C}_{27}, \mathrm{C}_{29}$ and $\mathrm{C}_{30}$ hopane traces are shown. Sand blank chromatograms shown below each sample trace are to the same absolute scaling. Relative retention times are shown in min. Compound abbreviations: $\mathrm{C}_{30^{-}} \alpha \beta=17 \alpha(\mathrm{H}), 21 \beta(\mathrm{H})$-hopane; 30-nor $=17 \alpha(\mathrm{H}), 21 \beta(\mathrm{H})$-30-norhomohopane; $\mathrm{C}_{30}-\beta \alpha=17 \beta$ $(\mathrm{H}), 21 \alpha(\mathrm{H})$-hopane; $\mathrm{C}_{29}-\alpha \beta=17 \alpha(\mathrm{H}), 21 \beta(\mathrm{H})$-30-norhopane; $\mathrm{C}_{29}$ Ts =18 $\alpha(\mathrm{H}), 21 \beta(\mathrm{H})$-30-norneohopane; $\mathrm{C}_{29}-\beta \alpha=17 \beta(\mathrm{H}), 21 \alpha(\mathrm{H})-30-$ norhopane; $\mathrm{Ts}=\mathrm{C}_{27} 18 \alpha$-trisnorhopane II; Tm $=\mathrm{C}_{27} 17 \alpha$-trisnorhopane; diasteranes $(20 S$ and $20 R$ epimers $)=13 \beta(\mathrm{H}), 17 \alpha(\mathrm{H})$ diasterane; $\alpha \alpha \alpha(20 S$ and $20 R$ epimers $)=5 \alpha(\mathrm{H}), 14 \alpha(\mathrm{H}), 17 \alpha(\mathrm{H})$ sterane; $\alpha \beta \beta(20 S$ and $20 R$ epimers $)=5 \alpha(\mathrm{H}), 14 \beta(\mathrm{H}), 17 \beta(\mathrm{H})$ sterane. (b) Typical Archean saturated hydrocarbon trace (from sample GKF 705.3; $\mathrm{m} / \mathrm{z}$ 85). Internal standard (1 ug 3-methylheneicosane) indicated by " $\xi$ ". Regular $n$-alkane peaks $\left(\mathrm{C}_{13}\right.$ to $\left.\mathrm{C}_{24}\right)$ indicated by “*”. Compound abbreviations: $\mathrm{Pr}=$ pristane; $\mathrm{Ph}=$ phytane.

$14 \alpha(\mathrm{H}), 17 \beta(\mathrm{H})$ and $5 \alpha(\mathrm{H}), 14 \beta(\mathrm{H}), 17 \beta(\mathrm{H})$ regular steranes (both $20 R$ and $20 S$ epimers) and rearranged $13 \beta(\mathrm{H}), 17 \alpha(\mathrm{H})$-diasteranes are the dominant isomers.

Sand blanks are largely free of hydrocarbons and contain very low abundances of terpenoid biomarkers and $n$-alkanes. Average sample to blank ratios were calculated for each compound class using the 17 samples and four sand blanks that were processed using the techniques described in Section 2. Overall, the average sample to blank ratio for cyclic terpenoid biomarkers is 11.58 $( \pm 7.77,1 \sigma \mathrm{SD})$. Separated by compound class, the sample to blank ratio is lower for cheilanthanes $(4.88 \pm 1.57,1 \sigma \mathrm{SD})$ and higher for steranes $(19.70 \pm 15.82,1 \sigma \mathrm{SD})$ and hopanes $(16.38 \pm$ $14.10,1 \sigma \mathrm{SD})$. This is primarily because the sand blanks are dominated by cheilanthane biomarkers. Overall, the average sample to blank ratio for $n$-alkanes is $3.67( \pm 2.81,1 \sigma \mathrm{SD})$. This value may be lower than the values for the cyclic biomarkers because $n$-alkanes are particularly abundant and widespread in the modern environment as a result of fossil fuel burning and petrochemical use. Sand blank alkane yields $(n=4)$ ranged from 83.7 to $805 \mathrm{ng}$, whereas sand blank biomarker yields ranged from 0.93 to $5.18 \mathrm{ng}$; these quantities are significantly lower than the sample bitumen yields. It is possible that further refinement of the methods could increase sample to blank ratio values by eliminating low levels of carry-over between samples and blanks and by reducing sample contact with hydrocarbon aerosols.

\section{Justification of methodology}

In developing the techniques described in Section 2 , we conducted several methodological experiments to test the efficacy of our approach. We sought throughout to minimize the number of sample processing steps and the amount of equipment required, as each handling step is an opportunity for contaminants to be introduced. This section presents the results of several of these methodological 
tests, demonstrating the necessity of, and best practices for, each sample preparation step.

\subsection{Sample preparation: cutting}

Because anthropogenic petroleum products are ubiquitous in the modern environment, particularly around machinery and in industrialized settings such as research labs, even cores obtained without the use of drill fluids and analyzed soon after recovery may contain foreign lipids on their surfaces. To determine if such compounds are present on the outsides of drill core pieces and, if so, how best to eliminate the contamination, we pursued an approach initiated by Brocks (2001) by comparing the extractable hydrocarbon contents of exterior and interior portions of the core samples.

We cut a quarter core piece (GKF 1326.25) into three pieces using a table saw with a diamond-edged blade according to the methods described in Section 2.3 (Fig. 3). After cutting, the three pieces were processed separately. The first, called "untreated," was left intact without further preparation. The second, called "rinsed," was sonicated for $30 \mathrm{~min}$ each in DCM-extracted DI water and DCM prior to further processing. This DCM was also retained and analyzed (called "rinse solvent"). The outer surfaces ( 3 to $5 \mathrm{~mm}$ ) of the third piece were cut off and separated into the flat sides, called "outside flat," and the curved surfaces, called "outside curve," The internal portion (diameter $\sim 9.5 \mathrm{~cm}$ ) was called "inside." The samples from these five treatments were then processed and analyzed in parallel according the methods described in Section 2.

Chromatograms depicting the saturated hydrocarbons from the bitumens of these six samples are presented in Fig. 4. Although all the fractions are dominated by $n$-alkanes, their distributions and abundances are markedly different between the treatments. The hydrocarbons from the inside piece are low in abundance and have a regular, condensate-like carbon number distribution that peaks at about $\mathrm{C}_{18}$. In contrast, all the outside surfaces, the rinsed piece and the untreated piece have higher abundances of hydrocarbons that show strong even/odd carbon number preferences. Such preferences should not persist in high maturity rocks (Peters et al., 2005). These bitumens also have abundant $n$-alkanes greater than $\mathrm{C}_{22}$ and some display bimodal distribution patterns. These features are especially pronounced in the rinse solvent.

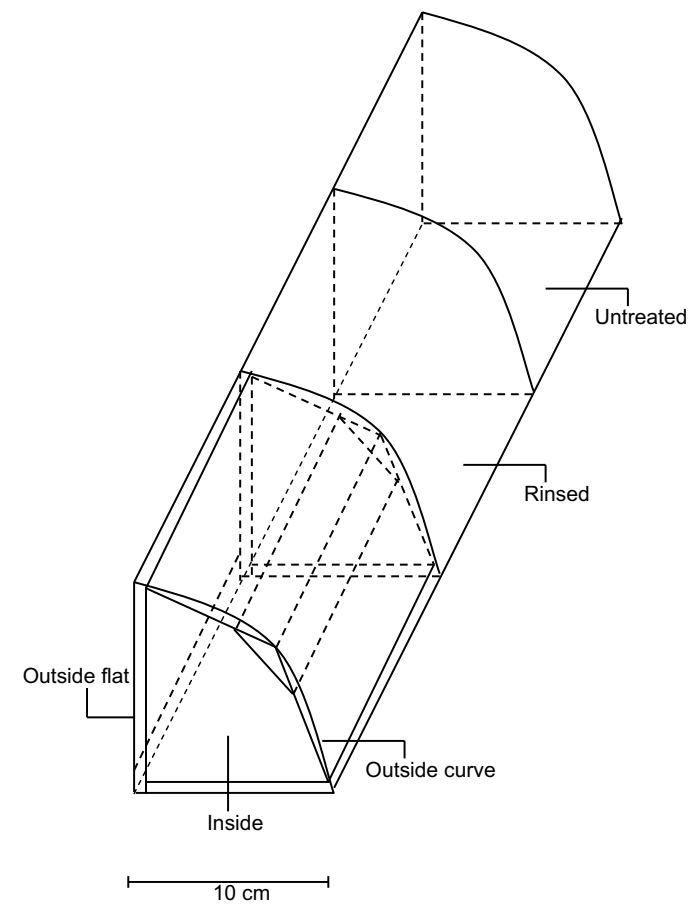

Fig. 3. Diagram of quarter core sample (GKF 1326.25) used in cutting experiment (described in Section 4.1). Bold and dashed lines represent cuts made with table saw.

Additionally, there are other compounds, including branched alkanes, in these pieces that are not present in the inside piece. The occurrence and abundance of these compounds differs between the treatments; the outside, rinsed and untreated pieces are extremely heterogeneous. In contrast, the compounds found in the Agouron and Deep Time Drilling Project inner cores are largely homogeneous and similar to those found in the inside piece of GKF 1326.25.

Cyclic terpenoid biomarker analyses of the bitumens from GKF 1326.25 show parallel patterns. Several series of hopanes and steranes are present in all of the samples. These include $17 \alpha(\mathrm{H})$, $21 \beta(\mathrm{H})$-hopanes, $2 \alpha$ - and $3 \beta$-methlyhopanes, $5 \alpha(\mathrm{H})$, $14 \alpha(\mathrm{H}), 17 \alpha(\mathrm{H})$ and $5 \alpha(\mathrm{H}), 14 \beta(\mathrm{H}), 17 \beta(\mathrm{H})$ regular steranes (both $20 S$ and 20R) and rearranged $13 \beta(\mathrm{H}), 17 \alpha(\mathrm{H})$-diasteranes. Although similar compounds are found in each of the treatments, the relative abundances of rearranged molecules are distinct. In general, the bitumen from the inside piece contains the highest abundance of thermodynamically-favored isomers. For example, $\mathrm{C}_{27}$ 
GKF 1326.25
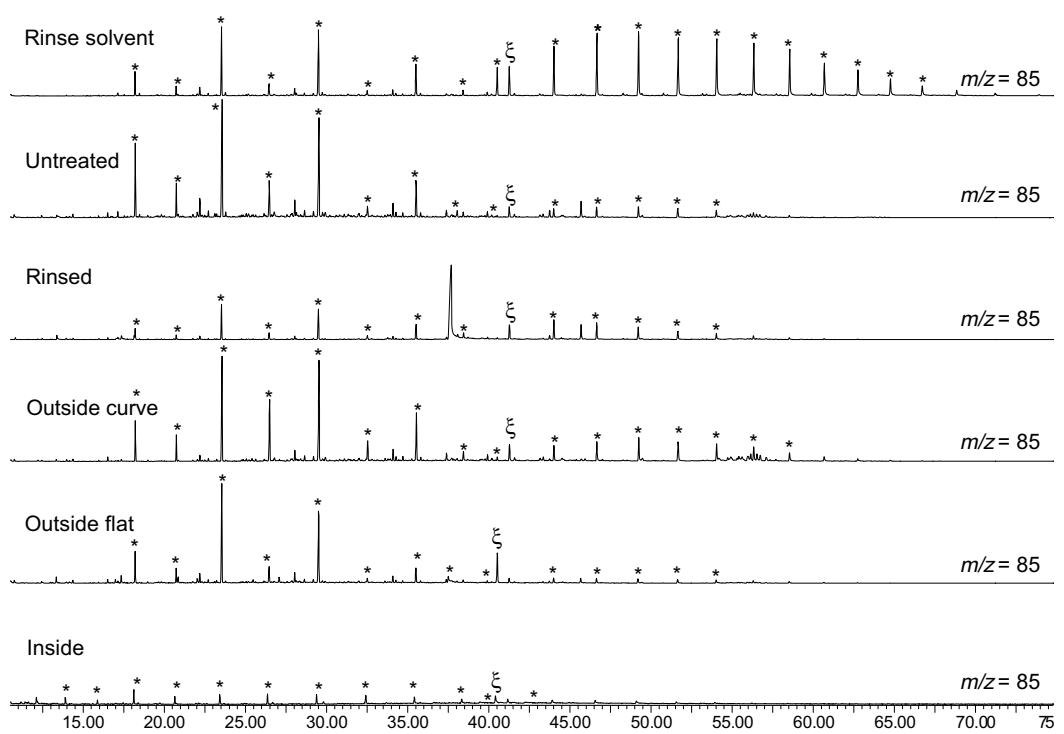

Fig. 4. Saturated hydrocarbon traces $(\mathrm{m} / \mathrm{z}$ 85) of five treatments of GKF 1326.25 and the solvent used to clean the "rinsed" piece. All chromatograms are shown to the same absolute scaling. Relative retention times are shown in min. Internal standard (1 ug 3methylheneicosane) indicated by " $\xi$ ". Regular $n$-alkane peaks indicated by "**”.

$18 \alpha$-trisnorhopane II (Ts) is more stable than $\mathrm{C}_{27}$ $17 \alpha$-trisnorhopane (Tm). In samples with a common organic matter source, the ratio of $\mathrm{Ts}$ to $\mathrm{Tm}$ increases with maturity. As shown in Table 2, the value of Ts/Tm is highest in the inside piece and significantly lower in the other treatments. The rinsed piece has the second highest value. The ratio of diasteranes to regular steranes also increases with thermal maturity (e.g. Peters et al., 2005). As shown in Table 2, the ratio of diasteranes to regular steranes $\left(\mathrm{C}_{27}-\mathrm{C}_{29}\right)$ is highest in the inside piece and second highest in the rinsed piece.

Our data reveal that the compounds found in the outside, untreated and rinsed pieces are not entirely syngenetic to the Archean sediment and are not from a single identifiable source. The hydrocarbons from bitumens associated with outer portions of the cores are highly heterogeneous and almost certainly include material introduced during drilling and/or storage. Only the signature from the inside piece likely represents original, syngenetic, highly mature Archean hydrocarbons. Although sonicating core pieces in DCM appears to move some of the exogenous compounds from the core to the rinse solvent, only cutting off the outer surfaces of the core ade- quately eliminates the foreign contaminants. Therefore, we believe that it is necessary to cleanly cut off the outer 3 to $5 \mathrm{~mm}$ of core material prior to analysis.

\subsubsection{Sample preparation: crushing}

After determining that it was necessary to remove the outer core surfaces, we conducted an experiment to see if the bitumen could be extracted from the inner core solely by sonication in organic solvent without further treatment. We sonicated an inner core piece twice in DCM for $30 \mathrm{~min}$ and processed the bitumen as described in Section 2. We were unable to detect biomarkers (data not shown). This result was expected because in high maturity sediments, bitumen is held tightly in a matrix of recalcitrant kerogen and mineral material. It is therefore largely inaccessible to organic solvents in whole rock form and only becomes extractable after the samples are crushed to a fine powder.

Rock crushing equipment typically used to prepare samples for organic geochemical analysis proved unsuitable for the extremely low blank requirements of Precambrian biomarker analysis. For example, samples crushed in an alumnia cera- 
Table 2

Cutting experiment biomarker ratios

\begin{tabular}{|c|c|c|c|c|c|}
\hline Biomarker ratio & Inside & Rinsed & Outside flat & Outside curve & Untreated \\
\hline $\mathrm{Ts} / \mathrm{Tm}^{\mathrm{a}}$ & 2.15 & 1.70 & 1.44 & 1.34 & 0.88 \\
\hline$\sum \mathrm{C}_{27-29} \mathrm{dia} /$ regular steranes ${ }^{\mathrm{b}}$ & 1.30 & 0.84 & 0.66 & 0.37 & 0.54 \\
\hline
\end{tabular}

a "Ts/Tm" is the ratio of $\mathrm{C}_{27} 18 \alpha$-trisnorhopane II (Ts) to $\mathrm{C}_{27} 17 \alpha$-trisnorhopane (Tm); $2 \sigma$ standard deviations $=0.08$.

b " $\sum \mathrm{C}_{27}-\mathrm{C}_{29}$ dia/regular steranes" is the ratio of the sum of $\mathrm{C}_{27}$ to $\mathrm{C}_{29}$ diasteranes to the sum of the $\mathrm{C}_{27}$ to $\mathrm{C}_{29}$ regular steranes; $2 \sigma$ standard deviations $=0.04$.

mic puck mill (SPEX 8505) were found to contain similar quantities of biomarkers to sand blanks processed alongside them. Because of its size and construction, this type of mill cannot readily be cleaned by submersion and sonication in organic solvents. This may result in less effective cleaning between samples and so can lead to cross-contamination due to sample carry over.

To avoid this problem, we developed a set of solid stainless steel tools that can be cleaned by sonication in solvent. The tools also maximize efficiency and minimize physical stress on the investigator. We designed a stainless steel mortar and pestle (Fig. 1a) that can be clamped down to an aluminum foil-covered board. Samples can be easily crushed to small pieces (ca. 1/4" diameter) without exposure to potentially contaminating surfaces and without sample loss. We then use a small stainless steel puck mill (Fig. 1b, $9 \mathrm{~cm}$ diameter $\times 5.4 \mathrm{~cm}$ height) to crush the samples to a fine powder.

\subsection{Extraction and fractionation}

We tested two methods for solvent extraction of crushed rock powder. Initially, a Dionex accelerated solvent extractor (ASE) was used. This instrument has the advantage of performing automated extraction under elevated temperature and pressure, potentially increasing solvent access to the rock matrix. However, we found that an ASE introduces low, but unpredictable, levels of contamination. For example, ASE-extracted sand blanks can contain high abundances of biomarkers of similar composition to those found in samples processed alongside them. This may be because there is plastic tubing in an ASE that is not optimally suited to trace hydrocarbon extraction. Additionally, carry over may occasionally occur between samples if small amounts of residual extract are not fully flushed through the machine.

In an effort to bypass this unpredictable source of contamination, we conducted an experiment to see if bitumen could effectively be liberated from high maturity powder by sonication. We divided the outside cuttings of one sample ("GKP 332.5 outside") and a sand blank into two equal parts. One half of each was extracted using an ASE; the other was extracted using two rounds of sonication in DCM. For this experiment, and in general, we chose to use two rounds of sonication because we found that subsequent extraction yield undetectable quantities of hydrocarbons. For both the sample and the blank, we found that extraction using the ASE produced approximately a twofold increase in biomarker yield (Table 3). Given the cost of unpredictable contamination introduced by use of an ASE, the decrease in yield with sonication extraction is relatively acceptable. When trace contaminants and sample carry over are critical concerns, high maturity bitumens can be effectively extracted using sonication methods as described in Section 2.4.

\subsection{Preparation of bitumen II}

As discussed above, studies of high maturity organic matter must take into account the possibility not only of laboratory contamination but also of migration of geologically younger hydrocarbons into the sediment. These mobile contaminants are most likely to be extractable from the whole rock powder as a primary extract (termed "bitumen I"). To assess the syngeneity of our primary extracts, we prepared a set of demineralized powders and re-extracted them to obtain a second bitumen, termed "bitumen II." Because the hydrocarbons in bitumen II are not originally solvent extractable and are considered more closely associated with the kerogen and mineral matrix of the rocks, they are less likely to be contaminated by external, migrated bitumen. If bitumen I and bitumen II extracts from a given rock sample have similar compositions, it would strongly suggest that they derive from the same pool of syngenetic organic matter. 
Table 3

Extraction experiment biomarker yields

\begin{tabular}{lllll}
\hline & $\begin{array}{l}\text { Sonication extraction }{ }^{\mathrm{a}} \text { GKF 332.5 } \\
\text { outside }\end{array}$ & $\begin{array}{l}\text { ASE }^{\mathrm{b}} \text { GKF 332.5 } \\
\text { outside }\end{array}$ & $\begin{array}{l}\text { Sonication extraction sand } \\
\text { blank }\end{array}$ & $\begin{array}{l}\text { ASE sand } \\
\text { blank }\end{array}$ \\
\hline $\begin{array}{l}\text { Extractable hopanes } \\
\text { (ng) }\end{array}$ & 2.39 & 5.49 & 0.50 & 1.83 \\
$\begin{array}{c}\text { Extractable steranes } \\
\text { (ng) }\end{array}$ & 4.81 & 9.08 & 1.05 & 2.61 \\
\hline
\end{tabular}

${ }^{\text {a }}$ Yield for samples extracted by two rounds of sonication for $30 \mathrm{~min}$ in DCM.

${ }^{\mathrm{b}}$ Yield for samples extracted using an accelerated solvent extractor.

In this study we demineralized and re-extracted seven samples. Hydrocarbon abundance, biomarker carbon number distributions, degree of isomerization and indicated thermal maturity are similar between the two sets of bitumens. A set of biomarker chromatograms from a representative sample (GKF 314.65) is shown in Fig. 5. The same compounds, in approximately the same relative abundances, are found in both bitumens. For example, $\mathrm{Ts} / \mathrm{Tm}$ and the degree of diasterane isomeriza- tion ("dia S/R") are nearly identical between bitumen I and bitumen II for this sample (Fig. 5).

Despite these similarities, there are systematic differences between bitumens I and II. These differences may be explained by a closer association of the latter with the rock mineral matrix. For example, the methyl rearrangement reactions that lead to diasteranes are thought to be catalyzed by clay minerals (Rubenstein et al., 1975; Sieskind et al., 1979). If bitumen II is more closely associated with

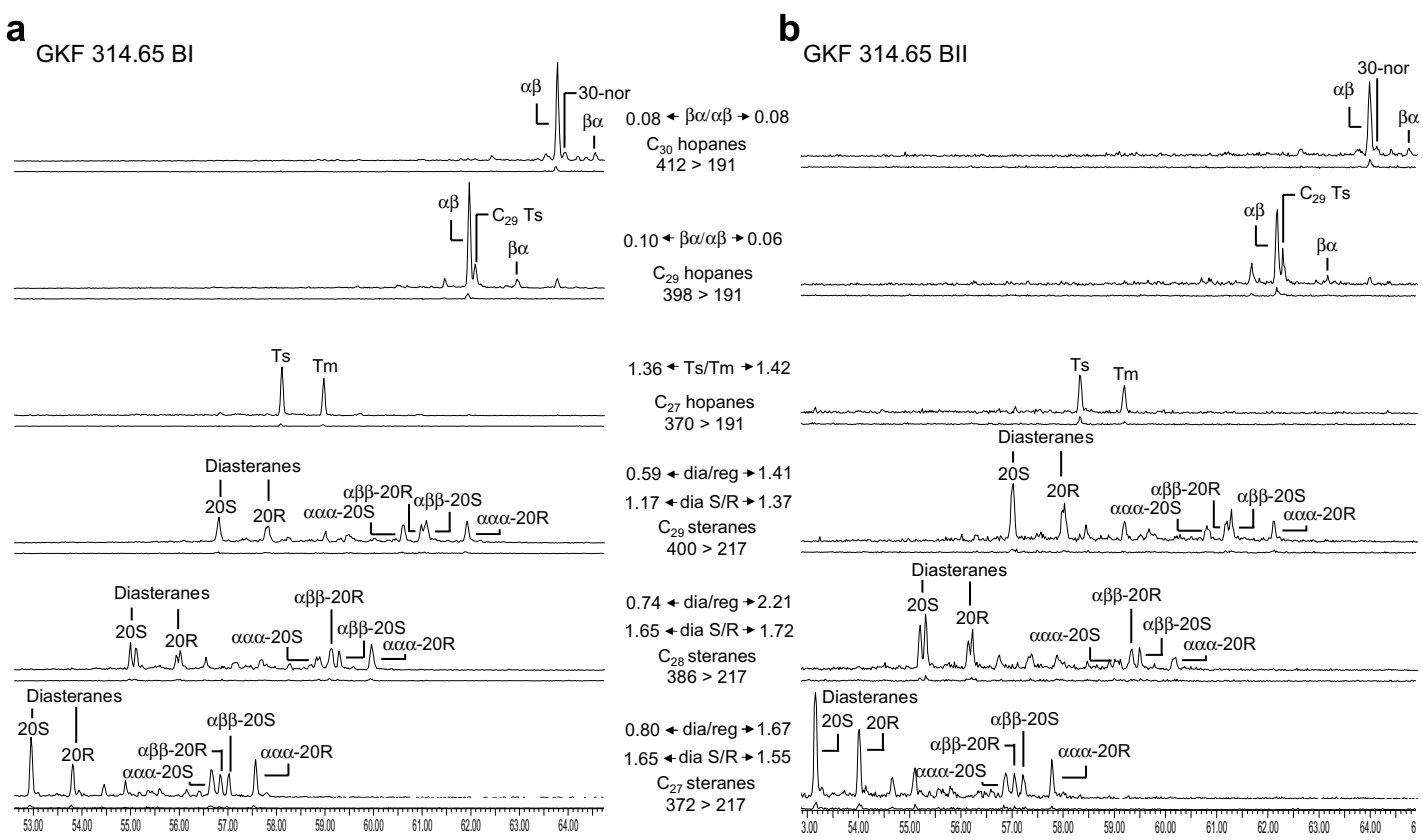

Fig. 5. Typical biomarker results (from sample GKF 314.65) from MRM-GC-MS-MS analysis of bitumen I (a) and bitumen II (b). Relative retention times are shown in min. $\mathrm{C}_{27}$ to $\mathrm{C}_{29}$ steranes and $\mathrm{C}_{27}$ hopane traces are shown; MRM transitions and relevant ratios for bitumen I ("BI") and bitumen II ("BII") are indicated between corresponding traces. Sand blank chromatograms are shown below each sample trace to the same absolute scaling. Compound abbreviations are as in Fig. 2; "dia S/R" is the ratio of $20 S$ to $20 R$ epimers of $13 \beta(\mathrm{H}), 17 \alpha(\mathrm{H})$ diasterane; "dia/reg" is the ratio of $13 \beta(\mathrm{H}), 17 \alpha(\mathrm{H})$ diasteranes $(20 S$ and $20 R$ epimers) to $5 \alpha(\mathrm{H}), 14 \alpha(\mathrm{H}), 17 \alpha(\mathrm{H})$ and $5 \alpha(\mathrm{H}), 14 \beta(\mathrm{H}), 17 \beta(\mathrm{H})$ regular steranes ( $20 S$ and $20 R$ epimers); "Ts/Tm" is the ratio of $\mathrm{C}_{27} 18 \alpha$-trisnorhopane II to $\mathrm{C}_{27} 17 \alpha$-trisnorhopane $(\mathrm{Tm}) ; " \beta \alpha / \alpha \beta$ " is the ratio of $17 \beta(\mathrm{H}), 21 \alpha(\mathrm{H})$ hopane (moretane) to $17 \alpha(\mathrm{H}), 21 \beta(\mathrm{H})$ hopane. 
the mineral matrix and clays, it would be likely to contain greater abundances of diasteranes than bitumen I. As shown in Fig. 5, diasterane/regular sterane ratio values are higher for bitumen II than bitumen I, a pattern seen for most samples in this study. It is especially important when extracting trace amounts of hydrocarbons to conduct such assessments of syngeneity (see Section 5).

\section{4. $G C-M S$ and $M R M-G C-M S-M S$}

Analysis of high maturity sediments with trace quantities of biomarkers is facilitated by recent improvements in instrumentation. Highly sensitive MS methods such as MRM-GC-MS-MS can reliably detect sub-ppb quantities of hydrocarbons. Certain molecules, including cyclic terpenoid biomarkers, fragment to known product ions in a mass spectrometer via predictable pathways determined by their chemical structures. Appropriate precursor-product $m / z$ values are summarized in Table 1 . The selectivity of MRM techniques enhances signal to noise ratio, allowing reliable detection of biomarkers at much lower abundances than is possible using other approaches such as GC-MS in full scan or selected ion monitoring modes.

\section{Discussion: data analysis and assessment of syngeneity}

The methods described in Section 2 have greatly improved our confidence in our analysis of high maturity Precambrian organic matter. These methods produce low levels of background contamination and relatively high sample biomarker yield. However, it is still important to scrutinize each analysis for evidence of contamination and to conduct more general tests for syngeneity over the entire data set. The detected compounds must be demonstrably Precambrian in origin and must be derived from their host rocks to be meaningfully interpreted as molecular fossils.

First order evidence for contamination of Precambrian rock extracts would include the presence of biomarkers for later-evolving organisms, particularly polyterpenoids of higher plants; none of these were detected. Indigenous organic matter with a single source should also show consistent patterns of molecular rearrangement and isomerization between structural homologues (e.g., members of a carbon number series). The full range of saturated and aromatic hydrocarbons and biomarker com- pounds should be considered for each individual analysis because an aberrant signal might be observed in only one or a few compound classes. For the purposes of our study, which included assessing microbial diversity from some of the earliest well-preserved sedimentary sequences in the geologic record, samples that bore any anomalous signals were excluded. Thus, although 70 samples from the Agouron cores were processed, only 33 were included in the final database (Waldbauer et al., 2007). Samples were excluded for a variety of reasons. Some have bimodally distributed $n$ alkane distributions, others contain biomarker abundances similar to a parallel blank, and others display inconsistent or characteristically immature ratios of rearranged biomarker compounds.

For example, the sample depicted in Fig. 6 (GKP 741.54) was excluded because its biomarker and $n$ alkane chromatograms display characteristics of immature organic matter. Unlike in syngenetic Archean bitumens, several thermodynamically unfavored isomers, including $\mathrm{C}_{27} 17 \alpha$-trisnorhopane (Tm) and $5 \alpha(\mathrm{H}), 14 \alpha(\mathrm{H}), 17 \alpha(\mathrm{H})$ regular steranes $(20 S$ and $20 R$ ) are present at greater abundance than more thermodynamically favored rearranged isomers (Fig. 6a). Additionally, the degree of $\alpha / \beta$ isomerization and $S / R$ epimerization is not consistent between sterane carbon numbers (Fig. 6a). Steranes from a single, syngenetic organic matter source that has been exposed to a set of temperature and pressure conditions should all be rearranged in the same manner. Finally, as shown in Fig. 6b, the saturated hydrocarbon fraction from this sample is not dominated by low molecular weight $n$-alkanes. Instead, it contains branched and unsaturated compounds at greater abundances than the internal standard (i.e., greater than $1 \mu \mathrm{g}$ ). As described in Section 3, these characteristics are not typical of Archean bitumen and suggest that this sample is contaminated.

In addition to this careful scrutiny of individual sample analyses, further intra- and inter-sample comparisons should be undertaken to demonstrate syngeneity. It is important not only that individual samples be free of contamination, but also that the bitumens are syngenetic to their host material. Comparisons between bitumens I and II, between stratigraphically correlated samples, and across significant stratigraphic boundaries, can assist with determination of syngeneity.

Samples may be demineralized and re-extracted (see Sections 2.5 and 4.3) to yield bitumen II 
a
GKF 741.54

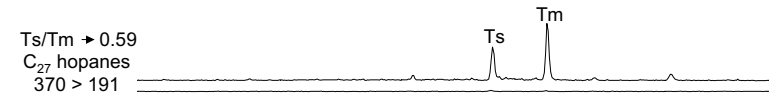

$\mathrm{dia} / \mathrm{reg} \rightarrow 0.44$ reg $\alpha \beta \beta S / R \rightarrow 2.67$ $\mathrm{C}_{29}$ steranes $400>217$

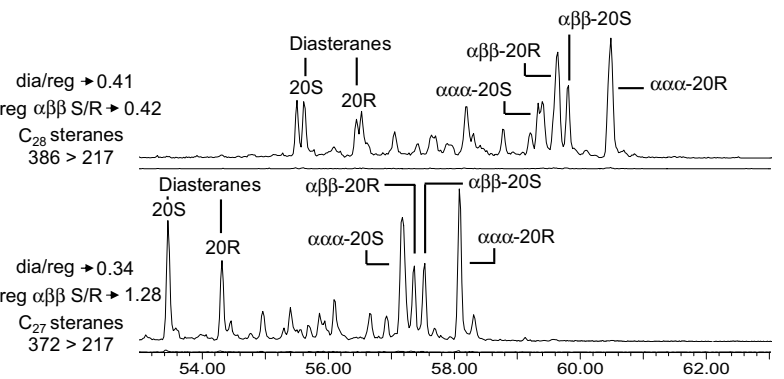

b GKF 741.54

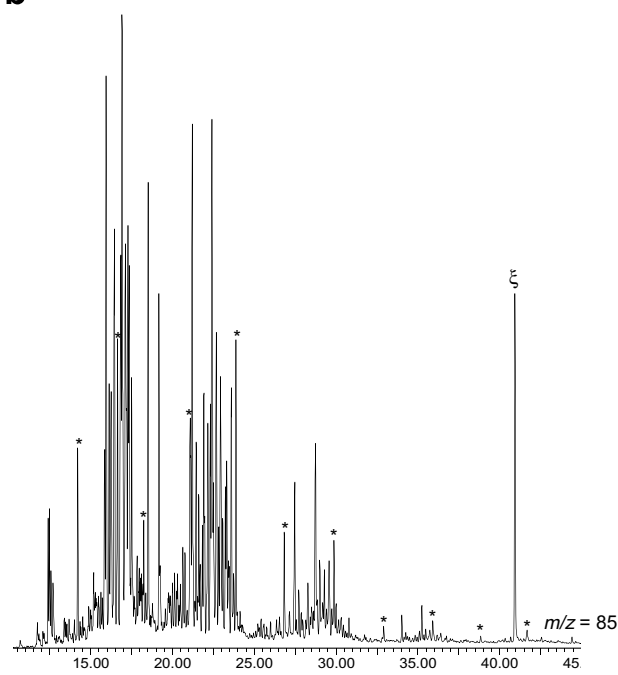

Fig. 6. Example of a potentially contaminated sample (GKF 741.54). Compound abbreviations and symbols are as in Figs. 2 and 5; "reg $\alpha \beta \beta \mathrm{S} / \mathrm{R}$ " is the ratio of $20 S$ to $20 R$ epimers of $5 \alpha(\mathrm{H}), 14 \beta(\mathrm{H}), 17 \beta(\mathrm{H})$ sterane. Relative retention times are shown in min. (a) Biomarker chromatograms of $\mathrm{C}_{27}$ to $\mathrm{C}_{29}$ steranes and $\mathrm{C}_{27}$ hopanes; sand blank traces are shown below each chromatogram to the same absolute scaling. (b) Chromatogram of saturated hydrocarbon fraction $(\mathrm{m} / \mathrm{z} 85)$.

fractions. Because bitumen II represents a pool of hydrocarbons that was not solvent-accessible in the crushed rock powder, it is less likely to be affected by mobile contaminants than bitumen I. Therefore, for a given sample, if bitumen I is similar in composition to bitumen II (with predictable differences as described in Section 4.3), it is likely to be derived from the same pool of syndepositional organic matter instead of from a separate source.

Comparisons between stratigraphically correlated samples from geographically distinct cores can also strongly support claims of syngeneity. If feasible, cores drilled at close proximity can be used to assess the lateral variability along the strike of a given unit. Such cores also allow for comparison of directly correlated samples, potentially strengthening claims of syngeneity. Given the resources necessary to obtain drill core material, it may be difficult to justify 're-sampling' the same strata; nevertheless, we have found inter-core comparisons to be valuable tests of syngeneity. For example, in the case of the Agouron Griqualand Drilling Project, cores GKF and GKP were drilled $24 \mathrm{~km}$ apart through different but correlatable portions of a depositional system. This allows for the comparison of chemostratigraphic patterns and variations between corre- lated samples through a variety of depositional systems. By making such comparisons between GKP and GKF, we observed strong correlations in biomarker content, especially in the deepwater facies (Waldbauer et al., 2007). Inter-core correlations are strong evidence both for depositional control of organic matter content and the syngeneity of molecular fossils.

Comparison of Archean samples to younger units across an unconformity may also support claims of syngeneity. In cases where Phanerozoic sediments unconformably overlie Precambrian material, a strong geochemical contrast should exist. This contrast would be overprinted and diminished if contaminants were introduced during drilling, storage, or handling. The Agouron Drilling project recovered approximately $200 \mathrm{~m}$ of a PermoCarboniferous diamictite that was deposited directly on top of the youngest Precambrian unit, creating a 2 billion year unconformity (Visser, 1989). Bitumens extracted from this diamictite are in fact geochemically distinct from the underlying Archean material. The younger material is much less thermally mature and substantially more heterogeneous. This clear contrast provides additional evidence that bitumens extracted from Archean units are in fact syngenetic and were not contaminated either by hydrocarbons 
from younger units or from the external environment.

All of the characteristics of each sample and its corresponding blank should be examined in tandem with complete data set analyses of syngeneity as described above. Given the low biomarker yields from Precambrian sediments and the ubiquitous environmental presence of petroleum-derived hydrocarbons, sample contamination can easily occur. Throughout data analysis, it remains up to the investigator to determine the best and most consistent course of action - a single set of criteria is unlikely to be applicable to all situations.

\section{Conclusions}

Key aspects of methods described in this paper would not have been feasible during the 1960s when the organic geochemistry of Precambrian rocks was first studied. Not only did researchers not initially recognize the prevalence of potential contaminants, but also the materials and instrumentation necessary to successfully process high maturity samples were not available. Materials such as high purity organic-free solvents were not commercially available and investigators had to distill their own (Hoering, 1967). Pure, isotopically labeled internal standards were similarly not available. Additionally, instrumentation such as highly sensitive GC-MSMS was not refined until the 1980s.

The procedures presented take advantage of these advances in technology. While we believe that the methods are well suited for the analysis of trace amounts of hydrocarbons, as described above, each data set should be carefully examined for any evidence of contamination or sample carry over. Application of the techniques allows researchers to do now that which was impossible in the mid-20th century, that is, study demonstrably Precambrian high maturity organic matter to learn about microbial evolution and early life on Earth.

\section{Acknowledgements}

Funding for this research came from the NSF (EAR0418619), the NASA Exobiology Program (Grant\# NNG05GN62G), the Agouron Institute and the NASA Astrobiology Institute. J.R.W. receives support through an NDSEG Fellowship from the Office of Naval Research. We would like to thank the Griqualand Project team members, the Agouron Institute and the members of the Deep
Time Drilling Project and the NASA Astrobiology Institute for the core samples. We specifically acknowledge Alex Birch, Francis McDonald, John Dunlop and Les Bonser for overseeing the core drilling and field operations. Thanks go to Roger Buick, Ariel Anbar, Joe Kirschvink and Dawn Sumner for on-site sample collection and core curation. For design and manufacture of our crushing tools, we would like to thank Andrew Gallant and the MIT Central Machine Shop. We would also like to thank Gordon Love for many useful discussions on biomarker analysis, Rick Kayser for lab. assistance and Carolyn Colonero for maintaining the mass spectrometers. Finally, we thank Kenneth Peters and an anonymous reviewer for thoughtful comments.

\section{Associate Editor-K. E. Peters}

\section{References}

Anbar, A.D., Duan, Y., Lyons, T.W., Arnold, G.L., Kendall, B., Creaser, R.A., Kaufman, A.J., Gordon, G.W., Scott, C., Garvin, J., Buick, R., 2007. A whiff of oxygen before the Great Oxidation Event? Science 317, 1903-1906.

Armstrong, R.A., Compton, W., Retief, E.A., Wilke, N.J., 1986. Ages and isotopic evolution of the Ventersdorp volcanics. In: Geocongress 1986. Geological Society of South Africa, South Africa, pp. 89-92.

Barghoorn, E.S., 1957. Origin of Life. Geological Society of America Memoir 67, 75-86.

Barghoorn, E.S., Meinschein, W.G., Schopf, J.W., 1965. Paleobiology of a Precambrian shale. Science 148, 461472.

Barton, E.S., Altermann, W., Williams, I.S., Smith, C.B., 1994. $\mathrm{U}-\mathrm{Pb}$ zircon age for a tuff in the Campbell Group, Griqualand West Sequence, South Africa; implications for early Proterozoic rock accumulation rates. Geology 22, 343-346.

Bekker, A., Holland, H.D., Wang, P.L., Rumble III, D., Stein, H.J., Hannah, J.L., Coetzee, L.L., Beukes, N.J., 2004. Dating the rise of atmospheric oxygen. Nature 427, 117-120.

Brocks, J.J., 2001. Molecular Fossils in Archean Rocks. Sydney University, Sydney.

Brocks, J.J., Buick, R., Logan, G.A., Summons, R.E., 2003a. Composition and syngeneity of molecular fossils from the 2.78 to 2.45 billion-year-old Mount Bruce Supergroup, Pilbara Craton, Western Australia. Geochimica et Cosmochimica Acta 67, 4289-4319.

Brocks, J.J., Buick, R., Summons, R.E., Logan, G.A., 2003b. A reconstruction of Archean biological diversity based on molecular fossils from the 2.78 to 2.45 billion-year-old Mount Bruce Supergroup, Hamersley Basin, Western Australia. Geochimica et Cosmochimica Acta 67, 4321-4335.

Brocks, J.J., Logan, G.A., Buick, R., Summons, R.E., 1999. Archean molecular fossils and the early rise of eukaryotes. Science 285, 1033-1036.

Brocks, J.J., Pearson, A., 2005. Building the biomarker tree of life. Review in Mineralogy and Geochemistry 59, 233-258. 
Brocks, J.J., Summons, R.E., 2003. Sedimentary hydrocarbons, biomarkers for early life. In: Holland, H.D., Turekian, K.K. (Eds.), Treatise on Geochemistry. Pergamon, Oxford, pp. 63 115.

Dutkiewicz, A., Volk, H., George, S.C., Ridley, J., Buick, R., 2006. Biomarkers from huronian oil-bearing fluid inclusions: An uncontaminated record of life before the Great Oxidation Event. Geology 34 (6), 437-440.

Eglinton, G., Scott, P.M., Belsky, T., Burlingame, A.L., Calvin, M., 1964. Hydrocarbons from a one-billion-year-old sediment. Science 145, 263-264.

Eigenbrode, J.L., 2004. Late Archean microbial ecology: An integration of molecular, isotopic and lithological studies. In: Earth Sciences. The Pennsylvania State University, State College.

Hayes, J.M., Kaplan, I.R., Wedeking, K.W., 1983. Precambrian organic geochemistry, preservation of the record. In: Schopf, J.W. (Ed.), Earth's Earliest Biosphere, Its Origin and Evolution. Princeton University Press, Princeton, pp. 93-134.

Hoering, T.C., 1966. Criteria for suitable rocks in Precambrian organic geochemistry. Carnegie Institution of Washington Yearbook 65, 365-372.

Hoering, T.C., 1967. The organic geochemistry of Precambrian rocks. In: Abelson, P.H. (Ed.), Researches in Geochemistry, vol. 2. John Wiley, New York, pp. 87-111.

Imbus, S.W., McKirdy, D.M., 1993. Organic geochemistry of Precambrian sedimentary rocks. In: Engel, M.H., Macko, S.A. (Eds.), Organic Geochemistry. Plenum Press, New York, pp. 657-684.

Kaufman, A.J., Johnston, D.T., Farquhar, J., Masterson, A.L., Lyons, T.W., Bates, S., Anbar, A., Arnold, G.L., Garvin, J., Buick, R., 2007. Late Archean biospheric oxygenation and atmospheric evolution. Science 317, 1900-1903.

Leventhal, J., Suess, S.E., Cloud, P., 1975. Nonprevalence of biochemical fossils in kerogen from pre-Phanerozoic sediments. Geology 72, 4706-4710.

McKirdy, D.M., 1974. Organic geochemistry in Precambrian research. Precambrian Research 1, 75-137.

Meinschein, W.G., 1965. Soudan Formation: organic extracts of early Precambrian rocks. Science 150, 601-605.
Nagy, B., 1970. Porosity and permeability of the early Precambrian Onverwacht chert: Origin of the hydrocarbon content. Geochimica et Cosmochimica Acta 34, 525-526.

Peters, K.E., Walters, C.C., Moldowan, J.M., 2005. The Biomarker Guide. Cambridge University Press, Cambridge, 1155 pp.

Rubenstein, I., Seiskind, O., Albrecht, P., 1975. Rearranged steranes in a shale: Occurrence and simulated formation. Journal of the Chemical Society, Perkin Transactions I, 18331836.

Sanyal, S.K., Kvenvolden, K.A., Marsden, S.S.J., 1971. Permeabilities of Precambrian Onverwacht cherts and other low permeability rocks. Nature 232, 325-327.

Schroder, S., Lacassi, J.P., Beukes, N.J., 2006. Stratigraphic and geochemical framework of the Agouron drill cores, Transvaal Supergroup (Neoarchean-Paleoproterozoic, South Africa). South African Journal of Geology 109, 23-54.

Sieskind, O., Joly, G., Albrecht, P., 1979. Simulation of the geochemical transformation of sterols: Superacid effects of clay minerals. Geochimica et Cosmochimica Acta 43, 16751679 .

Sumner, D.Y., Bowring, S.A., 1996. U-Pb geochronologic constraints on deposition of the Campbellrand Subgroup, Transvaal Supergroup, South Africa. Precambrian Research $79,25-35$

Visser, J.N.J., 1989. The Permo-Carboniferous Dwyka Formation of Southern Africa: Deposition by a predominantly subpolar marine ice sheet. Palaeogeography, Palaeoclimatology, Palaeoecology 70, 377-391.

Waldbauer, J.R., Sherman, L.S., Sumner, D.Y., Summons, R.E., 2007. Late Archean molecular fossils from the Transvaal Supergroup record the antiquity of microbial diversity and aerobiosis. Precambrian Research.

Walraven, F., Martini, J., 1995. Zircon Pb-evaporation age determinations of the Oak Tree Formation, Chuniespoort Group, Transvaal Sequence: Implications for TransvaalGriqualand West basin correlations. South African Journal of Geology 98, 58-67. 


\title{
Appendix C
}

\section{Proteorhodopsin photosystem gene expression enables photophosphorylation in a heterologous host}

\author{
Asunción Martínez, Alexander S. Bradley, Jacob R. Waldbauer, \\ Roger E. Summons and Edward F. DeLong
}

Reprinted with permission from Proceedings of the National Academy of Sciences (C) 2007 The National Academy of Sciences of the USA

Martínez, A., Bradley, A.S., Waldbauer, J.R., Summons, R.E. and DeLong, E.F. (2007)

Proteorhodopsin photosystem gene expression enables photophosphorylation in a heterologous host. Proceedings of the National Academy of Sciences 104: 5590-5595. 


\title{
Proteorhodopsin photosystem gene expression enables photophosphorylation in a heterologous host
}

\author{
A. Martinez*, A. S. Bradley ${ }^{\dagger}$, J. R. Waldbauer ${ }^{\ddagger}$, R. E. Summons ${ }^{\dagger}$, and E. F. DeLong*§ \\ *Department of Civil and Environmental Engineering, Division of Biological Engineering, and ${ }^{\dagger}$ Department of Earth, Atmospheric, and Planetary Sciences, \\ Massachusetts Institute of Technology, Cambridge, MA 02139; and ₹Joint Program in Chemical Oceanography, Woods Hole Oceanographic Institution \\ and Massachusetts Institute of Technology, Cambridge, MA 02139
}

Edited by W. Ford Doolittle, Dalhousie University, Halifax, NS, Canada, and approved February 9, 2007 (received for review December 22, 2006)

Proteorhodopsins (PRs) are retinal-containing proteins that catalyze light-activated proton efflux across the cell membrane. These photoproteins are known to be globally distributed in the ocean's photic zone, and they are found in a diverse array of Bacteria and Archaea. Recently, light-enhanced growth rates and yields have been reported in at least one PR-containing marine bacterium, but the physiological basis of light-activated growth stimulation has not yet been determined. To describe more fully PR photosystem genetics and biochemistry, we functionally surveyed a marine picoplankton large-insert genomic library for recombinant clones expressing PR photosystems in vivo. Our screening approach exploited transient increases in vector copy number that significantly enhanced the sensitivity of phenotypic detection. Two genetically distinct recombinants, initially identified by their orange pigmentation, expressed a small cluster of genes encoding a complete PR-based photosystem. Genetic and biochemical analyses of transposon mutants verified the function of gene products in the photopigment and opsin biosynthetic pathways. Heterologous expression of six genes, five encoding photopigment biosynthetic proteins and one encoding a PR, generated a fully functional PR photosystem that enabled photophosphorylation in recombinan Escherichia coli cells exposed to light. Our results demonstrate that a single genetic event can result in the acquisition of phototrophic capabilities in an otherwise chemoorganotrophic microorganism and they explain in part the ubiquity of PR photosystems among diverse microbial taxa.

photoheterotrophy | rhodopsin | lateral gene transfer | marine | metagenomics

D roteorhodopsins (PRs) are retinal-binding membrane proteins belonging to the rhodopsin family. Prokaryotic members of this family include photosensors (sensory rhodopsins) transmembrane proton pumps (bacteriorhodopsins, xanthorohodopsin, and PRs), and transmembrane chloride pumps (halorhodopsins). Originally discovered in Archaea, rhodopsins were later identified in Gammaproteobacteria of the SAR86 group during a cultivation-independent genomic survey. Dubbed proteorhodopsin, this photoprotein functions as a light-activated proton pump when expressed in Escherichia coli in the presence of exogenously added retinal (1). Since then, numerous molecular surveys have demonstrated that PR genes are ubiquitous in bacteria inhabiting the ocean's photic zone (2-9). An estimated $13 \%$ of bacteria in marine picoplankton populations, as well as a significant fraction of planktonic Euryarchaeota, contain a PR gene $(4,8)$. In a number of marine bacteria, retinal biosynthetic genes and PR are genetically linked, and their lateral transfer and retention appear to be relatively common events, indicating that the photosystem confers a significant fitness advantage ( 3 , $4,7,10,11)$. A recent report of light-stimulated growth in a PR-containing marine flavobacterium supports this hypothesis (11). Despite all of these observations however, the various specific functions and physiological roles of diverse marine microbial PRs remain to be fully described.

5590-5595 | PNAS | March 27, 2007 | vol. 104 | no. 13
To further characterize PR photosystem structure and function, we directly screened large-insert DNA libraries derived from marine picoplankton for visibly detectable PR-expressing phenotypes. In this report, we describe completely intact PRbased photosystems that can be functionally expressed in $E$. coli, without addition of exogenous photopigment (e.g., retinal or its precursors). Analyses of insertional mutants verified the functional annotation of each gene product in the photosystem biosynthetic pathway. We also show that light-activated, PRcatalyzed proton translocation, by the chemiosmotic potential it generates, activates photophosphorylation in E. coli.

Results

Screening a Fosmid Library for in Vivo PR Photosystem Expression. When E. coli expresses a PR apoprotein from an inducible promoter on a high-copy number plasmid, the cells acquire a red or orange pigmentation in the presence of exogenous all-trans retinal $(1,12)$. Retinal addition is required because $E$. coli lacks the ability to biosynthesize retinal or its precursor, $\beta$-carotene. Based on these observations, we screened for PR-containing clones on retinal-containing LB agar plating medium, which we expected would display an orange to red phenotype under these conditions. To enhance assay sensitivity, we used the copycontrol system present in our fosmid vector that allowed a controlled transition from one copy per cell to multiple (up to $100)$ vector copies upon addition of the inducer L-arabinose (13).

A fosmid library prepared from ocean surface water picoplankton containing 12,280 clones ( $\approx 440 \mathrm{Mb}$ of cloned DNA)

(14) was screened by using the above approach. Three orange colonies were identified as potential PR-expressing clones on the LB-retinal-L-arabinose agar plates. All three showed no pigmentation in the absence of the high-copy number inducer. Unexpectedly, these clones also displayed an orange phenotype in the absence of L-retinal when induced to high copy number. The sequence of one clone, HF10_19P19, revealed the presence of a $\mathrm{PR}$ gene near the fosmid vector junction (see below). Because the clones exhibited orange pigmentation in the absence of exogenous retinal, we expected that they must also be expressing retinal biosynthetic genes. Two clones, HF10_25F10 and

Author contributions: A.M. and E.F.D. designed research; A.M., A.S.B., and J.R.W. performed research: A.M A. S.B. J.R.W. R.E.S., and E.F.D. analyzed data; and A.M. and EF.D. wrote the paper.

The authors declare no conflict of interest.

This article is a PNAS direct submission.

Abbreviations: CCCP, carbonylcyanide $m$-chlorophenylhydrazone; $D C C D, N, N^{\prime}$-dicyclohexylcarbodiimide: FPP, farnesyl diphosphate; GGPP, geranylgeranyl pyrophosphate: IPP, isopentenyl diphosphate; PR, proteorhodopsin.

Data deposition: The sequences reported in this paper have been deposited in the GenBank database [accession nos. EF100190 (HF10_19P19) and EF100191 (HF10_25F10)].
dis

STo whom correspondence should be addressed at: Department of Civil and Environmenta Engineering, 48-427, Massachusetts Institute of Technology, 15 Vassar Street, Cambridge,

This article contains supporting information online at www.pnas.org/cgi/content/full/ 0611470104/DC1.

๑ 2007 by The National Academy of Sciences of the USA 
A

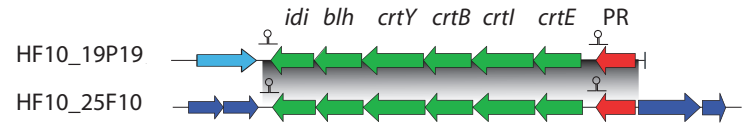

B

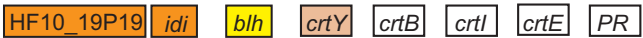

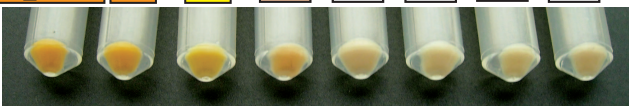

C

IPP
IPP $\delta$-isomerase

(idi)

D DMAPP

$\underset{\text { (ispA) }}{\text { FPP synthase }} F^{\mathrm{IPP}}$

huth $\mathrm{CH}_{2} \mathrm{O} \odot$ PP FP

$$
\underset{\text { crtE }}{\text { GGPP synthase }} \downarrow^{\text {IPP }}
$$

htw $\mathrm{CH}_{2} \mathrm{O} \odot \odot$ GGPP

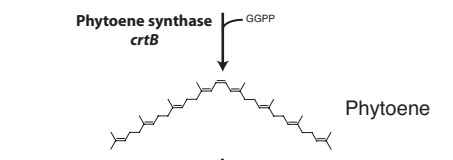

$$
\underset{\text { crtl }}{\text { Phytoene dehydrogenase }} \downarrow
$$

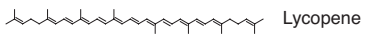

$$
\underset{\text { crtY }}{\text { Lycopene cyclase }}
$$

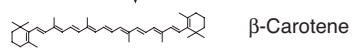

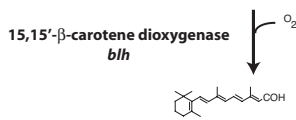

Retina
D
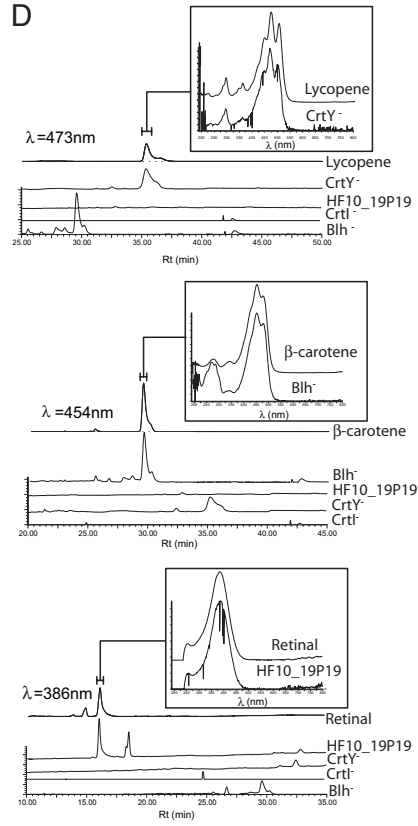

Fig. 1. Genetic and phenotypic analysis of PR photosystem transposon mutants. (A) Schematic representation of the PR gene clusters identified in this work. Predicted transcription terminators in the clusters are indicated. $(B)$ Color phenotype of intact cells of transposon-insertion mutants grown in liquid cultures with arabinose. (C) Retinal biosynthesis pathway. Names of genes encoding pathway enzymes are indicated. The genes that are present in $E$. coli are in parentheses. (D) HPLC profiles of wild-type and transposon mutant extracts. Detection wavelengths are indicated. Absorption spectrum of relevant peaks, including standards used for identification, are shown on the top for each panel.

HF10_19P19, were analyzed further for PR photosystem gene expression and function.

Genomic Analyses of Candidate PR Photosystem-Expressing Clones. The full DNA sequence of the two putative PR photosystemcontaining fosmids was obtained by sequencing a collection of transposon-insertion clones. The approach facilitated rapid DNA sequencing while simultaneously providing a set of precisely located insertion mutants for phenotypic analysis of specific gene functions (15).

Both PR photosystem-containing clones were derived from Alphaproteobacteria based on ORF content similarity to homologues in the National Center for Biotechnology Information nonredundant protein database [supporting information (SI) Tables 1 and 2]. The clones exhibited highest identity to other
PR-containing BAC clones from Alphaproteobacteria from the Mediterranean and Red Seas (8). This similarity was evident across the entire cloned insert, although some large-scale rearrangements were apparent. The HF10_19P19 PR-inferred protein sequence was most similar to a homologue from another environmental BAC, MedeBAC66A03 (67\% identity, 83\% similarity). The MedeBAC66A03 PR was previously reported to exhibit fast photocycle kinetics and light-activated proton translocation when expressed in $E$. coli in the presence of exogenous retinal (8). Clone HF10_25F10 PR was most similar in inferred protein sequence to another BAC clone, RED17H08 PR (93\% identity, $97 \%$ similarity) and was very similar to MedeBAC66A03 as well (62\% identity, $78 \%$ similarity). Both of the PR genes analyzed here encoded proteins with a glutamine residue at position 105 , a characteristic of blue light-absorbing

PNAS | March 27, 2007 | vol. 104 | no. 13 | 5591 

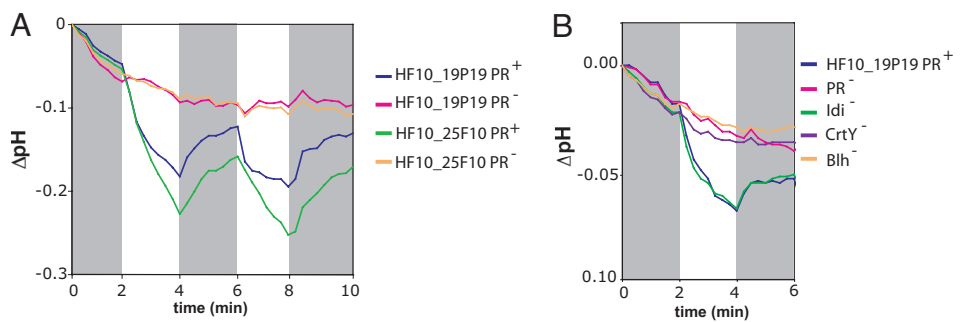

Fig. 2. Proton-pumping assays. $\mathrm{pH}$ measurements are expressed as $\mathrm{pH}$ change with respect to the $\mathrm{pH}$ at time 0 for each sample. Gray boxes indicate dark periods.

PRs (5) and consistent with the orange pigmentation observed in clones expressing them.

Adjacent to the PR gene in both clones was a predicted six-gene operon encoding putative enzymes involved in $\beta$-carotene and retinal biosynthesis (Fig. $1 A$ ). A similar arrangement was reported in MedeBAC66A03 and RED17H08 (8) and more recently in a wide variety of diverse marine bacterial groups (7). The genes encoded on these operons include $\mathrm{crtE}$ [putative geranylgeranyl pyrophosphate (GGPP) synthase], crtI (phytoene dehydrogenase), crtB (phytoene synthase), crtY (lycopene cyclase), blh $\left(15,15^{\prime}-\beta\right.$ carotene dioxygenase), and idi [isopentenyl diphosphate (IPP) $\delta$-isomerase]. The putative role of these proteins in the retina biosynthetic pathway (for review, see ref. 16) is indicated in Fig. $1 C$. The first reactions in the pathway are catalyzed by the IPP $\delta$-isomerase and farnesyl diphosphate (FPP) synthase. Both enzymes are part of the isoprenoid and ubiquinone metabolic pathways and are present in E. coli. crtE, crtB, crtI, and $\operatorname{crt} Y$ appear to encode all of the enzymes necessary to synthesize $\beta$-carotene from FPP. The $b l h$ gene found in MedeBAC66A03 was previously shown to encode a $15,15^{\prime}-\beta$-carotene dioxygenase that cleaves $\beta$-carotene, producing two molecules of all-trans-retinal (8).

Apart from the PR and putative $\beta$-carotene and retinal biosynthesis operon, no other genes were shared between the two PR-containing fosmids. With the exception of a gene encoding a putative deoxyribodipyrimidine photolyase in HF10_25F10, no other genes flanking the PR photosystem had an obvious, light-related function (SI Tables 1 and 2).

Genetic and Phenotypic Analysis of the PR Photosystem. To obtain direct evidence for the functional annotations of putative retinal biosynthesis genes, we analyzed different transposon mutant phenotypes that carried an insertion in predicted PR photosystem genes. The cell pigmentation and HPLC pigment analyses in selected mutants are shown in Fig. $1 B$ and $D$, respectively. HF10_19P19 cells carrying the intact vector were orange when grown in the presence of arabinose, consistent with expression of a blue-adapted retinal-PR complex. HPLC analysis revealed the presence of retinal in cell extracts, demonstrating that clone HF10_19P19 contained all genes required for retinal biosynthesis in $E$. coli. Neither lycopene nor $\beta$-carotene was observed in the intact clone extracts, indicating that there was little if any accumulation of pigment intermediates (Fig. $1 D$ ). Cells containing transposon insertions in the $i d i$ gene were also orange and contained retinal. The lack of phenotype in this mutant can be attributed to the presence of the endogenous idi gene in E. coli (17).

As expected, transposon insertion mutants disrupted in the PR gene itself were devoid of orange pigmentation, and HPLC analysis showed a low but detectable level of retinal in these extracts (data not shown). It is unclear at present whether the low levels of retinal were due to polar effects caused by the transposon insertion in downstream expression or whether they result from pathway inhibition due to product accumulation.

5592 | www.pnas.org/cgi/doi/10.1073/pnas.0611470104
Transposon-insertion mutants in $\operatorname{crt} E, \operatorname{crt} B$, and $\operatorname{crt} I$ showed no pigmentation, as expected for this biosynthetic pathway if it is interrupted before lycopene formation, the first colored product in the pathway. crtY-insertion mutants, however, were pink, suggesting that they were accumulating lycopene. Pigment analysis verified that $\operatorname{crt} Y$-insertion mutant extracts contained lycopene but not retinal or $\beta$-carotene (Fig. $1 B$ and $D$ ). Finally, blh-insertion mutants had a yellow phenotype, and HPLC analysis showed that these cells lacked detectable retinal but instead accumulated $\beta$-carotene. This finding demonstrates that the blh gene in HF10_19P19 encodes a $15,15^{\prime}-\beta$-carotene dioxygenase, similar to a homologue recently described by Sabehi et al. (8). Transposon insertions in all other predicted genes outside the PR cluster had no visibly obvious phenotype. Identical pigmentation phenotypes were observed with insertions in the corresponding genes of the other PR photosystem clone, HF10_25F10. Taken together, these results strongly support the functional assignments of PR-associated retinal biosynthetic pathway genes and demonstrate that they are necessary and sufficient to induce retinal biosynthesis in $E$. coli.

Light-Activated Proton Translocation. We assayed both HF10_19P19 and HF10_25F10 grown under high-copy number conditions for light-activated proton-translocating activity. Light-dependent decreases in $\mathrm{pH}$ were observed in $\mathrm{PR}^{+}$clones but not in mutants containing a transposon insertion in the $\mathrm{PR}$ gene $\left(\mathrm{PR}^{-}\right)$(Fig. $2 A$ ). In addition, no light-dependent proton-translocating activity was observed in insertion mutants unable to synthesize retinal $\left(\mathrm{CrtY}^{-}\right.$ or $\left.\mathrm{Blh}^{-}\right)$. In contrast, $\mathrm{Idi}^{-}$mutants had normal proton-pumping activity, confirming that this gene was not required under our growth conditions (Fig. $2 B$ ). These results demonstrate that both fosmids independently expressed a functional PR with lightactivated proton-translocating activity.

PR-Driven Proton Translocation Results in Photophosphorylation in E. coli. Analogous to earlier studies of haloarchaeal bacteriorhodopsins $(18,19)$, it was previously postulated that light-activated, PR-induced proton motive force could drive ATP synthesis as protons reenter the cell through the ATP synthase complex (Fig. $3 A)(1,12)$. This hypothesis was not previously tested, however, in either native or heterologously expressed PR-based photosystems. To this end, we measured light-induced changes in ATP levels in the PR-photosystem-containing clones and $\mathrm{PR}^{-}$mutant derivatives by using a luciferase-based assay. The assay measures total ATP, and so we expected to observe increases in ATP concentration only if PR-driven ATP biosynthesis exceeded endogenous turnover rates, under our experimental conditions. Control $\mathrm{pH}$ measurements indicated that $\mathrm{PR}^{+}$cells used in the ATP assay were indeed capable of light-activated proton translocation (Fig. 3B). ATP measurements performed after 5 min of illumination showed significant light-induced increases in cellular ATP levels in the $\mathrm{PR}^{+}$clone but not in a $\mathrm{PR}^{-}$mutant (Fig. 
A

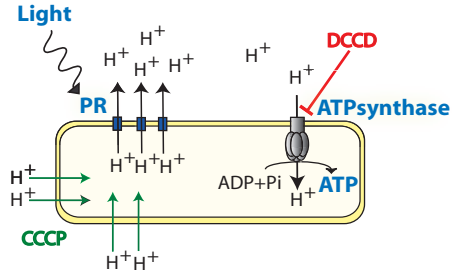

B
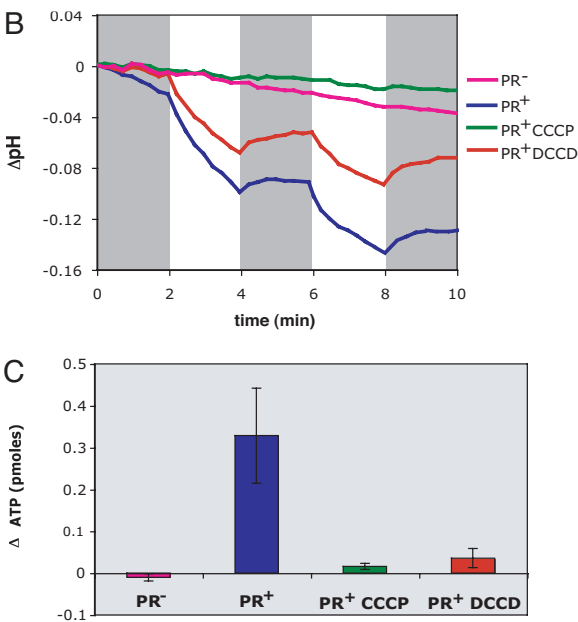

Fig. 3. Light-activated, PR-enabled photophosphorylation in E. coli. (A) Diagram of the proposed mechanism of PR-dependent ATP synthesis. The effects of the inhibitors used are indicated. (B) Proton-pumping assays with HF10_19P19 cells. pH measurements are expressed as the $\mathrm{pH}$ change respect to the $\mathrm{pH}$ at time 0 for each sample. CCCP, $25 \mu \mathrm{M}$; DCCD, $1 \mathrm{mM}$. Gray boxes indicate dark periods. (C) ATP assays with HF10_19P19 cells. Results are expressed as the difference between the ATP level in the light and the ATP leve in the dark, $\triangle A T P$, for each treatment.

$3 C$ ). The 0.3-pmol increase in ATP observed in the $\mathrm{PR}^{+}$cells exposed to light (Fig. 3C) represents a $29 \%$ increase over identical cell preparations maintained in the dark, which corresponds to a net gain after 5 min of illumination of $\approx 2.2 \times 10^{5}$ molecules of ATP per colony-forming unit (or viable cell) assayed. For comparison, oxidative phosphorylation, measured by ATP increases observed $5 \mathrm{~min}$ after the addition of $0.2 \%$ succinate to $\mathrm{PR}^{+}$cells in the dark, resulted in a net gain of $9 \times$ $10^{5}$ ATP molecules per live cell (data not shown).

Similar light-activated, PR-catalyzed photophosphorylation was also observed in cells containing the HF10_25F10 fosmid. Although the PR photosystem of this clone is similar to that of HF10_19P19, all of the genes flanking the two different photosystem gene suites are completely different and derive from different chromosomal contexts. Because the PR and retinal biosynthetic genes are the only shared genes on both clones, the results strongly suggest that these specific PR photosystem genes are both necessary and sufficient to drive photophosphorylation in $E$. coli cells.

To characterize more fully the light-driven photophosphorylation observed in $\mathrm{PR}^{+} E$. coli cells, we tested the effects of carbonylcyanide $m$-chlorophenylhydrazone (CCCP), an uncou- pler, and $N, N^{\prime}$-dicyclohexylcarbodiimide (DCCD), a covalent inhibitor of $\mathrm{H}^{+}$-ATP synthase, on light-driven ATP synthesis (Fig. 3). We used concentrations that inhibited aerobic growth of $E$ coli on succinate, an oxidative phosphorylation process requiring both proton-motive force and ATP synthase activity (20). Addition of CCCP, which permeabilizes the cell membrane to $\mathrm{H}^{+}$, completely abolished the light-driven decrease in $\mathrm{pH}$ and subsequently photophosphorylation. This result demonstrates that both processes depend on the establishment of an $\mathrm{H}^{+}$ electrochemical gradient. In contrast, addition of the $\mathrm{H}^{+}$-ATP synthase inhibitor DCCD did not affect external $\mathrm{pH}$ changes resulting from PR-catalyzed proton translocation, but it completely abolished photophosphorylation. This result indicates that $\mathrm{H}^{+}$-ATP synthase is indeed responsible for the lightactivated ATP increases we observed in $\mathrm{PR}^{+}$cells

\section{Discussion}

The results presented here demonstrate the utility of functionally screening large-insert DNA "metagenomic" libraries for new phenotypes and activities directly and without subcloning, an approach pioneered by soil microbiologists $(15,21,22)$. Although large-insert libraries increase the probability of capturing complete metabolic pathways in a single clone, their low copy number decreases the sensitivity of detecting heterologous gene expression. We show here that increasing fosmid copy number (13) can significantly enhance detectable levels of recombinant gene expression and therefore increases the detection rate of desired phenotypes in metagenomic libraries. The PR photosystem recombinants we characterized could be detected visually by pigment production and exhibited light-dependent proton translocation and subsequent photophosphorylation, only when the fosmid vector was induced to high copy number. The approach was not completely effective in detecting all targeted genotypes however. Even under "copy-up" conditions, we were unable to detect all PR-containing clones known to exist in our library (4, 7). Despite the limitations, this approach for functional screening of microbial community genomic libraries is useful for identifying specific activities or phenotypes, even in the absence of sequence information. Additionally, this approach provides useful material for downstream genetic and biochemical characterization and for testing hypotheses derived from bioinformatic analyses.

Genetic and biochemical characterization of PR photosystemcontaining clones reported here provided direct evidence that only six genes are required to enable light-activated proton translocation and photophosphorylation fully in a heterologous host. Sabehi et al. (8) demonstrated previously that coexpression of marine bacterial blh with PR, in the presence of the $\beta$-carotene biosynthetic genes from Erwinia herbicola, led to $\beta$-carotene cleavage and subsequent formation of retinal-bound PR. We show here that a set of six genetically linked genes known to be found in a wide variety of different marine bacterial taxa $(7,8$, $11)$ are both necessary and sufficient for the complete synthesis and assembly of a fully functional PR photoprotein in E. coli. and assembly of a fully functional PR photoprotein in E. coli.
These heterologously expressed marine bacterial photosystems exhibited light-dependent proton translocation activity in the absence of exogenously added retinal or $\beta$-carotene. One gene in the PR photosystem cluster was dispensable under our conditions: the $i d i$ gene that encodes IPP $\delta$-isomerase, an activity already present in E. coli (17), as is the FPP synthase, catalyzing the next two steps in the pathway (23). The presence of the $i d i$ gene in the cluster likely enables retinal production in the native organism because isomerization of IPP to dimethylallyl pyrophosphate can be a rate-limiting step in $\beta$-carotene biosynthesis $(24,25)$.

It was previously postulated that light-activated proton translocation catalyzed by PR elevates the proton-motive force, thereby driving ATP synthesis as protons reenter the cell through 
the $\mathrm{H}^{+}$-ATP synthase complex $(1,12)$. Although this capability has been demonstrated for haloarchaeal bacteriorhodopsins (18, 19, 26, 27), PR-based photophosphorylation has not been demonstrated previously. Our data demonstrate that illumination of cells expressing a native marine bacterial PR photosystem generates a proton-motive force that does indeed drive cellular ATP synthesis. Under our experimental conditions, 5 min of illumination resulted in a net gain of $2.2 \times 10^{5}$ ATP molecules per cell It should be quite possible to utilize the light to biochemical energy conversion enabled by the PR photosystem, for biosynthetic purposes.

The PR photosystem-catalyzed photophosphorylation described here is consistent with a proposed role for $\mathrm{PR}$ in marine microbial photoheterotrophy. A few previous studies were unable to detect light-enhanced growth rates or yields in PR-containing isolates grown in seawater or natural seawater incubations $(28,29)$. In one recent report, light stimulation of growth rate or yield in Pelagibacter ubique, a ubiquitous PR-containing marine planktonic bacterium, could not be detected. These negative results are somewhat difficul to interpret because natural seawater incubations are by necessity chemically undefined, and preferred growth substrates or other limiting nutrients in these experiments were unknown. In contrast, a significant enhancement of both growth rate and yield was recently reported in PR-expressing marine flavobacterium (11) albeit a direct link between PR and the light-induced growth stimulation was not conclusively demonstrated. Our direct observation of an intact PR photosystem gene expression and subsequent photophosphorylation, the recently reported light-enhanced growth rates and yields of PR-containing Flavobacteria (11), and the general ubiquity of PR photosystem genes in diverse microbial taxa of the ocean's photic zone (2-9) all strongly support a significant role for PR-based phototrophy in planktonic marine microorganisms.

In different physiological, ecological, phylogenetic, and genomic contexts, PR activity may benefit cells in a variety of ways, some not directly related to enhanced growth rates or yields. In some bacteria, the $\mathrm{H}^{+}$-ATP synthase functions as an ATPase under low respiratory conditions, hydrolyzing ATP and driving proton efflux to maintain the proton-motive force (30). In the light, PR activity could offset this effect and reverse conditions from ATP consumption to ATP production. PR contributions to cellular energy metabolism are likely to be particularly important in starved or substrate-limited cells. Similar to the situation for some Haloarchaea, which use bacteriorhodopsin under oxygen-limiting conditions $(18,19,26,27)$, low respiratory rates may trigger $\mathrm{PR}$ expression or activity in marine bacteria, as well. The PR-generated proton-motive force can also be directly coupled to other energy-requiring cellular activities, including flagellar motility or active transport of solutes into or out of the cell (32-34). This phenomenon was recently demonstrated by the coupling of PR activity to flagellar rotation in $E$. coli (31). Although a sensory function for some PR variants is also a possibility $(10,35)$, the PR photosystems described here, and the vast majority of others observed to date $(1,3,4,7,8,11)$ are not genetically linked to sensory transducers, the hallmark of all known sensory rhodopsins. Most all PRs characterized so far therefore appear to function as light-activated ion pumps.

The marine PR family is remarkably diverse, has a widespread phylogenetic distribution, and is functional in both ether-linked phytanyl and ester-linked fatty acid membrane systems of $\mathrm{Ar}$ chaea and Bacteria $(4,7,10)$. A recent survey found that one-third of PR clones examined were colocalized with photopigment biosynthetic operons (7). Operon arrangement and distribution, as well as phylogenetic relationships, suggest that lateral transfer of PR photosystem genes is relatively common among diverse marine microbes $(4,7,10)$. The observations reported here demonstrate that acquisition of just a few genes can lead to functional PR photosystem expression and photo- phosphorylation. The ability of a single lateral transfer event to confer phototrophic capabilities likely explains the ecological and phylogenetic prevalence of these photosystems in nature. In principle, any microorganism capable of synthesizing FPP (a widespread intermediate in isoprenoid biosynthesis) could readily acquire this capability, as we have observed in $E$. coli.

Apparently, many otherwise chemoorganotrophic microbes in the ocean's photic zone have acquired the ability to use light energy to supplement cellular energy metabolism. The broad array of PR-containing microbes reflects the photosystem's fundamental contribution to cellular bioenergetics, a simplicity and compactness that favors PR photosystem lateral mobility, and a remarkable plasticity that enables photoprotein assembly and function in a diversity of phylogenetic groups and cell membrane types. From a genetic, physiological, and ecological perspective, the transition from heterotrophy to PR-enabled photoheterotrophy seems to represent a relatively small evolutionary step for contemporary microorganisms.

\section{Materials and Methods}

Fosmid Library. The HOT_10m fosmid library screened in this work has been described previously (14). It contains DNA from a planktonic sample collected $10 \mathrm{~m}$ below the surface at the ALOHA station $\left(22^{\circ} 45^{\prime} \mathrm{N}, 158^{\circ} \mathrm{W}\right)$ of the Hawaii Ocean Time series (HOT) cloned into the copy-controlled pCC1FOS fosmid vector (Epicentre Biotechnologies, Madison, WI). The library host, E. coli EPI300 (Epicentre Biotechnologies), supports the copy-control option of pCC1FOS

Screening for PR Expression. High-density colony macroarrays [12,280 clones of the HOT_10m library (ref. 14)] were prepared on a Performa II filter (Genetix Ltd., Boston, MA) by using a Q-PixII robot (Genetix Ltd.). The filter was carefully laid over a 22-cm plate containing $250 \mathrm{ml}$ of LB agar supplemented with L-arabinose $(0.02 \%)$, the copy-up inducer, and all-trans-retinal $(20 \mu \mathrm{M})$, and the plate was incubated at $37^{\circ} \mathrm{C}$ for $24 \mathrm{~h}$. The filters were used to facilitate the visual detection of color against the white background. Colonies were inspected visually for the appearance of orange or red color. Fosmid DNA from positive clones was retransformed into fresh E. coli EPI300 and rescreened as above to verify that the color was conferred by the fosmid. The end DNA sequence of the positive clones was obtained by using primers $\mathrm{T} 7$ and EpiFos5R as described previously (14)

In Vitro Transposition and Full Fosmid Sequencing. Fosmid clones to be characterized were submitted to random in vitro transposition by using the EZ-Tn5 $<$ kan-2 $>$ insertion kit (Epicentre Biotechnologies) according to the manufacturer's instructions. The transposition reaction was transformed by electroporation into EPI300 cells, and clones containing fosmids with Tn5 insertions were selected in LB chloramphenicol, kanamycin $(12 \mu \mathrm{g} / \mathrm{ml}$ and $25 \mu \mathrm{g} / \mathrm{ml}$, respectively). The color phenotype of individual Tn5-insertion clones was analyzed on LB plates containing chloramphenicol, kanamycin, and $0.02 \% \mathrm{~L}$-arabinose as above. DNA sequencing off the Tn 5 ends was performed by using KAN-2 FP-1 and KAN-2 RP-1 primers, a BigDye version 3.1 cycle sequencing kit, and ABI Prism 3700 DNA analyzer (Applied Biosystems, Forest City, CA). The complete DNA sequence was assembled by using Sequencher version 4.5 (Gene Codes Corporation, Ann Arbor, MI) and annotated with FGENESB (Softberry, Mount Kisco, NY) and Artemis version 6 (The Wellcome Trust Sanger Institute, Cambridge, U.K.).

Carotenoid Extraction. Overnight cultures of the appropriate clones were diluted $1: 100$ into $50 \mathrm{ml}$ of $\mathrm{LB}$ chloramphenicol (12 $\mu \mathrm{g} / \mathrm{ml})$ and incubated for $3 \mathrm{~h}$ at $37^{\circ} \mathrm{C}$ with shaking $(200 \mathrm{rpm})$. At that point, L-arabinose was added to a $0.02 \%$ final concentration, and cultures were incubated for $16 \mathrm{~h}$. Cells were harvested by 
centrifugation and rinsed twice in salt solution. Cell pellets were kept frozen $\left(-20^{\circ} \mathrm{C}\right)$ in the dark. Frozen cells were extracted by sonication $(5 \mathrm{~min})$ in a cold $4: 1(\mathrm{vol} / \mathrm{vol})$ mixture of acetone methanol (OmniSolv; EMD Chemicals, Gibbstown, NJ) with 0.1 $\mathrm{mM}$ butylated hydroxytoluene added as an antioxidant. Cells were pelleted by centrifugation, and the supernatant was removed and filtered through ashed silica gel (230-400 mesh; EMD Chemicals). Extracts were then concentrated by evaporation under dry $\mathrm{N}_{2}$. All extraction steps were performed in darkness or low light to minimize carotenoid photooxidation.

HPLC Analysis. Chromatographic separation and analysis of carotenoids by high-performance liquid chromatography (HPLC) adapted a reverse-phase method from Barua and Olson (36). A $5-\mu \mathrm{m}$ Zorbax-ODS C18 column $(150 \times 4.6 \mathrm{~mm})$ (Agilent Technologies, Palo Alto, CA) was used at $30^{\circ} \mathrm{C}$ in a column oven with a Waters (Milford, MA) 2795 separations module operated with MassLynx 4.0 software. Separation was achieved with linear gradient at a flow rate of $0.8 \mathrm{ml} / \mathrm{min}: 100 \%$ solvent A to $100 \% \mathrm{~B}$ over 20 min followed by isocratic elution with B for an additional $20 \mathrm{~min}$, where $\mathrm{A}=$ methanol $/$ water $(3: 1 \mathrm{vol} / \mathrm{vol})$ and $\mathrm{B}=$ methanol/dichloromethane $(4: 1 \mathrm{vol} / \mathrm{vol})$. The column was equilibrated after each run with solvent A for $10 \mathrm{~min}$. The detector was a Waters 996 photodiode array detector scanning wavelengths from 190 to $800 \mathrm{~nm}$ with a resolution of $1.2 \mathrm{~nm}$ and sampling rate of one spectrum per s. Carotenoids were identified by comparing absorbance spectra and retention times with authentic standards.

Proton-Pumping Experiments. Clones to be analyzed for protonpumping activity were streaked on $15-\mathrm{cm} \mathrm{LB}$ agar plates containing $12 \mu \mathrm{g} / \mathrm{ml}$ chloramphenicol and $0.001 \%$ L-arabinose and incubated at $37^{\circ} \mathrm{C}$ for $48 \mathrm{~h}$. Cells were resuspended in $20 \mathrm{ml}$ of salt solution ( $\left.10 \mathrm{mM} \mathrm{NaCl} / 10 \mathrm{mM} \mathrm{MgCl}_{2} / 100 \mu \mathrm{M} \mathrm{CaCl}_{2}, \mathrm{pH} 7.0\right)$,

1. Beja O, Aravind L, Koonin EV, Suzuki MT, Hadd A, Nguyen LP, Jovanovich SB, Gates CM, Feldman RA, Spudich JL, et al. (2000) Science 289:1902-1906. 2. Venter JC, Remington K, Heidelberg JF, Halpern AL, Rusch D, Eisen JA, W D, Paulsen I, Nelson KE, Nelson W, et al. (2004) Science 304:66-74.

3. de la Torre JR, Christianson LM, Beja O, Suzuki MT, Karl DM, Heidelber J. DeLong EF (2003) Proc Natl Acad Sci USA 100:12830-12835.

4. Frigaard N-U, Martinez A, Mincer TJ, DeLong EF (2006) Nature 439:847-850. 5. Man D, Wan W, Sabehi G, ArJ, D Pos AF, Ma) Nan Spudich JL, Beja O (2003) EMBO J 22:1725-1731.

. Man-Aharonovich D, Sabehi G, Sineshchekov O, Spudich EN, Spudich JL Beja O (2004) Photochem Photobiol Sci 3:459-462.

McCarren J, DeLong EF, Environ Microbiol, in press.

8. Sabehi G, Ly A, Jung K-H, Partha R, Spudich JL, Isacson T, Hirschberg

Wagner M, Beja O (2005) PLOS Biol 3.273.

Sabehi G, Massana R, Bielawski JP, Rosenterg M, Delong EF, Bejo O (2003) Environ Microbiol 5:842-849.

. Sharma AK, Spudich JI, Doolittle WF (2006) Trends Microbiol 14:463-469.

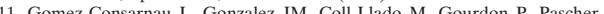
Neutze R, Pedros-Alio C, Pinhassi J (2007) Nature 445:210-213.

Beja O, Spulich EN, Spudich JL, Leclerc M, DeLong EF (2001) Nature 411:786-789.

13. Wild J, Hradecna Z, Szybalski W (2002) Genome Res 12:1434-1444.

14. Delong EF, Preston CM, Mincer T, Rich V, Hallam SJ, Frigard N-U, Martinez A, Sullivan MB, Edwards R, Brito BR, et al. (2006) Science 311:496503.

15. MacNeil IA, Tiong CL, Minor C, August PR, Grossman TH, Loiacono KA, Lynch BA, Phillips T, Narula S, Sundaramoorthi R, et al. (2001) J Mol Microbiol Biotechnol 3:301-308.

16. Armstrong GA (1997) Annu Rev Microbiol 51:629-659.

17. Hahn FM, Hurlburt AP, Poulter CD (1999) J Bacteriol 181:4499-4504. rinsed twice, and adjusted to an $A_{600}$ of $0.5-0.7$. Two milliliters of cell suspension was placed in an RPC-100 photosynthetic chamber (i-Works, Dover, $\mathrm{NH}$ ) connected to a $22^{\circ} \mathrm{C}$ circulating water bath $\mathrm{pH}$ was measured by using a Beckman (Fullerton, CA) $\Phi 360 \mathrm{pH}$ meter equipped with a Futura microelectrode. Light was provided by a 160 -watt halogen lamp placed $4 \mathrm{~cm}$ from the chamber. Irradiance within the chamber was 500-650 $\mu \mathrm{mol}$ $\mathrm{Q} \mathrm{m} \mathrm{m}^{-2} \mathrm{~s}^{-1}$

ATP Measurements. Cell suspensions were prepared as above. Three milliliters of cell suspension was placed in 5-ml screw-cap glass vials. The vials for the dark samples were wrapped in foil. glass vials. The vials for the dark samples were wrapped in foil.
Ten centimeters of water was used to minimize heat transfer to
the samples. Irradiance under these conditions was $650 \mu \mathrm{mol} Q$ the samples. Irradiance under these conditions was $650 \mu \mathrm{mol} Q$ (BactTiter Glo, Promega, Madison, WI) as follows. At each time point, 5 aliquots (20 $\mu$ leach) of every sample were dispensed into white 96-well assay plates [CoStar (Bethesda, MD) 3917]. One hundred microliters of BactTiterGlo reagent was added per well, and luminescence was measured after 10 min using a Victor3 plate reader (PerkinElmer, Waltham, MA) with a 1-s integration time. An ATP standard curve was used to calculate the concentration of ATP in the samples. For inhibitor experiments, cell suspensions were incubated in the dark for $20 \mathrm{~h}$ in the presence of $1 \mathrm{mM}$ DCCD or for $2 \mathrm{~h}$ in the presence of $25 \mu \mathrm{M}$ CCCP or the ethanol vehicle. Succinate was added to a $0.2 \%$ final concentration to measure ATP synthesis from respiration in the dark.

We thank Drew Endy for the use of the luminometer, and Jay McCarren for comments on the manuscript. This work was supported by a grant from the Gordon and Betty Moore Foundation (to E.F.D.), National Science Foundation (NSF) Microbial Observatory award (MCB0348001) and NSF Science and Technology Center Award EF0424599 (to E.F.D.).

18. Danon A, Stoeckenius W (1974) Proc Natl Acad Sci USA 71:1234-1238 19. Oesterhelt D, Stoeckenius W (1973) Proc Natl Acad Sci USA 70:2853-2857. 19. Oesterh D, Stoeckenius W (1973) Proc Nall Acad Sci USA 70.2853-285

21.

Rondon MR, Augus PR, Bettermann AD, Brady SF, Grossman TH, Liles MR, Loiacono KA, Lynch BA, MacNeil IA, Minor C, et al. (2000) Appl Environ Microbiol 66:2541-2547.

22. Gillespie DE, Brady SF, Bettermann AD, Cianciotto NP, Liles MR, Rondon MR, Clardy J, Goodman RM, Handelsman J (2002) Appl Environ Microbiol $68: 4301-4306$

23. Fujisaki S, Takahashi I, Hara H, Horiuchi K, Nishino T, Nishimura Y (2005) J Biochem (Tokyo) 137:395-400

24. Kajiwara S, Fraser PD, Kondo K, Misawa N (1997) Biochem J 324:421-426. 25. Sun Z, Cunningham FX, Jr, Gantt E (1998) Proc Natl Acad Sci USA 95:11482-11488

26. Brock TD, Petersen S (1976) Arch Microbiol 109:199-200

27. Hartmann R, Sickinger HD, Oesterhelt D (1980) Proc Natl Acad Sci USA 77:3821-3825

28. Giovannoni SJ, Bibbs L, Cho J-C, Stapels MD, Desiderio R, Vergin KL, Rappe MS, Laney S, Wilhelm LJ, Tripp HJ, et al. (2005) Nature 438:82-85.

9. Schwalbach MS, Brown M, Fuhrman J (2005) Aquatic Microb Ecol 39:235-245. 30. Booth IR (1985) Microbiol Rev 49:359-378.

31. Walter JM, Greenfield D, Bustamante C, Liphardt V (2007) Proc Natl Acad Sci USA 104:2408-2412.

32. Konings WN (2006) Antonie Van Leeuwenhoek 90:325-342.

33. Manson MD, Tedesco P, Berg HC, Harold FM, Van der Drift C (1977) Proc Natl Acad Sci USA 74:3060-3064,

34. Matsura S, Shioi J, Imae Y (1977) FEBS Lett 82:187-190.

35. Spudich JL (2006) Trends Microbiol 14:480-487.

36. Barua AB, Olson JA (1998) J Chromatog 707:69-79. 


\title{
Appendix D
}

\section{The Geological Succession of Primary Producers in the Oceans}

\author{
Andrew H. Knoll, Roger E. Summons, Jacob R. Waldbauer and \\ John E. Zumberge
}

Reprinted with permission from The Evolution of Primary Producers in the Sea (C) 2007 Elsevier Inc.

Knoll, A.H., Summons, R.E., Waldbauer, J.R. and Zumberge, J.E. (2007) The Geological Succession of Primary Producers in the Oceans. In The Evolution of Primary Producers in the Sea, P.G. Falkowski and A.H. Knoll, eds. Academic Press, p. 133-163. 
8

\title{
The Geological Succession of Primary Producers in the Oceans
}

\author{
ANDREW H. KNOLL, ROGER E. SUMMONS, \\ JACOB R. WALDBAUER, AND JOHN E. ZUMBERGE
}

I. Records of Primary Producers in Ancient Oceans

A. Microfossils

B. Molecular Biomarkers

II. The Rise of Modern Phytoplankton

A. Fossils and Phylogeny

B. Biomarkers and the Rise of Modern Phytoplankton

C. Summary of the Rise of Modern Phytoplankton

III. Paleozoic Primary Production
A. Microfossils
B. Paleozoic Molecular Biomarkers
C. Paleozoic Summary

IV. Proterozoic Primary Production
A. Prokaryotic Fossils
B. Eukaryotic Fossils
C. Proterozoic Molecular Biomarkers
D. Summary of the Proterozoic Record

V. Archean Oceans

VI. Conclusions
A. Directions for Continuing Research
References

In the modern oceans, diatoms, dinoflagellates, and coccolithophorids play prominent roles in primary production (Falkowski et al. 2004). The biological observation that these groups acquired photosynthesis via endosymbiosis requires that they were preceded in time by other photoautotrophs. The geological observation that the three groups rose to geobiological prominence only in the Mesozoic Era also requires that other primary producers fueled marine ecosystems for most of Earth history. The question, then, is: what did primary production in the oceans look like before the rise of modern phytoplankton groups?

In this chapter, we explore two records of past primary producers: morphological 
fossils and molecular biomarkers. Because these two windows on ancient biology are framed by such different patterns of preservational bias and diagenetic selectivity, they are likely to present a common picture of stratigraphic variation only if that view reflects evolutionary history.

\section{RECORDS OF PRIMARY PRODUCERS IN ANCIENT OCEANS}

\section{A. Microfossils}

Microfossils, preserved as organic cell walls or mineralized tests and scales, record the morphologies and (viewed via transmission electron microscopy) ultrastructures of ancient microorganisms. Such fossils can provide unambiguous records of phytoplankton in past oceans-diatom frustules, for example-and they commonly occur in large population sizes, with numerous occurrences that permit fine stratigraphic resolution and wide geographic coverage.

Set against this is a number of factors that limit interpretation. Not all photoautotrophs produce preservable cell walls or scales, and, of those that do, not all generate fossils that are taxonomically diagnostic. Thus, although many modern diatoms precipitate robust frustules of silica likely to enter the geologic record, others secrete weakly mineralized shells with a correspondingly lower probability of preservation. Similarly, whereas dinoflagellates as a group have left a clear record of dinocysts, many extant species do not produce preservable cysts and others form cysts that would not be recognized unambiguously as dinoflagellate in fossil assemblages. (The phylogenetic affinities of fossil dinocysts are established by the presence of an archeopyle, a distinctive excystment mechanism peculiar to but not universally found within dinoflagellates.) Especially in the early history of a group, character combinations that readily distinguish younger members may not be in place. Thus, stem group diatoms without well-developed frustules might well leave no morphologic record at all in sediments.

By virtue of their decay-resistant extracellular sheaths and envelopes, many cyanobacteria have a relatively high probability of entering the fossil record, and some benthic lineages are both readily preservable and morphologically distinctive (Knoll and Golubic 1992). On the other hand, important picoplankton such as Prochlorococcus are unlikely to leave recognizable body (or, as it turns out, molecular) fossils. A number of algal clades include good candidates for fossilization, especially groups with distinctive resting stages (phycomate prasinophytes, dinoflagellates) or mineralized skeletons (diatoms, coccolithophorids, coralline reds, caulerpalean and dasyclad greens). Other primary producers fossilize occasionally but only under unusual depositional or diagenetic circumstances (e.g., Butterfield 2000; Xiao et al. 2002, 2004; Foster and Afonin 2006), and still others rarely if ever produce morphologically interpretable fossils.

Diagenesis can obliterate fossils as well as preserve them: organic walls are subject to postdepositional oxidation and mineralized skeletons may dissolve in undersaturated pore waters. The result is that presence and absence cannot be weighted equally in micropaleontology. The presence of a fossil unambiguously shows that the cell from which it derived lived at a certain time in a particular place, but absence may reflect true absence, low probability of fossilization, or obfuscating depositional or diagenetic conditions. For older time intervals, tectonic destruction of the sedimentary record imposes an additional challenge; in particular, subduction inexorably destroys oceanic crust and the sediments that mantle it, so that deep-sea sediments are common only in Jurassic and younger ocean basins.

\section{B. Molecular Biomarkers}

The chemical constituents of biomass produced by living organisms can be incorporated 
into sediments and ultimately into sedimentary rocks that can survive for billions of years. Where these compounds are preserved in recognizable forms, they represent another opportunity for organisms to leave a trace of themselves in the fossil record. Organic biomarkers are the diagenetically altered remains of the products of cellular biosynthesis and may be aptly termed molecular fossils. Most biomarkers are derived from lipids and are potentially stable over billion-year time scales under ideal conditions (Brocks and Summons 2004).

Given the variety of organic compounds produced by cells and the vast quantities of sedimentary organic matter in a rock record that stretches back billions of years, biomarkers are a potentially rich source of information concerning the diversity and ecology of ancient communities. However, the process of organic matter incorporation into rocks and its transformation during deep burial imposes some strong constraints on the kinds of information that can be recovered millions of years after the fact. The classes of molecules that contain molecular sequence information, nucleic acids, and most proteins, do not survive long in the geologic environment. DNA can survive for at least a few hundred thousand years, especially in reducing environments such as euxinic sediments (e.g., Coolen et al. 2004) where heterotrophy is curtailed by a lack of electron acceptors, but it is not an option where the aim is to look at changes on million-year or longer time scales. Other kinds of biomolecules, however, prove remarkably resilient in the rock record.

Any molecule with a hydrocarbon skeleton has the potential to be preserved over long periods. For the most part, this refers to the hydrocarbon portions of membrane lipids, which are the major constituent of extractable organic matter (bitumen) in sedimentary rocks. Diagenesis quickly strips these compounds of their reactive polar functionalities, and over longer periods causes stereochemical and structural rearrangements, but hydrocarbon skeletons can remain recognizable as the products of particular biosynthetic pathways on time scales that approach the age of the Earth (e.g., Brocks and Summons 2004; Peters et al. 2005).

The character of the information contained in molecular fossils is variable. Some are markers for the presence and, to the extent they can be quantified relative to other inputs, abundance of particular organisms. The taxonomic specificity of such biomarkers ranges from species to domain level. Others are markers for the operation of a particular physiology or biosynthetic pathway that may have a broad and/or patchy taxonomic distribution. Still other kinds of biomarkers are most strongly associated with specific depositional settings, making their presence more indicative of paleoenvironmental conditions than of any particular biology. Interpretation of the molecular fossil record depends on our ability to recognize biomarker compounds, link them to biosynthetic precursors, and then make inferences about what the presence of those molecules in the rock record tells us about contemporary biology and geochemistry.

Turning to biomarkers that might establish a molecular fossil record of primary production in marine settings, several classes of compounds are promising for their combination of biochemical and/or taxonomic specificity. Pigments are natural candidates, representing markers of the photosynthetic machinery itself. Input of chlorophyll to sediments can result in several kinds of molecular fossils, including porphyrins and the pristane and phytane skeletons of the chlorophyll side chain (Figure 1). It was the recognition of vanadyl porphyrin as the molecular fossil of chlorophyll that led Alfred Treibs (1936) to make the first compelling chemical argument for the biogenic origin of petroleum. Other pigments, such as carotenoids, are subject to very selective preservation, generally requiring the presence of reduced sulfur species; the functional groups that confer many of their biophysical properties and taxonomic 


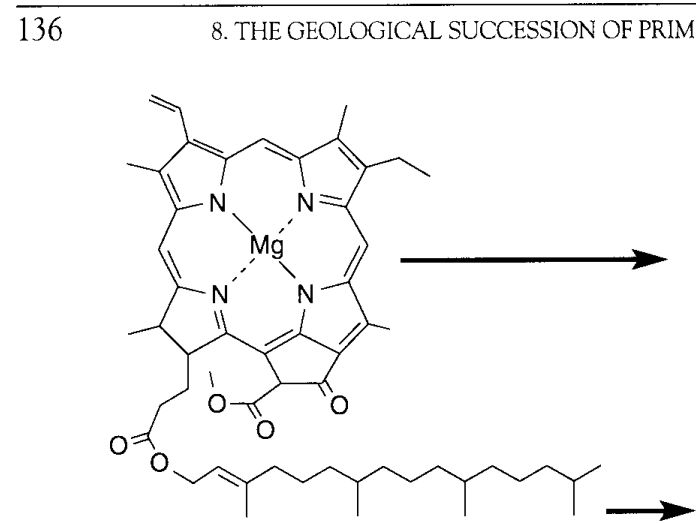

Chlorophyll a
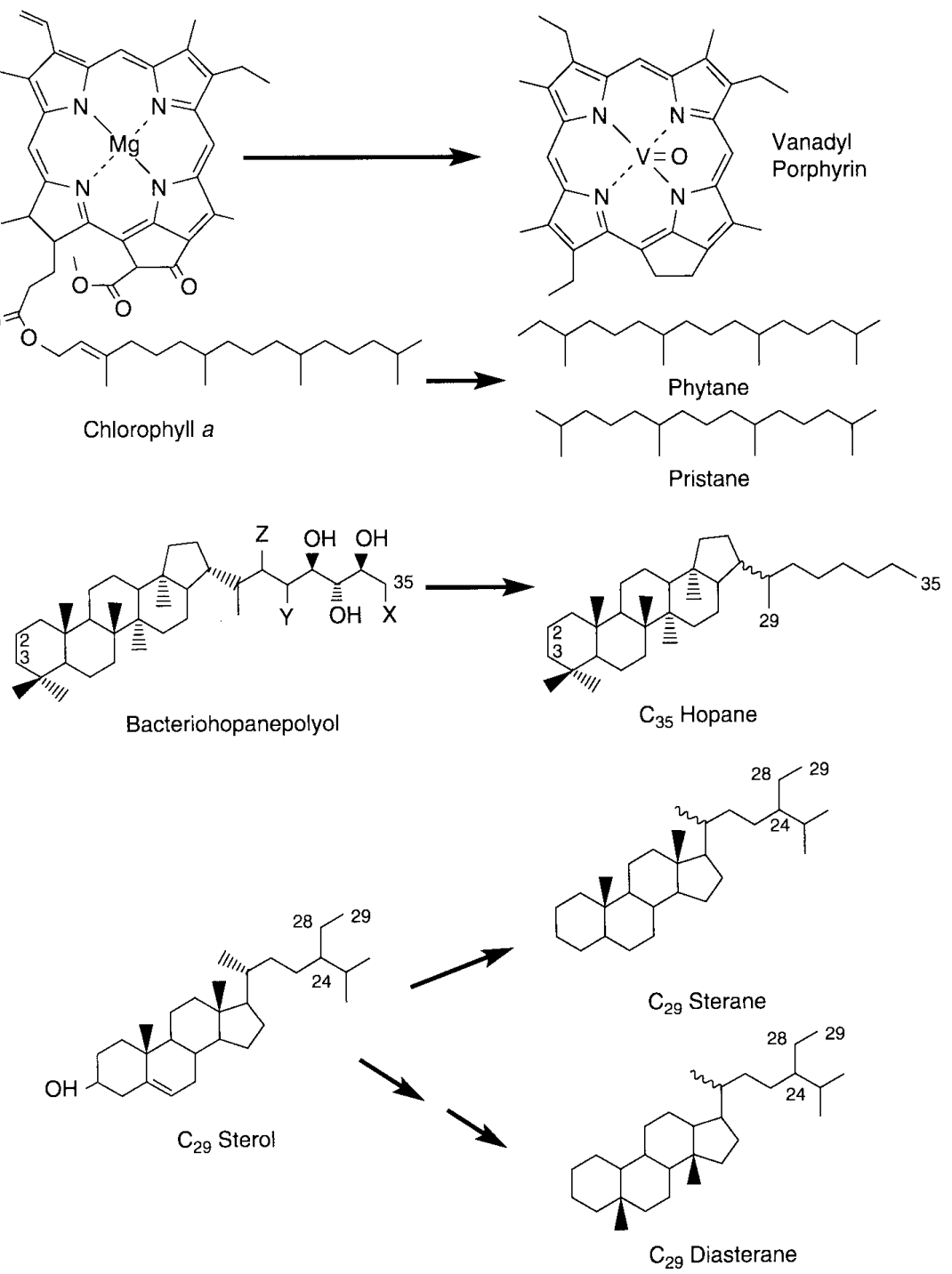

FIGURE 1. Structures of diagnostic phytoplankton lipids (left) and their fossil counterparts (right).

specificity are lost through chemical reduction processes early in diagenesis (e.g., Kohnen et al. 1991, 1993; Hebting et al. 2006).

Although the preservation of pigmentderived biomarkers is spotty and information is steadily lost over time as diagenesis proceeds, another class of extraordinarily durable molecules provides us with much of the molecular fossil record of primary producers, particularly in Paleozoic and older rocks. These are the polycyclic triterpenoids produced by the cyclization of the isoprenoid squalene and found in the membranes of both eukaryotes and bacteria. The main types are the steroids, which are ubiquitous among the Eucarya but known 
from only a very few bacteria, and the hopanoids, including the bacteriohopanepolyols (BHPs), produced by a wide variety of autotrophic and heterotrophic bacteria. These molecules have the great advantages of a durable polycyclic skeleton that is clearly a biological product and a wellcharacterized diagenetic fate involving a number of rearrangements that provide information about the postburial history of the organic matter. The structures of some commonly used biomarker lipids and their fossil counterparts are shown in Figure 1. Table 1 summarizes current knowledge of the biological affinities of hydrocarbons commonly found in marine sediment samples, excluding biomarkers derived from terrestrial organisms.

Molecular fossils suffer from some of the same limitations as body fossils. Not all ecologically and biogeochemically important groups leave distinctive molecular fingerprints, making them difficult to follow in time. Moreover, the structures of lipids are not nearly as diverse as body fossils; different, often distantly related or physiologically disparate organisms can produce similar patterns of lipids. Generally, biomarkers will reflect an average of inputs to sediments, which can be influenced by factors including bottom water oxygenation, sediment mineralogy, and grain surface area available for sorptive protection (Hedges and Keil 1995). These inputs are attenuated by remineralization of organic matter as it sinks: this reworking is $>95 \%$ complete by $3000 \mathrm{~m}$ depth (Martin et al. 1987). The high degree of water-column degradation of organic matter in deep basins means that, even where deep-water sediments survive subduction, they commonly contain little organic matter; hence, the biomarker record of open-ocean primary production is poor. The problem of interpreting absence can be acute, because in biomarker analysis, absence can only be defined in terms of detection limits and, hence, is conditionally dependent upon the analytical technology available.
Much of what is known about the diagenesis and preservation of biomarkers derives from studies of the origin and composition of petroleum (e.g., Peters et al. 2005). Petroleum geologists were initially interested in identifying the source rocks from which hydrocarbon accumulations originated. Information on the thermal histories of source rocks is also key for modeling hydrocarbon generation. Biomarkers provide a way to determine both parameters through complementary analyses of sedimentary bitumen in source rocks and their derived oil accumulations. Companies serving the petroleum exploration industry have developed and maintained databases of bitumen and oil composition that can be used to compare oils to bitumen in their source horizons and model the thermal histories of petroleum deposits. These databases can be employed as a predictive tool when exploring in frontier regions. An example is the commercial "Oils" database generated by GeoMark Research, which records geochemical analyses of more than 10,000 crude oil samples from every known petroliferous basin on the globe (www.geomarkresearch.com). The "Oils" data comprise the contents of $\mathrm{S}, \mathrm{Ni}$, and $\mathrm{V}$; the carbon isotopic compositions of bulk saturated and aromatic hydrocarbons; and quantitative analysis of approximately 100 individual hydrocarbons, including $n$-alkanes, acyclic isoprenoids, steroids, and triterpenoids. Abundances of the latter biomarkers, which have been determined using a rigorously reproducible analytical protocol, allow calculation of 23 diagnostic molecular ratios that can be used to predict paleoenvironmental features of an oil's source rock without direct knowledge of the rock itself (Zumberge 1987) or to evaluate hydrocarbon charge histories from field to basin scales (e.g., Zumberge et al. 2005). Averaging of data from numerous oil samples within a well, field, or an entire basin helps to overcome anomalies in individual hydrocarbon samples that reflect differences in maturity and losses from evaporation, water 


\section{8. THE GEOLOGICAL SUCCESSION OF PRIMARY PRODUCERS IN THE OCEANS}

TABLE 1. Hydrocarbon biomarkers prevalent in marine sediments and petroleum derived from marine sediments and their known source organisms

\begin{tabular}{|c|c|c|c|}
\hline Fossil hydrocarbon ${ }^{\alpha}$ & Functionalized precursors & Established sources ${ }^{b, c}$ & References $^{d}$ \\
\hline $\mathrm{C}_{27}-\mathrm{C}_{35}$ bacteriohopanes & $\begin{array}{l}\mathrm{C}_{35} \text { bacteriohopanepolyols } \\
\text { (BHPs) }\end{array}$ & $\begin{array}{l}\text { Bacteria although } \\
\text { nonspecific }\end{array}$ & $\begin{array}{l}\text { Rohmer et al. } 1984 \\
1992\end{array}$ \\
\hline 2-Methylhopanes & 2-Methyl-BHP & $\begin{array}{l}\text { Cyanobacteria although } \\
\text { also in methanotrophs } \\
\text { and other bacteria }\end{array}$ & $\begin{array}{l}\text { Zundel and Rohmer } \\
\text { 1985b, 1985c; Bisseret } \\
\text { et al. } 1985 \text {; Summons } \\
\text { et al. } 1999\end{array}$ \\
\hline 3-Methylhopanes & 3-Methyl-BHP & $\begin{array}{l}\text { Methanotrophs, other } \\
\text { proteobacteria }\end{array}$ & $\begin{array}{l}\text { Zundel and Rohmer } \\
1985 a\end{array}$ \\
\hline $\begin{array}{l}\text { Aryl isoprenoids, } \\
\text { isorenieratane }\end{array}$ & $\begin{array}{l}\text { Aromatic carotenoids, e.g., } \\
\text { isorenieratene, okenone }\end{array}$ & $\begin{array}{l}\text { Green and purple sulfur } \\
\text { bacteria }\end{array}$ & $\begin{array}{l}\text { Summons and Powell } \\
\text { 1987; Brocks et al. } 2005\end{array}$ \\
\hline Gammacerane & Tetrahymanol & $\begin{array}{l}\text { Purple nonsulfur bacteria, } \\
\text { some protists }\end{array}$ & $\begin{array}{l}\text { Ten Haven et al. } 1989 \\
\text { Kleemann et al. } 1990\end{array}$ \\
\hline $\begin{array}{l}\text { Tricyclic terpanes, } \\
\text { cheilanthanes }\end{array}$ & Unknown & $\begin{array}{l}\text { Unknown, probably } \\
\text { bacteria }\end{array}$ & $\begin{array}{l}\text { Moldowan et al. } \\
1983\end{array}$ \\
\hline$<\mathrm{C}_{20}$ acyclic isoprenoids & $\begin{array}{l}\text { Bacterial and algal } \\
\text { chlorophylls archaeol }\end{array}$ & $\begin{array}{l}\text { Photosynthetic bacteria } \\
\text { and protists Archaea } \\
\text { although nonspecific }\end{array}$ & Peters et al. 2005 \\
\hline$>C_{20}$ acyclic isoprenoids & $\begin{array}{l}\text { Glycerol ether lipids; also } \\
\text { found as free hydrocarbons }\end{array}$ & $\begin{array}{l}\text { Archaea although } \\
\text { nonspecific }\end{array}$ & Peters et al. 2005 \\
\hline Cholestane & $\begin{array}{l}\text { Cholesterol and related } \\
\mathrm{C}_{27} \text { sterols }\end{array}$ & $\begin{array}{l}\text { Photosynthetic protists, } \\
\text { metazoa }\end{array}$ & Volkman 2003 \\
\hline $\begin{array}{l}\text { Ergostane; } \\
\text { 24-methylcholestane }\end{array}$ & $\begin{array}{l}\text { Erogosterol and related } \\
\mathrm{C}_{28} \text { sterols }\end{array}$ & $\begin{array}{l}\text { Photosynthetic protists, } \\
\text { prevalent in diatoms }\end{array}$ & Volkman 2003 \\
\hline $\begin{array}{l}\text { Stigmastane; } \\
\text { 24-ethylcholestane }\end{array}$ & $\begin{array}{l}\text { Sitosterol, stigmasterol, } \\
\text { and related } \mathrm{C}_{29} \text { sterols }\end{array}$ & $\begin{array}{l}\text { Photosynthetic protists, } \\
\text { prevalent in chlorophytes }\end{array}$ & Volkman 2003 \\
\hline 24-n-Propylcholestane & 24-n-Propylcholesterol & Marine chrysophytes & Moldowan 1984 \\
\hline $\begin{array}{l}\text { Dinosterane, triaromatic } \\
\text { dinosteroids }\end{array}$ & Dinosterol, dinostanol & Dinoflagellates & $\begin{array}{l}\text { Summons et al. } 1987, \\
\text { 1992; Moldowan and } \\
\text { Talyzina } 1988\end{array}$ \\
\hline 24-Norcholestane & $\begin{array}{l}24-\text { Norcholesterol and } \\
\text { related 24-nor sterols }\end{array}$ & Diatoms & Rampen et al. 2005 \\
\hline $\begin{array}{l}\mathrm{C}_{20} \mathrm{C}_{25,} \text { and } \mathrm{C}_{30} \\
\text { highly branched } \\
\text { isoprenoids (HBIs) }\end{array}$ & $\begin{array}{l}\text { Mono- or polyunsaturated } \\
\text { HBIs }\end{array}$ & Diatoms & $\begin{array}{l}\text { Volkman et al. 1994; } \\
\text { Belt et al. 2000; } \\
\text { Sinninghe Damsté } \\
\text { et al. } 2004\end{array}$ \\
\hline $\begin{array}{l}n-C_{37}-C_{39} \text { alkenones } \\
\text { or alkanes }\end{array}$ & $n-\mathrm{C}_{37}-\mathrm{C}_{39}$ alkenones & Haptophytes & $\begin{array}{l}\text { Volkman et al. } 1980 \\
\text { Marlowe et al. } 1984\end{array}$ \\
\hline
\end{tabular}

${ }^{a}$ In most cases, the connection between the fossil hydrocarbon and organismic source is supported by detection of diagenetic intermediates in sediments.

${ }^{b}$ Extensive, systematic studies of lipids from microbial cultures are rare. Many lipid-organism relationships remain unknown.

"Genomic sequencing will also help identify the metabolic potential of source organisms to produce preservable compounds; such identification depends on (currently incomplete) knowledge of the biosynthetic pathways of biomarker molecules.

"Indicative but incomplete list. Reviews that provide extensive citation lists include Brocks and Summons 2004 and Peters et al. 2005. 
washing, or biodegradation. Although it is not widely appreciated, the global ubiquity of sedimentary bitumen and oil accumulations of all sizes means that hydrocarbons can also serve as a source of information on trends in the global carbon cycle (e.g., Andrusevich et al. 1998, 2000) and patterns in the evolution and environmental distributions of organisms, as discussed further later. The data illustrated in Figures 2, 3, 4, 5 , and 6 come from the "Oils" database and represent the averages of numerous samples from a global selection of prominent Cenozoic to Proterozoic petroleum systems, both marine and lacustrine.

Inspection of trends in the "Oils" database suggests that some aspects of the composi- tion of sedimentary hydrocarbons appear to be relatively invariant with age, changing instead with source rock lithology and sedimentary environment. These features are mostly reflected in the abundance patterns of bacterial and archaeal biomarkers. An example is depicted in Figure 2, which plots the relative abundance of a diagnostic bacteriohopane hydrocarbon (30-norhopane) as a function of paleolatitude. 30-Norhopane can arguably originate in several ways, but one particularly prolific source would be those BHPs with a hydroxyl substituent at position " $Z$ " (see Figure 1); this makes them prone to oxidative cleavage, leading to a $\mathrm{C}_{29}$ hydrocarbon (Rohmer et al. 1992). Such precursor BHPs occur commonly in proteobacteria,

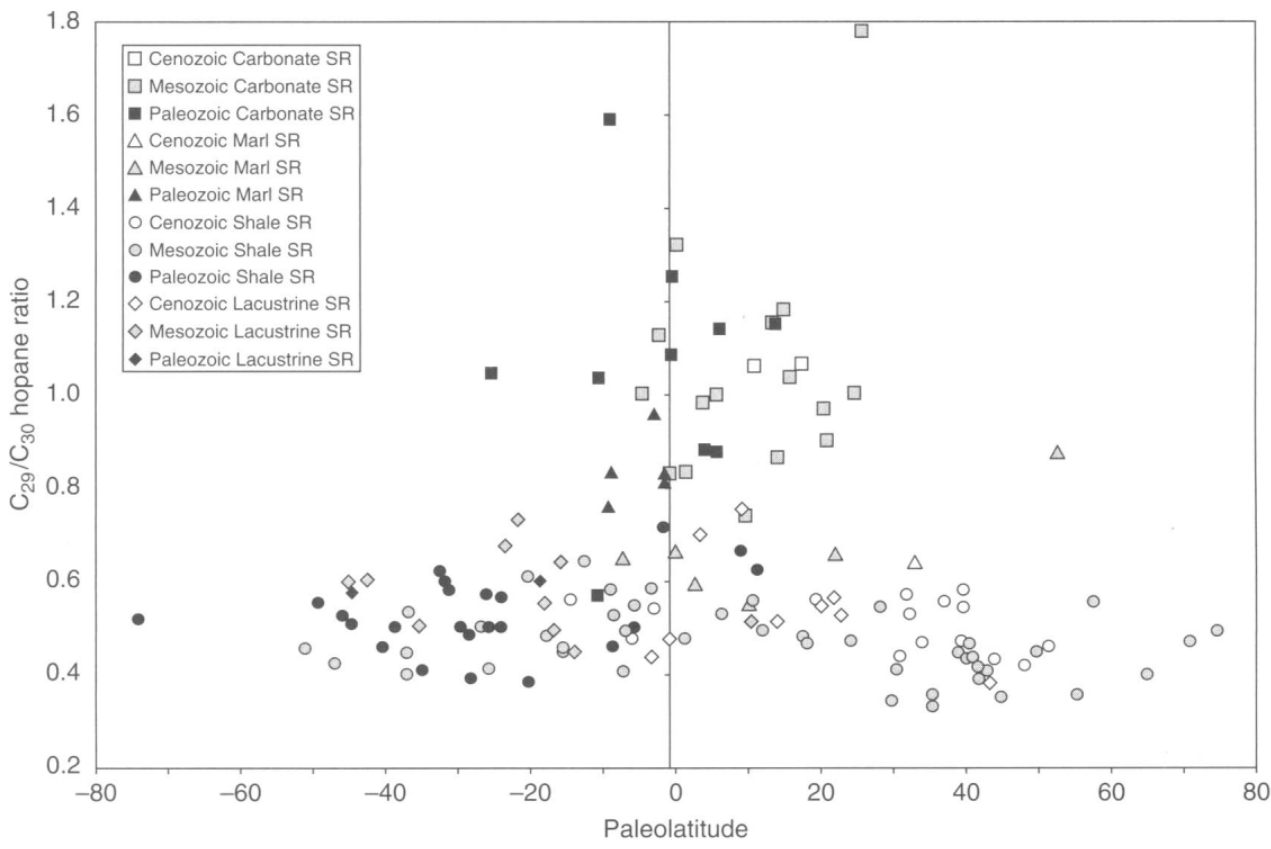

FIGURE 2. This figure depicts a diagnostic biomarker ratio derived from the averaged analyses of numerous oil samples representing important commercial petroleum accumulations plotted versus their paleolatitude. Samples are grouped according to geological era and classified according to the lithology and environment of the source rock, namely marine distal shales, marine marls, marine carbonates, or lacustrine sediments. The ratio of hopanes with 29 carbons to those with 30 carbons tends to be highest in carbonates and marls and in samples from low paleolatitude. Oils sourced from marine distal shales invariably show a $\mathrm{C}_{29} / \mathrm{C}_{31}$ hopane ratio $<0.7$, whereas marine carbonates tend to have values of 0.8 or more. This pattern holds irrespective of age over the duration of the Phanerozoic. 


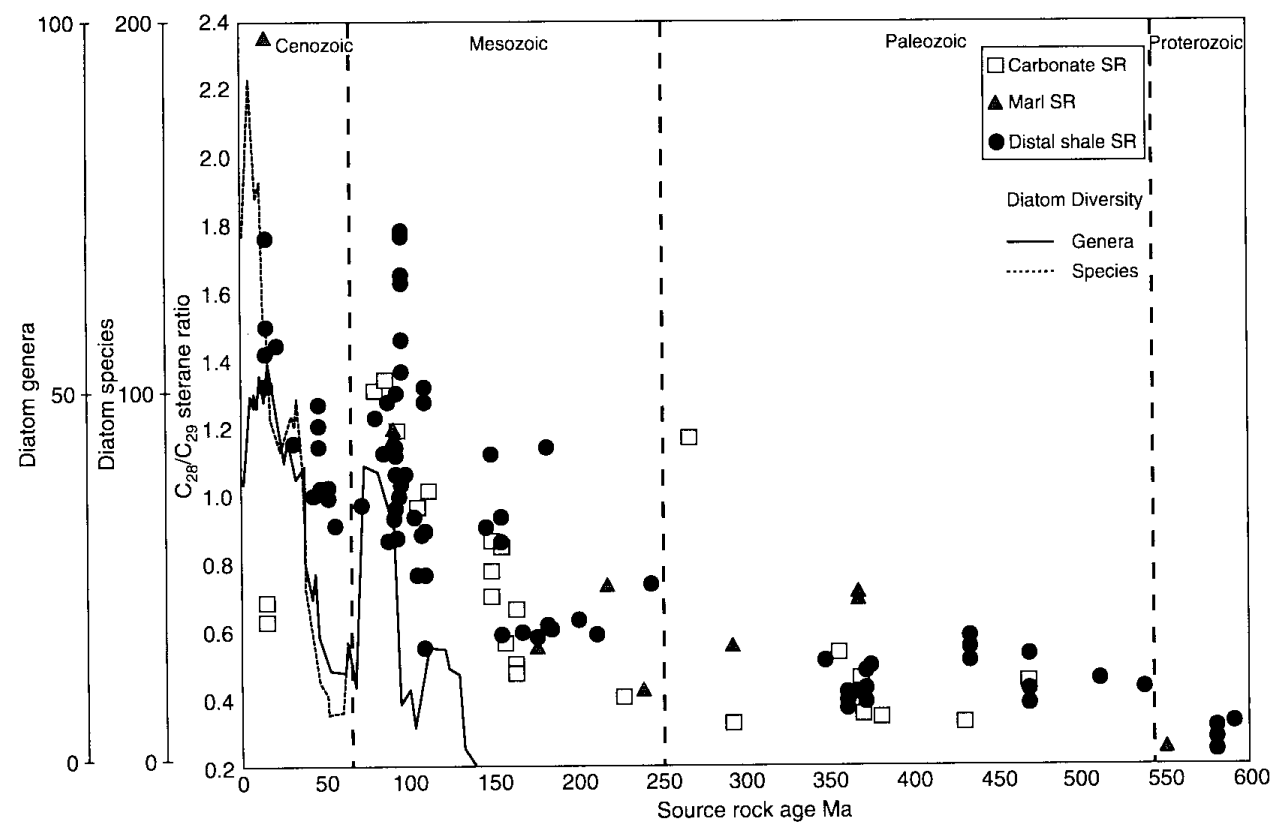

FIGURE 3. The secular increase in the abundance of $C_{28}$ relative to $C_{29}$ steranes over the Phanerozoic Eon, particularly during the last 250 million years, in petroleum systems from the GeoMark Oils database. Overlain are the fossil diatom species and genera diversity curves from Katz et al. (2004).

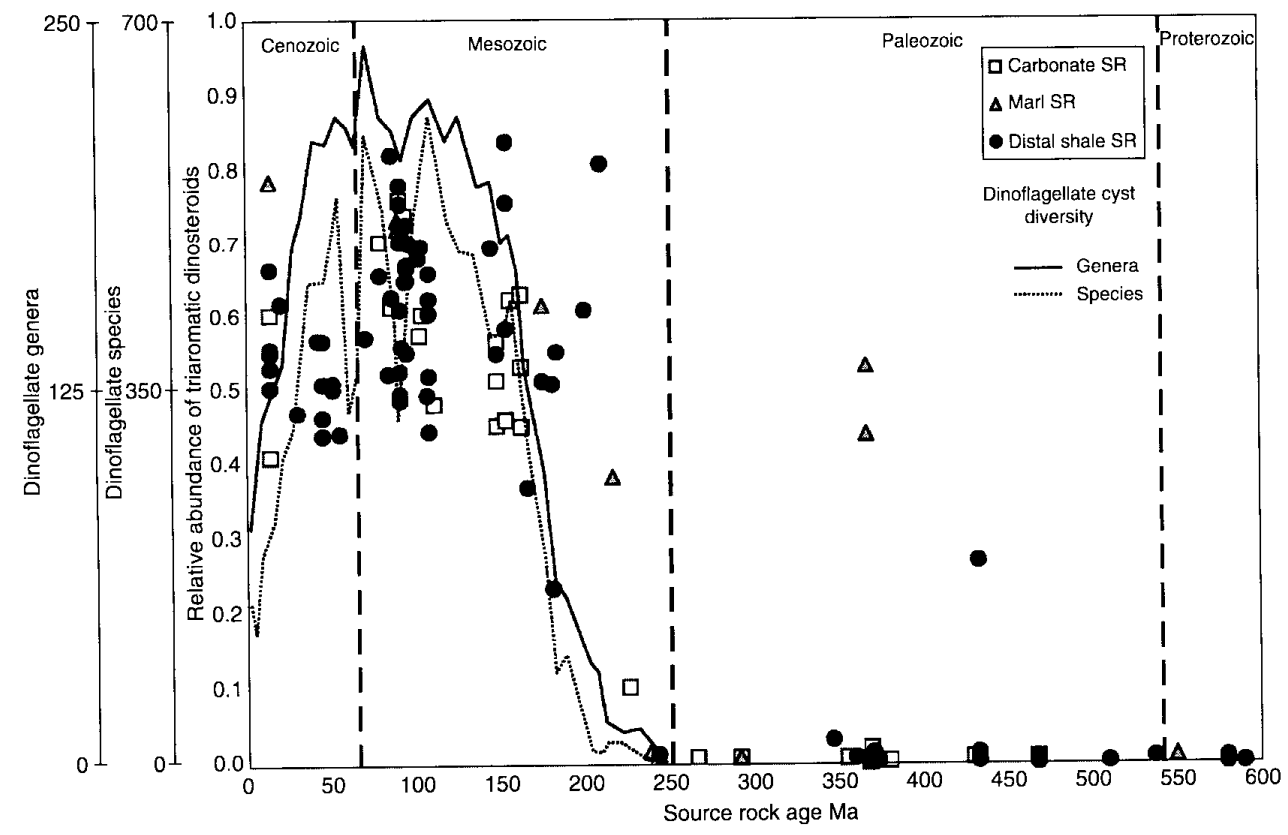

FIGURE 4. The relative abundance of aromatic dinosteranes over the Phanerozoic Eon from the GeoMark Oils database, showing marked increase during the early Mesozoic. Note concordance with genera- and species-level dinoflagellate fossil cyst diversity curves of Katz et al. (2004). Paleozoic occurrences of dinosteranes in petroleum sources are infrequent but merit further attention. 

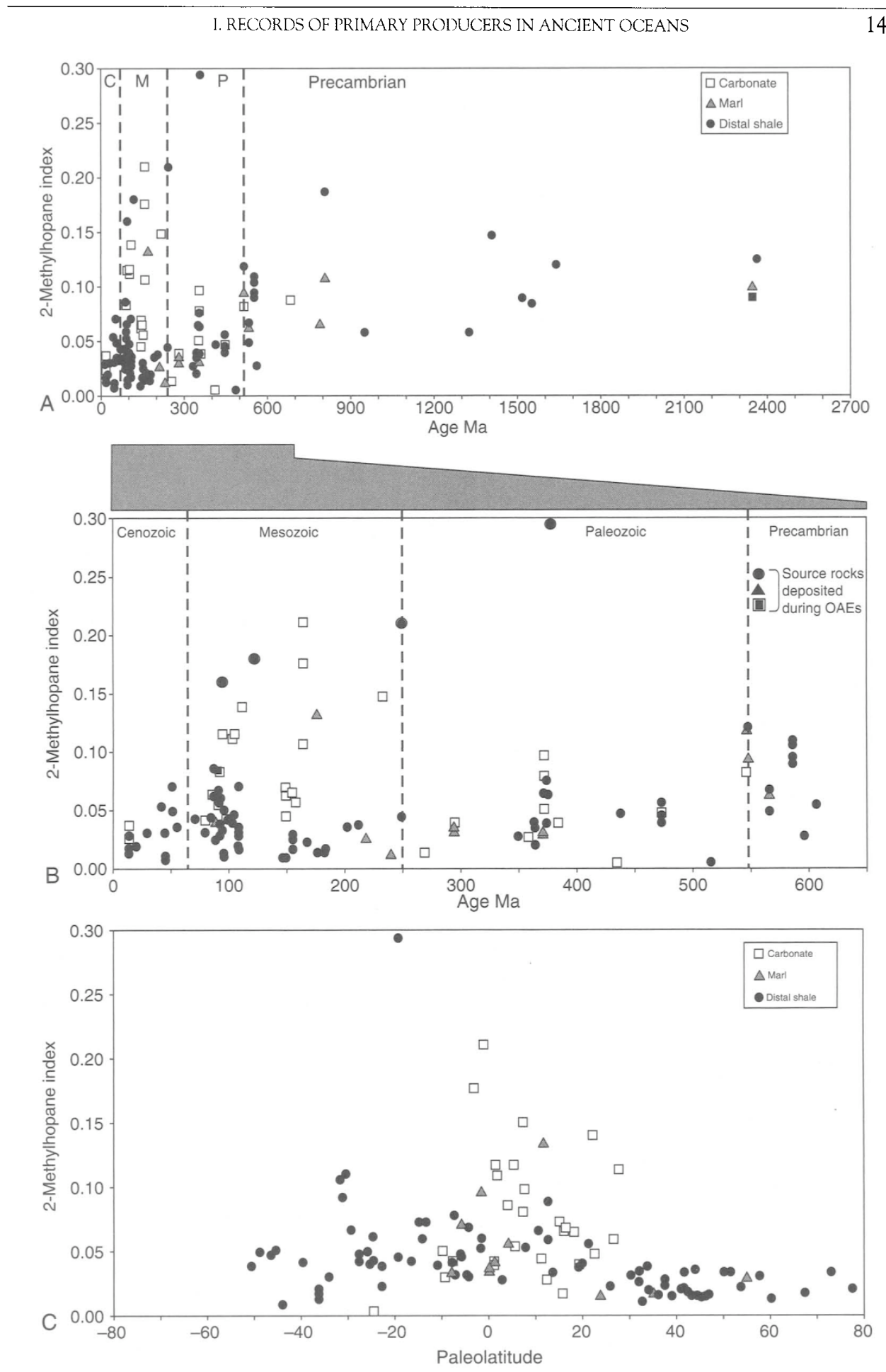

FIGURE 5. For legend see next page. 
FIGURE 5. (Cont'd) (A) $\mathrm{C}_{30}$ 2-methylhopane (2-MeH) index ( $=\mathrm{C}_{30} 2-\mathrm{MeH} /\left(\mathrm{C}_{30}\right.$ 2-MeH $+\mathrm{C}_{30}$ desmethyl hopane) through geologic time. Values are generally elevated throughout the Precambrian, with distal shales reaching below 0.05 only during the Phanerozoic. Compilation includes data from the GeoMark Oils database and Kuypers et al. (2004) (B) Expansion of the time scale of (A) to focus on the Phanerozoic. Source rocks deposited during oceanic anoxic events (OAEs) are highlighted and often show elevated 2-MeH indices. (C) 2-MeH indices of Phanerozoic petroleum systems by source rock lithology and paleolatitude. The highest 2-MeH values are generally found at low latitudes, often in carbonate depositional environments.

including methylotrophs. They appear to be especially common in carbonate-precipitating sedimentary environments; the ratio of $\mathrm{C}_{29} / \mathrm{C}_{30}$ hopane tends to be highest in oils from carbonates, intermediate in marls, and lowest in distal shales (Subroto et al. 1991). This relationship holds independent of the geological age of the source rocks. Carbonates accumulate predominantly in low latitudes, explaining the paleogeographic correspondence shown in Figure 2.

In contrast to the trends shown by biomarkers from prokaryotes, acyclic and cyclized terpenoids and steroids (see Figure 1) derived from planktonic algae and vascular plants show strong age-related trends (e.g., Summons and Walter 1990; Brocks and Summons 2004). For example, the oleanoid triterpenoids such as $\beta$-amyrin are important in the predation-defense mechanisms of flowering plants. Oleanane, a hydrocarbon derived from these triterpenoids, is sometimes abundant in oils from rocks deposited on continental margins, showing a marked increase in oils formed from Cenozoic rocks that reflects the Cretaceous radiation of the angiosperms (Moldowan et al. 1994). Low levels of oleanane in rocks as old as Jurassic age, however, suggests either that early flowering plants were sporadic inhabitants of Mesozoic environments or that oleanoid triterpenoid synthesis originated in their phylogenetic precursors (e.g., Peters et al. 2005). Observations concerning algal biomarkers are discussed later.

\section{THE RISE OF MODERN PHYTOPLANKTON}

\section{A. Fossils and Phylogeny}

In modern oceans, three algal groups dominate primary production on continental shelves: the diatoms, dinoflagellates, and

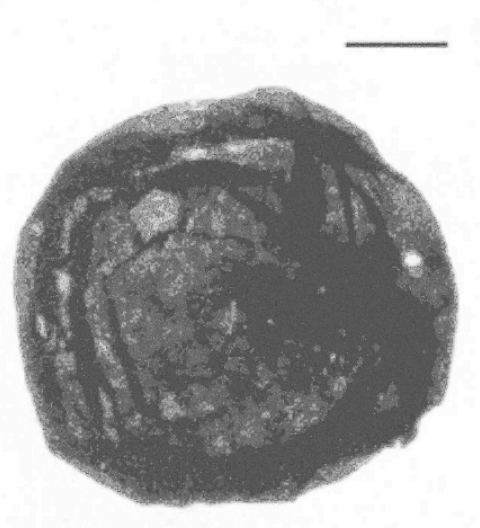

A

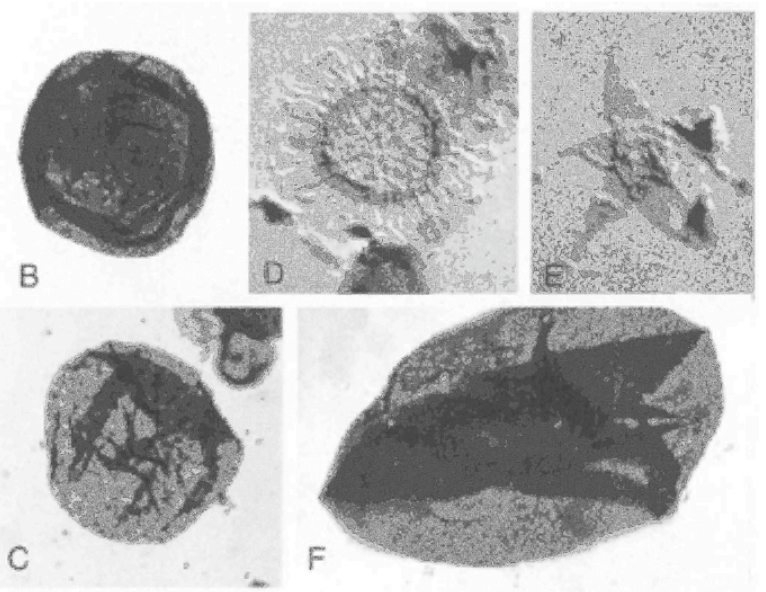

FIGURE 6. Proterozoic and Early Cambrian protistan microfossils. (A) and (B) are Neoproterozoic leiospherid acritarchs; (C-F) are Early Cambrian acritarchs (C-E) and a prasinophyte phycoma (F; Tasmanites). Scale bar $=40$ microns. 
coccolithophorids. As detailed elsewhere in this volume, fossils clearly suggest that these groups all rose to taxonomic and ecological prominence only during the Mesozoic Era (Delwiche, Chapter 10; de Vargas et al., Chapter 12; Kooistra et al., Chapter 11, this volume). Could there have been, however, an earlier "cryptic" evolutionary history for these groups? For example, might nonmineralizing stem group diatoms or haptophytes have been ecologically important but paleontologically uninterpretable in Paleozoic oceans? Might the significance of Paleozoic dinoflagellates be obscured by fossils that are abundant and diverse but lack archeopyles?

Several reports claim microfossil evidence for Paleozoic diatoms, dinoflagellates, and haptophytes, but such fossils are rare and subject to alternative interpretation as contaminants (the mineralized skeletons) or different taxa (organic fossils). Stratigraphic research indicates that the Paleozoic silica cycle differed substantially from that of the Cretaceous and Tertiary periods, with sponges and radiolarians dominating biological removal of silica from the oceans (Maliva et al. 1989). Similarly, sediments preserved in obducted slices of Paleozoic seafloor at best contain only limited evidence for pelagic carbonate deposition. Such observations cannot eliminate the possibility that rare diatoms, coccolithophorids, or calcareous dinoflagellates lived in Paleozoic oceans, but they clearly indicate that these groups did not perform the biogeochemical roles they have played since the Mesozoic Era.

Complementing this, molecular clock estimates for diatom and coccolithophorid diversification, calibrated by well-preserved fossils, suggest that these groups have no long Paleozoic "prehistory" (de Vargas et al., Chapter 12; Kooistra et al., Chapter 11, this volume). On the other hand, some molecular clock analyses suggest divergence times for the plastids in photosynthetic chromalveolates well back into the Proterozoic (Douzery et al. 2004; Yoon et al. 2004). If these estimates are even broadly cor- rect, they must be accommodated in one of two ways. Either the molecular clocks date divergence within a closely related group of unicellular red algae that subsequently and individually were incorporated as plastids in chromalveolate algae, or photosynthetic chromalveolates emerged from a single Proterozoic endosymbiosis (Cavalier-Smith 1999) but remained ecologically unimportant or paleontologically unrecognizable until much later.

\section{B. Biomarkers and the Rise of Modern Phytoplankton}

The rise to ecological prominence of the three chlorophyll $c$-containing eukaryotic plankton lineages left several imprints in the molecular fossil record, especially in the distributions of steranes with different sidechain alkylation patterns. A secular increase in the ratio of $\mathrm{C}_{28}$ to $\mathrm{C}_{29}$ steranes (24-methylcholestanes versus 24-ethylcholestanes), first noted by Grantham and Wakefield (1988), has been attributed to increasing production by chlorophyll $c$ algae, which dominate $\mathrm{C}_{28}$ sterane input, relative to green algae, which synthesize primarily $\mathrm{C}_{29}$ steroids (Volkman 2003). An updated plot of the $\mathrm{C}_{28} / \mathrm{C}_{29}$ sterane ratio versus geological age, based on averages from 123 petroleum systems worldwide, is shown in Figure 3 along with data for diatom diversity. The $C_{28} / C_{29}$ sterane ratio remains below 0.4 in the Neoproterozoic and, with one exception, below 0.7 through the Paleozoic. Corresponding to the diversification of diatoms in the later half of the Mesozoic, there is a rise in the $\mathrm{C}_{28} / \mathrm{C}_{29}$ sterane ratio to values as high as 1.8 , followed by an apparent drop in the Paleocene and Eocene. Values climb again in the Miocene, accompanying a second rise in the numbers of diatom genera and species.

Other biomarkers show marked increases in abundance in the Cretaceous that also likely reflect diatom radiation. These include 24-norcholestanes (Holba et al. 1998a, b), the so-called highly branched isoprenoids (HBIs; Sinninghe Damsté et al. 1999a, b; Belt 
et al. 2000; Allard et al. 2001), long-chain diols, and mid-chain hydroxyl methylalkanoates (Sinninghe Damsté et al. 2003). The secular increase in 24-norcholestane abundance (Moldowan et al. 1991; Holba et al. $1998 \mathrm{a}, \mathrm{b})$ was observed and linked to the diatom radiation well before a precursor sterol was recognized in a culture of the centric diatom Thalassiosira aff. antarctica (Rampen et al. 2005). In fact, in the study of Rampen et al. (2005) only one, that is T. aff. antarctica, of 100 different diatom taxa was found to produce 24-norcholesta-5, 22-dien-3 $\beta$-ol.

The detail of novel sterol production by diatoms provides an interesting window into the connections among biomarkers, taxonomy, and the physiological roles of lipids. In a recent study of diatom sterols, Suzuki et al. (2005) reported that environmental samples of diatoms collected from the North Pacific Ocean and the Bering Sea contained 24-norsterols. Further, samples of the diatom Coscinodiscus marginatus, initially devoid of 24-norcholesterol, contained significant amounts of this and the related steroid 27-nor-24-methylcholesta-5,22-dien$3 \beta$-ol after storage at $3^{\circ} \mathrm{C}$ for 30 days. These authors attributed the latter change to bacterial biodegradation. Due to the ubiquity of 24-norsteranes in Mesozoic sediments (Holba et al. 1998a, b), and no other evidence for selective side-chain biodegradation of sterols (biodegrading bacteria are unlikely to select for removal of $\mathrm{C}_{26}$ and $\mathrm{C}_{27}$ of the apparent precursor 24-methylcholesta5,22 -dien-3 $\beta$-ol and leave other sterols untouched), it seems far more likely that there is a direct, as opposed to diagenetic, source for the 24-norsteroids in sediments. The rarity of 24-norsterols in cultured diatoms (Rampen et al. 2005) more likely reflects the fact that sterol biosynthesis responds to physiological conditions and that laboratory culture conditions have as yet not mimicked the natural conditions, such as low temperature and, perhaps, low light under which 24-norsterols are produced by some diatoms. The enigma surrounding the origins of 24-norsteroids, and the ultimate detection of 24-norcholesta-5,22-dien-3 $\beta$-ol by Rampen et al. (2005) in a cold water diatom species, provide a timely reminder that biomarkers not only reflect the presence of particular algal taxa but also reflect the environmental conditions under which they thrive. A corollary to this is that cultured organisms might not always produce the same assemblage of lipids as their counterparts growing under natural conditions.

The other major class of diatom-specific biomarkers is the HBI. So far as is currently known, the occurrence of HBI is confined to four genera, namely Navicula, Haslea, and Pleurosigma within the pennates, and Rhizosolenia among the centrics (Volkman et al. 1994; Belt et al. 2000; Sinninghe Damsté et al. 2004). Both molecular phylogeny and fossils indicate that centric diatoms predate pennates (Kooistra et al. 2006). Therefore, the genus Rhizosolenia is considered the likely source of the first recorded fossil HBI at about 91 million years ago (Ma) — which predates recorded fossil tests of Rhizosolenid diatoms by about 20 million years (Sinninghe Damsté et al. 2004). This discrepancy in timing could reflect incomplete paleontological sampling, which systematically underestimates first appearances, HBI synthesis by a morphologically distinct stem group relative of the rhizosolenids, or both. Biosynthetic pathways are such a fundamental characteristic of organisms that they might be detectable through chemical fossils before the first classical fossils of a clade are ever recognizable.

Dinosteroid biomarkers, derived from the 4-methylsterols of dinoflagellates (Robinson et al. 1984), show an analogous pattern of secular increase in the Mesozoic, in accord with microfossil evidence for later Triassic dinoflagellate radiation. As in the case of diatom HBI, however, several reported occurrences of dinosteranes predate fossil cysts, in some cases by hundreds of millions of years (Summons et al. 1992; Moldowan and Talyzina 1998; Talyzina et al. 2000). These deserve close attention as they may 
establish a genuine pre-Mesozoic history of dinoflagellates, as predicted by molecular phylogenies and clocks. Some aspects of this putative history are considered further later. In Figure 4, data derived from the "Oils" database show the pattern of secular variation in triaromatic dinosteroid abundances, along with a recent compilation of dinoflagellate cyst diversity (Katz et al. 2004).

The third group of modern plankton, the haptophytes, produces distinctive lipids in the form of long-chain $\left(n-\mathrm{C}_{37}\right.$ to $\left.\mathrm{C}_{39}\right)$, unsaturated ketones known as alkenones (Volkman et al. 1980; Marlowe et al. 1984). These can be abundant and easily recognized in Recent and Cenozoic sediments that have not experienced extensive diagenesis, and they form the basis of a widely used paleotemperature proxy (Brassell et al. 1986). Their distinctiveness, however, lies in the carbonyl functionality and one to four unsaturations, which are easily reduced and inherently unstable over geological time scales (Prahl et al. 1989). Thus, the oldest reported detection is in Cretaceous sediments (Farrimond et al. 1986), and we would not expect to be able to recognize them in Paleozoic or older rocks.

The molecular and morphological records of eukaryotic predominance in shelf primary production are mirrored by indications of relatively low cyanobacterial contributions. Some cyanobacteria are known to biosynthesize BHPs and analogues with an extra methyl group attached to the 2 position of the A ring (2-MeBHP); the hydrocarbon cores of these molecules provide a potentially useful tracer for cyanobacterial input to sedimentary organic matter (Summons et al. 1999). A part from a few notable exceptions, values of the 2-methylhopane (2-MeHI) index fraction of hopanoids (methylated at the 2 position relative to their desmethyl counterparts) tend higher in Proterozoic samples than they are in Paleozoic and, especially, Jurassic and younger oils (Figure 5A). This is especially true of samples from shales. (Examination of the Phanerozoic record [Figure $5 \mathrm{~B}$ ] shows that higher
2-MeHI values are found in oils from carbonate lithologies, formed predominantly at low paleolatitudes [Figure 5C].) The exceptions are associated with widespread anoxia in the oceans (Figure 5B). Kuypers et al. (2004a, b) and Dumitrescu and Brassell (2005) studied biomarkers associated with Cretaceous oceanic anoxic events (OAEs) and found that the relative abundances of 2-methylhopanoids, as measured by the 2$\mathrm{MeHI}$, were distinctively enhanced, along with nitrogen $(\mathrm{N})$, isotopic evidence for cyanobacterial primary productivity (Kuypers et al. 2004b). Mass extinction at the Permian-Triassic boundary is also associated with widespread anoxia in shallow oceans (Wignall and Twitchett 2002). In Figure 5, the two data points for the Permian Triassic transition represent unpublished data from the Perth Basin, Australia, and the boundary stratotype in Meishan, China; in these sections, high 2-MeHI correlate with independent molecular, iron speciation, and sulfur isotopic evidence for intense euxinia (Grice et al. 2005). The highest 2$\mathrm{MeHI}$ value (0.29) recorded in the GeoMark Oils database comes from the Larapintine Petroleum System, Australia, which includes oils from the Late Devonian reef complex of the Canning Basin (Edwards et al. 1997) sourced from black shales deposited near to Frasnian-Famennian boundary, another event characterized by geological and geochemical evidence for pervasive euxinia (e.g., Bond et al. 2004). The samples from the Cretaceous OAEs, Permian-Triassic boundary, and Frasnian-Fammenian shale all contain abundant isorenieratane and aryl isoprenoids derived from the brown pigmented strains of the green sulfur bacteria (Chlorobiaceae), considered diagnostic for photic zone euxinia (e.g., Summons and Powell 1987; Koopmans et al. 1996; Kuypers et al. 2004a; Grice et al. 2005; van Breugel et al. 2005). Kuypers et al. (2004b) hypothesize that the $\mathrm{N}$ cycle was compromised while euxinic conditions prevailed during the Cretaceous OAEs, creating an unusual opportunity for the 
proliferation of $\mathrm{N}$-fixing cyanobacteria. The disparate occurrences described previously suggest a more general correlation between high 2-MeHI and photic zone euxinia, a topic we return to in our discussion of Proterozoic primary production.

Although cyanobacteria appear to have been minor contributors to primary production on most Mesozoic and Cenozoic continental shelves, they remain the dominant phytoplankton in open-ocean, oligotrophic environments today. Whether this is a recent or long-standing situation is difficult to discern given the paucity of the deepsea sedimentary records and the absence of 2Me-BHP in cyanobacterial picoplankton (Summons, unpublished data).

\section{Summary of the Rise of Modern Phytoplankton}

Fossils, molecular biomarkers, molecular clocks for individual clades, and the sedimentary silica record all tell a consistent story: the modern phytoplankton has Mesozoic roots. How we interpret this transformation depends in no small part on what we think came before.

\section{PALEOZOIC PRIMARY PRODUCTION}

\section{A. Microfossils}

Microfossils of presumptive eukaryotic phytoplankton are both abundant and diverse in Paleozoic marine rocks (Figure 6C-F). A number of forms, including Tasmanites, Pterospermella, and Cymatiosphaera, have morphologies and ultrastructures that relate them to prasinophyte phycomata (Tappan 1980). Indeed, in well-studied microfossil assemblages from Lower Cambrian shales, at least $20 \%$ of described morphotaxa and more than half of all individual fossils are likely prasinophytes (e.g., Volkova et al. 1983; Knoll and Swett 1987; Moczydlowska 1991). Others, with regularly distributed processes, tantalizingly resemble dinocysts, but lack archeopyles. Still other acritarchs (the group name given to closed, organic-walled microfossils of uncertain systematic relationships) (Evitt 1963) do not closely resemble known cysts of modern phytoplankton. Collectively, these microfossils show evidence of marked Cambrian and Ordovician radiations that parallel the two-stage diversification of marine animals (Knoll 1989). For reasons that remain obscure, acritarch diversity drops near the end of the Devonian and remains low for the remainder of the Paleozoic Era (Molyneux et al. 1996).

Moldowan and Talyzina (1998) innovatively attempted to break the phylogenetic impasse regarding Cambrian microfossils. Extracts from fossiliferous clays of the Lower Cambrian Lükati Formation, Estonia, contain low abundances of dinosterane and 4a-methyl-24-ethylcholestane, both known to originate from the sterols of dinoflagellates (Robinson et al. 1984; Summons et al. 1987). Moldowan and Talyzina (1998) divided the microfossil populations in a Lükati sample into three groups-tasmanitids (see Figure 6F; interpreted as prasinophyte phycomata), a low fluorescence group dominated by leiosphaerid acritarchs (also possible phycomata of prasinophytes like the extant Halosphaera), and a high fluorescence fraction containing abundant process-bearing acritarchs (e.g., see Figure 6D)-and analyzed the sterane content of these subassemblages. The tasmanitid and low fluorescence fractions contained relatively abundant $\mathrm{C}_{29}$ steranes but little or no dinoflagellate lipid. In contrast, the high fluorescence fraction contained relatively high abundances of dinosterane, suggesting that the dominant, process-bearing acritarchs are dinocysts sans archeopyles. It is not clear that sterols play a structural role in cyst walls, making selective adsorption a real possibility. Moreover, whereas dinosterane and 4a-methyl-24-ethylcholestane abundances in these samples are relatively high, their concentrations are absolutely low. Thus, the specific attribution of acritarch taxa to 
the dinoflagellates remains speculative. Nonetheless, these analyses do clearly suggest that dinoflagellates were present in coastal Cambrian oceans and may have left a morphological as well as biogeochemical record. Given the low abundances of dinoflagellate biomarkers, it is possible that the constituent dinoflagellates were largely heterotrophs, not primary producers.

\section{B. Paleozoic Molecular Biomarkers}

The molecular fossil record prior to the rise of the chlorophyll $c$ lineages broadly corroborates the microfossil evidence for the occurrence and potential ecological importance of other eukaryotic phytoplankton in the Paleozoic. In particular, the high abundance of $\mathrm{C}_{29}$ steranes relative to $\mathrm{C}_{27}$ and
$\mathrm{C}_{28}$ homologues suggests a greater role for green algae in marine primary production at this time. This signal is observed in globally distributed rocks and petroleum systems from the latest Neoproterozoic into the Paleozoic and wanes in the later Paleozoic, although the depositional bias for much of this time is toward low paleolatitudes (Figure 7). In a study of tasmanite oil shales from different locations in Tasmania, Revill et al. (1994) found that $C_{27}$ and $C_{29}$ steranes were present in roughly equal abundance and dominated over $C_{28}$. All the samples were shales with a high total organic carbon content, with the visible organic matter primarily comprising Tasmanites punctatus microfossils. These early Permian deposits, which were geographically localized, contained abundant dropstones and evidence

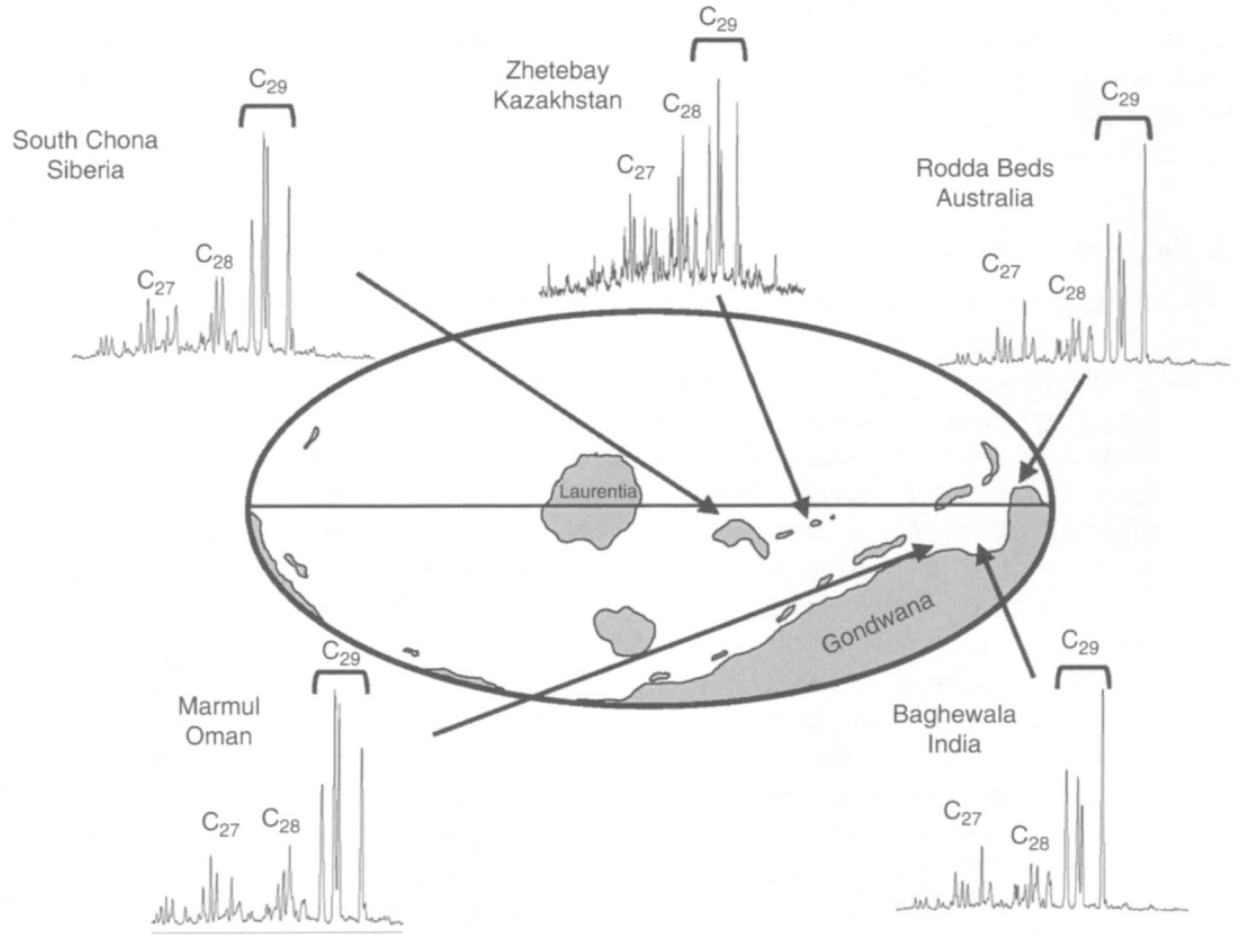

FIGURE 7. Dominance of $\mathrm{C}_{29}$ steranes in late Neoproterozoic/Early Cambrian petroleum systerns. Paleogeography shows concentration of depositional systems to low paleolatitudes during this time. Continental reconstruction redrawn after C. Scotese, available at www.scotese.com. 
of low-temperature minerals, which were clearly glacial in origin. Thus, the Tasmanites punctatus may have occupied an ecological niche similar to that occupied by modern seaice diatom communities (Revill et al. 1994).

Dinosteranes are generally below detection in the Paleozoic marine sediments and oils that have been examined and reported to date. In contrast, triaromatic dinosteroids have been found in significant abundance in several lower Paleozoic sedimentary rocks and petroleum samples. This speaks to the occurrence of either stem or crown group dinoflagellates in Paleozoic oceans. An additional factor in observed dinosterane abundances may be preservational bias. Saturated dinosteroids, dinosteranes, may only be preserved under strongly reducing conditions. A wider search for triaromatic dinosteroids, and authentication of the Paleozoic petroleum data through reanalysis and careful checking of pedigrees, may expose a more extensive pattern of occurrence, and hence, a richer early history for these plankton than is evident from the distribution of fossilized cysts.

\section{Paleozoic Summary}

Biomarker data for oils and some sediments suggest that dinoflagellates existed in Paleozoic oceans, but with few exceptions, lipids thought to be sourced by dinoflagellates occur in low abundances, raising the question whether Paleozoic dinoflagellates functioned to any great extent as primary producers. The same is true of possible stem-group heterokonts. Thus, although $\mathrm{Chl}$ a $+\mathrm{c}$ phytoplankton may well have existed in Paleozoic oceans, they do not appear to have played anything like the ecological role they have assumed since the Mesozoic Era began. In contrast, microfossil and biomarker molecules both suggest that green algae played a greater role in marine primary production than they have in the past 100 million years, and biomarkers also suggest a significant role for cyanobacterial production on continental shelves. Macro- fossils, predominantly of calcareous skeletons, further indicate that red and green algae were ecologically important in the shallow shelf benthos (Wray 1977).

\section{PROTEROZOIC PRIMARY PRODUCTION}

Fossils (whether morphological or molecular) are less abundant in Proterozoic rocks than they are in Phanerozoic samples, and Proterozoic sedimentary rocks, themselves, are less abundantly preserved than their younger counterparts. Nonetheless, fossils have been reported from hundreds of Proterozoic localities (Mendelson and Schopf 1992), allowing us to recognize at least broad patterns of stratigraphic and paleoenvironmental distribution. Indeed, Proterozoic micropaleontology has developed to the point where it has become predictive, in the sense that knowledge of age and environmental setting permits reasonable prediction about the fossil content of a given rock sample (e.g., Knoll et al. 2006).

\section{A. Prokaryotic Fossils}

By the earliest Proterozoic Eon, cyanobacteria must have been important contributors to primary production-there is no other plausible source for the $\mathrm{O}_{2}$ that began to accumulate in the atmosphere and surface oceans 2.45-2.32 billion years ago (Ga). Consistent with this observation, it has been appreciated since the early days of Precambrian paleontology that cyanobacteria-like microfossils are abundant and widespread constituents of Proterozoic fossil assemblages (Figure 8; Schopf 1968). Not all cyanobacteria have diagnostic morphologies, but some do and others are likely candidates for attribution given knowledge of taphonomy (processes of preservation) and depositional environments represented in the record. By mid-Proterozoic times, if not earlier, all major clades of cyanobacteria existed in marine and near-shore terrestrial 

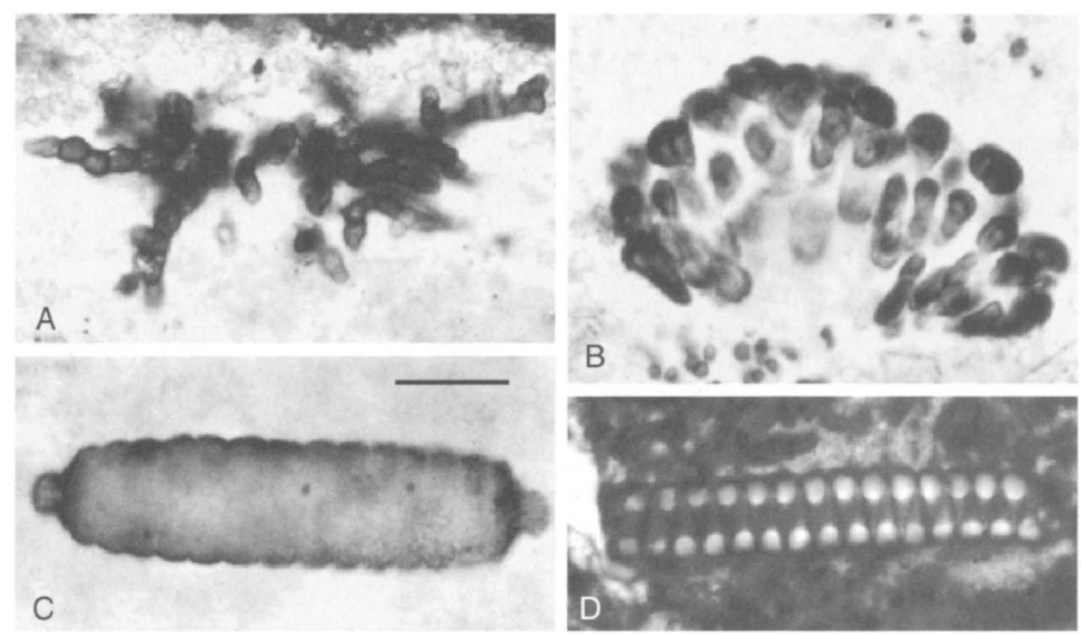

FIGURE 8. Cyanobacteria in Proterozoic sedimentary rocks. (A) 700-800-million-year-old endolithic pleurcapsalean fossil. (B) 1500-million-year-old mat building cyanobacterium closely related to modern Entophysalis. (C) 1500-million-year-old short trichome. (D) Spirulina-like fossil in latest Proterozoic (<600Ma) phosphorite. Scale bar $=15$ microns in (A-C), and $=25$ microns in (D).

environments, including those that differentiate akinetes and heterocysts (Tomitani $e t$ al. 2006). The best-characterized Proterozoic cyan-obacteria come from early diagenetic chert nodules in carbonate successions (e.g., Schopf 1968; Zhang 1981; Knoll et al. 1991; Sergeev et al. 1995, 2002; Golubic and SeongJoo 1999). These fossils are largely benthic and largely coastal marine. Stromatolites, however, indicate a much wider distribution of benthic cyanobacteria in the photic zone. (A role for cyanobacteria or of organisms in general is difficult to establish in the precipitated stromatolites found in Earth's oldest well-preserved sedimentary successions; however, the likelihood that cyanobacteria were major architects of Proterozoic stromatolites that accreted primarily by trapping and binding is high) (Grotzinger and Knoll 1999). Microfossils are less useful for evaluating the contributions of cyanobacteria to the phytoplankton of Proterozoic oceans because many were small, nondescript, and likely to settle on the seafloor in places where interpretable preservation was improbable. Given the distribution of planktonic clades on a phylogenetic tree calibrated by welldocumented fossils, however, it is likely that cyanobacteria were important constituents of the phytoplankton in Proterozoic oceans (Sanchez-Baracaldo et al. 2005; Tomitani et al. 2006; see later).

\section{B. Eukaryotic Fossils}

Two problems shadow attempts to understand the Proterozoic history of photosynthetic eukaryotes. Given the polyphyletic evolution of at least simple unicellular and multicellular characters, convergence complicates interpretation of many Proterozoic protistan fossils. In addition, given the oftobserved reality that stem group organisms display only a subset of the characters that collectively identify crown group members of clades, early fossils may challenge finer scale systematic attribution, even though they may be unambiguously eukaryotic.

Despite these problems, a small number of fossil populations provide calibration points for eukaryotic phylogenies. Bangiomorpha pubescens (Butterfield 2000) is a large 
population of multicellular microfossils found in tidal flat deposits of the ca. $1200 \mathrm{Ma}$ Hunting Formation, Arctic Canada. These erect filaments, preserved via rapid burial by carbonate mud and subsequent silicification, display patterns of thallus organization, cell division, and cell differentiation that ally them to the bangiophyte red algae. Complementing this, a moderate diversity of cellularly preserved florideophyte red algal thalli occurs in $<600 \mathrm{Ma}$ phosphorites of the Ediacaran Doushantuo Formation, China (Xiao et al. 2004). Shifting to another branch of the eukaryotic tree, several taxa of vase shaped microfossils in the ca. 750 Kwagunt Formation, Grand Canyon, Arizona, can be related to lobose testate amoebae, placing a minimum constraint on the timing of amoebozoan divergence (Porter et al. 2003).

Accepting the presence of red algae by $1200 \mathrm{Ma}$, one might expect to observe green algal fossils in younger Proterozoic rocks. Several candidate taxa have been described, of which Proterocladus, a branching coenocytic thallus organized much like living Cladophora, is most compelling (Butterfield et al. 1994). Palaeovaucheria clavata, described from $>1005 \pm 4 \mathrm{Ma}$ shales in Siberia (Herman 1990), as well as ca. 750-800 Ma shales from Spitsbergen (Butterfield 2004), has a branching filamentous morphology and pattern of reproductive cell differentiation very similar to that of the extant xanthophyte alga Vaucheria. Kooistra et al. (Chapter 11 , this volume) speculate that this similarity arose via convergence in a green algal clade; either interpretation places the origin of green algae earlier than $1000 \mathrm{Ma}$.

Fossils show that eukaryotic photoautotrophs were present in the benthos no later than the Mesoproterozoic Era (1600$1000 \mathrm{Ma})$, but what about the phytoplankton? Unicellular taxa occur in all three divisions of the Plantae, making it likely that such cells existed by the time that Bangiomorpha evolved. Of these, however, only the phycomate prasinophytes are likely to have left a tractable fossil record in marine sedimentary rocks. As noted previously, the three major types of ornamented phycoma known from living prasinophytes have fossil records that extend backward to the Early Cambrian, but there is little evidence of earlier origin. In contrast, extant Halosphaera develop smoothly spheroidal phycomata that could easily be represented among leiosphaerid acritarchs in Proterozoic rocks (see Figure 6A and B; Tappan 1980). Ultrastructural and microchemical studies (e.g., Javaux et al. 2004; Marshall et al. 2005) provide our best opportunity to test this hypothesis.

Chromalveolates may be recorded in a very different way. In 1986, Allison and Hilgert reported small $(7-40 \mu \mathrm{m}$ in maximum dimension), apparently siliceous ovoid scales in cherts of the Tindir Group, northwestern Canada, now judged to be $>635$ and $<710 \mathrm{Ma}$ (Kaufman et al. 1992). The scales resemble those formed by living prymnesiophytes and (at a smaller size range) chrysophytes, likely documenting early diversification somewhere within the chromalveolate branch of the eukaryotic tree.

Fossils of any kind are rare in rocks older than about 2000 million years, but unambiguous fossils of eukaryotes occur in shales as old as 1650-1850 Ma (Knoll et al. 2006); little is known about their systematic relationships or physiology. Compilations of total diversity (e.g., Knoll 1994; Vidal and Moczydlowska 1997), assemblage diversity (Knoll et al. 2006), and morphospace occupation (Huntley et al. 2006) through time agree that a moderate diversity of eukaryotic organisms existed in Mesoproterozoic oceans. By ca. $1200 \mathrm{Ma}$ if not earlier, this diversity included photosynthetic eukaryotes. Diversity appears to have increased modestly in the Neoproterozoic, but the major radiations within preservable seaweed and phytoplankton groups took place only at the end of the Proterozoic Era and during the ensuing Cambrian and Ordovician Periods (Knoll et al. 2006). 


\section{Proterozoic Molecular Biomarkers}

Rocks containing organic matter amenable to biomarker analysis grow increasingly rare as we sample more deeply into the Proterozoic, and many of those available have undergone extensive heating such that only the most recalcitrant molecules remain. Nevertheless, a molecular fossil record of primary production is emerging for this long interval of Earth history. A major feature of the record is the high relative abundance of 2-MeHI in organic-rich distal shales throughout the Proterozoic (see Figure 5), which, in conjunction with the microfossil record and geochemical evidence for oxic surface waters in oceans, provides strong evidence for the importance of cyanobacterial production. There has been such limited sampling of Paleo- and Mesoproterozoic sediments that it has not been possible to examine these for correlations with lithology, as has been accomplished for the Phanerozoic (see Figure 5C).

Steranes have been reported from a number of Proterozoic successions (e.g., Summons and Walter 1990; Hayes et al. 1992; Dutkiewicz et al. 2003) and are generally found in low abundance, reflecting at least in part the thermal maturity of their host rocks. These molecular fossils establish the presence of eukaryotes in Proterozoic oceans, but the scarcity of detailed records limits the inferences that can be drawn concerning ecological role or taxonomic affinities (because group-distinctive markers are generally below detection limits). The geologic record of steroid biosynthesis extends into the Late Archean, several hundred million years before the first recognized protistan fossils. However, there continue to be doubts about the syngeneity of these steroids because of the advanced maturity of all the sections studied so far and because of the potential for the bitumens found there to have migrated from younger sequences or to be contaminants from drill and handling (e.g., Brocks et al. 2003a, b). In contrast, the Roper and McArthur Basins of northern
Australia contain rocks of low-to-moderate thermal maturity, more consistent with the probability of finding genuinely syngenetic biomarkers. Given that studies of the Roper and McArthur Basin sediments and oils consistently show the presence of steroids (Summons et al. 1988a, b; Dutkiewicz et al. 2003) along with other evidence for the in situ (Summons et al. 1994) character of the bitumens, there seems little doubt that steroid biosynthesis operated as long ago as $1640 \mathrm{Ma}$. Preservation is a major limitation for both body and molecular fossil records at this point. Nonetheless, sterane abundances in rocks of this age appear to be low, independent of maturity level, and do not approach Phanerozoic abundances until the Neoproterozoic Era. Based on a few exceptionally well-preserved deposits of organic material in Mesoproterozoic shales, there appear to have been times and places where producer communities were very different from those that characterize later periods. Brocks et al. (2005) have reported biomarkers of anaerobic, sulfide-utilizing phototrophs in the carbonate facies of the Barney Creek Formation, Australia, suggesting that euxinic waters extended well into the photic zone. Molecular markers of eukaryotes and cyanobacteria in those portions of the Barney Creek Formation are exceptionally scarce, raising the possibility that, in at least some environments during the Proterozoic, production by anoxygenic photoautotrophs may have been quantitatively important. In fact, the scarcity of steroids and 2methylhopanoids in samples with most abundant biomarkers for phototrophic sulfur bacteria is also consistent with the highly euxinic conditions they require. The extent to which this scenario reflects global versus local conditions awaits further elucidation, but it is consistent with geochemical proxies for oceanic redox conditions, observed globally (e.g., Logan et al. 1995; Arnold et al. 2003; Shen et al. 2003; Gellatly and Lyons 2005).

In contrast to the scarcity of suitable organic-rich rocks in the Paleoproterozoic 
and Mesoproterozoic successions, the Neoproterozoic is replete with well-characterized organic matter in low maturity sections from Australia, North America, Oman (e.g., Grantham et al. 1987) and eastern Siberia (Summons and Powell 1992; see Summons and Walter 1990; Hayes et al. 1992, for reviews). Of particular note are the oldest commercial petroleum accumulations in Siberia and Oman. These late Neoproterozoic oils display striking biomarker patterns characterized by particularly abundant steroidal hydrocarbons. Predominance of $\mathrm{C}_{29}$ steranes over other homologues is a feature of oils from the South Oman Salt Basin that has received much attention since it was first reported by Grantham (1986). Examination of Neoproterozoic petroleum samples worldwide suggests that this is a globally significant feature (see Figure 7) that records the rise of green algae to ecological prominence. Further, samples that show the strong predominance of $\mathrm{C}_{29}$ steranes are also generally characterized by anomalously light carbon isotopic compositions, in the range of -33 to $-37 \%$ PDB. It is likely no coincidence that the oldest commercial petroleum deposits bear the prominent signature of a green algal contribution to petroleum-prone organic matter and that some green algae are known for their capacity to biosynthesize decay-resistant aliphatic biopolymers in their cell wall (algaenans; Derenne et al. 1991, 1992; Gelin et al. 1996, $1997,1999)$, a likely source of acyclic hydrocarbons in these oils (e.g., Höld et al. 1999).

\section{Summary of the Proterozoic Record}

Microfossil and biomarker records are consistent in showing that cyanobacteria and eukaryotic microorganisms were both present in Proterozoic oceans. Fossils indicate that the primary endosymbiotic event establishing the photosynthetic Plantae took place no later than ca. $1200 \mathrm{Ma}$, in broad agreement with molecular clock estimates appropriately ornamented by error estimates (Hackett et al., Chapter 7, this volume). Thus, eukaryotic algae contributed to primary production during at least the last 600 million years of the Proterozoic Era. Yet, preserved biomarkers are dominated by cyanobacteria and other photosynthetic bacteria, suggesting that eukaryotes played a limited quantitative role in primary production. Increasing amounts of $\mathrm{C}_{29}$ steranes appear in later Neoproterozoic samples, typified by the high sterane-to-hopane ratios and strong $\mathrm{C}_{29}$ sterane predominances in oils from the South Oman Salt Basin and eastern Siberia (e.g., Grantham 1986; Summons and Powell 1992); this suggests that green algae began to play an increasing role in primary production by $600-700 \mathrm{Ma}$. The timing of this transition is not well constrained but, in Oman, it begins prior to the Marinoan glaciation and extends to the Neoproterozoic-Cambrian boundary (Grosjean et al. 2005, and unpublished data), falsifying the hypothesis that the green algal proliferation was a response to the Acraman impact event in Australia (McKirdy et al. 2006). In short, algae may have emerged as major contributors to global primary production only during the late Neoproterozoic to Early Paleozoic interval distinguished by marked increases in fossil diversity.

\section{ARCHEAN OCEANS}

We evaluate the Archean geobiological record cautiously, as available data are sparse. Sedimentary rocks are limited in volume, especially for the early Archean, and most surviving strata have been altered by at least moderate metamorphism. Thus, any interpretation must be provisional.

The expectation from both phylogeny and the Proterozoic biogeochemical record is that prokaryotic primary producers are likely to have governed early marine ecosystems. Cyanobacteria have the ecological advantage of obtaining electrons from ubiquitous water molecules, but there is no reason to believe that cyanobacteria were the primordial photoautotrophs (see Blankenship 
et al., Chapter 3, this volume). Indeed, the question of when cyanobacteria, with their coupled photosystems, evolved remains contentious. In an early ocean dominated by anoxygenic photobacteria, the availability of electron donors $\left(\mathrm{Fe}^{++}, \mathrm{H}_{2}, \mathrm{H}_{2} \mathrm{~S}\right)$ would have limited primary production (Kharecha et al. 2005).

The four principal lines of evidence used to contract an evolutionary history of Proterozoic oceans apply equally to the Archean record: microfossils, biomarker molecules, sedimentary textures that record microbe/ sediment interactions on the ancient seafloor (e.g., stromatolites), and stable isotopic signatures (Knoll 2003b). Few microfossils have been reported from Archean cherts and shales. Somewhat poorly preserved fossils occur in latest Archean cherts from South Africa (Lanier 1986; Klein et al. 1987; Altermann and Schopf 1995); these could include cyanobacteria, but other alternatives cannot be rejected. More controversial are the nearly $3500 \mathrm{Ma}$ carbonaceous microstructures interpreted as bacterial, and possibly cyanobacterial, trichomes by Schopf (1993). Recently, not only their systematic interpretation but their fundamental interpretation as biogenic has been called into question (Brasier et al. 2002, 2005, 2006). Debate about these structures continues (e.g., Schopf et al. $2002 a, b$, in response to Brasier et al. 2002), but few believe that these structures, whatever their origin, provide phylogenetic or physiological insights into early life.

The stromatolite record is similar. At least 40 occurrences of stromatolites have been reported from Archean rocks (Schopf 2006) - not a lot given that the record is 1 billion years long. Those younger than about $3000 \mathrm{Ma}$ include structures that accreted by the trapping and binding of fine particles; such textures are more or less uniformly associated with microbial activity. Bedding surfaces on siliciclastic rocks of comparable age similarly include textures attributable to microbial mat communities (Noffke et al. 2006). Older stromatolites are largely precipitated structures whose bio- genicity is harder to establish. Conoidal forms in ca. $3450 \mathrm{Ma}$ rocks from Western Australia (Hofmannetal. 1999; Allwood et al. 2006) and "roll-up structures" (sediment sheets that were ripped up and rolled into a cylinder by currents, suggesting microbially mediated cohesion of poorly lithified laminae) in comparably old rocks from South Africa (Tice and Lowe 2004) may well require biological participation to form, but the taxonomic and physiological nature of the participants remains uncertain (see Tice and Lowe 2006, for an argument that anoxygenic photobacteria fueled Early Archean mat ecosystems).

No biogeochemically informative organic molecules are known from Early Archean rocks. Late Archean biomarkers have been reported; controversy surrounding their identification and interpretation has two distinct aspects. The first relates to their provenance and whether or not all the organic matter present in ancient sediments is coeval, as recognized by Brocks et al. $(2003 a, b)$. This question can best be addressed through studies of cores recently drilled and curated under controlled conditions. For example, the Agouron-Griqualand Paleoproterozoic Drilling Project (AGPDP) and the NASA Astrobiology Institute Drilling Project (ABDP) have recovered fresh cores from South Africa and the Pilbara Craton of Western Australia, respectively, which are being studied for a range of paleobiologic proxies, including analyses of preserved organic matter. One aim of this research is to control or eliminate contamination by hydrocarbons from younger sediments; a second aim is to test for relationships between extractable hydrocarbons and rock properties that could not exist in the case of contamination.

The second aspect of the controversy revolves around the degree to which biosynthetic pathways may have evolved over long time scales. It is fair to state that there must have been evolution in the structure and function of lipids over geological time. However, key enzymes in the biosynthetic 
pathways leading to sterols (Summons et al. 2006) and other triterpenoids in extant organisms are highly conserved. The known geological record of molecular fossils, especially steranes and triterpanes, is notable for the limited number of structural motifs that are recorded. With a few exceptions, the carbon skeletons are the same as those found in the lipids of extant organisms, and no demonstrably extinct structures have been reported. Furthermore, the patterns of occurrence of sterane and triterpane isomers are rigid over billion-year time scales and correlate strongly with environments of deposition, suggesting that diagenetic pathways connecting functional lipids to their fossil biomarker counterparts are also conserved. We also have evidence, through the occurrence of rearranged steranes (diasteranes) and unconventional steroids such as the 2-alkyl and 3-alkyl steranes (Summons and Capon 1988, 1991; van Kaam-Peters et al. 1998) and their aromatic counterparts (Dahl et al. 1995), that fossil steranes originated from precursors that carried a 3-hydroxyl group and unsaturation in the tetracyclic ring system, as extant sterols do. Thus, there is no evidence for major changes in the known record of chemical fossils that could be attributed to the inception, evolution, or alternative lipid biosynthetic pathways to the 24-alkylated steroids or hopanoids (Kopp et al. 2005). Accordingly, if biomarkers that have been identified are confirmed to be indigenous to late Archean rocks, this will constitute robust evidence for the presence of algae and bacteria early in Earth history. The fact that molecular oxygen is an absolute requirement for the biosynthesis of algal sterols also implies that oxygenic photosynthesis must have been present at the time (Summons et al. 2006).

The carbon isotopic abundances of Early Archean carbonates and organic matter are comparable to those of younger rocks, indicating fractionation like that imparted by Rubisco-based autotrophy. Indeed, $\mathrm{C}$-isotopic signatures that are consistent with carbon fixation by Rubisco extend backward to nearly $3800 \mathrm{Ma}$ metamorphosed sediments from southwestern Greenland (Rosing and Frei 2004). The question is whether these signatures require such an interpretation. Other biochemical pathways for carbon fixation exist and at least some of them impart isotopic signatures that are equally consistent with Archean data (e.g., Knoll and Canfield 1998). It has been known for 2 decades that abiotic syntheses of organic matter, like that demonstrated by Miller (1953), fractionate C-isotopes (Chang et al. 1982); the degree of fractionation appears to vary widely as a function of initial conditions. More recently, McCollom and Seewald (2006) have shown that Fischer-Tropsch-type (FTT) synthesis can produce organic compounds depleted in ${ }^{13} \mathrm{C}$ relative to their carbon source to a degree similar to that associated with biological carbon fixation. In these experiments, formic acid was reacted with native iron at $250^{\circ} \mathrm{C}$ and 325 bar, and a series of $n$-alkanes were produced that were depleted by $\sim 36 \%$ relative to the reactant carbon. This isotopic discrimination is in the range observed for the difference in $\delta^{13} \mathrm{C}$ values of coexisting carbonate minerals and organic matter in some Archean deposits. This finding emphasizes the importance of understanding the depositional context (e.g., sedimentary versus hydrothermal) of this very ancient carbonaceous matter when assessing its biogenicity.

We conclude that the origin of life predates the known record of preserved sedimentary rocks, but the nature of that life-and, in particular, the nature of primary producers in the oceans-remains uncertain. All known geobiological records from Archean rocks are consistent with an early evolution of cyanobacteria, but few if any require such an interpretation (Knoll 2003a). Indeed, Kopp et al. (2005) have hypothesized that cyanobacteria originated only in association with the initial accumulation of free oxygen in the atmosphere, 2320-2450 Ma (Holland 2006). Careful geobiological analyses of well-preserved Archean rocks remain a priority for continuing research. 


\section{CONCLUSIONS}

In combination, paleontological and organic geochemical data suggest that the second half of Earth history can be divided into three major eras, with respect to marine photosynthesis. Limited data from Paleoproterozoic and Mesoproterozoic rocks suggest that cyanobacteria and other photosynthetic bacteria dominated primary production at that time, with anoxygenic photosynthetic bacteria playing an important role, at least locally, in water masses subtended by a euxinic oxygen-minimum zone. Indeed, available data suggest that cyanobacteria continued as principal photoautotrophs well into the Phanerozoic Eon and long after photosynthesis originated in eukaryotic cells. $C_{29}$ sterane abundances indicate that green algae joined, but did not entirely displace, cyanobacteria as major primary producers during the latest Proterozoic and Cambrian; the second phase of primary production history thus initiated persisted until the Mesozoic radiation of modern phytoplankton dominants. Later Triassic oceans may have been the first in which cyanobacteria played a relatively minor role in continental shelf production. (Of course, they remain important today in the open gyre systems little recorded by pre-Jurassic sedimentary rocks.) The degree to which $\mathrm{Chl} \mathrm{a}+\mathrm{c}$ algae participated in Neoproterozoic and Paleozoic marine ecosystems remains unresolved, but if present their role must have been much smaller than it has been during the past 200 million years.

The observation that the oceans have experienced two major shifts over the past billion years in the composition of primary producers, and the corollary that at least some clades emerged as ecologically dominant primary producers long after their evolutionary origin, invites discussion of possible drivers. The importance of cyanobacteria in Proterozoic primary production can be attributed to at least two circumstances, their early diversification and envi- ronmental circumstances in Proterozoic oceans. Prior to the proliferation of eukaryotic algae, cyanobacteria would, of course, have had an open playing field, flourishing in oxygenated surface waters from coastline to mid-ocean gyres, although ceding deeper, at least intermittently euxinic parts of the photic zone to green and purple photosynthetic bacteria. Why, however, does it appear that cyanobacteria continued as dominant features of the photosynthetic biota on continental shelves long after red and green algae entered the oceans? At least in part, the answer may have to do with the nutrient structure of oceans in which, beneath an oxygenated surface layer, the oxygen minimum zone (Brocks et al. 2005), if not the entire deep ocean (Canfield 1998), had a high propensity for developing euxinia. Under these conditions, one would expect little fixed $\mathrm{N}$ to resurface during upwelling (Anbar and Knoll 2002; Fennel et al. 2005), providing strong selective advantage for cyanobacteria able to fix $\mathrm{N}$ and scavenge low concentrations of fixed $\mathrm{N}$ effectively from seawater.

Increasing oxygenation of the oceans during the Neoproterozoic Era (Canfield et al. 2006; Fike et al. 2006) would have begun to alleviate the $\mathrm{N}$ budget, as the midlevel waters that source upwelling would have been increasingly likely to remain oxic, limiting denitrification and anammox reactions that strip fixed $\mathrm{N}$ from ascending anoxic water masses. More ammonium would have been returned to the surface, and nitrate would have begun to accumulate for the first time. In consequence, eukaryotes would have spread more completely across benthic environments and into the phytoplankton, as recorded in the geological record (Knoll et al. 2006).

Dinoflagellates, diatoms, and coccolithophorids exhibit many features that collectively account for their ecological success in modern oceans (Delwiche, Chapter 10; de Vargas et al., Chapter 12; Kooistra et al., Chapter 11, this volume). Why, then, do we not see evidence for similar success in Paleozoic seas? 
One possibility is that the secondary endosymbioses that led to these groups took place only at the beginning of the Mesozoic Era or shortly earlier. Such a scenario is consistent with cladespecific molecular clocks for diatoms and coccolithophorids but is inconsistent with the hypothesis that secondary endosymbiosis involving red algal photosymbionts occurred only once, in the early history of the chromalveolates (Hackett et al., Chapter 7, this volume). Regardless of the timing of clade origination, however, we need to consider environmental factors, for the simple reason that it is hard to conceive of biological barriers would have prevented secondary endosymbiosis long before the Mesozoic began.

Black shale distributions may provide perspective on this issue. Multiregional to globally widespread black shales are essentially absent from Cenozoic successions but occur at about seven discrete stratigraphic horizons in the Mesozoic record (Jones and Jenkyns 2001). In contrast, there are at least seven black shale horizons in the Devonian record alone and many more in other parts of the Paleozoic, especially the Cambrian and Ordovician (Berry and Wilde 1978). Prior to the dawn of the Cambrian, most shales were carbonaceous (e.g., Knoll and Swett 1990; Abbott and Sweet 2000). If the redox structure of the oceans influenced the selective environment of green versus $\mathrm{Chl}$ a+c phytoplankton, then it may be that only in Mesozoic oceans did environmental conditions routinely favor the latter. As noted previously, fossils and biomarkers indicate that greens and cyanobacteria transiently reestablished themselves as principal primary producers during the Mesozoic OAEs; green sulfurbacteriaalsoproliferated duringepisodes of photic zone euxinia. Moreover, unlike chromalveolate photoautotrophs, both green algae and cyanobacteria show a pronounced preference for ammonium over nitrate in metabolism (Litchman, Chapter 16, this volume). Thus, the long-term redox evolution of the oceans may govern the composition of marine primary producers through time.

Whatever their drivers, the two observed transitions in the marine photosynthetic biota provide an important framework and stimulus for continuing paleobiological investigations of animal evolution. Latest Proterozoic and Cambrian phytoplankton radiation may not simply be a response to animal evolution (e.g., Peterson and Butterfield 2005) but also a driver. Welldocumented (Bambach 1993) increases in body size among Mesozoic (versus Paleozoic) marine invertebrates may reflect the Mesozoic radiation of larger net plankton, while the so-called Mesozoic Marine Revolution among mostly Cretaceous and Cenozoic marine animals may specifically reflect the rise to ecological prominence of diatoms (see Finkel, Chapter 15, this volume). Vermeij (1977) first documented the major evolutionary changes in skeletonized marine fauna during this interval and ascribed it to a late Mesozoic radiation of predators able to penetrate shells. Bambach (1993), however, argued that the required radiation of top predators could only occur as a consequence of increased primary production and, hence, increase nutrient status in the oceans. Bambach (1993) suggested that evolving angiosperms increased nutrient fluxes to the oceans, and although this likely did occur (see Knoll 2003c), the evolution of a high-quality food source and efficient nutrient transporter in the form of diatoms likely played at least an equal role.

\section{A. Directions for Continuing Research}

Over the past decade, both paleontologists and organic geochemists have made inroads into problems of photosynthetic history. Nonetheless, there continue to be more questions than answers. Future research will require more and independent studies of fossils and hydrocarbon distribution on Archean and Proterozoic rocks. However, it will also require phylogenetic, biosynthetic, and functional studies of sterols and BHPs (especially 2-Me-BHP) in living organisms that will increase our ability to interpret ancient records. In comparable fashion, continuing research on Protero- 
zoic and Paleozoic microfossils will need to stress wall ultrastructure (Arouri et al. 1999, 2000; Talzyina 2000; Javaux et al. 2004) and microchemical analysis (e.g., FTIR, hydropyrolysis, $\mathrm{x}$-ray and, perhaps, Raman spectroscopy; Love et al. 1995; Schopf et al. 2002a, b; Boyce et al. 2003; Marshall et al., 2005) interpreted in light of corresponding analyses of living cells and younger, taxonomically unambiguous fossils.

Finally, as noted previously, the evolution of photosynthetic organisms did not take place in a passive or unchanging ocean nor did it occur in an ecological vacuum. Improved understanding of Earth's redox history and the evolutionary record of animals (and land plants) (Falkowski et al. 2004) will provide the framework needed to interpret the evolutionary history of marine photoautotrophs as it continues to emerge.

\section{Acknowledgments}

AHK, JW, and RES acknowledge research support from NSF grant 0420592. AHK also acknowledges NSF grant OCE-0084032 (Project EREUPT, P. Falkowski, PI). RES is further supported by NASA Exobiology Program (Grant No. NNG05GN62G), NSF (EAR0418619), and PRF grant (41553-AC2) from the American Chemical Society. JRW receives support from an NDSEG Graduate Fellowship from the Office of Naval Research.

\section{References}

Abbott, S.T., and Sweet, I.P. (2000). Tectonic control on third-order sequences in a siliciclastic ramp-style basin: an example from the Roper Superbasin (Mesoproterozoic), northern Australia. Austral. J. Earth Sci. 47: 637-657.

Allard, W.G., Belt, S.T., Massé, G., Naumann, R., Robert, J.-M., and Rowland, S. J. (2001). Tetraunsaturated sesterterpenoids (Haslenes) from Haslea ostrearia and related species. Phytochemistry 56: 795-800.

Allison, C.W., and Hilgert, J.W. (1986). Scale microfossils from the Early Cambrian of northwest Canada. J. Paleontol. 60: 973-1015.

Allwood, A.C., Walter, M.R., Kamber, B.S., Marshall,C.P., and Burch, I.W. (2006). Stromatolite reef from the
Early Archaean era of Australia. Nature 441: 714718.

Altermann, W., and Schopf, J.W. (1995). Microfossils from the Neoarchean Campbell Group, Griqualand West sequence of the Transvaal Supergroup, and their paleoenvironmental and evolutionary implications. Precambrian Res. 75: 65-90.

Anbar, A.D., and Knoll, A.H. (2002). Proterozoic ocean chemistry and evolution: a bioorganic bridge? Science 297: $1137-1142$.

Andrusevich, V.E., Engel, M.H., Zumberge, J.E., and Brothers, L.A. (1998). Secular, episodic changes in stable isotopic composition of crude oils. Chem. Geol. 152: 59-72.

Andrusevich, V.E., Engel, M.H., and Zumberge, J.E. (2000). Effects of paleolatitude on the stable carbon isotope composition of crude oils. Geology 28: 847-850.

Arnold, G.L., Anbar, A.D., Barling, J., and Lyons, T.W. (2003). Molybdenum isotope evidence for widespread anoxia in mid-Proterozoic oceans. Science 304: $87-90$.

Arouri, K., Greenwood P.F, and Walter, M.R. (1999). A possible chlorophycean affinity of some Neoproterozoic acritarchs. Org. Geochem. 30: 1323-1337.

Arouri, K., Greenwood, P.F., and Walter, M.R. (2000). Biological affinities of Neoproterozoic acritarchs from Australia: microscopic and chemical characterisation. Org. Geochem. 31: 75-89.

Bambach, R.K. (1993). Seafood through time-changes in biomass, energetics, and productivity in the marine ecosystem. Paleobiology 19: 372-397.

Belt, S.T., Allard, W.G., Massé, G., Robert, J.-M., and Rowland S. J. (2000). Highly branched isoprenoids (HBIs): identification of the most common and abundant sedimentary isomers. Geochim. Cosmochim. Acta 64: 3839-3851.

Berry, W.B.N., and Wilde, P. (1978). Progressive ventilation of the oceans-an explanation for the distribution of the Lower Paleozoic Black Shales. Am. J. Sci. 278: $257-275$.

Bisseret, P., Zundel, M,, and Rohmer, M. (1985). Prokaryotic triterpenoids. 2. 2B-Methylhopanoids from Methylobacterium organophilum and Nostoc muscorum, a new series of prokaryotic triterpenoids. Eur. J. Biochem. 150: 29-34.

Blankenship, R., Sadekar, S., and Raymond, J. (2007). The evolutionary transition from anoxygenic to oxygenic photosynthesis. Evolution of Primary Producers in the sea. P.G. Falkowski and A.H. Knoll, eds. Boston, Elsevier, pp. 21-35.

Bond, D., Wignall, P.B., and Racki, G. (2004). Extent and duration of marine anoxia during the Frasnian-Famennian (Late Devonian) mass extinction in Poland, Germany, Austria and France. Geol. Mag. 141: 173-193.

Boyce, C.K., Cody, G.D., Fogel, M.L., Hazen, R.M., and Knoll, A.H. (2003). Chemical evidence for cell wall lignification and the evolution of tracheids in Early Devonian plants. Int. J. Plant Sci. 164: 691-702. 
Brasier, M., McLoughlin, N., Green, O., and Wacey, D. (2006). A fresh look at the fossil evidence for early Archaean cellular life. Phil. Trans. R. Soc. B 361: 887-902.

Brasier, M.D., Green, O.R., Jephcoat, A.P., Kleppe, A.K., van Kranendonk, M.J., Lindsay, J.F., Steele, A., and Grassineau, N.V. (2002). Questioning the evidence for Earth's oldest fossils. Nature 416: 76-81.

Brasier, M.D., Green, O.R., Lindsay, J.F., McLoughlin, N., Steele, A., and Stoakes, C. (2005). Critical testing of Earth's oldest putative fossil assemblage from the similar to $3.5 \mathrm{Ga}$ Apex Chert, Chinaman Creek, western Australia. Precambrian Res. 140: 55-102.

Brassell, S.C., Eglinton, G., Marlowe, I.T.,Pflaumann, U., and Sarnthein, M. (1986). Molecular stratigraphy: a new tool for climatic assessment. Nature 320: 129-133.

Brocks, J.J., Love, G.D., Snape, C.E., Logan, G.A., Summons, R.E., and Buick, R. (2003a) Release of bound aromatic hydrocarbons from late Archean and Mesoproterozoic kerogens via hydropyrolysis. Geochim. Cosmochim. Acta 67: 1521-1530.

Brocks, J.J., Love, G.D., Summons, R.E., Knoll, A.H., Logan, G.A., and Bowden, S. (2005). Biomarker evidence for green and purple sulfur bacteria in an intensely stratified Paleoproterozoic ocean. Nature 437: 866-870.

Brocks, J.J., and Summons, R.E. (2004). Sedimentary hydrocarbons, biomarkers for early life. Treatise in Geochemistry. Vol. 8. H.D. Holland and K. Turekian, eds. Amsterdam, Elsevier, pp. 65-115.

Brocks, J.J., Summons, R.E., Logan, G.A., and Buick, R. (2003b). Molecular fossils in Archean rocks II: composition, thermal maturity and integrity of hydrocarbons extracted from sedimentary rocks of the 2.78 to 2.45 billion years old Mount Bruce Supergroup, Pilbara Craton, Western Australia. Geochim. Cosmochim. Acta 67: 4289-4319.

Butterfield, N.J. (2000). Bangiomorpha pubescens n. gen., n. sp.: implications for the evolution of sex, multicellularity and the Mesoproterozoic/Neoproterozoic radiation of eukaryotes. Paleobiology 26: 386-404.

Butterfield, N.J. (2004). A vaucheriacean alga from the middle Neoproterozoic of Spitsbergen: implications for the evolution of Proterozoic eukaryotes and the Cambrian explosion. Paleobiology 30: 231-252.

Butterfield, N.J., Knoll, A.H., and Swett, N. (1994). Paleobiology of the Neoproterozoic Svanbergfjellet Formation, Spitsbergen. Fossils Strata 34: 1-84.

Canfield, D.E. (1998). A new model for Proterozoic ocean chemistry. Nature 396: 450-453.

Canfield, D.E., Poulton, S.W., and Narbonne, G.M. (2007) Late Neoproterozoic deep-ocean oxygenation and the rise of animal life. Science 315: 92-95.

Cavalier-Smith, T. (1999). Principles of protein and lipid targeting in secondary symbiogenesis: euglenoid, dinoflagellate, and sporozoan plastid origins and the eukaryote family tree. J. Euk. Microbiol. 46: 347-366.

Chang, S., Des Marais, D., Mack, R., Miller, S.L., and Strathearn, G.E. (1982). Prebiotic organic syntheses and the origin of life. Earth's Earliest Biosphere: Its Origin and Evolution. J.W. Schopf, ed. Princeton, NJ, Princeton University Press, p. 53-92.

Coolen, M.J.L., Muyzer, G., Rijpstra, W.I.C., Schouten, S., Volkman, J.K., and Sinninghe Damsté, J.S. (2004). Combined DNA and lipid analyses of sediments reveal changes in Holocene haptophyte and diatom populations in an Antarctic lake. Earth Planet. Sci. Lett. 223: 225-239.

DahlJ.,Moldowan J.M.,SummonsR.E.,McCaffreyM.A., Lipton P.A., Watt D.S., and Hope J.M. (1995). Extended 3 $\beta$-alkyl steranes and 3-alkyl triaromatic steroids in oils and rock extracts. Geochim. Cosmochim. Acta 59: 3717-3729.

Delwiche, C.F. (2007) The origin and evolution of dinoflagellates. Evolution of Primary Producers in the Sea. P.G. Falkowski and A.H. Knoll, eds. Boston, Elsevier, pp. 195-205.

Derenne, S., Largeau, C., Casadevall, E., Berkaloff, C., and Rousseau, B. (1991).Chemical evidence of kerogen formation in source rocks and oil shales via selective preservation of thin resistant outer walls of microalgae: origin of ultralaminae. Geochim. Cosmochim. Acta 55: 1041-1050.

Derenne, S., Le Berre, F., Largeau, C., Hatcher, P. G., Connan, J., and Raynaud, J.-F. (1992). Formation of ultralaminae in marine kerogens via selective preservation of thin resistant outer walls of microalgae. Org.Geochem. 19: 345-350.

de Vargas, C., Aubrey, M.-P., Probert, I., and Young, J. (2006). Origin and evolution of coccolithophores: From coastal hunters to oceanic farmers. Evolution of Primary Producers in the Sea. P.G. Falkowski and A.H. Knoll, eds. Boston, Elsevier, pp. 251-285.

Douzery, E.J.P., Snell, E.A., Bapteste, E., Delsuc, F., and Philippe, H. (2004). The timing of eukaryotic evolution: does a relaxed molecular clock reconcile proteins and fossils? Proc. Nat. Acad. Sci. U S A 101: 15386-15391.

Dumitrescu, M., and Brassell, S.C. (2005). Biogeochemical assessment of sources of organic matter and paleoproductivity during the early Aptian oceanic anoxic event at Shatsky Rise, ODP Leg 198. Org. Geochem. 36: 1002-1022.

Dutkiewicz, A., Volk, H., Ridley, J., and George, S. (2003). Biomarkers, brines, and oil in the Mesoproterozoic, Roper Superbasin, Australia. Geology 31: 981-984.

Edwards, D.S., Summons, R.E., Kennard, J.M., Nicoll, R.S., Bradshaw, J., Bradshaw, M., Foster C.B., O'Brien, G.W., and Zumberge, J.E. (1997). Geochemical characterisation of Palaeozoic petroleum systems in northwestern Australia. Austral. Petrol. Production Explorationists Assoc. J. 37: 351-379.

Evitt, W.R. (1963). A discussion and proposals concerning fossil dinoflagellates, hystrichospheres, and acritarchs: 2. Proc. Nat. Acad. Sci., U S A 49: 298-302. 
Farrimond, P., Eglinton, G., and Brassell, S.C. (1986). Alkenones in Cretaceous black shales, Blake-Bahama Basin, western North Atlantic. Org. Geochem. 10: 897-903.

Falkowski, P.G., Katz, M.E., Knoll, A.H., Quigg, A., Raven, J.A., Schofield, O., and Taylor, F.J.R. (2004). The evolution of modern eukaryotic phytoplankton. Science 305: 354-360.

Fennel, K., Follows, M., and Falkowski, P.G. (2005). The co-evolution of the nitrogen, carbon and oxygen cycles in the Proterozoic ocean. Am. J. Sci. 305: $526-545$.

Fike, D.A., Grotzinger, J.P., Pratt, L.M., and Summons, R.E. (2006). Oxidation of the Ediacaran Ocean. Nature 444: 744-747.

Finkel, Z.V. (2007). Does phytoplankton cell size matter? The evolution of modern marine food webs. Evolution of Primary Producers in the Sea. P.G. Falkowski and A.H. Knoll, eds. Boston, Elsevier, pp. 333-350.

Foster, C.B., and Afonin, S.A. (2006). Syndesmorion gen. nov. A coenobial alga of Chlorococcalean affinity from the continental Permian-Triassic deposits of Dalongkou section, Xinjiang Province, China. Rev. Palaeobot. Palynol. 138: 1-8.

Gelin, F., Boogers, I., Noordeloos, A.A.M., Sinninghe Damsté, J.S., Hatcher, P.G., and de Leeuw, J.W. (1996). Novel, resistant microalgal polyethers: An important sink of organic carbon in the marine environment? Geochim. Cosmochim. Acta 60: 1275-1280.

Gelin, F., Boogers, I., Noordeloos, A.A.M., Sinninghe Damsté, J.S., Riegman, R., and de Leeuw, J.W. (1997). Resistant biomacromolecules in marine microalgae of the classes Eustigmatophyceae and Chlorophyceae: geochemical implications. Org. Geochem. 26: $659-675$.

Gelin, F., Volkman, J.K., Largeau, C., Derenne, S., Sinninghe Damsté, J.S., and de Leeuw, J. W. (1999). Distribution of aliphatic, nonhydrolyzable biopolymers in marine microalgae. Org. Geochem. 30: 147-159.

Gellatly, A.M., and Lyons, T.M. (2005). Trace sulfate in mid-Proterozoic carbonates and the sulfur isotope record of biospheric evolution. Geochim. Cosmochim. Acta 69: 3813-3829.

Golubic, S., and Seong-Joo, L. (1999). Early cyanobacterial fossil record: preservation, palaeoenvironments and identification. Eur. J. Phycol. 34: 339-348.

Grantham, P.J. (1986). The occurrence of unusual $\mathrm{C}_{27}$ and $C_{2 \xi}$ sterane predominances in two types of Oman crude oil. Org. Geochem. 9: 1-10.

Grantham, P.J., Lijmbach, G.W.M., Posthuma, J., Hughes Clarke, M.W., and Willink, R.J. (1987). Origin of crude oils in Oman. J. Petroleum Geol. 11: 61-80.

Grantham, P.J., and Wakefield, L.L. (1988). Variations in the sterane carbon number distributions of marine source rock derived crude oils through geological time. Org. Geochem. 12: 61-73.

Grice, K., Cao, C., Love, G.D., Bottcher, M.E., Twitchett, R.J., Grosjean, E., Summons, R.E.,
Turgeon, S.C., Dunning, W., and Jin, Y. (2005). Photic zone euxinia during the Permian-Triassic superanoxic event. Science 307: 706-709.

Grosjean, E., Stalvies, C., Love, G.D., Lewis, A.N., Hebting, Y., Fike, D.A., Taylor, P.N., Newall, M.J., Grotzinger, J.P., Farrimond, P., and Summons, R.E. (2005). New oil-source correlations in the South Oman Salt Basin. Organic Geochemistry: Challenges for the 21st Century. 22 IMOG Seville, Spain, Abstract OOS-1.

Grotzinger, J.P., and Knoll, A.H. (1999). Proterozoic stromatolites: evolutionary mileposts or environmental dipsticks? Ann. Rev. Earth Planet. Sci. 27: 313-358.

Hackett, J.D., Yoon, H.S., Butterfield, N.J., Sanderson, M.J., and Bhattacharya, D. (2007). Plastid endosymbiosis: Sources and timing of the major events. Evolution of the Primary Producers in the Sea. P.G. Falkowski and A.H. Knoll, eds. Boston, Elsevier, pp. 109-131.

Hayes, J.M., Summons, R.E., Strauss, H., Des Marais, D.J., and Lambert, I.B. (1992). Proterozoic biogeochemistry. The Proterozoic Biosphere: A Multidisciplinary Study. J.W. Schopf and C. Klein, eds. Cambridge, UK, Cambridge University Press, pp. 81-133.

Hebting, Y, Schaeffer, P., Behrens, A., Adam, P., Schmitt, G., Schneckenburger, P., Bernasconi, S. M., and Albrecht, P. (2006). Biomarker evidence for a major preservation pathway of sedimentary organic carbon. Science 312: 1627-1631.

Hedges, J.I., and Keil, R.G. (1995). Sedimentary organic matter preservation: an assessment and speculative synthesis. Mar. Chem. 49: 81-115.

Herman, N. (1990). Organic World One Billion Years Ago. Nauka, Leningrad.

Hofmann,H.J., Grey, K.,Hickman, A.H., and Thorpe, R.I. (1999). Origin of 3.45 Ga coniform stromatolites in Warrawoona Group, Western Australia. Geol. Soc. Am. Bull. 111: 1256-1262.

Holba, A.G., Dzou, L.I.P., Masterson, W.D., Hughes, W.B., Huizinga, B.J., Singletary, M.S., Moldowan, J.M., Mello, M.R., and Tegelaar, E. (1998a). Application of 24-norcholestanes for constraining source age of petroleum. Org. Geochem. 29: 1269-1283.

Holba, A.G., Tegelaar, E.W., Huizinga, B.J., Moldowan, J.M., Singletary, M.S., McCaffrey, M.A., and Dzou, L.I.P. (1998b). 24-norcholestanes as age-sensitive molecular fossils. Geology 26: 783-786.

Höld, I.M., Schouten, S., Jellema, J., and Sinninghe Damsté, J.S. (1999). Origin of free and bound midchain methyl alkanes in oils, bitumens and kerogens of the marine, Infracambrian Huqf Formation (Oman). Org. Geochem. 30: 1411-1428.

Holland, H.D. (2006). The oxygenation of the atmosphere and oceans. Phil. Trans. R. Soc. B. 361: 903-915.

Huntley, J.W., Xiao, S.H., and Kowalewski, M. (2006). 1.3 billion years of acritarch history: an empirical morphospace approach. Precambrian Res. 144: 52-68. 
160 8. THE GEOLOGICAL SUCCESSION OF PRIMARY PRODUCERS IN THE OCEANS

Javaux, E.J., Knoll, A.H., and Walter, M.R. (2004). TEM evidence for eukaryotic diversity in mid-Proterozoic oceans. Geobiology 2: 121-132.

Jones, C.E., and Jenkyns, H.C. (2001). Seawater strontium isotopes, oceanic anoxic events, and seafloor hydrothermal activity in the Jurassic and Cretaceous. Am. J. Sci. 301: 112-149.

Katz, M.E., Finkel, Z.V., Grzebyk, D., Falkowski, P.G., and Knoll, A.H. (2004). Evolutionary trajectories and biogeochemical impacts of marine eukaryotic phytoplankton. Ann. Rev. Ecol. Syst. 35: 523-556.

Kaufman, A.J., Knoll, A.H., and Awramik, S.M. (1992). Bio- and chemostratigraphic correlation of Neoproterozoic sedimentary successions: the upper Tindir Group, northwestern Canada as a test case. Geology 20: 181-185.

Kharecha, P., Kasting, J., and Siefert, J. (2005). A coupled atmosphere-ecosystem model of the early Archean Earth. Geobiology 3: 53-76.

Kleemann, G., Poralla, K., Englert, G., Kjøsen, H., Liaaen-Jensen, S., Neunlist, S., and Rohmer, M. (1990). Tetrahymanol from the phototrophic bacterium Rhodopseudomonas palustris: first report of a gammacerane triterpene from a prokaryote. ]. Gen. Microbiol. 136: 2551-2553.

Klein, C., Beukes, N.J., and Schopf, J.W. (1987). Filamentous microfossils in the early Proterozoic Transvaal Supergroup - their morphology, significance, and paleoenvironmental setting. Precambrian Res. 36: 81-94.

Knoll, A.H. (1989). Evolution and extinction in marine realm: some constraints imposed by microplankton. Phil. Trans. R. Soc. B 325: 279-290.

Knoll, A.H. (1994). Proterozoic and Early Cambrian protists: evidence for accelerating evolutionary tempo. Proc. Nat. Acad. Sci. U S A 91: 6743-6750.

Knoll, A.H. (2003a). The geological consequences of evolution. Geobiology 1: 3-14.

Knoll, A.H. (2003b). Life on a Young Planet. Princeton, NJ, Princeton University Press.

Knoll, A.H. (2003c). Biomineralization and evolutionary history. Rev. Mineral. Geochem. 54: 329-356.

Knoll, A.H., and Canfield, D.E. (1998). Isotopic inferences on early ecosystems. Paleontol. Soc. Pap. 4: 212-243.

Knoll, A.H., and Golubic, S. (1992). Living and Proterozoic cyanobacteria. Early Organic Evolution: Implications for Mineral Energy Resources. M. Schidlowski, S. Golubic, M.M. Kimberley, D.M. McKirdy, and P.A. Trudinger, eds. Berlin, Springer-Verlag, pp. 450-462.

Knoll, A.H., Javaux, E.J., Hewitt, D., and Cohen, P. (2006). Eukaryotic organisms in Proterozoic oceans. Phil. Trans. R. Soc. B 361: 1023-1038.

Knoll, A.H., and Swett, K. (1987). Micropaleontology across the Precambrian-Cambrian boundary in Spitsbergen. J. Paleontol. 61: 898-926.

Knoll, A.H., and Swett, K. (1990). Carbonate deposition during the late Proterozoic era: an example from Spitsbergen. Am. J. Sci. 290-A: 104-132.
Knoll, A.H., Swett, K., and Mark, J. (1991). The Draken Conglomerate Formation: paleobiology of a Proterozoic tidal flat complex. J. Paleontol. 65: 531-569.

Kohnen, M.E.L., Sinninghe Damsté, J.S., Baas, M., Kock-van Dalen, A.C., and de Leeuw, J.W. (1993). Sulphur-bound steroid and phytane carbon skeletons in geomacromolecules: implications for the mechanism of incorporation of sulphur into organic matter. Geochim. Cosmochim. Acta 57: 25152528.

Kohnen, M.E.L., Sinninghe Damsté, J.S., and de Leeuw, J.W. (1991). Biases from natural sulphurization in palaeoenvironmental reconstruction based on hydrocarbon biomarker distributions. Nature 349: 775-778.

Kooistra, W.H.C.F., Gersonde, R., Medlin, L.K., and Mann, D.G. (2007). The origin and evolution of the diatoms: Their adaptation to a planktonic existence. Evolution of Primary Producers in the Sea. P. G. Falkowski and A.H. Knoll, eds. Boston, Elsevier, pp. 207-249.

Koopmans, M.P., Van Kaam-Peters, H.M.E., Schouten, S., de Leeuw, J.W., Sinninghe Damsté, J.S., Koster, J., Kenig, F, and Hartgers, W.A. (1996). Diagenetic and catagenetic products of isorenieratene: molecular indicators for photic zone anoxia: Geochim. Cosmochim. Acta 60: 4467-4496.

Kopp, R.E., Kirschvink, J.L., Hilburn, I.A., and Nash, C.Z. (2005). The Paleoproterozoic snowball Earth: a climate disaster triggered by the evolution of oxygenic photosynthesis. Proc. Nat. Acad. Sci. US A 102: 11131-11136.

Kuypers, M.M.M., Lourens, L.J., Rijpstra, W.I.C., Pancost, R.D., Nijenhuis, I.A., and Sinninghe Damsté, J.S. (2004a). Orbital forcing of organic carbon burial in the proto-North Atlantic during oceanic anoxic event 2. Earth Planet. Sci. Lett. 228: 465-482.

Kuypers, M.M.M., van Breugel, Y., Schouten, S., Erba, E., Sinninghe Damsté, J.S. (2004b). $\mathrm{N}_{2}$-fixing cyanobacteria supplied nutrient $\mathrm{N}$ for Cretaceous oceanic anoxic events. Geology 32: 853-856.

Lanier, W.P. (1986). Approximate growth rates of Early Proterozoic microstromatolites as deduced by biomass productivity. Palaios 1: 525-542.

Litchman, E. (2007). Resource competition and the ecological success of phytoplankton. Evolution of Primary Producers in the Sea. P.G. Falkowski and A.H. Knoll, eds. Boston, Elsevier, pp. 351-375.

Logan, G.A., Hayes, J.M., Hieshima, G.B., and Summons, R.E. (1995). Terminal Proterozoic reorganisation of biogeochemical cycles. Nature 376: 53-56.

Love, G.D., Snape, C.E., Carr, A.D., and Houghton, R.C. (1995). Release of covalently-bound alkane biomarkers in high yields from kerogen via catalytic hydropyrolysis. Org. Geochem. 23: 981-986.

Maliva, R., Knoll, A.H., and Siever, R. (1989). Secular change in chert distribution: a reflection of evolving 
biological participation in the silica cycle. Palaios 4: 519-532.

Marlowe, I.T., Green, J.C., Neal, A.C., Brassell, S.C., Eglinton, G., and Course, P.A. (1984). Long chain (n-C37-C39) alkenones in the Prymnesiophyceae. Distribution of alkenones and other lipids and their taxonomic significance. Br. Phycol. I. 19: 203-216.

Marshall, C.P., Javaux, E.J., Knoll, A.H., and Walter, M.R. (2005). Combined micro-Fourier transform infrared (FTIR) spectroscopy and Micro-Raman spectroscopy of Proterozoic acritarchs: a new approach to palaeobiology. Precambrian Res. 138: 208-224.

Martin, J.H., Knauer, G.A., Karl, D.M., and Broenkow, W.W. (1987). VERTEX: carbon cycling in the northeast Pacific. Deep-Sea Res. 34: 267-285.

McCollom, T.M., and Seewald, J.S. (2006). Carbon isotope composition of organic compounds produced by abiotic synthesis under hydrothermal conditions. Earth Planet. Sci. Lett. 243: 74-84.

McKirdy, D.M., Webster, L.J., Arouri, K.R., Grey, K., and Gostin, V.A. (2006). Contrasting sterane signatures in Neoproterozoic marine rocks of Australia before and after the Acraman asteroid impact. Org. Geochem. 37: 189-207.

Mendelson, C.V., and Schopf, J.W. (1992). Proterozoic and selected Early Cambrian microfossils and microfossil-like objects. The Proterozoic Biosphere: A Multidisciplinary Study. J.W. Schopf and C. Klein, eds. Cambridge, UK, Cambridge University Press, pp. 867-951.

Miller, S.L. (1953). A production of amino acids under possible primitive Earth conditions. Science 117: 527-528.

Moczydlowska, M. (1991). Acritarch biostratigraphy of the Lower Cambrian and the Precambrian-Cambrian boundary in southeast Poland. Fossils Strata 29: 1-127.

Moldowan, J.M. (1984). C30-steranes, novel markers for marine petroleums and sedimentary rocks. Geochim. Cosmochim. Acta 48: 2767-2768.

Moldowan, J.M., Dahl, J.E.P., Huizinga, B.J., Fago, F.J., Hickey, L.J., Peakman, T.M., and Taylor, D.W. (1994). The molecular fossil record of oleanane and its relation to angiosperms. Science 265: 768-771.

Moldowan, J.M., Lee, C.Y., Watt, D.S., Jeganathan, A., Slougui, N.E., and Grallegos, E.J. (1991). Analysis and occurrence of $\mathrm{C}_{26}$-steranes in petroleum and source rocks. Geochim. Cosmochim. Acta 55: 1065-1081.

Moldowan, J.M., Seifert, W.K., and Gallegos, E.J. (1983). Identification of an extended series of tricyclic terpanes in petroleum. Geochimicha et Cosmochimica Acta 47: 1531-1534.

Moldowan, J.M., and Talyzina, N.M. (1998). Biogeochemical evidence for dinoflagellate ancestors in the early Cambrian. Science 281: 1168-1170.

Molyneux, S.G., Le Hérissé, A., and Wicander, R. (1996). Paleozoic phytoplankton. Palynology: Principles and Applications. J. Jansonius and D.C. McGregor, eds.
Tulsa, OK, American Association of Stratigraphic Palynologists Press, pp. 493-529.

Noffke,N.,Eriksson,K.A.,Hazen, R.M., andSimpson,E.L (2006). A new window into Early Archean life: microbial mats in Earth's oldest siliciclastic tidal deposits (3.2 Ga Moodies Group, South Africa). Geology 34: 253-256.

Peters, K.E., Walters, C.C., and Moldowan, J.M. (2005). The Biomarker Guide, 2nd ed. Cambridge, UK, Cambridge University Press.

Peterson, K.J., and Butterfield, N.J. (2005). Origin of the Eumetazoa: testing ecological predictions of molecular clocks against the Proterozoic fossil record. Proc. Nat. Acad. Sci. U S A 102: 9547-9552.

Porter, S.M, Meisterfeld, R., and Knoll, A.H. (2003). Vase-shaped microfossils from the Neoproterozoic Chuar Group, Grand Canyon: a classification guided by modern testate amoebae. J. Paleontol. 77: 409-429.

Prahl, F.G., de Lange, G.J., Lyle, M., and Sparrow, M.A. (1989). Postdepositional stability of long-chain alkenones under contrasting redox conditions. Nature 341: 434-437.

Rampen, S.W., Schouten, S., Abbas, B., Panoto, F.E., Muyzer, G., Campbell, C.N., Fehling, J., and Sinninghe Damsté, J.S. (2005). The origin of 24norcholestanes and their use as age-diagnostic biomarkers. Organic Geochemistry: Challenges for the 21st Century. 22 IMOG Seville, Spain, Abstract PB1-10.

Revill, A.T., Volkman, J.K., O'Leary, T., Summons, R.E., Boreham, C.J., Banks, M.R., and Denwer, K. (1994). Depositional setting, hydrocarbon biomarkers and thermal maturity of tasmanite oil shales from Tasmania, Australia. Geochim. Cosmochim. Acta 58: 3803-3822.

Robinson, N., Eglinton, G., and Brassell, S.C. (1984) Dinoflagellate origin for sedimentary $4 \alpha$-methylsteroids and $5 \alpha(\mathrm{H})$-stanols. Nature 308: 439-442.

Rohmer, M., Bisseret, P., and Neunlist, S. (1992). The hopanoids: prokaryotic triterpenoids and precursors of ubiquitous molecular fossils. Biological Markers in Sediments and Petroleum. J.M. Moldowan, P. Albrecht, and R.P. Philp, eds. New York, Prentice Hall, pp. $1-17$.

Rohmer, M., Bouvier-Nave, P., and Ourisson, G. (1984). Distribution of hopanoid triterpenes in prokaryotes. J. Gen. Microbiol. 130: 1137-1150.

Rosing, M.T., and Frei, R. (2004). U-rich Archaean seafloor sediments from Greenland-indications of $>3700 \mathrm{Ma}$ oxygenic photosynthesis. Earth Planet. Sci. Lett. 217: 237-244.

Sanchez-Baracaldo, P., Hayes, P.K., and Blank, C.E. (2005). Morphological and habitat evolution in the Cyanobacteria using a compartmentalization approach. Geobiology 3: 145-165.

Schopf, J.W. (1968). Microflora of the Bitter Springs Formation, Late Precambrian, central Australia. J. Paleontol. 42: 651-688. 
162 8. THE GEOLOGICAL SUCCESSION OF PRIMARY PRODUCERS IN THE OCEANS

Schopf, J.W. (1993). Microfossils of the early Archean Apex chert-new evidence of the antiquity of life. Science 260: 640-646.

Schopf, J.W. (2006). Fossil evidence of Archaean life. Phil. Trans. R. Soc. B 361: 869-885.

Schopf, J.W., Kudryavtsev, A.B., Agresti, D.G., Wdowiak, T.J., and Czaja, A.D. (2002a). LaserRaman imagery of Earth's earliest fossils. Nature 416: 73-76.

Schopf,J.W.,Kudryavtsev,A.B.,Agresti,D.G.,Wdowiak, T.J., and Czaja, A.D. (2002b). Laser-Raman spectroscopy: images of the Earth's earliest fossils? Reply. Nature 420: 477.

Sergeev, V.N., Gerasimenko, L.M., and Zavarzin, G.A. (2002). The Proterozoic history and present state of cyanobacteria. Microbiology 71: 623-637.

Sergeev, V.N., Knoll, A.H., and Grotzinger, J.P. (1995). Paleobiology of the Mesoproterozoic Billyakh Group, northern Siberia. Paleontol. Soc. Mem. 39: 1-37.

Shen, Y., Knoll, A.H., and Walter, M.R. (2003). Evidence for low sulphate and deep water anoxia in a midProterozoic marine basin. Nature 423: 632-635.

Sinninghe Damsté, J.S., Muyzer, G., Abbas, B., Rampen, S.W., Masse, G., Allard, W.G., Belt, S.T., Robert, J.M., Rowland, S.J., Moldowan, J.M., Barbanti, S.M., Fago, F.J., Denisevich, P., Dahl, J., Trindade, L.A.F, and Schouten, S. (2004). The rise of the rhizosolenid diatoms. Science 304: 584-587.

Sinninghe Damsté, J.S., Rampen, S., Rijpstra, W.I.C., Abbas, B., Muyzer, G., and Schouten, S. (2003). A diatomaceous origin for long-chain diols and midchain hydroxy methyl alkanoates widely occurring in Quaternary marine sediments: Indicators for high-nutrient conditions. Geochim. Cosmochim. Acta 67: $1339-1348$

Sinninghe Damsté, J.S., Rijpstra, W.I.C., Schouten, S., Peletier, H., van der Maarel, M.J., and Gieskes, W.W.C. (1999a). A $C_{25}$ highly branched isoprenoid alkene and $\mathrm{C}_{25}$ and $\mathrm{C}_{27}$ n-polyenes in the marine diatom Rhizosolenia setigera. Org. Geochem. 30: 95-100.

Sinninghe Damsté, J.S., Schouten, S., Rijpstra, W.I.C., Hopmans, E.C., Peletier, H., Gieskes, W.W.C., and Geenevasen, J.A.J. (1999b). Structural identification of the C25 highly branched isoprenoid pentaene in the marine diatom Rhizosolenia setigera. Org. Geochem. 30: 1581-1583.

Subroto, E.A., Alexander, R., and Kagi, R.I. (1991). 30-Norhopanes: their occurrence in sediments and crude oils. Chem. Geol. 93: 179-192.

Summons, R.E., Bradley, A.S., Jahnke, L.L., and Waldbauer, J.R. (2006). Steroids, triterpenoids and molecular oxygen. Phil. Trans. R. Soc. B 361: 951-968

Summons, R.E., Brassell, S.C., Eglinton, G., Evans, E., Horodyski, R.J., Robinson, N., and Ward, D.M. (1988b). Distinctive hydrocarbon biomarkers from fossiliferous sediments of the Late Proterozoic Walcott Member, Chuar Group, Grand Canyon, Arizona. Geochim. Cosmochim. Acta 52: 2625-2637.
Summons, R.E., and Capon, R.J. (1988). Fossil steranes with unprecedented methylation in ring-A. Geochimicha et Cosmochimica Acta 52: 2733-2736.

Summons, R.E., and Capon, R.J. (1991). Identification and significance of 3-ethyl steranes in sediments and petroleum. Geochim. Cosmochim. Acta 55: 2391-2395.

Summons, R.E.,Jahnke,L.L.,Hope,J.M., and Logan,G.A. (1999). 2-Methylhopanoids as biomarkers for cyanobacterial oxygenic photosynthesis. Nature 400: 554-557.

Summons, R.E., and Powell, T.G. (1987). Identification of aryl isoprenoids in source rocks and crude oils: biological markers for the green sulphur bacteria. Geochim. Cosmochim. Acta 51: 557-566.

Summons, R.E., and Powell, T.G. (1992). Hydrocarbon composition of the Late Proterozoic oils of the Siberian Platform: implications for the depositional environment of the source rocks. Early Organic Evolution: Implications for Mineral and Energy Resources. M. Schidlowski, S. Golubic, M.M. Kimberley, D.M. McKirdy, and P.A. Trudinger, eds. Berlin, SpringerVerlag, pp. 296-507.

Summons, R.E., Powell, T.G., and Boreham, C.J. (1988a). Petroleum geology and geochemistry of the Middle Proterozoic McArthur Basin, Northern Australia: III. Composition of extractable hydrocarbons. Geochim. Cosmochim. Acta 52: 1747-1763.

Summons, R.E., Taylor D., and Boreham, C.J. (1994). Geochemical tools for evaluating petroleum generation in Middle Proterozoic sediments of the McArthur Basin, Northern Territory, Australia. Austral. Petroleum Explor. Assoc. J. 34: 692-706.

Summons, R.E., Thomas, J., Maxwell, J.R., and Boreham, C. J. (1992). Secular and environmental constraints on the occurrence of dinosterane in sediments. Geochim. Cosmochim. Acta 56: 2437-2444.

Summons, R.E., Volkman, J.K., and Boreham, C.J. (1987). Dinosterane and other steroidal hydrocarbons of dinoflagellate origin in sediments and petroleum. Geochim. Cosmochim. Acta 51: 3075-3082.

Summons, R.E., and Walter M.R. (1990). Molecular fossils and microfossils of prokaryotes and protists from Proterozoic sediments. Am. J. Sci. 290-A: 212-244.

Suzuki, N., Yasuo, N., Nakajo, T., and Shine, H. (2005). Possible origin of 24-norcholesterol in marine environment. Organic Geochemistry: Challenges for the 21st Century. 22 IMOG Seville, Spain, Abstract OB2-2.

Talyzina, N.M. (2000). Ultrastructure and morphology of Chuaria circularis (Walcott, 1899) Vidal and Ford (1985) from the Neoproterozoic Visingso Group, Sweden. Precambrian Res. 102: 123-134.

Talyzina, N.M., Moldowan, J.M., Johannisson, A., and Fago, FJ. (2000). Affinities of Early Cambrian acritarchs studied by using microscopy, fluorescence flow cytometry and biomarkers. Rev. Palaeobot. Palynol. 108: 37-53.

Tappan, H. (1980). The Paleobiology of Plant Protists. San Francisco, Freeman. 
Ten Haven, H.L., Rohmer, M., Rullkotter, J., and Bisseret, P. (1989). Tetrahymanol, the most likely precursor of gammacerane, occurs ubiquitously in marine sediments. Geochim. Cosmochim. Acta 53: 3073-3079.

Tice, M.M., and Lowe, D.R. (2004). Photosynthetic microbial mats in the 3,416-Myr-old ocean. Nature 431: $549-552$

Tice, M.M., and Lowe, D.R. (2006). Hydrogen-based carbon fixation in the earliest known photosynthetic organisms. Geology 34: 37-40.

Tomitani, A., Knoll, A.H., Cavanaugh, C.M., and Ohno, T. (2006). The evolutionary diversification of cyanobacteria: molecular phylogenetic and paleontological perspectives. Proc. Nat. Acad. Sci. U S A 103: 5442-5447.

Treibs, A. (1936). Chlorophyll and hemin derivatives in organic mineral substances. Angezw. Chem. 49: 682-686.

van Breugel, Y., Schouten, S., Paetzel, M., Ossebaar, J., Sinninghe Damsté, J.S. (2005). Reconstruction of $\delta^{13} \mathrm{C}$ of chemocline $\mathrm{CO} 2(\mathrm{aq})$ in past oceans and lakes using the $\delta^{13} \mathrm{C}$ of fossil isorenieratene. Earth Planet. Sci. Lett. 235: 421-434.

van Kaam-Peters, H.M.E., Köster, J., van der Gaast, S.J., Dekker, M., de Leeuw, J.W., and Sinninghe Damsté, J.S. (1998). The effect of clay minerals on diasterane/ sterane ratios. Geochim. Cosmochim. Acta 62: 2923-2929.

Vermeij, G.J. (1977). The Mesozoic marine revolution; evidence from snails, predators and grazers. Paleobiology 3: 245-258.

Vidal, G., and Moczydlowska, M. (1997). Biodiversity, speciation, and extinction trends of Proterozoic and Cambrian phytoplankton. Paleobiology 23: 230-246.

Volkman, J.K. (2003). Sterols in microorganisms. Appl. Microbiol. Biotechnol. 60: 496-506.

Volkman, J.K., Barrett, S.M., and Dunstan, G.A. (1994). $\mathrm{C}_{25}$ and $\mathrm{C}_{36}$ highly branched isoprenoid alkanes in laboratory cultures of two marine diatoms. Org. Geochem. 21: 407-413.

Volkman, J.K., Eglinton, G., Corner, E.D.S., and Sargent, J.R. (1980). Novel unsaturated straight-chain $C_{37}$ $C_{39}$ methyl and ethyl ketones in marine sediments and a coccolithophore Emiliania huxleyi. Advances in Organic Geochemistry 1979. A.G. Douglas and J.R. Maxwell, eds. London, Pergamon, pp. 219-227.

Volkova, N.G., Kirjanov, V.V., Piscun, L. V., Paškeviciene, L.T., and Jankauskas, T.V. (1983). Plant microfos- sils. Upper Precambrian and Cambrian Palaeontology of the East European Platform. A. Urbanek and A.Y. Rozanov, eds. Warsaw, Wydawnictwa Geologiczne. pp. 7-46.

Wignall, P.B., and Twitchett, R.J. (2002). Extent, duration and nature of the Permian-Triassic superanoxic event. Geol. Soc. Am. Spec. Paper 356: 395-413.

Wray, J.L. (1977). Calcareous Algae. Amsterdam, Elsevier, Amsterdam.

Xiao, S., Knoll, A.H., Yuan, X., and Pueschel, C.M. (2004). Phosphatized multicellular algae in the Neoproterozoic Doushantuo Formation, China, and the early evolution of florideophyte red algae. Am.J. Bot. 91: 214-227.

Xiao, S., Yuan, X., Steiner, M., and Knoll, A.H. (2002). Macroscopic carbonaceous compressions in a terminal Proterozoic shale: a systematic reassessment of the Miaohe biota, South China. J. Paleontol. 76: 345-374.

Yoon, H.S., Hackett, J.D., Ciniglia, C., Pinto, G., and Bhattacharya, D. (2004). A molecular timeline for the origin of photosynthetic eukaryotes. Mol. Biol. Evol. 21: 809-818.

Zhang, Y. (1981). Proterozoic stromatolite microfloras of the Gaoyuzhuang Formation (Early Sinian, Riphean), Hebei, China. J. Paleontol. 55: 485-506.

Zumberge, J.E. (1987). Prediction of source rock characteristics based on terpane biomarkers in crude oils: a multivariate statistical approach. Geochim. Cosmochim. Acta 51: 1625-1637.

Zumberge, J.E., Russell, J.A., and Reid, S.A. (2005). Charging of Elk Hills reservoirs as determined by oil geochemistry. AAPG Bull. 89: 1347-1371.

Zundel, M., and Rohmer, M. (1985a). Hopanoids of the methylotrophic bacteria Methylococcus capsulatus and Methylomonas sp. as possible precursors of $\mathrm{C}_{29}$ and $\mathrm{C}_{30}$ hopanoid chemical fossils. FEMS Microbiol. Lett. 28: 61-64.

Zundel, M., and Rohmer, M. (1985b). Prokaryotic triterpenoids 3 . The biosynthesis of $2 B$-methylhopanoids and 315-methylhopanoids of Methylobacterium organophilum and Acetobacter pasteurianus ssp. pasteu rianus. Eur. J. Biochem. 150: 35-39.

Zundel, M., and Rohmer, M. (1985c). Prokaryotic triterpenoids. 1.3ß-Methylhopanoids from Acetobacter sp. and Methylococcus capsulatus. Eur. J. Biochem. 150: 23-27. 


\title{
Appendix E
}

\section{The carbon cycle and associated redox processes through time}

\author{
John M. Hayes and Jacob R. Waldbauer
}

Reprinted with permission from Philosophical Transactions of the Royal Society $B$ (C) 2006 The authors

Hayes, J.M. and Waldbauer, J.R. (2006) The carbon cycle and associated redox processes through time. Philosophical Transactions of the Royal Society B 361: 931-950. 


\title{
The carbon cycle and associated redox processes through time
}

\author{
John M. Hayes ${ }^{1, *}$ and Jacob R. Waldbauer ${ }^{2}$ \\ ${ }^{1}$ Department of Geology and Geophysics, Woods Hole Oceanographic Institution, Woods Hole, \\ MA 02543, USA \\ ${ }^{2}$ foint Program in Chemical Oceanography, Woods Hole Oceanographic Institution, and Massachusetts \\ Institute of Technology, Cambridge, MA 02139, USA
}

Earth's biogeochemical cycle of carbon delivers both limestones and organic materials to the crust. In numerous, biologically catalysed redox reactions, hydrogen, sulphur, iron, and oxygen serve prominently as electron donors and acceptors. The progress of these reactions can be reconstructed from records of variations in the abundance of ${ }^{13} \mathrm{C}$ in sedimentary carbonate minerals and organic materials. Because the crust is always receiving new $\mathrm{CO}_{2}$ from the mantle and a portion of it is being reduced by photoautotrophs, the carbon cycle has continuously released oxidizing power. Most of it is represented by $\mathrm{Fe}^{3+}$ that has accumulated in the crust or been returned to the mantle via subduction. Less than $3 \%$ of the estimated, integrated production of oxidizing power since $3.8 \mathrm{Gyr}$ ago is represented by $\mathrm{O}_{2}$ in the atmosphere and dissolved in seawater. The balance is represented by sulphate. The accumulation of oxidizing power can be estimated from budgets summarizing inputs of mantle carbon and rates of organic-carbon burial, but levels of $\mathrm{O}_{2}$ are only weakly and indirectly coupled to those phenomena and thus to carbon-isotopic records. Elevated abundances of ${ }^{13} \mathrm{C}$ in carbonate minerals $c a 2.3 \mathrm{Gyr}$ old, in particular, are here interpreted as indicating the importance of methanogenic bacteria in sediments rather than increased burial of organic carbon.

Keywords: carbon cycle; carbon isotopes; atmospheric oxygen; methanogenesis; subduction; mantle

Together, biological and geological processesoxygenic photosynthesis and the burial of organic carbon-get credit for producing and maintaining the $\mathrm{O}_{2}$ in Earth's breathable atmosphere. This view of the carbon cycle as the engine of environmental evolution is based on sedimentary records. The disappearance of mass-independent fractionation of the isotopes of sulphur is the most reliable indicator for the accumulation and persistence of traces of $\mathrm{O}_{2}$ in the atmosphere beginning at about $2.4 \mathrm{Gyr}$ ago $(\mathrm{Ga})\left(P_{\mathrm{O}_{2}} \geq 10^{-5} \mathrm{~atm}\right.$; Pavlov \& Kasting 2002; Farquhar \& Wing 2003; Bekker et al. 2004). Biomarkers derived from lipids associated with cyanobacteria first appear in sedimentary rocks with an age of $2.7 \mathrm{Gyr}$ (Summons et al. 1999; Brocks et al. 2003; Eigenbrode 2004). These provide strong evidence for oxygenic photoautotrophy at that time (i.e. for production of $\mathrm{O}_{2}$ as opposed to its accumulation, persistence, and global distribution). Abundances of oxidized and reduced minerals in ancient soil profiles and sediments generally indicate a transition from weakly reducing to weakly oxidizing conditions at Earth's surface soon after 2.47 Ga (Bekker et al. 2004; Canfield 2005; Catling \& Claire 2005).

But what about examining the engine itself? Records of the burial of organic carbon, which should be provided by abundances of ${ }^{13} \mathrm{C}$ in

*Author for correspondence (jhayes@whoi.edu).

One contribution of 14 to a Discussion Meeting Issue 'Major steps in cell evolution'. sedimentary carbonates and organic material (Broecker 1970), could indicate the pace and the mechanism of oxidation. For events and processes during the past $500 \mathrm{Myr}$, carbon-isotopic records have been interpreted with considerable success (e.g. Garrels \& Lerman 1981; Holland 1984; Berner 1991, 2004; Kump \& Arthur 1999). The same approach has been extended to Precambrian records (e.g. Schidlowski et al. 1975; Hayes 1983, 1994; Knoll et al. 1986; Derry et al. 1992; Karhu \& Holland 1996; Halverson et al. 2005), but conclusions have usually been qualitative rather than quantitative. The carbon-isotopic record can be described as 'consistent with' some postulated event or process, but understanding of the carbon cycle has not been complete enough to allow resolution of uncertainties or elaboration of details.

Two recent findings may change this. First, new evidence (Saal et al. 2002) has led to wide agreement (cf. Resing et al. 2004) on the rate at which C is delivered to the crust from the mantle at mid-ocean ridges. This significant reduction in uncertainties about the input allows a new approach to carbon budgets. Second, a previously overlooked output of carbon from the ocean has been recognized and quantified. Whereas earlier concepts limited the outputs to sedimentary carbonates and organic matter, the new view includes a very large flow of carbon that is taken up during the weathering of seafloor basalts. This changes the structure of the mass balances. The potential role of this production of 'carbonated basalts' in the carbon 


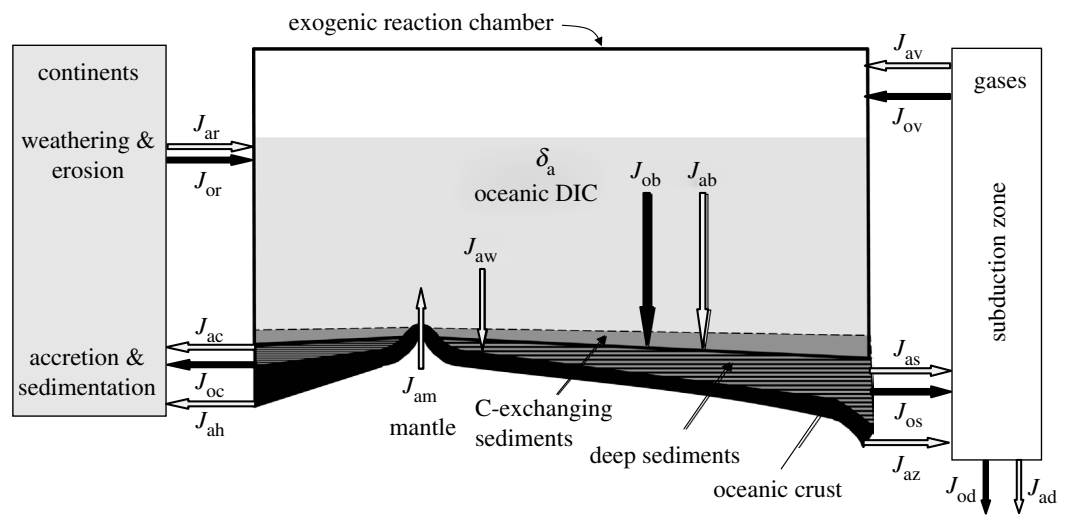

Figure 1. A schematic of the biogeochemical cycle of carbon. The arrows represent fluxes of carbon that are explained in the text.

cycle was first identified by Staudigel et al. (1989). Subsequently, Alt \& Teagle (1999, 2003) have examined amounts and isotopic compositions of these carbonate minerals and discussed their importance in the modern carbon cycle. Walker (1990), Sleep \& Zahnle (2001), and Nakamura \& Kato (2004) have called attention to carbonation of submarine basalts as an important phenomenon during the Archaean. Bjerrum \& Canfield (2004) have introduced a systematic treatment of this problem.

Our purpose here, based on those developments, is to demonstrate a new approach to studies of the carbon cycle and its effects on the global environment. Among those effects, we focus on oxidation of Earth's surface over the past $4 \mathrm{Gyr}$.

\section{THE STRUCTURE OF THE CARBON CYCLE}

A geochemical view of the carbon cycle is shown in figure 1 . The scheme chosen highlights processes that control the isotopic composition of inorganic carbon dissolved in the ocean. That carbon pool is in rough equilibrium with the atmosphere and with carbonate minerals derived from seawater. It is the senior author of our best records of how the carbon cycle has operated over the course of Earth history. To interpret those records, we must consider the fluxes indicated in figure 1. They represent processes that are linked by balances of mass and electrons. The related equations are, at present, analytical tools rather than components of an elaborate model.

The reaction chamber in which isotopic variations are shaped is comprised of the atmosphere, hydrosphere, and C-exchanging sediments and soils. Carbon flows into that chamber from the mantle and by recycling of carbon within the crust. It leaves through burial in sediments and during weathering of seafloor basalts. If the amount of $\mathrm{C}$ in the reactor is constant, the inputs will be balanced by the outputs:

$J_{\mathrm{am}}+J_{\mathrm{ar}}+J_{\mathrm{or}}+J_{\mathrm{av}}+J_{\mathrm{ov}}=J_{\mathrm{ab}}+J_{\mathrm{ob}}+J_{\mathrm{aw}}$.

The terms in this equation represent fluxes of carbon in mol per time. The subscripts ' $a$ ' and 'o' refer to inorganic and organic carbon. In detail, $J_{\mathrm{am}}$ is the total input of C from the mantle via outgassing of magmas at seafloor hydrothermal vents and at hot-spot and islandarc volcanoes; $J_{\text {ar }}$ and $J_{\text {or }}$ are, respectively, returns of carbonate and organic $\mathrm{C}$ by exposure and weathering of deposits on continents and shelves; and $J_{\mathrm{av}}$ and $J_{\mathrm{ov}}$ are returns, principally by arc volcanism, of carbonate and organic $\mathrm{C}$ remobilized during subduction. Among the outputs, $J_{\mathrm{ab}}$ and $J_{\mathrm{ob}}$ are the net burials of carbonate-and organic-C in marine sediments, deriving mainly from processes in surface waters but often bearing strong secondary imprints; and $J_{\text {aw }}$ is carbonate being taken up at the seafloor during the weathering of basalts. These, and all additional definitions pertinent to this discussion, are summarized in table 1.

A second mass balance equates incoming and outgoing ${ }^{13} \mathrm{C}$. Its elaboration leads to a key indicator of variations in the operation of the carbon cycle. For simplicity and generality, it is often written in this form:

$J_{\mathrm{i}} \delta_{\mathrm{i}}=J_{\mathrm{ab}} \delta_{\mathrm{ab}}+J_{\mathrm{ob}} \delta_{\mathrm{ob}}+J_{\mathrm{aw}} \delta_{\mathrm{aw}}$,

where $J_{\mathrm{i}}$ represents the summed inputs to the exogenic reaction chamber, $\delta_{\mathrm{i}}$ represents their weighted-average isotopic composition and the remaining $\delta$ terms represent the isotopic compositions of the indicated fluxes. In detail, a fully correct form would be

$$
\begin{gathered}
J_{\mathrm{am}} \delta_{\mathrm{am}}+J_{\mathrm{ar}} \delta_{\mathrm{ar}}+J_{\mathrm{or}} \delta_{\mathrm{or}}+J_{\mathrm{av}} \delta_{\mathrm{av}}+J_{\mathrm{ov}} \delta_{\mathrm{ov}} \\
=J_{\mathrm{ab}} \delta_{\mathrm{ab}}+J_{\mathrm{ob}} \delta_{\mathrm{ob}}+J_{\mathrm{aw}} \delta_{\mathrm{aw}} .
\end{gathered}
$$

Because values for many of the terms on the left side of this equation are inaccessible, analyses usually proceed from equation (1.2), incorporating the assumptions that $J_{\mathrm{i}}=J_{\mathrm{ab}}+J_{\mathrm{ob}}+J_{\mathrm{aw}}$ and that $\delta_{\mathrm{i}}=\delta_{\mathrm{am}}$. When historical variations are considered, the latter has two components: (i) that mixing of recycling inputs eventually yields an unbiased sample and (ii) that $\delta_{\mathrm{am}}$ is constant. Over rock-cycle time-scales of $300 \mathrm{Myr}$ or more, the first requirement is probably met. Second, the constancy and uniformity of $\delta_{\text {am }}$ are well supported. Independent of age of emplacement or location, diamonds from peridotitic xenoliths have $\delta=-5 \%$ o (Pearson et al. 2004). The same value is found in carbonatites and mantle-derived basalts (Kyser 1986; Mattey 1987). 
Table 1. Definitions.

\begin{tabular}{|c|c|}
\hline term & definition \\
\hline \multicolumn{2}{|c|}{ main variables } \\
\hline$A_{\mathrm{Ox}}$ & rate of accumulation of oxidants in crust ( $\mathrm{mol} \mathrm{O}_{2}$ equivalent) per time \\
\hline$J$ & flux of carbon (mol per time) \\
\hline$L$ & flux of oxidant or reductant (mol $\mathrm{O}_{2}$ equivalent) per time \\
\hline$M$ & molar quantity of C (no subscript: total crustal carbon, all forms) \\
\hline$f$ & fraction of C buried in organic form $\left(=J_{\mathrm{ob}} /\left(J_{\mathrm{ab}}+J_{\mathrm{aw}}+J_{\mathrm{ob}}\right)\right)$ \\
\hline$j_{\mathrm{x}}$ & flux of substance $\mathrm{x}$ (mol per time) \\
\hline$\Delta_{\mathrm{g}}$ & $\begin{array}{l}\text { isotopic difference between DIC in surface seawater and diagenetically stabilized carbonate minerals in } \\
\text { sedimentary rocks }(\%) \text {; see equation }(1.4)\end{array}$ \\
\hline$\Delta_{\mathrm{m}}$ & $\begin{array}{l}\text { isotopic difference between DIC in surface seawater and carbonate in weathered oceanic basalts (\%); see } \\
\text { equation (1.6) }\end{array}$ \\
\hline$\gamma$ & fraction of crustal C recycling during $\tau \cdot \gamma=\left(1-\mathrm{e}^{-k \tau}\right)$, where $k=(\ln 2) /($ half-mass age $)$ \\
\hline$\delta$ & $\delta^{13} \mathrm{C}$ relative to the Vienna PeeDee Belemnite standard (Zhang \& Li 1990) \\
\hline$\varepsilon$ & $\begin{array}{l}\text { isotopic fractionation between DIC in surface seawater and sedimentary organic carbon (\%o); see equation } \\
(1.5)\end{array}$ \\
\hline$\varphi$ & $\mathrm{Fe}^{3+} / \Sigma \mathrm{Fe}$ \\
\hline$\lambda$ & fraction of buried carbonate accounted for by ocean-crustal carbonates $\left(=J_{\mathrm{aw}} /\left(J_{\mathrm{aw}}+J_{\mathrm{ab}}\right)\right)$ \\
\hline$\tau$ & time-step in numerical integrations $\left(10^{7}\right.$ years $)$; figures 5,7 and 9 \\
\hline \multicolumn{2}{|c|}{ subscripts appended to $J, L$ and $\delta$} \\
\hline \multicolumn{2}{|c|}{ first or only part } \\
\hline $\mathrm{Ox}$ & oxidant \\
\hline Red & reductant \\
\hline a & carbonate carbon \\
\hline $\mathrm{i}$ & input to exogenic reaction chamber; see equation (1.3) \\
\hline o & organic carbon \\
\hline \multicolumn{2}{|c|}{ second part, pertaining to a directional flux } \\
\hline $\mathrm{b}$ & burial, transfer from exogenic reaction chamber to deep sediments \\
\hline c & transfer from deep sediments to continental crust \\
\hline $\mathrm{d}$ & transfer from subduction zone to mantle \\
\hline $\mathrm{h}$ & transfer from ocean crust to continental crust \\
\hline $\mathrm{m}$ & transfer from mantle to exogenic reaction chamber \\
\hline $\mathrm{r}$ & (returns) transfer from continent to exogenic reaction chamber \\
\hline s & (subduction) transfer from deep sediments to subduction zone \\
\hline $\mathrm{v}$ & (volcanism) transfer from subduction zone to exogenic reaction chamber \\
\hline $\mathrm{w}$ & (weathering) transfer from exogenic reaction chamber to ocean crust \\
\hline $\mathrm{x}$ & transfer from exogenic reaction chamber to space \\
\hline $\mathrm{z}$ & transfer from ocean crust to subduction zone \\
\hline
\end{tabular}

The task now is to provide a useful approach to interpreting observed variations in $\delta_{\mathrm{ab}}$, the carbonisotopic composition of sedimentary carbonates. As has become conventional, we define the fraction of input $\mathrm{C}$ buried in organic form as $f \equiv J_{\mathrm{ob}} / J_{\mathrm{i}}$. Following Bjerrum \& Canfield (2004), we define the fraction of total carbonate accounted for by ocean-crustal carbonates as $\lambda \equiv J_{\text {aw }} /\left(J_{\text {aw }}+J_{\mathrm{ab}}\right)$.

Our approach to the isotopic variables differs from previous expositions. As the reference point, we choose the isotopic composition of total dissolved inorganic carbon (DIC) in marine surface waters, $\delta_{\mathrm{a}}$. The isotopic compositions of the outputs are related to $\delta_{\mathrm{a}}$ by the following expressions:

$\delta_{\mathrm{ab}}=\delta_{\mathrm{a}}-\Delta_{\mathrm{g}}$,

$\delta_{\mathrm{ob}}=\delta_{\mathrm{a}}-\varepsilon$,

$\delta_{\text {aw }}=\delta_{\mathrm{a}}-\Delta_{\mathrm{m}}$,

where $\Delta_{\mathrm{g}}$ is the globally averaged isotopic difference between surface DIC and diagenetically stabilized sedimentary carbonates. At present, for example, comparison of pre-industrial $\delta_{\mathrm{a}}$ (Quay et al. 2003) and average sedimentary carbonate (Shackleton 1987) indicates $\Delta_{\mathrm{g}} \approx 2 \%$. Local variations in $\Delta_{\mathrm{g}}$ can affect specific sedimentary records. The difference in isotopic composition between sedimentary organic carbon and DIC, $\varepsilon$, is principally (though not exclusively) due to isotopic discrimination during biotic carbon fixation. The sign chosen in equation (1.5), with $\varepsilon>0$ corresponding to depletion of ${ }^{13} \mathrm{C}$ in biomass, is conventional in marine biogeochemistry. Values of $\Delta_{\mathrm{m}}$ will be positive when ocean-crustal carbonates are depleted in ${ }^{13} \mathrm{C}$ relative to surface waters. Reports of $\delta_{\text {aw }}$, required to evaluate $\Delta_{\mathrm{m}}$, are rare. Alt \& Teagle (2003) find $\delta_{\mathrm{aw}}=$ $1.7 \pm 0.4 \%$ o for ocean-crustal carbonates that have formed during the past $160 \mathrm{Myr}$. The average value of $\delta_{\mathrm{ab}}$ during the same interval (Veizer et al. 1999) is $1.7 \%$. At present, therefore, $\Delta_{\mathrm{m}} \approx \Delta_{\mathrm{g}} \approx 2 \%$. Other reports occasionally indicate lower values of $\delta_{\text {aw }}$ and thus suggest larger values of $\Delta_{\mathrm{m}}$ but, based on associated sedimentary features, the authors uniformly attribute the depletion of ${ }^{13} \mathrm{C}$ to infrequent additions of $\mathrm{C}$ derived from oxidation of organic material.

Substitution of equations (1.4)-(1.6) in equation (1.3) and simplification of the result yields

$\delta_{\mathrm{ab}}-\delta_{\mathrm{i}}=f\left(\varepsilon-\Delta_{\mathrm{g}}\right)+\lambda(1-f)\left(\Delta_{\mathrm{m}}-\Delta_{\mathrm{g}}\right)$.

Phil. Trans. R. Soc. B (2006) 
For $\Delta_{\mathrm{g}}=0$, equation (1.7) is equivalent to equation (1.4) of Bjerrum \& Canfield (2004). If, in addition, either or both $\Delta_{\mathrm{m}}$ and $\lambda$ are zero, equation (1.7) becomes $\delta_{\mathrm{ab}}-\delta_{\mathrm{i}}=f \varepsilon$, the expression found in numerous prior discussions of isotopic fractionation in the carbon cycle. Does this expression indicate that $\Delta_{\mathrm{m}}$ and $\Delta_{\mathrm{g}}$ can be as effective as $f$ and $\varepsilon$ in controlling $\delta_{\mathrm{ab}}-\delta_{\mathrm{i}}$ ? Probably not. Their leverage is relatively small. In most circumstances, the first term, $f\left(\varepsilon-\Delta_{\mathrm{g}}\right)$, will be at least four times larger than the second, $\lambda(1-f)\left(\Delta_{\mathrm{m}}-\Delta_{\mathrm{g}}\right)$.

Rearrangement of equation (1.7) yields an expression for $f$, the fraction of carbon buried in organic form:

$f=\frac{\delta_{\mathrm{ab}}-\delta_{\mathrm{i}}-\lambda\left(\Delta_{\mathrm{m}}-\Delta_{\mathrm{g}}\right)}{\varepsilon-\Delta_{\mathrm{g}}-\lambda\left(\Delta_{\mathrm{m}}-\Delta_{\mathrm{g}}\right)}$.

Commonly (e.g. Hayes et al. 1999), $f$ is estimated from $\left(\delta_{\mathrm{ab}}-\delta_{\mathrm{i}}\right) /\left(\delta_{\mathrm{ab}}-\delta_{\mathrm{ob}}\right)$. This is precisely equivalent to $\left(\delta_{\mathrm{ab}}-\delta_{\mathrm{i}}\right) /\left(\varepsilon-\Delta_{\mathrm{g}}\right)$. The numerator and denominator in that fraction lack the correction terms, $-\lambda\left(\Delta_{\mathrm{m}}-\Delta_{\mathrm{g}}\right)$, which are prominent in equation (1.8). If $\Delta_{\mathrm{m}} \approx \Delta_{\mathrm{g}}$, as at present, $\lambda\left(\Delta_{\mathrm{m}}-\Delta_{\mathrm{g}}\right)=0$ and the terms are inconsequential, independent of the importance of basalt carbonation. Such cases are summarized graphically in figure $2 a$. The vertical line at $\delta_{\mathrm{ab}}-\delta_{\mathrm{i}}=6.5 \%$ indicates $\delta_{\mathrm{ab}}=1.5 \%$, near Earth's observed, long-term average value. The lines correspond (left to right) to $\varepsilon=22,26$, 30 and $34 \%$. The shaded area indicates that, depending on the value of $\varepsilon, \delta_{\mathrm{ab}}=1.5 \%$ corresponds to $0.2 \leq f \leq 0.32$. This range encompasses the values most frequently noted in previous discussions of the carbon cycle. In particular, $\delta_{\mathrm{ab}}-\delta_{\mathrm{ob}}=28 \%$, corresponding to $\varepsilon=30$ and $\Delta_{\mathrm{g}}=2 \%$, is representative of much of the Phanerozoic (Hayes et al. 1999).

The lower $f$ values marked by shading in the other frames of figure 2 correspond to the same value of $\delta_{\mathrm{ab}}$, but are based on different estimates of $\Delta_{\mathrm{g}}, \Delta_{\mathrm{m}}$ and $\lambda$. Negative values of $\Delta_{\mathrm{g}}$ are observed when sedimentary carbonates are strongly affected by methanogenic diagenesis (Irwin et al. 1977). In Phanerozoic strata, which have formed in the presence of relatively abundant $\mathrm{O}_{2}$ and $\mathrm{SO}_{4}{ }^{2-}$, this phenomenon is restricted to concretions or other zones, in which supplies of sedimentary organic matter have been large enough that methanogenesis has eventually become prominent. When concentrations of $\mathrm{O}_{2}$ and $\mathrm{SO}_{4}{ }^{2-}$ in seawater were significantly lower, methanogenesis must have been more important. Accordingly, the effects of negative values of $\Delta_{g}$, corresponding to globally important levels of methanogenic diagenesis, are explored in figure $2 b, c$. Figure $2 d$ shows that variations in $\Delta_{\mathrm{m}}$ are probably least important in affecting estimates of $f$. Under steady-state conditions, inversion of the oceanic ${ }^{13} \mathrm{C}$ gradient - enrichment of ${ }^{13} \mathrm{C}$ in bottom waters $\left(\Delta_{\mathrm{m}}=-5 \%\right.$, figure $\left.2 d\right)$-is practically required to produce $\delta_{\mathrm{ab}}-\delta_{\mathrm{i}}<0$.

\section{REDOX BALANCES IN THE CARBON CYCLE}

Figure 3 duplicates the plan of figure 1, but depicts flows of oxidants and reductants generated by the carbon cycle. The biological cycle of production and respiration is at its centre. Redox partners for $\mathrm{C}$ are generalized as Red and Ox. The focus on oxidants

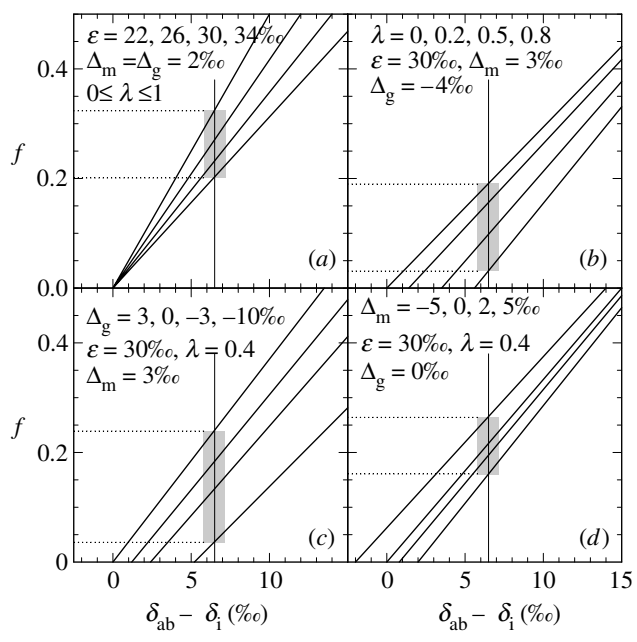

Figure 2. Graphs indicating relationships between $f$ and $\delta_{\mathrm{ab}}-\delta_{\mathrm{i}}$ (1.7). Slopes and intercepts vary in response to varying values of $\varepsilon, \lambda, \Delta_{\mathrm{m}}$, and $\Delta_{\mathrm{g}}$. In each frame, one of these has been assigned four different values and the others have been held constant. The values assigned are indicated in each frame. For the parameter that varies, the sequence of values corresponds to the lines as seen from left to right.

rather that oxygen is necessary. The carbon-burial flux provides information about the consumption of electron donors, but not about the identity of those donors. Over the course of Earth history, the electrondonating role of Red has been played by $\mathrm{H}_{2}, \mathrm{Fe}^{2+}, \mathrm{S}^{2-}$ and $\mathrm{H}_{2} \mathrm{O}$ (at least). Correspondingly, the oxidized forms of these substances have accumulated in Earth's crust. The rate of accumulation will be set by the difference between the rate at which carbon-cycling produces oxidants and the rate at which those oxidants are consumed.

To quantify rates, we begin by viewing the $J_{\mathrm{o}}$ terms, introduced above as fluxes of carbon, as fluxes of reducing power. The $L$ terms shown in figure 3 represent flows of oxidizing or reducing power carried by other elements or by mixtures of $\mathrm{C}$ and other elements, ( $\mathrm{mol} \mathrm{O}_{2}$ equivalent) per time. Because reduction of $\mathrm{CO}_{2}$ to organic carbon and oxidation of $\mathrm{H}_{2} \mathrm{O}$ to $\mathrm{O}_{2}$ are both four-electron processes, values of the $J_{\mathrm{o}}$ and $L$ terms are numerically equivalent. Production of $1 \mathrm{~mol}$ of organic carbon could be balanced by oxidation of $2 \mathrm{~mol}$ of $\mathrm{H}_{2}, 8 \mathrm{~mol}$ of $\mathrm{Fe}^{2+}$, $0.5 \mathrm{~mol}$ of $\mathrm{S}^{2-}$ (to $\mathrm{SO}_{4}{ }^{2-}$ ), or $2 \mathrm{~mol}$ of $\mathrm{H}_{2} \mathrm{O}$. All would be equivalent to $1 \mathrm{~mol}$ of $\mathrm{O}_{2}$. To emphasize that it pertains to the net effect of multiple processes, rather than to a specific flux, we use $A_{\mathrm{Ox}}$ to designate the rate at which oxidants accumulate in the crust and exogenic reaction chamber.

To begin, the biological carbon cycle releases oxidants at the rate at which organic carbon is buried in deep sediments $\left(J_{\mathrm{ob}}\right)$. The rate of accumulation is then moderated by effects of two types. Within the crust itself, a portion of the oxidants is consumed by the geological carbon cycle. Two pathways are shown in figure 3. The first is oxidative weathering of organic carbon exposed on and eroded from the continents 


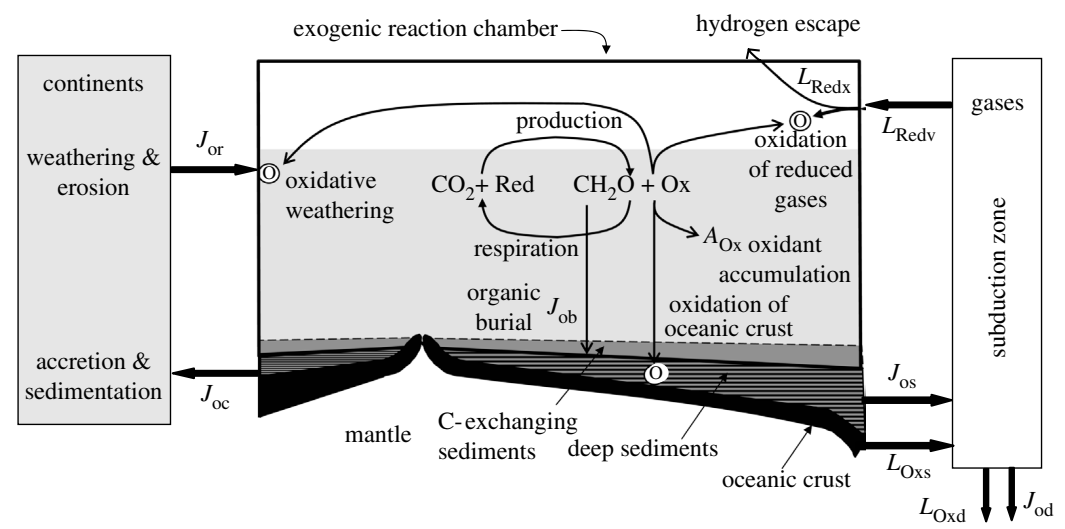

Figure 3. A schematic of flows of oxidants and reductants generated by the carbon cycle. Sites of oxidation are marked by the letter $\mathrm{O}$.

$\left(J_{\mathrm{or}}\right)$. The second is oxidation of reduced gases produced at subduction zones. The corresponding flux is designated as $L_{\text {Redv }}$. It includes not only volcanic gases $\left(\mathrm{H}_{2}, \mathrm{CO}, \mathrm{SO}_{2}\right)$, but also products ranging from methane to petroleum, which are delivered to the exogenic reaction chamber by thermal processes in subduction zones and deep basins.

The export of oxidants and reductants also moderates $A_{\text {ox }}$. This occurs in subduction zones and at the top of the atmosphere. Within subduction zones, electrons are transferred between subducted organic materials $\left(J_{\mathrm{os}}\right)$ and oxidants (metal oxides and $\mathrm{SO}_{4}{ }^{2-}$, $\left.L_{\mathrm{Oxs}}\right)$ in the descending slab. Products will include not only those returned to the crust (noted above), but also unconsumed oxidants $\left(L_{\text {Oxd }}\right)$ and reduced carbon (diamond, graphite, $J_{\text {od }}$ ) exported to the mantle. Subduction of carbonate decreases the mass of carbon in the crust, but, because carbon is supplied from the mantle as $\mathrm{CO}_{2}$, does not export either oxidizing or reducing power from the crust. Subduction of sulphide is similarly inconsequential. Mantle $S$ occurs as sulphide even under oxidizing conditions (e.g. $\Delta \mathrm{FMQ} \sim 2$; Luth 2004).

At the top of the atmosphere, $\mathrm{H}_{2}$ can be lost to space, thus exporting reducing power and effectively increasing $A_{\mathrm{Ox}}$. This occurs when reduced gases of either volcanic or biological origin reach high altitudes. Depending on atmospheric conditions, a portion of the reducing power carried by these gases can be lost as hydrogen escapes to space (Catling et al. 2001).

Summation provides an estimate of the rate at which oxidants will accumulate:

$$
\begin{aligned}
A_{\mathrm{Ox}}= & \text { (net release of oxidants within crust by C cycle }) \\
& +(\text { reductant export })-(\text { net oxidant export }) \\
& A_{\mathrm{Ox}} \geq\left(J_{\mathrm{ob}}-J_{\mathrm{or}}-L_{\mathrm{Redv}}\right)+L_{\mathrm{Redx}}-\left(L_{\mathrm{Oxd}}-J_{\mathrm{od}}\right) .
\end{aligned}
$$

The second expression denotes $A_{\mathrm{Ox}}$ as a minimum value because the net release of oxidants by the $\mathrm{C}$ cycle may exceed $J_{\mathrm{ob}}-J_{\mathrm{or}}-L_{\mathrm{Redv}}$. This would occur if a portion of the organic carbon returning from continents $\left(J_{\mathrm{or}}\right)$ was not reoxidized but instead simply reburied. The efficiency of reoxidation will depend on the nature of $\mathrm{Ox}$ (e.g. $\mathrm{O}_{2}$ versus $\mathrm{SO}_{4}{ }^{2-}$ ) and the reactivity of $J_{\text {or }}$ (e.g. graphitic kerogen versus hydrocarbons).

Equation (2.1) ignores a category of processes often considered in oxygen budgets. Sediments and oceanic crust assimilated by continents will incorporate portions of the Red and Ox from the exogenic reaction chamber. Oxidized and reduced forms of sulphur and iron are prime examples. Surging flows of these reactants to the sediments or from continents can, for example, significantly affect levels of $\mathrm{O}_{2}$ in the atmosphere and ocean. These and related phenomena, especially in the sulphur cycle, have already been elegantly treated by others (Berner 2004; Canfield 2004).

Imbalances yielding a net release or consumption of oxidizing power by the carbon cycle are of two types. The more obvious are marked by isotopic signals indicating rapid variations of $f$ and consequent departures from steady state. At such times, the system will evolve dynamically (Rothman et al. 2003). At other times, when $f$ varies slowly, the system will evolve quasistatically through a succession of steady states. The persistence of small imbalances between fluxes can lead to the accumulation of crustal inventories of carbon, chiefly on the continents. In this case, the fluxes designated in equation (2.1) should be represented as time-dependent variables. Then, at any time, $t$,

$$
\begin{aligned}
\int_{0}^{t} A_{\mathrm{Ox}}(t) \mathrm{d} t= & \int_{0}^{t}\left[J_{\mathrm{ob}}(t)-J_{\mathrm{or}}(t)-L_{\mathrm{Redv}}(t)\right] \mathrm{d} t \\
& +\int_{0}^{t} L_{\mathrm{Redx}}(t) \mathrm{d} t-\int_{0}^{t}\left[L_{\mathrm{Oxd}}(t)-J_{\mathrm{od}}(t)\right] \mathrm{d} t .
\end{aligned}
$$

The first term on the right-hand side is the integrated difference between burial and reoxidation of organic carbon. It quantifies the accumulation of organic carbon in continents and marine sediments. The second and third terms quantify the effects of loss of $\mathrm{H}_{2}$ to space and of subduction of oxidized and reduced materials. Notably, this summation of oxidation has no isotopic dimensions. $J_{\mathrm{ob}}$ is related to $f$ and thus to the isotopic record, but it is $J_{\mathrm{ob}}-J_{\mathrm{or}}-L_{\mathrm{Redv}}$ that matters, 
and it is further altered by effects of subduction and escape of $\mathrm{H}_{2}$ to space.

The first term in equation (2.2) is closely related to a principle dating from the nineteenth century ( J. J. Ebelmen's work from 1845 to 1855 , reviewed by Berner \& Maasch 1996) and elaborated in modern detail most influentially by Garrels (e.g. Garrels \& Perry 1974) and Berner (2004 and earlier references cited therein). Specifically, the amount of organic carbon stored in the crust should balance the oxidizing power represented by the crustal inventories of $\mathrm{Fe}^{3+}$, $\mathrm{SO}_{4}{ }^{2-}$ and $\mathrm{O}_{2}$. To this, space science and plate tectonics have added the second and third terms.

It is difficult to reconstruct the histories of the variables in equation (2.2). A boundary value for the first integral, however, can be obtained by consideration of crust-mantle carbon budgets and variations in $f$, the organic-carbon burial fraction.

\section{INPUTS OF MANTLE CARBON}

Fluxes and inventories throughout the carbon cycle depend on inputs of carbon from the mantle. These occur at mid-ocean ridges, arc volcanoes, hotspots and in plume events. The present strengths of these sources will be considered sequentially. An estimate of variations over the course of Earth history will follow.

\section{(a) Mid-ocean ridges}

Each year, $21 \mathrm{~km}^{3}$ of basalt is added to the oceanic crust at spreading centres (Crisp 1984; also consistent with a plate-creation rate of $3.4 \mathrm{~km}^{2} \mathrm{yr}^{-1}$ (Rowley 2002) and a plate thickness of 5-7 km (White et al. 1992; Kadko 1994)). Sampled after cooling at the seafloor, its $\mathrm{CO}_{2}$ content is dependent on the hydrostatic pressure. At the depths of mid-ocean ridges, the result is commonly about 200 p.p.m. (the routinely reported concentrations refer to weights of $\mathrm{CO}_{2}$ ). The question is how much $\mathrm{CO}_{2}$ was in the parent magma. The difference will have been transferred, by way of hydrothermal circulation, to the ocean.

Recently, Saal et al. (2002) have shown that, in undegassed MORB (mid-ocean ridge basalt), concentrations of $\mathrm{CO}_{2}$ vary with those of $\mathrm{Nb}$. The weight ratio is $\mathrm{CO}_{2}: \mathrm{Nb}=239 \pm 46(2 \sigma)$. Since $\mathrm{Nb}$ is not lost during degassing, the initial $\mathrm{CO}_{2}$ content of a sample of MORB can be estimated from its content of $\mathrm{Nb}$. The average for normal MORBs (i.e. those in which traceelement abundances have not been affected by proximity to plumes or recently subducted continental materials) on the East Pacific Rise is 3.45 p.p.m. Nb (Su \& Langmuir 2003). The estimated, average, initial content of $\mathrm{CO}_{2}$ is thus $3.45 \times 239=825$ p.p.m. If 200 p.p.m. remain in the cooled basalt, the difference transmitted to the exogenic reservoir is 625 p.p.m. Given a rock density of $2.8 \mathrm{~g} \mathrm{~cm}^{-3}$, this corresponds to $0.8 \mathrm{Tmol} \mathrm{C} \mathrm{yr}^{-1}\left(\mathrm{Tmol}=\right.$ teramol $\left.=10^{12} \mathrm{~mol}\right)$. If the $\mathrm{Nb}$ average quoted for all MORBs (5.02 p.p.m.; Su \& Langmuir 2003) is instead used as the basis for the calculation, the result is $1.3 \mathrm{Tmol} \mathrm{C} \mathrm{yr}^{-1}$. These values bracket a third estimate, namely $0.9 \mathrm{Tmol} \mathrm{C} \mathrm{yr}^{-1}$, reported in the original publication (Saal et al. 2002) and reached using a slightly different approach.
Numerous alternative estimates have been based on $\mathrm{CO}_{2}:{ }^{3} \mathrm{He}$ ratios in hydrothermal fluids and gases. These have been reviewed by Resing et al. (2004), who settle on a range of $0.5-2 \mathrm{Tmol} \mathrm{C} \mathrm{yr}^{-1}$. Preferring the approach based on chemical analyses of the rocks, we adopt $1 \mathrm{Tmol} \mathrm{C} \mathrm{yr}^{-1}$ as the present magnitude of the mid-ocean ridge component of $J_{\mathrm{am}}$.

\section{(b) Arc volcanoes}

The annual magma volume is $0.5 \mathrm{~km}^{3}$ (Carmichael 2002). Carbon dioxide is abundant in the gases, but its isotopic composition often deviates from the mantle value and the $\mathrm{CO}_{2}$ is regarded as deriving from subducted sedimentary carbonates and organic carbon as well from the mantle (Sano \& Marty 1995; Sano \& Williams 1996; Shaw et al. 2003,2004). The total flux of $\mathrm{CO}_{2}$ from arc volcanism is approximately $1.6 \mathrm{Tmol} \mathrm{yr}^{-1}$ (Hilton et al. 2002). Of this, approximately $13 \%$ is from the mantle (Shaw et al. 2003), yielding an arc-volcanic component of $J_{\mathrm{am}}$ of $0.2 \mathrm{Tmol} \mathrm{C} \mathrm{yr}^{-1}$. The remaining $1.4 \mathrm{Tmol} \mathrm{yr}^{-1}$ is recycling crustal C. Wallace (2005) obtains a similar result by a different method.

\section{(c) Oceanic islands and plumes}

In a collection of estimates, this is the most uncertain. The annual volume of magma is approximately $3 \mathrm{~km}^{3}$ (though possibly as small as $1.9 \mathrm{~km}^{3}$ ), combining igneous provinces and hotspot volcanoes on continents with those in the ocean (Crisp 1984). A more recent, separate tabulation of large igneous provinces by Marty \& Tolstikhin (1998) finds a total of $95.5 \times$ $10^{6} \mathrm{~km}^{3}$ in the past $250 \mathrm{Myr}$, for a rate of $0.4 \mathrm{~km}^{3} / \mathrm{yr}$. It is broadly agreed that these magmas are volatile-rich compared to MORB. Basing their estimate on ${ }^{3} \mathrm{He}$ budgets, Marty \& Tolstikhin (1998) suggest that the total output from oceanic islands and plumes 'is at best similar to that of spreading centres'. Given our estimate above, this suggests an input of somewhat less than $1 \mathrm{Tmol} \mathrm{C} \mathrm{yr}^{-1}$.

Since mid-ocean ridge magmas are roughly 10 times more voluminous, the estimated equal flux of mantle $\mathrm{CO}_{2}$ from oceanic islands and plumes calls for a 10-fold enrichment of $\mathrm{CO}_{2}$, and thus perhaps of $\mathrm{Nb}$, in the parent magmas. Observed enrichments of $\mathrm{Nb}$ (Hofmann 2004) range from at least 13-fold (Mangaia, Pitcairn, Tahaa) to 2-fold (Mauna Loa). Moreover, Pineau et al. (2004) have suggested that $\mathrm{CO}_{2}: \mathrm{Nb}$ ratios might range to values more than 3-fold higher than that found by Saal et al. (2002). In sum, the estimate of $1 \mathrm{Tmol} \mathrm{C} \mathrm{yr}^{-1}$, equal to that at the spreading centres, is plausible but highly uncertain.

Together, mid-ocean ridges, arc volcanoes and emissions at island volcanoes and during plume events provide an annual input from the mantle of approximately $2.2 \mathrm{Tmol} \mathrm{C}$.

\section{INVENTORIES AND ACCUMULATION} OF CARBON

Total quantities of carbonate carbon in the crust, estimated from stratigraphic inventories (Holser et al. 1988; Wedepohl 1995; Hunt 1996; Des Marais 2001; Berner 2004; Arvidson et al. in press), commonly range 
from 2800 to $6500 \mathrm{Emol}\left(\mathrm{Emol}=\mathrm{examol}=10^{18} \mathrm{~mol}\right)$. The same reports provide estimates of the total quantity of organic carbon ranging from 675 to 1300 Emol. Only one of these (Arvidson et al. in press) includes carbonate associated with basalt. It also yields the lowest ratio of organic to total carbon, namely 0.13 . The other reports yield organic fractions ranging from 0.15 to 0.20 , and seem to be influenced by isotopic mass balances, which suggest higher relative quantities of organic carbon.

Wilkinson \& Walker (1989) took an alternative approach and focused exclusively on sedimentary carbonates. Examining mass-age data, they found that the best fit could be provided by a constantmass, constant-burial system with first-order recycling and including $9600 \mathrm{Emol}$ carbonate carbon. An alternative fit emphasizing data from younger sequences yielded a result of $7900 \mathrm{Emol}$. If we arbitrarily adopt an organic-carbon fraction of 0.15 , the corresponding inventories of organic carbon are 1700 and $1400 \mathrm{Emol}$, for total carbon inventories of 11300 and 9300 Emol.

The most detailed inventory (Holser et al. 1988) provides total crustal $\mathrm{C}=7640 \mathrm{Emol}$. Favouring the approach using mass-age data, particularly that based on younger sequences, we weight it equally with the stratigraphic compilations and estimate total crustal $\mathrm{C}=8500$ Emol.

The time required to accumulate this inventory depends on assumptions about the input flux. If the total flux of $2.2 \mathrm{Tmol} \mathrm{C} \mathrm{yr}^{-1}$ estimated above were constant, the time required would be 3.86 Gyr. More commonly, it is believed that, earlier in Earth history, such fluxes were higher. Here, we follow Sleep \& Zahnle (2001) and Lowell \& Keller (2003). These authors scale input fluxes to estimates of hightemperature heat flow, chiefly at spreading centres. For the case in which continents grew episodically from 10 to $80 \%$ of current area between 3200 and $2500 \mathrm{Myr}$ ago $(\mathrm{Ma})$, high-temperature heat flow is calculated to decrease from $9 \times$ the current level at $3800 \mathrm{Ma}$, to $4.9 \times$ at $3200 \mathrm{Ma}$, to $2.3 \times$ at $2500 \mathrm{Ma}$, and then to decline exponentially. The resulting scaled carbon flux is shown in figure 4 .

Integration of that flux beginning at $3800 \mathrm{Ma}$, the end of the late, heavy bombardment, yields the totals depicted graphically in figure 5 . The sum exceeds $8500 \mathrm{Emol}$, the estimated present crustal inventory, after only $575 \mathrm{Myr}$, at $3225 \mathrm{Ma}$. In fact, with continents just beginning to form and crustal storage reservoirs thus sharply restricted, returns of carbon to the mantle would probably have become nearly equal to inputs from the mantle well before then.

Together, figure 5 and equations (1.1)-(2.2) provide a new context for considering the development of the carbon cycle. Before turning to the isotopic records, however, we must first review available information regarding the fates of carbon in subduction zones.

\section{CARBON CYCLING AT SUBDUCTION ZONES}

Processes in subduction zones are crucial to redox balances in the carbon cycle. Figure 6 shows flows of carbon (in oxidized and reduced form) and other

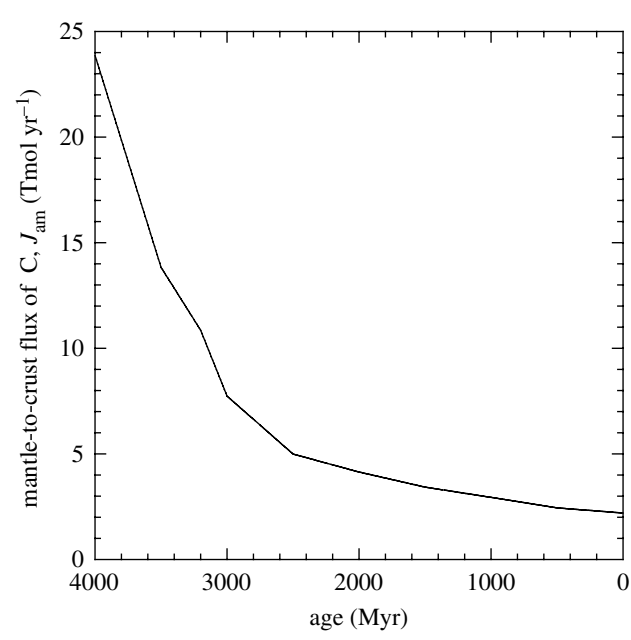

Figure 4. Estimated values of $J_{\mathrm{am}}$ as a function of time. The value at $0 \mathrm{Ma}$ is documented in the text. The scaling relationship at earlier times derives from Sleep \& Zahnle (2001) and Lowell \& Keller (2003).

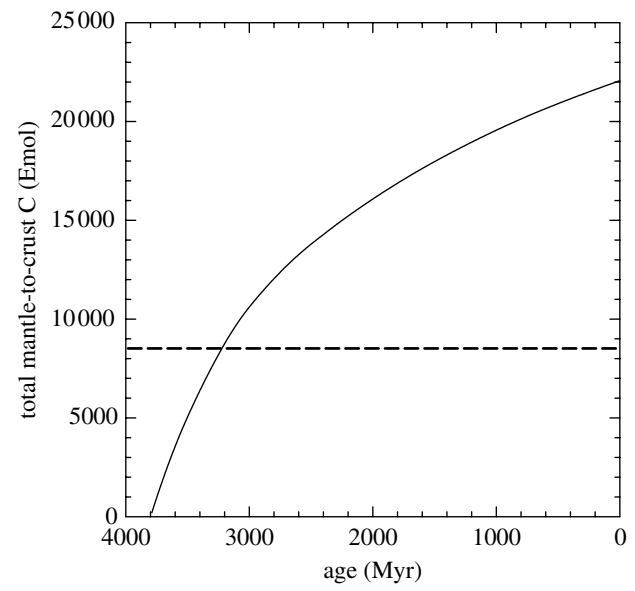

Figure 5. Total amount of mantle carbon delivered to the crust as a function of time. Specifically, total mantle-to-crust $\mathrm{C}=\sum_{3800}^{t} J_{\mathrm{am}}(t) \tau$, where $t$ is the age, $\mathrm{Myr}$, and $\tau$, the timestep, is $10^{7}$ years. The broken line at $8500 \mathrm{Emol} \mathrm{C}$ represents the best estimate of the present crustal inventory of C (table 2).

products of carbon cycling into, through and out of subduction zones. Reducing power produced by the carbon cycle is carried into subduction zones by organic carbon $\left(J_{\mathrm{os}}\right)$. Oxidizing power is carried by sulphate and by ferric iron. The crossing paths and redox reactions suggest the diverse transformations involved. While many different sequences of reactions are possible, the equations in figure 6 summarize the required balances of mass and electrons. All of the carbon that enters the subduction zone must be transferred in some form to either the crust or mantle. And the balance between inputs of reductants and oxidants must be reflected by materials leaving the subduction zone. Resolution and dissection of 


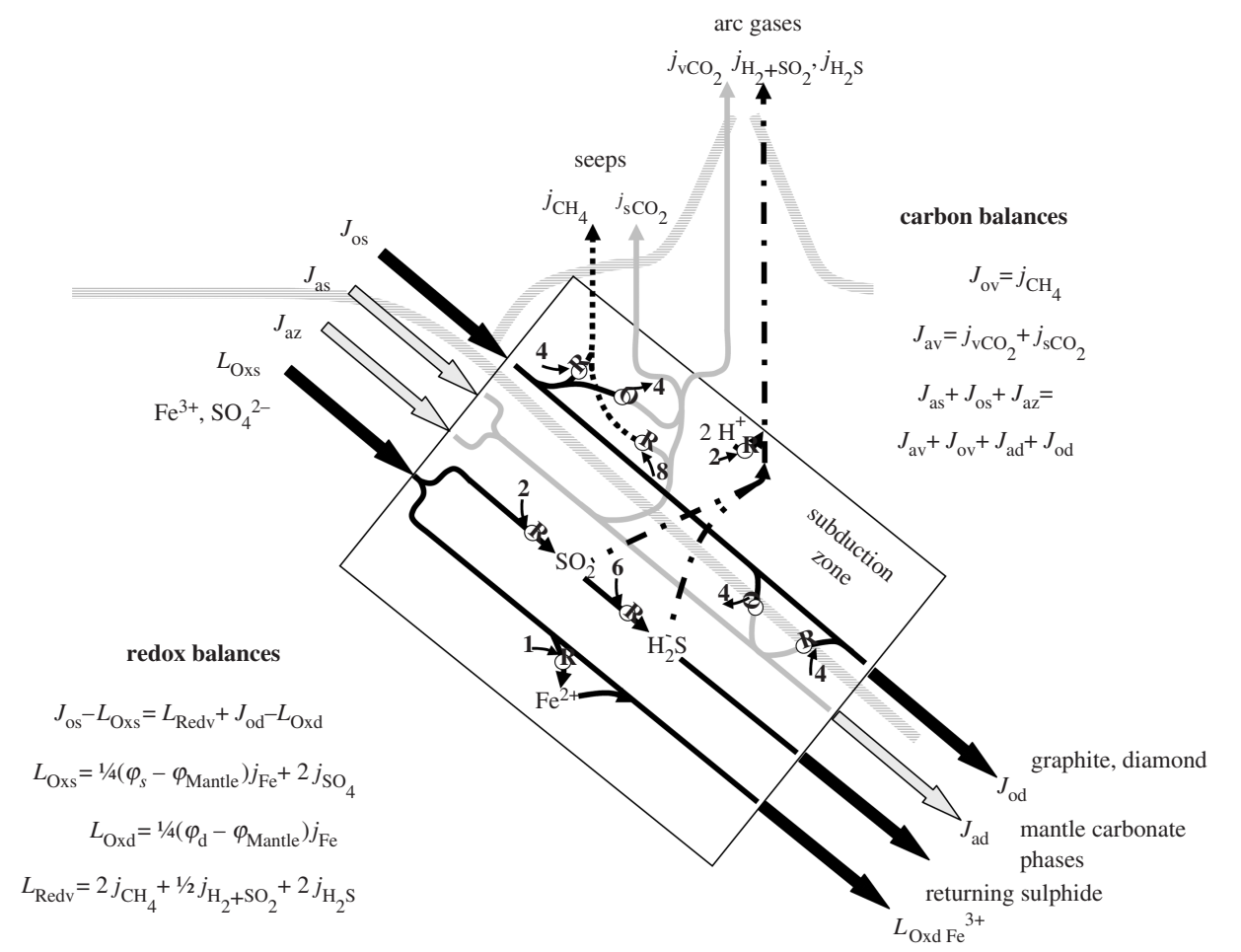

Figure 6. Schematic of fluxes of carbon and reducing power in a subduction zone. Terms denote $\mathrm{Fe}^{3+} / \Sigma \mathrm{Fe}(\varphi)$, and fluxes of carbon $\left(J\right.$, mol per time), specific substances $\left(J\right.$, mol per time) and oxidizing or reducing power $\left(L, \mathrm{~mol}_{2}\right.$ equivalent per time). Subscripts are defined in table 1 . Chemical reductions and oxidations are marked by circled letters ( $\mathrm{R}$ or $\mathrm{O})$. Adjacent numbers indicate number of electrons gained or lost.

processes within subduction zones are not currently possible, but available evidence bears on two key questions. When a slab is subducted, (i) what happens to the carbon and (ii) what happens to the reducing power carried by the organic matter?

\section{(a) Carbon fluxes}

The downgoing flux, $\left(J_{\text {ad }}+J_{\text {od }}\right)$, might be quantified directly if we knew how much material was being incorporated by the mantle and if samples of it were returned to the surface, so that their carbon contents could be determined. Although $J_{\text {od }}$ and $J_{\text {ad }}$ are sampled by diamond-bearing rocks and mantle xenoliths, it is not clear how representative these samples are, nor do they allow for quantification of the total downgoing $\mathrm{C}$ flux.

More headway can be made by considering the fluxes from arc volcanism and other volatile emissions from subduction zones and then estimating the downgoing flux from the difference between the trench input and surface output. The magnitudes of both are rather uncertain; here, we summarize the best estimates. The three inputs are the organic and carbonate components of subducted sediment ( $J_{\mathrm{os}}$ and $J_{\mathrm{as}}$ ) and the carbonated basalt in the subducting crust $\left(J_{\mathrm{az}}\right)$. The global subducting-sediment budget of Plank \& Langmuir (1998) puts $J_{\text {as }}$ at $0.9 \mathrm{Tmol} \mathrm{C} \mathrm{yr}^{-1}$. Estimates of $J_{\text {os }}$ are very poorly constrained, mostly because it is small in comparison to fluxes to and from the continents and shelves. For the modern C cycle, Holser et al. (1988) estimate $J_{\mathrm{oc}} / J_{\mathrm{os}} \approx 40$. We adopt their value, $J_{\mathrm{os}}=$ $0.2 \mathrm{Tmol} \mathrm{C} \mathrm{yr}{ }^{-1}$, suggesting a mean $\mathrm{C}_{\mathrm{org}}$ content for subducting sediment of $0.13 \mathrm{wt} \%$.

The third flux, $J_{\mathrm{az}}$, likely delivers most of the carbon to subduction zones. It derives from $J_{\mathrm{aw}}$, for which estimates range over more than an order of magnitude from less than 1 to more than $3 \mathrm{Tmol} \mathrm{C} \mathrm{yr}^{-1}$ (Bach et al. 2003). The alternate fate for carbon buried by basalt carbonation is represented by $J_{\text {ah }}$, the portion of carbonated basalt actually accreted onto continents. Since this is relatively small, it follows that carbonate can be stored in ocean crust along passive margins for hundreds of millions of years, as in much of the Atlantic basin today. The idea that $J_{\text {az }}$ approaches $J_{\text {aw }}$ incorporates an assumption that, following tectonic rearrangements, such material is eventually subducted. The resulting rough estimate of the total subduction

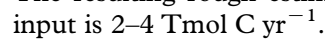

For the volatile outputs from arcs, the most complete budget has been compiled by Hilton et al. (2002), who considered subduction inputs to and volatile emissions from 26 arc systems worldwide. The authors calculated a global volcanic arc $\mathrm{CO}_{2}$ flux of 1.6 $\mathrm{Tmol} \mathrm{C} \mathrm{yr}^{-1}$ and emphasized the importance of 
distinguishing between sources of volatiles. For $\mathrm{CO}_{2}$, these are carbonate and organic $\mathrm{C}$ in the subducting slab and $\mathrm{CO}_{2}$ in the mantle wedge. Since the mantle component amounts to $0.2 \mathrm{Tmol} \mathrm{C} \mathrm{yr}^{-1}$ (noted above), $j_{\mathrm{vCO}_{2}}=1.4 \mathrm{Tmol} \mathrm{C} \mathrm{yr}^{-1}$. The portion due to subducted carbonates can be estimated from the isotopic composition of the non-mantle component. For all of the arcs, it exceeded the input of sedimentary carbonate. In all cases but one, inclusion of carbonated basalt eliminated the shortfall. Notably, these results are at odds with calculated phase equilibria that indicate carbonated basalts should undergo little devolatilization along most subduction geotherms (Kerrick \& Connolly 2001). The other component of $J_{\text {av }}$ is $j_{\mathrm{SCO}_{2}}$, emission of $\mathrm{CO}_{2}$ from seeps, especially in fore-arc and back-arc regions. This flux is unconstrained, and may be as large as $j_{\mathrm{vCO}_{2}}$ (Hilton et al. 2002). Ingebritsen \& Manning (2002) have pointed to diffuse fluid flow through tectonically active crust as potentially a major flux of subduction-derived volatiles. This degassing pathway may be sufficient to reconcile the crust-mantle water balance, and could well constitute a significant return of slab-derived $\mathrm{CO}_{2}$ to the crust. Accordingly, $J_{\text {av }}$ is between 1.4 and 2.8 $\mathrm{Tmol} \mathrm{C} \mathrm{yr}^{-1}$

The other return flux of carbon from subduction zones to the exogenic chamber is $J_{\text {ov }}$ the reduced carbon from high-and low-temperature seeps, primarily $\mathrm{CH}_{4}$. The related geological forms are diverse, and include mud volcanoes and seeps associated with gas hydrates (Milkov \& Etiope 2005; Milkov 2005). Their output of $\mathrm{CH}_{4}$, estimated at $2.1 \mathrm{Tmol} \mathrm{yr}^{-1}$ (Milkov \& Etiope 2005), derives from microbial methanogenesis and thermal processes in sediments and sedimentary rocks, as well as from subducted carbon. Since the carbon in $J_{\mathrm{ov}}\left(=\mathrm{CH}_{4}\right)$ has oxidation number equal to -4 and the organic carbon in $J_{\mathrm{os}}$ has oxidation number equal to zero, redox balance provides the stronger constraint. If $J_{\mathrm{os}}$ is approximately $0.2 \mathrm{Tmol} \mathrm{C} \mathrm{yr}{ }^{-1}, J_{\mathrm{ov}}$ cannot be larger than $0.1 \mathrm{Tmol} \mathrm{C} \mathrm{yr}^{-1}$. Total volcanic and seep fluxes of carbon $\left(J_{\mathrm{av}}+J_{\mathrm{ov}}\right)$ are between 1.5 and $2.9 \mathrm{Tmol} \mathrm{C} \mathrm{yr}^{-1}$.

From inputs of $2-4 \mathrm{Tmol} \mathrm{C} \mathrm{yr}^{-1}$ and recycling fluxes of $1.5-2.9 \mathrm{Tmol} \mathrm{C} \mathrm{yr}^{-1}$, we estimate that the fraction recycled is approximately 0.6 . By difference, the flux returning to the mantle is $0.8-1.6 \mathrm{Tmol} \mathrm{C} \mathrm{yr}^{-1}$.

\section{(b) Reducing power}

Any reduced $\mathrm{C}$ sent to the mantle - graphite, diamond, elemental C-leaves oxidant behind in the surface environment, thus contributing to $A_{\mathrm{Ox}}$. It is also possible that organic $\mathrm{C}$ would be oxidized within the subduction zone by reaction with sulphate or an oxidized metal, such as $\mathrm{Fe}^{3+}$. When this occurs, does the reduction product $\left(\mathrm{Fe}^{2+}\right.$, for example) become an organic-carbon proxy that also contributes to $A_{\mathrm{Ox}}$ ? No, the reaction instead amounts to a last-minute reversal of processes within the carbon cycle. Within the exogenic reaction chamber, $\mathrm{Fe}^{3+}$ will have been produced within the downgoing slab by hydrothermal alteration and by oxidation of $\mathrm{Fe}^{2+}$ at the expense of $\mathrm{O}_{2}$. Or the sulphate will have been produced by processes within the carbon cycle. In either case, the oxidizing power will be represented by an equivalent quantity of organic carbon. For the carbon cycle, therefore, the oxidation of organic carbon by oxidized metals or sulphur within subduction zones is a functional equivalent of biological respiration. Details follow.

If the $\mathrm{Fe}^{3+}$ or sulphate is a product of aerobic oxidation, it carries the oxidizing power of $\mathrm{O}_{2}$ produced during photosynthesis. The same organism that produced the $\mathrm{O}_{2}$ produced an equivalent amount of organic carbon. The oxidation of that organic material within the subduction zone amounts to a reversal of the overall process.

If the $\mathrm{Fe}^{3+}$ was produced at the expense of sulphate during hydrothermal alteration, that sulphate can similarly be traced to photosynthetic $\mathrm{O}_{2}$ and carbon. The chain of chemical events has an additional link, but the oxidation of organic carbon within the subduction zone is again simply a reversal of the process.

If the sulphate was produced by anaerobic, photosynthetic bacteria, those organisms will have produced an equivalent amount of organic carbon. The oxidation of that organic material within the subduction zone amounts to a reversal of the overall process.

Finally, if the $\mathrm{Fe}^{3+}$ was produced by serpentinization, an equivalent quantity of $\mathrm{H}_{2}$ will also have been produced and used by microbiota to produce an equivalent amount of organic carbon, either directly, through chemosynthesis, or indirectly, through consumption of $\mathrm{O}_{2}$, photosynthetic production of organic matter, etc. Again, the oxidation of organic $\mathrm{C}$ within the subduction zone amounts simply to a reversal.

If the subducted organic carbon returned to the surface as $\mathrm{CO}_{2}$ after reducing an inorganic substance to some oxidation state lower than that of its input-if $\mathrm{Fe}^{3+} / \Sigma \mathrm{Fe}$ in the downgoing slab were driven to values lower than $\mathrm{Fe}^{3+} / \Sigma \mathrm{Fe}$ in unaltered MORB - that would contribute to $A_{\mathrm{Ox}}$. Failing that, $J_{\mathrm{od}}>0$ provides the only route by which processes in subduction zone can yield a net export of oxidizing power by the carbon cycle.

In magnitude, $J_{\mathrm{od}}$ is constrained to be less than $J_{\mathrm{os}}$. Exchange of $\mathrm{C}$ between reduced and oxidized pools is not excluded. Some of the $\mathrm{C}$ in $J_{\text {od }}$ might derive from $J_{\text {as }}$ or $J_{\mathrm{az}}$. Estimation of $J_{\text {od }}$ requires knowledge of $J_{\text {os }}$ and $L_{\text {Redv }}$. The rate of subduction of organic matter, as discussed above, is here taken as $0.2 \mathrm{Tmol} \mathrm{yr}^{-1}$, though better estimates are clearly warranted. $L_{\text {Redv }}$ has two components: the reduced gas flux from arc volcanoes, and the reduced gases from other seeps, represented by $j_{\mathrm{CH}_{4}}$.

The reduced component of the volcanic gas flux is represented by $j_{\mathrm{H}_{2}}+\mathrm{SO}_{2}$ and $j_{\mathrm{H}_{2} \mathrm{~S}}$. In the present context, the question is how it relates to $J_{\mathrm{os}}$, the reducing power delivered by subduction of organic carbon. The hydrogen abundance in volcanic gases is set by redox equilibrium with water, such that $\mathrm{H}_{2}: \mathrm{H}_{2} \mathrm{O}$ ratios are maintained near $c a 0.01$ at most eruptive temperatures (Giggenbach 1996). Taking the arc magmatic-water flux at $17 \mathrm{Tmol} \mathrm{yr}^{-1}$ (Wallace 2005) results in a hydrogen component of $0.17 \mathrm{Tmol} \mathrm{yr}^{-1}$. The $\mathrm{SO}_{2}$ efflux from arcs, some of which may derive from sources other than reduction of subducted sulphate, is of similar magnitude, $0.16-0.28 \mathrm{Tmol} \mathrm{yr}^{-1}$ (Halmer et al. 2002; Wallace 
2005). Other reduced species in arc gases are relatively minor. The ratio of $\mathrm{H}_{2} \mathrm{~S}: \mathrm{SO}_{2}$ is generally between 1 and 0.05 (Halmer et al. 2002), and some of the hydrogen sulphide released at volcanic arcs likely derives from volatilization of sulphide in the downgoing slab rather than reduction of sulphate by organic carbon. The reducing power carried by other volatiles, such as $\mathrm{CO}, \mathrm{COS}$ and $\mathrm{CS}_{2}$, is orders of lower magnitude. Since $\mathrm{H}_{2}$ and $\mathrm{SO}_{2}$ represent twoelectron reductions, the total flux is halved to convert to moles $\mathrm{O}_{2}$ equivalent, whereas $\mathrm{H}_{2} \mathrm{~S}$ produced from sulphate represents eight electrons or $2 \mathrm{~mol} \mathrm{O}_{2}$ equiv. The resulting estimate from gas chemistry is $0.2 \mathrm{Tmol}_{2}$ equiv. $\mathrm{yr}^{-1}$ from $\mathrm{H}_{2}$ and $\mathrm{SO}_{2}$ and $0.4-0.02 \mathrm{Tmol} \mathrm{O}_{2}$ equiv. $\mathrm{yr}^{-1}$ from $\mathrm{H}_{2} \mathrm{~S}$. By itself, the flux of $\mathrm{H}_{2}$ and $\mathrm{SO}_{2}$ is already equivalent to the reducing power carried by subducted organic carbon. The obvious presence of additional reduced outputs, namely volcanic $\mathrm{H}_{2} \mathrm{~S}$ and hydrocarbons at seeps $\left(j_{\mathrm{CH}_{4}}\right)$, shows that better knowledge of redox budgets for subduction zones is needed. With due regard for the uncertainties imposed by the present budgets, it also suggests that most or all reducing power carried by subducted organic carbon is returned to the exogenic reaction chamber and that $J_{\text {od }}$ is small at present.

\section{CYCLING OF CARBON AND ITS REDOX PARTNERS OVER TIME}

Over time, the crust has accumulated carbon. Integrated inputs from the mantle have exceeded integrated returns to the mantle. As a means of exploring the balance, we can accept the fluxes and reservoirs proposed thus far as hypotheses and consider how $8500 \mathrm{Emol} \mathrm{C}$ might have accumulated and what electron donors were probably associated with the production of organic carbon.

\section{(a) The crust-mantle carbon balance}

Values of $\left(J_{\mathrm{ad}}+J_{\mathrm{od}}\right) / J_{\mathrm{am}}$ control the accumulation of crustal $\mathrm{C}$. When they are less than 1, the crustal inventory will grow. Ideally, a geologic record would exist, but proxies for $\left(J_{\mathrm{ad}}+J_{\mathrm{od}}\right)$ are rare. The low $\delta^{13} \mathrm{C}$ values of some diamonds, particularly those of eclogite paragenesis, strongly suggest derivation from crustal organic carbon (Pearson et al. 2004), though this has been disputed (Deines et al. 2001). Recently, it is been suggested that organic carbon can be subducted beyond $250 \mathrm{~km}$ and contribute to sublithospheric diamonds (Tappert et al. 2005). The stability of carbonated eclogite at high pressures and temperatures (Dasgupta et al. 2004) indicates that eclogitization may be an important route for the subduction of carbon (both oxidized and reduced) into the mantle. This is particularly interesting in light of recent suggestions that eclogitization is a geologically recent phenomenon (Bjørnerud \& Austrheim 2004). A hotter upper mantle earlier in Earth history would more efficiently devolatilize downgoing slabs at shallower depths, removing both carbon and water. Shallow decarbonation, combined with drying of the slab and consequent inhibition of the formation of eclogite, may have

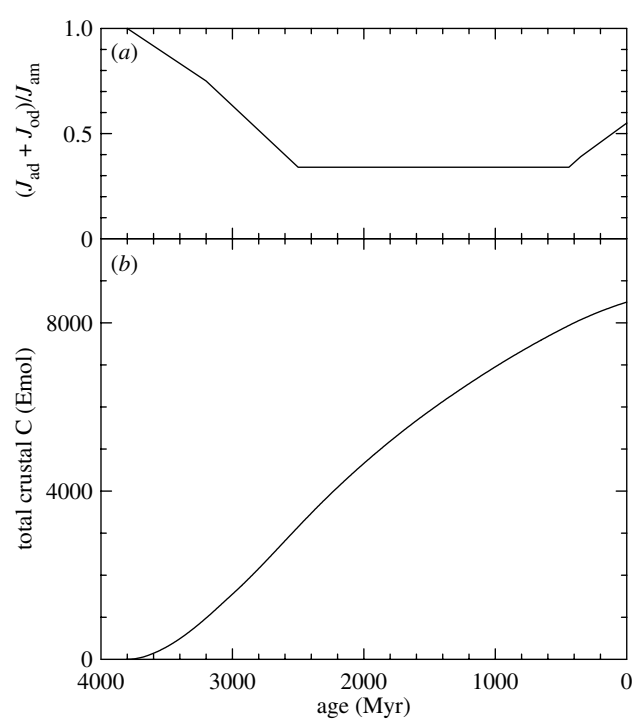

Figure 7. Growth of the crustal inventory of carbon over time. (a) Outputs to the mantle relative to inputs from the mantle as a function of time. The ratio is assumed to be near unity when continents were non-existent and to have declined as they increased in size. Values have been adjusted to provide the observed total of $8500 \mathrm{Emol} \mathrm{C}$ by $0 \mathrm{Ma}$. (b) The integral obtained by applying the return fraction specified in panel (a) to the fluxes shown in figure 4 . In detail, total crustal $\mathrm{C}=$ $\sum_{3800}^{t} J_{\mathrm{am}}(t)\left[1-\left(\left(J_{\mathrm{ad}}(t)+J_{\mathrm{od}}(t)\right) / J_{\mathrm{am}}(t)\right)\right] \tau$.

meant that $J_{\text {od }}$ and $J_{\text {ad }}$ were small early in Earth history (Des Marais 1985). Much depends on the tectonic regime, and when the present style of plate tectonics began, which has been the subject of much debate (Van Kranendonk 2004; Stern 2005).

One view takes the slow growth of continents together with the steep early declines in the rate at which carbon is delivered from the mantle (figure 4) as constraining variations in $\left(J_{\text {ad }}+J_{\text {od }}\right) / J_{\text {am }}$ quite strongly. The scenario associated with figure 4 provides $80 \%$ of the present continental area by $2.5 \mathrm{Ga}$. By comparison, Veizer \& Mackenzie (2004) point to evidence suggesting that only $25 \%$ of continental crust accumulated between 4.0 and $2.6 \mathrm{Ga}$, another $35 \%$ in the interval to $1.7 \mathrm{Ga}$, and the final $40 \%$ thereafter. Because the continents house the major reservoirs of crustal carbon, $\left(J_{\mathrm{ad}}+J_{\mathrm{od}}\right) / J_{\mathrm{am}}$ must approach 1 (no net crustal storage) in the early Archaean and decline to lower values only as continents grow. The time course of $\left(J_{\mathrm{ad}}+J_{\mathrm{od}}\right) / J_{\mathrm{am}}$ shown in figure $7 a$ fits these criteria while eventually yielding a crustal inventory of $8500 \mathrm{Emol} \mathrm{C}$ (figure $7 b$ ) and a modern $\left(J_{\mathrm{ad}}+J_{\mathrm{od}}\right) / J_{\mathrm{am}}=0.55$. The latter value is in the middle of the range estimated above $(0.36-0.73)$.

\section{(b) The accumulation of organic carbon} and oxidized electron donors

Reconstruction of redox budgets requires estimates of $f$. These derive from interpretation of the carbon isotopic records, with due attention to the possible 


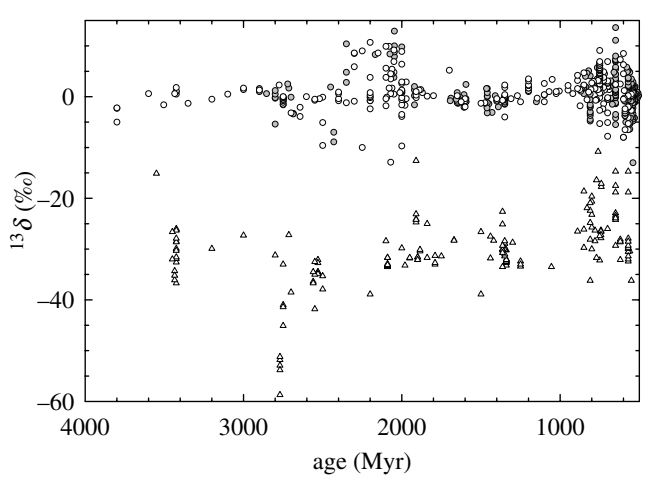

Figure 8. Isotopic compositions of sedimentary carbonates and of sedimentary organic carbon as a function of time. Each point represents the average isotopic composition of an entire stratigraphic unit. Carbonates (Shields \& Veizer 2002) are represented by circles. Filled symbols represent geologic units which are well dated. Open symbols represent units for which the dates are approximate. Samples of organic material (Strauss \& Moore 1992) are represented by triangles. The ages assigned in the original compilation have been revised to agree with those assigned by Shields \& Veizer (2002).

importance of $\Delta_{\mathrm{m}}, \Delta_{\mathrm{g}}$ and $\lambda$. Figure 8 summarizes observations of $\delta_{\mathrm{ab}}$ and $\delta_{\mathrm{ob}}$. The latter represent chemically isolated samples of kerogen (Strauss \& Moore 1992). The ratios of $\mathrm{H}: \mathrm{C}$ in Precambrian kerogens are frequently well below 0.5 (Hayes et al. 1983). Accordingly, the kerogens have been extensively dehydrogenated by processes that frequently involve the loss of ${ }^{13} \mathrm{C}$-depleted hydrocarbons and thus enrichment of ${ }^{13} \mathrm{C}$ in the residual kerogen. Because our objective is to reconstruct probable isotopic compositions of organic carbon at the time it was removed from the exogenic reaction chamber (i.e. as it flowed through the arrow representing $J_{\mathrm{ob}}$ in figure 1), we will base our estimates of $f$ mainly on the lower values of $\delta_{\mathrm{ob}}$ in each time interval

\section{(c) 4400-3800 Ma}

In parallel with the isotopic records summarized in figure 8, the integrations in figures 5 and 7 have begun at $3800 \mathrm{Ma}$. This point in time roughly marks the end of the late, heavy bombardment and the beginning of Earth's sedimentary record. Catling et al. (2001) have considered how earlier events might have set the stage for carbon cycling. In particular, they estimate that impacts of asteroids between 4400 and $3800 \mathrm{Ma}$ probably delivered $1000 \mathrm{Emol}$ of reduced carbon to Earth's surface. To whatever extent such material was not incorporated by the mantle, Earth's crust will have begun with an inventory of primordial organic carbon. When biological cycling of carbon began, the processes would have been

production :

$\left(\mathrm{CO}_{2}\right)_{\text {from outgassing }}+\mathrm{Red} \rightarrow\left(\mathrm{C}_{\text {org }}\right)_{\text {from production }}+\mathrm{Ox}$,

recycling : $\left(\mathrm{C}_{\text {org }}\right)_{\text {primordial }}+\mathrm{Ox} \rightarrow\left(\mathrm{CO}_{2}\right)_{\text {crustal }}+\mathrm{Red}$,

net :

$\left(\mathrm{CO}_{2}\right)_{\text {from outgassing }}+\left(\mathrm{C}_{\text {org }}\right)_{\text {primordial }}$

$\rightarrow\left(\mathrm{C}_{\text {org }}\right)_{\text {from production }}+\left(\mathrm{CO}_{2}\right)_{\text {crustal }}$.

Phil. Trans. R. Soc. B (2006)
In these equations, Red and $\mathrm{Ox}$ are reduced and oxidized forms of a redox partner, such as $\mathrm{Fe}$ or $\mathrm{S}$. Reactions between surviving, asteroidal organic material (termed 'primordial') and oxidants produced during carbon cycling might have been biologically catalysed and occurred within the exogenic reaction chamber or they might have been thermal and occurred only at depth within accumulating sediments. In either case, the resulting $\mathrm{CO}_{2}$ became part of the crustal inventory. Such reactions would have two effects: (i) an exchange of biotic organic carbon for primordial organic carbon and (ii) consumption of oxidants. Only when production acted to increase the inventory of $\mathrm{C}_{\text {org }}$ (here taken to begin at $3.8 \mathrm{Ga}$ ) would oxidants begin to accumulate.

\section{(d) $3800-2800 \mathrm{Ma}$}

For this interval, the isotopic record of sedimentary carbonates compiled by Shields \& Veizer (2002) includes 24 stratigraphic units. With an average value of $\delta_{\mathrm{ab}}=-0.1 \%$, they are consistently enriched in ${ }^{13} \mathrm{C}$ relative to mantle carbon. The standard deviation of the population is $1.9 \%$. For the same interval, figure 8 yields an estimate of $\delta_{\mathrm{ob}}=-36 \%$. Nakamura \& Kato (2004) measured values of $\delta_{\text {aw }}$ in basalts from the Warrawoona Group (3425 Myr, Western Australia). Their average result, $-0.3 \pm 1.2 \%$, does not differ significantly from $\delta_{\mathrm{ab}}$ in sedimentary carbonates in this time interval. Accordingly, $\Delta_{\mathrm{m}} \approx \Delta_{\mathrm{g}}$, the $\lambda\left(\Delta_{\mathrm{m}}-\Delta_{\mathrm{g}}\right)$ terms in equation (1.8) are small, and $f=\left(\delta_{\mathrm{ab}}-\delta_{\mathrm{i}}\right) /$ $\left(\varepsilon-\Delta_{\mathrm{g}}\right)=\left(\delta_{\mathrm{ab}}-\delta_{\mathrm{i}}\right) /\left(\delta_{\mathrm{ab}}-\delta_{\mathrm{ob}}\right)=0.14$. Observed values of $\delta_{\mathrm{ab}}$ and $\delta_{\mathrm{ob}}$ then indicate that $14 \%$ of the carbon being delivered to the exogenic reaction chamber by outgassing of $\mathrm{CO}_{2}$ from the mantle was being buried as reduced, organic carbon.

The net production of organic carbon during any increment of time can be estimated:

$$
\begin{aligned}
\text { net } \mathrm{C}_{\text {org }}= & \left(\mathrm{C}_{\text {org }} \text { produced by reduction of } J_{\mathrm{am}}\right) \\
& +\left(\mathrm{C}_{\text {org }} \text { produced by reduction of recycling } \mathrm{C}\right) \\
& -\left(\mathrm{C}_{\text {org }} \text { oxidized during recycling }\right) .
\end{aligned}
$$

The recycling carbon (i.e. $J_{\mathrm{or}}+J_{\mathrm{ar}}+J_{\mathrm{ov}}+J_{\mathrm{av}}$ ) will be some portion of the total crustal carbon. If a representative half-mass age is taken as $300 \mathrm{Myr}$, the fraction recycling in a $10 \mathrm{Myr}$ interval will be $2.3 \%$ (see table 1). In any $10 \mathrm{Myr}$ interval, therefore, $2.3 \%$ of crustal carbon will be recycled, essentially mixing with the $10 \mathrm{Myr}$ input of mantle carbon. Of that total, a portion controlled by $\left(J_{\mathrm{ad}}+J_{\mathrm{od}}\right) / J_{\mathrm{am}}$ will be returned to the mantle. Of the remainder, a fraction $f$ will be buried in organic form. Assuming that all recycling organic carbon is oxidized, an integrable form of equation (6.1) is then

$$
\text { net } \mathrm{C}_{\mathrm{org}}=f\left[J_{\mathrm{am}} \tau\left(1-\frac{J_{\mathrm{ad}}+J_{\mathrm{od}}}{J_{\mathrm{am}}}\right)+\gamma M\right]-\gamma M_{\mathrm{o}},
$$

where $\gamma$ is the fraction of crustal carbon recycling in a time interval of length $\tau$ (e.g. 0.023 for $\tau=10^{7}$ years and a half-mass age of $300 \mathrm{Myr}$ ), and, at the beginning of the time-step, $M$ is the total crustal inventory of carbon in all forms (figure $7 b$ ) and $M_{\mathrm{o}}$ is the total inventory of organic carbon. Stepwise summation 


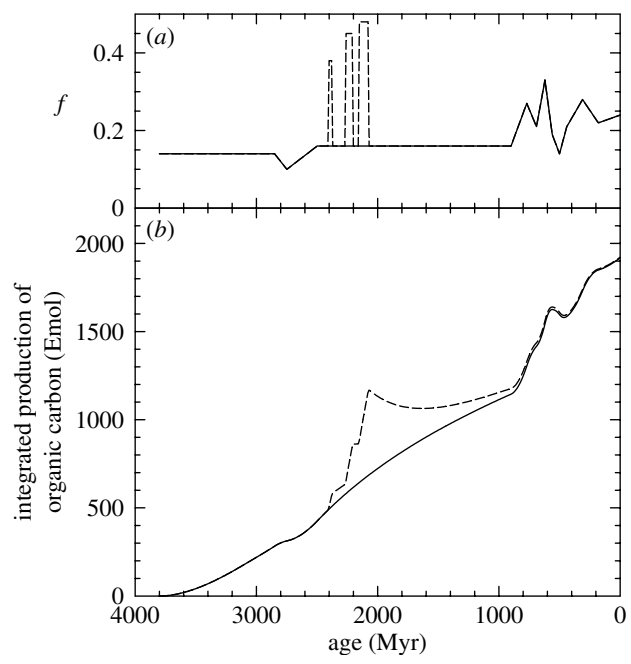

Figure 9. Integrated net production of organic carbon, equivalent to the net release of oxidizing power $\left(\mathrm{Emol} \mathrm{O}_{2}\right)$, over time. (a) Estimated values of $f$. For ages greater than $890 \mathrm{Myr}$, they are based on the isotopic compositions shown in figure 8 . For younger ages, they are consistent with the isotopic compositions shown in figure 8 and are based in detail on records summarized by Hayes et al. (1999). The broken lines between 2080 and 2400 Myr represent isotopic excursions reviewed by Melezhik et al. (1999) and discussed in the text. (b) The integral obtained when the $f$ values shown in figure $9 a$ and the carbon-return ratios shown in figure $7 a$ are substituted in equation (6.2), with $\gamma=0.023$ and $M=M_{\mathrm{o}}=0$ at $3800 \mathrm{Ma}$.

provides an estimate of the integrated production of organic carbon, the first integral on the right-hand side of equation (2.2).

The calculation is based on (i) values of $\left(J_{\mathrm{ad}}+\right.$ $\left.J_{\text {od }}\right) / J_{\text {am }}$ chosen to provide the observed crustal inventory of C (figure $7 a$ ); (ii) values of $J_{\text {am }}$ provided by figure 4 ; and (iii) values of $f$ shown in figure $9 a$ and estimated from the observations summarized in figure 8 . Setting $M=M_{\mathrm{o}}=0$ at $3800 \mathrm{Ma}$ and summing Net $\mathrm{C}_{\text {org }}$ yields the result shown in figure $9 b$. Because a sharp drop in $\delta_{\mathrm{ob}}$ suggests a major change in carbon cycling at approximately $2770 \mathrm{Ma}$ (Hayes 1983, 1994), we will focus first on the interval $3800-2800 \mathrm{Ma}$. By $2800 \mathrm{Ma}, M_{\mathrm{o}}=$ oxidant release $=300 \mathrm{Emol} \mathrm{O}_{2}$ equiv.

To place this result in context, consider the inventories summarized in table 2. For all of the materials considered, a range of estimates can be found in the literature. Those included here are representative high and low values. The value of $300 \mathrm{Emol} \mathrm{O}_{2}$ equiv. greatly exceeds the oxidizing power represented by $\mathrm{O}_{2}$ in the modern atmosphere and ocean and approaches the low estimate of the amount of oxidizing power represented by total crustal deposits of sulphate and sulphate dissolved in seawater. These comparisons decisively eliminate two otherwise-interesting possibilities, namely that $\mathrm{O}$ or $\mathrm{S}$ could have served as the dominant redox partner for $\mathrm{C}$ at this stage of Earth history. In the first case, levels of $\mathrm{O}_{2}$ would have risen high enough to inhibit the mass-independent fractionation of
Table 2. Crustal inventories of reduced and oxidized products of carbon cycling ${ }^{\mathrm{a}}$.

\begin{tabular}{|c|c|c|c|}
\hline \multirow[b]{2}{*}{ product } & \multicolumn{2}{|c|}{$\begin{array}{l}\text { estimated crustal } \\
\text { total Emol } \\
\mathrm{O}_{2} \text { equivalent }\end{array}$} & \multirow[b]{2}{*}{ reference } \\
\hline & high & low & \\
\hline \multirow{3}{*}{$\begin{array}{l}\mathrm{O}_{2} \\
\mathrm{Fe}^{3+\mathrm{b}}\end{array}$} & 37.2 & 37.2 & Keeling et al. (1993) \\
\hline & 1860 & & Goldschmidt (1954) \\
\hline & & 1020 & $\begin{array}{l}\text { Ronov \& Yaroshevsky } \\
\quad(1976)\end{array}$ \\
\hline \multirow[t]{2}{*}{$\mathrm{SO}_{4}^{2-}$} & 480 & & Garrels \& Perry (1974) \\
\hline & & 332 & Holser et al. (1988) \\
\hline \multirow{3}{*}{$\begin{array}{l}\text { total } \\
\quad \text { oxidants } \\
\text { organic C }\end{array}$} & 2377 & 1389 & \\
\hline & 1280 & & Des Marais (2001) \\
\hline & & 675 & Arvidson et al. (in press) \\
\hline
\end{tabular}

${ }^{a}$ A similar compilation has been prepared by Catling et al. (2001). It includes an estimate of $1000 \mathrm{Emol}$ organic $\mathrm{C}$ delivered to the crust by impacts of asteroids between 4400 and $3800 \mathrm{Ma}$. The entries above pertain to the present. To whatever extent primordial organic pertain to the present. To whatever extent primordial organic
material has survived in the crustal inventory, it is included in the 'organic C' category.

Calculated from data summarized by Lécuyer \& Rickard (1999). Represents $\mathrm{Fe}^{3+}$ in excess over mantle $\mathrm{Fe}^{3+} / \Sigma \mathrm{Fe}=0.12$ (Bezos \& Humler 2005)

sulphur isotopes. In the second, concentrations of dissolved sulphate would have climbed to levels that lead to large differences between the ${ }^{34} S$ contents of sedimentary sulphides and sulphates, a signal that is not observed until later (Hayes et al. 1992).

The remaining oxidized product listed in table 2 is ferric iron. Its crustal inventory is large enough to offer plenty of headroom. Iron formations are, moreover, important components of the Archaean sedimentary record. The present context, however, requires that iron served as the electron donor in primary production while the environment remained strictly anaerobic. An organism capable of utilizing $\mathrm{Fe}^{2+}$ directly has recently been isolated (Jiao et al. 2005). In principle, we need look no further, but alternatives deserve consideration. These are: (i) the true value of $f$ was really near zero; (ii) $f$ was 0.14 but no oxidants accumulated; or (iii) $f$ was 0.14 and the electron donor was $\mathrm{H}_{2}$, for which the immediate oxidized product $\left(\mathrm{H}_{2} \mathrm{O}\right)$ was invisible, although iron was probably the ultimate source of the electrons.

The true value of $f$ could be approximately zero if the basalt-carbonation correction had been wrongly excluded and the correct value of $\lambda\left(\Delta_{\mathrm{m}}-\Delta_{\mathrm{g}}\right)$ was $4.9 \%$. If $\lambda$ were 0.9 , this would require that the initial average value of $\delta_{\text {aw }}$ had been $-5.5 \%$ and that Nakamura \& Kato (2004) found instead -0.3\%o, because the isotopic compositions of all of their samples had been affected by post-depositional carbon-isotopic exchange. However, among 42 samples of four different types, the most negative value of $\delta_{\text {aw }}$ is $-3.4 \%$ and there is no correlation between $\delta$ and the abundance of carbonate. Evidence for the required alteration is therefore lacking, making it likely that $f$ truly approached 0.14 .

If $f$ approached 0.14 but no oxidants accumulated ((ii), above), ( $\left.J_{\mathrm{ad}}+J_{\mathrm{od}}\right) / J_{\mathrm{am}}$ must have been 1 , 
indicating rapid and quantitative return of all carbon to the mantle, even as 3.5 million $\mathrm{km}^{2}$ of continental crust (Lowe 1992) formed prior to $3.2 \mathrm{Ga}$. Moreover, as substantial quantities of organic carbon were forming as required by $f=0.14$ (and thus $J_{\mathrm{ob}} / J_{\mathrm{am}} \geq 0.14$ ), the putatively non-accumulating oxidant, which could not be either $\mathrm{O}_{2}$ or sulphate and which had to be present only at trace levels, was able to reoxidize all of the organic material completely even as the carbon itself returned quantitatively to the mantle. The required combination of circumstances is practically impossible.

The third alternative, in which $f$ was approximately 0.14 and $\mathrm{H}_{2}$ served as the electron donor, fits well with multiple lines of evidence. Anaerobic organisms capable of producing organic material from $\mathrm{CO}_{2}$ and $\mathrm{H}_{2}$ are abundant and include both chemoautotrophs (methanogens, acetogens) and photoautotrophs (photosynthetic bacteria; Tice \& Lowe 2004 have already postulated that such organisms were important members of near-shore microbial communities $3.4 \mathrm{Ga}$ ). The potential versatility of microbial ecosystems based on such organisms is great enough to provide both producer and consumer assemblages and thus to stabilize carbon cycling and yield globally consistent isotopic fractionations. Fermentative consumption would remobilize organic $\mathrm{C}$ as a mixture of $\mathrm{CO}_{2}$ and $\mathrm{CH}_{4}$, providing needed greenhouse warming (Kasting \& Catling 2003) and setting the stage for the isotopic transient observed at $2.8-2.7 \mathrm{Ga}$ (Hayes 1994).

The required net input of $\mathrm{H}_{2}$ would be $600 \mathrm{Emol}$ ( $=300 \mathrm{Emol}_{2}$ equiv.). Owing to the postulated declines in $J_{\text {am }}$ (figure 4) and increases in [1- $\left(J_{\text {ad }}+\right.$ $\left.\left.J_{\text {od }}\right) / J_{\text {am }}\right]$ (equation (6.2)), the required rate would rise only slowly from $0.5 \mathrm{Tmol} \mathrm{yr}^{-1}$ at $3500 \mathrm{Ma}$ to $0.9 \mathrm{Tmol} \mathrm{yr}^{-1}$ at $2800 \mathrm{Ma}$. If, as is likely, conditions at the seafloor favoured serpentinization reactions (Sleep et al. 2004), a flux of $0.7 \mathrm{Tmol} \mathrm{H}_{2} \mathrm{yr}^{-1}$ could be provided by alteration of less than $10^{15} \mathrm{~g} \mathrm{Mg-rich}$ (komatiitic) basalt per year. The present rate of generation of ocean crust is about $6 \times 10^{16} \mathrm{~g} \mathrm{yr}^{-1}$, so the requirement poses no problem. In the overall sequence of electron transfers, it is only the immediate oxidized product of carbon fixation $\left(\mathrm{H}_{2} \mathrm{O}\right)$ which is 'invisible'. The reducing power represented by the organic matter is balanced by $\mathrm{Fe}^{3+}$, which is accumulating or being subducted.

To summarize, from both geological and biological points of view, there are highly plausible mechanisms by which substantial amounts of organic carbon could be produced and buried in accord with the isotopic records, with complementary releases of oxidizing power, without any requirement for generation of $\mathrm{O}_{2}$ during the interval $3800-2800 \mathrm{Ga}$. The most likely redox partner for $\mathrm{C}$ during this interval is $\mathrm{Fe}$, either directly via phototrophic oxidation of dissolved $\mathrm{Fe}^{2+}$, and/or indirectly, with $\mathrm{H}_{2}$ shuttling electrons from $\mathrm{Fe}^{2+}$ in seafloor basalts to phototrophic and chemoautotrophic producers.

\section{(e) 2800-1800 Ma}

The next billion years begin with the apparent onset of oxygenic photosynthesis (Hayes 1983, 1994; Summons et al. 1999), which includes a period during which the abundance of ${ }^{13} \mathrm{C}$ in sedimentary carbonates was sometimes markedly enriched (Karhu \& Holland 1996; Melezhik et al. 1999), and ends with the likely onset of sulphidic conditions in the deep sea (Canfield 1998).

Estimates of $f$ derived from the isotopic records provide scant evidence that the development of oxygenic photosynthesis, for all its magnificence as a biochemical innovation, provided a significant increase in the net release of oxidizing power. If anything, $J_{\mathrm{ob}}$ initially declined, a feature marked by the notch visible at $2.8 \mathrm{Ga}$ in figure $9 a$. By $2500 \mathrm{Ma}, f$ appears to have risen to 0.16 , a value only slightly higher than that estimated for the early Archaean. At $2450 \mathrm{Ma}$, when traces of $\mathrm{O}_{2}$ quench the mass-independent fractionation of sulphur isotopes, the estimated minimal total release of oxidizing power by the $\mathrm{C}$ cycle is $460 \mathrm{Emol} \mathrm{O}_{2}$ equiv. Although uncertainties in that total are very large, it is far short of the capacities of crustal $\mathrm{Fe}$ and $\mathrm{S}$ to supply electrons (table 2). Levels of $\mathrm{O}_{2}$ in the environment would have been sharply limited by the strength of those sinks.

What, then, happened between 2400 and $2000 \mathrm{Ma}$ ? Contents of ${ }^{13} \mathrm{C}$ in carbonates are frequently elevated, occasionally exceeding $+10 \%$ o (figure 8 ). Accepting these compositions as representative of the global pool of oceanic DIC would imply a near-trebling of $f$. Writing three years after Karhu \& Holland (1996) published 'Carbon isotopes and the rise of atmospheric oxygen', Melezhik et al. (1999) drew on additional data to define three apparently separate pulses of isotopic enrichment. That dissection of the record yields the three spikes in $f$ that are represented by broken lines in figure $9 a$ (alternative stratigraphic correlations yield only two spikes; Bekker et al. 2003). The isotopic signal has been associated with other lines of geochemical evidence indicating that the atmosphere became more oxidizing at about the same time. The ensemble has become known as 'The Great Oxidation Event' (Holland 2002).

One view of the scale of the event is provided by the broken line in figure $9 b$. If the isotopic signals represent pulses of carbon burial, the integrated production of organic carbon by $2000 \mathrm{Ma}$ would be $600 \mathrm{Emol}$ greater. As shown by table 2, that is more than enough to provide the present inventories of $\mathrm{SO}_{4}{ }^{2-}$ and $\mathrm{O}_{2}$. But there are problems with interpreting these isotopic variations in terms of carbon burial.

(i) There is no parallel isotopic enrichment in the organic carbon, as would be expected if the carbonate represented the oceanic DIC that was the carbon source for primary producers. Karhu \& Holland (1996) searched for a signal in the organic-carbon record and found its absence 'rather odd'. If the record were not so fragmentary and noisy (preservation is a much greater problem for organic carbon than for carbonate minerals) that the problem could be set aside (Melezhik et al. 1999), this alone would be fatal to the interpretation.

(ii) Evidence is lacking for the large deposits of organic carbon that should have formed (Melezhik et al. 1999; Aharon 2005). 
(iii) The sequence of isotopic signals is reversed from that expected. If the oxygenation that cut off mass-independent fractionation of sulphur isotopes was due to an organic-carbon-burial event, the disappearance of the mass-independent fractionation of sulphur isotopes should not precede the first carbon-isotopic enrichments.

(iv) It is difficult to envision supplies of nutrients great enough to sustain the levels of productivity required to supply the organic carbon. Aharon (2005) has discussed supplies of phosphate very insightfully, concluding that efficient stripping of $P$ from organic matter prior to burial provides the only solution. To achieve the projected values of $f$, the $\mathrm{C}: \mathrm{P}$ ratio in buried organic matter would have to be 4000 and the organicpreservation rate would have to be $6.6 \%$, about fivefold higher than that observed in the Black Sea.

(v) Stratigraphic correlations are not secure enough to demonstrate that the various isotopic excursions are coeval, and thus necessarily linked to variations in a global reservoir (Aharon 2005).

(vi) Most of the units displaying the isotopic enrichments are dolostones with substantial isotopic variability. In their list of 12 'major localities,' Melezhik et al. (1999) report withinunit ranges of 3-13\% and an average range of $7 \%$. Consistent with this, for the interval 2350-2000 Ma (inclusive), Shields \& Veizer (2002) report $589 \delta$-values between -2.5 and $+2.5 \%$ and 424 between +5 and $+13 \%$. That is, 'normal' isotopic abundances are more common than elevated abundances.

(vii) When complete chemical analyses of the carbonates are reported, they often include substantial concentrations of $\mathrm{SiO}_{2}$ (to $40 \%$; Melezhik et al. 1999). Stratigraphic columns indicate that some of the isotopically enriched carbonates are closely interbedded with shales (Buick et al. 1998), others are described as 'nodular' (Bekker et al. 2003).

The latter features (vi) and (vii) are more characteristic of diagenetic carbonates than of marine limestones faithfully carrying records of oceanic DIC. When it is recalled that values of ${ }^{13} \delta$ to $+13 \%$ are common in much younger dolomites that have been affected by methanogenic diagenesis (Klein et al. 1999; Mazzullo 2000), that alternative interpretation demands attention. In fact, attribution of the isotopic signals to methanogenic diagenesis has already been favoured by several sets of authors (Yudovich et al. 1991; Dix et al. 1995).

In all likelihood, the diagenetic alternative has failed to win popularity because an alternative does not appear to be needed. Multiple, convergent lines of evidence-independent of carbon-isotopic signalsindicate that thresholds of environmental oxidation were crossed during this time interval (Bekker et al. 2004). When the carbon-isotopic record fits into this picture at least roughly, why not include it? Even more to the point, given the apparent reality of the oxidation, how else should the carbon-isotopic enrichments be explained? They are temporally associated, dramatic and numerous (Bekker et al. 2003), globally distributed, and have the right polarity (enrichment rather than depletion).

\section{(f) Mechanisms of oxygenation}

The problem lies not in accepting the isotopic signals as markers of the oxidation but in assuming that they represent the cause. This is a key distinction. Either the steady functioning of the carbon cycle catalysed biological and ecological developments that shifted the relative strengths of oxygen sources and sinks (Holland 1978) or dramatic changes in carbon fluxes were required. If the former, the carbon-isotopic enrichments are environmental reporters comparable to the mass-independent fractionations of sulphur isotopes. If the latter, they point to increased burial of organic carbon but not necessarily to permanent changes in oxidation.

Flux-driven changes are subject to reversal. Buried organic material will be recycled by erosion and volcanism. As indicated by the downward trend of the broken line in figure $9 b$ after $2000 \mathrm{Ma}$, oxidants will be consumed. Even if a rise in $\mathrm{O}_{2}$ were driven by increases in $J_{\mathrm{ob}}$, some additional change would be required to stabilize the transition and make it permanent. Geophysical phenomena such as rifting and increased sedimentation can increase $J_{\mathrm{ob}}$, but are inherently cyclical. What they accomplish in one epoch will be undone in another. Increased burial of organic carbon is a half answer. It can push $\mathrm{O}_{2}$ levels higher, but maintaining them will require some further change, a second half to the answer.

Unidirectional change, a permanent strengthening of the source of oxidants, is in the realm of evolutionary biology. Physiological and ecological changes could stabilize higher levels of $\mathrm{O}_{2}$. Do they serve as the second half of the answer? If so, they are the biological results of geophysical stimuli and we need an explanation for the linkage. Or are physiological and ecological changes answers in themselves? If so, advances in oxygenation have largely biological origins and combinations of disparate phenomena are not required.

Kinetic factors are also pertinent. In discussing the carbon cycle, it is common to distinguish between the fast, biological cycle of photosynthesis and respiration and the slow, geological cycle in which erosion, weathering and volcanism are balanced by carbon burial. In global chemical terms, $\mathrm{O}_{2}$ is a highly reactive, transient intermediate that is produced and consumed within the exogenic reaction chamber. Its steady-state abundance must depend on the relative strengths of sources and sinks in that system. In contrast, sedimentary carbonates and organic materials are outputs from that system. Their relative abundances are controlled by geophysical factors that vary over relatively long time-scales.

\section{(g) Causes of the carbon-isotopic transient}

We can suggest a sequence of biologically driven environmental changes, associated with a rise in levels of $\mathrm{O}_{2}$, that would produce the observed isotopic signals without requiring enhanced burial of organic carbon. 


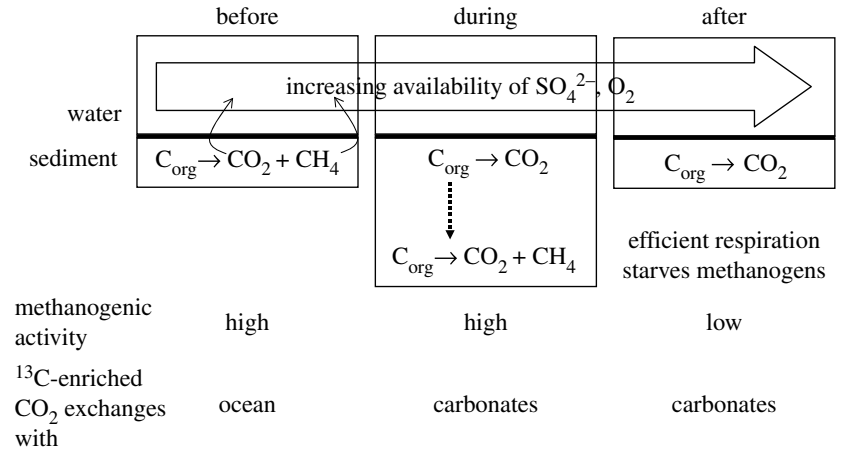

Figure 10. Summary of a sequence of conditions that could account for the abundance of ${ }^{13} \mathrm{C}$-enriched sedimentary carbonates 2400-2080 Myr ago.

They would also account for the absence of congruent variations in the organic-carbon isotopic record.

Especially prior to $2.3 \mathrm{Ga}$, methanogenic diagenesis must have been common. If electrons for biosynthesis were supplied mainly by $\mathrm{Fe}^{2+}$, the insolubility of the oxidized product, $\mathrm{Fe}^{3+}$, meant that electron acceptors were rare in most surface environments (Walker 1987). When respiring heterotrophs are excluded, the recycling of organic carbon in microbial communities will be managed by fermenters, with methanogens playing a vital role. This will have occurred in the same zones that are now occupied by aerobes. These methanogenic communities will have exchanged $\mathrm{CO}_{2}$ freely and directly with the oceanic water column, and, as a result, the isotopically enriched pools of $\mathrm{CO}_{2}$ that are characteristic of methanogenic diagenesis in modern environments will not have developed. This situation is summarized in the 'before' segment of figure 10 .

The development of oxygenic photosynthesis by at least $2700 \mathrm{Ma}$ began to change that situation, however slowly. Particularly near oxygen-producing communities (many of the isotopically enriched dolostones are associated with stromatolites; Melezhik et al. 1999), methanogens will eventually have been pushed to deeper levels in the sediment. When this occurred, apparently beginning at about $2400 \mathrm{Ma}$, isotopically enriched pools of porewater DIC will have developed. As carbon was exchanged with the carbonate minerals in the sediment (a process catalysed by the organic acids produced during fermentation), the isotopic signal characteristic of methanogenesis will have been recorded for the first time. Within about $400 \mathrm{Myr}$, a second event shut the signal off. Fundamentally, it must have been some weakening of the supply of organic material to methanogenic communities. As suggested in the 'after' segment of figure 10, it could have been caused by a further rise in the availability of electron acceptors. Alternatively, the tight association between stromatolitic producers and methanogenic consumers, indicated by many of the ${ }^{13} \mathrm{C}$-enriched sequences, might have been destabilized by environmental changes.

As in the Neoproterozoic, the isotopic excursions are associated with apparently extreme glaciations (Young et al. 2001; Tajika 2003). The effects of those glaciations on global redox geochemistry, if any, are unclear. It has conversely been proposed that the glaciations were caused by global oxidation which destroyed a methane greenhouse and that this occurred promptly after the development of oxygenic photosynthesis (Kopp et al. 2005). The second part of this does not fit with biomarker records that place the advent of oxygenic photosynthesis at or before $2.7 \mathrm{Ga}$ (Summons et al. 1999; Brocks et al. 2003; Eigenbrode 2004). It also does not fit with the inescapably slow effects of carbon cycling. Releases of oxidizing power have been continuous (figure $9 b$ ). But the oxidized products accumulated by $2.5 \mathrm{Ga}$ cannot have accounted for more than a fraction of the inventory of ferric iron and almost none of the sulphate (cf. table 2). In those circumstances, by what mechanism did the development of oxygenic photosynthesis promptly sustain steady-state concentrations of $\mathrm{O}_{2}$ high enough to destroy the methane greenhouse? Until that question can be answered, the evidence for (i) a $400 \mathrm{Ma}$ delay between the evolutionary event and its environmental impact and (ii) the continuing capacity of $\mathrm{Fe}$ and $\mathrm{S}$ as redox buffers should overrule speculation.

Oxidation of $\mathrm{S}$ by $\mathrm{O}_{2}$ would have produced sulphate in surface environments. However, by $1800 \mathrm{Ma}$, the estimated integrated release of oxidizing power (figure $9 b$ ) was only $820 \mathrm{Emol} \mathrm{O}_{2}$ equiv. This is still well below the low estimate of the amount required to build the crustal inventory of $\mathrm{Fe}^{3+}$ (table 2). As suggested by Canfield (1998), therefore, it is likely that production of sulphate mainly provided a route, via sulphate-reducing bacteria, to delivery of excess sulphide to deep ocean waters and the consequent cessation of deposition of banded iron formations.

\section{(h) 1800 Ma-present}

Carbon-isotopic records from the Neoproterozoic onward are good enough that values of $f$ can be estimated with some confidence (Hayes et al. 1999). The results are crudely summarized in figure $9 a$. When these values of $f$ are applied to the carbon fluxes estimated here, the projected final total release of oxidizing power is $1920 \mathrm{Emol} \mathrm{O}_{2}$ equiv. This is close to the average estimate of the total release represented by the sum of crustal Fe ${ }^{3+}, \mathrm{SO}_{4}{ }^{2-}$ and $\mathrm{O}_{2}$. 
The agreement is notable, but discrepancies within the redox accounts are more impressive. Estimates of crustal inventories of oxidants vary by a factor of 1.7 . Similarly, the estimated crustal inventories of organic carbon fall short (perhaps by two times) of the summed inventories of oxidized products and of the estimated total burial of organic carbon (figure $9 b$ ). At face value, this requires that reduced products have been exported from the crust more efficiently than oxidized products. Unless organic carbon could be exported to the mantle more efficiently than $\mathrm{Fe}^{3+}$, we are practically required to attribute such an imbalance to loss of $\mathrm{H}_{2}$ from the top of the atmosphere. The redox imbalance and the likely importance of $\mathrm{H}_{2}$ loss have been incisively examined and elaborated by Catling et al. (2001) and by Catling \& Claire (2005). They associate loss of $\mathrm{H}_{2}$ mainly with atmospheric $\mathrm{CH}_{4}$ and thus with the Archaean. By contrast, Tian et al. (2005) have noted the possible importance of the low exobase temperature of an anoxic atmosphere in limiting the rate of $\mathrm{H}_{2}$ escape from the early Earth. They contend that higher levels of $\mathrm{O}_{2}$ in the atmosphere after $2.4 \mathrm{Ga}$ would have increased exobase temperatures and promoted escape of hydrogen. Resolution of the history of hydrogen escape will constrain the second integral on the righthand side of equation (2.2), and be a key step in reconstructing the time course of $A_{\mathrm{Ox}}$.

The dearth of reduced products becomes particularly notable when the likely integrated effects of subduction, represented by the third integral in equation (2.2), are considered. Lécuyer \& Rickard (1999) contend that the net rate at which subduction is transferring $\mathrm{Fe}^{3+}$ from the crust to the mantle is presently $7 \mathrm{Tmol} \mathrm{yr}^{-1}$, or $1.8 \mathrm{Tmol} \mathrm{O}_{2}$ equiv. $\mathrm{yr}^{-1}$. If the continents are at steady state with respect to organic carbon, so that, in equation (6.2), $f \gamma M+\gamma M_{\mathrm{o}}=0$, the rate at which the carbon cycle is releasing oxidizing power is given by (cf. equation (6.2))

$f\left[J_{\mathrm{am}}-\left(J_{\mathrm{ad}}+J_{\mathrm{od}}\right)\right]=0.24(2.2-1.2)$

$=0.2 \mathrm{Tmol} \mathrm{O}_{2}$ equiv. $\mathrm{yr}^{-1}$,

where the values substituted are centre points of the ranges estimated in earlier sections of this paper. Since the net rate of subduction of $\mathrm{Fe}^{3+}$ proposed by Lécuyer \& Rickard (1999) greatly exceeds this value, it is either incorrect or represents a temporary situation. Attention to subduction of $\mathrm{Fe}^{3+}$ is, however, a very good idea. The continuing release of oxidizing power, coupled with the minimal variations in atmospheric levels of $\mathrm{O}_{2}$ during the Phanerozoic (Berner 2004) and balanced exchange of oxidizing power by the cycles of carbon and sulphur during the same interval (Canfield 2004), requires a continuing sink. It seems inescapable that this is supplied by oxidation of $\mathrm{Fe}^{2+}$ at the seafloor.

Bounds can be placed on the extent of this transfer over Earth history, since iron returning to the mantle with a higher $\mathrm{Fe}^{3+} / \Sigma \mathrm{Fe}$ than it emerged with will result in oxidation of the upper mantle. There is a growing consensus that the redox state of the upper mantle, as measured by its oxygen fugacity, has not changed greatly over Earth history. The recent constraint of $\mathrm{Li} \&$ Lee (2004), from the V/Sc systematics of MORBs, indicates that the oxygen fugacity of the mantle source has increased by at most $0.3 \log$ unit since $3.5 \mathrm{Ga}$. Using the relation of Kress \& Carmichael (1991), this translates into a maximal $6 \%$ increase in the ferric iron content of the mantle, if all of that increase were attributable to addition of $\mathrm{Fe}^{3+}$. Taking the mass of the upper mantle to be $1.34 \times 10^{27} \mathrm{~g}$ (Ballentine et al. 2002), the mantle $\mathrm{Fe}$ content to be $6.3 \mathrm{wt} \%$ (Palme \& O'Neill 2003) and the mantle $\mathrm{Fe}^{3+} / \Sigma \mathrm{Fe}$ to be 0.12 (Bezos \& Humler 2005), this yields a maximal input of $1.1 \times 10^{22} \mathrm{~mol}$ of ferric iron since $3.5 \mathrm{Ga}$, or a maximum average input rate of $3 \mathrm{Tmol} \mathrm{Fe}^{3+} \mathrm{yr}^{-1}$ ( $0.75 \mathrm{Tmol} \mathrm{O}_{2}$ equiv.). This is comfortably within potential loads imposed by $A_{\mathrm{Ox}}$.

The agreement between the projected release of oxidizing power and the average estimate of oxidized products is far from comforting. Because considerable amounts of $\mathrm{Fe}^{3+}$ must have been exported to the mantle, the total release of oxidizing power by the carbon cycle should exceed considerably the inventory of $\mathrm{Fe}^{3+}$ remaining in the crust. Part of any shortfall can be accommodated by recalling that the present estimates are minima. Finite values of $J_{\text {od }}$ and a failure to reoxidize organic carbon in $J_{\mathrm{or}}$ will increase $A_{\mathrm{Ox}}$.

The reburial of organic carbon can be treated quantitatively. If a portion, $x$, of $J_{\text {or }}$ is simply reburied, two things will happen: (i) since it does not re-enter the exogenic system as $\mathrm{CO}_{2}$, that $\mathrm{C}$ will be unavailable for reduction. The organic burial flux and thus the overall production of oxidizing power will be decreased by an amount $f x \mathcal{F}_{\text {or }}$; (ii) the oxidizing power consumed by oxidation of $J_{\text {or }}$ will be reduced by an amount $x f_{\text {or }}$. The first term is a loss of oxidizing power, the second is a gain. The difference is $(1-f) \times \mathcal{f}_{\text {or. }}$. Unfortunately, this term cannot simply be added to equation (6.2). Although it would account for the carbon and redox balances in a single increment of time, the proper treatment of subsequent increments would require that the reburied material be followed, with appropriate adjustments being made to sedimentary and continental inventories. Even during the Phanerozoic, the presence of detrital coal and kerogen in marine sediments (Sackett et al. 1974) shows that $x$ is not zero. On the other hand, it is known that microorganisms in weathering profiles incorporate carbon even from refractory kerogens (Petsch et al. 2001) and that the great bulk of organic carbon in marine sediments is not recycled, detrital organic material. Under anaerobic conditions, however, reburial of organic carbon is likely to have been more important.

Can the carbon cycle operate at a point of redox neutrality, neither releasing nor consuming oxidants, even when isotopic compositions indicate significant rates of burial of organic carbon? The answer is in equation (6.3). Even if burial and recycling are balanced, redox neutrality still requires $J_{\mathrm{am}}=J_{\mathrm{ad}}+J_{\text {od }}$. In other words, inputs from the mantle are equal to outputs to the mantle. This is not a likely coincidence. Carbon flows into subduction zones not just from $J_{\mathrm{am}}$ but from multiple sources. If the portion of $J_{\mathrm{as}}+J_{\mathrm{os}}+$ $J_{\mathrm{az}}$ equal to $J_{\mathrm{am}}$ is to be returned to the mantle while the balance flows to $J_{\text {av }}$ and $J_{\text {ov }}$, a very clever demon will be required to operate the carbon valve in the subduction zone. Notably, $f$ (and thus $\delta_{\mathrm{ab}}-\delta_{\mathrm{i}}$ ) is not a measure of 
$J_{\mathrm{am}}-\left(J_{\mathrm{ad}}+J_{\mathrm{od}}\right)$. No particular value of $\delta_{\mathrm{ab}}$ can be recognized as indicating redox neutrality. This yields two fundamental insights. First, caution is required when interpreting the isotopic record in terms of redox variations. Second, the conditions required for redox neutrality are precise and improbable.

\section{CONCLUDING REMARKS}

We have taken a new approach to mass and redox balances involving the $\mathrm{C}$ cycle. Its principal strength is a realistic acknowledgement of the importance of inputs from and outputs to the mantle. This broader context has invited consideration of processes that may otherwise be overlooked or misconstrued. Its weakness is that our knowledge of some of the key processes is presently imprecise. We need to learn more about inputs of carbon, hydrogen escape and processes in subduction zones to better evaluate each component of $A_{\text {Ox }}$. Even now, however, the resulting uncertainties do not cripple the approach. The key finding is dramatic: the persistence of $\delta_{\mathrm{ab}}$ at values near zero rather than $-5 \%$ can be interpreted only in terms of continuous and substantial releases of oxidizing power by the carbon cycle. Two points follow.

The oxidation of the crust has been more continuous than episodic. Increases in steady-state levels of oxidants derive either from combinations of geological and biological changes or from biological changes alone. The scientific problem of reconstructing Earth's oxidation is more biological than geochemical

Continuous sinks for oxidizing power are required, at least since atmospheric levels of $\mathrm{O}_{2}$ stabilized at current levels approximately $550 \mathrm{Ma}$. Since exchanges of oxidizing power between the carbon and sulphur cycles have been balanced during that same time interval, this can have been provided only by the oxidation of iron.

Together, these affect our view of the carbon cycle. It should not be seen as the competent and versatile manager of an electron-transfer market, effortlessly balancing offers and bids. Instead, it is a wrong-way passenger on a downgoing escalator. It continuously receives new $\mathrm{CO}_{2}$ from the mantle, relentless supplies of solar energy and nutrients leached from deposits that have retained their organic carbon. To hold its place, it must constantly produce new organic matter. While doing so, it releases oxidants that threaten to reverse its accomplishments. The stability of $\delta_{\mathrm{ab}}$ is an indicator of the success of its evolutionary strategies. The stability of $P_{\mathrm{O}_{2}}$ is evidence of an equally competent sink.

The seeds from which the ideas discussed have grown were planted when J.M.H. looked out of Alvin's viewports and observed the $\mathrm{H}_{2}$-rich waters of the Lost City hydrothermal vent system. The generosity of Deborah Kelley and Jeffrey Karson, the co-chief scientists of the Lost City cruise, is greatly appreciated. Since then, we have benefited from helpful and stimulating exchanges with Stan Hart, Don Anderson, David Rowley and Alberto Saal. We are very grateful for critical reviews of this manuscript provided by David Catling and Jennifer Eigenbrode. J.M.H. receives support as a member of the Astrobiology team led by S. D'Hondt, University of Rhode Island. J.R.W. is supported by an NDSEG Graduate Fellowship from the Office of Naval Research.

Phil. Trans. R. Soc. B (2006)

\section{REFERENCES}

Aharon, P. 2005 Redox stratification and anoxia of the early Precambrian oceans: implications for carbon isotope excursions and oxidation events. Precambrian Res. 137, 207-222.

Alt, J. C. \& Teagle, D. A. H. 1999 The uptake of carbon during alteration of ocean crust. Geochim. Cosmochim. Acta 63, 1527-1535. (doi:10.1016/S0016-7037(99) 00123-4)

Alt, J. C. \& Teagle, D. A. H. 2003 Hydrothermal alteration of upper oceanic crust formed at a fast-spreading ridge: mineral, chemical, and isotopic evidence from ODP Site 801. Chem. Geol. 201, 191-211. (doi:10.1016/S00092541(03)00201-8)

Arvidson, R. S., Mackenzie, F. T. \& Guidry, M. In press. MAGic: a Phanerozoic model for the geochemical cycling of major rock-forming components. Am. F. Sci.

Bach, W., Peucker-Ehrenbrink, B., Hart, S. R. \& Blusztajn, J. S. 2003 Geochemistry of hydrothermally altered oceanic crust: DSDP/ODP Hole 504B-implications for seawater-crust exchange budgets and $\mathrm{Sr}$ - and $\mathrm{Pb}$-isotopic evolution of the mantle. Geochem. Geophys. Geosyst. G3 4, 1-29.

Ballentine, C. J., van Keken, P. E., Porcelli, D. \& Houri, E. H. 2002 Numerical models, geochemistry and the zeroparadox noble-gas mantle. Phil. Trans. R. Soc. A 360, 2611-2631. (doi:10.1098/rsta.2002.1083)

Bekker, A., Karhu, J. A., Eriksson, K. A. \& Kaufman, A. J. 2003 Chemostratigraphy of Paleoproterozoic carbonate successions of the Wyoming Craton: tectonic forcing of biogeochemical change? Precambrian Res. 120, 279-325. (doi:10.1016/S0301-9268(02)00164-X)

Bekker, A., Holland, H. D., Wang, P.-L., Rumble III, D., Stein, H. J., Hannah, J. L., Coetzee, L. L. \& Beukes, N. J. 2004 Dating the rise of atmospheric oxygen. Nature 427, 117-120. (doi:10.1038/nature02260)

Berner, R. A. 1991 A model for atmospheric $\mathrm{CO}_{2}$ over Phanerozoic time. Am. F. Sci. 291, 339-376.

Berner, R. A. 2004 The Phanerozoic carbon cycle. New York, NY: Oxford University Press.

Berner, R. A. \& Maasch, K. A. 1996 Chemical weathering and controls on atmospheric $\mathrm{O}_{2}$ and $\mathrm{CO}_{2}$ : fundamental principles were enunciated by J. J. Ebelmen in 1845 . Geochim. Cosmochim. Acta 60, 1633-1637. (doi:10.1016/ 0016-7037(96)00104-4)

Bezos, A. \& Humler, E. $2005 \mathrm{The}^{3+} / \Sigma \mathrm{Fe}$ ratios of MORB glasses and their implications for mantle melting. Geochim. Cosmochim. Acta 69, 711-725. (doi:10.1016/j.gca.2004. 07.026)

Bjerrum, C. J. \& Canfield, D. E. 2004 New insights into the burial history of organic carbon on the early Earth. Geochem. Geophys. Geosyst. 5, 1-9. (doi:10.1029/2004 GC000713)

Bjørnerud, M. G. \& Austrheim, H. 2004 Inhibited eclogite formation: the key to the rapid growth of strong and Buoyant Archean continental crust. Geol. Soc. Am. 32, 765-768. (doi:10.1130/G20590.1)

Brocks, J. J., Buick, R., Logan, G. A. \& Summons, R. E. 2003 Composition and syngeneity of molecular fossils from the 2.78 to 2.45 billion-year-old Mount Bruce Supergroup, Pilbara Craton, Western Australia. Geochim. Cosmochim. Acta 67, 4289-4319. (doi:10.1016/S00167037(03)00208-4)

Broecker, W. S. 1970 A boundary condition on the evolution of atmospheric oxygen. F. Geophys. Res. 75, 3553-3557.

Buick, I. S., Uken, R., Gibson, R. L. \& Wallmach, T. 1998 High $\delta^{13} \mathrm{C}$ Paleoproterozoic carbonates from the transvaal Supergroup, South Africa. Geology 26, 875-878. (doi:10.1130/ 0091-7613(1998)026<0875:HCPCFT > 2.3.CO;2) 
Canfield, D. E. 1998 A new model for Proterozoic ocean chemistry. Nature 396, 450-453. (doi:10.1038/24839)

Canfield, D. E. 2004 The evolution of the earth surface sulfur reservoir. Am. F. Sci. 304, 839-861.

Canfield, D. E. 2005 The early history of atmospheric oxygen: homage to Robert M. Garrels. Annu. Rev. Earth Planet. Sci. 33, 1-36. (doi:10.1146/annurev.earth.33. 092203.122711)

Carmichael, I. S. E. 2002 The 'andesite aqueduct' perspectives on the evolution of intermediate magmatism in westcentral (105-99 ${ }^{\circ}$ W) Mexico. Contrib. Mineral. Petrol. 143, 641-663.

Catling, D. C. \& Claire, M. W. 2005 How Earth's atmosphere evolved to an oxic state: a status report. Earth Planet. Sci. Lett. 237, 1-20. (doi:10.1016/j.epsl.2005.06.013)

Catling, D. C., Zahnle, K. J. \& McKay, C. P. 2001 Biogenic methane, hydrogen escape, and the irreversible oxidation of the early earth. Science 293, 839-843. (doi:10.1126/ science.1061976)

Crisp, J. A. 1984 Rates of magma emplacement and volcanic output. f. Volcanol. Geotherm. Res. 20, 177-211. (doi:10. 1016/0377-0273(84)90039-8)

Dasgupta, R., Hirschmann, M. \& Withers, A. C. 2004 Deep global cycling of carbon constrained by the solidus of anhydrous, carbonated eclogite under upper mantle conditions. Earth Planet. Sci. Lett. 227, 73-85. (doi:10. 1016/j.eps1.2004.08.004)

Deines, P., Viljoen, F. \& Harris, J. W. 2001 Implications of the carbon isotope and mineral inclusion record for the formation of diamonds in the mantle underlying a mobile belt: Venetia, South Africa. Geochim. Cosmochim. Acta 65, 813-838. (doi:10.1016/S0016-7037(00)00569-X)

Derry, L. A., Kaufman, A. J. \& Jacobsen, S. B. 1992 Sedimentary cycling and environmental change in the Late Proterozoic: evidence from stable and radiogenic isotopes. Geochim. Cosmochim. Acta 56, 1317-1329. (doi:10.1016/0016-7037(92)90064-P)

Des Marais, D. J. 1985 Carbon exchange between the mantle and the crust, and its effect upon the atmosphere: today compared to Archean time. In The carbon cycle and atmospheric $\mathrm{CO}_{2}$ : natural variations Archean to present (ed. E. T. Sundquist \& W. S. Broecker), Geophysical Monograph 32, pp. 602-611. Washington, DC: American Geophysical Union.

Des Marais, D. J. 2001 Isotopic evolution of the biogeochemical carbon cycle during the Precambrian. Rev. Mineral. Geochem. 43, 555-578.

Dix, G. R., Thomson, M. L., Longstaffe, F. J. \& McNutt, R. H. 1995 Systematic decrease of high delta13C values with burial in late Archaean $(2.8 \mathrm{Ga})$ diagenetic dolomite: evidence for methanogenesis from the Crixas Greenstone Belt, Brazil. Precambrian Res. 70, 253-268. (doi:10.1016/ 0301-9268(94)00044-R)

Eigenbrode, J. 2004 Late archean microbial ecology: an integration of molecular, isotopic, and lithologic studies. $\mathrm{Ph} . \mathrm{D}$. thesis, Earth Sciences, the Pennsylvania State University.

Farquhar, J. \& Wing, B. A. 2003 Multiple sulfur isotopes and the evolution of the atmosphere. Earth Planet. Sci. Lett. 213, 1-13. (doi:10.1016/S0012-821X(03)00296-6)

Garrels, R. M. \& Lerman, A. 1981 Phanerozoic cycles of sedimentary carbon and sulfur. Geology 78, 4652-4655.

Garrels, R. M. \& Perry Jr, E. A. 1974 Cycling of carbon, sulfur, and oxygen through geologic time. Sea 5, 303-336.

Giggenbach, W. F. 1996 Chemical composition of volcanic gases. In Monitoring and mitigation of volcano hazards (ed. S. Scarpa \& R. I. Tilling), pp. 221-256. Berlin: Springer.

Goldschmidt, V. M. 1954 Geochemistry. Oxford: Clarendon Press.
Halmer, M. M., Schmincke, H.-U. \& Graf, H.-F. 2002 The annual volcanic gas input into the atmosphere, in particular into the stratosphere: a global data set for the past 100 years. F. Volcanol. Geotherm. Res. 115, 511-528. (doi:10.1016/S0377-0273(01)00318-3)

Halverson, G. P., Hoffman, P. F., Schrag, D. P., Maloof, A. C. \& Rice, A. H. H. 2005 Toward a Neoproterozoic composite carbon-isotope record. Geol. Soc. Am. 117, 1181-1207. (doi:10.1130/B25630.1)

Hayes, J. M. 1983 Geochemical evidence bearing on the origin of aerobiosis, a speculative interpretation. In The Earth's earliest biosphere: its origin and evolution (ed. J. W. Schopf), pp. 291-301. Princeton, NJ: Princeton University Press.

Hayes, J. M. 1994 Global methanotrophy at the Archean-Proterozoic transition. In Early life on Earth. Nobel Symposium (ed. S. Bengtson), pp. 220-236. New York, NY: Columbia University Press.

Hayes, J. M., Kaplan, I. R. \& Wedeking, K. W. 1983 Precambrian organic geochemistry, preservation of the record. In The Earth's earliest biosphere: its origin and evolution (ed. J. W. Schopf), pp. 93-134. Princeton, NJ: Princeton University Press.

Hayes, J. M., Lambert, I. B. \& Strauss, H. 1992 The sulfurisotopic record. In The Proterozoic biosphere, a multidisciplinary study (ed. J. W. Schopf \& C. Klein), pp. 129-132. Cambridge, UK: Cambridge University Press.

Hayes, J. M., Strauss, H. \& Kaufman, A. J. 1999 The abundance of ${ }^{13} \mathrm{C}$ in marine organic matter and isotopic fractionation in the global biogeochemical cycle of carbon during the past $800 \mathrm{Ma}$. Chem. Geol. 161, 103-125. (doi:10.1016/S0009-2541(99)00083-2)

Hilton, D. R., Fischer, T. \& Marty, B. 2002 Noble gases and volatile recycling at subduction zones. MSA Special Volume: Noble Gasses 47, 319-370.

Hofmann, A. W. 2004 Sampling mantle heterogeneity through oceanic basalts: isotopes and trace elements. In Treatise on geochemistry, vol. 2 (ed. H. D. Holland \& K. K. Turekian), pp. 61-101. Oxford: Elsevier.

Holland, H. D. 1978 The chemistry of the atmosphere and oceans. New York, NY: Wiley.

Holland, H. D. 1984 The chemical evolution of the atmosphere and oceans. Princeton, NJ: Princeton University Press.

Holland, H. D. 2002 Volcanic gases, black smokers, and the great oxidation event. Geochim. Cosmochim. Acta 66, 3811-3826. (doi:10.1016/S0016-7037(02)00950-X)

Holser, W. T., Schidlowski, M., MacKenzie, F. T. \& Maynard, J. B. 1988 Geochemical cycles of carbon and sulfur. In Chemical cycles in the evolution of the Earth (ed. C. B. Gregor, R. M. Garrels, F. T. Mackenzie \& J. B. Maynard), pp. 105-173. New York, NY: Wiley.

Hunt, J. M. 1996 Petroleum geochemistry and geology. New York, NY: Freeman.

Ingebritsen, S. E. \& Manning, C. E. 2002 Diffuse fluid flux through orogenic belts: implications for the world ocean. Proc. Natl Acad. Sci. USA 99, 9113-9116. (doi:10.1073/ pnas.132275699)

Irwin, H., Curtis, C. D. \& Coleman, M. L. 1977 Isotopic evidence for source of diagenetic carbonates formed during burial of organic rich sediments. Nature 269, 209-213. (doi:10.1038/269209a0)

Jiao, Y., Kappler, A., Croal, L. R. \& Newman, D. K. 2005 Isolation and characterization of a genetically tractable photoautotrophic $\mathrm{Fe}(\mathrm{II})$-oxidizing bacterium, Rhodopseudomonas palustris strain TIE-1. Appl. Environ. Microbiol. 71, 4487-4496. (doi:10.1128/AEM.71.8.4487-4496.2005)

Kadko, D. 1994 An assessment of the effect of chemical scavenging within hydrothermal plumes upon ocean

Phil. Trans. R. Soc. B (2006) 
geochemistry. Earth Planet. Sci. Lett. 120, 361-374. (doi:10.1016/0012-821X(93)90250-D)

Karhu, J. A. \& Holland, H. D. 1996 Carbon isotopes and the rise of atmospheric oxygen. Geology 24, 867-870. (doi:10. 1130/0091-7613(1996)024<0867:CIATRO > 2.3.CO;2)

Kasting, J. F. \& Catling, D. 2003 Evolution of a habitable planet. Annu. Rev. Astron. Astrophys. 41, 429-463. (doi:10.1146/annurev.astro.41.071601.170049)

Keeling, R. F., Najjar, R. P., Bender, M. L. \& Tans, P. P. 1993 What atmospheric oxygen measurements can tell us about the global carbon cycle. Global Biogeochemical Cycles 7, 36-67.

Kerrick, D. M. \& Connolly, J. A. D. 2001 Metamorphic devolatilization of subducted oceanic metabasalts: implications for seismicity, arc magmatism and volatile recycling. Earth Planet. Sci. Lett. 189, 19-29. (doi:10. 1016/S0012-821X(01)00347-8)

Klein, J. S., Mozley, P., Campbell, A. \& Cole, R. 1999 Spatial distribution of carbon and oxygen isotopes in laterally extensive carbonate-cemented layers: implications for mode of growth and subsurface identification. F. Sediment. Res. 69, 184-191.

Knoll, A. H., Hayes, J. M., Kaufman, A. J., Swett, K. \& Lambert, I. B. 1986 Secular variation in carbon isotope ratios from Upper Proterozoic successions of Svalbard and East Greenland. Nature 321, 832-838. (doi:10.1038/ 321832a0)

Kopp, R. E., Kirschvink, J. L., Hilburn, I. A. \& Nash, C. Z. 2005 The Paleoproterozoic snowball Earth: a climate disaster triggered by the evolution of oxygenic photosynthesis. Proc. Natl Acad. Sci. USA 102, 11 131-11 136. (doi:10.1073/pnas.0504878102)

Kress, V. C. \& Carmichael, I. S. E. 1991 The compressibility of silicate liquids containing $\mathrm{Fe}_{2} \mathrm{O}_{3}$ and the effect of composition, temperature, oxygen fugacity and pressure on their redox states. Contrib. Mineral. Petrol. 108, 82-92. (doi:10.1007/BF00307328)

Kump, L. R. \& Arthur, M. A. 1999 Interpreting carbon-isotope excursions: carbonates and organic matter. Chem. Geol. 161, 181-198. (doi:10.1016/S0009-2541(99)00086-8)

Kyser, T. K. 1986 Stable isotope variations in the mantle. Stable isotopes in high temperature geological processes. Rev. Mineral. 16, 141-164.

Lécuyer, C. \& Ricard, Y. 1999 Long-term fluxes and budget of ferric iron: implication for the redox states of the Earth's mantle and atmosphere. Earth Planet. Sci. Lett. 165, 197-211. (doi:10.1016/S0012-821X(98)00267-2)

Li, Z.-X. A. \& Lee, C.-T. A. 2004 The constancy of upper mantle $\mathrm{fO}_{2}$ through time inferred from $\mathrm{V} / \mathrm{Sc}$ ratios in basalts. Earth Planet. Sci. Lett. 228, 483-493. (doi:10. 1016/j.epsl.2004.10.006)

Lowe, D. R. 1992 Major events in the geological development of the Precambrian Earth. In The Proterozoic biosphere (ed. J. W. Schopf \& C. Klein), pp. 67-76. Cambridge, UK: Cambridge University Press.

Lowell, R. P. \& Keller, S. M. 2003 High-temperature seafloor hydrothermal circulation over geologic time and Archean banded iron formations. Geophys. Res. Lett. 30, 1-44. (doi:10.1029/2002GL016536)

Luth, R. W. 2004 Mantle volatiles-distribution and consequences. In Treatise on geochemistry, vol. 2 (ed. H. D. Holland \& K. K. Turekian), pp. 319-361. Oxford: Elsevier.

Marty, B. \& Tolstikhin, I. N. $1998 \mathrm{CO}_{2}$ fluxes from midocean ridges, arcs and plumes. Chem. Geol. 145, 233-248. (doi:10.1016/S0009-2541(97)00145-9)

Mattey, D. P. 1987 Carbon isotopes in the mantle. Terra Cognita 7, 31-37.

Mazzullo, S. J. 2000 Organogenic dolomitization in peritidal to deep-sea sediments. F. Sediment. Res. 70, 10-23.

Phil. Trans. R. Soc. B (2006)
Melezhik, V. A., Fallick, A. E., Medvedev, P. V. \& Makarikhin, V. V. 1999 Extreme ${ }^{13} \mathrm{C}_{\text {cab }}$ enrichment in ca. 2.0 Ga magnesite-stromatolite-dolomite-'red beds' association in a global context: a case for the world-wide signal enhanced by a local environment. Earth Sci. Rev. 48, 71-120. (doi:10.1016/S0012-8252(99)00044-6)

Milkov, A. V. 2005 Molecular and stable isotope compositions of natural gas hydrates: a revised global dataset and basic interpretations in the context of geological settings. Org. Geochem. 36, 681-702. (doi:10.1016/j.orggeochem. 2005.01.010)

Milkov, A. V. \& Etiope, G. 2005 Global methane emission through mud volcanoes and its past and present impact on the Earth's climate- a comment. Int. F. Earth Sci. (Geol. Rundsch.) 94, 490-492. (doi:10.1007/s00531-005-0480-5)

Nakamura, K. \& Kato, Y. 2004 Carbonatization of oceanic crust by the seafloor hydrothermal activity and its significance as a $\mathrm{CO}_{2}$ sink in the Early Archean. Geochim. Cosmochim. Acta 68, 4595-4618. (doi:10.1016/j.gca.2004. 05.023)

Palme, H. \& O’Neill, H. S. C. 2003 Cosmochemical estimates of mantle composition. In Treatise on geochemistry, vol. 2 (ed. H. D. Holland \& K. K. Turekian), pp. 1-38. Oxford: Elsevier.

Pavlov, A. A. \& Kasting, J. F. 2002 Mass-independent fractionation of sulfur isotopes in Archean sediments: strong evidence for an anoxic Archean atmosphere. Astrobiology 2, 27-41. (doi:10.1089/1531107027536 21321)

Pearson, D. G., Canil, D. \& Shirey, S. B. 2004 Mantle samples included in volcanic rocks: xenoliths and diamonds. In Treatise on geochemistry, vol. 2 (ed. H. D. Holland \& K. K. Turekian), pp. 172-221. Oxford: Elsevier.

Petsch, S. T., Eglinton, T. I. \& Edwards, K. J. $2001{ }^{14}$ C-Dead living biomass: evidence for microbial assimilation of ancient organic carbon during shale weathering. Science 292, 1127-1131. (doi:10.1126/science.1058332)

Pineau, F., Cartigny, P. \& Javoy, M. 2004 Carbon flux and $\mathrm{C} / \mathrm{Nb}$ ratios in the mantle in ridge context, Eos Trans. $A G U$, vol. 85, Fall Meet. Suppl., Abstract V31C-1453P.

Plank, T. \& Langmuir, C. H. 1998 The chemical composition of subducting sediment and its consequences for the crust and mantle. Chem. Geol. 145, 325-394. (doi:10.1016/ S0009-2541(97)00150-2)

Quay, P., Sonnerup, P., Westby, T., Stutsman, J. \& McNichol, A. 2003 Changes in the ${ }^{13} \mathrm{C} /{ }^{12} \mathrm{C}$ of dissolved inorganic carbon in the ocean as a tracer of anthropogenic $\mathrm{CO}_{2}$ uptake. Global Biogeochem. Cycles 17, 1-4. (doi:10.1029/ 2001GB001817)

Resing, J. A., Lupton, J. E., Feely, R. A. \& Lilley, M. D. 2004 $\mathrm{CO}_{2}$ and ${ }^{3} \mathrm{He}$ in hydrothermal plumes: implications for mid-ocean ridge $\mathrm{CO}_{2}$ flux. Earth Planet. Sci. Lett. 226, 449-464. (doi:10.1016/j.epsl.2004.07.028)

Ronov, A. B. \& Yaroshevsky, A. A. 1976 A new model for the chemical structure of Earth's crust. Geochem. Int. 13, 89-121.

Rothman, D. H., Hayes, J. M. \& Summons, R. E. 2003 Dynamics of the Neoproterozoic carbon cycle. Proc. Natl Acad. Sci. USA 100, 8124-8129. (doi:10.1073/pnas. 0832439100)

Rowley, D. B. 2002 Rate of plate creation and destruction: $180 \mathrm{Ma}$ to present. Geol. Soc. Am. Bull. 114, 927-933. (doi:10.1130/0016-7606(2002)114<0927:ROPCAD > 2.0. $\mathrm{CO} ; 2)$

Saal, A. E., Hauri, E. H., Langmuir, C. H. \& Perfit, M. R. 2002 Vapour undersaturation in primitive mid-oceanridge basalt and the volatile content of Earth's upper mantle. Nature 419, 451-446. (doi:10.1038/nature01073) 
Sackett, W. M., Poag, C. W. \& Eadie, B. J. 1974 Kerogen recycling in the Ross Sea, Antarctica. Science 185, 1045-1047.

Sano, Y. \& Marty, B. 1995 Origin of carbon in fumarolic gas from island arcs. Chem. Geol. 119, 265-274. (doi:10.1016/ 0009-2541(94)00097-R)

Sano, Y. \& Williams, S. N. 1996 Fluxes of mantle and subducted carbon along convergent plate boundaries. Geophys. Res. Lett. 23, 2749-2752. (doi:10.1029/96GL02260)

Schidlowski, M., Eichmann, R. \& Junge, C. E. 1975 Precambrian sedimentary carbonates: carbon and oxygen isotope geochemistry and implications for the terrestrial oxygen budget. Precambrian Res. 2, 1-69. (doi:10.1016/ 0301-9268(75)90018-2)

Shackleton, N. J. 1987 The carbon isotope record of the Cenozoic: history of organic carbon burial and of oxygen in the ocean and atmosphere. In Marine petroleum source rocks (ed. J. Brooks \& A. J. Fleet). Geological Society Special Publication No. 26, pp. 423-434. Oxford: Blackwell.

Shaw, A. M., Hilton, D. R., Fischer, T. P., Walker, J. A. \& Alvarado, G. E. 2003 Contrasting $\mathrm{He}-\mathrm{C}$ relationships in Nicaragua and Costa Rica: insights into $\mathrm{C}$ cycling through subduction zones. Earth Planet. Sci. Lett. 214, 499-513. (doi:10.1016/S0012-821X(03)00401-1)

Shaw, A. M., Hilton, D. R., Macpherson, C. G. \& Sinton, J. M. 2004 The $\mathrm{CO}_{2}-\mathrm{He}-\mathrm{Ar}-\mathrm{H}_{2} \mathrm{O}$ systematics of the Manus back-arc basin: resolving source composition from degassing and contamination effects. Geochim. Cosmochim. Acta 68, 1837-1856. (doi:10.1016/j.gca.2003.10.015)

Shields, G. \& Veizer, J. 2002 Precambrian marine carbonate isotope database: version 1.1. Geochem. Geophys. Geosyst. G3 3, 1-12.

Sleep, N. H. \& Zahnle, K. 2001 Carbon dioxide cycling and implications for climate. F. Geophys. Res. 106, 1373-1399. (doi:10.1029/2000JE001247)

Sleep, N. H., Meibom, A., Fridriksson, T., Coleman, R. G. \& Bird, D. K. $2004 \mathrm{H}_{2}$-rich fluids from serpentinization: geochemical and biotic implications. Proc. Natl Acad. Sci USA 101, 12 818-12 823. (doi:10.1073/pnas.0405289101)

Staudigel, H., Hart, S. R., Schmincke, H.-U. \& Smith, B. M. 1989 Cretaceous ocean crust at DSDP Sites 417 and 418: carbon uptake from weathering versus loss by magmatic outgassing. Geochim. Cosmochim. Acta 53, 3091-3094. (doi:10.1016/0016-7037(89)90189-0)

Stern, R. J. 2005 Evidence from ophiolites, blueschists, and ultrahigh-pressure metamorphic terranes that the modern episode of subduction tectonics began in Neoproterozoic time. Geol. Soc. Am. 33, 557-560. (doi:10.1130/G21365.1)

Strauss, H. \& Moore, T. B. 1992 Abundances and isotopic compositions of carbon and sulfur species in whole rock and kerogen samples. In The Proterozoic biosphere (ed. J. W. Schopf \& C. Klein), pp. 709-798. Cambridge, UK: Cambridge University Press.

Su, Y., \& Langmuir, C.H. 2003 Global MORB chemistry compilation at the segment scale. MS thesis, Department of Earth and Environmental Sciences, Columbia University, Database available at http://www.petdb.org.
Summons, R. E., Jahnke, L. L., Hope, J. M. \& Logan, G. A 1999 2-Methylhopanoids as biomarkers for cyanobacterial oxygenic photosynthesis. Nature 400, 554-557. (doi:10. $1038 / 23005$ )

Tajika, E. 2003 Faint young sun and the carbon cycle: implications for the Proterozoic global glaciations. Earth Planet. Sci. Lett. 214, 443-453. (doi:10.1016/S0012821X(03)00396-0)

Tappert, R., Stachel, T., Harris, J. W., Muehlenbachs, K., Lugwig, T. \& Grey, G. P. 2005 Subducting oceanic crust: the source of deep diamonds. Geol. Soc. Am. Bull. 33, 565-568.

Tian, F., Toon, O. B., Pavlov, A. A. \& De Sterck, H. 2005 A hydrogen-rich early earth atmosphere. Science 308, 1014-1017. (doi:10.1126/science.1106983)

Tice, M. M. \& Lowe, D. R. 2004 Photosynthetic microbial mats in the 3416-Myr-old ocean. Nature 431, 549-552. (doi:10.1038/nature02888)

Van Kranendonk, M. 2004 Archean tectonics 2004: a review. Precambrian Res. 131, 143-151. (doi:10.1016/j.precamres. 2003.12.008)

Veizer, J. \& Mackenzie, F. T. 2004 Evolution of sedimentary rocks. In Treatise on geochemistry, vol. 7 (ed. H. D. Holland \& K. K. Turekian), pp. 369-407. Oxford: Elsevier.

Veizer, J. et al. $1999{ }^{87} \mathrm{Sr} /{ }^{86} \mathrm{Sr}$, and $\delta^{18} \mathrm{O}$ evolution of Phanerozoic seawater. Chem. Geol. 161, 59-88. (doi:10. 1016/S0009-2541(99)00081-9)

Walker, J. C. G. 1987 Was the Archean biosphere upside down? Nature 329, 710-712. (doi:10.1038/329710a0)

Walker, J. C. G. 1990 Precambrian evolution of the climate system. Palaeogeogr. Palaeoclimatol. Palaeoecol. 82, 261-289.

Wallace, P. J. 2005 Volatiles in subduction zone magmas: concentrations and fluxes based on melt inclusion and volcanic gas data. F. Volcanol. Geotherm. Res. 140, 217-240. (doi:10.1016/j.jvolgeores.2004.07.023)

Wedepohl, K. H. 1995 The composition of the continental crust. Geochim. Cosmochim. Acta 59, 1217-1232. (doi:10. 1016/0016-7037(95)00038-2)

White, R. S., McKenzie, D. P. \& O'Nions, R. K. 1992 Oceanic crustal thickness from seismic measurements and rare earth element inversions. F. Geophys. Res. 97, 683-714.

Wilkinson, B. H. \& Walker, J. C. G. 1989 Phanerozoic cycling of sedimentary carbonate. Am. F. Sci. 289, 525-548.

Young, G. M., Long, D. G. F., Fedo, C. M. \& Nesbitt, H. W. 2001 Paleoproterozoic Huronian basin: product of a Wilson cycle punctuated by glaciations and a meteorite impact. Sediment. Geol. 141-142, 233-254. (doi:10.1016/ S0037-0738(01)00076-8)

Yudovich, Y. E., Makarikhin, V. V., Medvedev, P. V. \& Sukhanov, N. V. 1991 Carbon isotope anomalies in carbonates of the Karelian Complex. Geochem. Int. 28, 56-62.

Zhang, Q.-L. \& Li, W.-J. 1990 A calibrated measurement of the atomic weight of carbon. Chinese Sci. Bull. 35, 290-296. 


\title{
Appendix F
}

\section{Steroids, triterpenoids and molecular oxygen}

\author{
Roger E. Summons, Alexander S. Bradley, Linda L. Jahnke and \\ Jacob R. Waldbauer
}

Reprinted with permission from Philosophical Transactions of the Royal Society $B$ (C) 2006 The authors

Summons, R.E., Bradley, A.S., Jahnke, L.L. and Waldbauer, J.R. (2006) Steroids, triterpenoids and molecular oxygen. Philosophical Transactions of the Royal Society B 361: $951-968$. 


\title{
Steroids, triterpenoids and molecular oxygen
}

\author{
Roger E. Summons ${ }^{1, *}$, Alexander S. Bradley ${ }^{1}$, Linda L. Jahnke ${ }^{2}$ \\ and Jacob R. Waldbauer ${ }^{3}$ \\ ${ }^{1}$ Department of Earth, Atmospheric and Planetary Sciences, Massachusetts Institute of Technology, \\ 77 Massachusetts Avenue E34-246, Cambridge, MA 02139-4307, USA \\ ${ }^{2}$ Planetary Biology Branch, NASA Ames Research Center, Moffett Field, CA 94035, USA \\ ${ }^{3}$ Joint Program in Chemical Oceanography, Woods Hole Oceanographic Institution and MIT, \\ Cambridge, MA 02139, USA
}

There is a close connection between modern-day biosynthesis of particular triterpenoid biomarkers and presence of molecular oxygen in the environment. Thus, the detection of steroid and triterpenoid hydrocarbons far back in Earth history has been used to infer the antiquity of oxygenic photosynthesis. This prompts the question: were these compounds produced similarly in the past? In this paper, we address this question with a review of the current state of knowledge surrounding the oxygen requirement for steroid biosynthesis and phylogenetic patterns in the distribution of steroid and triterpenoid biosynthetic pathways.

The hopanoid and steroid biosynthetic pathways are very highly conserved within the bacterial and eukaryotic domains, respectively. Bacteriohopanepolyols are produced by a wide range of bacteria, and are methylated in significant abundance at the $\mathrm{C} 2$ position by oxygen-producing cyanobacteria. On the other hand, sterol biosynthesis is sparsely distributed in distantly related bacterial taxa and the pathways do not produce the wide range of products that characterize eukaryotes. In particular, evidence for sterol biosynthesis by cyanobacteria appears flawed. Our experiments show that cyanobacterial cultures are easily contaminated by sterol-producing rust fungi, which can be eliminated by treatment with cycloheximide affording sterol-free samples.

Sterols are ubiquitous features of eukaryotic membranes, and it appears likely that the initial steps in sterol biosynthesis were present in their modern form in the last common ancestor of eukaryotes. Eleven molecules of $\mathrm{O}_{2}$ are required by four enzymes to produce one molecule of cholesterol. Thermodynamic arguments, optimization of function and parsimony all indicate that an ancestral anaerobic pathway is highly unlikely.

The known geological record of molecular fossils, especially steranes and triterpanes, is notable for the limited number of structural motifs that have been observed. With a few exceptions, the carbon skeletons are the same as those found in the lipids of extant organisms and no demonstrably extinct structures have been reported. Furthermore, their patterns of occurrence over billion year time-scales correlate strongly with environments of deposition. Accordingly, biomarkers are excellent indicators of environmental conditions even though the taxonomic affinities of all biomarkers cannot be precisely specified. Biomarkers are ultimately tied to biochemicals with very specific functional properties, and interpretations of the biomarker record will benefit from increased understanding of the biological roles of geologically durable molecules.

Keywords: Archaean; biomarker hydrocarbons; steroids; sterols; triterpenoids; hopanes aerobic biosynthesis

\section{INTRODUCTION}

(a) The advent of oxygenic photosynthesis and an oxygen-rich atmosphere

The compositional and evolutionary history of the atmosphere-ocean system may be reconstructed from the chemistry and habit of sedimentary minerals, basalt weathering profiles and secular change in stable isotopes, along with numerical modelling and theoretical considerations (Cloud 1972). According to the paradigm of Cloud, Holland and Walker, oxygen was a

* Author for correspondence (rsummons@mit.edu).

One contribution of 14 to a Discussion Meeting Issue 'Major steps in cell evolution'. trace component of the early atmosphere and rose, within weakly constrained bounds, to within one-tenth of the present atmospheric level (PAL) by about 540 Myr ago (Ma) (Cloud 1972; Walker et al. 1983). It is further hypothesized that oxygen played a role in the deposition of Archaean and Palaeoproterozoic banded iron formations, and that the fluxes of reduced minerals and volcanic gases into the ocean and atmosphere acted as a buffer to keep atmospheric oxygen concentrations low for a protracted period. An apparently 'sudden' development of oxidized soil profiles about $2300 \mathrm{Ma}$, together with carbon, sulphur and iron isotopic indicators, suggest that the oxygen rise was not uniform but occurred in a stepwise manner (Holland 1984; Holland \& Beukes 1990; Des Marais et al. 1992; 
Karhu \& Holland 1996; Rasmussen \& Buick 1999; Des Marais 2000; Farquhar et al. 2000a,b; Holland 2002; Rouxel et al. 2005). The 'step' which occurred just prior to $2300 \mathrm{Ma}$ was to greater than $10^{-5} \mathrm{PAL}$ and accompanied by dramatic environmental changes indicated by large excursions in the $\delta^{13} \mathrm{C}$ content of marine carbonates, and possible 'snowball Earth' events (Kirschvink et al. 2000; Bekker et al. 2004). A second very significant rise in the Late Neoproterozoic also probably took place in stages punctuated by multiple ice ages with dramatic swings in carbon and sulphur isotopes indicating a radical reorganization of the biogeochemical cycles (Hoffman \& Schrag 2002; Rothman et al. 2003; Halverson et al. 2005; Hurtgen et al. 2005).

\section{(b) Isotopic and molecular evidence pertaining to timing}

Carbon and sulphur isotopes, molecular biomarkers, and possibly iron isotopes suggest that the advent of oxygenic photosynthesis preceded the Early Palaeoproterozoic 'Great Oxidation Event' by $400 \mathrm{Myr}$ or more. Certain 2-methylhopanoid biomarkers that we associate with modern-day oxygen-producing cyanobacteria and steroids that require oxygen-utilizing enzymes for their biosynthesis can be found in rocks as old as $2715 \mathrm{Myr}$. This is consistent with the notion that oxygen production from oxygenic photosynthesis is indeed an ancient process. The inventory of organic carbon preserved in Middle to Late Archaean black shales and carbonates, indicating prolific primary productivity in diverse palaeoenvironments, is another signal that would be consistent with an early appearance of oxygenic photosynthesis since electron donors other than water might have been limiting in supply and/or spatial distribution in an anaerobic surface ocean (Walker et al. 1983; Des Marais 2000; Rosing \& Frei 2004). Molecular hydrogen, a feasible alternative electron donor produced by sub-sea basalt weathering, was likely abundant in the deep ocean, available for carbon fixation and possibly for phototrophic carbon fixation (Sleep et al. 2004; Tice \& Lowe 2004; Hayes \& Waldbauer 2006). Sulphur isotopic data indicate that sulphate reducing bacteria were active by $2.7 \mathrm{Gyr}$ ago (Ga) which, indirectly, suggest that oxygenic photosynthesis was extant (Shen et al. 2001; Rouxel et al. 2005).

Analyses of hydrocarbons from Fortescue and Hamersley group sediments of the Pilbara Craton, western Australia (Brocks et al. 1999; Summons et al. 1999; Eigenbrode et al. 2001, 2004; Eigenbrode 2004) have uncovered many of the same kinds of carbon skeletons that are prevalent in Phanerozoic sediments and petroleum (Peters et al. 2004). Of particular interest are the steroid and triterpenoid carbon skeletons that are apparently derived from sterols and bacteriohopanepolyols (BHP), respectively. The presence of these types of hydrocarbons in Late Archaean sediments, which are considered by many researchers to be diagnostic for Eukarya and Bacteria, has been further used as an evidence for the antiquity of both of these domains (Brocks et al. 1999).

\section{(c) Hopanoid biomarkers}

In bacteria, the functional forms of hopanoids are amphiphilic BHP. These comprise a $\mathrm{C}_{30}$ pentacyclic triterpene hydrocarbon skeleton, derived from squalene via the enzyme squalene-hopene cyclase, and are linked via a $\mathrm{C}-\mathrm{C}$ bond to a $\mathrm{C}_{5}$ sugar moiety derived from ribose. The polar moieties of BHP may be augmented with sugars, amino acids or other functionalized units. These substituents on BHP evidently serve a functional role and also provide a chemical mechanism for their preservation in the geological record. They facilitate intermolecular condensation reactions, and cross-linking by reduced sulphur compounds, that result in incorporation into kerogen (Wakeham et al. 1995). The apolar ring system of hopanoids may be modified by unsaturation or by addition of an extra methyl group at either position 2 or position 3 located in the A-ring. Although oxygen is not required for hopanoid biosynthesis, the vast majority of known hopanoid producers are aerobic or microaerophilic bacteria (Rohmer et al. 1984). These include the cyanobacteria and $\alpha$ - and $\beta$-proteobacteria. Notable exceptions include Geobacter sp. (Fischer et al. 2005) although, logically, there may be many more obligate or facultative anaerobes which make BHP, given that surveys of bacterial taxa have been limited. Recent studies have provided molecular isotopic evidence for biosynthesis of hopanoids in anaerobic environments (Thiel et al. 1999, 2003; Pancost et al. 2000).

Cyanobacteria are presently the only known bacteria to synthesize abundant 2-methylhopanoids with an extended polyhydroxylated side chain (i.e. 2Me-BHP) (Rohmer et al. 1984; Summons et al. 1999). Labelling experiments demonstrate that the 2-methyl substituent originates from L-methionine, presumably via $S$-adenosylmethionine, with preservation of all three hydrogens and consistent with methylation of a $\Delta^{2}$-hopanoid (Zundel \& Rohmer 1985). Other details of the biosynthesis and the specific functions of $2 \mathrm{Me}-\mathrm{BHP}$ remain to be elucidated. Cyanobacteria containing $2 \mathrm{Me}-\mathrm{BHP}$ are distributed broadly throughout cyanobacterial phylogeny including Gloeobacter violaceus (a deeply branching cyanobacterium lacking thylakoids) and the $\mathrm{N}_{2}$-fixing Nostoc spp. (a heterocystous filament-former). While marine cyanobacteria were poorly represented in the initial survey (Summons et al. 1999), more recent work does not suggest that $2 \mathrm{Me}-\mathrm{BHP}$ are widely represented in those marine cyanobacteria that have been brought into culture.

\section{(d) Steroid biomarkers}

Sterols are derived from the same squalene precursor as hopanoids but, in marked contrast to BHP, are known to have an oxygen-dependent biosynthesis beginning with the formation of the first intermediate, 2,3oxidosqualene. Enzymes involved in sterol biosynthesis are highly specific in their substrate requirements and the mechanism by which oxidosqualene cyclase (OSC) converts 2,3-oxidosqualene to either of two protosterols, lanosterol or cycloartenol, depends on precise control of multiple intermediates along the cyclization cascade (Abe et al. 1993; Wendt et al. 1997, 2000). The unravelling of the intimate details of these exquisitely 
stereospecific reactions, based on more than 50 years concerted research since the process was postulated (Woodward \& Bloch 1953), is considered one of the classic accomplishments of molecular science.

Sterols are characteristic of all Eukarya. They are not found in Archaea and the proven occurrences in Bacteria are sparsely distributed and yield a limited array of products. Methylococcus capsulatus, Gemmata obscuriglobus and some members of the myxobacteria are proven steroid-producing bacteria (Bird et al. 1971; Kohl et al. 1983; Bode et al. 2003; Pearson et al. 2003; Volkman 2003, 2005).

There are at least 11 original studies and numerous reviews citing sterol occurrence in cyanobacteria. Prominent among these, a crystalline mixture of sterols was isolated from a culture of Phormidium luridum and identified by gas chromatography-mass spectrometry (GC-MS) to contain $\mathrm{C}_{29} \Delta 7, \mathrm{C}_{29} \Delta 5, \mathrm{C}_{29} \Delta 7,22$, $\mathrm{C}_{29} \Delta 5,7,22$ and $\mathrm{C}_{29} \Delta 5,22$ with minor amounts of cholesterol (DeSousa \& Nes 1968). When it was conducted, this work stood in marked contrast to earlier studies asserting the absence of sterols from cyanobacteria (Levin \& Bloch 1964) while other, more recent studies have failed to detect them (Rohmer et al. 1979, 1984).

The reports of cyanobacterial sterols apply to a taxonomically diverse range of cultured organisms including Spirulina platensis, Nostoc sp. and Calothrix sp. with $\mathrm{C}_{29} \Delta 5, \mathrm{C}_{29} \Delta 7, \mathrm{C}_{29} \Delta 5,22$ (Paoletti et al. 1976), Anabaena sp. (x3), Nodularia sp. and Nostoc sp. with $\mathrm{C}_{29} \Delta 5, \mathrm{C}_{29} \Delta 7, \mathrm{C}_{29} \Delta 5,22, \mathrm{C}_{29} \Delta 5,7,22, \mathrm{C}_{29: 0}$ and cholesterol (Kohlhase \& Pohl 1988), and Anabaena hallensis with $\mathrm{C}_{29} \Delta 5, \mathrm{C}_{28} \Delta 5, \mathrm{C}_{29: 0}$ (Hai et al. 1996). Lanosterol has been identified in Chlorogloeopsis fritschii (Sallal et al. 1987) and there are numerous reports of sterols in natural samples such as cyanobacterial mats.

Given the variety of organisms investigated, and apart from the $C$. fritschii results, there is a striking similarity in the sterols identified as well as their relative abundances. It has been noted that the strong predominance of unsaturated $\mathrm{C}_{29}$ sterols-phytosterols-is similar to that found in green algae and vascular plants. Moreover, of the more than a dozen cyanobacterial genomes completed to date (ranging from Gloeobacter to Nostoc) none contains genes encoding sterol synthesis enzymes.

\section{(e) Alternative views about oxygen and} biomarkers

An alternate hypothesis concerning the history of environmental oxidation argues for a relatively late (just prior to $2.3 \mathrm{Ga}$ ) evolution of cyanobacteria whose oxygen-producing capability destroyed a methane greenhouse thereby directly triggering the $2.3-2.2 \mathrm{Ga}$ Makganeyene glaciation. It was recently proposed (Kopp et al. 2005) that all the 'sudden' indicators of environmental oxidation, such as red bed appearance and the large attenuation in mass-independent sulphur isotope fractionation around this time period, record the inception of oxygenic photosynthesis, and that there was essentially no time lag between the origin of organisms capable of oxygenic photosynthesis and their rise to ecological dominance and impact on global geochemistry.

\section{(f) The present study}

The validity of biomarker methodologies for drawing inferences about biota and ocean redox in the Archaean rests upon a number of foundations.

(i) The fossilized lipids, hydrocarbons, reported in Archaean rocks must actually be 'Archaean' in age and indigenous to the samples in which they were found.

(ii) Membrane lipid compositions and biosynthetic pathways must be features of cell biology that are conserved over evolutionary time, such that information about past life can be drawn from knowledge of modern organisms.

(iii) The distribution of lipid biosynthetic pathways should be phylogenetically informative, such that the presence of particular molecules in the rock record provides information about contemporaneous biodiversity.

These premises all deserve critical appraisal. The issue of syngeneity has been addressed by Brocks et al. (2003) who concluded that Archaean biomarkers were 'probably syngenetic' with the host rocks. However, they could not totally exclude bitumen migration from younger sediments and, thus, additional research to address this point using freshly drilled and carefully curated drill cores is underway (Buick et al. 2004; Summons et al. 2004).

To address (ii) and (iii) above, we draw on the inventory of protein sequences of key biosynthetic enzymes for fresh insights about the biosynthetic oxygen requirement for sterol synthesis and the status of sterols as markers that are specific for Eukarya. We also investigated the sterol contents of some cyanobacteria purported to contain them, including their capacity for biosynthesis. Lastly, we review studies of the biosynthesis of 2-methylhopanoids and the phylogenetic distribution that pertains to oxygen availability.

\section{MATERIAL AND METHODS}

(a) Protein sequence and structure analysis

Protein sequences and structures of sterol synthesis enzymes in various organisms were obtained from databases using the basic local alignment search tool (Altschul et al. 1997). Except as noted below, sequence and structure data were retrieved from databases searched through the National Center for Biotechnology Information and Joint Genome Institute Web servers. OSC sequences for Acanthamoeba castellanii and Hartmannella vermiformis were retrieved from the Protist EST Programme database (http://amoebidia.bcm. umontreal.ca/public/pepdb/welcome.php); for Cyanidioschyzon merolae from the C. merolae Genome Project (http:// merolae.biol.s.u-tokyo.ac.jp/) and for G. obscuriglobus from the Institute for Genomic Research (http://www.tigr.org/tdb/ ufmg/index.shtml). Sequences were aligned using ClustalX and alignments manually edited in GeneDoc, and protein crystal structures were visualized with $\mathrm{Cn} 3 \mathrm{D}$ and RasMol.

\section{(b) Cyanobacterial lipid analysis}

(i) Culture conditions

Cyanobacteria were grown within an illuminated incubator (12-12 light-dark cycle) with $300 \mathrm{ml}$ of BG-11 or D-media with addition of filter-sterilized cycloheximide $\left(100 \mathrm{mg} \mathrm{l}^{-1}\right)$. 
Anabaena cylindrica (ATCC 27899), P. luridum (UTEX 426), Fischerella sp. (ATCC 29538), C. fristschii (ATCC 27193), Gloeobacter sp. TS and Gloeocapsa sp. were grown at $30^{\circ} \mathrm{C}$, and the Yellowstone isolates Phormidium sp. RC and OSS4 at $35^{\circ} \mathrm{C}$. Cultures were harvested by centrifugation using Corex centrifuge bottles, which were solvent-rinsed with methylene chloride and methanol prior to use. Cell pellets were frozen, lyophilized and stored at $-20^{\circ} \mathrm{C}$.

(ii) Lipid analysis

Total lipid was prepared from the stored cultures by a modified Bligh-Dyer extraction (Jahnke 1992) of lyophilized biomass. A $300 \mathrm{ml}$ aliquot of BG-11 and medium D were processed in parallel as control blanks. After addition of an internal standard (epiandrosterone or cholesterol-D4), small aliquots $(c a 0.1 \mathrm{mg}$ ) of the total lipid extracts were hydrolysed in $1 \mathrm{ml}$ of $0.1 \mathrm{~N} \mathrm{HCl}:$ methanol $(1: 1)$ at $60^{\circ} \mathrm{C}$ for $2 \mathrm{~h}$. After removal of solvent, and azeotropic drying with dichloromethane, the products were derivatized with $25 \mu \mathrm{l}$ each of bis(trimethylsilyl)trifluoroacetamide and pyridine with heating at $70^{\circ} \mathrm{C}$ for $30 \mathrm{~min}$ and analysed by GC-MS. Sterols were identified by comparison with literature spectra and the spectra of authentic compounds.

In a corollary set of analyses, lyophilized biomass from previous experimental protocols, which had been stored at $4{ }^{\circ} \mathrm{C}$, were extracted in a similar manner. PCR amplification of DNA from P. luridum, C. fristschii, Phormidium RC and Phormidium OSS4 using a fungal primer (ITS-4B) specific to Basidomycetes showed positive bands. Laboratory maintained stock cultures of these same cyanobacteria were negative using this primer set (C. Raleigh \& K. Cullings 2000 , personal communication).

\section{RESULTS AND DISCUSSION}

We first discuss the sterol biosynthetic pathway, with particular emphasis on oxygen utilization and molecular evolution of the synthesis enzymes. Three phases of sterol biosynthesis are explored: the epoxidation of squalene, the cyclization of oxidosqualene to protosterols and modification of the sterol skeleton, principally by oxidative demethylation (figure 1). Second, we present evidence from lipid analyses of several cyanobacteria that previous reports of sterol synthesis by these organisms may have been compromised by contamination.

\section{(a) Squalene monooxygenase}

(i) Mechanism and oxygen requirement

The epoxidation of squalene is the first oxygendependent step in the sterol pathway, and the point at which the synthesis of steroids diverges from that of hopanoids. Early work showed that sterol biosynthesis in yeast does not occur in fermentative cells and only initiates at micromolar levels of $\mathrm{O}_{2}$ (Jahnke \& Klein 1983). The stereospecific conversion of squalene to (3S)-2,3-oxidosqualene is catalysed by the enzyme squalene monooxygenase (SQMO; also known as squalene epoxidase) (figure 2). SQMO is a flavoprotein that requires $\mathrm{O}_{2}$ and flavine-adenine dinucleotide (FAD) to effect oxygenation, nicotinamide adenine dinucleotide phosphate, reduced form (NADPH)-cytochrome P450 reductase (itself a flavoprotein) to regenerate $\mathrm{FAD}$ and a soluble protein factor for squalene transport (Lee et al. 2004). Epoxidation proceeds by the reaction of oxygen with the bound dihydroflavin $\left(\mathrm{Fl}_{\mathrm{red}} \mathrm{H}_{2}\right)$ to give a 4a-hydroperoxyflavin ( $\mathrm{FlH}(4 \mathrm{a})-\mathrm{OOH})$. The oxygen transfer occurs by nucleophilic attack by the 2,3 double bond of squalene on the terminal oxygen of the 4a-hydroperoxide, yielding oxidized flavin $\left(\mathrm{Fl}_{\mathrm{ox}}\right)$ and 2,3-oxidosqualene (Bruice et al. 1983; Torres \& Bruice 1999). The FAD is regenerated by NADPH-cytochrome P450 reductase (Laden et al. 2000).

This reaction depends on the electrophilic character of the hydroxy group of the hydroperoxide, since the attack comes from the 2,3-olefin of squalene. The epoxidation also benefits from the relatively weak $\mathrm{O}-\mathrm{O}$ bond in the hydroperoxide ( $47 \mathrm{kcal} \mathrm{mol}^{-1}$; Blanksby \& Ellison 2003), making the oxygen transfer energetically feasible. It has been suggested (Raymond \& Blankenship 2004) that the squalene epoxide oxygen might be derived from a source besides $\mathrm{O}_{2}$, such as water. Such a scheme would presumably proceed through hydroxylation of a cofactor followed by squalene epoxidation using the water-derived hydroxyl. This scheme is excluded on two grounds: first, such a hydroxyl would itself have a nucleophilic character, preventing attack by the squalene olefin; second, the $\mathrm{C}-\mathrm{O}$ bond of the hydroxyl is much stronger $\left(96 \mathrm{kcal} \mathrm{mol}^{-1}\right.$; Blanksby \& Ellison 2003), providing a much higher energy barrier to oxygen transfer. Furthermore, the next enzyme in the pathway, OSC, requires the $3 S$ (and rejects the $3 R$ ) form of 2,3-oxidosqualene, and hydroperoxide epoxidation affords the required stereoselectivity. In this chemical context, an $\mathrm{O}_{2}$-independent route to oxidosqualene is highly disfavoured.

\section{(ii) Evolutionary conservation}

SQMO contains several motifs responsible for substrate and cofactor binding that are conserved in all known sequences of the protein, including those of prokaryotes (Lee et al. 2000, 2004; Pearson et al. 2003). All known SQMOs (and data are available for animals, fungi, plants, amoeboid and kinetoplastid protists, and bacteria) use the epoxidation mechanism described above. But might there be alternative, chemically and evolutionarily unrelated methods to produce oxidosqualene?

There are enzymes that catalyse the epoxidation of olefins without FAD. These are cytochrome P450 oxygenases that use $\mathrm{Fe}$ and $\mathrm{O}_{2}$ and transfer oxygen to a variety of unsaturated substrates, such as arachidonate during eicosanoid biosynthesis. As discussed below with regard to sterol demethylases (which are $\mathrm{P} 450$ cytochromes), the $\mathrm{O}_{2}$ requirement of such enzymes is at least as stringent as that of the flavoproteins. It is noteworthy that no organism is known to have replaced SQMO with a cytochrome P450, though there is no

Figure 1. (Opposite.) Generalized synthetic pathway of sterols. Sterol precursor squalene is oxidatively converted to oxidosqualene, which is cyclased to one of two protosterols: cycloartenol or lanosterol. The protosterol undergoes subsequent modifications including oxidative demethylations and desaturations to result in the terminal sterol product. Enzymes are labelled with EC number where available, or gene abbreviation. Terminal sterols yield derived steranes after burial and diagenesis. Enzymes labelled in bold are discussed in the text. Those requiring molecular oxygen are noted.

Phil. Trans. R. Soc. B (2006) 


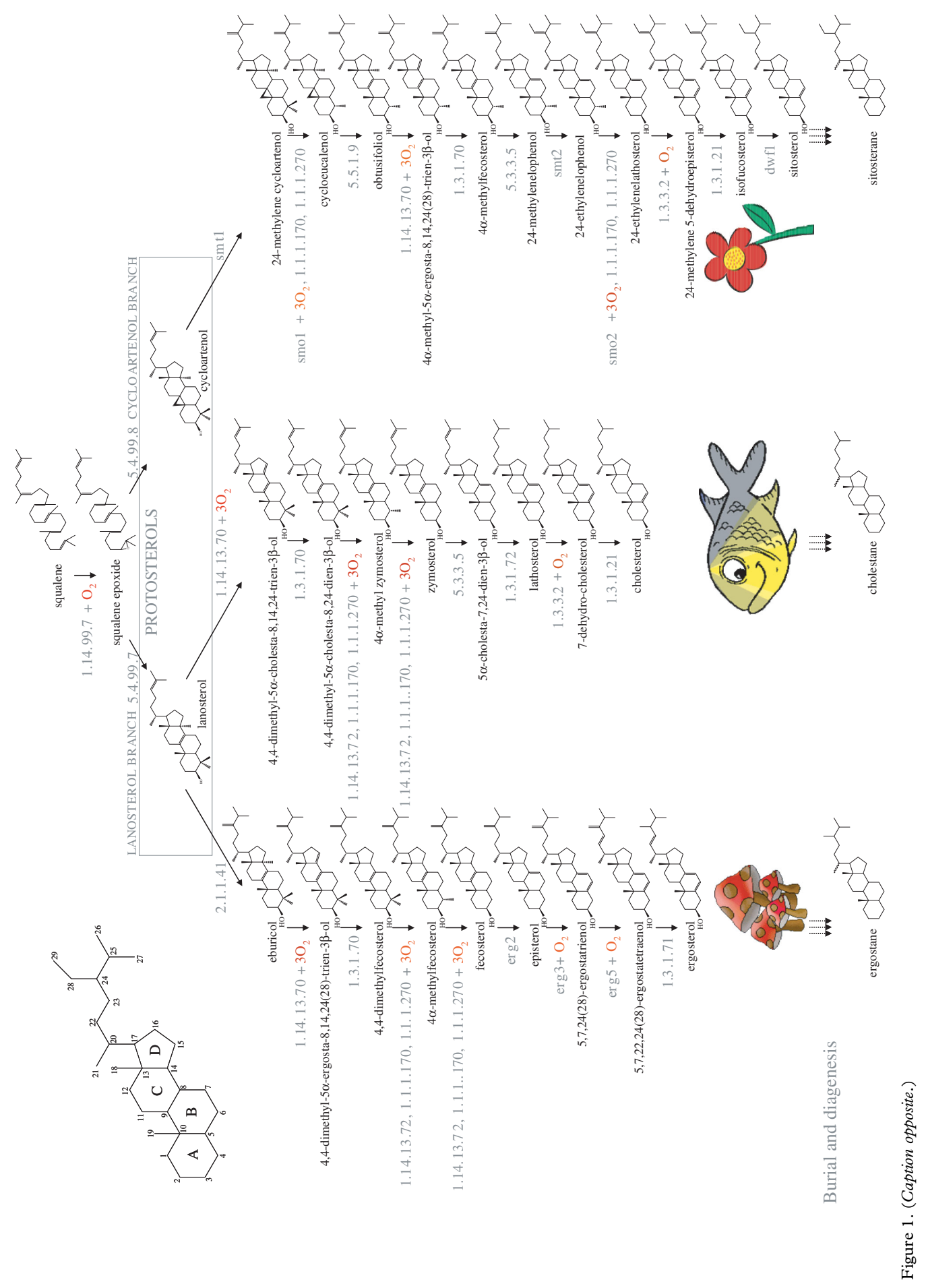

Phil. Trans. R. Soc. B (2006) 


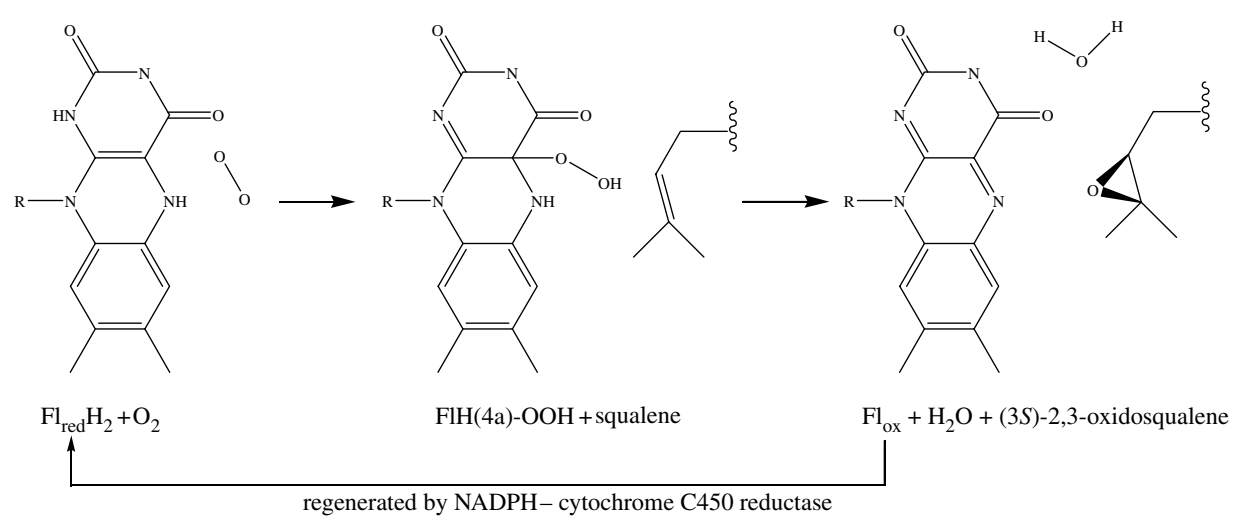

Figure 2. Mechanism for the epoxidation of squalene by squalene monooxygenase (SQMO).

recognized chemical or enzymological prohibition against this. In fact, a secondary squalene epoxidase activity for CYP17, a P450 cytochrome whose primary role is the hydroxylation of pregnenolone and progesterone in corticosteroid hormone synthesis in vertebrates, has recently been demonstrated in mouse tumour cells (Liu et al. 2005). Were lipid biochemistry sufficiently plastic, one might expect a gene replacement to have taken place, wherein the flavoprotein SQMO would be supplanted by a P450 cytochrome with multiple activities, thereby reducing the number of enzymes required to constitute the complete pathway. In fact, such gene replacement is not observed. The universal retention of the FAD-dependent oxygenase even when other (though equally $\mathrm{O}_{2}$-requiring) mechanisms are available is an example of the evolutionary conservatism of biosynthetic pathways.

\section{(b) Oxidosqualene cyclase}

(i) Structure and function of oxidosqualene cyclases

The tetracyclic core that characterizes all sterols is created through the cyclization of squalene $(3 S)-2,3-$ epoxide by the enzyme oxidosqualene cyclase (OSC). This is the second step in sterol biosynthesis after the epoxidation of squalene, and one of the most complex biochemical reactions catalysed by a single enzyme. The cyclization is executed with a remarkable degree of specificity and stereochemical control. The products are either lanosterol or cycloartenol (figure 1), the two 'protosterols' that are subsequently modified to functional products such as cholesterol or phytosterols.

Several decades of work have elucidated much of the mechanism of cyclization by OSC and identified specific residues responsible for particular steps in the cyclization cascade. The detailed chemistry of this enzyme was recently reviewed (Wendt et al. 2000; Wendt 2005) and the crystal structure of human OSC determined at $2.1 \AA$ resolution (Thoma et al. 2004) allowing visualization of the enzyme and interactions with the substrate in unprecedented detail. Figure 3 shows two views of the active site of human OSC with its product, lanosterol. Briefly, the key steps in the cyclization (and residues responsible) include: (i) Positioning of squalene 2,3-epoxide in a prefolded configuration within the active site (Y98, Y704).

(ii) Protonation of the epoxide group by the catalytic acid (D455, C456, C533).

(iii) Ring formation, during which cation intermediates are stabilized by cation $-\pi$ interactions with aromatic residues of the active site (W387, F444, W581, F696) and the cation migrates out to C20.

(iv) Skeletal rearrangement via hydride- and methylshifts (largely spontaneous) as the proton migrates back to a region of high $\pi$-electron density around the $\mathrm{B} / \mathrm{C}$ rings.

(v) Deprotonation by basic residues to quench the final protosteryl cation (Y503, H232)-the position of the deprotonation determines the OSC product (lanosterol versus cycloartenol). D455 is ultimately reprotonated by E459.

The sequences of genes encoding OSCs are now available for many organisms, principally animals, plants and fungi, but also several protists. Alignment of these sequences shows very high degree of conservation across the breadth of eukaryotic diversity (at least five of the kingdom-level divisions of Adl et al. (2005)). The active-site residues mentioned above are absolutely conserved, i.e. $100 \%$ identity at the amino acid level. Alternative mechanisms for oxidosqualene cyclization do not appear to have arisen in any of the eukaryotic lineages sampled to date. Together with the conservation of function seen in the squalene epoxidases, this strongly suggests that, at least, the initial steps in sterol biosynthesis were present, generally in their modern form, in the last common ancestor of all eukaryotes.

(ii) Oxidosqualene cyclase product profiles and eukaryote phylogeny

There are two main types of OSCs, based on the end product of the cyclization: lanosterol synthases and cycloartenol synthases. The cyclization process in the two types of enzymes is identical until the final deprotonation step. A deprotonation from C9 forms

Phil. Trans. R. Soc. B (2006) 
the 8,9-double bond of lanosterol whereas a deprotonation from C19 allows the cycloartenol cyclopropyl ring to close. Thus far, lanosterol synthase has been found only among the opisthokonts (animals + fungi + choanozoa), trypanosomatids (Trypanosoma, Leishmania) and dinoflagellates (Giner et al. 1991). All other eukaryotes that have been examined in this regard (at least members of the higher plants, green and red algae, amoebozoa, diatoms, euglenids and heterolobosea) make cycloartenol as their protosterol.

Site-directed mutagenesis experiments, notably those of Matsuda and co-workers (Meyer et al. 2000, 2002; Joubert et al. 2001; Segura et al. 2002; Lodeiro et al. 2004) have indicated the key residues that control the product profile of OSCs, including T381, C449 and V453. From analysis of the crystal structure, each appears to affect the position of the catalytic base dyad $\mathrm{H} 232-\mathrm{Y} 503$. By controlling the position from which the protosterol cation is deprotonated, these residues determine which product will be formed by the cyclase. The second-sphere residue C449 is particularly interesting: the $\mathrm{H} 449 \mathrm{~N}$ mutant of Arabidopsis thaliana OSC is the most efficient lanosterol synthase to be generated from a wild-type (WT) cycloartenol synthase (88\% lanosterol yield). Given its distance from the substrate (approx. 7.4 $\AA$ ), its control of the product profile is likely indirect. Interestingly, mutagenesis experiments have yet to induce cycloartenol formation from a WT lanosterol synthase. Natural OSCs generally conform to the residue-product relations found in mutagenesis experiments (figure $3 a$ ), with exceptions that may be evolutionarily informative. From the protein alignment, patterns in differential conservation of these product-controlling residues can be discerned: opisthokont lanosterol synthases are T381/C,Q449/V453, while cycloartenol synthases are all Y381/H449/I453. Two exceptions to this pattern emerge: the lanosterol-producing OSCs of the bacteria M. capsulatus and G. obscuriglobus (discussed further below) and the trypanosomatid lanosterol synthases.

The trypanosomatids make lanosterol as their protosterol, despite the presence of a tyrosine at position 381. Several lines of evidence indicate that the trypanosomatids ancestrally possessed a cycloartenol synthase. First, the T381Y mutation has been shown to decrease the efficiency of the Saccharomyces cerevisiae lanosterol synthase, and induce the formation of parkeol and lanostene-3,9-diol (and not cycloartenol) as secondary products. The fixation of a T381Y mutation in a lanosterol synthase is thus unlikely. Second, euglenids Euglena gracilis (Anding et al. 1971) and Astasia longa (Rohmer \& Brandt 1973) and heterolobosea (Naegleria sp.; Raederstorff \& Rohmer 1987), more deeply branching than the trypanosomatids within the Excavate kingdom (Simpson et al. 2005), have been shown to make sterols via the cycloartenol pathway. Third, post-cyclization modification of sterols in the kinetoplastids follows a cycloartenol-type route; the $14 \alpha$-demethylase of Trypanosoma brucei has recently been shown to be specific for the cycloartenol pathway intermediate obtusifoliol (Lepesheva et al. 2004). Taken together, this evidence suggests the following hypotheses: the trypanosomatids began with a cycloartenol synthase
(Y381/H449/I453) which underwent two mutations, $\mathrm{H} 449 \mathrm{Q}$ and I453V (each requiring a single nucleotide change) to yield a lanosterol synthase. Downstream modification of the protosterol remained essentially as the cycloartenol pathway, but at least some of the enzymes must have adapted to different substrates; in particular smt 1 and smo1 (figure 1). It is worth noting that the Y381T/H449Q/I453V triple mutant of A. thaliana OSC is a reasonably efficient lanosterol producer; second-sphere mutations may have further enhanced the specificity of the trypanosomatid OSC.

Lanosterol synthesis appears to have arisen at least twice among the eukaryotes: once in an ancestor of the opisthokonts and once in an ancestor of the trypanosomatids after the divergence of the euglenids. Dinoflagellates have also been reported to make lanosterol (Giner et al. 1991), but no sequence information is currently available for their cyclases; they may represent a third instance of innovation, or may have acquired a lanosterol synthase laterally. It is as yet unclear which type, lanosterol synthase or cycloartenol synthase, was the ancestral form of OSC. Given the hypothesized independent originations of lanosterol synthase and the apparent difficulty in mutating a WT lanosterol synthase to produce cycloartenol, it is tempting to infer cycloartenol synthase as the more ancient of the two forms. If, on the other hand, eukaryotic phylogeny is rooted in the branch leading to the opisthokonts (Arisue et al. 2005) there is no strong parsimony argument either way.

(iii) Prokaryotic oxidosqualene cyclases

While sterol synthesis is nearly ubiquitous among the Eukarya, only three instances are known among prokaryotes, all bacteria: $M$. capsulatus (a $\gamma$-proteobacterium), G. obscuriglobus (a planctomycete) and a paraphyletic group of myxobacteria ( $\delta$-proteobacteria). These organisms are not closely related and the reason for the sparse appearance of sterol synthesis in phylogenetically distant bacterial taxa remains unclear. If it is the result of vertical inheritance from a common, sterol-synthesizing bacterial ancestor, dozens of parallel losses of the entire pathway in many lineages would be required. Alternatively, sterol biosynthesis genes may have been acquired by bacteria via lateral transfer from eukaryotes, potentially followed by one or more intra-bacterial transfer events. Such a eukaryote-tobacteria gene transfer has been proposed to account for the similarly sparse occurrence of glutaminyl-tRNA synthetase among the Bacteria (Lamour et al. 1994; Brown \& Doolittle 1999). At present, however, there is not sufficient evidence to draw clear conclusions concerning the evolutionary relationships between eukaryotic and prokaryotic OSCs.

Of bacterial groups, sterol synthesis is most widely distributed among the myxobacteria. Of 11 genera ( 88 total strains) tested by Bode et al. (2003) only four were found to produce sterols. The sterol-producing genera do not form a monophyletic group in the myxobacterial phylogeny of Shimkets \& Woese (1992), implicating some combination of multiple gains, multiple losses and transfer events to explain the distribution of this capability. The most complete bacterial sterol synthesis pathway is in the myxobacterium Nannocystis excedens, 


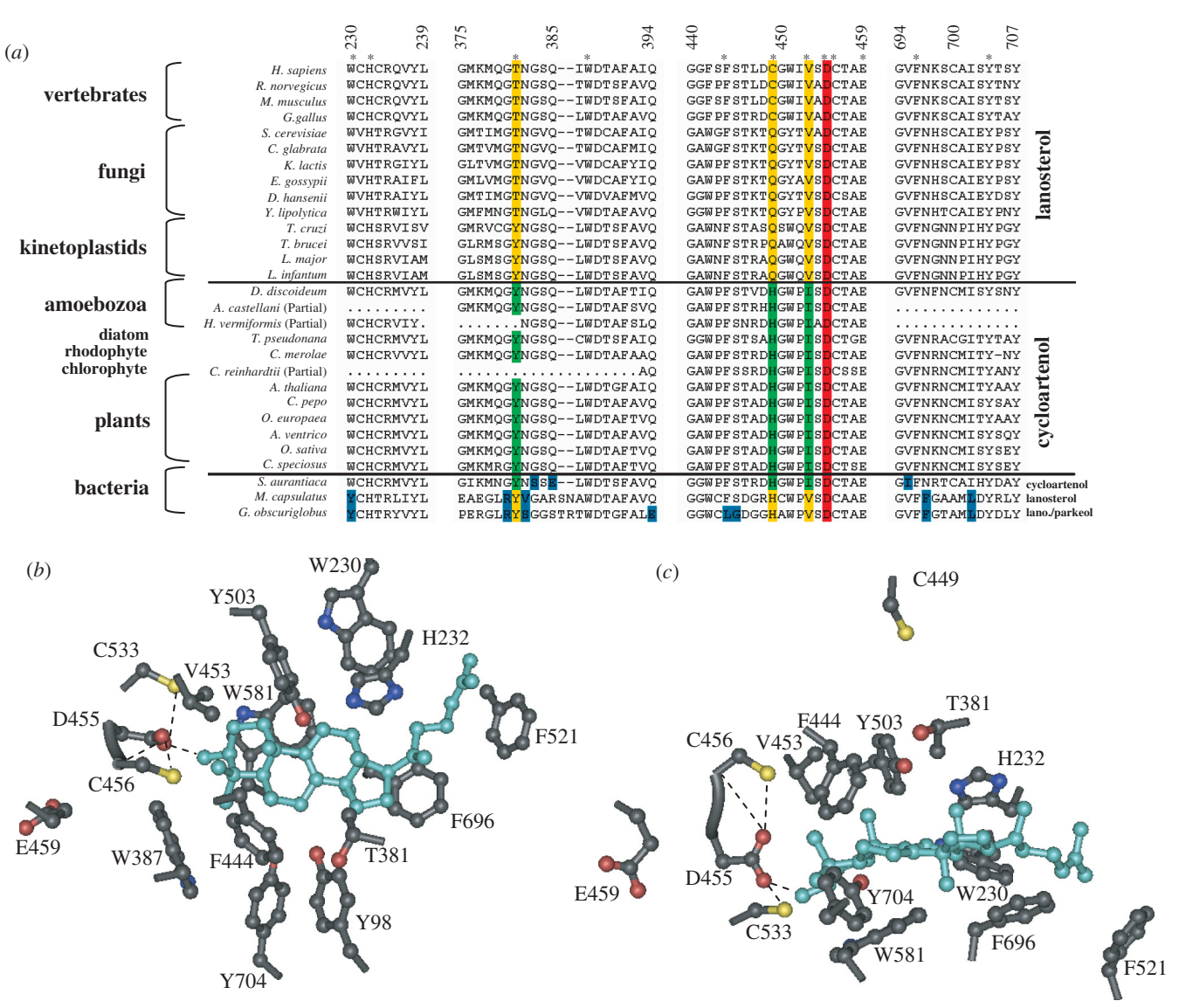

Figure 3. (a) Alignment of oxidosqualene cyclase (OSC) protein sequences. The catalytic acid, D455, is highlighted in red. Positions 381, 449 and 453 are differentially conserved between lanosterol synthases (yellow) and cycloartenol synthases (green). Highly conserved residues that are substituted in bacterial OSCs are highlighted in blue. Residues that are shown in the structures in $(b)$ and $(c)$ are indicated by asterisks. Numbering (throughout this figure and in text) refers to Homo sapiens OSC. (b) View of the active site of H. sapiens OSC with lanosterol shown in light blue. The hydrogen-bonding network around the catalytic acid D455 is indicated by dashed lines. H232 and Y503 constitute the catalytic base dyad and effect the final deprotonation. (c) View of the active site in the molecular plane of lanosterol. Note the distance of second-sphere residue C449 from the substrate. $(b)$ and $(c)$ are drawn from the crystal structure determined by Thoma et al. (2004).

which can demethylate lanosterol at C4 and C14 and progress as far as lathosterol (figure 1). Interestingly, the myxobacteria cyclize oxidosqualene to both lanosterol and cycloartenol; Cystobacter sp. produce both protosterols (Bode et al. 2003). If OSC was laterally acquired by the myxobacteria, it is unclear from the phylogenetic distribution alone which type they initially got from eukaryotes.

Three bacterial OSC sequences are presently available: the lanosterol synthase of $M$. capsulatus, the OSC of G. obscuriglobus, which produces a mixture of lanosterol and parkeol and the cycloartenol synthase of the myxobacterium Stigmatella aurantiaca. Overall, these enzymes are quite similar to the eukaryotic cyclases and make use of the same catalytic groups to effect the same chemistry. Of the three, the Stigmatella OSC is most like eukaryotic enzymes (figure 3). It shows the standard Y381/H449/I453 pattern of eukaryotic cycloartenol synthases, and fewer differences among highly conserved residues than the other two known sequences. The $M$. capsulatus and $G$. obscuriglobus OSCs are another exception to the pattern of differential conservation of residues 381, 449 and 453. These cyclases are $\mathrm{Y} 381 / \mathrm{H} 449 / \mathrm{V} 453$, making 453 the only position to be consistently differentially conserved between WT lanosterol and cycloartenol synthases. Both the $M$. capsulatus and G. obscuriglobus OSCs do, however, have modifications to other activesite residues (not yet explored in mutagenesis experiments) that may contribute to their unusual product profiles. In particular, the W230Y, G380R and N382 $(\mathrm{V}, \mathrm{S})$ substitutions (residues otherwise conserved across the Eukarya and in Stigmatella) could all affect the configuration of the active site near the deprotonating base, altering the enzyme product. Further, Gemmata alone has F444L and S445G substitutions; these are residues positioned close to T381 and C449 and may contribute to the Gemmata OSC's high yield 
<smiles>[R]C(C)[C@H]1CC[C@]2(C)C(C)=C(C)CC[C@]12C</smiles>

(a)<smiles>CS[Te]1CCC(C=O)CC1</smiles><smiles>[CH2-]C</smiles><smiles>[R]C(C)[C@H]1CC[C@]2(CO)C(C)=C(C)CC[C@]12C</smiles>

(h)

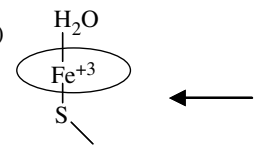

(b)<smiles>C[As]1CCCC1</smiles><smiles>[R]C(C)[C@H]1CC[C@]2(C)[C@@H](C)C(C)=C(C)CC[C@]12C</smiles>

(g)

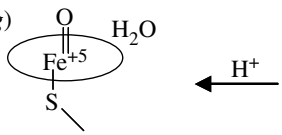<smiles>[R]C(C)[C@H]1CC[C@]2(C)C(C)=C(C)CC[C@]12C</smiles>

(c)
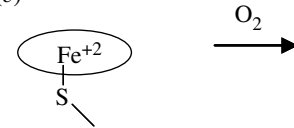<smiles>[R]C(C)[C@H]1CC[C@]2(C)C(C)=C(C)CC[C@]12C</smiles>

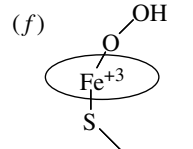<smiles>[R]C(C)[C@H]1CC[C@]2(C)C(C)=C(C)CC[C@]12C</smiles>

(d)

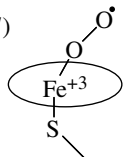

$1 e^{-}$<smiles>[R]C(C)C1CC[C@]2(C)C(C)=C(C)CC[C@]12C</smiles>

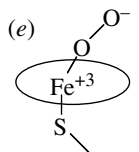

Figure 4. Steps in the oxidation of the $14 \alpha$ angular methyl group of sterols by the CYP51 active site and molecular oxygen. The methyl group is oxidized three times by the cycle $(a-h)$, each using one molecule of $\mathrm{O}_{2}$. Note that the Fe (IV) + Por + complex is written $\mathrm{Fe}(\mathrm{V})$ for simplicity. After the third oxidation the methyl group is removed.

of parkeol, a protosterol otherwise very minor among WT cyclases.

(iv) Oxygen requirements of cyclization

The cyclization of oxidosqualene to the sterol hydrocarbon skeleton is not oxidative and does not require an external electron acceptor. OSC is, however, highly specific for $(3 S)-2,3$-oxidosqualene; neither $(3 R)-2,3-$ oxidosqualene, squalene epoxidized at other positions, nor unoxygenated squalene are suitable substrates. OSC has likely always acted on oxidosqualene, so the ability to epoxidize squalene was a prerequisite for production of the 6,6,6,5-ring structure. Indeed, the catalytic acid group of OSC (the conserved DCxxE motif) may not be acidic enough to protonate the olefin of squalene, but can manage the epoxide. That the sterol cyclization chemistry was present in its modern (oxidosqualene-dependent) form in the eukaryotic last common ancestor is supporting evidence that early eukaryotic life had the means to oxygenate squalene.

\section{(c) Sterol demethylases}

According to Bloch's (1987) postulate, the sequential departure of the three nuclear methyl groups from the protosterol lanosterol, in the order $14 \alpha$-methyl, $4 \alpha-$ methyl, and $4 \beta$-methyl, leads to an improvement in the fitness of the molecule, reaching a maximum with cholesterol. While it is now known that the order of removal of the methyl groups does vary in plants, compared to the above order in fungi and animals, there are some lines of experimental evidence that functional fitness improves (Bloch 1983).

Demethylation at the $14 \alpha$ carbon is catalysed by an oxidative demethylase of the cytochrome P450 superfamily in plants, animals and fungi, and a few bacteria. Oxidation of each of the two C-4 methyl groups is carried out by an unrelated enzyme of the sterol desaturase family. The functions of these enzymes are essential for eukaryotes, perhaps because demethylation of $\alpha$ face of sterols is required to allow proper sterol-fatty acid interaction to achieve the maximum membrane microviscosities (Dahl et al. 1980). In animals, removal of the $14 \alpha$-methyl group is the first step in the sterol synthetic pathway following cyclization, and the CYP51 substrate is lanosterol (figure 1). In filamentous fungi, lanosterol is methylated at C-24 before being demethylated at C-14 and the CYP51 substrate is eburicol (24-methylene-24,25-dihydrolanosterol). Several steps occur along the cycloartenol pathway in plants before $14 \alpha$-demethylation, including one demethylation at $\mathrm{C}-4$ and the opening of the cyclopropyl ring so that plant CYP51 operates on obtusifoliol.

\section{(i) $C$-14 demethylases}

The removal of the $14 \alpha$-methyl group of sterols (figure 4 ) is performed by sterol $14 \alpha$-demethylase (CYP51). CYP51 belongs to the cytochrome P450 enzyme 
superfamily, which includes more than 2000 members in all three domains of life. All P450s are used in oxidative reactions on various molecules and all require molecular oxygen as a substrate. The active site of $\mathrm{P} 450$ s contains a Fe-protoporphyrin IX (haem). CYP51 is the only cytochrome P450 that performs the same function in different biological domains. Some researchers have suggested that CYP51 is the ancestral P450 (Nelson \& Strobel 1989; Aoyama et al. 1994; Rezen et al. 2004). CYP51 operates on one of four substrates-lanosterol (in animals and fungi), dihydrolanosterol (animals), obtusifoliol (plants and kinetoplastids), or eburicol (fungi). These substrates differ only in the nature of the side chain and the presence or absence of a second methyl group at C-4.

The small differences between substrates probably account for a general lack of substrate specificity among the demethylases. Most CYP51s can demethylate any of the four substrates, although a few plant CYP51s are obtusifoliol-specific. In humans, a defect in CYP51 causes Antley-Bixler syndrome. In yeast, $14 \alpha-$ demethylase inhibition is fatal, and this makes the enzyme an attractive target for fungicides. Fungicides may take advantage of the differences between fungal and animal CYP51 active sites to selectively inhibit the fungal enzyme.

Rezen et al. (2004) found that CYP51s separate phylogenetically into plant (obtusifoliol), animal (lanosterol) and fungal (eburicol) groups. Bacterial CYP51s including those of M. capsulatus and Mycobacterium tuberculosis both fall within the plant lineage. The purified $M$. tuberculosis protein will demethylate lanosterol in vitro although obtusifoliol is preferred. However, despite the phylogenetic placement of its CYP51, M. capsulatus synthesizes lanosterol. It has yet to be demonstrated whether lanosterol is the substrate for M. capsulatus CYP51. Jackson et al. (2002) note that the M. capsulatus CYP51 is novel in that it is linked to a ferredoxin domain, but that the CYP51 in M. tuberculosis is part of an operon in which it is followed by ferredoxin. The M. capsulatus gene may be the result of a lateral transfer event from $M$. tuberculosis followed by a mutation. Perhaps the best example of the broad substrate specificity of these enzymes is Streptomyces coelicolor, which contains a gene with lowlevel homology to bacterial CYP51s and that demethylates eburicol but not lanosterol (Lamb et al. 2003). However, this gene is not a CYP51, and the conserved sites differ significantly from CYP51. Streptomyces coelicolor does not contain sterols, and the in vivo function of this protein is unknown.

The particulars of amino acid residue participation in substrate binding and catalysis of CYP51 are unknown. Podust and co-workers (Podust et al. $2001 b$ ) noted that two channels with access to the haem may serve to transport substrate and product to and from the active site. They (Podust et al. 2001a) also performed modelling experiments based on the crystal structure of $M$. tuberculosis CYP51 in the presence of the azole inhibitors fluconazole and 4-phenylimidazole. They found that the inhibitors coordinate themselves with the haem iron in the large $\left(2600 \AA^{3}\right)$ cavity opposite the cysteine (C394) that binds the haem to the protein. Amino acids surrounding this cavity were

Phil. Trans. R. Soc. B (2006) considered likely to be involved in binding and/or catalysis and showed a high degree of conservation across CYP51s from many organisms. Only approximately $10 \%$ (41 conserved residues) of CYP51s are absolutely conserved across all domains of life (with the exception of five residues in the kinetoplastids) and most of these are not at the active site. Half of these sites have been examined by site-directed mutagenesis experiments, which have shown that most of them are essential for CYP51 function. Naturally occurring azole-resistant strains of Candida albicans contain the mutation $\mathrm{Y} 132 \mathrm{H}$, which does not directly interact with substrate and may reflect a more complicated interaction between protein and substrate, and Bellamine et al. (2004) report from several site-directed mutagenesis experiments that azole and substrate binding are uncoupled. Among the residues identified by Podust et al. $(2001 a, b)$ as participating in the active site, there are several positions which differ between CYP51 subfamilies operating on different substrates.

Other site-directed mutagenesis studies on 10 residues that were believed to be strictly conserved among CYP51s (Lepesheva \& Waterman 2004) found that most mutants lost all demethylase activity, although one (A197G) showed enhanced demethylase activity. Based on a combination of mutagenesis experiments and the crystal structure of CYP51 they postulated potential substrate binding sites: $\mathrm{D} 90$ as a binding site for the sterol $3 \beta-\mathrm{OH}, \mathrm{L} 172$ and R194 associating with the side chain, and F82 binding the sterol A or B ring. Kinetoplastid genomes revealed differences from the 'strictly conserved' at five of the CYP51 residues in which mutations produced total or near total loss of function in M. tuberculosis (D90A, L127M，G175S/C，R194C，D195H/R). Lepesheva et al. (2004) showed that the kinetoplastid CYP51s were obtusifoliol-specific and that mutation of these five residues back to the conserved state did not improve the ability of the kinetoplastid enzyme to metabolize lanosterol. A clearer understanding of the chemical role of these conserved residues will be necessary to discern the evolutionary implications of these changes.

The general mechanism of substrate oxidation by P450 enzymes is known from several decades of work on the camphor-oxidizing P450 of Pseudomonas putida. It consists of eight steps (Groves \& Han 1995; Meunier et al. 2004):

(i) substrate binding and subsequent displacement of the haem $\mathrm{Fe}$ (III)

(ii) electron transfer to $\mathrm{Fe}$ (III) from a reductase cofactor to yield $\mathrm{Fe}(\mathrm{II})$ and a haem with a negative charge;

(iii) binding of molecular oxygen to the ferrous iron yielding a $\mathrm{Fe}$ (III)-dioxygen bond;

(iv) transfer of a second electron to the haem to yield a negatively charged $\mathrm{Fe}$ (III)-peroxo complex which is a very strong nucleophile;

(v) protonation of the $\mathrm{Fe}(\mathrm{III})$-peroxo complex yielding a $\mathrm{P} 450-\mathrm{Fe}$ (III)-OOH which also behaves as a nucleophile. This protonation likely involves the action of an acidic residue (D251 in P450cam) near the active site; 
(vi) a second protonation of the distal oxygen atom and cleavage of the $\mathrm{O}-\mathrm{O}$ bond, yielding a molecule of water and the formation of the reactive electrophilic iron-oxo species $\mathrm{Fe}(\mathrm{IV})$;

(vii) transfer of the oxygen atom from the iron-oxo complex to the substrate;

(viii) dissociation of the product.

In CYP51, this process operates three times on the $14 \alpha$-methyl group of the sterol which successively converts the $14 \alpha$-methyl group to $14 \alpha$-hydroxymethyl, $14 \alpha$-carboaldehyde and $14 \alpha$-formyl intermediates, subsequently eliminating formic acid and leaving a $\Delta 14,15$ double bond in the sterol. It is significant that the initial hydroxylation of the methyl, which requires the abstraction of $\mathrm{H}^{*}$, is achieved with the high redox potential associated with the $\mathrm{Fe}(\mathrm{IV})$ complex, which is achieved only through the action of molecular oxygen. This complex has an effective oxidation state of $\mathrm{Fe}(\mathrm{V})$ due to the additional charge on the porphyrin. See Meunier et al. (2004) for a detalied review.

(ii) C-4 demethylases

Demethylation of a C-4 methyl group requires the action of a suite of three enzymes working sequentially (Gachotte et al. 1998, 1999; Benveniste 2004):

(i) C-4 $\alpha$-methyl oxidase (ERG25 in yeast and smo1/smo2 in plants), which operates on the $4 \alpha$-methyl carbon three times with molecular oxygen to produce a $4 \alpha$-carboxylic acid (Darnet \& Rahier 2003, 2004);

(ii) $4 \alpha$-carboxysterol-C-4-dehydrogenase/C-4-decarboxylase ( $4 \alpha-C D$ or ERG26 in yeast) which decarboxylates the $4 \alpha$-acid and produces a 3-oxosteroid (Gachotte et al. 1998; Rondet et al. 1999);

(iii) NADPH-dependent sterone reductase (ERG27) which stereospecifically reduces the 3 -oxosteroid to a $3 \beta-\mathrm{OH}$.

These enzymes always act on the $4 \alpha$-methyl group. In the first demethylation, the $4 \alpha$-methyl is removed, and the $4 \beta$-methyl group rearranges to the $4 \alpha$ position. In both animals and fungi, the two methyl groups are sequentially removed following $14 \alpha$-demethylation. The C-4 demethylase enzymatic suite converts 4,4-

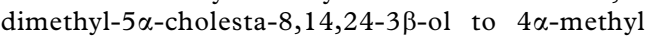
zymosterol, which is subsequently converted by the same suite of enzymes to zymosterol (Benveniste 2004). In plants, the first methyl group is removed at the level of 24-methylene cycloartenol, which is converted to cycloeucalenol. This pathway goes through several subsequent steps, including the removal of the $14 \alpha$-methyl group before being demethylated at C-4 a second time at the level of 24ethylenelophenol, which is converted to 24-ethylenelathosterol. Plant genomes show the presence of two distinct sterol methyl oxidases (smo1 and smo2) (Darnet \& Rahier 2004), and gene silencing experiments in $A$. thaliana have demonstrated that each of these operate with high substrate specificity - smol on 24-methylene cycloartenol and smo2 on 24-ethylenelophenol (Benveniste 2004).
Other taxa which synthesize C-4 desmethyl sterols include the bacteria $M$. capsulatus and N. excedens, and the kinetoplastids. Genome sequences of M. capsulatus and three kinetoplastid species are available in GenBank and a comparison of the sequenced genome to known sequences for erg 25 , smo 1 and smo2 revealed no significant homologues to any of these genes among these organisms. It is possible that these organisms are using an alternative enzyme for C-4 sterol demethylation.

(iii) Energetic constraints and oxygen usage

The mechanism of sterol demethylation functionalizes a methyl group attached to a quaternary carbon by attaching an oxygen atom. CYP51 achieves this by the homolytic cleavage of a $\mathrm{C}-\mathrm{H}$ bond to create a methyl radical, which subsequently reacts with the oxygen distally attached to the haem of the P450 (Meunier et al. 2004). This is an energetically expensive process which is overcome in part by using the most powerful oxidizing agent available: molecular $\mathrm{O}_{2}$.

The $\mathrm{C}-\mathrm{H}$ bond dissociation energy $(D)$ of a methyl group attached to a quaternary carbon is $401 \mathrm{~kJ} \mathrm{~mol}^{-1}$ (March 1992). However, bond energies of the transition state in the demethylase enzymes are unknown and so this does not indicate actual activation energy of the methyl group, but gives an indication of the stability of the radical. This dissociation energy is higher (indicating a less stable radical) than that of secondary $\left(401 \mathrm{~kJ} \mathrm{~mol}^{-1}\right)$ and tertiary $\left(401 \mathrm{~kJ} \mathrm{~mol}^{-1}\right)$ carbons, but not as high as that of the $\mathrm{C}-\mathrm{H}$ bond in primary $\left(419 \mathrm{~kJ} \mathrm{~mol}^{-1}\right)$ carbons, methane $\left(438 \mathrm{~kJ} \mathrm{~mol}^{-1}\right)$ or benzene $\left(464 \mathrm{~kJ} \mathrm{~mol}^{-1}\right)$.

Microbial degradation of both benzene and methane takes place readily in the presence of oxygen, but is also possible under many conditions as mildly exergonic processes with sulphate as a terminal electron acceptor. This indicates that abstraction of $\mathrm{C}-\mathrm{H}$ bonds stronger than those in sterol methyl groups is possible without molecular oxygen. Although thermodynamically feasible, an enzyme that demethylated sterols anaerobically would be energetically much more expensive.

Sterol demethylases have evolved at least twice, and in each case require three molecules of molecular oxygen to catalyse the reaction. Constructed phylogenetic trees of CYP51 cluster those demethylases into groups that parallel the families of sterol cyclases, suggesting that oxidative sterol demethylation is an ancient and conserved pathway that has existed at least since the time of the split between plants, kinetoplastids and opisthokonts (Rezen et al. 2004). Nature may contain at least one undescribed sterol demethylase (the C-4 demethylase in M. capsulatus and kinetoplastids). As this undescribed enzyme occurs in obligate aerobes, it is likely to require molecular oxygen.

On strict chemical grounds it may be possible to devise biosynthetic routes to sterols that proceed anaerobically as has been recently proposed (Raymond \& Blankenship 2004). However, any postulated anaerobic pathway for sterol synthesis must replace five enzymes which use a combined 11 or 12 molecules of $\mathrm{O}_{2}$ with anaerobic enzymes capable of performing the equivalent process, and further postulate that all of these enzymes have been lost or are unknown. Any 

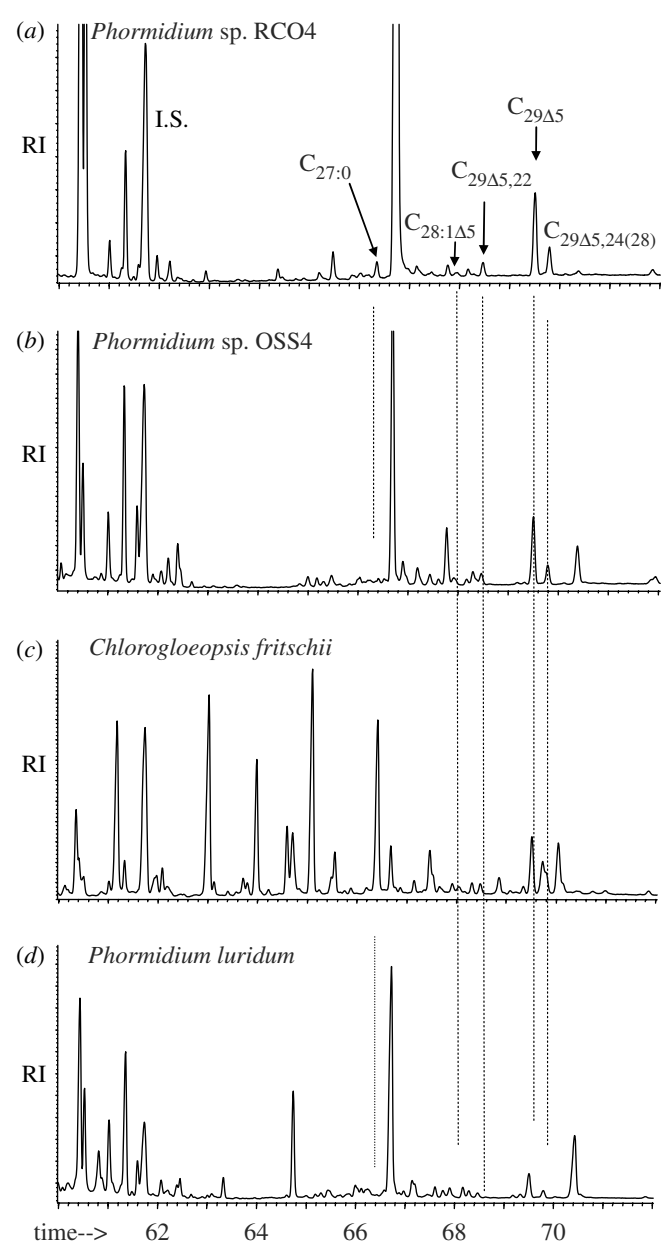

Figure 5. Total ion chromatograms for showing sterols in the total lipid extracts of four of the investigated cyanobacterial cultures $(a-d)$. Phormidium sp. RCO4 and OSS 4 are isolates from Yellowstone National Park. All organisms were grown for a biomarker and isotopic investigation of mat-forming communities (Jahnke et al. 2004). RI, relative intensity; I.S., internal standard.

hypothesis that proposes enzymes existed in the past, and for which all evidence has been lost, is not testable.

(d) On the occurrence of sterols in cyanobacteria In connection with earlier studies of 2-methylhopanoids and other biomarkers in strains of cultured cyanobacteria (Summons et al. 1999; Jahnke et al. 2004), we checked for the presence of sterols in the total lipid extracts of some of the genera previously reported to contain them. Samples were hydrolysed with acid in order to render conjugated sterols in the free form and then converted to trimethylsilyl derivatives for GC-MS. As figure 5 shows, our cultured samples contained an abundance of sterols. Moreover, the distributions were similar in all samples with many of the same compounds reported by previous workers
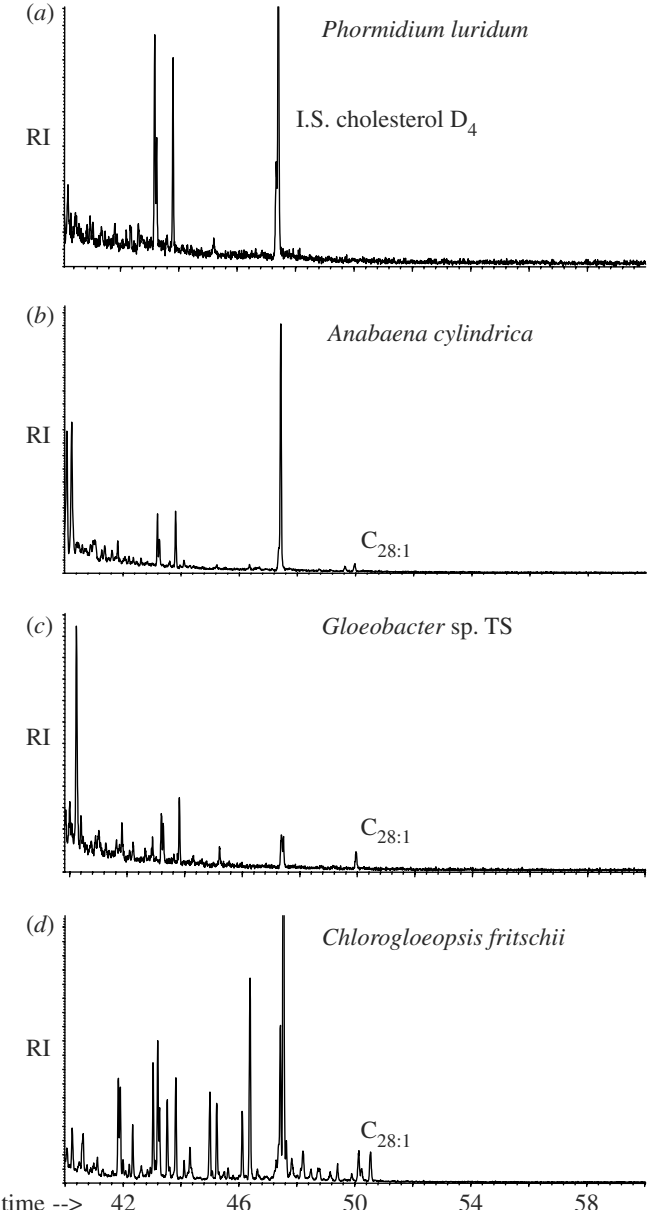

Figure 6. Gas chromatography-mass spectrometry data, depicted as the $m / z \quad 129$ ion which is diagnostic for trimethylsilyl (TMS) sterols, for some cyanobacteria cultured in the presence of cycloheximide $(a-d)$. The only detectable sterol is ergosterol which was also present in blank analyses of the BG-11 culture medium and attributable to that source.

including a strong predominance of $\mathrm{C}_{29} \Delta 5, \mathrm{C}_{29} \Delta 5,22$ and $\mathrm{C}_{29} \Delta 5,24(28)$ (figure 5).

These results prompted us to examine other options, one of which was to re-culture the organisms in media with defined sterol contents and in the presence of cycloheximide, a compound known to inhibit the growth of eukaryotes by blocking protein synthesis. After several sub-cultures in the presence of cycloheximide we could only identify traces of a $\mathrm{C}_{27}$ and a $\mathrm{C}_{28}$ sterol that were subsequently found to be components of the BG-11 medium (figure 6). After re-culturing of these cyanobacteria in the original media, and in the absence of cycloheximide, they continued to be free of detectable sterols.

The results of these experiments indicated that our original cultures were contaminated by an organism that produced $\mathrm{C}_{29}$ sterols in abundance. The source of contamination was investigated using, firstly,

Phil. Trans. R. Soc. B (2006) 
a universal gene probe for eukaryotes which was positive and, secondly, a probe specific for Basidomycetes (rust fungi) which was also positive. Unlike other fungi which produce ergosterol $\left(\mathrm{C}_{28} \Delta 5\right)$ or cholesterol $\left(\mathrm{C}_{27} \Delta 5\right)$ as their major sterols, Uredospores such as Uromyces phaseoli (bean rust), flax rust and Cronartium fusiforme (fusiform rust) all produce $\mathrm{C}_{29}$ sterols like their host plants. In these organisms, the principal sterols are $\mathrm{C}_{29} \Delta 7$ and $\mathrm{C}_{29} \Delta 7,24$ (28) (Jackson \& Frear 1968; Lin et al. 1972; Carmack et al. 1976).

Lack of sterols in cyanobacteria is further evidenced by their genome sequences, which reveal that the only genes significantly homologous to sterol synthases are squalene-hopene synthases. As microbial genome sequencing progresses, diverse new sterol producers may be discovered on the basis of gene content; the utility of this approach has already been demonstrated (Pearson et al. 2003). Such searches will be aided by the high degree of conservation among sterol synthesis genes, which makes them readily recognizable on the basis of sequence similarity. It is worth noting that, among the 258 prokaryotic genomes sequenced and available as of this writing, only one previously unknown sterol producer, G. obscuriglobus (Pearson et al. 2003), has been discovered.

\section{RELATING BIOMARKERS TO BIOLOGICAL AND GEOCHEMICAL EVOLUTION}

\section{(a) Membrane function of sterols and} evolutionary and ecological adaptation

The function of sterols in cellular membranes has been a topic of long-standing interest among biochemists and cell biologists. Fifty years after the central steps in the sterol synthesis pathway were elucidated by Bloch and co-workers, understanding of the structural and functional roles of these cardinal eukaryotic lipids remains incomplete and an active area of research. It was recognized early on that sterols modulate the micro-scale fluidic properties of the membrane-its density, viscosity, and so on - and that sterols that differ by only the addition of a methyl group or a double bond can produce measurably different effects. The principal mechanism for this structural effect of sterols is their induction of a liquid-ordered phase in membranes, a state intermediate between high-temperature liquiddisordered and low temperature solid-ordered (gel) phases. Further, the liquid-ordered and liquid-disordered phases can coexist in the same membrane, allowing for spatial heterogeneity and the notion of the membrane as a 'fluid mosaic' with discrete domains (lipid rafts) (Simons \& Vaz 2004). A functional role for sterols has been suggested in the reduction of permeability of membranes to cations, particularly protons and sodium, hence assisting in energy conservation (Haines 2001). This is likely due to enhanced exclusion of water clusters or chains from the membrane, though the precise mechanism remains under investigation (Tepper \& Voth 2005).

The foregoing discussion of the structural and functional characteristics of steroids could apply essentially equally well to hopanoids, which are membrane constituents of many bacteria. The two classes of lipids share the basic polycyclic skeleton side chain structure and are of nearly identical molecular dimensions (approx. $8 \AA$ by $19 \AA$ ). Hopanoids have been demonstrated to influence membrane ordering and fluid properties similarly to steroids (Kannenberg et al. 1983). On the basis of such comparisons, hopanoids have been termed 'sterol surrogates' and 'functionally equivalent' in membranes (Ourisson et al. 1987). This begs the question: why should functionally equivalent molecules be so strongly differentially conserved across the breadth of the diversity of cellular life?

While steroids and hopanoids are structurally similar, they differ in a key respect: steroids have their polar group attached directly to the ring structure at C3, while the polar functions in hopanoids are attached to the side chain. As a result, the ring structure of steroids sits near the edge of the lipid bilayer, but that of hopanoids is nearer the centre. This suggests that the two types of terpenoid may move quite differently in membranes, particularly with regard to their ability to translocate from one leaflet of the bilayer to the other, a phenomenon known as 'lipid flip-flop'.

Flip-flop is an important property of membrane lipids because in order for a membrane to deform -i.e. to curve inward or outward-one leaflet of the bilayer must become longer while the other becomes shorter. This curvature is effected by flipping lipid molecules from one leaflet to the other. Sterols have among the shortest $t_{1 / 2}$ values for transbilayer flip-flop of any membrane lipid (Holthuis \& Levine 2005), meaning that steroid-containing membranes are readily deformed. This was elegantly demonstrated (Bacia et al. 2005) in a cell-free system of giant unilamellar vesicles, where it was found that not only does the addition of sterols to lipid vesicles induce domain formation and budding, but the type of sterol controls the direction of curvature. Cholesterol and lophenol induce positive (outward) curvature, while lanosterol and cholesteryl sulphonate cause inward (negative) budding. Varying the proportions of a mixture of cholesterol and cholesteryl sulphonate controlled both domain size and budding behaviour. Eukaryotic cells have highly specific structural requirements for their membrane sterols: changing even the position of unsaturation in the ring system or the stereochemistry of the hydroxyl group attachment can result in an incompetent cell envelope (Xu et al. 2005). Taken together, this evidence suggests that a membrane with a well-regulated sterol composition is a powerful tool for export and import across the cell membrane.

Eukaryotes, both unicellular and multicellular, make extensive use of endo- and exocytosis. The innovation of sterol biosynthesis, in allowing rapid membrane deformation, may have been a key step in the evolution of these processes. In eukaryotes, phagocytosis and membrane biogenesis are closely coupled. When part of a membrane is drawn in to engulf a particle, lipid synthesis is stimulated (through the sterol regulatory element binding protein transcriptional regulators) to just the degree to replace the consumed membrane segment (Castoreno et al. 2005). With a flexible, deformable membrane, many mechanisms to generate curvature are possible, including protein scaffolding, helix insertion and active 
cytoskeletal remodelling (McMahon \& Gallop 2005). Much of the dynamic character of the eukaryotic membrane system can be attributed to these curvature mechanisms. Though experimental quantification of the transbilayer movement of hopanoids (particularly their flip-flop half-time) is lacking, the absence of endoand exocytosis among the bacteria may indicate that hopanoids are not functionally equivalent to sterols in this regard.

The ability to perform this type of transmembrane transport had profound evolutionary and ecological consequences. In essence, the invention of endocytosis is the dawn of predation. Prior to endocytosis, heterotrophy proceeded largely through the dissolved phase, and no stratified trophic relationships existed. Once large particles (including other cells) could be imported and enzymatically degraded intracellularly, the predator-prey dynamic, that shaped much of evolutionary history, was established.

(i) Possible role of $\mathrm{O}_{2}$ in the biosynthesis of 2-methylbacteriohopanepolyols

Precise details of the biosynthetic pathway leading to 2-methylbacteriohopanepolyols (2Me-BHP) are not known although the methyl group is known to be transferred intact from L-methionine (Zundel \& Rohmer 1985). Genomes of sequenced cyanobacteria and $M$. capsulatus (which produces $3 \mathrm{Me}-\mathrm{BHP}$ ) reveal that they contain homologues of sterol-methyltransferases found in plants. The transfer of a methyl group to the hopanoid ring structure is presumably preceded by desaturation at the 2-3 position. Aerobic sterol desaturases are present in the genomes of several cyanobacteria, with unknown function. It has yet to be demonstrated that either sterol-methyltransferases or sterol desaturases are involved in the methylation of the hopanoid A-ring, but their potential should be investigated.

(ii) Alternative oxidants on the early Earth

Oxidizing power may have been scarce on the Earth's surface before the oxygenation of the atmosphere. Under such conditions, it is possible that cellular life made use of oxidants that have since been supplanted by nearly ubiquitous $\mathrm{O}_{2}$. Postulated scenarios for the use of such alternative oxidants should uphold criteria of geochemical and biochemical viability; nitrogen oxides fail the first test, while water, as discussed above for SQMO, generally fails the second. One feasible alternative is hydrogen peroxide. Significant $\mathrm{H}_{2} \mathrm{O}_{2}$ is generated by the reaction of water with pyrite under anaerobic, UV-illuminated conditions (Blankenship \& Hartman 1998; Borda et al. 2003), a plausible scenario on the early Earth. The use of peroxide as a 'transitional' redox partner (both as an oxidant and as a reductant) in biogeochemical evolution has been discussed previously (Kasting et al. 1985; Blankenship \& Hartman 1998; Borda et al. 2003). Hydrogen peroxide may have been a suitable oxidant for biosynthetic oxygenation reactions, such as those described above in sterol synthesis. Such a scenario has, at minimum, three prerequisites to be fulfilled: (i) $\mathrm{H}_{2} \mathrm{O}_{2}$ must have been produced in geochemically significant quantities and have been available to micro-organisms in a variety of habitats. (ii) The enzymes of the pathway of interest must be able to use $\mathrm{H}_{2} \mathrm{O}_{2}$ as an oxidant, and themselves be stable in concentrations of peroxide thought likely to arise. (iii) Cells must be able to use exogenous peroxide anabolically to produce the biochemicals at question. Even if only the first condition can be demonstrated, further chemistry (such as the iron-catalysed HaberWeiss reaction) should be considered as sources for redox partners for metabolism. Exploration of these possibilities will likely lead to new insights into the coevolution of the redox chemistry of the geosphere and biosphere, and highlight the importance of understanding the evolutionary biochemistry of reactive oxygen species.

\section{SUMMARY AND FUTURE DIRECTIONS}

The sequences of key enzymes in steroid biosynthesis are very highly conserved within the eukaryotic domain and it appears likely that the initial steps of the pathway were present in their modern form in the last common ancestor of eukaryotes.

Steroid biosynthesis is an oxygen intensive process with, for example, 11 molecules of $\mathrm{O}_{2}$ being required for the synthesis of one molecule of cholesterol. It is also energetically expensive. While one can postulate anaerobic alternatives to some steps in the pathway, these would be even more energy intensive. Any postulate for an ancestral anaerobic pathway to sterols must explain the replacement of five enzymes with anaerobic equivalents and, further, that all of these have been lost or are unknown.

Previous reports of sterol biosynthesis in cyanobacteria appear to be erroneous. It seems that cyanobacterial cultures are easily contaminated by fungi related to rusts. The sterol biosynthetic capability of other Bacteria is patchily distributed, characterized by pathways that are either anomalous or incomplete, and likely gained from Eukarya by lateral gene transfer.

The generally accepted hypothesis that hopanoids are sterol surrogates in bacteria deserves re-visiting with investigations of the localization and functional roles of BHP. In particular, studies of the phylogenetic distribution, biosynthesis and functional role of $2 \mathrm{Me}-$ BHP in cyanobacteria would be particularly valuable. As a starting point, one could hypothesize that the biosynthesis of $2 \mathrm{Me}-\mathrm{BHP}$ in cyanobacteria involves an oxygen-dependent desaturase and a methyltransferase analogous to those employed in sterol biosynthesis.

Understanding early steps in cellular evolution will be aided by more detailed studies of hydrocarbons in Archaean and Proterozoic rocks. Further studies of the membrane function of sterols and their role in evolutionary and ecological adaptation will also be valuable.

We thank Tsegereda Embaye (NASA) and Janet Hope (Geoscience Australia) for technical assistance in the analysis of cyanobacterial lipids. We also thank Yanek Hebting (MIT), Ken Cullings and Chris Raleigh (NASA Ames) for helpful discussion. Lee Kump, Katja Meyer, Ann Pearson and John Volkman provided insightful and constructive reviews that improved the manuscript. This work was supported by the NASA Exobiology Program (Award NNG05GN62G) and NSF (Award EAR 0418619). 


\section{REFERENCES}

Abe, I., Rohmer, M. \& Prestwich, G. D. 1993 Enzymatic cyclization of squalene and oxidosqualene to sterols and triterpenes. Chem. Rev. 93, 2189-2206. (doi:10.1021/ cr00022a009)

Adl, S. M. et al. 2005 The new higher level classification of eukaryotes with emphasis on the taxonomy of protists. 7. Eukaryot. Microbiol. 52, 399-451. (doi:10.1111/j.15507408.2005.00053.x)

Altschul, S. F., Madden, T. L., Schaffer, A. A., Zhang, J., Zhang, Z., Miller, W. \& Lipman, D. J. 1997 Gapped BLAST and PSI-BLAST: a new generation of protein database search programs. Nucl. Acids Res. 25, 3389-3402.

Anding, C., Brandt, R. D. \& Ourisson, G. 1971 Sterol biosynthesis in Euglena gracilis Z. Sterol precursors in light-grown and dark-grown Euglena gracilis Z. Eur. 7. Biochem. 24, 259-263. (doi:10.1111/j.1432-1033. 1971.tb19679.x)

Aoyama, Y., Funae, Y., Noshiro, M., Horiuchi, T. \& Yoshida, Y. 1994 Occurrence of a P450 showing high homology to yeast lanosterol 14-demethylase (P450(14DM)) in the rat liver. Biochem. Biophys. Res. Commun. 201, 1320-1326. (doi:10.1006/bbrc.1994.1848)

Arisue, N., Hasegawa, M. \& Hashimoto, T. 2005 Root of the Eukaryota tree as inferred from combined maximum likelihood analyses of multiple molecular sequence data. Mol. Biol. Evol. 22, 409-420. (doi:10.1093/molbev/ msi023)

Bacia, K., Schwille, P. \& Kurzchalia, T. 2005 Sterol structure determines the separation of phases and the curvature of the liquid-ordered phase in model membranes. Proc. Natl Acad. Sci. USA 102, 3272-3277. (doi:10.1073/pnas. 0408215102)

Bekker, A., Holland, H. D., Wang, P. L., Rumble, D., Stein, H. J., Hannah, J. L., Coetzee, L. L. \& Beukes, N. J. 2004 Dating the rise of atmospheric oxygen. Nature 427, 117-120. (doi:10.1038/nature02260)

Bellamine, A., Lepesheva, G. \& Waterman, M. 2004 Fluconazole binding and sterol demethylation in three CYP51 isoforms indicate differences in active site topology. F. Lipid Res. 45, 2000-2007. (doi:10.1194/jlr. M400239-JLR200)

Benveniste, P. 2004 Biosynthesis and accumulation of sterols. Ann. Rev. Plant Biol. 55, 429-457.

Bird, C. W., Lynch, J. M., Pirt, F. J. \& Reid, W. W. 1971 Steroids and squalene in Methylococcus capsulatus grown on methane. Nature 230, 473. (doi:10.1038/230473a0)

Blankenship, R. E. \& Hartman, H. 1998 The origin and evolution of oxygenic photosynthesis. Trends Biochem. Sci. 23, 94. (doi:10.1016/S0968-0004(98)01186-4)

Blanksby, S. J. \& Ellison, G. B. 2003 Bond dissociation energies of organic molecules. Accounts Chem. Res. 36, 255-263. (doi:10.1021/ar020230d)

Bloch, K. 1983 Sterol structure and membrane function CRC Crit. Rev. Biochem. 14, 47-92.

Bloch, K. 1987 Summing up. Annu. Rev. Biochem. 56, 1-18. (doi:10.1146/annurev.bi.56.070187.000245)

Bode, H. B., Zeggel, B., Silakowski, B., Wenzel, S. C., Reichenbach, H. \& Muller, R. 2003 Steroid biosynthesis in prokaryotes: identification of myxobacterial steroids and cloning of the first bacterial 2,3(S)-oxidosqualene cyclase from the myxobacterium Stigmatella aurantiaca. Mol. Microbiol. 47, 471-481. (doi:10.1046/j.1365-2958. 2003.03309.x)

Borda, M. J., Elsetinow, A. R., Strongin, D. R. \& Schoonen, M. A. 2003 A mechanism for the production of hydroxyl radical at surface defect sites on pyrite. Geochim. Cosmochim. Acta 67, 935-939. (doi:10.1016/S00167037(02)01222-X)

Phil. Trans. R. Soc. B (2006)
Brocks, J. J., Logan, G. A., Buick, R. \& Summons, R. E. 1999 Archean molecular fossils and the early rise of eukaryotes. Science (Washington, DC) 285, 1033-1036. (doi:10.1126/ science.285.5430.1033)

Brocks, J. J., Buick, R., Logan, G. A. \& Summons, R. E. 2003 Composition and syngeneity of molecular fossils from the 2.78 to 2.45 billion-year-old Mount Bruce Supergroup, Pilbara Craton, Western Australia. Geochim. Cosmochim. Acta 67, 4289-4319. (doi:10.1016/S0016-7037(03) 00208-4)

Brown, J. R. \& Doolittle, W. F. 1999 Gene descent, duplication, and horizontal transfer in the evolution of glutamyl- and glutaminyl-tRNA synthetases. F. Mol. Evol. 49, 485. (doi:10.1007/PL00006571)

Bruice, T. C., Noar, J. B., Ball, S. S. \& Venkataram, U. V. 1983 Monooxygen donation potential of 4a-hydroperoxyflavins as compared with those of a percarboxylic acid and other hydroperoxides. Monooxygen donation to olefin, tertiary amine, alkyl sulfide, and iodide ion. F. Am. Chem. Soc. 105, 2452-2463. (doi:10.1021/ja00346a057)

Buick, R., Anbar, A. D., Mojzsis, S. J., Kaufman, A. J, Kieft, T. L., Lyons, T. W. \& Humayun, M. 2004 The case for scientific drilling of Precambrian sedimentary sequences: a mission to early Earth. American Geophysical Union, Fall Meeting 2001, abstract \#P22B-0544.

Carmack, C. L., Weete, J. D. \& Kelly, W. D. 1976 Hydrocarbons, fatty acids and sterols of Cronartium fusiforme. Physiol. Plant Pathol. 8, 43-49.

Castoreno, A. B., Wang, Y., Stockinger, W., Jarzylo, L. A., Du, H., Pagnon, J. C., Shieh, E. C. \& Nohturfft, A. 2005 Transcriptional regulation of phagocytosis-induced membrane biogenesis by sterol regulatory element binding proteins. Proc. Natl Acad. Sci. USA 102, 13 129-13 134. (doi:10.1073/pnas.0506716102)

Cloud, P. E. 1972 A working model of the primitive Earth. Am. F. Sci. 272, 537-548.

Dahl, C., Dahl, J. \& Bloch, K. 1980 Effect of alkylsubstituted precursors of cholesterol on artificial and natural membranes and on the viability of Mycoplasma capricolum. Biochemistry 19, 1462-1467. (doi:10.1021/ bi00548a031)

Darnet, S. \& Rahier, A. 2003 Enzymological properties of sterol-C4-methyl-oxidase of yeast sterol biosynthesis. Biochim. Biophys. Acta Mol. Cell Biol. Lipids 1633, 106-117.

Darnet, S. \& Rahier, A. 2004 Plant sterol biosynthesis: identification of two distinct families of sterol 4a-methyl oxidases. Biochem. F. 378, 889-898.

Des Marais, D. J. 2000 Evolution: when did photosynthesis emerge on Earth? Science 289, 1703-1705.

Des Marais, D. J., Strauss, H., Summons, R. E. \& Hayes, J. M. 1992 Carbon isotope evidence for the stepwise oxidation of the Proterozoic environment. Nature 359, 605-609. (doi:10.1038/359605a0)

DeSousa, N. J. \& Nes, W. R. 1968 Sterols: isolation from a blue-green alga. Science 162, 363-364.

Eigenbrode, J. L. 2004 Late Archean microbial ecology: an integration of molecular, isotopic and lithological studies. Ph.D. thesis, Earth Sciences, the Pennsylvania State University.

Eigenbrode, J. L., Freeman, K. H., Brocks, J. J., Summons, R. E. \& Logan, G. A. 2001 Late Archean biomarkers of carbonate and shale lithofacies from the Hamersley Basin, Pilbara Craton, Western Australia. In 11th V.M. Goldschmidt Conference, Hot Springs, VA, USA, p. 3461.

Eigenbrode, J. L., Freeman, K. H. \& Summons, R. E. 2004 Biogeochemical processes in Late Archean marine biosphere revealed by isotopic and molecular records. American Geophysical Union, Fall Meeting 2004, abstract \#B33B-0260 
Farquhar, J., Bao, H. \& Thiemens, M. $2000 a$ Atmospheric influence of Earth's earliest sulfur cycle. Science 289, 756-758. (doi:10.1126/science.289.5480.756)

Farquhar, J., Savarino, J., Jackson, T. L. \& Thiemens, M. $2000 b$ Evidence of atmospheric sulphur in the Martian regolith from sulphur isotopes in meteorites. Nature 404, 50-52. (doi:10.1038/35003517)

Fischer, W. W., Summons, R. E. \& Pearson, A. 2005 Targeted genomic detection of biosynthetic pathways: anaerobic production of hopanoid biomarkers by a common sedimentary microbe. Geobiology 3, 33-40. (doi:10.1111/ j.1472-4669.2005.00041.x)

Gachotte, D., Barbuch, R., Gaylor, J., Nickel, E. \& Bard, M. 1998 Characterization of the Saccharomyces cerevisiae ERG26 gene encoding the C-3 sterol dehydrogenase (C-4 decarboxylase) involved in sterol biosynthesis. Proc. Natl Acad. Sci. USA 95, 13794-13799.

Gachotte, D., Sen, S. E., Eckstein, J., Barbuch, R., Krieger, M., Ray, B. D. \& Bard, M. 1999 Characterization of the Saccharomyces cerevisiae ERG27 gene encoding the 3-keto reductase involved in C-4 sterol demethylation. Proc. Natl Acad. Sci. USA 96, 12655-12660.

Giner, J.-L., Wünsche, L., Andersen, R. A. \& Djerassi, C. 1991 Dinoflagellates cyclize squalene oxide to lanosterol. Biochem. Syst. Ecol. 19, 142-145.

Groves, J. T. \& Han, Y.-Z 1995 Models and mechanisms of cytochrome P450 action. In Cytochrome P450: structure, mechanism, and biochemistry (ed. P. R. Ortiz de Montellano), 2nd edn, pp. 1-45. New York: Plenum Press.

Hai, T., Schneider, B., Schmidt, J. \& Adam, G. 1996 Sterols and triterpenoids from the cyanobacterium Anabaena hallensis. Phytochemistry 41, 1083. (doi:10.1016/00319422(95)00778-4)

Haines, T. H. 2001 Do sterols reduce proton and sodium leaks through lipid bilayers? Prog. Lipid Res. 40, 299-324. (doi:10.1016/S0163-7827(01)00009-1)

Halverson, G. P., Hoffman, P. F., Schrag, D. P., Maloof, A. C. \& Rice, A. H. N. 2005 Toward a Neoproterozoic composite carbon-isotope record. Geol. Soc. Am. Bull. 117, 1181-1207. (doi:10.1130/B25630.1)

Hayes, J. M. \& Waldbauer, J. R. 2006 The carbon cycle and associated redox processes through time. Phil. Trans. $R$. Soc. B 361, 931-950. (doi:10.1098/rstb.2006.1840)

Hoffman, P. F. \& Schrag, D. P. 2002 The snowball Earth hypothesis: testing the limits of global change. Terra Nova 14, 129-155. (doi:10.1046/j.1365-3121.2002.00408.x)

Holland, H. D. 1984 The chemical evolution of the atmosphere and oceans. Princeton, NJ: Princeton University Press.

Holland, H. D. 2002 Volcanic gases, black smokers, and the Great Oxidation Event. Geochim. Cosmochim. Acta 66, 3811-3826. (doi:10.1016/S0016-7037(02)00950-X)

Holland, H. D. \& Beukes, N. J. 1990 A palaeoweathering profile from Griqualand West, South Africa: evidence for a dramatic rise in atmospheric oxygen between 2.2 and 1.9 bybp. Am. F. Sci. 290-A, 1-34.

Holthuis, J. \& Levine, T. 2005 Lipid traffic: floppy drives and a superhighway. Nat. Rev. Mol. Cell Biol. 6, 209-220. (doi:10.1038/nrm1591)

Hurtgen, M. T., Arthur, M. A. \& Halverson, G. P. 2005 Neoproterozoic sulfur isotopes, the evolution of microbial sulfur species, and the burial efficiency of sulfide as sedimentary pyrite. Geology 33, 41-44. (doi:10.1130/ G20923.1)

Jackson, L. L. \& Frear, D. S. 1968 Lipids of rust fungi-II. Stigmast-7-enol and stigmasta-7,24(28)-dienol in flax rust uredospores. Phytochemistry 7, 651. (doi:10.1016/S00319422(00)88242-4)

Jackson, C. J., Lamb, D. C., Marczylo, T. H., Warrilow, A. G. S., Manning, N. J., Lowe, D. J., Kelly, D. E. \& Kelly, S. L. 2002 A novel sterol $14 \alpha$-demethylase/ferredoxin

Phil. Trans. R. Soc. B (2006) fusion protein (MCCYP51FX) from Methylococcus capsulatus represents a new class of the c cytochrome P450 superfamily. F. Biol. Chem. 277, 46 959-46 965. (doi:10. 1074/jbc.M203523200)

Jahnke, L. L. 1992 The effects of growth temperature on the methyl sterol and phospholipid fatty acid composition of Methylococcus capsulatus (Bath). FEMS Microbiol. Lett. 15, 209-212.

Jahnke, L. L. \& Klein, H. P. 1983 Oxygen requirements for formation and activity of the squalene epoxidase in Saccharomyces cerevisiae. F. Bacteriol. 155, 488-492.

Jahnke, L. L., Embaye, T., Hope, J., Turk, K. A., Van Zuilen, M., Des Marais, D. J., Farmer, J. D. \& Summons, R. E. 2004 Lipid biomarker and carbon isotopic signatures for stromatolite-forming, microbial mat communities and Phormidium cultures from Yellowstone National Park. Geobiology 2, 31-47. (doi:10.1111/j.1472-4677.2004.00021.x)

Joubert, B. M., Nguyen, L. N., Matsuda, S. P. T. \& Buckner, F. S. 2001 Cloning and functional characterization of a Trypanosoma brucei lanosterol $14 \alpha$-demethylase gene. Mol. Biochem. Parasitol. 117, 115-117. (doi:10.1016/S01666851(01)00337-1)

Kannenberg, E., Blume, A., McElhaney, R. N. \& Poralla, K. 1983 Monolayer and calorimetric studies of phosphatidylcholines containing branched-chain fatty acids and of their interactions with cholesterol and with a bacterial hopanoid in model membranes. Biochim. Biophys. Acta 733, 111-116.

Karhu, J. A. \& Holland, H. D. 1996 Carbon isotopes and the rise of atmospheric oxygen. Geology 24, 867-870. (doi:10. 1130/0091-7613(1996)024<0867:CIATRO > 2.3.CO;2)

Kasting, J. F., Holland, H. D. \& Pinto, J. P. 1985 Oxidant abundances in rainwater and the evolution of atmospheric oxygen. F. Geophys. Res. 90, 10 497-10 510.

Kirschvink, J. L., Galdos, E. J., Bertani, L. E., Beukes, N. J., Jens, G., Maepa, L. N. \& Steinberger, R. E. 2000 Paleoproterozoic snowball Earth: extreme climatic and geochemical global change and its biological consequences. Proc. Natl Acad. Sci. USA 97, 1400-1405. (doi:10.1073/pnas.97.4.1400)

Kohl, W., Gloe, A. \& Reichenbach, H. 1983 Steroids from the myxobacterium Nannocyctis excedens. F. Gen. Microbiol. 129, 1629-1635.

Kohlhase, M. \& Pohl, P. 1988 Saturated and unsaturated sterols of nitrogen-fixing blue-green algae (cyanobacteria). Phytochemistry 27, 1735-1740. (doi:10.1016/00319422(88)80434-5)

Kopp, R. E., Kirschvink, J. L., Hilburn, I. A. \& Nash, C. Z. 2005 The Paleoproterozoic snowball Earth: a climate disaster triggered by the evolution of oxygenic photosynthesis. Proc. Natl Acad. Sci. USA 102, 11 131-11 136. (doi:10.1073/pnas.0504878102)

Laden, B. P., Tang, Y. \& Porter, T. D. 2000 Cloning, heterologous expression, and enzymological characterization of human squalene monooxygenase. Arch. Biochem. Biophys. 374, 381-388. (doi:10.1006/abbi.1999.1629)

Lamb, D. C., Ikeda, H., Nelson, D. R., Ishikawa, J., Skaug, T., Jackson, C., Omura, S., Waterman, M. R. \& Kelly, S. L. 2003 Cytochrome P450 complement (CYPome) of the avermectin-producer Streptomyces avermitilis and comparison to that of Streptomyces coelicolor A3(2). Biochem. Biophys. Res. Commun. 307, 610-619. (doi:10. 1016/S0006-291X(03)01231-2)

Lamour, V., Quevillon, S., Diriong, S., N'Guyen, V. C., Lipinski, M. \& Mirande, M. 1994 Evolution of the GlxtRNA synthetase family: the glutaminyl enzyme as a case of horizontal gene transfer. Proc. Natl Acad. Sci. USA 91, 8670-8674

Lee, H. K., Denner-Ancona, P., Sakakibara, J., Ono, T. \& Prestwich, G. D. 2000 Photoaffinity labeling and 
site-directed mutagenesis of rat squalene epoxidase. Arch. Biochem. Biophys. 381, 43-52. (doi:10.1006/abbi.2000. 1966)

Lee, H. K., Zheng, Y. F., Xiao, X. Y., Bai, M., Sakakibara, J., Ono, T. \& Prestwich, G. D. 2004 Photoaffinity labeling identifies the substrate-binding site of mammalian squalene epoxidase. Biochem. Biophys. Res. Commun. 315, 1-9. (doi:10.1016/j.bbrc.2004.01.012)

Lepesheva, G. I. \& Waterman, M. R. 2004 CYP51-the omnipotent P450. Mol. Cell. Endocrinol. 215, 165-170. (doi:10.1016/j.mce.2003.11.016)

Lepesheva, G. I., Nes, W. D., Zhou, W., Hill, G. C. \& Waterman, M. R. 2004 CYP51 from Trypanosoma brucei is obtusifoliol-specific. Biochemistry 43, $10789-10799$. (doi:10.1021/bi048967t)

Levin, E. Y. \& Bloch, K. 1964 Absence of sterols in bluegreen algae. Nature 202, 90-91.

Lin, H.-K., Langenbach, R. J. \& Knoche, H. W. 1972 Sterols of Uromyces phaseoli uredospores. Phytochemistry 11, 2319-2322. (doi:10.1016/S0031-9422(00)88399-5)

Liu, Y., Yao, Z.-X. \& Papadopoulos, V. 2005 Cytochrome P450 17 $\alpha$-hydroxylase/17,20 lyase (CYP17) function in cholesterol biosynthesis: identification of squalene monooxygenase (epoxidase) activity associated with CYP17 in Leydig cells. Mol. Endocrinol. 19, 1918-1931. (doi:10. 1210/me.2004-0271)

Lodeiro, S., Segura, M., Stahl, M., Schulz-Gasch, T. \& Matsuda, S. 2004 Oxidosqualene cyclase second-sphere residues profoundly influence the product profile. ChemBioChem 5, 1581-1585. (doi:10.1002/cbic.200400086)

March, J. 1992 Advanced organic chemistry: reactions, mechanisms, and structure, 4th edn. New York: Wiley.

McMahon, H. T. \& Gallop, J. L. 2005 Membrane curvature and mechanisms of dynamic cell membrane remodelling. Nature 438, 590-596. (doi:10.1038/nature04396)

Meunier, B., de Visser, S. P. \& Shaik, S. 2004 Mechanism of oxidation reactions catalyzed by cytochrome P450 enzymes. Chem. Rev. (Washington, DC, United States) 104, 3947-3980.

Meyer, M., Segura, M., Wilson, W. \& Matsuda, S. 2000 Oxidosqualene cyclase residues that promote formation of cycloartenol, lanosterol, and parkeol. Angew. Chem. Int. Ed. 39, 4090-4092. (doi:10.1002/1521-3773(20001117)39: $22<4090:$ AID-ANIE4090>3.0.CO;2-8)

Meyer, M. M., Xu, R. \& Matsuda, S. P. T. 2002 Directed evolution to generate cycloartenol synthase mutants that produce lanosterol. Org. Lett. 4, 1395-1398. (doi:10. 1021/o10257225)

Nelson, D. \& Strobel, H. 1989 Secondary structure prediction of 52 membrane-bound cytochromes P450 shows a strong structural similarity to P450cam. Biochemistry 28, 656-660. (doi:10.1021/bi00428a036)

Ourisson, G., Rohmer, M. \& Poralla, K. 1987 Prokaryotic hopanoids and other polyterpenoid sterol surrogates. Annu. Rev. Microbiol. 41, 301-333. (doi:10.1146/ annurev.mi.41.100187.001505)

Pancost, R. D., Sinninghe Damste, J. S., de Lint, S., van der Maarel, M. J. E. C., Gottschal, J. C. \& Party, M. S. S. 2000 Biomarker evidence for widespread anaerobic methane oxidation in Mediterranean sediments by a consortium of methanogenic Archaea and Bacteria. Appl. Environ. Microbiol. 66, 1126-1132. (doi:10.1128/ AEM.66.3.1126-1132.2000)

Paoletti, C., Pushparaj, B., Florenzano, G., Capella, P. \& Lerker, G. 1976 Unsaponifiable matter of green and bluegreen algal lipids as a factor of biochemical differentiation of their biomass. II. Terpenic alcohol and sterol fractions. Lipids 11, 266-271.
Pearson, A., Budin, M. \& Brocks, J. J. 2003 Phylogenetic and biochemical evidence for sterol synthesis in the bacterium Gemmata obscuriglobus. Proc. Natl Acad. Sci. USA 100, 15 352-15 357. (doi:10.1073/pnas.2536559100)

Peters, K. E., Walters, C. C. \& Moldowan, J. M. 2004 The biomarker guide, 2nd edn. Cambridge, UK: Cambridge University Press.

Podust, L. M., Poulos, T. L. \& Waterman, M. R. $2001 a$ Crystal structure of cytochrome P450 $14 \alpha$-sterol demethylase (CYP51) from Mycobacterium tuberculosis in complex with azole inhibitors. Proc. Natl Acad. Sci. USA 98, 3068-3073. (doi:10.1073/pnas.061562898)

Podust, L. M., Stojan, J., Poulos, T. L. \& Waterman, M. R. $2001 b$ Substrate recognition sites in $14 \alpha$-sterol demethylase from comparative analysis of amino acid sequences and X-ray structure of Mycobacterium tuberculosis CYP51. f. Inorg. Biochem. 87, 227-235. (doi:10.1016/S01620134(01)00388-9)

Raederstorff, D. \& Rohmer, M. 1987 Sterol biosynthesis via cycloartenol and other biochemical features related to photosynthetic phyla in the amoebae Naegleria lovaniensis and Naegleria gruberi. Eur. F. Biochem. 164, 427-434. (doi:10.1111/j.1432-1033.1987.tb11075.x)

Rasmussen, B. \& Buick, R. 1999 Redox state of the Archean atmosphere: evidence from detrital heavy minerals in $c a$ 3250-2750 Ma sandstones from the Pilbara Craton, Australia. Geology 27, 115-118. (doi:10.1130/00917613(1999)027<0115:RSOTAA > 2.3.CO;2)

Raymond, J. \& Blankenship, R. E. 2004 Biosynthetic pathways, gene replacement and the antiquity of life. Geobiology 2, 199-203. (doi:10.1111/j.1472-4677.2004. 00037.x)

Rezen, T., Debeljak, N., Kordis, D. \& Rozman, D. 2004 New aspects on lanosterol $14 \alpha$-demethylase and cytochrome P450 evolution: lanosterol/cycloartenol diversification and lateral transfer. F. Mol. Evol. 59, 51-58.

Rohmer, M. \& Brandt, R. 1973 Sterols and their precursors in Astasia longa Pringsheim. Eur. F. Biochem. 36, 446-454. (doi:10.1111/j.1432-1033.1973.tb02929.x)

Rohmer, M., Bouvier, P. \& Ourisson, G. 1979 Molecular evolution of biomembranes: structural equivalents and phylogenetic precursors of sterols. Proc. Natl Acad. Sci. USA 76, 847-851.

Rohmer, M., Bouvier-Nave, P. \& Ourisson, G. 1984 Distribution of hopanoid triterpanes in prokaryotes. 7. Gen. Microbiol. 130, 1137-1150.

Rondet, S., Taton, M. \& Rahier, A. 1999 Identification, characterization, and partial purification of 4 alphacarboxysterol-C3-dehydrogenase/C4-decarboxylase from Zea mays. Arch. Biochem. Biophys. 366, 249-260.

Rosing, M. T. \& Frei, R. 2004 U-rich Archaean seafloor sediments from Greenland-indications of $>3700 \mathrm{Ma}$ oxygenic photosynthesis. Earth Planet. Sci. Lett. 217, 237-244. (doi:10.1016/S0012-821X(03)00609-5)

Rothman, D. H., Hayes, J. M. \& Summons, R. E. 2003 Dynamics of the Neoproterozoic carbon cycle. Proc. Natl Acad. Sci. USA 100, 8124-8129. (doi:10.1073/pnas. 0832439100)

Rouxel, O. J., Bekker, A. \& Edwards, K. J. 2005 Iron isotope constraints on the Archean and Paleoproterozoic ocean redox state. Science 307, 1088-1091. (doi:10.1126/ science.1105692)

Sallal, A. K., Ghannoum, M. A., Al-Hasan, R. H., Nimer, N. A. \& Radwan, S. S. 1987 Lanosterol and diacylglycerophosphocholines in lipids from whole cells and thylakoids of the cyanobacterium Chlorogloeopsis fritschii. Arch. Microbiol. (Hist. Arch.) 148, 1-7. (doi:10.1007/ BF00429638)

Segura, M., Lodeiro, S., Meyer, M., Patel, A. \& Matsuda, S. 2002 Directed evolution experiments reveal mutations a

Phil. Trans. R. Soc. B (2006) 
cycloartenol synthase residue His 477 that dramatically alter catalysis. Org. Lett. 4, 4459-4462. (doi:10.1021/ ol0269897)

Shen, Y., Buick, R. \& Canfield, D. E. 2001 Isotopic evidence for microbial sulphate reduction in the Early Archaean era. Nature 410, 77-81. (doi:10.1038/ 35065071)

Shimkets, L. \& Woese, C. R. 1992 A phylogenetic analysis of the myxobacteria: basis for their classification. Proc. Natl Acad. Sci. USA 89, 9459-9463.

Simons, K. \& Vaz, W. L. C. 2004 Model systems, lipid rafts and cell membranes. Annu. Rev. Biophys. Biomol. Struct. 33, 269-295. (doi:10.1146/annurev.biophys.32.110601. 141803)

Simpson, A. G. B., Inagaki, Y. \& Roger, A. J. 2005 The evolutionary relationships amongst excavates: a concatened protein analysis. F. Eukaryot. Microbiol. 52, 7S-27S. (doi:10.1111/j.1550-7408.2005.05202003_1_68.x)

Sleep, N. H., Meibom, A., Fridriksson, T., Coleman, R. G. \& Bird, D. K. 2004 H2-rich fluids from serpentinization: geochemical and biotic implications. Proc. Natl Acad. Sci. USA 101, $12818-12823 . \quad$ (doi:10.1073/pnas. 0405289101)

Summons, R. E., Jahnke, L. L., Hope, J. M. \& Logan, G. A. 1999 2-Methylhopanoids as biomarkers for cyanobacterial oxygenic photosynthesis. Nature 400, 554-556. (doi:10. 1038/23005)

Summons, R. E., Waldbauer, J. R., Buick, R., Dunlop, J. S. \& Bonser, L. C. 2004 Hydrocarbon analysis of Hamersley Basin deep drill cores: preliminary results. American Geophysical Union, Fall Meeting 2004, abstract \#B32B-05.

Tepper, H. L. \& Voth, G. A. 2005 Protons may leak through pure lipid bilayers via a concerted mechanism. Biophys. F. 88, 3095-3108. (doi:10.1529/biophysj.104.056184)

Thiel, V., Peckmann, J., Seifert, R., Wehrung, P., Reitner, J. \& Michaelis, W. 1999 Highly isotopically depleted isoprenoids: molecular markers for ancient methane venting. Geochim. Cosmochim. Acta 63, 2959-3966. (doi:10.1016/ S0016-7037(99)00177-5)

Thiel, V., Blumenberg, M., Pape, T., Seifert, R. \& Michaelis, W. 2003 Unexpected occurrence of hopanoids at gas seeps in the Black Sea. Org. Geochem. 34, 81-87. (doi:10.1016/ S0146-6380(02)00191-2)

Thoma, R., Schulz-Gasch, T., D’Arcy, B., Benz, J., Aebi, J., Dehmlow, H., Hennig, M., Stihle, M. \& Ruf, A. 2004 Insight into steroid scaffold formation from the structure of human oxidosqualene cyclase. Nature 432, 118. (doi: 10 . 1038/nature02993)
Tice, M. M. \& Lowe, D. R. 2004 Photosynthetic microbial mats in the 3164-Myr old ocean. Nature 431, 549-552. (doi:10.1038/nature02888)

Torres, R. A. \& Bruice, T. C. 1999 Theoretical investigation of the [1,2]-sigmatropic hydrogen migration in the mechanism of oxidation of 2-aminobenzoyl-CoA by 2-aminobenzoyl-CoA monooxygenase/reductase. Proc. Natl Acad. Sci. USA 96, 14 748-14 752. (doi:10.1073/ pnas.96.26.14748)

Volkman, J. K. 2003 Sterols in microorganisms. Appl. Microbiol. Biotechnol. 60, 495-506.

Volkman, J. K. 2005 Sterols and other triterpenoids: source specificity and evolution of biosynthetic pathways. Org. Geochem. 36, 139-159. (doi:10.1016/j.orggeochem.2004. 06.013)

Wakeham, S. G., Sinninghe Damste, J. S., Kohnen, M. E. L. \& De Leeuw, J. W. 1995 Organic sulfur compounds formed during early diagenesis in Black Sea sediments. Geochim. Cosmochim. Acta 59, 521-533. (doi:10.1016/ 0016-7037(94)00361-O)

Walker, J. C. G., Klein, C., Schidlowski, M., Schopf, J. W., Stevenson, D. J. \& Walter, M. R. 1983 Environmental evolution of the Archean-Early Proterozoic Earth. In Earth's earliest biosphere: its origin and evolution, pp. 260-290.

Wendt, K. U. 2005 Enzyme mechanisms for triterpene cyclization: new pieces of the puzzle. Angew. Chem. Int. Ed. 44, 3966-3971. (doi:10.1002/anie.200500804)

Wendt, K. U., Poralla, K. \& Schulz, G. E. 1997 Structure and function of a squalene cyclase. Science 277, 1811-1815. (doi:10.1126/science.277.5333.1811)

Wendt, K. U., Schulz, G. E., Corey, E. J. \& Liu, D. R. 2000 Enzyme mechanisms for polycyclic triterpene formation. Angew. Chem. Int. Ed. 39, 2812-2833. (doi:10.1002/15213773(20000818)39:16<2812::AID-ANIE2812>3.3.CO; 2-R)

Woodward, R. B. \& Bloch, K. 1953 The cyclization of squalene in cholesterol synthesis. F. Am. Chem. Soc. 75, 2023-2024. (doi:10.1021/ja01104a535)

Xu, F., Rychnovsky, S. D., Belani, J. D., Hobbs, H. H., Cohen, J. C. \& Rawson, R. B. 2005 Dual roles for cholesterol in mammalian cells. Proc. Natl Acad. Sci. USA 102, 14 551-14 556. (doi:10.1073/pnas.05035 90102)

Zundel, M. \& Rohmer, M. 1985 Prokaryotic triterpenoids. 3. The biosynthesis of 2 beta-methylhopanoids and 3 beta-methylhopanoids of Methylobacterium organophilum and Acetobacter pasteurianus ssp. pasteurianus. Eur. F. Biochem. 150, 35-39. (doi:10.1111/j.1432-1033.1985. tb08984.x) 